\title{
Catalytic, Regioselective Hydrocarbofunctionalization of Unactivated Alkenes with Diverse C-H Nucleophiles
}

Kin S. Yang, John A. Gurak, Jr., Zhen Liu, and Keary M. Engle*

The Scripps Research Institute, Department of Chemistry, 10550 N. Torrey Pines Rd., La Jolla, CA 92037, USA

\section{Table of Contents}

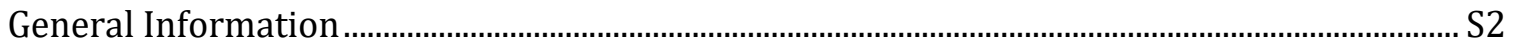

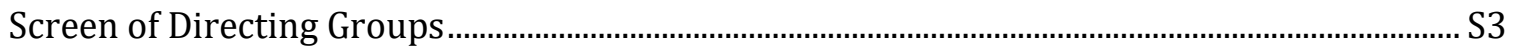

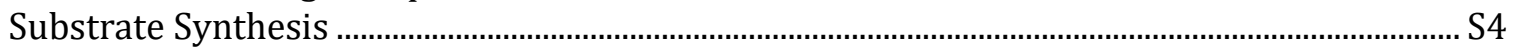

General Procedure for Evaluating Carbon Nucleophile Scope for Hydrofunctionalization ... S8

General Procedure for Evaluating Alkene Scope for Hydrofunctionalization...........................S22

Reaction via 6-Membered Palladacycle............................................................................................... 30

Plausible Reaction Mechanism via 6-Membered Palladacycle .....................................................S32

General Procedure for Evaluating Hydrofunctionalization of Dienes ...........................................S33

Reaction of 2-methylcyclohexane-1,3-dione and Methyl Cyanoacetates with Diene ............S36

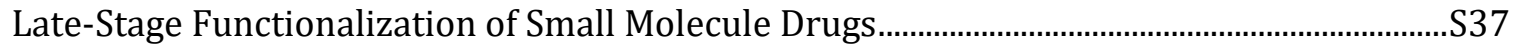

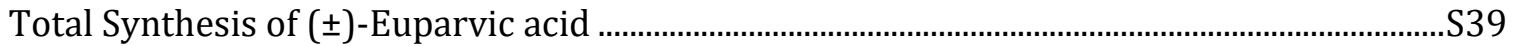

Procedures for Preliminary Mechanistic Studies............................................................................ 40

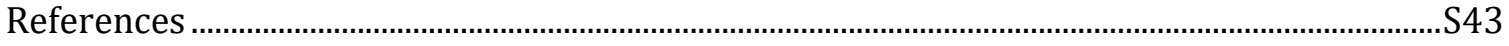

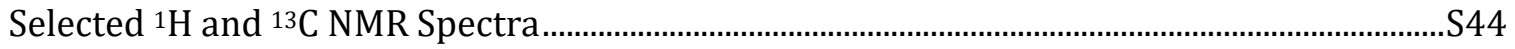

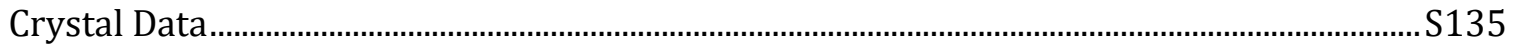

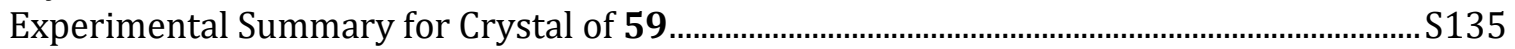

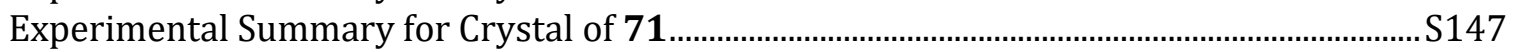

Experimental Summary for Crystal of 80

Experimental Summary for Crystal of 81 ............................................................................... 


\section{General Information}

Unless stated otherwise, all materials were used as received from commercial sources without further purification. All glassware and stirring bars were dried in an oven at $100^{\circ} \mathrm{C}$ overnight unless otherwise stated. DCM, MeCN, and DMF were purchased from Aldrich and used as received without additional drying. 1 Dram reaction vials and caps were purchased from ChemGlass (Cat\#: CG-4904-05) with TFE septa. Ambient temperature refers to 21-24 ${ }^{\circ} \mathrm{C}$. Elevated temperatures were maintained by an Ika heating block for 1 dram vials or a silicon oil bath for larger vessels. Thin-layer chromatography (TLC) was performed using EMD Millipore $250 \mathrm{~mm}$ silica gel F-254 plates (250 $\mu \mathrm{m}$ ) with F-254 fluorescent indicator and visualized by UV fluorescence quenching, iodine, Seebach's stain, or potassium permanganate stain. SiliCycle SiliaFlash P60 silica gel (particle size 40-63 $\mu \mathrm{m}$ ) was used for flash chromatography. ${ }^{1} \mathrm{H}$ and ${ }^{13} \mathrm{C}$ NMR spectra were recorded on a Bruker DRX equipped with a $5 \mathrm{~mm}$ DCH cryoprobe (600 MHz and $150 \mathrm{MHz}$, respectively). ${ }^{1} \mathrm{H}$ spectra were reported relative to $\mathrm{Me}_{4} \mathrm{Si}(\delta 0.0)$ or residual solvent signals unless otherwise stated. ${ }^{13} \mathrm{C}$ NMR spectra were calibrated to residual solvent signals $\left(\mathrm{CDCl}_{3}\right.$ at $77.23 \mathrm{ppm}$, DMSO at $39.52 \mathrm{ppm},\left(\mathrm{CD}_{3}\right)_{2} \mathrm{CO}$ at 29.84, and $\mathrm{CD}_{3} \mathrm{OD}$ at 49.00). High-resolution mass spectra (HRMS) were recorded on an Agilent LC/MSD TOF mass spectrometer by electrospray ionization (Positive mode) time of flight experiments.

\section{Chemical and Supplier:}

The following chemicals were purchased from:

Alfa Aesar: Vinyl acetic acid

Oakwood: 8-Aminoquinoline, EDC, HATU

Fisher: Pyridine and acetic acid

Combi-Blocks: Quercetin, (+)-(1R,4S)-N-boc-4-aminocyclopent-2-enecarboxylic acid, 4-hydroxycoumarin, 2,4dihydroxyquinoline

TCI: Phentolamine mesylate

Apidogen: Zearalenone

AstaTech: 4-Benzyl-2-phenyl-2-oxazoline-5-one

All other chemicals were purchased from Aldrich and used as received. 
Screen of Directing Groups

Table S1

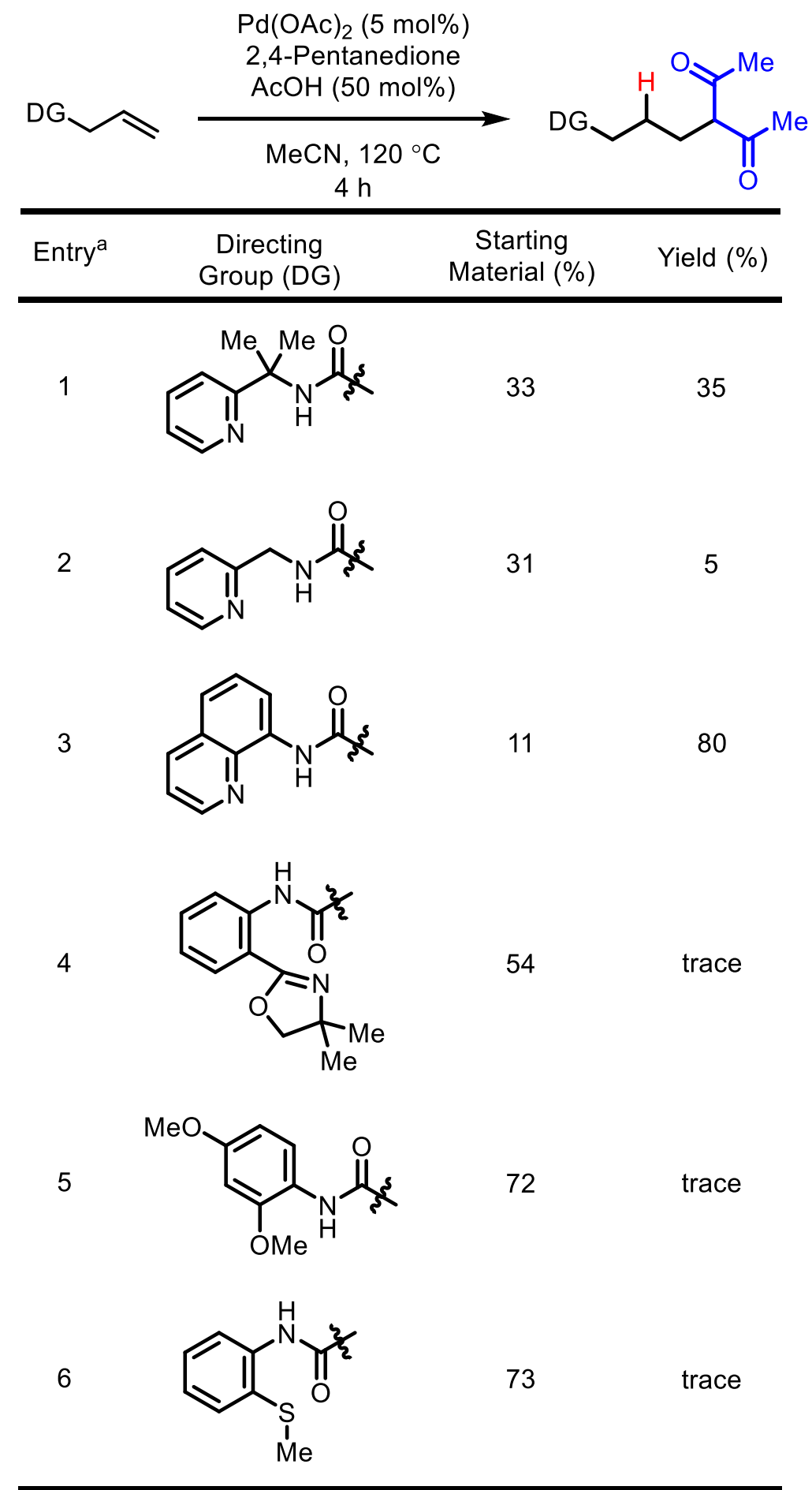

${ }^{a}$ Reaction conditions: $0.1 \mathrm{mmol}$ alkene, $5 \mathrm{~mol} \% \mathrm{Pd}(\mathrm{OAc})_{2}$, $50 \mathrm{~mol} \% \mathrm{AcOH}, 1.5$ equiv 2,4-pentanedione, $0.05 \mathrm{~mL}$ MeCN, $120^{\circ} \mathrm{C}, 4 \mathrm{~h}$. Yields based on ${ }^{1} \mathrm{H}$ NMR analysis of crude reaction mixture with 1,3,5-triisopropyl benzene as an internal standard. 
<smiles>C=CCC(=O)Nc1cccc2cccnc12</smiles>

1<smiles>C=CC(C(=O)Nc1cccc2cccnc12)C(C)(C)O</smiles>

S5<smiles>C=CC(C)C(=O)Nc1cccc2cccnc12</smiles>

76<smiles>CCC=CCC(=O)Nc1cccc2cccnc12</smiles>

s6<smiles>C=CC(C(=O)Nc1cccc2cccnc12)C(C)C</smiles>

S2<smiles>C=CC(C)(C)C(=O)Nc1cccc2cccnc12</smiles>

S3<smiles>C=CC(CCC(=C)C)C(=O)Nc1cccc2cccnc12</smiles>

S4<smiles>C=CCC(=O)Nc1ccccc1C1=NC(C)(C)CO1</smiles><smiles>C=CCC(=O)Nc1ccc(OC)cc1OC</smiles>

S17<smiles>CC/C=C\CC(=O)Nc1cccc2cccnc12</smiles>

s7<smiles>O=C(Nc1cccc2cccnc12)C1C=CCCC1</smiles>

58<smiles>C=CCC(=O)NCc1ccccn1</smiles>

S15

Alkenes 1, 76, S2-S8, and S15-S18 were synthesized as reported (1).<smiles>CC=CCC(=O)O</smiles>

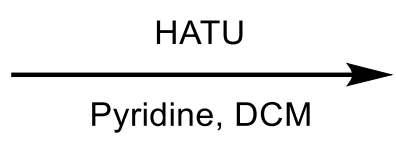<smiles>CC=CCC(=O)Nc1cccc2cccnc12</smiles>

(E)- $\boldsymbol{N}$-(quinolin-8-yl)pent-3-enamide (S9): trans-Pent-3-enoic acid (1.20 g, $12.0 \mathrm{mmol}$ ) was charged into a 100 mL RB flask containing $20 \mathrm{~mL}$ DCM. 8-Aminoquinoline (1.15 g, $8.0 \mathrm{mmol})$, pyridine (1.29 mL, $16.0 \mathrm{mmol})$, and HATU ( $4.56 \mathrm{~g}, 12.0 \mathrm{mmol}$ ) were added sequentially, and the reaction was stirred at ambient temperature for $36 \mathrm{~h}$. The deep brown solution was diluted with EtOAc $(100 \mathrm{~mL})$, washed with sat. $\mathrm{NaHCO}_{3}(100 \mathrm{~mL}, \times 2)$ and brine $(100 \mathrm{~mL}, \times 1)$, dried over $\mathrm{Na}_{2} \mathrm{SO}_{4}$, and purified by column chromatography (5-10\% EtOAc in Hexanes) to afford $1.28 \mathrm{~g}(71 \%)$ yield of $\mathbf{S 9}$ as a yellow oil. ${ }^{1} \mathbf{H} \mathbf{N M R}\left(600 \mathrm{MHz}, \mathrm{CDCl}_{3}\right) \delta 10.01(\mathrm{~s}, 1 \mathrm{H}), 8.80(\mathrm{dd}, J=4.2,1.7 \mathrm{~Hz}, 1 \mathrm{H}), 8.77(\mathrm{dd}, J=7.5,1.5 \mathrm{~Hz}$, $1 \mathrm{H}), 8.15(\mathrm{dd}, J=8.3,1.7 \mathrm{~Hz}, 1 \mathrm{H}), 7.56-7.47(\mathrm{~m}, 2 \mathrm{H}), 7.44(\mathrm{dd}, J=8.2,4.2 \mathrm{~Hz}, 1 \mathrm{H}), 5.87-5.72(\mathrm{~m}, 2 \mathrm{H}), 3.30-3.25(\mathrm{~m}$, 2H), 1.85-1.78 (m, 3H). ${ }^{13} \mathrm{C}$ NMR $\left(150 \mathrm{MHz}, \mathrm{CDCl}_{3}\right) \delta 170.28,148.42,138.74,136.51,134.68,131.53,128.14,127.61$, 123.70, 121.75, 121.67, 116.56, 42.31, 18.32. HRMS calcd. for $\mathrm{C}_{14} \mathrm{H}_{15} \mathrm{~N}_{2} \mathrm{O}+[\mathrm{M}+\mathrm{H}]+:$ 227.11789, Found: 227.1177.<smiles>Nc1cccc2cccnc12</smiles><smiles>CC(C)C(C)O</smiles><smiles>O=C(C/C=C/c1ccccc1)Nc1cccc2cccnc12</smiles>

79

(E)-4-phenyl- $\boldsymbol{N}$-(quinolin-8-yl)but-3-enamide (79): trans-Styrylacetic acid (2.16 g, $13.3 \mathrm{mmol}$ ) was charged into a $250 \mathrm{~mL} \mathrm{RB}$ flask containing $50 \mathrm{~mL}$ dry DMF at room temperature. 8-Aminoquinoline (1.74 g, $12.1 \mathrm{mmol}), \mathrm{EDCl}$ $(2.55 \mathrm{~g}, 13.3 \mathrm{mmol})$, and HOAt $(1.81 \mathrm{~g}, 13.3 \mathrm{mmol})$ were added sequentially, and the reaction was stirred at room temperature for $16 \mathrm{~h}$. The yellow solution was diluted with EtOAc $(100 \mathrm{~mL})$, washed with sat. $\mathrm{NaHCO}_{3}(100 \mathrm{~mL}, \times 2)$, DI water $(100 \mathrm{~mL}, \times 2)$, and brine $(100 \mathrm{~mL}, \times 1)$, dried over $\mathrm{Na}_{2} \mathrm{SO}_{4}$, and purified by column chromatography (33\% EtOAc in Hexanes) to afford $2.82 \mathrm{~g}(81 \%)$ yield of $\mathbf{7 9}$ as a pale yellow solid. ${ }^{\mathbf{1}} \mathbf{H} \mathbf{~ N M R}\left(600 \mathrm{MHz}, \mathrm{CDCl}_{3}\right) \delta 10.07(\mathrm{~s}$, 
$1 \mathrm{H}), 8.77(\mathrm{dd}, J=7.5,1.5 \mathrm{~Hz}, 1 \mathrm{H}), 8.73(\mathrm{dd}, J=4.2,1.7 \mathrm{~Hz}, 1 \mathrm{H}), 8.14(\mathrm{dd}, J=8.2,1.7 \mathrm{~Hz}, 1 \mathrm{H}), 7.57-7.52(\mathrm{~m}, 1 \mathrm{H}), 7.50$ $(\mathrm{dd}, J=8.3,1.5 \mathrm{~Hz}, 1 \mathrm{H}), 7.48-7.44(\mathrm{~m}, 2 \mathrm{H}), 7.42(\mathrm{dd}, J=8.2,4.2 \mathrm{~Hz}, 1 \mathrm{H}), 7.34(\mathrm{dd}, J=8.4,6.9 \mathrm{~Hz}, 2 \mathrm{H}), 7.29-7.24(\mathrm{~m}$, $1 \mathrm{H}), 6.72(\mathrm{dt}, J=15.8,1.4 \mathrm{~Hz}, 1 \mathrm{H}), 6.50(\mathrm{dt}, J=15.9,7.3 \mathrm{~Hz}, 1 \mathrm{H}), 3.50(\mathrm{dd}, J=7.3,1.4 \mathrm{~Hz}, 2 \mathrm{H}) .{ }^{13} \mathrm{C} \mathrm{NMR}(150 \mathrm{MHz}$, $\left.\mathrm{CDCl}_{3}\right) \delta 169.50,148.44,138.68,137.16,136.51,135.14,134.57,128.77(\times 2), 128.11,127.88,127.58,126.67(\times 2)$, 122.58, 121.82, 121.79, 116.63, 42.55. HRMS calcd. for $\mathrm{C}_{19} \mathrm{H}_{17} \mathrm{~N}_{2} \mathrm{O}+[\mathrm{M}+\mathrm{H}]^{+}:$289.13354, Found: 289.1335.
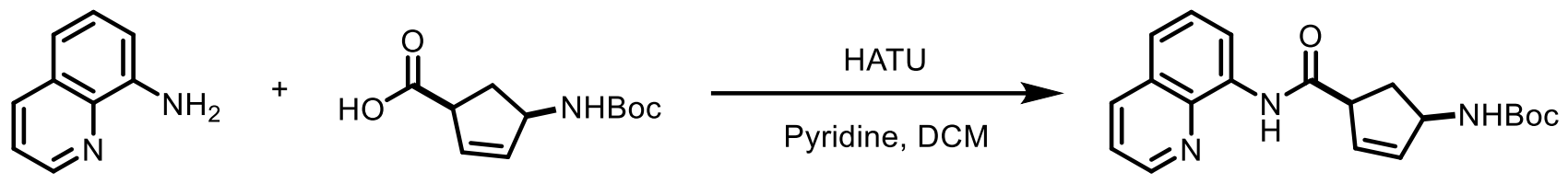

S10

tert-butyl ((cis)-4-(quinolin-8-ylcarbamoyl)cyclopent-2-en-1-yl)carbamate (S10): (cis)-4-((tertbutoxycarbonyl)amino)cyclopent-2-ene-1-carboxylic acid (795 mg, $2.05 \mathrm{mmol}$ ) was charged into a $100 \mathrm{~mL}$ RB flask containing $7 \mathrm{~mL}$ DCM. 8-Aminoquinoline (288 mg, $2.0 \mathrm{mmol})$, collidine (0.54 mL, $4.0 \mathrm{mmol})$, and HATU (0.760 g, 2.0 mmol) were added sequentially, and the reaction was stirred at ambient temperature for $24 \mathrm{~h}$. The deep brown solution was diluted with EtOAc $(50 \mathrm{~mL})$, washed with sat. $\mathrm{NaHCO}_{3}(50 \mathrm{~mL}, \times 2)$ and brine $(50 \mathrm{~mL}, \times 1)$, dried over $\mathrm{Na}_{2} \mathrm{SO}_{4}$, and purified by column chromatography (20-30\% EtOAc in Hexanes) to afford $600 \mathrm{mg}$ (85\%) yield of $\mathbf{S 1 0}$ as a yellow soild. ${ }^{1} \mathrm{H}$ NMR $\left(600 \mathrm{MHz}, \mathrm{CDCl}_{3}\right) \delta 9.89(\mathrm{~s}, 1 \mathrm{H}), 8.84(\mathrm{dd}, J=4.3,1.6 \mathrm{~Hz}, 1 \mathrm{H}), 8.72(\mathrm{dd}, J=6.7,2.3 \mathrm{~Hz}, 1 \mathrm{H})$, $8.18(\mathrm{dd}, J=8.3,1.7 \mathrm{~Hz}, 1 \mathrm{H}), 7.58-7.50(\mathrm{~m}, 2 \mathrm{H}), 7.47(\mathrm{dd}, J=8.3,4.2 \mathrm{~Hz}, 1 \mathrm{H}), 6.09-6.00(\mathrm{~m}, 2 \mathrm{H}), 5.56(\mathrm{~d}, J=9.5 \mathrm{~Hz}$, $1 \mathrm{H}), 4.91(\mathrm{t}, J=9.3 \mathrm{~Hz}, 1 \mathrm{H}), 3.71-3.65(\mathrm{~m}, 1 \mathrm{H}), 2.64(\mathrm{dt}, J=14.1,8.6 \mathrm{~Hz}, 1 \mathrm{H}), 2.01(\mathrm{dt}, J=14.1,3.1 \mathrm{~Hz}, 1 \mathrm{H}), 1.44(\mathrm{~s}$, 9H). ${ }^{13} \mathrm{C}$ NMR $\left(150 \mathrm{MHz}, \mathrm{CDCl}_{3}\right) \delta 172.73,155.50,148.65,148.51,138.74,136.72,136.10,134.36,131.94,128.20$, 127.57, 122.10, 121.88, 117.02, 116.73, 79.40, 56.00, 53.17, 35.80, 28.69. HRMS calcd. for $\mathrm{C}_{20} \mathrm{H}_{24} \mathrm{~N}_{3} \mathrm{O}_{3}+[\mathrm{M}+\mathrm{H}]^{+}$: 354.18122, Found: 354.1811.

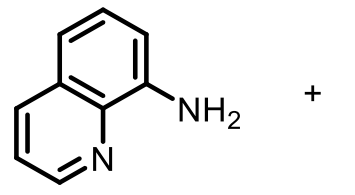<smiles>CC(C)/C=C/CC(=O)O</smiles>
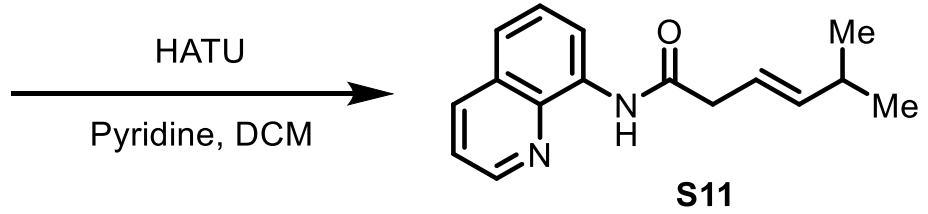

(E)-5-methyl- $\boldsymbol{N}$-(quinolin-8-yl)hex-3-enamide (S11): (E)-5-methylhex-3-enoic acid was synthesized as reported by Maruoka (2). (E)-5-methylhex-3-enoic acid (400 mg, $3.1 \mathrm{mmol}$ ) was charged into a $100 \mathrm{~mL}$ RB flask containing 6 mL DCM. 8-Aminoquinoline (288 mg, $2.0 \mathrm{mmol})$, pyridine $(0.32 \mathrm{~mL}, 4.0 \mathrm{mmol})$, and HATU (1.14 g, $3.0 \mathrm{mmol})$ were added sequentially, and the reaction was stirred at ambient temperature for $24 \mathrm{~h}$. The deep brown solution was diluted with EtOAc $(50 \mathrm{~mL})$, washed with sat. $\mathrm{NaHCO}_{3}(50 \mathrm{~mL}, \times 2)$ and brine $(50 \mathrm{~mL}, \times 1)$, dried over $\mathrm{Na}_{2} \mathrm{SO}_{4}$, and purified by column chromatography (10-15\% EtOAc in Hexanes) to afford $370 \mathrm{mg}(73 \%)$ yield of $\mathbf{S 1 1}$ as a yellow oil. ${ }^{1} \mathrm{H}$ NMR $\left(600 \mathrm{MHz}, \mathrm{CDCl}_{3}\right) \delta 10.14(\mathrm{~s}, 1 \mathrm{H}), 8.80-8.75(\mathrm{~m}, 2 \mathrm{H}), 8.15(\mathrm{dd}, J=8.3,1.7 \mathrm{~Hz}, 1 \mathrm{H}), 7.57-7.47(\mathrm{~m}, 2 \mathrm{H})$, 7.44 (dd, $J=8.2,4.2 \mathrm{~Hz}, 1 \mathrm{H}$ ), 5.82 (ddt, $J=15.4,6.7,1.2 \mathrm{~Hz}, 1 \mathrm{H}$ ), 5.71 (dtd, $J=15.5,7.2,1.2 \mathrm{~Hz}, 1 \mathrm{H}$ ), 3.26 (dt, $J=7.1$, $0.9 \mathrm{~Hz}, 2 \mathrm{H}), 2.51-2.40(\mathrm{~m}, J=7.0 \mathrm{~Hz}, 1 \mathrm{H}), 1.13(\mathrm{~d}, J=6.8 \mathrm{~Hz}, 6 \mathrm{H}) .{ }^{13} \mathrm{C}$ NMR $\left(150 \mathrm{MHz}, \mathrm{CDCl}_{3}\right) \delta 170.33,148.28,144.71$, 138.82, 136.46, 134.72, 128.14, 127.63, 121.75, 121.66, 119.65, 116.46, 42.34, 31.63, 22.53 (×2). HRMS calcd. for $\mathrm{C}_{16} \mathrm{H}_{19} \mathrm{~N}_{2} \mathrm{O}+[\mathrm{M}+\mathrm{H}]^{+}: 255.14919$, Found: 255.1491.
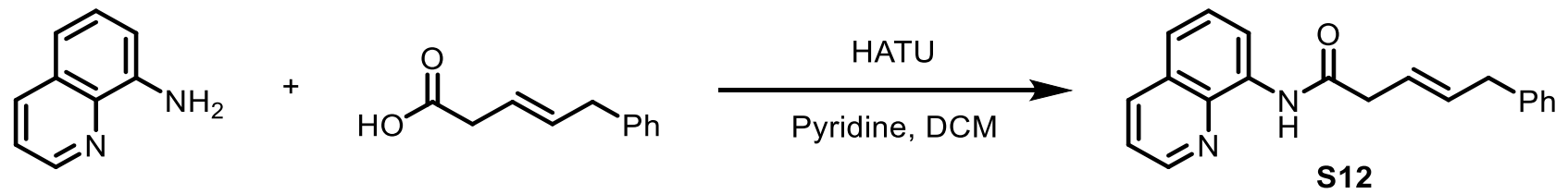

(E)-5-phenyl- $\boldsymbol{N}$-(quinolin-8-yl)pent-3-enamide (S12): (E)-5-phenylpent-3-enoic acid was synthesized as reported by Maruoka (2). (E)-5-phenylpent-3-enoic acid (529 mg, $3.0 \mathrm{mmol}$ ) was charged into a $100 \mathrm{~mL}$ RB flask containing $20 \mathrm{~mL}$ DCM. 8-Aminoquinoline (288 mg, $2.0 \mathrm{mmol})$, pyridine (0.32 mL, $4.0 \mathrm{mmol})$, and HATU (1.14 g, 3.0 mmol) were added sequentially, and the reaction was stirred at ambient temperature for $24 \mathrm{~h}$. The deep brown solution was diluted with EtOAc $(50 \mathrm{~mL})$, washed with sat. $\mathrm{NaHCO}_{3}(50 \mathrm{~mL}, \times 2)$ and brine $(50 \mathrm{~mL}, \times 1)$, dried over $\mathrm{Na}_{2} \mathrm{SO}_{4}$, and purified by column chromatography (10\% EtOAc in Hexanes) to afford $310 \mathrm{mg}$ (51\%) yield of $\mathbf{S 1 2}$ as a 
yellow oil. ${ }^{1} \mathrm{H}$ NMR $\left(600 \mathrm{MHz}, \mathrm{CDCl}_{3}\right) 6(\mathrm{dd}, J=8.2,4.2 \mathrm{~Hz}, 1 \mathrm{H}), 7.34-7.26(\mathrm{~m}, 4 \mathrm{H}), 7.25-7.18(\mathrm{~m}, 1 \mathrm{H}), 6.02-5.94(\mathrm{~m}$, $1 \mathrm{H}), 5.88-5.79(\mathrm{~m}, 1 \mathrm{H}), 3.52(\mathrm{~d}, J=6.7 \mathrm{~Hz}, 2 \mathrm{H}), 3.32(\mathrm{dq}, J=7.2,1.1 \mathrm{~Hz}, 2 \mathrm{H}) .{ }^{13} \mathrm{C} \mathbf{N M R}\left(150 \mathrm{MHz}, \mathrm{CDCl}_{3}\right) \delta 169.94$, 148.39, 140.12, 138.75, 136.53, 135.58, 134.65, 128.93 (×2), 128.69 (×2), 128.16, 127.62, 126.38, 123.95, 121.78, 121.75, 116.61, 42.27, 39.32. HRMS calcd. for $\mathrm{C}_{20} \mathrm{H}_{19} \mathrm{~N}_{2} \mathrm{O}+[\mathrm{M}+\mathrm{H}]^{+}: 303.14919$, Found: 303.1491.

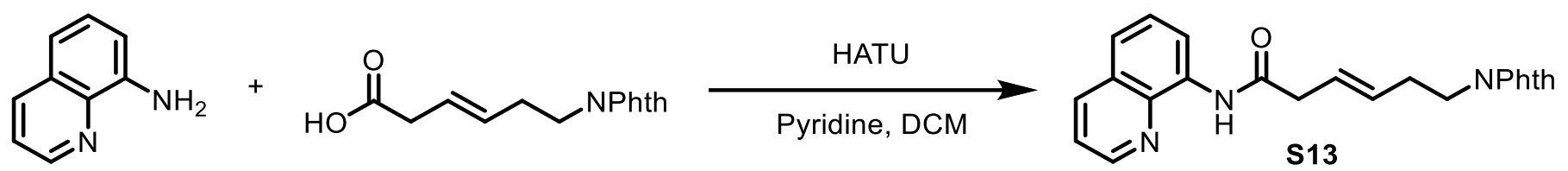

(E)-6-(1,3-dioxoisoindolin-2-yl)- $\boldsymbol{N}$-(quinolin-8-yl)hex-3-enamide (S13): (E)-6-(1,3-dioxoisoindolin-2-yl)hex3-enoic acid was synthesized as reported by Maruoka (2). (E)-6-(1,3-dioxoisoindolin-2-yl)hex-3-enoic acid (820 mg, $3.0 \mathrm{mmol}$ ) was charged into a $100 \mathrm{~mL} \mathrm{RB}$ flask containing $10 \mathrm{~mL}$ DCM. 8-Aminoquinoline (288 mg, $2.0 \mathrm{mmol}$ ), pyridine $(0.32 \mathrm{~mL}, 4.0 \mathrm{mmol})$, and HATU $(1.14 \mathrm{~g}, 3.0 \mathrm{mmol})$ were added sequentially, and the reaction was stirred at ambient temperature for $16 \mathrm{~h}$. The deep brown solution was diluted with EtOAc ( $50 \mathrm{~mL}$ ), washed with sat. $\mathrm{NaHCO}_{3}$ $(50 \mathrm{~mL}, \times 2)$ and brine $(50 \mathrm{~mL}, \times 1)$, dried over $\mathrm{Na}_{2} \mathrm{SO}_{4}$, and purified by column chromatography (5-10\% EtOAc in Hexanes) to afford $485 \mathrm{mg}(61 \%)$ yield of $\mathbf{S 1 3}$ as an off-white solid. ${ }^{\mathbf{1}} \mathbf{H} \mathbf{N M R}\left(600 \mathrm{MHz}, \mathrm{CDCl}_{3}\right) \delta 9.96(\mathrm{~s}, 1 \mathrm{H}), 8.81$ $(\mathrm{dd}, J=4.2,1.7 \mathrm{~Hz}, 1 \mathrm{H}), 8.69$ (dd, $J=7.1,1.9 \mathrm{~Hz}, 1 \mathrm{H}), 8.14(\mathrm{dd}, J=8.3,1.7 \mathrm{~Hz}, 1 \mathrm{H}), 7.76(\mathrm{dd}, J=5.4,3.0 \mathrm{~Hz}, 2 \mathrm{H}), 7.63$ (dd, $J=5.5,3.0 \mathrm{~Hz}, 2 \mathrm{H}), 7.54-7.48(\mathrm{~m}, 2 \mathrm{H}), 7.44(\mathrm{dd}, J=8.2,4.2 \mathrm{~Hz}, 1 \mathrm{H}), 5.90-5.77(\mathrm{~m}, 2 \mathrm{H}), 3.88-3.83(\mathrm{~m}, 2 \mathrm{H}), 3.26$ $(\mathrm{d}, J=6.5 \mathrm{~Hz}, 2 \mathrm{H}), 2.56(\mathrm{q}, J=6.9 \mathrm{~Hz}, 2 \mathrm{H}) .{ }^{13} \mathrm{C}$ NMR $\left(150 \mathrm{MHz}, \mathrm{CDCl}_{3}\right) \delta 169.56,168.51(\times 2), 148.50,138.71,136.45$, 134.55, 134.01 (×2), 132.29, 132.25, 128.11, 127.55, 125.82, $123.36(\times 2), 121.82,121.71,116.56,42.22,37.65,32.07$. HRMS calcd. for $\mathrm{C}_{23} \mathrm{H}_{20} \mathrm{~N}_{3} \mathrm{O}_{3}+[\mathrm{M}+\mathrm{H}]+:$ 386.14992, Found: 386.1500 .<smiles>CC(C)=CCCCCCC=CCC(=O)O</smiles>
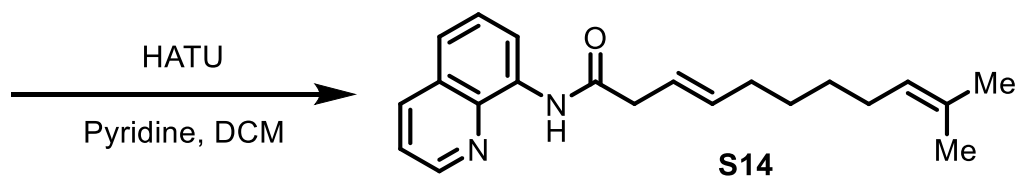

(E)-10-methyl- $\boldsymbol{N}$-(quinolin-8-yl)undeca-3,9-dienamide (S14): (E)-10-methylundeca-3,9-dienoic acid was synthesized as reported by Maruoka (2). (E)-10-methylundeca-3,9-dienoic acid (795 mg, $4.05 \mathrm{mmol}$ ) was charged into a $100 \mathrm{~mL}$ RB flask containing $10 \mathrm{~mL}$ DCM. 8-Aminoquinoline (389 mg, $2.70 \mathrm{mmol}$ ), pyridine $(0.44 \mathrm{~mL}, 5.40$ $\mathrm{mmol}$ ), and HATU (1.54 g, $4.05 \mathrm{mmol}$ ) were added sequentially, and the reaction was stirred at ambient temperature for $18 \mathrm{~h}$. The deep brown solution was diluted with EtOAc $(50 \mathrm{~mL})$, washed with sat. $\mathrm{NaHCO}_{3}(50 \mathrm{~mL}, \times 2)$ and brine ( $50 \mathrm{~mL}, \times 1)$, dried over $\mathrm{Na}_{2} \mathrm{SO}_{4}$, and purified by column chromatography (5-10\% EtOAc in Hexanes) to afford 573 mg (66\%) yield of $\mathbf{S 1 4}$ as a yellow oil. ${ }^{\mathbf{1}} \mathbf{H} \mathbf{~ N M R}\left(600 \mathrm{MHz}, \mathrm{CDCl}_{3}\right) \delta 10.07(\mathrm{~s}, 1 \mathrm{H}), 8.81-8.75(\mathrm{~m}, 2 \mathrm{H}), 8.15(\mathrm{dd}, J=8.3$, $1.7 \mathrm{~Hz}, 1 \mathrm{H}), 7.57-7.47(\mathrm{~m}, 2 \mathrm{H}), 7.44(\mathrm{dd}, J=8.2,4.2 \mathrm{~Hz}, 1 \mathrm{H}), 5.83(\mathrm{dtt}, J=15.4,6.5,1.1 \mathrm{~Hz}, 1 \mathrm{H}), 5.74(\mathrm{dtt}, J=15.4,7.1$, $1.3 \mathrm{~Hz}, 1 \mathrm{H}), 5.11$ (tdt, $J=7.2,2.9,1.5 \mathrm{~Hz}, 1 \mathrm{H}), 3.27(\mathrm{dd}, J=7.0,1.1 \mathrm{~Hz}, 2 \mathrm{H}), 2.17(\mathrm{q}, J=6.8 \mathrm{~Hz}, 2 \mathrm{H}), 2.01(\mathrm{q}, J=7.3 \mathrm{~Hz}$, 2H), $1.67(\mathrm{~d}, J=1.3 \mathrm{~Hz}, 3 \mathrm{H}), 1.59(\mathrm{~s}, 3 \mathrm{H}), 1.54-1.47(\mathrm{~m}, 2 \mathrm{H}), 1.46-1.36(\mathrm{~m}, 2 \mathrm{H}) .{ }^{13} \mathbf{C}$ NMR $\left(150 \mathrm{MHz}, \mathrm{CDCl}_{3}\right) \delta 170.30$, $148.34,138.80,137.44,136.48,134.72,131.59,128.14,127.62,124.89,122.45,121.75,121.67,116.54,42.43,32.92$, 29.70, 29.16, 28.16, 25.95, 17.91. HRMS calcd. for $\mathrm{C}_{21} \mathrm{H}_{27} \mathrm{~N}_{2} \mathrm{O}+[\mathrm{M}+\mathrm{H}]+$ : 323.21179, Found: 323.2116 .

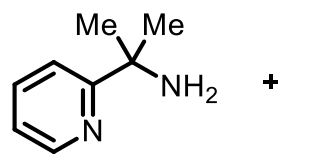<smiles>C=CCC(=O)O</smiles>

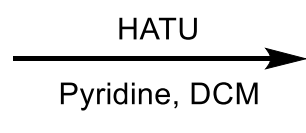<smiles>C=CCC(=O)NC(C)(C)c1ccccn1</smiles>

S19

N-(2-(pyridin-2-yl)propan-2-yl)but-3-enamide (S19): Vinyl acetic acid (461 mg, $5.36 \mathrm{mmol}$ ) was charged into a $100 \mathrm{~mL}$ RB flask containing $10 \mathrm{~mL}$ DCM. 2-(pyridin-2-yl)propan-2-amine (3) (as reported by Affron and Bull) (486.3 $\mathrm{mg}, 3.57 \mathrm{mmol})$, collidine (865 mg, $7.14 \mathrm{mmol})$, and HATU (1.63 g, $4.28 \mathrm{mmol}$ ) were added sequentially, and the reaction was stirred at ambient temperature for $5 \mathrm{~d}$. The deep brown solution was diluted with EtOAc (50 $\mathrm{mL})$, washed with sat. $\mathrm{NaHCO}_{3}(50 \mathrm{~mL}, \times 2)$ and brine $(50 \mathrm{~mL}, \times 1)$, dried over $\mathrm{Na}_{2} \mathrm{SO}_{4}$, purified by column chromatography (30\% EtOAc in Hexanes), and dried in vacuo to afford $346 \mathrm{mg}(47 \%)$ yield of $\mathbf{S 1 9}$ as an oil. $1 \mathbf{H} \mathbf{~ N M R ~ ( 6 0 0 ~ M H z , ~}$ $\left.\mathrm{CDCl}_{3}\right) \delta 8.51(\mathrm{ddd}, \mathrm{J}=5.0,1.8,1.0 \mathrm{~Hz}, 1 \mathrm{H}), 7.85(\mathrm{~s}, 1 \mathrm{H}), 7.71(\mathrm{td}, \mathrm{J}=7.8,1.8 \mathrm{~Hz}, 1 \mathrm{H}), 7.39(\mathrm{dd}, \mathrm{J}=8.1,1.1 \mathrm{~Hz}, 1 \mathrm{H}), 7.19$ 
(ddd, J = 7.4, 4.9, $1.0 \mathrm{~Hz}, 1 \mathrm{H}$ ), $6.02(\mathrm{ddt}, \mathrm{J}=17.3,10.4,7.1 \mathrm{~Hz}, 1 \mathrm{H}), 5.28-5.21(\mathrm{~m}, 2 \mathrm{H}), 3.07$ (dt, J = 7.1, $1.3 \mathrm{~Hz}, 2 \mathrm{H}$ ), $1.75(\mathrm{~s}, 6 \mathrm{H}) .{ }^{13} \mathrm{C}$ NMR $\left(150 \mathrm{MHz}, \mathrm{CDCl}_{3}\right) \delta 170.04,164.77,147.84,137.27,132.16,122.05,119.66,119.19,56.69$, 43.11, 27.69. HRMS calcd. for $\mathrm{C}_{12} \mathrm{H}_{17} \mathrm{~N}_{2} \mathrm{O}+[\mathrm{M}+\mathrm{H}]+:$ 205.13354 Found: 205.1393.

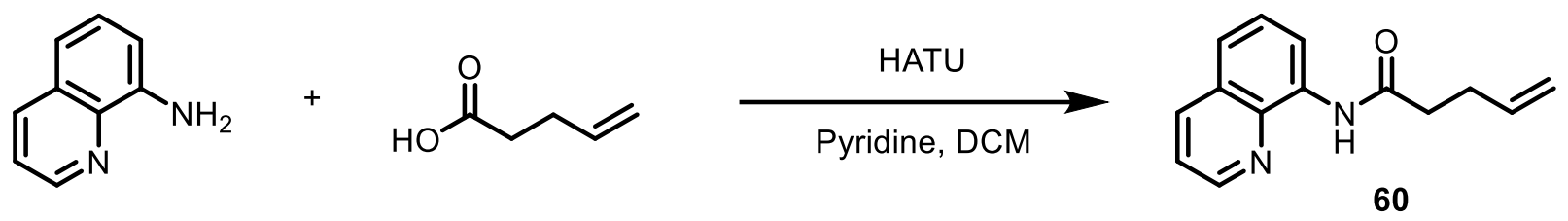

$\boldsymbol{N}$-(quinolin-8-yl)pent-4-enamide (60): Pent-4-enoic acid (1.50 g, $15 \mathrm{mmol}$ ) was charged into a $100 \mathrm{~mL}$ RB flask containing $20 \mathrm{~mL}$ DCM. 8-Aminoquinoline (1.44 g, $10 \mathrm{mmol})$, pyridine (1.58 g, $20 \mathrm{mmol})$, and HATU (5.7 g, $15 \mathrm{mmol})$ were added sequentially, and the reaction was stirred at ambient temperature for $18 \mathrm{~h}$. The deep brown solution was diluted with EtOAc $(200 \mathrm{~mL})$, washed with sat. $\mathrm{NaHCO}_{3}(100 \mathrm{~mL}, \times 2)$ and brine $(50 \mathrm{~mL}, \times 1)$, dried over $\mathrm{Na}_{2} \mathrm{SO}_{4}$, and purified by column chromatography (10-15\% EtOAc in Hexanes) to afford $1.61 \mathrm{~g}(71 \%)$ yield of $\mathbf{6 0}$ as a yellow oil. ${ }^{1} \mathrm{H}$ NMR $\left(600 \mathrm{MHz}, \mathrm{CDCl}_{3}\right) \delta 9.83(\mathrm{~s}, 1 \mathrm{H}), 8.80(\mathrm{dd}, J=4.2,1.7 \mathrm{~Hz}, 1 \mathrm{H}), 8.79(\mathrm{dd}, J=7.5,1.5 \mathrm{~Hz}, 1 \mathrm{H}), 8.16(\mathrm{dd}, J=$ 8.2, $1.7 \mathrm{~Hz}, 1 \mathrm{H}), 7.57-7.52(\mathrm{~m}, 1 \mathrm{H}), 7.50(\mathrm{dd}, J=8.2,1.5 \mathrm{~Hz}, 1 \mathrm{H}), 7.46(\mathrm{dd}, J=8.2,4.2 \mathrm{~Hz}, 1 \mathrm{H}), 5.94(\mathrm{ddt}, J=16.8,10.3$, $6.4 \mathrm{~Hz}, 1 \mathrm{H}), 5.16(\mathrm{dq}, J=17.1,1.6 \mathrm{~Hz}, 1 \mathrm{H}), 5.05(\mathrm{dq}, J=10.3,1.4 \mathrm{~Hz}, 1 \mathrm{H}), 2.71-2.65(\mathrm{~m}, 2 \mathrm{H}), 2.62-2.54(\mathrm{~m}, 2 \mathrm{H}) .{ }^{13} \mathrm{C}$ NMR $\left(150 \mathrm{MHz}, \mathrm{CDCl}_{3}\right) \delta 171.16,148.33,138.55,137.08,136.58,134.70,128.15,127.65,121.80,121.63,116.68$, 115.93, 37.55, 29.71. HRMS calcd. for $\mathrm{C}_{14} \mathrm{H}_{15} \mathrm{~N}_{2} \mathrm{O}+[\mathrm{M}+\mathrm{H}]+:$ 227.11789, Found: 227.1179.
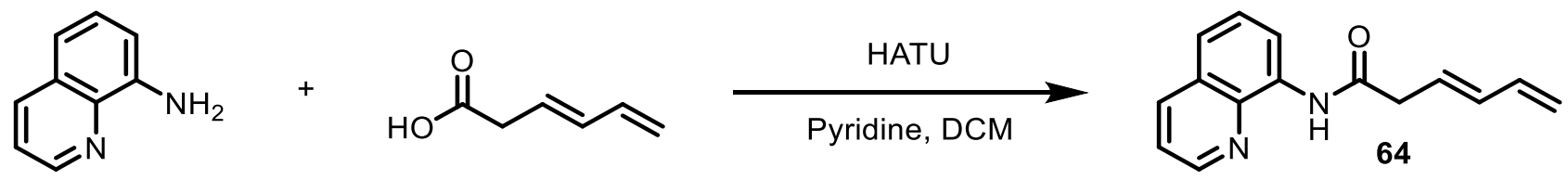

(E)- $\boldsymbol{N}$-(quinolin-8-yl)hexa-3,5-dienamide (64): (E)-10-methylundeca-3,9-dienoic acid (4) (1 g, 8.9 mmol) was charged into a $100 \mathrm{~mL}$ RB flask containing $15 \mathrm{~mL}$ DCM. 8-Aminoquinoline $(1.15 \mathrm{mg}, 8.0 \mathrm{mmol})$, collidine $(2.2 \mathrm{~mL}, 8.0$ $\mathrm{mmol}$ ), and HATU (3.4 g, $8.9 \mathrm{mmol})$ were added sequentially, and the reaction was stirred at ambient temperature for $24 \mathrm{~h}$. The deep brown solution was diluted with EtOAc $(100 \mathrm{~mL})$, washed with sat. $\mathrm{NaHCO}_{3}(50 \mathrm{~mL}, \times 2)$ and brine $(50 \mathrm{~mL}, \times 1)$, dried over $\mathrm{Na}_{2} \mathrm{SO}_{4}$, and purified by column chromatography (20\% EtOAc in Hexanes) to afford $1.50 \mathrm{~g}$ (78\%) yield of 64 as a yellow oil. ${ }^{1} \mathbf{H}$ NMR $\left(600 \mathrm{MHz}, \mathrm{CDCl}_{3}\right) \delta 9.96(\mathrm{~s}, 1 \mathrm{H}), 8.79(\mathrm{dd}, J=4.2,1.7 \mathrm{~Hz}, 1 \mathrm{H}), 8.76(\mathrm{dd}, J=$ $7.4,1.6 \mathrm{~Hz}, 1 \mathrm{H}), 8.15(\mathrm{dd}, J=8.2,1.7 \mathrm{~Hz}, 1 \mathrm{H}), 7.56-7.52(\mathrm{~m}, 1 \mathrm{H}), 7.50(\mathrm{dd}, J=8.2,1.6 \mathrm{~Hz}, 1 \mathrm{H}), 7.45(\mathrm{dd}, J=8.3,4.2 \mathrm{~Hz}$, $1 \mathrm{H}), 6.44(\mathrm{dt}, J=16.8,10.2 \mathrm{~Hz}, 1 \mathrm{H}), 6.40-6.32(\mathrm{~m}, 1 \mathrm{H}), 6.04-5.96(\mathrm{~m}, 1 \mathrm{H}), 5.25(\mathrm{dd}, J=16.9,1.6 \mathrm{~Hz}, 1 \mathrm{H}), 5.13(\mathrm{dd}, J=$ $10.1,1.6 \mathrm{~Hz}, 1 \mathrm{H}), 3.38$ (dd, $J=7.3,1.3 \mathrm{~Hz}, 2 \mathrm{H}) .{ }^{13} \mathrm{C}$ NMR $\left(150 \mathrm{MHz}, \mathrm{CDCl}_{3}\right) \delta 169.42,148.46,138.67,136.59,136.53$, 135.87, 134.57, 128.12, 127.59, 126.28, $121.81(\times 2), 117.56,116.65,42.13$. HRMS calcd. for $\mathrm{C}_{15} \mathrm{H}_{15} \mathrm{~N}_{2} \mathrm{O}+[\mathrm{M}+\mathrm{H}]+$ : 239.11789, Found: 239.1180.<smiles>Nc1cccc2cccnc12</smiles><smiles>C=C/C(C)=C/CC(=O)O</smiles>
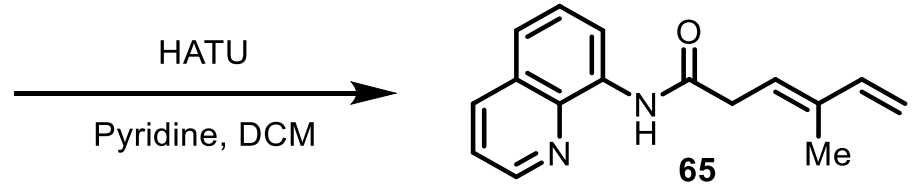

(E)-4-methyl- $\boldsymbol{N}$-(quinolin-8-yl)hexa-3,5-dienamide (65): (E)-10-methylundeca-3,9-dienoic acid (5) (1.39 g, 11.0 mmol) was charged into a $100 \mathrm{~mL}$ RB flask containing $20 \mathrm{~mL}$ DCM. 8-Aminoquinoline (1.44 g, $10.0 \mathrm{mmol}$ ), pyridine $(1.76 \mathrm{~mL}, 22 \mathrm{mmol})$, and HATU $(4.18 \mathrm{~g}, 11.0 \mathrm{mmol})$ were added sequentially, and the reaction was stirred at ambient temperature for $24 \mathrm{~h}$. The deep brown solution was diluted with EtOAc $(100 \mathrm{~mL})$, washed with sat. $\mathrm{NaHCO}_{3}(100$ $\mathrm{mL}, \times 2)$ and brine $(100 \mathrm{~mL}, \times 1)$, dried over $\mathrm{Na}_{2} \mathrm{SO}_{4}$, and purified by column chromatography (5-10\% EtOAc in Hexanes) to afford $1.86 \mathrm{~g}(74 \%)$ yield of 65 as a yellow oil. ${ }^{1} \mathbf{H} \mathbf{~ N M R}\left(600 \mathrm{MHz}, \mathrm{CDCl}_{3}\right) \delta 10.02(\mathrm{~s}, 1 \mathrm{H}), 8.77(\mathrm{dd}, J=$ $4.2,1.7 \mathrm{~Hz}, 1 \mathrm{H}), 8.75(\mathrm{dd}, J=7.4,1.6 \mathrm{~Hz}, 1 \mathrm{H}), 8.15(\mathrm{dd}, J=8.2,1.7 \mathrm{~Hz}, 1 \mathrm{H}), 7.57-7.47(\mathrm{~m}, 2 \mathrm{H}), 7.44(\mathrm{dd}, J=8.3,4.2 \mathrm{~Hz}$, $1 \mathrm{H}), 6.54(\mathrm{dd}, J=17.3,10.8 \mathrm{~Hz}, 1 \mathrm{H}), 5.92-5.85(\mathrm{~m}, 1 \mathrm{H}), 5.27(\mathrm{~d}, J=17.6 \mathrm{~Hz}, 1 \mathrm{H}), 5.10(\mathrm{~d}, J=10.7 \mathrm{~Hz}, 1 \mathrm{H}), 3.45(\mathrm{~d}, J=$ $7.5 \mathrm{~Hz}, 2 \mathrm{H}), 1.93-1.89(\mathrm{~m}, 3 \mathrm{H}) .{ }^{13} \mathrm{C}$ NMR $\left(150 \mathrm{MHz}, \mathrm{CDCl}_{3}\right) \delta 169.45,148.47,140.90,138.92,138.73,136.49,134.63$, $128.12,127.60,123.83,121.79,121.75,116.60,112.94,38.01,12.41$. HRMS calcd. for $\mathrm{C}_{16} \mathrm{H}_{17} \mathrm{~N}_{2} \mathrm{O}+[\mathrm{M}+\mathrm{H}]^{+}$: 253.13354, Found: 253.1334. 
General Procedure for Evaluating Carbon Nucleophile Scope for Hydrofunctionalization<smiles>C=CCC(=O)Nc1cccc2cccnc12</smiles>

Unless otherwise stated, the procedure was as follows. To a 1 dram ( $4 \mathrm{~mL}$ ) vial equipped with a magnetic stir bar were added $\mathrm{Pd}(\mathrm{OAc})_{2}(2.2 \mathrm{mg}$., $0.01 \mathrm{mmol}$ or $4.4 \mathrm{mg}, 0.02 \mathrm{mmol})$, alkene 1 (42.5 mg, $\left.0.2 \mathrm{mmol}\right)$, acetic acid (6.0 mg, $0.1 \mathrm{mmol})$, carbon nucleophile $(0.3 \mathrm{mmol})$, and MeCN $(0.1 \mathrm{~mL})$. The vial was sealed with an unpunctured TFE septum-covered screw cap, and placed in a heating block that was pre-heated to $120^{\circ} \mathrm{C}$. After the designated reaction time, the dark black reaction was purified either by flash column chromatography directly or an aqueous workup followed by flash column chromatography to produce the desired product. 

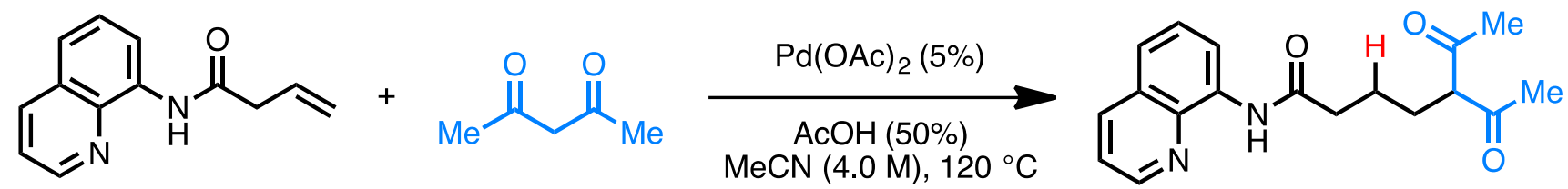

5-acetyl-6-oxo- $\boldsymbol{N}$-(quinolin-8-yl)heptanamide (2): The reaction was carried out according to a slightly modified procedure using alkene 1 (42.5 mg, $0.2 \mathrm{mmol})$, pentane-2,4-dione $(30.0 \mathrm{mg}, 0.3 \mathrm{mmol})$, acetic acid $(6.0 \mathrm{mg}, 0.1$ $\mathrm{mmol}), \mathrm{Pd}(\mathrm{OAc})_{2}(2.2 \mathrm{mg}, 0.01 \mathrm{mmol})$, and $\mathrm{MeCN}(0.05 \mathrm{~mL})$. The reaction was run for $4 \mathrm{~h}$, and the product was purified by flash column chromatography ( $\mathrm{SiO}_{2}, 40-50 \%$ EtOAc in Hexanes) to afford $55.5 \mathrm{mg}(89 \%)$ of 2 as a pale yellow solid. Note: The NMR spectra show an approximate $1: 1$ mixture of two tautomers. ${ }^{1} \mathbf{H} \mathbf{~ N M R}\left(600 \mathrm{MHz}^{-} \mathrm{CDCl}_{3}\right)$ 反 9.91-9.71 (m, 1H), 8.85-8.79 (m, 1H), 8.79-8.72 (m, 1H), 8.21-8.14 (m, 1H), 7.58-7.49 (m, 2H), 7.49-7.44 (m, 1H), $3.71(\mathrm{t}, J=7.2 \mathrm{~Hz}, 0.5 \mathrm{H}), 2.68-2.56(\mathrm{~m}, 2 \mathrm{H}), 2.41-2.34(\mathrm{~m}, 1 \mathrm{H}), 2.22-2.17(\mathrm{~m}, 6 \mathrm{H}), 2.03-1.96(\mathrm{~m}, 1 \mathrm{H}), 1.95-1.87(\mathrm{~m}$, 1H), 1.81-1.72 (m, 1H). ${ }^{13}$ C NMR $\left(150 \mathrm{MHz}, \mathrm{CDCl}_{3}\right) \delta 204.33,191.52,171.07,170.89,148.39,138.51,136.66,136.61$, 134.58, 128.19, 128.16, 127.66, 127.61, 121.87, 121.76, 116.69, 116.66, 110.06, 68.92, 37.65, 37.43, 29.39, 27.85, 27.37, 26.22, 23.52, 23.17. HRMS calcd. for $\mathrm{C}_{18} \mathrm{H}_{21} \mathrm{~N}_{2} \mathrm{O}_{3}+[\mathrm{M}+\mathrm{H}]^{+}:$313.15467, Found: 313.1544 .
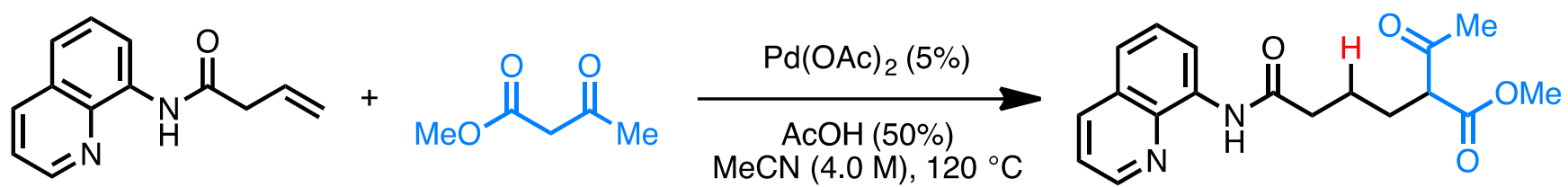

Methyl-2-acetyl-6-oxo-6-(quinolin-8-ylamino)hexanoate (3): The reaction was carried out according to a slightly modified procedure using alkene $1(42.5 \mathrm{mg}, 0.2 \mathrm{mmol})$, methyl-3-oxobutanoate (34.8 $\mathrm{mg}, 0.3 \mathrm{mmol})$, acetic acid (6.0 mg, $0.1 \mathrm{mmol}), \mathrm{Pd}(\mathrm{OAc})_{2}(2.2 \mathrm{mg}, 0.01 \mathrm{mmol})$, and $\mathrm{MeCN}(0.05 \mathrm{~mL})$. The reaction was run for $4 \mathrm{~h}$, and the product was purified by flash column chromatography $\left(\mathrm{SiO}_{2}, 40-50 \%\right.$ EtOAc in Hexanes) to afford $50.7 \mathrm{mg}(77 \%)$ of 3 as a yellow oil. ${ }^{1} \mathrm{H}$ NMR $\left(600 \mathrm{MHz} \mathrm{CDCl}_{3}\right) \delta 9.79(\mathrm{~s}, 1 \mathrm{H}), 8.81(\mathrm{dd}, J=4.2,1.7 \mathrm{~Hz}, 1 \mathrm{H}), 8.75(\mathrm{dd}, J=7.3,1.6 \mathrm{~Hz}, 1 \mathrm{H})$, $8.16(\mathrm{dd}, J=8.3,1.7 \mathrm{~Hz}, 1 \mathrm{H}), 7.57-7.48(\mathrm{~m}, 2 \mathrm{H}), 7.46(\mathrm{dd}, J=8.2,4.2 \mathrm{~Hz}, 1 \mathrm{H}), 3.74(\mathrm{~s}, 3 \mathrm{H}), 3.53(\mathrm{t}, J=7.3 \mathrm{~Hz}, 1 \mathrm{H}), 2.60$ (t, $J=7.4 \mathrm{~Hz}, 2 \mathrm{H}), 2.26(\mathrm{~s}, 3 \mathrm{H}), 2.00(\mathrm{~m}, 2 \mathrm{H}), 1.81(\mathrm{~m}, 2 \mathrm{H}) .{ }^{13} \mathbf{C}$ NMR $\left(150 \mathrm{MHz}, \mathrm{CDCl}_{3}\right) \delta 203.02,170.98,170.27$, 148.37, 138.51, 136.59, 134.61, 128.15, 127.61, 121.84, 121.71, 116.65, 59.63, 52.69, 37.71, 29.24, 27.86, 23.49. HRMS calcd. for $\mathrm{C}_{18} \mathrm{H}_{21} \mathrm{~N}_{2} \mathrm{O}_{4}+[\mathrm{M}+\mathrm{H}]+:$ 329.14958, Found: 329.1495 .
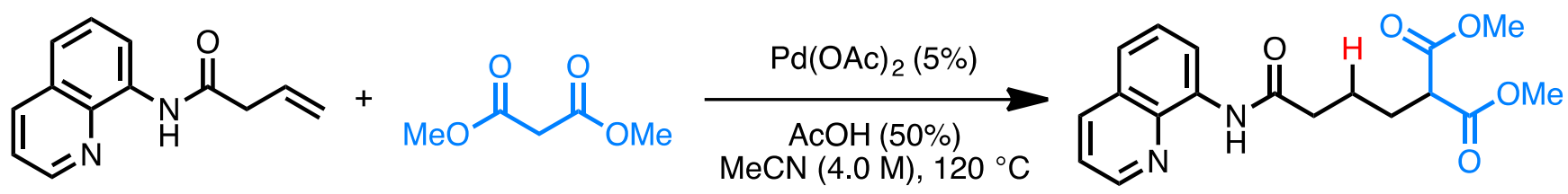

Dimethyl-2-(4-oxo-4-(quinolin-8-ylamino)butyl)malonate (4): The reaction was carried out according to a slightly modified procedure using alkene $1(42.5 \mathrm{mg}, 0.2 \mathrm{mmol})$, dimethyl malonate (39.6 mg, $0.3 \mathrm{mmol})$, acetic acid $(6.0 \mathrm{mg}, 0.1 \mathrm{mmol}), \mathrm{Pd}(\mathrm{OAc})_{2}(2.2 \mathrm{mg}, 0.01 \mathrm{mmol})$, and $\mathrm{MeCN}(0.05 \mathrm{~mL})$. The reaction was run for $7 \mathrm{~h}$, and the product was purified by flash column chromatography $\left(\mathrm{SiO}_{2}, 30-40 \%\right.$ EtOAc in Hexanes) to afford $49.1 \mathrm{mg}(71 \%)$ of 4 as pale yellow oil. ${ }^{1} \mathrm{H}$ NMR $\left(600 \mathrm{MHz}, \mathrm{CDCl}_{3}\right) \delta 9.80(\mathrm{~s}, 1 \mathrm{H}), 8.81(\mathrm{dd}, J=4.2,1.7 \mathrm{~Hz}, 1 \mathrm{H}), 8.76(\mathrm{dd}, J=7.3,1.6 \mathrm{~Hz}, 1 \mathrm{H}), 8.17$ $(\mathrm{dd}, J=8.2,1.7 \mathrm{~Hz}, 1 \mathrm{H}), 7.57-7.48(\mathrm{~m}, 2 \mathrm{H}), 7.46(\mathrm{dd}, J=8.2,4.2 \mathrm{~Hz}, 1 \mathrm{H}), 3.75(\mathrm{~s}, 6 \mathrm{H}), 3.46(\mathrm{t}, J=7.5 \mathrm{~Hz}, 1 \mathrm{H}), 2.61(\mathrm{t}, J$ $=7.5 \mathrm{~Hz}, 2 \mathrm{H}), 2.10-2.02(\mathrm{~m}, 2 \mathrm{H}), 1.90-1.82(\mathrm{~m}, 2 \mathrm{H}) .{ }^{13} \mathrm{C}$ NMR $\left(150 \mathrm{MHz}, \mathrm{CDCl}_{3}\right) \delta 170.97,169.87(\times 2), 148.36,138.52$, $136.60,134.64,128.15,127.63,121.83,121.69,116.67,52.79(\times 2), 51.74,37.71,28.60,23.53$. HRMS calcd. for $\mathrm{C}_{18} \mathrm{H}_{21} \mathrm{~N}_{2} \mathrm{O}_{5}+[\mathrm{M}+\mathrm{H}]+:$ 345.14450, Found345.1446.
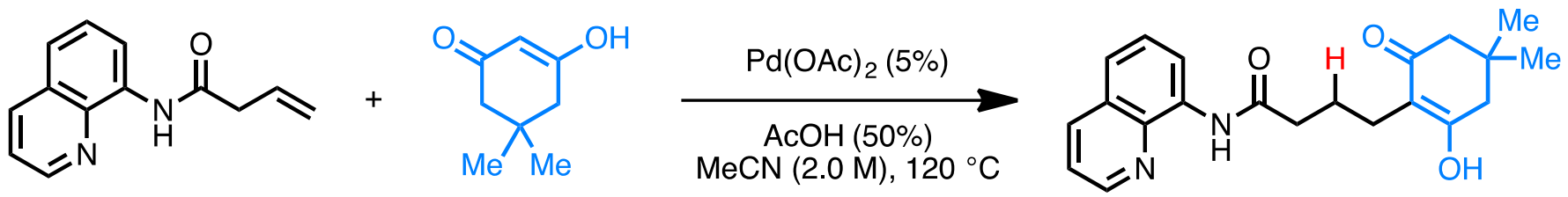

4-(2-hydroxy-4,4-dimethyl-6-oxocyclohex-1-en-1-yl)- $\boldsymbol{N}$-(quinolin-8-yl)butanamide (5): The reaction was carried out according to the general procedure with slight modification using alkene $\mathbf{1}(42.5 \mathrm{mg}, 0.2 \mathrm{mmol}$ ), 
dimedone (42 mg, $0.3 \mathrm{mmol}$ ), acetic acid ( $6.0 \mathrm{mg}, 0.1 \mathrm{mmol}), \mathrm{Pd}(\mathrm{OAc})_{2}(2.2 \mathrm{mg}, 0.01 \mathrm{mmol})$, and $\mathrm{MeCN}(0.1 \mathrm{~mL})$. The reaction was run for $4 \mathrm{~h}$, and the solution was diluted with EtOAc $(20 \mathrm{~mL})$. The organic layer was washed with sat. aq. $\mathrm{NaHCO}_{3}(15 \mathrm{~mL})$, brine $(10 \mathrm{~mL})$, dried over $\mathrm{Na}_{2} \mathrm{SO}_{4}$ and concentrated. The residue purified by rapid flash column chromatography $\left(\mathrm{SiO}_{2}, 50 \%\right.$ EtOAc in Hexanes) to afford $57.6 \mathrm{mg}(82 \%)$ of $\mathbf{5}$ as a yellow oil. Column chromatography was completed within 5 minutes since we observed some oxidation product on prolonged exposure to silica. ${ }^{\mathbf{1}} \mathbf{H}$ NMR $\left(600 \mathrm{MHz}, \mathrm{CDCl}_{3}\right) \delta 10.67(\mathrm{~s}, 1 \mathrm{H}), 10.02(\mathrm{~s}, 1 \mathrm{H}), 8.82(\mathrm{dd}, J=4.2,1.7 \mathrm{~Hz}, 1 \mathrm{H}), 8.77(\mathrm{dd}, J=6.1,2.9 \mathrm{~Hz}, 1 \mathrm{H}), 8.19(\mathrm{dd}, J$ $=8.2,1.7 \mathrm{~Hz}, 1 \mathrm{H}), 7.59-7.51(\mathrm{~m}, 2 \mathrm{H}), 7.49(\mathrm{dd}, J=8.3,4.2 \mathrm{~Hz}, 1 \mathrm{H}), 2.60-2.54(\mathrm{~m}, 2 \mathrm{H}), 2.52(\mathrm{t}, J=6.8 \mathrm{~Hz}, 2 \mathrm{H}), 2.41(\mathrm{~s}$, 2H), $2.25(\mathrm{~s}, 2 \mathrm{H}), 1.90-1.82(\mathrm{~m}, 2 \mathrm{H}), 1.08(\mathrm{~s}, 6 \mathrm{H}) .{ }^{13} \mathrm{C} \mathrm{NMR}\left(150 \mathrm{MHz}, \mathrm{CDCl}_{3}\right) \delta 198.70,174.39,172.30,148.64,138.55$, 136.69, 133.87, 128.15, 127.50, 122.64, 122.04, 117.47, 112.67, 50.94, 43.45, 35.39, 31.99, $28.61(\times 2), 24.48,20.28$. HRMS calcd. for $\mathrm{C}_{21} \mathrm{H}_{25} \mathrm{~N}_{2} \mathrm{O}_{3}+[\mathrm{M}+\mathrm{H}]+:$ 353.18597, Found: 353.1859.
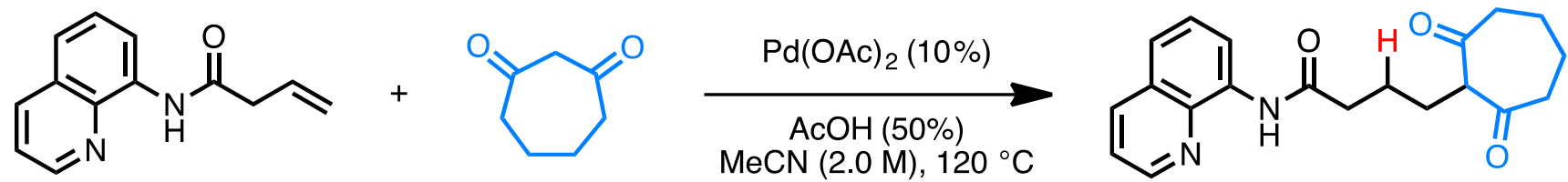

4-(2,7-dioxocycloheptyl)-N-(quinolin-8-yl)butanamide (6): The reaction was carried out according to the general procedure using alkene $1(42.5 \mathrm{mg}, 0.2 \mathrm{mmol})$, cycloheptane-1,3-dione (37.8 $\mathrm{mg}, 0.3 \mathrm{mmol})$, acetic acid (6.0 $\mathrm{mg}, 0.1 \mathrm{mmol}), \mathrm{Pd}(\mathrm{OAc})_{2}(4.4 \mathrm{mg}, 0.02 \mathrm{mmol})$, and $\mathrm{MeCN}(0.1 \mathrm{~mL})$. The reaction was run for $4 \mathrm{~h}$, and the product was purified by flash column chromatography (SiO2, 40-50\% EtOAc in Hexanes) to afford $48.7 \mathrm{mg}$ (72\%) of 6 as an offwhite solid. ${ }^{1} \mathbf{H}$ NMR $(600 \mathrm{MHz}, \mathrm{CDCl} 3) \delta 9.79(\mathrm{~s}, 1 \mathrm{H}), 8.81(\mathrm{dd}, \mathrm{J}=4.2,1.7 \mathrm{~Hz}, 1 \mathrm{H}), 8.75(\mathrm{dd}, \mathrm{J}=7.3,1.6 \mathrm{~Hz}, 1 \mathrm{H}), 8.16$ $(\mathrm{dd}, \mathrm{J}=8.2,1.6 \mathrm{~Hz}, 1 \mathrm{H}), 7.57-7.48(\mathrm{~m}, 2 \mathrm{H}), 7.46(\mathrm{dd}, \mathrm{J}=8.2,4.2 \mathrm{~Hz}, 1 \mathrm{H}), 3.86(\mathrm{t}, \mathrm{J}=6.9 \mathrm{~Hz}, 1 \mathrm{H}), 2.65-2.55(\mathrm{~m}, 4 \mathrm{H})$, 2.55-2.47 (m, 2H), 2.13-2.03 (m, 2H), 1.99-1.92 (m, 2H), 1.91-1.82 (m, 2H), 1.79-1.70 (m, 2H). ${ }^{13}$ C NMR (150 MHz, CDCl3) $\delta 206.97(\times 2), 171.28,148.37,138.53,136.57,134.67,128.15,127.61,121.83,121.65,116.63,66.83,44.23$ (×2), 38.03, 26.08, $25.75(\times 2)$, 23.24. HRMS calcd. for $\mathrm{C}_{20} \mathrm{H}_{23} \mathrm{~N}_{2} \mathrm{O}_{3}+[\mathrm{M}+\mathrm{H}]+:$ 339.17032, Found: 339.1703 .
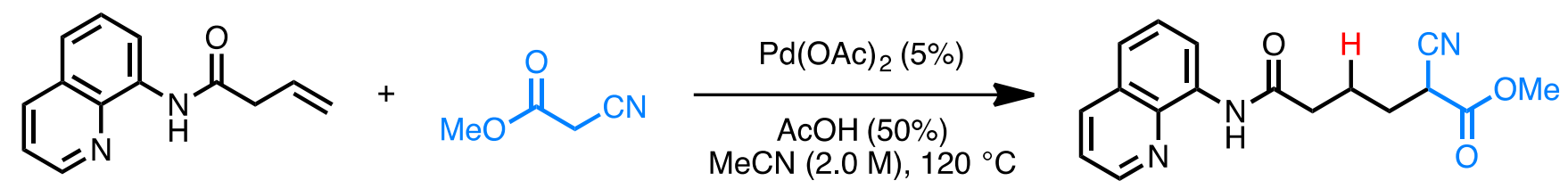

Methyl-2-cyano-6-oxo-6-(quinolin-8-ylamino)hexanoate (7): The reaction was carried out according to the general procedure using alkene $1(42.5 \mathrm{mg}, 0.2 \mathrm{mmol})$, methyl-2-cyanoacetate (29.7 $\mathrm{mg}, 0.3 \mathrm{mmol})$, acetic acid (6.0 $\mathrm{mg}, 0.1 \mathrm{mmol}), \mathrm{Pd}(\mathrm{OAc})_{2}(2.2 \mathrm{mg}, 0.01 \mathrm{mmol})$, and MeCN $(0.1 \mathrm{~mL})$. The reaction was run for $4 \mathrm{~h}$, and the product was purified by flash column chromatography $\left(\mathrm{SiO}_{2}, 40-50 \%\right.$ EtOAc in Hexanes) to afford $46.8 \mathrm{mg}$ (75\%) of 7 as an offwhite solid. ${ }^{1} \mathrm{H}$ NMR $\left(600 \mathrm{MHz}, \mathrm{CDCl}_{3}\right) \delta 9.82(\mathrm{~s}, 1 \mathrm{H}), 8.81(\mathrm{dd}, J=4.2,1.7 \mathrm{~Hz}, 1 \mathrm{H}), 8.75(\mathrm{dd}, J=7.3,1.7 \mathrm{~Hz}, 1 \mathrm{H}), 8.17$ (dd, $J=8.3,1.7 \mathrm{~Hz}, 1 \mathrm{H}), 7.57-7.49(\mathrm{~m}, 2 \mathrm{H}), 7.47$ (dd, $J=8.2,4.2 \mathrm{~Hz}, 1 \mathrm{H}), 3.83(\mathrm{~s}, 3 \mathrm{H}), 3.61(\mathrm{dd}, J=8.0,5.9 \mathrm{~Hz}, 1 \mathrm{H})$, $2.67(\mathrm{td}, J=7.1,3.0 \mathrm{~Hz}, 2 \mathrm{H}), 2.22-2.07(\mathrm{~m}, 2 \mathrm{H}), 2.07-1.99(\mathrm{~m}, 2 \mathrm{H}) .{ }^{13} \mathbf{C}$ NMR $\left(150 \mathrm{MHz}, \mathrm{CDCl}_{3}\right) \delta 170.30,166.59$, 148.42, 138.49, 136.63, 134.48, 128.17, 127.61, 121.89, 121.86, 116.72, 116.44, 53.78, 37.48, 36.91, 29.50, 22.89. HRMS calcd. for $\mathrm{C}_{17} \mathrm{H}_{18} \mathrm{~N}_{3} \mathrm{O}_{3}+[\mathrm{M}+\mathrm{H}]+: 312.13427$, Found: 312.1343 .
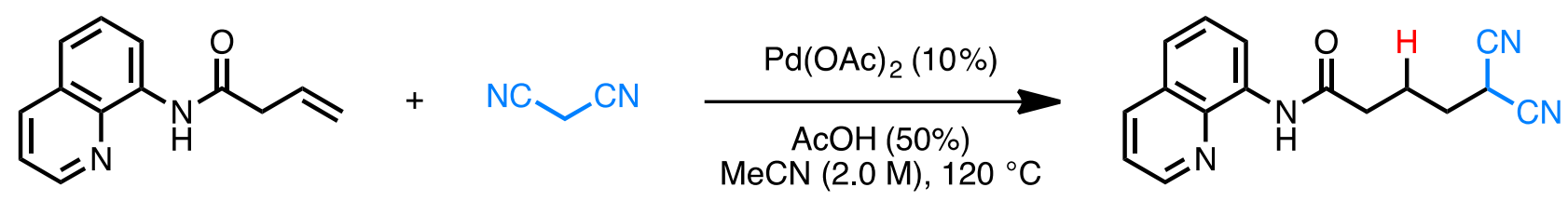

5,5-dicyano- $\boldsymbol{N}$-(quinolin-8-yl)pentanamide (8): The reaction was carried out according to the general procedure using alkene 1 ( $42.5 \mathrm{mg}, 0.2 \mathrm{mmol})$, malononitrile (19.8 mg, $0.3 \mathrm{mmol}$ ), acetic acid (6.0 mg, $0.1 \mathrm{mmol}), \mathrm{Pd}(\mathrm{OAc})_{2}(4.4$ $\mathrm{mg}, 0.02 \mathrm{mmol})$, and MeCN $(0.1 \mathrm{~mL})$. The reaction was run for $4 \mathrm{~h}$, and the product was purified by flash column chromatography $\left(\mathrm{SiO}_{2}, 25 \%\right.$ EtOAc in Hexanes) to afford $21.0 \mathrm{mg}(37 \%)$ of $\mathbf{8}$ as a yellow residue. ${ }^{1} \mathbf{H} \mathbf{~ N M R}(600 \mathrm{MHz}$, $\left.\mathrm{CDCl}_{3}\right) \delta 9.84(\mathrm{~s}, 1 \mathrm{H}), 8.81(\mathrm{dd}, J=4.2,1.7 \mathrm{~Hz}, 1 \mathrm{H}), 8.73(\mathrm{dd}, J=6.8,2.2 \mathrm{~Hz}, 1 \mathrm{H}), 8.18(\mathrm{dd}, J=8.3,1.7 \mathrm{~Hz}, 1 \mathrm{H}), 7.58-$ $7.51(\mathrm{~m}, 2 \mathrm{H}), 7.48(\mathrm{dd}, J=8.3,4.2 \mathrm{~Hz}, 1 \mathrm{H}), 3.90(\mathrm{t}, J=7.0 \mathrm{~Hz}, 1 \mathrm{H}), 2.72(\mathrm{t}, J=6.7 \mathrm{~Hz}, 2 \mathrm{H}), 2.28-2.21(\mathrm{~m}, 2 \mathrm{H}), 2.17-2.09$ $(\mathrm{m}, 2 \mathrm{H}) .{ }^{13} \mathrm{C}$ NMR $\left(150 \mathrm{MHz}, \mathrm{CDCl}_{3}\right) \delta{ }^{13} \mathrm{C} \mathrm{NMR}\left(151 \mathrm{MHz}, \mathrm{CDCl}_{3}\right) \delta 169.78,148.48,138.45,136.68,134.27,128.17$, 
127.58, 122.09, 121.96, 116.79, $112.65(\times 2), 36.07,30.53,22.79,22.44$. HRMS calcd. for $\mathrm{C}_{16} \mathrm{H}_{15} \mathrm{~N}_{4} \mathrm{O}+[\mathrm{M}+\mathrm{H}]^{+}$: 279.12404, Found: 279.1239.
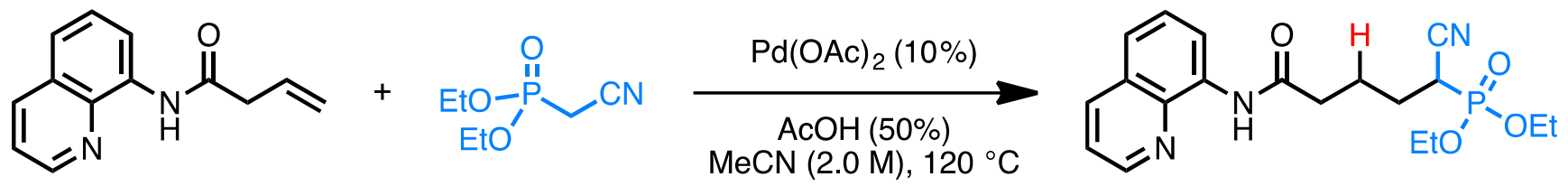

Diethyl-(1-cyano-5-oxo-5-(quinolin-8-ylamino)pentyl)phosphonate (9): The reaction was carried out according to the general procedure using alkene $1(42.5 \mathrm{mg}, 0.2 \mathrm{mmol})$, diethyl-(cyanomethyl)phosphonate (53.2 $\mathrm{mg}, 0.3 \mathrm{mmol}$ ), acetic acid (6.0 mg, $0.1 \mathrm{mmol}), \mathrm{Pd}(\mathrm{OAc})_{2}(4.4 \mathrm{mg}, 0.02 \mathrm{mmol})$, and $\mathrm{MeCN}(0.1 \mathrm{~mL})$. The reaction was run for $4 \mathrm{~h}$, and then was diluted with $50 \mathrm{~mL}$ of EtOAc. The organic layer was washed with sat. $\mathrm{NaHCO}_{3}(50 \mathrm{~mL} \times 2)$, dried over $\mathrm{Na}_{2} \mathrm{SO}_{4}$, and concentrated under reduced pressure. The product was purified by flash column chromatography $\left(\mathrm{SiO}_{2}, 33 \%\right.$ EtOAc in Hexanes) to afford $41.2 \mathrm{mg}(53 \%)$ of $\mathbf{9}$ as a yellow oil. ${ }^{\mathbf{1}} \mathbf{H} \mathbf{~ N M R}(600 \mathrm{MHz}$, $\left.\mathrm{CDCl}_{3}\right) \delta 9.82(\mathrm{~s}, 1 \mathrm{H}), 8.81(\mathrm{dd}, J=4.2,1.7 \mathrm{~Hz}, 1 \mathrm{H}), 8.75(\mathrm{dd}, J=7.3,1.7 \mathrm{~Hz}, 1 \mathrm{H}), 8.17(\mathrm{dd}, J=8.2,1.7 \mathrm{~Hz}, 1 \mathrm{H}), 7.57-$ 7.50 (m, 2H), 7.47 (dd, $J=8.3,4.2 \mathrm{~Hz}, 1 \mathrm{H}), 4.30-4.19(\mathrm{~m}, 4 \mathrm{H}), 3.01$ (ddd, $J=23.7,10.0,4.6 \mathrm{~Hz}, 1 \mathrm{H}), 2.72-2.60(\mathrm{~m}, 2 \mathrm{H})$, 2.23-2.16 (m, 1H), 2.15-2.07 (m, 1H), 2.07-1.99 (m, 2H), $1.38(\mathrm{td}, J=7.1,1.9,0.6 \mathrm{~Hz}, 6 \mathrm{H}) .{ }^{13} \mathrm{C}$ NMR $\left(150 \mathrm{MHz}^{-} \mathrm{CDCl}_{3}\right)$ $\delta 170.32,148.41,138.50,136.62,134.52,128.16,127.62,121.88,121.82,116.72,116.30(\mathrm{~d}, J=9.5 \mathrm{~Hz}), 64.36(\mathrm{~d}, J=$ $6.8 \mathrm{~Hz}), 64.03(\mathrm{~d}, J=6.7 \mathrm{~Hz}), 37.01,30.24(\mathrm{~d}, J=143.2 \mathrm{~Hz}), 26.85(\mathrm{~d}, J=4.3 \mathrm{~Hz}), 23.99(\mathrm{~d}, J=12.2 \mathrm{~Hz}), 16.63$ (d, $J=$ $2.7 \mathrm{~Hz}$ ), 16.59 (d, $J=3.1 \mathrm{~Hz}$ ). HRMS calcd. for $\mathrm{C}_{19} \mathrm{H}_{25} \mathrm{~N}_{3} \mathrm{O}_{4} \mathrm{P}+[\mathrm{M}+\mathrm{H}]+:$ 390.15772, Found: 390.1578.
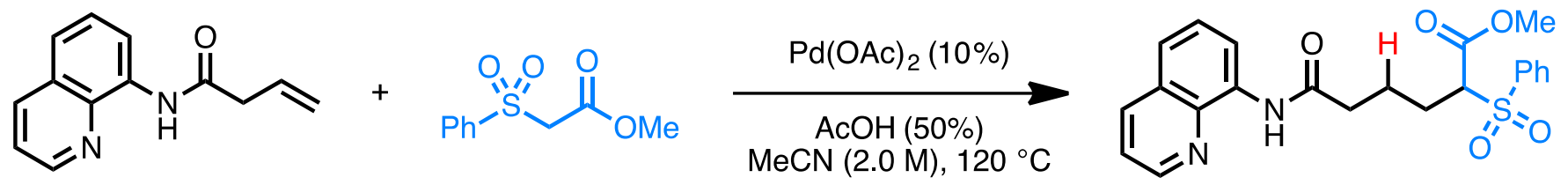

Methyl-6-oxo-2-(phenylsulfonyl)-6-(quinolin-8-ylamino)hexanoate (10): The reaction was carried out according to the general procedure using alkene $1(42.5 \mathrm{mg}, 0.2 \mathrm{mmol})$, methyl-2-(phenylsulfonyl)acetate $(64.2 \mathrm{mg}$, $0.3 \mathrm{mmol})$, acetic acid $(6.0 \mathrm{mg}, 0.1 \mathrm{mmol}), \mathrm{Pd}(\mathrm{OAc})_{2}(4.4 \mathrm{mg}, 0.02 \mathrm{mmol})$, and $\mathrm{MeCN}(0.1 \mathrm{~mL})$. The reaction was run for $4 \mathrm{~h}$, and the product was purified by flash column chromatography ( $\mathrm{SiO}_{2}, 40-50 \%$ EtOAc in Hexanes) to afford $40.2 \mathrm{mg}(47 \%)$ of 10 as a pale yellow oil. ${ }^{1} \mathbf{H} \mathbf{~ N M R}\left(600 \mathrm{MHz}, \mathrm{CDCl}_{3}\right) \delta 9.77(\mathrm{~s}, 1 \mathrm{H}), 8.81(\mathrm{dd}, J=4.2,1.6 \mathrm{~Hz}, 1 \mathrm{H}), 8.71$ (dd, $J=6.9,2.1 \mathrm{~Hz}, 1 \mathrm{H}), 8.17(\mathrm{dd}, J=8.3,1.7 \mathrm{~Hz}, 1 \mathrm{H}), 7.89-7.85(\mathrm{~m}, 2 \mathrm{H}), 7.68-7.59(\mathrm{~m}, 1 \mathrm{H}), 7.56-7.49(\mathrm{~m}, 4 \mathrm{H}), 7.47$ $(\mathrm{dd}, J=8.2,4.2 \mathrm{~Hz}, 1 \mathrm{H}), 4.04(\mathrm{dd}, J=10.9,4.0 \mathrm{~Hz}, 1 \mathrm{H}), 3.68(\mathrm{~s}, 3 \mathrm{H}), 2.58(\mathrm{dd}, J=8.1,7.0 \mathrm{~Hz}, 2 \mathrm{H}), 2.23-2.07(\mathrm{~m}, 2 \mathrm{H})$, $1.85(\mathrm{p}, J=7.8 \mathrm{~Hz}, 2 \mathrm{H}) .{ }^{13} \mathrm{C}$ NMR $\left(150 \mathrm{MHz}, \mathrm{CDCl}_{3}\right) \delta 170.37,166.46,148.40,138.48,137.25,136.62,134.51,134.49$, $129.51(\times 2), 129.29(\times 2), 128.15,127.60,121.89,121.80,116.69,70.83,53.28,37.34,26.62,23.03$. HRMS calcd. for $\mathrm{C}_{22} \mathrm{H}_{23} \mathrm{~N}_{2} \mathrm{O}_{5} \mathrm{~S}+[\mathrm{M}+\mathrm{H}]^{+}:$427.13222, Found: 427.1323.<smiles>C=CCC(=O)Nc1cccc2cccnc12</smiles>
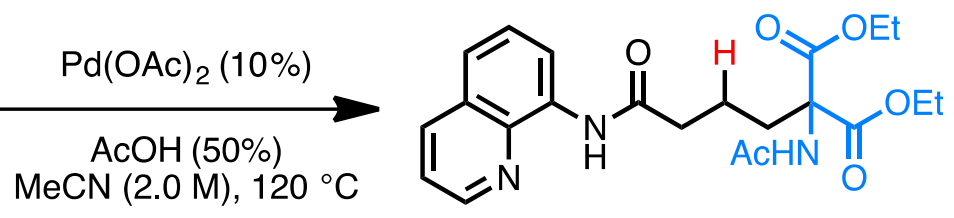

Diethyl-2-acetamido-2-(4-oxo-4-(quinolin-8-ylamino)butyl)malonate (11): The reaction was carried out according to the general procedure using alkene 1 ( $42.5 \mathrm{mg}, 0.2 \mathrm{mmol})$, diethyl-2-acetamidomalonate $(65.1 \mathrm{mg}, 0.3$ $\mathrm{mmol})$, acetic acid (6.0 mg, $0.1 \mathrm{mmol}), \mathrm{Pd}(\mathrm{OAc})_{2}(4.4 \mathrm{mg}, 0.02 \mathrm{mmol})$, and $\mathrm{MeCN}(0.1 \mathrm{~mL})$. The reaction was run for $24 \mathrm{~h}$, and the product was purified by flash column chromatography $\left(\mathrm{SiO}_{2}, 60-70 \%\right.$ EtOAc in Hexanes) to afford 31.2 mg (36\%) of 11 as a yellow solid. ${ }^{1} \mathbf{H}$ NMR $\left(600 \mathrm{MHz} \mathrm{CDCl}_{3}\right) \delta 9.77(\mathrm{~s}, 1 \mathrm{H}), 8.81(\mathrm{dd}, J=4.2,1.7 \mathrm{~Hz}, 1 \mathrm{H}), 8.74(\mathrm{dd}, J=$ 7.3, $1.6 \mathrm{~Hz}, 1 \mathrm{H}), 8.16(\mathrm{dd}, J=8.3,1.7 \mathrm{~Hz}, 1 \mathrm{H}), 7.55-7.49(\mathrm{~m}, 2 \mathrm{H}), 7.46(\mathrm{dd}, J=8.2,4.2 \mathrm{~Hz}, 1 \mathrm{H}), 6.86(\mathrm{~s}, 1 \mathrm{H}), 4.25(\mathrm{q}$, $4 \mathrm{H}), 2.57(\mathrm{t}, J=7.7 \mathrm{~Hz}, 2 \mathrm{H}), 2.49-2.43(\mathrm{~m}, 2 \mathrm{H}), 2.06(\mathrm{~s}, 3 \mathrm{H}), 1.71-1.62(\mathrm{~m}, 2 \mathrm{H}), 1.26(\mathrm{t}, J=7.1 \mathrm{~Hz}, 6 \mathrm{H}) .{ }^{13 C} \mathbf{N M R}(150$ $\left.\mathrm{MHz}, \mathrm{CDCl}_{3}\right) \delta 171.00,169.35,168.19(\times 2), 148.37,138.50,136.57,134.64,128.13,127.59,121.84,121.67,116.61$, 66.61, $62.86(\times 2), 37.72,32.04,23.33,20.04,14.20(\times 2)$. HRMS calcd. for $\mathrm{C}_{22} \mathrm{H}_{28} \mathrm{~N}_{3} \mathrm{O}_{6}+[\mathrm{M}+\mathrm{H}]^{+}:$430.19726, Found: 430.1972 . 


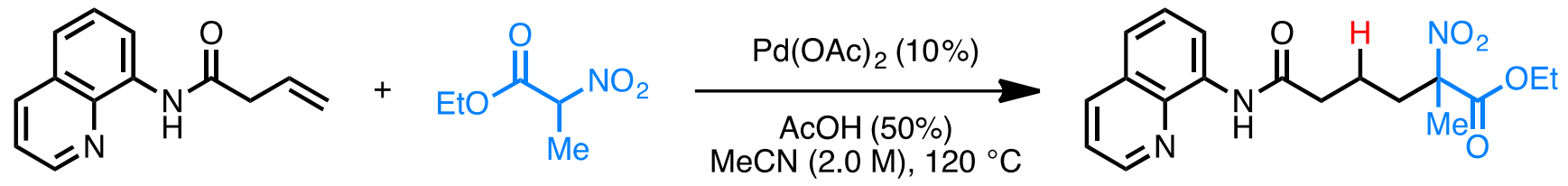

Ethyl-2-methyl-2-nitro-6-oxo-6-(quinolin-8-ylamino)hexanoate (12): The reaction was carried out according to the general procedure using alkene $1(42.5 \mathrm{mg}, 0.2 \mathrm{mmol})$, ethyl-2-nitropropanoate ( $44.1 \mathrm{mg}, 0.3 \mathrm{mmol})$, acetic acid ( $6.0 \mathrm{mg}, 0.1 \mathrm{mmol}), \mathrm{Pd}(\mathrm{OAc})_{2}(4.4 \mathrm{mg}, 0.02 \mathrm{mmol})$, and $\mathrm{MeCN}(0.1 \mathrm{~mL})$. The reaction was run for $4 \mathrm{~h}$, and the product was purified by flash column chromatography $\left(\mathrm{SiO}_{2}, 30 \%\right.$ EtOAc in Hexanes) to afford $64.6 \mathrm{mg}(90 \%)$ of 12 as a colorless oil. ${ }^{1} \mathrm{H}$ NMR $\left(600 \mathrm{MHz}, \mathrm{CDCl}_{3}\right) \delta 9.80(\mathrm{~s}, 1 \mathrm{H}), 8.81(\mathrm{dd}, J=4.2,1.7 \mathrm{~Hz}, 1 \mathrm{H}), 8.75(\mathrm{dd}, J=7.2,1.8 \mathrm{~Hz}, 1 \mathrm{H})$, 8.17 (dd, $J=8.3,1.7 \mathrm{~Hz}, 1 \mathrm{H}$ ), 7.57-7.49 (m, 2H), 7.47 (dd, $J=8.2,4.2 \mathrm{~Hz}, 1 \mathrm{H}), 4.27(\mathrm{q}, 2 \mathrm{H}), 2.70-2.57$ (m, $2 \mathrm{H}), 2.38$ (ddd, $J=14.0,12.2,4.7 \mathrm{~Hz}, 1 \mathrm{H}), 2.31$ (ddd, $J=14.0,12.2,4.7 \mathrm{~Hz}, 1 \mathrm{H}), 1.95-1.84(\mathrm{~m}, 1 \mathrm{H}), 1.84(\mathrm{~s}, 3 \mathrm{H}), 1.80-1.69$ (m, $1 \mathrm{H}), 1.29(\mathrm{t}, J=7.1 \mathrm{~Hz}, 3 \mathrm{H}) .{ }^{13} \mathrm{C}$ NMR $\left(150 \mathrm{MHz}, \mathrm{CDCl}_{3}\right) \delta 170.41,167.50,148.41,138.49,136.62,134.50,128.16$, 127.60, 121.88, 121.83, 116.70, 92.77, 63.07, 37.34, 37.33, 21.43, 19.87, 14.05. HRMS calcd. for $\mathrm{C}_{18} \mathrm{H}_{22} \mathrm{~N}_{3} \mathrm{O}_{5}+[\mathrm{M}+\mathrm{H}]^{+}$: 360.15540, Found: 360.1553.

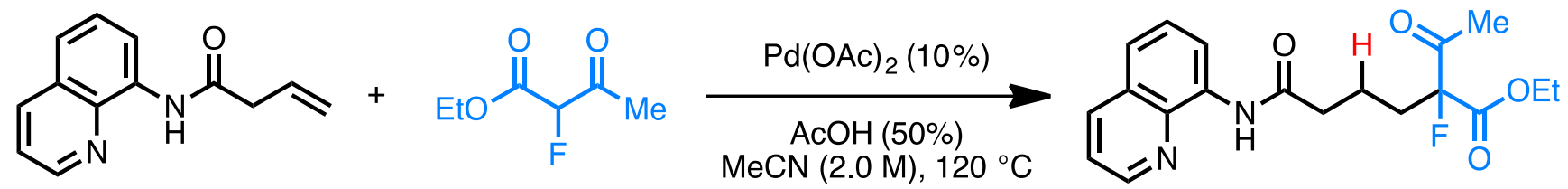

Ethyl-2-acetyl-2-fluoro-6-oxo-6-(quinolin-8-ylamino)hexanoate (13): The reaction was carried out according to the general procedure using alkene $1(42.5 \mathrm{mg}, 0.2 \mathrm{mmol})$, ethyl-2-fluoro-3-oxobutanoate ( $44.4 \mathrm{mg}, 0.3 \mathrm{mmol})$, acetic acid (6.0 mg, $0.1 \mathrm{mmol}), \mathrm{Pd}(\mathrm{OAc})_{2}(4.4 \mathrm{mg}, 0.02 \mathrm{mmol})$, and $\mathrm{MeCN}(0.1 \mathrm{~mL})$. The reaction was run for $4 \mathrm{~h}$, and the product was purified by flash column chromatography $\left(\mathrm{SiO}_{2}, 30 \%\right.$ EtOAc in Hexanes) to afford $51.8 \mathrm{mg}(72 \%)$ of 13 as a colorless oil. ${ }^{1} \mathrm{H}$ NMR $\left(600 \mathrm{MHz}, \mathrm{CDCl}_{3}\right) \delta 9.79(\mathrm{~s}, 1 \mathrm{H}), 8.80(\mathrm{dd}, J=4.2,1.7 \mathrm{~Hz}, 1 \mathrm{H}), 8.76(\mathrm{dd}, J=7.3,1.6 \mathrm{~Hz}$, $1 \mathrm{H}), 8.17(\mathrm{dd}, J=8.2,1.7 \mathrm{~Hz}, 1 \mathrm{H}), 7.57-7.48(\mathrm{~m}, 2 \mathrm{H}), 7.46(\mathrm{dd}, J=8.2,4.2 \mathrm{~Hz}, 1 \mathrm{H}), 4.28(\mathrm{qd}, J=7.1,3.0 \mathrm{~Hz}, 2 \mathrm{H}), 2.61$ $(\mathrm{t}, J=7.4 \mathrm{~Hz}, 2 \mathrm{H}), 2.34(\mathrm{~d}, J=4.7 \mathrm{~Hz}, 3 \mathrm{H}), 2.40-2.15(\mathrm{~m}, 2 \mathrm{H}), 1.97-1.84(\mathrm{~m}, 2 \mathrm{H}), 1.31(\mathrm{t}, J=7.1 \mathrm{~Hz}, 3 \mathrm{H}) .{ }^{13} \mathbf{C}$ NMR (150 $\mathrm{MHz}, \mathrm{CDCl}_{3}$ ) $\delta 202.15(\mathrm{~d}, J=29.1 \mathrm{~Hz}), 170.64,166.32$ (d, $\left.J=26.3 \mathrm{~Hz}\right), 148.37,138.51,136.60,134.58,128.15,127.62$, $121.85,121.73,116.69,100.49$ (d, $J=197.9 \mathrm{~Hz}), 62.94,37.50,33.38$ (d, $J=20.9 \mathrm{~Hz}) 26.03,19.18,19.16,14.25$. HRMS calcd. for $\mathrm{C}_{19} \mathrm{H}_{22} \mathrm{FN}_{2} \mathrm{O}_{4}+[\mathrm{M}+\mathrm{H}]+:$ 361.15581, Found: 361.1558.
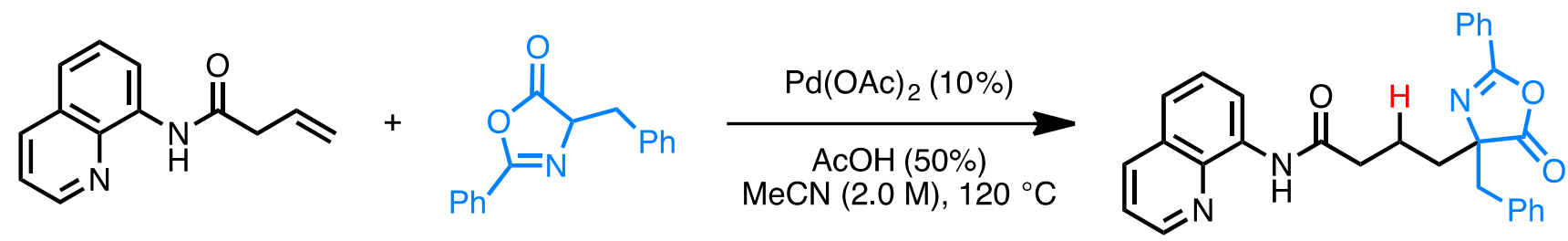

4-(4-benzyl-5-oxo-2-phenyl-4,5-dihydrooxazol-4-yl)- $\boldsymbol{N}$-(quinolin-8-yl)butanamide (14): The reaction was carried out according to the general procedure using alkene $\mathbf{1}(42.5 \mathrm{mg}, 0.2 \mathrm{mmol})$, 4-benzyl-2-phenyloxazol-5(4H)one (75.3 mg, $0.3 \mathrm{mmol}$ ), acetic acid (6.0 mg, $0.1 \mathrm{mmol}), \mathrm{Pd}(\mathrm{OAc})_{2}(4.4 \mathrm{mg}, 0.02 \mathrm{mmol})$, and $\mathrm{MeCN}(0.1 \mathrm{~mL})$. The reaction was run for $4 \mathrm{~h}$, and the product was purified by flash column chromatography $\left(\mathrm{SiO}_{2}, 30-35 \%\right.$ EtOAc in Hexanes) to afford $41.8 \mathrm{mg}$ (45\%) of $\mathbf{1 4}$ as a yellow oil. ${ }^{\mathbf{1}} \mathbf{H}$ NMR $\left(600 \mathrm{MHz}, \mathrm{CDCl}_{3}\right) \delta 9.79(\mathrm{~s}, 1 \mathrm{H}), 8.77(\mathrm{dd}, J=4.2,1.7$ $\mathrm{Hz}, 1 \mathrm{H}), 8.74(\mathrm{dd}, J=7.3,1.7 \mathrm{~Hz}, 1 \mathrm{H}), 8.15(\mathrm{dd}, J=8.3,1.7 \mathrm{~Hz}, 1 \mathrm{H}), 7.88-7.83(\mathrm{~m}, 2 \mathrm{H}), 7.56-7.47(\mathrm{~m}, 3 \mathrm{H}), 7.45(\mathrm{dd}, J=$ 8.2, $4.2 \mathrm{~Hz}, 1 \mathrm{H}), 7.41(\mathrm{td}, J=7.7,1.1 \mathrm{~Hz}, 2 \mathrm{H}), 7.18-7.10(\mathrm{~m}, 5 \mathrm{H}), 3.25(\mathrm{~d}, J=13.4 \mathrm{~Hz}, 1 \mathrm{H}), 3.17(\mathrm{~d}, J=13.4 \mathrm{~Hz}, 1 \mathrm{H})$, 2.65-2.54 (m, 2H), 2.21-2.13 (m, 2H), 1.93-1.83 (m, 1H), 1.82-1.72 (m, 1H). ${ }^{13} \mathbf{C ~ N M R}\left(150 \mathrm{MHz}, \mathrm{CDCl}_{3}\right) \delta 179.81$, 170.80, 160.30, 148.32, 138.52, 136.57, 134.59, 134.45, 132.76, $130.40(\times 2), 128.85(\times 2), 128.33(\times 2), 128.13$, $128.09(\times 2), 127.63,127.41,125.85,121.81,121.68,116.69,74.87,43.91,37.81,36.94,20.55$. HRMS calcd. for $\mathrm{C}_{29} \mathrm{H}_{26} \mathrm{~N}_{3} \mathrm{O}_{3}+[\mathrm{M}+\mathrm{H}]+:$ 464.19687, Found: 464.1967. 
<smiles>C=CCC(=O)Nc1cccc2cccnc12</smiles>
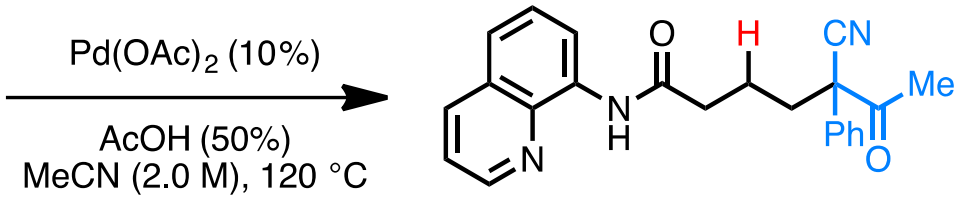

5-cyano-6-oxo-5-phenyl- $\boldsymbol{N}$-(quinolin-8-yl)heptanamide (15): The reaction was carried out according to the general procedure using alkene $1(42.5 \mathrm{mg}, 0.2 \mathrm{mmol}), 3$-oxo-2-phenylbutanenitrile ( $47.8 \mathrm{mg}, 0.3 \mathrm{mmol})$, acetic acid $(6.0 \mathrm{mg}, 0.1 \mathrm{mmol}), \mathrm{Pd}(\mathrm{OAc})_{2}(4.4 \mathrm{mg}, 0.02 \mathrm{mmol})$, and $\mathrm{MeCN}(0.1 \mathrm{~mL})$. The reaction was run for $4 \mathrm{~h}$, and the product was purified by flash column chromatography $\left(\mathrm{SiO}_{2}, 30 \%\right.$ EtOAc in Hexanes) to afford $55.7 \mathrm{mg}(75 \%)$ of 15 as a yellow oil. ${ }^{1} \mathrm{H}$ NMR $\left(600 \mathrm{MHz}, \mathrm{CDCl}_{3}\right) \delta 9.76(\mathrm{~s}, 1 \mathrm{H}), 8.80(\mathrm{dd}, J=4.2,1.7 \mathrm{~Hz}, 1 \mathrm{H}), 8.73(\mathrm{dd}, J=7.4,1.8 \mathrm{~Hz}, 1 \mathrm{H}), 8.16$ $(\mathrm{dd}, J=8.3,1.7 \mathrm{~Hz}, 1 \mathrm{H}), 7.56-7.35(\mathrm{~m}, 8 \mathrm{H}), 2.67-2.53(\mathrm{~m}, 2 \mathrm{H}), 2.39$ (ddd, $J=13.9,12.1,4.5 \mathrm{~Hz}, 1 \mathrm{H}), 2.27(\mathrm{~s}, 3 \mathrm{H}), 2.33-$ $2.22(\mathrm{~m}, 1 \mathrm{H}), 1.99-1.88(\mathrm{~m}, 1 \mathrm{H}), 1.82-1.71(\mathrm{~m}, 1 \mathrm{H}) .{ }^{13} \mathrm{C}$ NMR $\left(150 \mathrm{MHz}, \mathrm{CDCl}_{3}\right) \delta 198.75,170.54,148.36,138.49$, $136.59,134.55,133.61,129.82(\times 2), 129.25,128.14,127.61,126.54(\times 2), 121.83,121.72,119.34,116.69,60.07$, 37.45, 35.56, 26.89, 21.40. HRMS calcd. for $\mathrm{C}_{23} \mathrm{H}_{22} \mathrm{~N}_{3} \mathrm{O}_{2}+[\mathrm{M}+\mathrm{H}]^{+}:$372.17065, Found: 372.1705 .
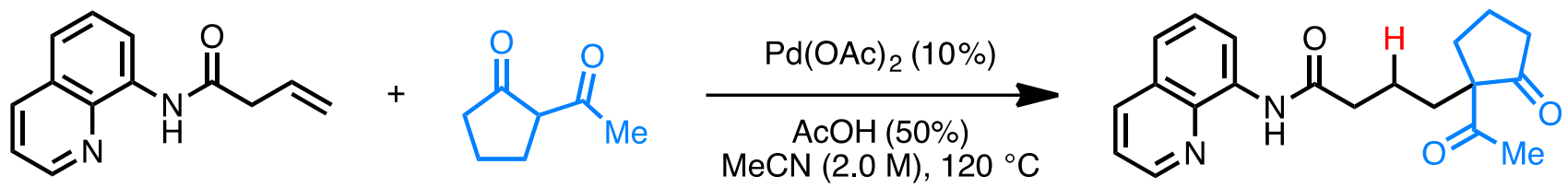

4-(1-acetyl-2-oxocyclopentyl)- $\boldsymbol{N}$-(quinolin-8-yl)butanamide (16): The reaction was carried out according to the general procedure using alkene $1(42.5 \mathrm{mg}, 0.2 \mathrm{mmol}), 2$-acetylcyclopentan-1-one $(39.0 \mathrm{mg}, 0.3 \mathrm{mmol})$, acetic acid $(6.0 \mathrm{mg}, 0.1 \mathrm{mmol}), \mathrm{Pd}(\mathrm{OAc})_{2}(4.4 \mathrm{mg}, 0.02 \mathrm{mmol})$, and $\mathrm{MeCN}(0.1 \mathrm{~mL})$. The reaction was run for $4 \mathrm{~h}$, and the product was purified by flash column chromatography $\left(\mathrm{SiO}_{2}, 30-40 \%\right.$ EtOAc in Hexanes) to afford $56.8 \mathrm{mg}(84 \%)$ of 16 as a pale yellow solid. ${ }^{1} \mathbf{H}$ NMR $\left(600 \mathrm{MHz}, \mathrm{CDCl}_{3}\right) \delta 9.78(\mathrm{~s}, 1 \mathrm{H}), 8.81(\mathrm{dd}, J=4.2,1.7 \mathrm{~Hz}, 1 \mathrm{H}), 8.75(\mathrm{dd}, J=7.3,1.7 \mathrm{~Hz}, 1 \mathrm{H})$, 8.17 (dd, $J=8.3,1.7 \mathrm{~Hz}, 1 \mathrm{H}$ ), 7.57-7.48 (m, 2H), 7.46 (dd, $J=8.2,4.2 \mathrm{~Hz}, 1 \mathrm{H}$ ), 2.74 (dddd, $J=12.6,6.9,4.2,1.5 \mathrm{~Hz}, 1 \mathrm{H}$ ), $2.58(\mathrm{dt}, J=8.4,6.9 \mathrm{~Hz}, 2 \mathrm{H}), 2.36-2.21(\mathrm{~m}, 2 \mathrm{H}), 2.25(\mathrm{~s}, 3 \mathrm{H}), 2.23-2.12(\mathrm{~m}, 1 \mathrm{H}), 1.97-1.90(\mathrm{~m}, 1 \mathrm{H}), 1.89-1.80(\mathrm{~m}, 1 \mathrm{H})$, 1.80-1.60 (m, 4H). ${ }^{13} \mathrm{C}$ NMR $\left(150 \mathrm{MHz}, \mathrm{CDCl}_{3}\right) \delta 216.08,204.40,170.77,148.38,138.50,136.63,134.56,128.17$, 127.63, 121.86, 121.77, 116.70, 69.36, 38.71, 37.79, 34.85, 30.80, 26.17, 21.12, 19.73. HRMS calcd. for $\mathrm{C}_{20} \mathrm{H}_{23} \mathrm{~N}_{2} \mathrm{O}_{3}+$ $[\mathrm{M}+\mathrm{H}]+:$ 339.17032, Found: 339.1704 .
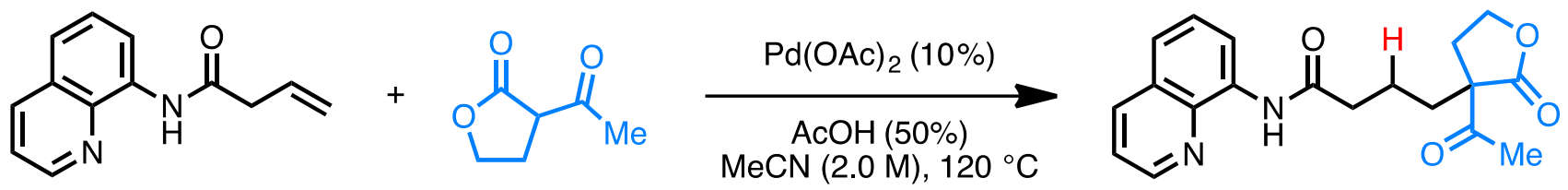

4-(3-acetyl-2-oxotetrahydrofuran-3-yl)- $\boldsymbol{N}$-(quinolin-8-yl)butanamide (17): The reaction was carried out according to the general procedure using alkene $1(42.5 \mathrm{mg}, 0.2 \mathrm{mmol}), 3$-acetyldihydrofuran-2(3H)-one (38.4 mg, $0.3 \mathrm{mmol})$, acetic acid $(6.0 \mathrm{mg}, 0.1 \mathrm{mmol}), \mathrm{Pd}(\mathrm{OAc})_{2}(4.4 \mathrm{mg}, 0.02 \mathrm{mmol})$, and $\mathrm{MeCN}(0.1 \mathrm{~mL})$. The reaction was run for $4 \mathrm{~h}$, and the product was purified by flash column chromatography ( $\mathrm{SiO}_{2}, 40 \%$ EtOAc in Hexanes) to afford 59.2

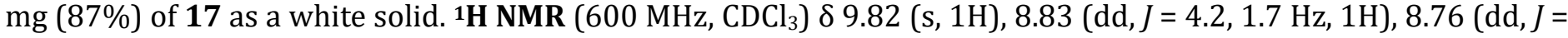
$7.1,1.9 \mathrm{~Hz}, 1 \mathrm{H}), 8.20$ (dd, $J=8.3,1.7 \mathrm{~Hz}, 1 \mathrm{H}), 7.60-7.53(\mathrm{~m}, 2 \mathrm{H}), 7.49$ (dd, $J=8.2,4.2 \mathrm{~Hz}, 1 \mathrm{H}), 4.35(\mathrm{td}, J=8.9,2.9 \mathrm{~Hz}$, $1 \mathrm{H}), 4.20(\mathrm{td}, J=9.2,7.1 \mathrm{~Hz}, 1 \mathrm{H}), 3.01(\mathrm{ddd}, J=13.0,7.1,2.9 \mathrm{~Hz}, 1 \mathrm{H}), 2.65(\mathrm{qt}, J=15.4,6.9 \mathrm{~Hz}, 2 \mathrm{H}), 2.41(\mathrm{~s}, 3 \mathrm{H}), 2.40-$ $2.30(\mathrm{~m}, 1 \mathrm{H}), 2.17(\mathrm{dt}, J=13.0,9.1 \mathrm{~Hz}, 1 \mathrm{H}), 1.99-1.88(\mathrm{~m}, 1 \mathrm{H}), 1.78-1.69(\mathrm{~m}, 2 \mathrm{H}) .{ }^{13} \mathbf{C ~ N M R}\left(150 \mathrm{MHz}, \mathrm{CDCl}_{3}\right) \delta 202.58$, 175.64, 170.44, 148.41, 138.49, 136.66, 134.46, 128.18, 127.61, 121.90, 121.87, 116.72, 66.70, 61.75, 37.27, 34.62, 29.32, 25.78, 20.86. HRMS calcd. for $\mathrm{C}_{19} \mathrm{H}_{21} \mathrm{~N}_{2} \mathrm{O}_{4}+[\mathrm{M}+\mathrm{H}]+:$ 341.14958, Found: 341.1495.

\section{Gram scale:}
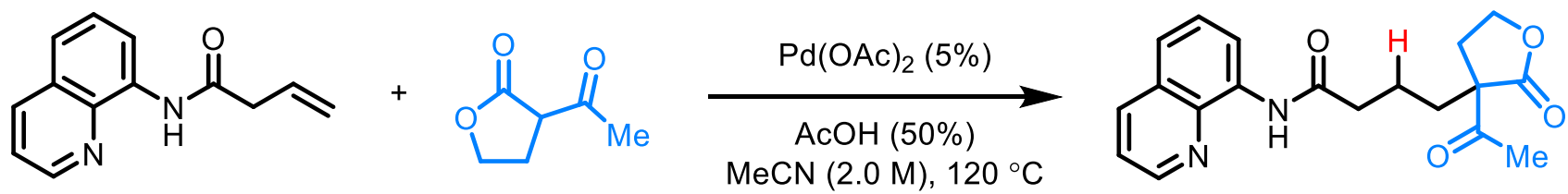
Alkene 1 (1.06 g, $5 \mathrm{mmol}$ ), 3-acetyldihydrofuran-2(3H)-one (769 mg, $6 \mathrm{mmol}$ ), acetic acid (150 mg, $2.5 \mathrm{mmol})$, $\mathrm{Pd}(\mathrm{OAc})_{2}(56 \mathrm{mg}, 0.25 \mathrm{mmol}$ ), and $\mathrm{MeCN}(2.5 \mathrm{~mL}$ ) were added to a $48 \mathrm{~mL}$ sealed tube. The resulting light yellow colored mixture was sealed and submerged into a pre-heated $120^{\circ} \mathrm{C}$ silicon oil bath. The reaction was run for $36 \mathrm{~h}$, and the product was purified by flash column chromatography $\left(\mathrm{SiO}_{2}, 40-50 \%\right.$ EtOAc in Hexanes) to afford $1.35 \mathrm{~g}$ (80\%) of $\mathbf{1 7}$ as a white solid. Characterization data as reported above.<smiles>C=CCC(=O)Nc1cccc2cccnc12</smiles>
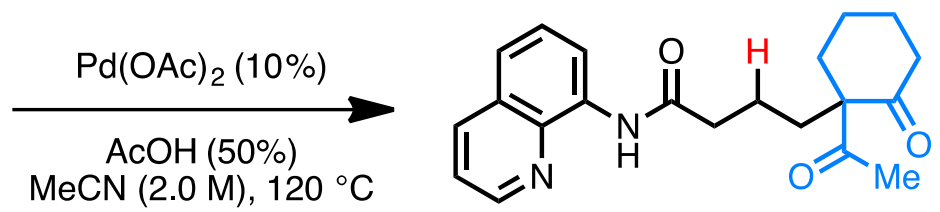

4-(1-acetyl-2-oxocyclohexyl)- $\boldsymbol{N}$-(quinolin-8-yl)butanamide (18): The reaction was carried out according to the general procedure using alkene $1(42.5 \mathrm{mg}, 0.2 \mathrm{mmol}), 2$-acetylcyclohexan-1-one (42.6 $\mathrm{mg}, 0.3 \mathrm{mmol})$, acetic acid $(6.0 \mathrm{mg}, 0.1 \mathrm{mmol}), \mathrm{Pd}(\mathrm{OAc})_{2}(4.4 \mathrm{mg}, 0.02 \mathrm{mmol})$, and $\mathrm{MeCN}(0.1 \mathrm{~mL})$. The reaction was run for $5 \mathrm{~h}$, and the product was purified by flash column chromatography $\left(\mathrm{SiO}_{2}, 40 \%\right.$ EtOAc in Hexanes) to afford $39.4 \mathrm{mg}$ (56\%) of 18 as a yellow oil. ${ }^{1} \mathrm{H}$ NMR $\left(600 \mathrm{MHz}, \mathrm{CDCl}_{3}\right) \delta 9.78(\mathrm{~s}, 1 \mathrm{H}), 8.81(\mathrm{dd}, J=4.2,1.7 \mathrm{~Hz}, 1 \mathrm{H}), 8.75(\mathrm{dd}, J=7.4,1.6 \mathrm{~Hz}, 1 \mathrm{H}), 8.16$ $(\mathrm{dd}, J=8.3,1.7 \mathrm{~Hz}, 1 \mathrm{H}), 7.56-7.48(\mathrm{~m}, 2 \mathrm{H}), 7.46(\mathrm{dd}, J=8.2,4.2 \mathrm{~Hz}, 1 \mathrm{H}), 2.59(\mathrm{td}, J=7.0,1.3 \mathrm{~Hz}, 2 \mathrm{H}), 2.55-2.45(\mathrm{~m}$, $2 \mathrm{H}), 2.38-2.23(\mathrm{~m}, 1 \mathrm{H}), 2.15(\mathrm{~s}, 3 \mathrm{H}), 2.02(\mathrm{ddd}, J=14.0,12.6,4.5 \mathrm{~Hz}, 1 \mathrm{H}), 2.01-1.95(\mathrm{~m}, 1 \mathrm{H}), 1.83-1.74(\mathrm{~m}, 2 \mathrm{H}), 1.75-$ $1.62(\mathrm{~m}, 3 \mathrm{H}), 1.65-1.48(\mathrm{~m}, 2 \mathrm{H}) .{ }^{13} \mathrm{C}$ NMR $\left(150 \mathrm{MHz}, \mathrm{CDCl}_{3}\right) \delta 210.31,207.03,171.12,148.37,138.53,136.59,134.65$, $128.16,127.62,121.84,121.66,116.66,67.87,41.87,38.09,34.36,33.66,27.42,26.52,22.41,20.11$. HRMS calcd. for $\mathrm{C}_{21} \mathrm{H}_{25} \mathrm{~N}_{2} \mathrm{O}_{3}+[\mathrm{M}+\mathrm{H}]^{+}:$353.18597, Found: 353.1859.
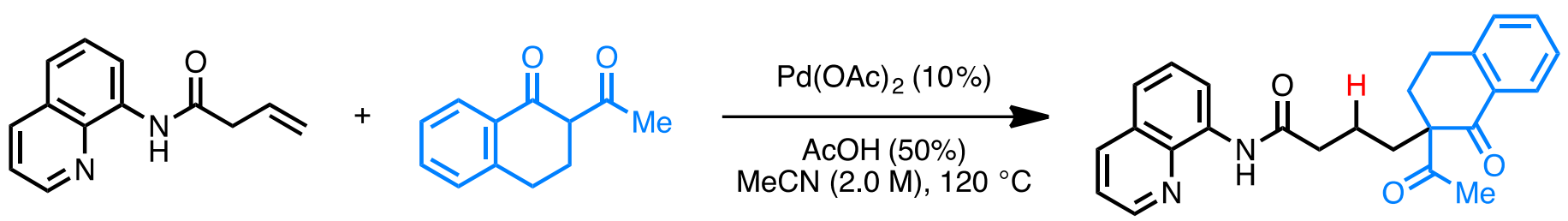

4-(2-acetyl-1-oxo-1,2,3,4-tetrahydronaphthalen-2-yl)- $\boldsymbol{N}$-(quinolin-8-yl)butanamide (19): The reaction was carried out according to the general procedure using alkene 1 (42.5 mg, $0.2 \mathrm{mmol}$ ), 2-acetyl-3,4-dihydronaphthalen$1(2 \mathrm{H})$-one (56.4 mg, $0.3 \mathrm{mmol}$ ), acetic acid (6.0 mg, $0.1 \mathrm{mmol}$ ), $\mathrm{Pd}(\mathrm{OAc})_{2}(4.4 \mathrm{mg}, 0.02 \mathrm{mmol})$, and $\mathrm{MeCN}(0.1 \mathrm{~mL})$. The reaction was run for $4 \mathrm{~h}$, and the product was purified by flash column chromatography $\left(\mathrm{SiO}_{2}, 33 \% \mathrm{EtOAc} \mathrm{in}\right.$ Hexanes) to afford $64.0 \mathrm{mg}(80 \%)$ of 19 as a yellow oil. ${ }^{1} \mathrm{H}$ NMR $\left(600 \mathrm{MHz}, \mathrm{CDCl}_{3}\right) \delta 9.79(\mathrm{~s}, 1 \mathrm{H}), 8.80(\mathrm{dd}, J=4.2,1.7$ $\mathrm{Hz}, 1 \mathrm{H}), 8.74(\mathrm{dd}, J=7.4,1.7 \mathrm{~Hz}, 1 \mathrm{H}), 8.16(\mathrm{dd}, J=8.2,1.7 \mathrm{~Hz}, 1 \mathrm{H}), 8.04(\mathrm{dd}, J=7.9,1.4 \mathrm{~Hz}, 1 \mathrm{H}), 7.55-7.44(\mathrm{~m}, 4 \mathrm{H})$, $7.30(\mathrm{t}, 1 \mathrm{H}), 7.21(\mathrm{~d}, J=7.6 \mathrm{~Hz}, 1 \mathrm{H}), 3.14-3.05(\mathrm{~m}, 1 \mathrm{H}), 2.94(\mathrm{dt}, J=17.5,5.0 \mathrm{~Hz}, 1 \mathrm{H}), 2.65(\mathrm{dt}, J=13.7,5.0 \mathrm{~Hz}, 1 \mathrm{H})$, $2.61(\mathrm{td}, J=7.3,2.8 \mathrm{~Hz}, 2 \mathrm{H}), 2.17(\mathrm{~s}, 3 \mathrm{H}), 2.15-2.00(\mathrm{~m}, 3 \mathrm{H}), 1.85-1.74(\mathrm{~m}, 2 \mathrm{H}) .{ }^{13} \mathrm{C}$ NMR $\left(150 \mathrm{MHz}, \mathrm{CDCl}_{3}\right) \delta 206.22$, 197.76, 171.08, 148.36, 143.89, 138.52, 136.58, 134.65, 134.06, 132.16, 129.12, 128.15, 128.13, 127.62, 126.95, 121.82, 121.66, 116.67, 64.05, 38.24, 33.87, 29.19, 27.24, 25.99, 20.60. HRMS calcd. for $\mathrm{C}_{25} \mathrm{H}_{25} \mathrm{~N}_{2} \mathrm{O}_{3}+[\mathrm{M}+\mathrm{H}]^{+}$: 401.18597, Found: 401.1859.
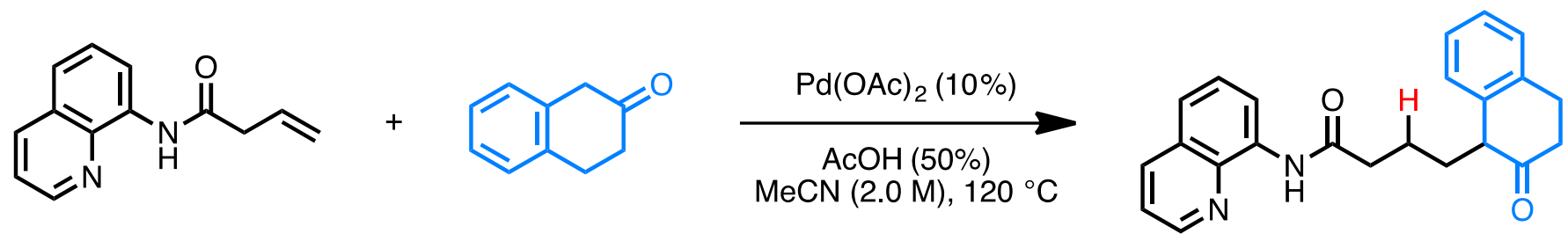

4-(2-oxo-1,2,3,4-tetrahydronaphthalen-1-yl)- $\boldsymbol{N}$-(quinolin-8-yl)butanamide (20): The reaction was carried out according to the general procedure using alkene $1(42.5 \mathrm{mg}, 0.2 \mathrm{mmol}), 3,4$-dihydronaphthalen-2(1H)-one $(43.8 \mathrm{mg}$, $0.3 \mathrm{mmol})$, acetic acid (6.0 mg, $0.1 \mathrm{mmol}), \mathrm{Pd}(\mathrm{OAc})_{2}(4.4 \mathrm{mg}, 0.02 \mathrm{mmol})$, and $\mathrm{MeCN}(0.1 \mathrm{~mL})$. The reaction was run for $4 \mathrm{~h}$, and the product was purified by flash column chromatography ( $\mathrm{SiO}_{2}, 40 \%$ EtOAc in Hexanes) to afford 36.3 mg (51\%) of 20 as a yellow oil. ${ }^{1} \mathbf{H}$ NMR $\left(600 \mathrm{MHz}^{\left.\mathrm{C} C \mathrm{CDl}_{3}\right)} \delta 9.77(\mathrm{~s}, 1 \mathrm{H}), 8.80(\mathrm{dd}, J=4.2,1.7 \mathrm{~Hz}, 1 \mathrm{H}), 8.75(\mathrm{dd}, J=\right.$ 7.4, $1.6 \mathrm{~Hz}, 1 \mathrm{H}), 8.16(\mathrm{dd}, J=8.3,1.7 \mathrm{~Hz}, 1 \mathrm{H}), 7.56-7.48(\mathrm{~m}, 2 \mathrm{H}), 7.45(\mathrm{dd}, J=8.2,4.2 \mathrm{~Hz}, 1 \mathrm{H}), 7.25-7.16(\mathrm{~m}, 4 \mathrm{H}), 3.49$ $(\mathrm{t}, J=6.8 \mathrm{~Hz}, 1 \mathrm{H}), 3.18(\mathrm{ddd}, J=15.4,9.5,5.7 \mathrm{~Hz}, 1 \mathrm{H}), 3.02(\mathrm{dt}, J=15.7,6.0 \mathrm{~Hz}, 1 \mathrm{H}), 2.66(\mathrm{dt}, J=17.3,5.6 \mathrm{~Hz}, 1 \mathrm{H})$, 
2.61-2.51 (m, 3H), 2.07-1.97 (m, 2H), 1.92-1.78 (m, 2H). ${ }^{13}$ C NMR (150 MHz, $\left.\mathrm{CDCl}_{3}\right) \delta 212.61,171.27,148.34$, 138.53, 137.22, 136.68, 136.58, 134.67, 128.28, 128.17, 128.15, 127.63, 127.23, 127.06, 121.81, 121.63, 116.64, 53.81, 38.11, 37.90, 31.49, 28.10, 23.36. HRMS calcd. for $\mathrm{C}_{23} \mathrm{H}_{23} \mathrm{~N}_{2} \mathrm{O}_{2}+[\mathrm{M}+\mathrm{H}]+:$ 359.17540, Found: 359.1753.
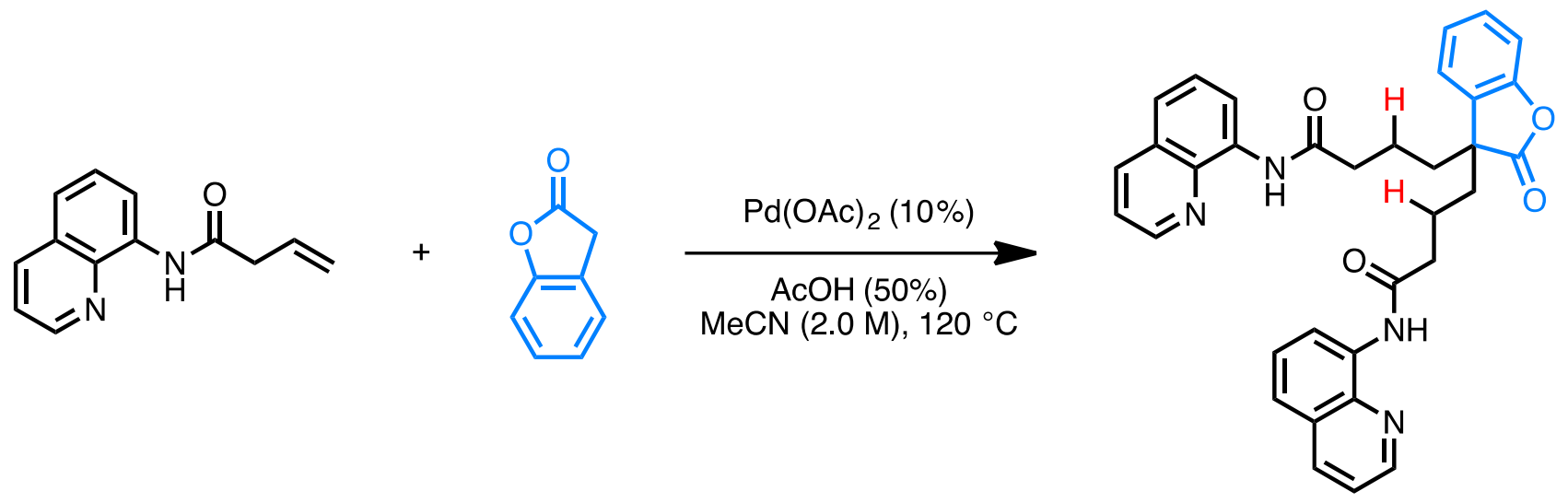

4,4'-(2-oxo-2,3-dihydrobenzofuran-3,3-diyl)bis( $N$-(quinolin-8-yl)butanamide) (21): The reaction was carried out according to the general procedure using alkene $1(42.5 \mathrm{mg}, 0.2 \mathrm{mmol})$, benzofuran-2(3H)-one $(40.2 \mathrm{mg}, 0.3$ mmol), acetic acid (6.0 mg, $0.1 \mathrm{mmol}), \mathrm{Pd}(\mathrm{OAc})_{2}(4.4 \mathrm{mg}, 0.02 \mathrm{mmol})$, and $\mathrm{MeCN}(0.1 \mathrm{~mL})$. The reaction was run for $4 \mathrm{~h}$, and the product was purified by flash column chromatography $\left(\mathrm{SiO}_{2}, 33 \%\right.$ EtOAc in Hexanes) to afford $95.0 \mathrm{mg}$ $(85 \%)$ of 21 as an off-white solid. ${ }^{1} \mathbf{H}$ NMR $\left(600 \mathrm{MHz}, \mathrm{CDCl}_{3}\right) \delta 9.70(\mathrm{~s}, 2 \mathrm{H}), 8.78(\mathrm{dd}, J=4.2,1.7 \mathrm{~Hz}, 2 \mathrm{H}), 8.70(\mathrm{dd}, J=$ $7.1,1.9 \mathrm{~Hz}, 2 \mathrm{H}), 8.15(\mathrm{dd}, J=8.2,1.7 \mathrm{~Hz}, 2 \mathrm{H}), 7.55-7.46(\mathrm{~m}, 4 \mathrm{H}), 7.44(\mathrm{dd}, J=8.2,4.2 \mathrm{~Hz}, 2 \mathrm{H}), 7.33-7.22(\mathrm{~m}, 2 \mathrm{H}), 7.20-$ $7.10(\mathrm{~m}, 2 \mathrm{H}), 2.57-2.48(\mathrm{~m}, 2 \mathrm{H}), 2.48-2.41(\mathrm{~m}, 2 \mathrm{H}), 2.13-2.01(\mathrm{~m}, 4 \mathrm{H}), 1.64-1.50(\mathrm{~m}, 4 \mathrm{H}) .{ }^{13} \mathrm{C} \mathrm{NMR}\left(150 \mathrm{MHz}, \mathrm{CDCl}_{3}\right)$ $\delta 180.02,170.75(\times 2), 153.46,148.34(\times 2), 138.49(\times 2), 136.55(\times 2), 134.55(\times 2), 129.74,129.07,128.12(\times 2)$, $127.59(\times 2), 124.87,123.77,121.82(\times 2), 121.68(\times 2), 116.63(\times 2), 111.06,52.45,37.91(\times 2), 37.77(\times 2), 20.86(\times 2)$. HRMS calcd. for $\mathrm{C}_{34} \mathrm{H}_{31} \mathrm{~N}_{4} \mathrm{O}_{4}+[\mathrm{M}+\mathrm{H}]+:$ 559.23398, Found: 559.2338.
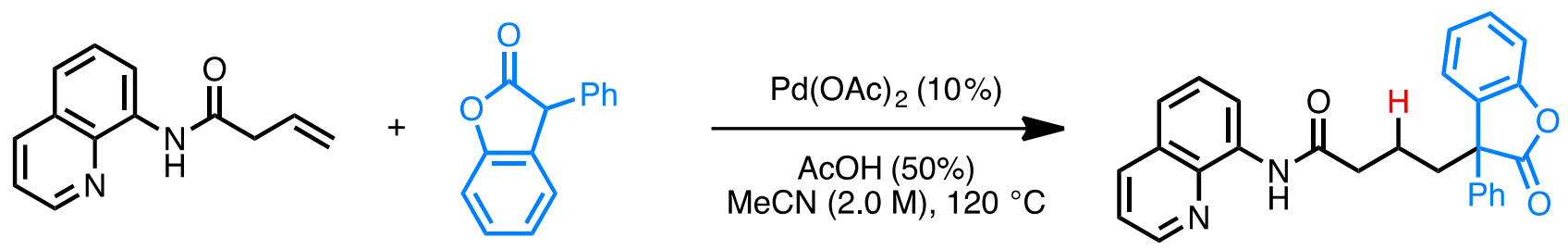

4-(2-oxo-3-phenyl-2,3-dihydrobenzofuran-3-yl)- $\boldsymbol{N}$-(quinolin-8-yl)butanamide (22): The reaction was carried out according to the general procedure using alkene $\mathbf{1}(42.5 \mathrm{mg}, 0.2 \mathrm{mmol})$, 3-phenylbenzofuran-2(3H)-one $(63.0$ $\mathrm{mg}, 0.3 \mathrm{mmol})$, acetic acid ( $6.0 \mathrm{mg}, 0.1 \mathrm{mmol}), \mathrm{Pd}(\mathrm{OAc})_{2}(4.4 \mathrm{mg}, 0.02 \mathrm{mmol})$, and $\mathrm{MeCN}(0.1 \mathrm{~mL})$. The reaction was run for $4 \mathrm{~h}$, and the product was purified by flash column chromatography ( $\mathrm{SiO}_{2}, 25 \%$ EtOAc in Hexanes) to afford $68.6 \mathrm{mg}(82 \%)$ of 22 as an orange oil. $1 \mathbf{H}$ NMR $\left(600 \mathrm{MHz}, \mathrm{CDCl}_{3}\right) \delta 9.73(\mathrm{~s}, 1 \mathrm{H}), 8.79(\mathrm{dd}, J=4.2,1.7 \mathrm{~Hz}, 1 \mathrm{H}), 8.71(\mathrm{dd}$, $J=7.1,1.9 \mathrm{~Hz}, 1 \mathrm{H}), 8.15(\mathrm{dd}, J=8.2,1.7 \mathrm{~Hz}, 1 \mathrm{H}), 7.54-7.48(\mathrm{~m}, 2 \mathrm{H}), 7.45(\mathrm{dd}, J=8.2,4.2 \mathrm{~Hz}, 1 \mathrm{H}), 7.43-7.39(\mathrm{~m}, 2 \mathrm{H})$, 7.39-7.30 (m, 4H), 7.29-7.26 (m, 1H), $7.23(\mathrm{td}, J=7.6,1.1 \mathrm{~Hz}, 1 \mathrm{H}), 7.19(\mathrm{~d}, J=8.1 \mathrm{~Hz}, 1 \mathrm{H}), 2.62-2.56(\mathrm{~m}, 1 \mathrm{H}), 2.56-$ $2.49(\mathrm{~m}, 2 \mathrm{H}), 2.45$ (ddd, $J=13.5,12.3,4.6 \mathrm{~Hz}, 1 \mathrm{H}), 1.75-1.66(\mathrm{~m}, 1 \mathrm{H}), 1.66-1.58(\mathrm{~m}, 1 \mathrm{H}) .{ }^{13} \mathbf{C}$ NMR $\left(150 \mathrm{MHz} \mathrm{CDCl}_{3}\right)$ $\delta 178.27,170.68,153.45,148.35,138.77,138.49,136.58,134.54,130.01,129.42,129.05(\times 2), 128.13(\times 2), 127.59$, $126.91(\times 2), 125.61,124.77,121.84,121.72,116.64,111.33,55.85,38.44,37.74,21.24$. HRMS calcd. for $\mathrm{C}_{27} \mathrm{H}_{23} \mathrm{~N}_{2} \mathrm{O}_{3}+$ $[\mathrm{M}+\mathrm{H}]^{+}:$423.17032, Found: 423.1704.
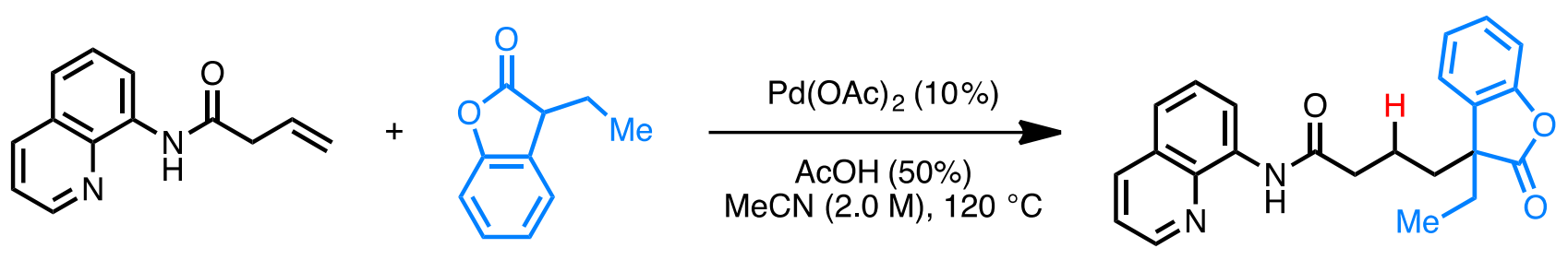

4-(3-ethyl-2-oxo-2,3-dihydrobenzofuran-3-yl)- $\mathbf{N}$-(quinolin-8-yl)butanamide (23): The reaction was carried out according to the general procedure using alkene $\mathbf{1}$ (42.5 mg, $0.2 \mathrm{mmol})$, 3-ethylbenzofuran-2(3H)-one (48.6 mg, 
$0.3 \mathrm{mmol})$, acetic acid (6.0 mg, $0.1 \mathrm{mmol}), \mathrm{Pd}(\mathrm{OAc})_{2}(4.4 \mathrm{mg}, 0.02 \mathrm{mmol})$, and $\mathrm{MeCN}(0.1 \mathrm{~mL})$. The reaction was run for $4 \mathrm{~h}$, and the product was purified by flash column chromatography $\left(\mathrm{SiO}_{2}, 20-25 \%\right.$ EtOAc in Hexanes) to afford $62.5 \mathrm{mg}(83 \%)$ of $\mathbf{2 3}$ as a white solid. ${ }^{1} \mathrm{H}$ NMR $\left(600 \mathrm{MHz}, \mathrm{CDCl}_{3}\right) \delta 9.71(\mathrm{~s}, 1 \mathrm{H}), 8.79(\mathrm{dd}, J=4.2,1.7 \mathrm{~Hz}, 1 \mathrm{H}), 8.71(\mathrm{dd}$, $J=7.2,1.8 \mathrm{~Hz}, 1 \mathrm{H}), 8.15(\mathrm{dd}, J=8.2,1.7 \mathrm{~Hz}, 1 \mathrm{H}), 7.54-7.46(\mathrm{~m}, 2 \mathrm{H}), 7.45(\mathrm{dd}, J=8.2,4.2 \mathrm{~Hz}, 1 \mathrm{H}), 7.29$ (ddd, $J=8.0$, $7.4,1.6 \mathrm{~Hz}, 1 \mathrm{H}), 7.22-7.14(\mathrm{~m}, 2 \mathrm{H}), 7.12(\mathrm{~d}, J=8.0 \mathrm{~Hz}, 1 \mathrm{H}), 2.56-2.41(\mathrm{~m}, 2 \mathrm{H}), 2.12-1.97(\mathrm{~m}, 3 \mathrm{H}), 1.93-1.83(\mathrm{~m}, 1 \mathrm{H})$, $1.64-1.51(\mathrm{~m}, 2 \mathrm{H}), 0.69(\mathrm{t}, J=7.4 \mathrm{~Hz}, 3 \mathrm{H}) .{ }^{13} \mathrm{C}$ NMR $\left(150 \mathrm{MHz}, \mathrm{CDCl}_{3}\right) \delta 180.10,170.82,153.62,148.35,138.48$, 136.56, 134.56, 129.93, 128.88, 128.13, 127.58, 124.63, 123.58, 121.83, 121.68, 116.61, 110.89, 53.25, 37.82, 37.54, 31.79, 21.01, 9.03. HRMS calcd. for $\mathrm{C}_{23} \mathrm{H}_{23} \mathrm{~N}_{2} \mathrm{O}_{3}+[\mathrm{M}+\mathrm{H}]+:$ 375.17032, Found: 375.1704 .
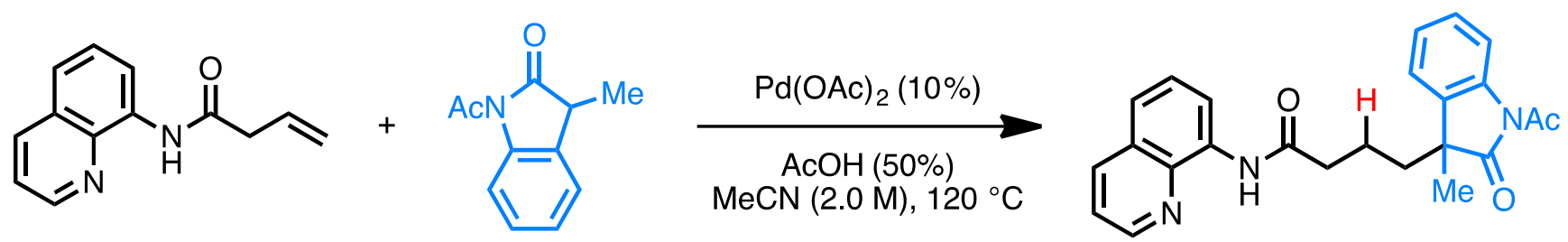

4-(1-acetyl-3-methyl-2-oxoindolin-3-yl)- $N$-(quinolin-8-yl)butanamide (24): The reaction was carried out according to the general procedure using alkene 1 ( $42.5 \mathrm{mg}, 0.2 \mathrm{mmol}), 1$-acetyl-3-methylindolin-2-one (56.0 mg, $0.3 \mathrm{mmol}$ ), acetic acid (6.0 mg, $0.1 \mathrm{mmol}), \mathrm{Pd}(\mathrm{OAc})_{2}(4.4 \mathrm{mg}, 0.02 \mathrm{mmol})$, and $\mathrm{MeCN}(0.1 \mathrm{~mL})$. The reaction was run for $4 \mathrm{~h}$, and the product was purified by flash column chromatography $\left(\mathrm{SiO}_{2}, 25-30 \%\right.$ EtOAc in Hexanes) to afford $66.8 \mathrm{mg}(83 \%)$ of 24 as a colorless oil. ${ }^{1} \mathrm{H}$ NMR $\left(600 \mathrm{MHz}, \mathrm{CDCl}_{3}\right) \delta 9.70(\mathrm{~s}, 1 \mathrm{H}), 8.78(\mathrm{dd}, J=4.2,1.7 \mathrm{~Hz}, 1 \mathrm{H}), 8.71$ $(\mathrm{dd}, J=7.2,1.8 \mathrm{~Hz}, 1 \mathrm{H}), 8.24(\mathrm{~d}, J=8.2 \mathrm{~Hz}, 1 \mathrm{H}), 8.16(\mathrm{dd}, J=8.3,1.7 \mathrm{~Hz}, 1 \mathrm{H}), 7.55-7.47(\mathrm{~m}, 2 \mathrm{H}), 7.45(\mathrm{dd}, J=8.3,4.2$ $\mathrm{Hz}, 1 \mathrm{H}$ ), 7.30 (ddd, $J=8.1,7.3,1.6 \mathrm{~Hz}, 1 \mathrm{H}), 7.25-7.18(\mathrm{~m}, 2 \mathrm{H}), 2.70(\mathrm{~s}, 3 \mathrm{H}), 2.53-2.40$ (m, 2H), 2.09 (ddd, $J=13.4$, 12.0, $4.9 \mathrm{~Hz}, 1 \mathrm{H}), 1.96$ (ddd, $J=13.4,12.2,4.9 \mathrm{~Hz}, 1 \mathrm{H}), 1.59-1.44(\mathrm{~m}, 2 \mathrm{H}), 1.45(\mathrm{~s}, 3 \mathrm{H}) .{ }^{13} \mathbf{C ~ N M R}\left(150 \mathrm{MHz}, \mathrm{CDCl}_{3}\right) \delta$ 181.72, 171.20, 170.85, 148.32, 139.65, 138.49, 136.60, 134.54, 132.86, 128.47, 128.14, 127.62, 125.63, 122.58, 121.83, 121.70, 116.85, 116.64, 49.07, 38.86, 37.93, 26.98, 25.11, 20.96. HRMS calcd. for $\mathrm{C}_{24} \mathrm{H}_{24} \mathrm{~N}_{3} \mathrm{O}_{3}+[\mathrm{M}+\mathrm{H}]+$ : 402.18122, Found: 402.1810.

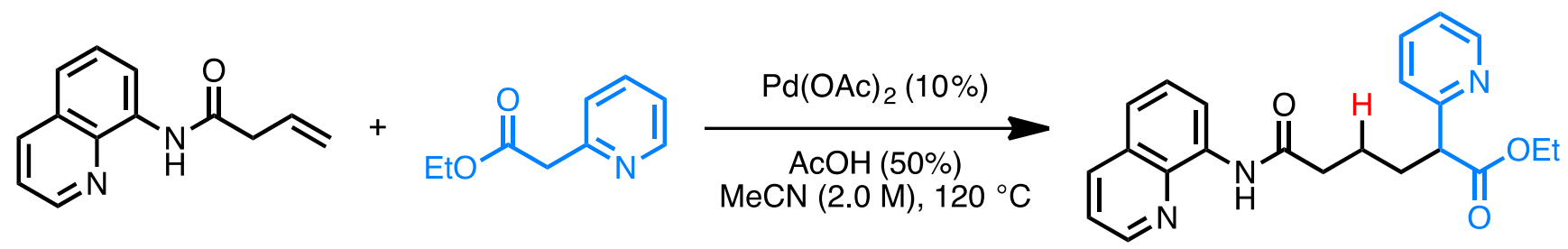

Ethyl-6-oxo-2-(pyridin-2-yl)-6-(quinolin-8-ylamino)hexanoate (25): The reaction was carried out according to the general procedure using alkene $1(42.5 \mathrm{mg}, 0.2 \mathrm{mmol})$, ethyl-2-(pyridin-2-yl)acetate $(49.6 \mathrm{mg}, 0.3 \mathrm{mmol})$, acetic acid (6.0 mg, $0.1 \mathrm{mmol}), \mathrm{Pd}(\mathrm{OAc})_{2}(4.4 \mathrm{mg}, 0.02 \mathrm{mmol})$, and $\mathrm{MeCN}(0.1 \mathrm{~mL})$. The reaction was run for $4 \mathrm{~h}$, and the product was purified by flash column chromatography $\left(\mathrm{SiO}_{2}, 40 \%\right.$ EtOAc in Hexanes) to afford $22.6 \mathrm{mg}(30 \%)$ of 25 as a brown oil. ${ }^{1} \mathbf{H}$ NMR $\left(600 \mathrm{MHz}, \mathrm{CDCl}_{3}\right) \delta 9.78(\mathrm{~s}, 1 \mathrm{H}), 8.80(\mathrm{dd}, J=4.2,1.7 \mathrm{~Hz}, 1 \mathrm{H}), 8.75(\mathrm{dd}, J=7.4,1.5 \mathrm{~Hz}, 1 \mathrm{H})$, 8.56 (ddd, $J=4.9,1.9,0.9 \mathrm{~Hz}, 1 \mathrm{H}), 8.15(\mathrm{dd}, J=8.3,1.7 \mathrm{~Hz}, 1 \mathrm{H}), 7.64(\mathrm{td}, J=7.7,1.9 \mathrm{~Hz}, 1 \mathrm{H}), 7.54-7.47(\mathrm{~m}, 2 \mathrm{H}), 7.45$ $(\mathrm{dd}, J=8.2,4.2 \mathrm{~Hz}, 1 \mathrm{H}), 7.33(\mathrm{dt}, J=7.8,1.1 \mathrm{~Hz}, 1 \mathrm{H}), 7.17(\mathrm{ddd}, J=7.5,4.8,1.1 \mathrm{~Hz}, 1 \mathrm{H}), 4.22-4.16(\mathrm{~m}, 1 \mathrm{H}), 4.15-4.10$ (m, 1H), $3.87(\mathrm{t}, J=7.6 \mathrm{~Hz}, 1 \mathrm{H}), 2.66-2.53(\mathrm{~m}, 2 \mathrm{H}), 2.31-2.20(\mathrm{~m}, 1 \mathrm{H}), 2.14-2.05(\mathrm{~m}, 1 \mathrm{H}), 1.90-1.72(\mathrm{~m}, 2 \mathrm{H}), 1.21(\mathrm{t}$, $J=7.1 \mathrm{~Hz}, 3 \mathrm{H}) .{ }^{13} \mathrm{C}$ NMR $\left(150 \mathrm{MHz}, \mathrm{CDCl}_{3}\right) \delta 173.06,171.37,158.72,149.71,148.33,138.52,136.93,136.56,134.68$, $128.14,127.62,122.89,122.41,121.79,121.60,116.63,61.17,54.03,38.00,31.94,23.75,14.37$. HRMS calcd. for $\mathrm{C}_{22} \mathrm{H}_{24} \mathrm{~N}_{3} \mathrm{O}_{3}+[\mathrm{M}+\mathrm{H}]^{+}:$378.18122, Found: 378.1813.

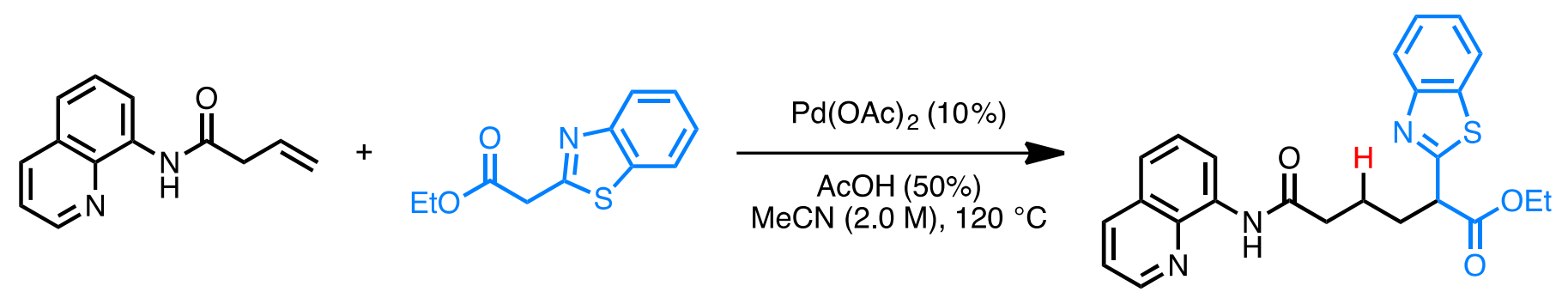


Ethyl-2-(benzo[d]thiazol-2-yl)-6-oxo-6-(quinolin-8-ylamino)hexanoate (26): The reaction was carried out according to the general procedure using alkene $1(42.5 \mathrm{mg}, 0.2 \mathrm{mmol})$, ethyl-2-(benzo[d] thiazol-2-yl)acetate $(66.4$ $\mathrm{mg}, 0.3 \mathrm{mmol})$, acetic acid (6.0 mg, $0.1 \mathrm{mmol}), \mathrm{Pd}(\mathrm{OAc})_{2}(4.4 \mathrm{mg}, 0.02 \mathrm{mmol})$, and $\mathrm{MeCN}(0.1 \mathrm{~mL})$. The reaction was run for $4 \mathrm{~h}$, and the product was purified by flash column chromatography ( $\mathrm{SiO}_{2}, 35 \%$ EtOAc in Hexanes) to afford $46.9 \mathrm{mg}(54 \%)$ of $\mathbf{2 6}$ as a yellow oil. ${ }^{1} \mathrm{H}$ NMR $\left(600 \mathrm{MHz}, \mathrm{CDCl}_{3}\right) \delta 9.78(\mathrm{~s}, 1 \mathrm{H}), 8.77(\mathrm{dd}, J=4.2,1.7 \mathrm{~Hz}, 1 \mathrm{H}), 8.75(\mathrm{dd}$, $J=7.4,1.5 \mathrm{~Hz}, 1 \mathrm{H}), 8.15(\mathrm{dd}, J=8.2,1.7 \mathrm{~Hz}, 1 \mathrm{H}), 8.01(\mathrm{~d}, J=8.0 \mathrm{~Hz}, 1 \mathrm{H}), 7.85(\mathrm{~d}, J=7.9 \mathrm{~Hz}, 1 \mathrm{H}), 7.55-7.42(\mathrm{~m}, 4 \mathrm{H})$, 7.37 (ddd, $J=8.2,7.2,1.2 \mathrm{~Hz}, 1 \mathrm{H}), 4.30-4.17(\mathrm{~m}, 3 \mathrm{H}), 2.68-2.58(\mathrm{~m}, 2 \mathrm{H}), 2.43-2.34(\mathrm{~m}, 1 \mathrm{H}), 2.30-2.20(\mathrm{~m}, 1 \mathrm{H}), 1.97-$ $1.87(\mathrm{~m}, 2 \mathrm{H}), 1.26(\mathrm{t}, J=7.1 \mathrm{~Hz}, 3 \mathrm{H}) .{ }^{13} \mathrm{C}$ NMR $\left(150 \mathrm{MHz}, \mathrm{CDCl}_{3}\right) \delta 171.24,170.93,168.13,152.86,148.32,138.48$, 136.54, 135.57, 134.61, 128.11, 127.60, 126.22, 125.36, 123.36, 121.79, 121.77, 121.65, 116.63, 61.95, 51.12, 37.64, 33.01, 23.51, 14.32. HRMS calcd. for $\mathrm{C}_{24} \mathrm{H}_{24} \mathrm{~N}_{3} \mathrm{O}_{3} \mathrm{~S}+[\mathrm{M}+\mathrm{H}]+:$ : 434.15329, Found: 434.1533.

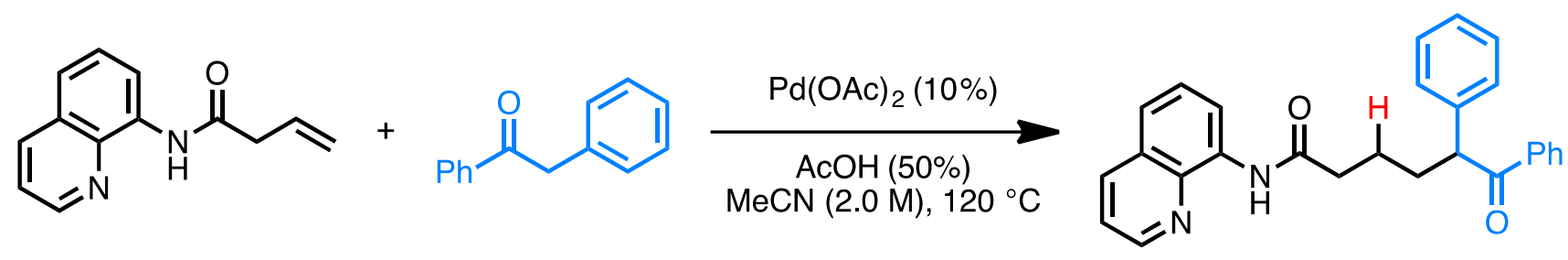

6-oxo-5,6-diphenyl- $\boldsymbol{N}$-(quinolin-8-yl)hexanamide (27): The reaction was carried out according to the general procedure using alkene 1 ( $42.5 \mathrm{mg}, 0.2 \mathrm{mmol}$ ), 1,2-diphenylethan-1-one (58.8 $\mathrm{mg}, 0.3 \mathrm{mmol}$ ), acetic acid (6.0 $\mathrm{mg}, 0.1$ $\mathrm{mmol}), \mathrm{Pd}(\mathrm{OAc})_{2}(4.4 \mathrm{mg}, 0.02 \mathrm{mmol})$, and $\mathrm{MeCN}(0.1 \mathrm{~mL})$. The reaction was run for $4 \mathrm{~h}$, and the product was purified by preparative TLC (15\% EtOAc in Hexanes) to afford $10.6 \mathrm{mg}(13 \%)$ of $\mathbf{2 7}$ as a pale yellow oil. ${ }^{\mathbf{H}} \mathbf{H} \mathbf{~ N M R}(600 \mathrm{MHz}$, $\left.\mathrm{CDCl}_{3}\right) \delta 9.77(\mathrm{~s}, 1 \mathrm{H}), 8.79(\mathrm{dd}, J=4.2,1.7 \mathrm{~Hz}, 1 \mathrm{H}), 8.74(\mathrm{dd}, J=7.4,1.6 \mathrm{~Hz}, 1 \mathrm{H}), 8.15(\mathrm{dd}, J=8.2,1.7 \mathrm{~Hz}, 1 \mathrm{H}), 7.98-$ $7.93(\mathrm{~m}, 2 \mathrm{H}), 7.56-7.42(\mathrm{~m}, 4 \mathrm{H}), 7.37(\mathrm{t}, J=7.8 \mathrm{~Hz}, 2 \mathrm{H}), 7.34-7.31(\mathrm{~m}, 2 \mathrm{H}), 7.28(\mathrm{dd}, J=8.5,6.8 \mathrm{~Hz}, 2 \mathrm{H}), 7.22-7.16$ $(\mathrm{m}, 1 \mathrm{H}), 4.63(\mathrm{t}, J=7.2 \mathrm{~Hz}, 1 \mathrm{H}), 2.65-2.51(\mathrm{~m}, 2 \mathrm{H}), 2.35-2.27(\mathrm{~m}, 1 \mathrm{H}), 2.04-1.94(\mathrm{~m}, 1 \mathrm{H}), 1.90-1.81(\mathrm{~m}, 1 \mathrm{H}), 1.81-$ $1.74(\mathrm{~m}, 1 \mathrm{H}) .{ }^{13} \mathrm{C}$ NMR $\left(150 \mathrm{MHz}, \mathrm{CDCl}_{3}\right) \delta 199.90,171.52,148.32,139.56,138.52,136.97,136.56,134.67,133.06$, $129.17(\times 2), 128.90(\times 2), 128.70(\times 2), 128.45(\times 2), 128.13,127.61,127.32,121.79,121.60,116.64,53.85,38.28$, 33.71, 24.00. HRMS calcd. for $\mathrm{C}_{27} \mathrm{H}_{25} \mathrm{~N}_{2} \mathrm{O}_{2}+[\mathrm{M}+\mathrm{H}]^{+}:$409.19105, Found: 409.1912.<smiles>C=CCC(=O)Nc1cccc2cccnc12</smiles>
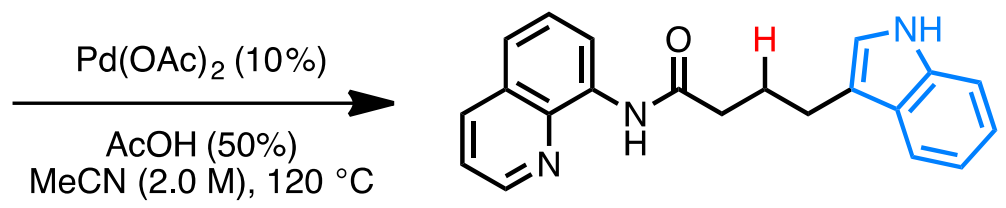

4-(1H-indol-3-yl)- $\mathbf{N}$-(quinolin-8-yl)butanamide (29): The reaction was carried out according to the general procedure using alkene 1 ( $42.5 \mathrm{mg}, 0.2 \mathrm{mmol}$ ), indole (35.2 $\mathrm{mg}, 0.3 \mathrm{mmol}$ ), acetic acid ( $6.0 \mathrm{mg}, 0.1 \mathrm{mmol}), \mathrm{Pd}(\mathrm{OAc})_{2}$ ( $4.4 \mathrm{mg}, 0.02 \mathrm{mmol}$ ), and MeCN $(0.1 \mathrm{~mL})$. The reaction was run for $4 \mathrm{~h}$, and the product was purified by flash column chromatography $\left(\mathrm{SiO}_{2}, 30 \%\right.$ EtOAc in Hexanes) to afford $55.0 \mathrm{mg}(83 \%)$ of $\mathbf{2 9}$ as an off-white solid. ${ }^{\mathbf{1}} \mathbf{H}$ NMR (600 $\left.\mathrm{MHz}_{\mathrm{CDCl}}\right) \delta 9.79(\mathrm{~s}, 1 \mathrm{H}), 8.83-8.77(\mathrm{~m}, 2 \mathrm{H}), 8.16(\mathrm{dd}, J=8.3,1.7 \mathrm{~Hz}, 1 \mathrm{H}), 7.96(\mathrm{~s}, 1 \mathrm{H}), 7.66(\mathrm{dq}, J=7.9,0.9 \mathrm{~Hz}, 1 \mathrm{H})$, 7.57-7.52 (m, 1H), 7.50 (dd, $J=8.2,1.4 \mathrm{~Hz}, 1 \mathrm{H}), 7.45$ (dd, $J=8.2,4.2 \mathrm{~Hz}, 1 \mathrm{H}), 7.36(\mathrm{dt}, J=8.2,0.9 \mathrm{~Hz}, 1 \mathrm{H}), 7.19$ (ddd, $J=8.1,7.0,1.2 \mathrm{~Hz}, 1 \mathrm{H}), 7.11(\mathrm{ddd}, J=8.0,7.0,1.0 \mathrm{~Hz}, 1 \mathrm{H}), 7.07(\mathrm{dd}, J=2.2,1.1 \mathrm{~Hz}, 1 \mathrm{H}), 2.93(\mathrm{td}, J=7.4,1.0 \mathrm{~Hz}, 2 \mathrm{H}$ ), $2.64(\mathrm{t}, J=7.4 \mathrm{~Hz}, 2 \mathrm{H}), 2.26(\mathrm{p}, J=7.5 \mathrm{~Hz}, 2 \mathrm{H}) .{ }^{13} \mathbf{C} \mathbf{N M R}\left(150 \mathrm{MHz}, \mathrm{CDCl}_{3}\right) \delta 171.94,148.30,138.57,136.60,136.57$, $134.79,128.16,127.73,127.68,122.18,121.78$ (×2), 121.55, 119.46, 119.23, 116.65, 115.99, 111.26, 37.85, 26.12, 24.79. HRMS calcd. for $\mathrm{C}_{21} \mathrm{H}_{20} \mathrm{~N}_{3} \mathrm{O}+[\mathrm{M}+\mathrm{H}]+: 330.16009$, Found: 330.1602 .
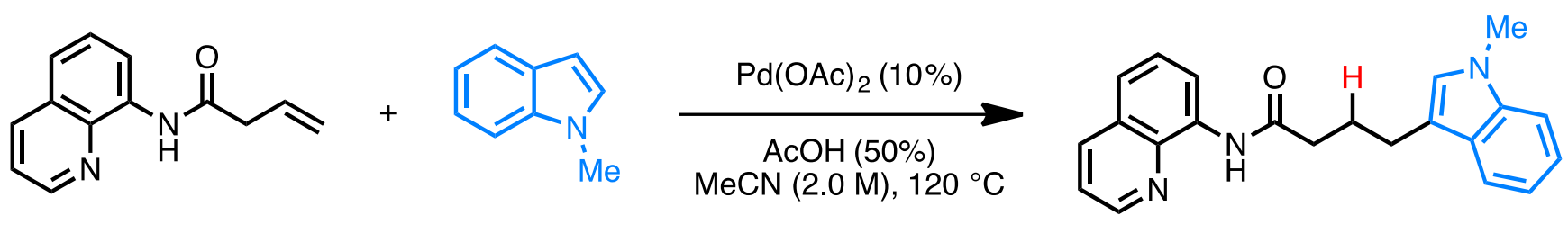

4-(1-methyl-1H-indol-3-yl)- $\mathbf{N}$-(quinolin-8-yl)butanamide (30): The reaction was carried out according to the general procedure using alkene 1 ( $42.5 \mathrm{mg}, 0.2 \mathrm{mmol}), 1$-methylindole (39.3 mg, $0.3 \mathrm{mmol}$ ), acetic acid (6.0 mg, 0.1 $\mathrm{mmol}), \mathrm{Pd}(\mathrm{OAc})_{2}(4.4 \mathrm{mg}, 0.02 \mathrm{mmol})$, and $\mathrm{MeCN}(0.1 \mathrm{~mL})$. The reaction was run for $4 \mathrm{~h}$, and the product was purified by flash column chromatography $\left(\mathrm{SiO}_{2}, 30-40 \%\right.$ EtOAc in Hexanes) to afford $61.6 \mathrm{mg}(90 \%)$ of 30 as a yellow oil. ${ }^{\mathbf{1}} \mathbf{H}$ 
NMR $\left(600 \mathrm{MHz}, \mathrm{CDCl}_{3}\right) \delta 9.78(\mathrm{~s}, 1 \mathrm{H}), 8.83-8.78(\mathrm{~m}, 2 \mathrm{H}), 8.16(\mathrm{dd}, J=8.2,1.6 \mathrm{~Hz}, 1 \mathrm{H}), 7.64(\mathrm{~d}, J=7.9 \mathrm{~Hz}, 1 \mathrm{H}), 7.54(\mathrm{t}$, $J=7.9 \mathrm{~Hz}, 1 \mathrm{H}), 7.50(\mathrm{dd}, J=8.2,1.4 \mathrm{~Hz}, 1 \mathrm{H}), 7.45(\mathrm{dd}, J=8.2,4.2 \mathrm{~Hz}, 1 \mathrm{H}), 7.28(\mathrm{~d}, J=8.2 \mathrm{~Hz}, 1 \mathrm{H}), 7.21$ (ddd, $J=8.1$, 6.9, $1.1 \mathrm{~Hz}, 1 \mathrm{H}), 7.09$ (ddd, $J=8.0,6.9,1.0 \mathrm{~Hz}, 1 \mathrm{H}), 6.92(\mathrm{~s}, 1 \mathrm{H}), 3.74(\mathrm{~s}, 3 \mathrm{H}), 2.92(\mathrm{t}, J=7.3 \mathrm{~Hz}, 2 \mathrm{H}), 2.62(\mathrm{t}, J=7.4 \mathrm{~Hz}$, $2 \mathrm{H}), 2.24(\mathrm{p}, J=7.4 \mathrm{~Hz}, 2 \mathrm{H}) .{ }^{13} \mathrm{C}$ NMR $\left(150 \mathrm{MHz}, \mathrm{CDCl}_{3}\right) \delta 172.00,148.28,138.57,137.31,136.58,134.80,128.17$, 128.11, 127.69, 126.77, 121.77, 121.69, 121.53, 119.31, 118.84, 116.64, 114.31, 109.32, 37.83, 32.81, 26.34, 24.67. HRMS calcd. for $\mathrm{C}_{22} \mathrm{H}_{22} \mathrm{~N}_{3} \mathrm{O}_{3}+[\mathrm{M}+\mathrm{H}]+: 344.17574$, Found: 344.1757.

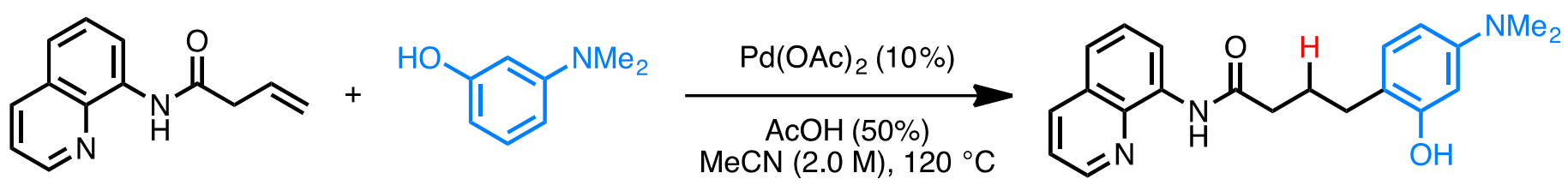

4-(4-(dimethylamino)-2-hydroxyphenyl)- $\boldsymbol{N}$-(quinolin-8-yl)butanamide (31): The reaction was carried out according to the general procedure using alkene $1(42.5 \mathrm{mg}, 0.2 \mathrm{mmol}), 3$-(dimethylamino)phenol (41.1 $\mathrm{mg}, 0.3$ mmol), acetic acid (6.0 mg, $0.1 \mathrm{mmol}), \mathrm{Pd}(\mathrm{OAc})_{2}(4.4 \mathrm{mg}, 0.02 \mathrm{mmol})$, and $\mathrm{MeCN}(0.1 \mathrm{~mL})$. The reaction was run for $4 \mathrm{~h}$, and the product was purified by flash column chromatography $\left(\mathrm{SiO}_{2}, 35 \%\right.$ EtOAc in Hexanes) to afford $37.7 \mathrm{mg}$ $(54 \%)$ of 31 as a brown solid. ${ }^{1} \mathbf{H}$ NMR $\left(600 \mathrm{MHz} \mathrm{CDCl}_{3}\right) \delta 9.91(\mathrm{~s}, 1 \mathrm{H}), 8.83(\mathrm{dd}, J=7.2,1.8 \mathrm{~Hz}, 1 \mathrm{H}), 8.81(\mathrm{dd}, J=4.2$, $1.7 \mathrm{~Hz}, 1 \mathrm{H}), 8.18(\mathrm{dd}, J=8.3,1.7 \mathrm{~Hz}, 1 \mathrm{H}), 7.95(\mathrm{~s}, 1 \mathrm{H}), 7.59-7.51(\mathrm{~m}, 2 \mathrm{H}), 7.47(\mathrm{dd}, J=8.2,4.2 \mathrm{~Hz}, 1 \mathrm{H}), 6.96(\mathrm{~d}, J=8.3$ $\mathrm{Hz}, 1 \mathrm{H}), 6.37(\mathrm{~d}, J=2.5 \mathrm{~Hz}, 1 \mathrm{H}), 6.29(\mathrm{dd}, J=8.3,2.6 \mathrm{~Hz}, 1 \mathrm{H}), 2.91(\mathrm{~s}, 6 \mathrm{H}), 2.71(\mathrm{t}, J=7.3 \mathrm{~Hz}, 2 \mathrm{H}), 2.65-2.60(\mathrm{~m}, 2 \mathrm{H})$, 2.05-1.99 (m, 2H). ${ }^{13} \mathrm{C}$ NMR $\left(150 \mathrm{MHz}, \mathrm{CDCl}_{3}\right) \delta 173.29,156.15,151.24,148.45,138.55,136.68,134.27,130.72$, $128.16,127.67,122.12,121.89,117.31,115.62,105.37,101.54,41.00(\times 2), 35.62,28.57,26.71$. HRMS calcd. for $\mathrm{C}_{21} \mathrm{H}_{24} \mathrm{~N}_{3} \mathrm{O}_{2}+[\mathrm{M}+\mathrm{H}]+:$ 350.18630, Found: 350.1862 .

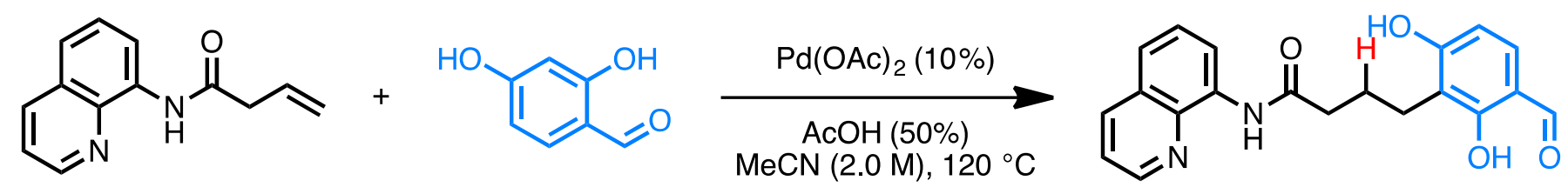

4-(3-formyl-2,6-dihydroxyphenyl)- $\mathbf{N}$-(quinolin-8-yl)butanamide (32): The reaction was carried out according to the general procedure using alkene 1 ( $42.5 \mathrm{mg}, 0.2 \mathrm{mmol}$ ), 2,4-dihydroxybenzaldehyde ( $41.4 \mathrm{mg}, 0.3 \mathrm{mmol}$ ), acetic acid $(6.0 \mathrm{mg}, 0.1 \mathrm{mmol}), \mathrm{Pd}(\mathrm{OAc})_{2}(4.4 \mathrm{mg}, 0.02 \mathrm{mmol})$, and $\mathrm{MeCN}(0.1 \mathrm{~mL})$. The reaction was run for $4 \mathrm{~h}$, and the product was purified by flash column chromatography $\left(\mathrm{SiO}_{2}, 35 \%\right.$ EtOAc in Hexanes) to afford $42.2 \mathrm{mg}(60 \%)$ of 32 as a white solid. ${ }^{1} \mathrm{H}$ NMR $\left(600 \mathrm{MHz}, \mathrm{CDCl}_{3}\right) \delta 11.81(\mathrm{~s}, 1 \mathrm{H}), 10.28(\mathrm{~s}, 1 \mathrm{H}), 10.04(\mathrm{~s}, 1 \mathrm{H}), 9.67(\mathrm{~s}, 1 \mathrm{H}), 8.85-8.79(\mathrm{~m}$, $2 \mathrm{H}), 8.20(\mathrm{dd}, J=8.3,1.7 \mathrm{~Hz}, 1 \mathrm{H}), 7.58(\mathrm{dd}, J=4.5,0.7 \mathrm{~Hz}, 2 \mathrm{H}), 7.49(\mathrm{dd}, J=8.2,4.2 \mathrm{~Hz}, 1 \mathrm{H}), 7.32(\mathrm{~d}, J=8.5 \mathrm{~Hz}, 1 \mathrm{H})$, $6.62(\mathrm{~d}, J=8.5 \mathrm{~Hz}, 1 \mathrm{H}), 2.90(\mathrm{t}, J=7.1 \mathrm{~Hz}, 2 \mathrm{H}), 2.66-2.61(\mathrm{~m}, 2 \mathrm{H}), 2.09-2.02(\mathrm{~m}, 2 \mathrm{H}) .{ }^{13} \mathbf{C} \mathbf{~ N M R}\left(150 \mathrm{MHz}^{2} \mathrm{CDCl}\right) \delta$ 194.60, 173.99, 164.36, 162.55, 148.63, 138.55, 136.72, 133.88, 133.73, 128.17, 127.56, 122.63, 122.04, 117.53, 114.68, 114.10, 110.50, 35.35, 24.18, 21.01. HRMS calcd. for $\mathrm{C}_{20} \mathrm{H}_{19} \mathrm{~N}_{2} \mathrm{O}_{4}+[\mathrm{M}+\mathrm{H}]^{+}:$351.13393, Found: 351.1340.

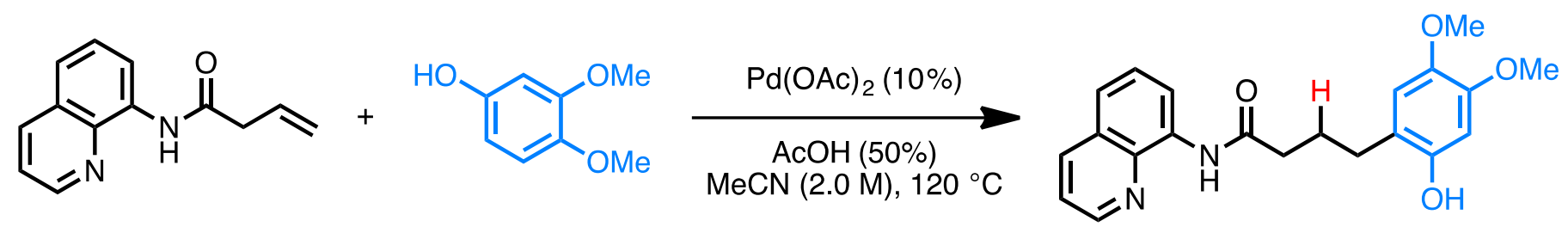

4-(2-hydroxy-4,5-dimethoxyphenyl)- $\boldsymbol{N}$-(quinolin-8-yl)butanamide (33): The reaction was carried out according to the general procedure using alkene 1 (42.5 mg, $0.2 \mathrm{mmol}), 3,4$-dimethoxyphenol (46.2 $\mathrm{mg}, 0.3 \mathrm{mmol}$ ), acetic acid (6.0 mg, $0.1 \mathrm{mmol}), \mathrm{Pd}(\mathrm{OAc})_{2}(4.4 \mathrm{mg}, 0.02 \mathrm{mmol})$, and $\mathrm{MeCN}(0.1 \mathrm{~mL})$. The reaction was run for $4 \mathrm{~h}$, and the product was purified by flash column chromatography $\left(\mathrm{SiO}_{2}, 40 \%\right.$ EtOAc in Hexanes) to afford $42.3 \mathrm{mg}(58 \%)$ of 33 as a yellow solid. ${ }^{1} \mathbf{H}$ NMR $\left(600 \mathrm{MHz} \mathrm{CDCl}_{3}\right) \delta 9.94(\mathrm{~s}, 1 \mathrm{H}), 8.85-8.80(\mathrm{~m}, 2 \mathrm{H}), 8.19(\mathrm{dd}, J=8.2,1.7 \mathrm{~Hz}, 1 \mathrm{H}), 8.09$ (s, 1H), 7.60-7.53 (m, 2H), $7.48(\mathrm{dd}, J=8.2,4.2 \mathrm{~Hz}, 1 \mathrm{H}), 6.62(\mathrm{~s}, 1 \mathrm{H}), 6.56(\mathrm{~s}, 1 \mathrm{H}), 3.85(\mathrm{~s}, 3 \mathrm{H}), 3.83(\mathrm{~s}, 3 \mathrm{H}) 2.75(\mathrm{t}, J=$ $7.2 \mathrm{~Hz}, 2 \mathrm{H}), 2.64-2.58(\mathrm{~m}, 2 \mathrm{H}), 2.08-2.01(\mathrm{~m}, 2 \mathrm{H}) .{ }^{13} \mathrm{C}$ NMR $\left(150 \mathrm{MHz}, \mathrm{CDCl}_{3}\right) \delta 173.30,149.58,148.74,148.50$, 142.76, 138.54, 136.72, 134.14, 128.17, 127.65, 122.29, 121.94, 117.61, 117.37, 113.80, 101.98, 56.88, 56.10, 35.40, 28.75, 26.74. HRMS calcd. for $\mathrm{C}_{21} \mathrm{H}_{23} \mathrm{~N}_{2} \mathrm{O}_{4}+[\mathrm{M}+\mathrm{H}]+:$ : 367.16523, Found: 367.1653. 

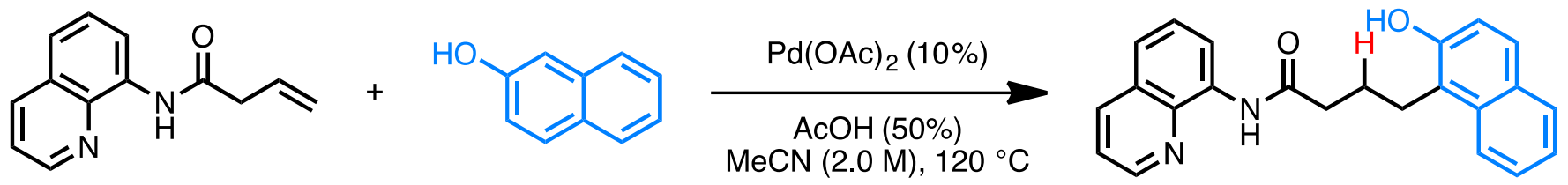

4-(2-hydroxynaphthalen-1-yl)- $\boldsymbol{N}$-(quinolin-8-yl)butanamide (34): The reaction was carried out according to the general procedure using alkene $\mathbf{1}(42.5 \mathrm{mg}, 0.2 \mathrm{mmol}), 2$-naphthol $(43.2 \mathrm{mg}, 0.3 \mathrm{mmol})$, acetic acid $(6.0 \mathrm{mg}, 0.1$ $\mathrm{mmol}), \mathrm{Pd}(\mathrm{OAc})_{2}(4.4 \mathrm{mg}, 0.02 \mathrm{mmol})$, and $\mathrm{MeCN}(0.1 \mathrm{~mL})$. The reaction was run for $4 \mathrm{~h}$, and the product was purified by flash column chromatography $\left(\mathrm{SiO}_{2}, 25-35 \%\right.$ EtOAc in Hexanes) to afford $58.6 \mathrm{mg}(82 \%)$ of $\mathbf{3 4}$ as a brown oil. ${ }^{1} \mathbf{H}$ NMR $\left(600 \mathrm{MHz}, \mathrm{CDCl}_{3}\right) \delta 10.00(\mathrm{~d}, J=8.5 \mathrm{~Hz}, 1 \mathrm{H}), 8.99(\mathrm{~s}, 1 \mathrm{H}), 8.88(\mathrm{dd}, J=7.1,1.8 \mathrm{~Hz}, 1 \mathrm{H}), 8.83(\mathrm{dd}, J=4.2,1.7 \mathrm{~Hz}$, $1 \mathrm{H}), 8.20(\mathrm{dd}, J=8.3,1.7 \mathrm{~Hz}, 1 \mathrm{H}), 7.92(\mathrm{~d}, J=8.5 \mathrm{~Hz}, 1 \mathrm{H}), 7.79(\mathrm{~d}, J=7.9 \mathrm{~Hz}, 1 \mathrm{H}), 7.68(\mathrm{~d}, J=8.8 \mathrm{~Hz}, 1 \mathrm{H}), 7.62-7.54$ (m, 2H), 7.51-7.45 (m, 2H), 7.32 (ddd, $J=8.0,6.7,1.1 \mathrm{~Hz}, 1 \mathrm{H}), 7.25(\mathrm{~d}, J=8.8 \mathrm{~Hz}, 1 \mathrm{H}), 3.28(\mathrm{t}, J=7.2 \mathrm{~Hz}, 2 \mathrm{H}), 2.67-$ $2.62(\mathrm{~m}, 2 \mathrm{H}), 2.23-2.16(\mathrm{~m}, 2 \mathrm{H}) .{ }^{13} \mathrm{C}$ NMR $\left(150 \mathrm{MHz}, \mathrm{CDCl}_{3}\right) \delta 173.70,153.38,148.53,138.57,136.72,134.10,133.36$, 129.42, 128.89, 128.32, 128.18, 127.65, 126.37, 122.78, 122.69, 122.41, 121.96, 119.81, 117.95, 117.49, 35.43, 25.39, 23.87. HRMS calcd. for $\mathrm{C}_{23} \mathrm{H}_{21} \mathrm{~N}_{2} \mathrm{O}_{2}+[\mathrm{M}+\mathrm{H}]+:$ 357.15975, Found: 357.1596 .<smiles>C=CCC(=O)Nc1cccc2cccnc12</smiles><smiles>Oc1ccc2cc(Br)ccc2c1</smiles>

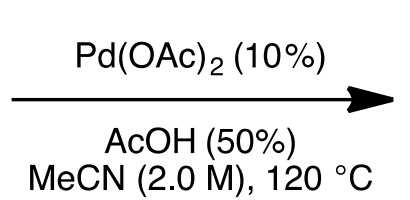<smiles>Ic1cccc2cccnc12</smiles><smiles>NC(=O)CCc1c(O)ccc2cc(Br)ccc12</smiles>

4-(6-bromo-2-hydroxynaphthalen-1-yl)- $\mathbf{N}$-(quinolin-8-yl)butanamide (35): The reaction was carried out according to the general procedure using alkene 1 (42.5 mg, $0.2 \mathrm{mmol}), 6$-bromo-2-naphthol $(67.0 \mathrm{mg}, 0.3 \mathrm{mmol})$, acetic acid ( $6.0 \mathrm{mg}, 0.1 \mathrm{mmol}), \mathrm{Pd}(\mathrm{OAc})_{2}(4.4 \mathrm{mg}, 0.02 \mathrm{mmol})$, and $\mathrm{MeCN}(0.1 \mathrm{~mL})$. The reaction was run for $4 \mathrm{~h}$, and the product was purified by flash column chromatography $\left(\mathrm{SiO}_{2}, 25 \%\right.$ EtOAc in Hexanes) to afford $44.6 \mathrm{mg}(69 \%)$ of 35 as an off-white solid. ${ }^{1} \mathbf{H}$ NMR $\left(600 \mathrm{MHz}, \mathrm{CDCl}_{3}\right) \delta 10.00(\mathrm{~s}, 1 \mathrm{H}), 9.15(\mathrm{~s}, 1 \mathrm{H}), 8.86(\mathrm{dd}, J=6.7,2.3 \mathrm{~Hz}, 1 \mathrm{H}), 8.83$ $(\mathrm{dd}, J=4.2,1.7 \mathrm{~Hz}, 1 \mathrm{H}), 8.20(\mathrm{dd}, J=8.3,1.7 \mathrm{~Hz}, 1 \mathrm{H}), 7.92(\mathrm{~d}, J=2.1 \mathrm{~Hz}, 1 \mathrm{H}), 7.78(\mathrm{~d}, J=9.0 \mathrm{~Hz}, 1 \mathrm{H}), 7.62-7.55(\mathrm{~m}$, $3 \mathrm{H}), 7.52(\mathrm{dd}, J=9.0,2.1 \mathrm{~Hz}, 1 \mathrm{H}), 7.49(\mathrm{dd}, J=8.2,4.2 \mathrm{~Hz}, 1 \mathrm{H}), 7.25(\mathrm{~d}, J=8.8 \mathrm{~Hz}, 2 \mathrm{H}), 3.24(\mathrm{t}, J=7.2 \mathrm{~Hz}, 1 \mathrm{H}), 2.66-$ $2.61(\mathrm{~m}, 2 \mathrm{H}), 2.18-2.13(\mathrm{~m}, 2 \mathrm{H}) .{ }^{13} \mathrm{C}$ NMR $\left(150 \mathrm{MHz}, \mathrm{CDCl}_{3}\right) \delta{ }^{13} \mathrm{C}$ NMR $\left(151 \mathrm{MHz}, \mathrm{CDCl}_{3}\right) \delta 173.59,153.77,148.56$, 138.55, 136.74, 134.01, 131.93, 130.72, 130.61, 129.51, 128.18, 127.63, 127.42, 124.58, 122.50, 121.99, 120.98, 118.31, 117.52, 116.42, 35.28, 25.34, 23.88. HRMS calcd. for $\mathrm{C}_{23} \mathrm{H}_{20} \mathrm{BrN}_{2} \mathrm{O}_{2}+[\mathrm{M}+\mathrm{H}]^{+}:$435.07027, Found: 435.0703.

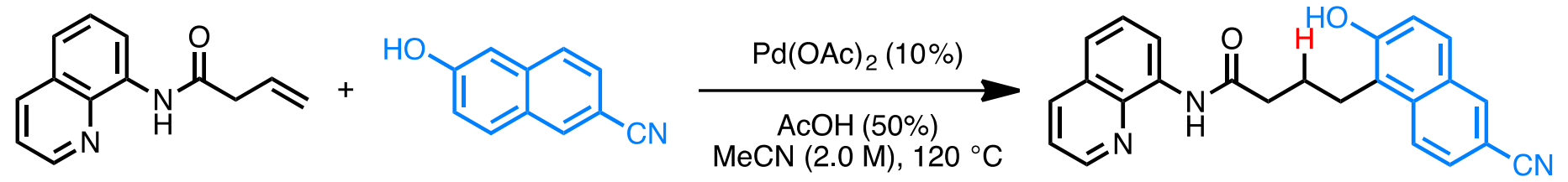

4-(6-cyano-2-hydroxynaphthalen-1-yl)- $\boldsymbol{N}$-(quinolin-8-yl)butanamide (36): The reaction was carried out according to the general procedure using alkene 1 (42.5 mg, $0.2 \mathrm{mmol}), 6$-cyano-2-naphthol (50.8 $\mathrm{mg}, 0.3 \mathrm{mmol})$, acetic acid $(6.0 \mathrm{mg}, 0.1 \mathrm{mmol}), \mathrm{Pd}(\mathrm{OAc})_{2}(4.4 \mathrm{mg}, 0.02 \mathrm{mmol})$, and $\mathrm{MeCN}(0.1 \mathrm{~mL})$. The reaction was run for $4 \mathrm{~h}$, and the product was purified by flash column chromatography $\left(\mathrm{SiO}_{2}, 30 \%\right.$ EtOAc in Hexanes) to afford $54.9 \mathrm{mg}(72 \%)$ of 36 as an off-white solid. ${ }^{1} \mathrm{H}$ NMR $\left(600 \mathrm{MHz}, \mathrm{CDCl}_{3}\right) \delta 10.04(\mathrm{~s}, 1 \mathrm{H}), 9.70(\mathrm{~s}, 1 \mathrm{H}), 8.86(\mathrm{dd}, J=5.9,3.1 \mathrm{~Hz}, 1 \mathrm{H}), 8.83$ $(\mathrm{dd}, J=4.2,1.7 \mathrm{~Hz}, 1 \mathrm{H}), 8.21(\mathrm{dd}, J=8.2,1.7 \mathrm{~Hz}, 1 \mathrm{H}), 8.15(\mathrm{~d}, J=1.8 \mathrm{~Hz}, 1 \mathrm{H}), 7.96(\mathrm{~d}, J=8.8 \mathrm{~Hz}, 1 \mathrm{H}), 7.72(\mathrm{~d}, J=8.9$ $\mathrm{Hz}, 1 \mathrm{H}), 7.63-7.57(\mathrm{~m}, 3 \mathrm{H}), 7.50(\mathrm{dd}, J=8.2,4.2 \mathrm{~Hz}, 1 \mathrm{H}), 7.35(\mathrm{~d}, J=8.8 \mathrm{~Hz}, 1 \mathrm{H}), 3.25(\mathrm{t}, J=7.3 \mathrm{~Hz}, 2 \mathrm{H}), 2.70-2.64(\mathrm{~m}$, 2H), 2.18-2.12 (m, 2H). ${ }^{13}$ C NMR $\left(150 \mathrm{MHz}, \mathrm{CDCl}_{3}\right) \delta 173.58,156.45,148.62,138.55,136.78,135.17,134.90,133.89$, 129.02, 128.20, 127.61, 127.03, 123.81, 122.66, 122.05, 121.75, 120.02, 118.58, 117.56, 105.84, 35.11, 25.16, 23.85. HRMS calcd. for $\mathrm{C}_{24} \mathrm{H}_{20} \mathrm{~N}_{3} \mathrm{O}_{2}+[\mathrm{M}+\mathrm{H}]+:$ 382.15500, Found: 382.1549. 


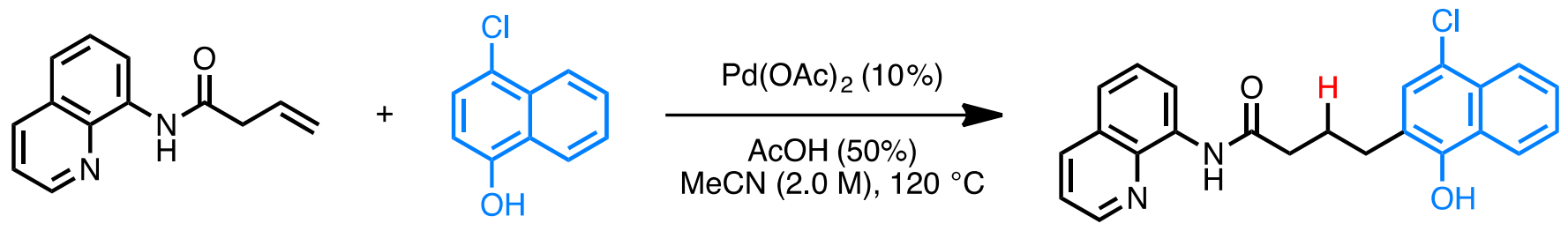

4-(4-chloro-1-hydroxynaphthalen-2-yl)- $\boldsymbol{N}$-(quinolin-8-yl)butanamide (37): The reaction was carried out according to the general procedure using alkene 1 (42.5 mg, $0.2 \mathrm{mmol}$ ), 4-chloro-1-naphthol (53.6 mg, $0.3 \mathrm{mmol})$, acetic acid (6.0 mg, $0.1 \mathrm{mmol}), \mathrm{Pd}(\mathrm{OAc})_{2}(4.4 \mathrm{mg}, 0.02 \mathrm{mmol})$, and $\mathrm{MeCN}(0.1 \mathrm{~mL})$. The reaction was run for $4 \mathrm{~h}$, and the product was purified by flash column chromatography ( $\mathrm{SiO}_{2}, 20 \%$ EtOAc in Hexanes) to afford $46.9 \mathrm{mg}(60 \%)$ of 37 as an off-white solid. ${ }^{1} \mathrm{H}$ NMR $\left(600 \mathrm{MHz}^{\mathrm{CDCl}}\right)_{3} \delta 10.03(\mathrm{~s}, 1 \mathrm{H}), 9.65(\mathrm{~s}, 1 \mathrm{H}), 8.88(\mathrm{dd}, J=6.8,2.1 \mathrm{~Hz}, 1 \mathrm{H}), 8.83$ $(\mathrm{dd}, J=4.2,1.7 \mathrm{~Hz}, 1 \mathrm{H}), 8.47-8.42(\mathrm{~m}, 1 \mathrm{H}), 8.20(\mathrm{dd}, J=8.3,1.7 \mathrm{~Hz}, 1 \mathrm{H}), 8.17-8.12(\mathrm{~m}, 1 \mathrm{H}), 7.62-7.51(\mathrm{~m}, 4 \mathrm{H}), 7.49$ $(\mathrm{dd}, J=8.2,4.2 \mathrm{~Hz}, 1 \mathrm{H}), 7.33(\mathrm{~s}, 1 \mathrm{H}), 2.96(\mathrm{t}, J=7.1 \mathrm{~Hz}, 2 \mathrm{H}), 2.66-2.61(\mathrm{~m}, 2 \mathrm{H}), 2.16-2.10(\mathrm{~m}, 2 \mathrm{H}) .{ }^{13} \mathbf{C}$ NMR $(150 \mathrm{MHz}$, $\left.\mathrm{CDCl}_{3}\right) \delta 173.66,150.34,148.59,138.55,136.74,133.94,130.68,128.38,128.18,127.61,127.06,126.79,125.80$, $124.09,123.36,122.59,122.05,122.02,120.28,117.56,34.98,28.92,26.48$. HRMS calcd. for $\mathrm{C}_{23} \mathrm{H}_{20} \mathrm{ClN}_{2} \mathrm{O}_{2}+[\mathrm{M}+\mathrm{H}]^{+}$: 391.12078, Found: 391.1208.

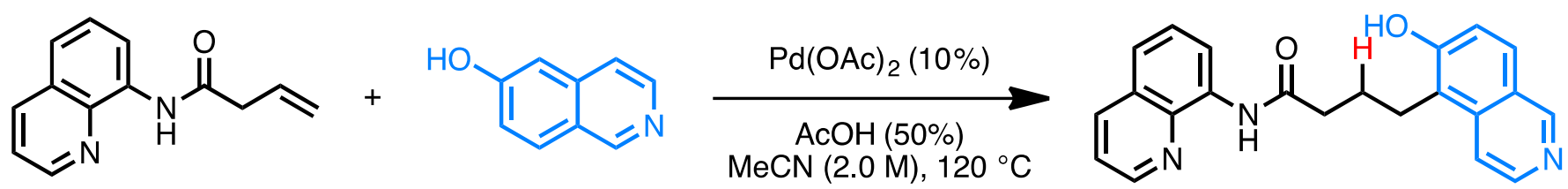

4-(6-hydroxyisoquinolin-5-yl)- $\mathbf{N}$-(quinolin-8-yl)butanamide (38): The reaction was carried out according to the general procedure using alkene 1 ( $42.5 \mathrm{mg}, 0.2 \mathrm{mmol}$ ), 6-hydroxyquinoline ( $43.6 \mathrm{mg}, 0.3 \mathrm{mmol}$ ), acetic acid (6.0 $\mathrm{mg}, 0.1 \mathrm{mmol}), \mathrm{Pd}(\mathrm{OAc})_{2}(4.4 \mathrm{mg}, 0.02 \mathrm{mmol})$, and $\mathrm{MeCN}(0.1 \mathrm{~mL})$. The reaction was run for $4 \mathrm{~h}$, and the product was purified by flash column chromatography $\left(\mathrm{SiO}_{2}, 70 \%\right.$ EtOAc in Hexanes) to afford $37.8 \mathrm{mg}(53 \%)$ of 38 as an offwhite solid. ${ }^{1} \mathrm{H}$ NMR $\left(600 \mathrm{MHz}, \mathrm{CDCl}_{3}\right) \delta 10.02(\mathrm{~d}, J=9.2 \mathrm{~Hz}, 1 \mathrm{H}), 9.27(\mathrm{~s}, 1 \mathrm{H}), 8.87(\mathrm{dd}, J=6.7,2.2 \mathrm{~Hz}, 1 \mathrm{H}), 8.83(\mathrm{dd}$, $J=4.2,1.7 \mathrm{~Hz}, 1 \mathrm{H}), 8.76(\mathrm{dd}, J=4.1,1.5 \mathrm{~Hz}, 1 \mathrm{H}), 8.25(\mathrm{~d}, J=7.8 \mathrm{~Hz}, 1 \mathrm{H}), 8.21(\mathrm{dd}, J=8.3,1.7 \mathrm{~Hz}, 1 \mathrm{H}), 7.94(\mathrm{~d}, J=9.0$ $\mathrm{Hz}, 1 \mathrm{H}), 7.63-7.54(\mathrm{~m}, 2 \mathrm{H}), 7.50(\mathrm{dd}, J=8.2,4.2 \mathrm{~Hz}, 1 \mathrm{H}), 7.47(\mathrm{~d}, J=9.1 \mathrm{~Hz}, 1 \mathrm{H}), 7.38(\mathrm{dd}, J=8.5,4.1 \mathrm{~Hz}, 1 \mathrm{H}), 3.25(\mathrm{t}$, $J=7.2 \mathrm{~Hz}, 2 \mathrm{H}), 2.69-2.63(\mathrm{~m}, 2 \mathrm{H}), 2.18-2.13(\mathrm{~m}, 2 \mathrm{H}) .{ }^{13} \mathbf{C}$ NMR $\left(150 \mathrm{MHz}, \mathrm{CDCl}_{3}\right) \delta 173.49,153.64,148.57,147.07$, $144.80,138.56,136.76,133.99,130.97,129.65,128.26,128.19,127.65,123.43,122.54,122.00,121.12,117.72$, 117.54, 35.25, 25.53, 23.62. HRMS calcd. for $\mathrm{C}_{22} \mathrm{H}_{20} \mathrm{~N}_{3} \mathrm{O}_{2}+[\mathrm{M}+\mathrm{H}]^{+}:$358.15500, Found: 358.1550.<smiles>C=CCC(=O)Nc1cccc2cccnc12</smiles>
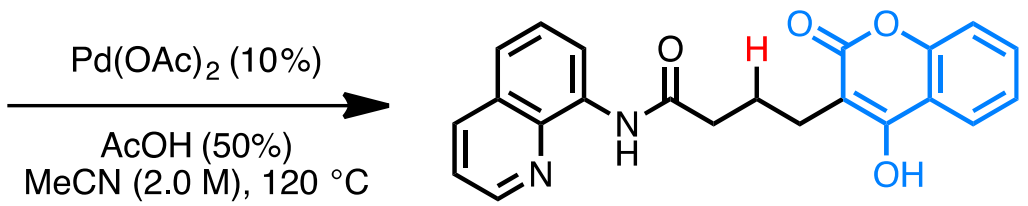

4-(4-hydroxy-2-oxo-2H-chromen-3-yl)- $\mathbf{N}$-(quinolin-8-yl)butanamide (39): The reaction was carried out according to the general procedure using alkene $1(42.5 \mathrm{mg}, 0.2 \mathrm{mmol})$, 4-hydroxycoumarin ( $48.6 \mathrm{mg}, 0.3 \mathrm{mmol})$, acetic acid (6.0 mg, $0.1 \mathrm{mmol}), \mathrm{Pd}(\mathrm{OAc})_{2}(4.4 \mathrm{mg}, 0.02 \mathrm{mmol})$, and $\mathrm{MeCN}(0.1 \mathrm{~mL})$. The reaction was run for $4 \mathrm{~h}$, and the product was purified by flash column chromatography $\left(\mathrm{SiO}_{2}, 30-40 \%\right.$ EtOAc in Hexanes) to afford $59.0 \mathrm{mg}(79 \%)$ of 39 as a white solid. ${ }^{1} \mathrm{H}$ NMR $\left(600 \mathrm{MHz}^{\mathrm{CDCl}}\right) \delta 11.96(\mathrm{~s}, 1 \mathrm{H}), 10.16(\mathrm{~s}, 1 \mathrm{H}), 8.86-8.79(\mathrm{~m}, 2 \mathrm{H}), 8.21(\mathrm{dd}, J=8.3$, $1.7 \mathrm{~Hz}, 1 \mathrm{H}), 8.00(\mathrm{dd}, J=7.9,1.6 \mathrm{~Hz}, 1 \mathrm{H}), 7.64-7.57(\mathrm{~m}, 2 \mathrm{H}), 7.55-7.48(\mathrm{~m}, 2 \mathrm{H}), 7.34-7.27(\mathrm{~m}, 2 \mathrm{H}), 2.88(\mathrm{t}, J=6.5 \mathrm{~Hz}$, 2H), 2.64-2.59 (m, 2H), 2.17-2.08 (m, 2H). ${ }^{13}$ C NMR (150 MHz, $\left.\mathrm{CDCl}_{3}\right) \delta 174.56,164.57,162.53,152.89,148.78$, 138.58, 136.74, 133.61, 131.58, 128.18, 127.46, 123.91 (×2), 123.06, 122.16, 117.71, 117.18, 116.48, 103.25, 35.39, 24.21, 22.64. HRMS calcd. for $\mathrm{C}_{22} \mathrm{H}_{19} \mathrm{~N}_{2} \mathrm{O}_{4}+[\mathrm{M}+\mathrm{H}]+:$ 375.13393, Found: 375.1338.<smiles>C=CCC(=O)Nc1cccc2cccnc12</smiles>

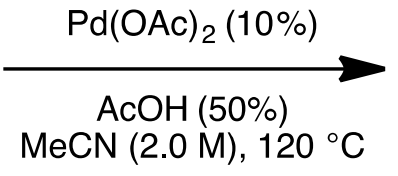<smiles>O=C(CCc1c(O)c2ccccc2[nH]c1=O)Nc1cccc2cccnc12</smiles> 
4-(4-hydroxy-2-oxo-1,2-dihydroquinolin-3-yl)- $N$-(quinolin-8-yl)butanamide (40): The reaction was carried out according to the general procedure using alkene 1 ( $42.5 \mathrm{mg}, 0.2 \mathrm{mmol})$, 4-hydroxyquinolin-2(1H)-one $(48.4 \mathrm{mg}$, $0.3 \mathrm{mmol})$, acetic acid (6.0 mg, $0.1 \mathrm{mmol}), \mathrm{Pd}(\mathrm{OAc})_{2}(4.4 \mathrm{mg}, 0.02 \mathrm{mmol})$, and $\mathrm{MeCN}(0.1 \mathrm{~mL})$. The reaction was run for $15 \mathrm{~h}$, and the product was purified by flash column chromatography $\left(\mathrm{SiO}_{2}, 5 \% \mathrm{MeOH}\right.$ in EtOAc) to afford $40.3 \mathrm{mg}$ (54\%) of 40 as a yellow solid. ${ }^{1} \mathbf{H}$ NMR $\left(600 \mathrm{MHz}^{\mathrm{CDCl}}\right) \delta 11.13(\mathrm{~s}, 1 \mathrm{H}), 10.10(\mathrm{~s}, 1 \mathrm{H}), 8.89-8.81(\mathrm{~m}, 2 \mathrm{H}), 8.78(\mathrm{~s}$, $1 \mathrm{H}), 8.21(\mathrm{dd}, J=8.2,1.7 \mathrm{~Hz}, 1 \mathrm{H}), 8.10(\mathrm{dd}, J=8.1,1.4 \mathrm{~Hz}, 1 \mathrm{H}), 7.63-7.57(\mathrm{~m}, 2 \mathrm{H}), 7.50(\mathrm{dd}, J=8.3,4.2 \mathrm{~Hz}, 1 \mathrm{H}), 7.47$ (ddd, $J=8.3,7.2,1.5 \mathrm{~Hz}, 1 \mathrm{H}$ ), 7.22 (ddd, $J=8.2,7.1,1.1 \mathrm{~Hz}, 1 \mathrm{H}), 7.09(\mathrm{~d}, J=8.1 \mathrm{~Hz}, 1 \mathrm{H}), 2.94(\mathrm{t}, J=6.7 \mathrm{~Hz}, 2 \mathrm{H}), 2.65-$ $2.60(\mathrm{~m}, 2 \mathrm{H}), 2.17-2.09(\mathrm{~m}, 2 \mathrm{H}) .{ }^{13} \mathbf{C}$ NMR $\left(150 \mathrm{MHz}, \mathrm{CDCl}_{3}\right) \delta 174.52,164.53,160.44,148.69,138.59,136.95,136.71$, 133.82, 130.41, 128.17, 127.53, 124.11, 122.80, 122.20, 122.09, 117.62, 116.83, 114.60, 109.34, 35.47, 24.36, 22.20. HRMS calcd. for $\mathrm{C}_{22} \mathrm{H}_{20} \mathrm{~N}_{3} \mathrm{O}_{3}+[\mathrm{M}+\mathrm{H}]+:$ 374.14992, Found: 374.1499.<smiles>C=CCC(=O)Nc1cccc2cccnc12</smiles>

4-(2-hydroxyphenyl)- $\boldsymbol{N}$-(quinolin-8-yl)butanamide (41): The reaction was carried out according to the general procedure using alkene 1 (42.5 mg, $0.2 \mathrm{mmol}$ ), phenol (28.2 mg, $0.3 \mathrm{mmol})$, acetic acid (6.0 mg, $0.1 \mathrm{mmol}), \mathrm{Pd}(\mathrm{OAc}){ }_{2}$ ( $4.4 \mathrm{mg}, 0.02 \mathrm{mmol}$ ), and MeCN $(0.1 \mathrm{~mL}$ ). The reaction was run for $4 \mathrm{~h}$, and the product was purified by preparative TLC (35\% EtOAc in Hexanes) to afford $4.0 \mathrm{mg}(7 \%)$ of $\mathbf{4 1}$ as a clear oil. ${ }^{1} \mathbf{H} \mathbf{~ N M R}\left(600 \mathrm{MHz}, \mathrm{CDCl}_{3}\right) \delta 9.93(\mathrm{~s}, 1 \mathrm{H})$, 8.87-8.80 (m, 2H), 8.19 (dd, J = 8.3, $1.7 \mathrm{~Hz}, 1 \mathrm{H}), 8.10(\mathrm{~s}, 1 \mathrm{H}), 7.60-7.52(\mathrm{~m}, 2 \mathrm{H}), 7.48(\mathrm{dd}, \mathrm{J}=8.2,4.2 \mathrm{~Hz}, 1 \mathrm{H}), 7.17-$ $7.09(\mathrm{~m}, 2 \mathrm{H}), 6.93(\mathrm{dd}, \mathrm{J}=8.0,1.2 \mathrm{~Hz}, 1 \mathrm{H}), 6.84(\mathrm{td}, \mathrm{J}=7.3,1.2 \mathrm{~Hz}, 1 \mathrm{H}), 2.80(\mathrm{t}, \mathrm{J}=7.5 \mathrm{~Hz}, 2 \mathrm{H}), 2.68-2.63(\mathrm{~m}, 2 \mathrm{H})$, 2.10-2.02 (m, 2H). ${ }^{13}$ C NMR $\left(150 \mathrm{MHz}, \mathrm{CDCl}_{3}\right) \delta 173.11,155.50,148.47,138.53,136.71,134.18,130.36,128.17$, 127.99, 127.66, 127.25, 122.22, 121.91, 120.21, 117.35, 116.89, 35.58, 29.54, 26.11. HRMS calcd. for $\mathrm{C}_{19} \mathrm{H}_{19} \mathrm{~N}_{2} \mathrm{O}_{2}+$ $[\mathrm{M}+\mathrm{H}]^{+}:$307.14410, Found: 307.1441.<smiles>C=CCC(=O)Nc1cccc2cccnc12</smiles>

4-(4-(dimethylamino)phenyl)- $\mathbf{N}$-(quinolin-8-yl)butanamide (42): The reaction was carried out according to the general procedure using alkene $1(42.5 \mathrm{mg}, 0.2 \mathrm{mmol}), N, N$-dimethylaniline ( $36.4 \mathrm{mg}, 0.3 \mathrm{mmol})$, acetic acid $(6.0 \mathrm{mg}$, $0.1 \mathrm{mmol}), \mathrm{Pd}(\mathrm{OAc})_{2}(4.4 \mathrm{mg}, 0.02 \mathrm{mmol})$, and $\mathrm{MeCN}(0.1 \mathrm{~mL})$. The reaction was run for $4 \mathrm{~h}$, and the product was purified by preparative TLC (35\% EtOAc in Hexanes) to afford $6.5 \mathrm{mg}(10 \%)$ of 42 as a yellow oil. ${ }^{1} \mathbf{H ~ N M R}(600 \mathrm{MHz}$, $\left.\mathrm{CDCl}_{3}\right) \delta 9.78(\mathrm{~s}, 1 \mathrm{H}), 8.82-8.77(\mathrm{~m}, 2 \mathrm{H}), 8.16(\mathrm{dd}, J=8.2,1.7 \mathrm{~Hz}, 1 \mathrm{H}), 7.59-7.48(\mathrm{~m}, 2 \mathrm{H}), 7.45(\mathrm{dd}, J=8.2,4.2 \mathrm{~Hz}, 1 \mathrm{H})$, 7.14-7.10 (m, 2H), 6.73-6.68 (m, 2H), $2.91(\mathrm{~s}, 6 \mathrm{H}), 2.68(\mathrm{t}, J=7.5 \mathrm{~Hz}, 2 \mathrm{H}), 2.57(\mathrm{t}, J=7.4 \mathrm{~Hz}, 2 \mathrm{H}), 2.12(\mathrm{p}, J=7.5 \mathrm{~Hz}$, 2H). ${ }^{13}$ C NMR $\left(150 \mathrm{MHz}, \mathrm{CDCl}_{3}\right) \delta 171.91,149.37,148.28,138.58,136.56,134.80,129.84,129.39(\times 2), 128.16$, 127.67, 121.77, 121.51, 116.64, $113.24(\times 2), 41.12(\times 2), 37.65,34.41,27.56$. HRMS calcd. for $\mathrm{C}_{21} \mathrm{H}_{24} \mathrm{~N}_{3} \mathrm{O}+[\mathrm{M}+\mathrm{H}]+$ : 334.19139, Found: 334.1913. 
General Procedure for Evaluating Alkene Scope for Hydrofunctionalization

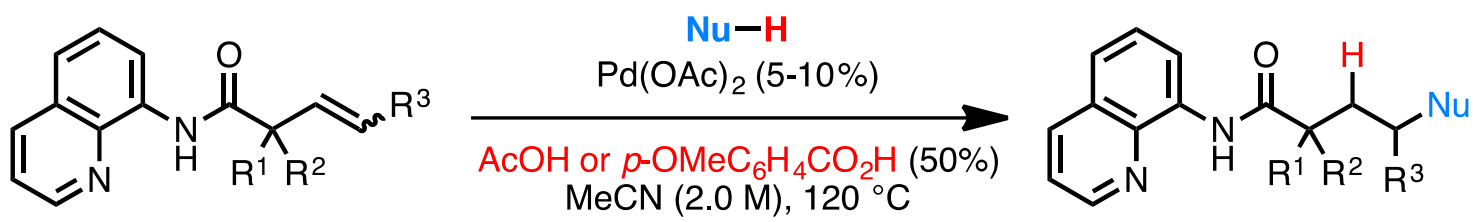

Unless otherwise stated, the procedure was as follows. To a 1 dram ( $4 \mathrm{~mL}$ ) vial equipped with a magnetic stir bar were added $\mathrm{Pd}(\mathrm{OAc})_{2}(2.2 \mathrm{mg}$., $0.01 \mathrm{mmol}$ or $4.4 \mathrm{mg}, 0.02 \mathrm{mmol})$, alkene $(0.2 \mathrm{mmol})$, acid $(0.1 \mathrm{mmol})$, carbon nucleophile $(0.3 \mathrm{mmol})$, and $\mathrm{MeCN}(0.1 \mathrm{~mL})$. The vial was sealed with an unpunctured TFE septum-covered screw cap and placed in a heating block that was pre-heated to $120^{\circ} \mathrm{C}$. After the designated reaction time, the dark black reaction was purified either by flash column chromatography directly or an aqueous workup followed by flash column chromatography to produce the desired product. 


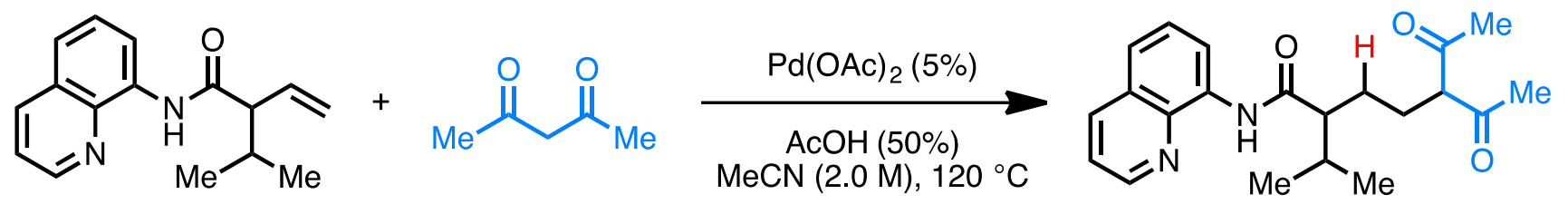

5-acetyl-2-isopropyl-6-oxo- $\boldsymbol{N}$-(quinolin-8-yl)heptanamide (43): The reaction was carried out according to the general procedure using alkene $\mathbf{S} 2(50.8 \mathrm{mg}, 0.2 \mathrm{mmol}), 2,4$-pentanedione $(30.0 \mathrm{mg}, 0.3 \mathrm{mmol})$, acetic acid $(6.0 \mathrm{mg}$, $0.1 \mathrm{mmol}), \mathrm{Pd}(\mathrm{OAc})_{2}(2.2 \mathrm{mg}, 0.01 \mathrm{mmol})$, and $\mathrm{MeCN}(0.1 \mathrm{~mL})$. The reaction was run for $4 \mathrm{~h}$, and the product was purified by flash column chromatography $\left(\mathrm{SiO}_{2}, 40 \%\right.$ EtOAc in Hexanes) to afford $63.8 \mathrm{mg}(90 \%)$ of 43 as a yellow oil. Note: The NMR spectra show an approximate $1: 1$ mixture of two tautomers. ${ }^{1} \mathbf{H}$ NMR $\left(600 \mathrm{MHz}, \mathrm{CDCl}_{3}\right) \delta 9.86-$ $9.82(\mathrm{~m}, 1 \mathrm{H}), 8.85-8.78(\mathrm{~m}, 2 \mathrm{H}), 8.21-8.14(\mathrm{~m}, 1 \mathrm{H}), 7.58-7.50(\mathrm{~m}, 2 \mathrm{H}), 7.49-7.45(\mathrm{~m}, 1 \mathrm{H}), 3.66(\mathrm{t}, J=7.2 \mathrm{~Hz}, 0.5 \mathrm{H})$, 2.35-2.19 (m, 2H), $2.17(\mathrm{~s}, 1 \mathrm{H}), 2.14(\mathrm{~d}, J=1.4 \mathrm{~Hz}, 4 \mathrm{H}), 2.09-1.96(\mathrm{~m}, 1 \mathrm{H}), 1.96-1.85(\mathrm{~m}, 1 \mathrm{H}), 1.78-1.63(\mathrm{~m}, 1 \mathrm{H})$, 1.57-1.52 (m, 0H), 1.09-0.95 (m, 6H). ${ }^{13}$ C NMR (150 MHz, CDCl $) \delta 204.59,204.41,191.40,173.69,173.59,148.47$, $148.45,138.63,138.60,136.64,136.58,134.49,134.45,128.20,128.18,127.66,127.60,121.87,121.80,121.75$, $116.71,116.68,110.29,69.11,56.41,56.16,31.60,31.52,30.95,29.73,28.81,27.92,26.57,26.32,23.06,21.11,20.96$, 20.63, 20.60. HRMS calcd. for $\mathrm{C}_{21} \mathrm{H}_{27} \mathrm{~N}_{2} \mathrm{O}_{3}+[\mathrm{M}+\mathrm{H}]+:$ 355.20162, Found: 355.2016.
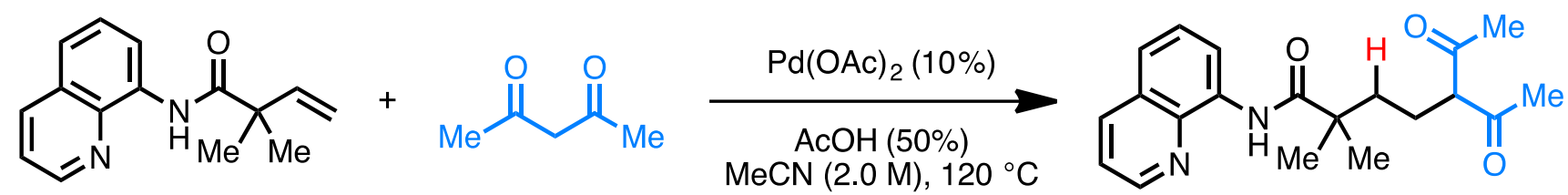

5-acetyl-2,2-dimethyl-6-oxo- $\boldsymbol{N}$-(quinolin-8-yl)heptanamide (44): The reaction was carried out according to the general procedure using alkene $\mathbf{S} 3(48.0 \mathrm{mg}, 0.2 \mathrm{mmol}), 2,4$-pentanedione $(30.0 \mathrm{mg}, 0.3 \mathrm{mmol})$, acetic acid $(6.0 \mathrm{mg}$, $0.1 \mathrm{mmol}), \mathrm{Pd}(\mathrm{OAc})_{2}(4.4 \mathrm{mg}, 0.02 \mathrm{mmol})$, and $\mathrm{MeCN}(0.1 \mathrm{~mL})$. The reaction was run for $28 \mathrm{~h}$, and the product was purified by flash column chromatography $\left(\mathrm{SiO}_{2}, 40-50 \%\right.$ EtOAc in Hexanes) to afford $51.6 \mathrm{mg}(76 \%)$ of 44 as a yellow oil. Note: The NMR spectra show an approximate $1: 1$ mixture of two tautomers. ${ }^{\mathbf{H}} \mathbf{H} \mathbf{~ N M R}\left(600 \mathrm{MHz}^{\mathrm{CDCl}} \mathrm{CD}_{3} \delta\right.$ 10.29-10.24 (m, 1H), 8.83-8.81 (m, 1H), 8.81-8.76 (m, 1H), 8.21-8.12 (m, 1H), 7.58-7.47 (m, 2H), 7.50-7.44 (m, 1H), $3.61(\mathrm{t}, J=7.2 \mathrm{~Hz}, 0.5 \mathrm{H}), 2.31-2.21(\mathrm{~m}, 1 \mathrm{H}), 2.15(\mathrm{~s}, 3 \mathrm{H}), 2.11(\mathrm{~s}, 3 \mathrm{H}), 1.95-1.88(\mathrm{~m}, 1 \mathrm{H}), 1.81-1.75(\mathrm{~m}, 1 \mathrm{H}), 1.67-$ 1.59 (m, 1H), 1.48 (s, 3H), 1.43 (s, 3H). ${ }^{13} \mathrm{C}$ NMR $\left(150 \mathrm{MHz}, \mathrm{CDCl}_{3}\right) \delta 204.45,191.31,175.97,175.92,148.53,148.51$, 138.98, 138.91, 136.62, 136.56, 134.63, 128.19, 128.16, 127.68, 127.61, 121.87, 121.84, 121.70, 121.67, 116.50, 116.46, 110.06, 69.37, 43.82, 42.39, 38.94, 29.28, 25.89, 25.82, 23.96, 23.39, 22.94. HRMS calcd. for $\mathrm{C}_{20} \mathrm{H}_{25} \mathrm{~N}_{2} \mathrm{O}_{3}+$ $[\mathrm{M}+\mathrm{H}]+:$ 341.18597, Found: 341.1860 .
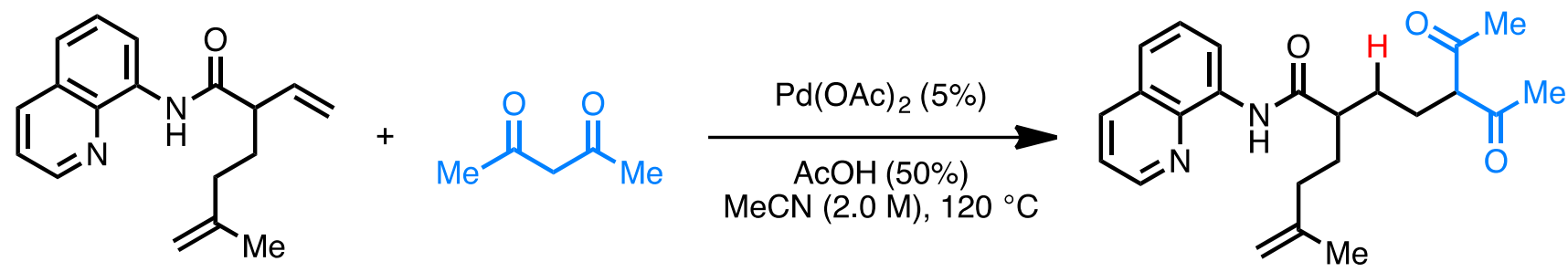

5-acetyl-2-(3-methylbut-3-en-1-yl)-6-oxo- $\boldsymbol{N}$-(quinolin-8-yl)heptanamide (45): The reaction was carried out according to the general procedure using alkene $\mathbf{S 4}(56.0 \mathrm{mg}, 0.2 \mathrm{mmol}), 2,4$-pentanedione $(30.0 \mathrm{mg}, 0.3 \mathrm{mmol})$, acetic acid (6.0 mg, $0.1 \mathrm{mmol}), \mathrm{Pd}(\mathrm{OAc})_{2}(2.2 \mathrm{mg}, 0.01 \mathrm{mmol})$, and $\mathrm{MeCN}(0.1 \mathrm{~mL})$. The reaction was run for $4 \mathrm{~h}$, and the product was purified by flash column chromatography $\left(\mathrm{SiO}_{2}, 30-40 \%\right.$ EtOAc in Hexanes) to afford 64.2 mg (84\%) of 45 as a yellow oil. Note: The NMR spectra show an approximate 1:1 mixture of two tautomers. ${ }^{\mathbf{1}} \mathbf{H} \mathbf{~ N M R}(600 \mathrm{MHz}$, $\left.\mathrm{CDCl}_{3}\right) \delta$ 9.90-9.86 (m, 1H), 8.84-8.77 (m, 2H), 8.21-8.15 (m, 1H), 7.58-7.51 (m, 2H), 7.50-7.45 (m, 1H), 4.79-4.70 $(\mathrm{m}, 2 \mathrm{H}), 3.65(\mathrm{t}, J=7.2 \mathrm{~Hz}, 0.5 \mathrm{H}), 2.57-2.46(\mathrm{~m}, 1 \mathrm{H}), 2.35-2.24(\mathrm{~m}, 1 \mathrm{H}), 2.19-2.17(\mathrm{~m}, 2 \mathrm{H}), 2.15-2.13(\mathrm{~m}, 4 \mathrm{H}), 2.20-$ $2.08(\mathrm{~m}, 2 \mathrm{H}), 2.04-1.85(\mathrm{~m}, 2 \mathrm{H}), 1.81-1.61(\mathrm{~m}, 2 \mathrm{H}), 1.76-1.70(\mathrm{~m}, 2 \mathrm{H}), 1.54-1.48(\mathrm{~m}, 1 \mathrm{H}) .{ }^{13} \mathbf{C ~ N M R}\left(150 \mathrm{MHz}^{-} \mathrm{CDCl}_{3}\right)$ $\delta$ 204.40, 204.31, 191.35, 173.99, 173.91, 148.48, 148.45, 145.02, 144.95, 138.62, 136.64, 136.58, 134.48, 134.45, $128.21,128.18,127.66,127.59,121.90,121.86,116.80,116.77,111.18,111.14,110.10,68.97,48.50,48.38,35.74$, 
35.62, 33.85, 31.31, 31.16, 30.89, 29.71, 28.98, 26.30, 25.96, 23.05, 22.48. HRMS calcd. for $\mathrm{C}_{23} \mathrm{H}_{29} \mathrm{~N}_{2} \mathrm{O}_{3}+[\mathrm{M}+\mathrm{H}]^{+}$: 381.21727, Found: 381.2174.

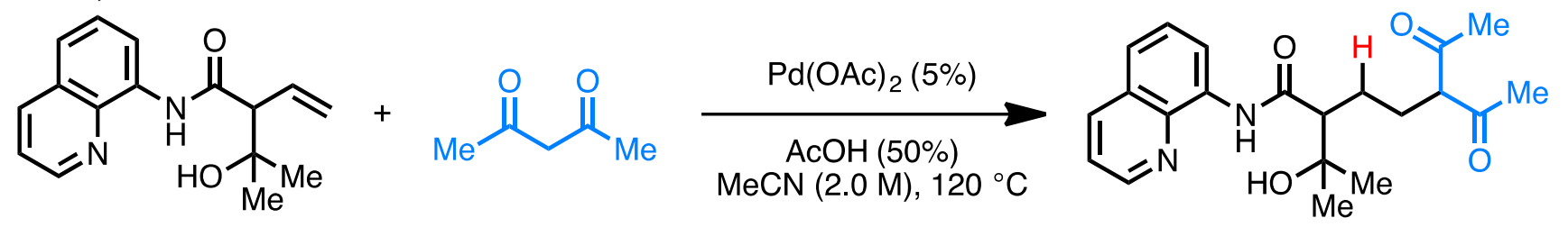

5-acetyl-2-(2-hydroxypropan-2-yl)-6-oxo- $\boldsymbol{N}$-(quinolin-8-yl)heptanamide (46): The reaction was carried out according to the general procedure using alkene $\mathbf{S} 5(54.0 \mathrm{mg}, 0.2 \mathrm{mmol}), 2,4$-pentanedione $(30.0 \mathrm{mg}, 0.3 \mathrm{mmol})$, acetic acid (6.0 mg, $0.1 \mathrm{mmol}), \mathrm{Pd}(\mathrm{OAc})_{2}(2.2 \mathrm{mg}, 0.01 \mathrm{mmol})$, and $\mathrm{MeCN}(0.1 \mathrm{~mL})$. The reaction was run for $4 \mathrm{~h}$, and the product was purified by flash column chromatography $\left(\mathrm{SiO}_{2}, 70 \%\right.$ EtOAc in Hexanes) to afford $58.4 \mathrm{mg}(79 \%)$ of 46 as a yellow oil. Note: The NMR spectra show an approximate 1:1 mixture of two tautomers. ${ }^{1} \mathbf{H}$ NMR $(600 \mathrm{MHz}$, $\left.\mathrm{CDCl}_{3}\right) \delta 10.11-10.05(\mathrm{~m}, 1 \mathrm{H}), 8.84-8.81(\mathrm{~m}, 1 \mathrm{H}), 8.81-8.74(\mathrm{~m}, 1 \mathrm{H}), 8.22-8.16(\mathrm{~m}, 1 \mathrm{H}), 7.59-7.53(\mathrm{~m}, 2 \mathrm{H}), 7.51-$ $7.45(\mathrm{~m}, 1 \mathrm{H}), 3.68(\mathrm{t}, J=6.9 \mathrm{~Hz}, 0.5 \mathrm{H}), 3.38(\mathrm{~s}, 0.5 \mathrm{H}), 3.28(\mathrm{~s}, 0.5 \mathrm{H}), 2.53-2.43(\mathrm{~m}, 1 \mathrm{H}), 2.36-2.22(\mathrm{~m}, 1 \mathrm{H}), 2.17(\mathrm{~s}$, $1 \mathrm{H}), 2.14-2.11(\mathrm{~m}, 4 \mathrm{H}), 2.11-2.04(\mathrm{~m}, 0.5 \mathrm{H}), 2.02-1.96(\mathrm{~m}, 0.5 \mathrm{H}), 1.95-1.85(\mathrm{~m}, 1 \mathrm{H}), 1.84-1.77(\mathrm{~m}, 0.5 \mathrm{H}), 1.71-1.63$ $(\mathrm{m}, 0.5 \mathrm{H}), 1.39(\mathrm{~s}, 1 \mathrm{H}), 1.35(\mathrm{~s}, 1 \mathrm{H}), 1.33(\mathrm{~s}, 1 \mathrm{H}), 1.29(\mathrm{~s}, 2 \mathrm{H}) .{ }^{13} \mathbf{C}$ NMR $\left(150 \mathrm{MHz}, \mathrm{CDCl}_{3}\right) \delta 204.27,204.16,191.33$, 174.02, 173.95, 148.71, 148.68, 138.75, 136.69, 136.64, 134.06, 133.98, 128.26, 128.23, 127.57, 127.49, 122.47, 122.39, 121.99, 117.32, 117.23, 110.07, 71.71, 71.68, 68.94, 58.39, 58.06, 29.94, 29.83, 29.81, 28.84, 28.77, 27.45, 27.33, 26.69, 26.46, 25.79, 23.03. HRMS calcd. for $\mathrm{C}_{21} \mathrm{H}_{27} \mathrm{~N}_{2} \mathrm{O}_{4}+[\mathrm{M}+\mathrm{H}]^{+}:$371.19653, Found: 371.1964.
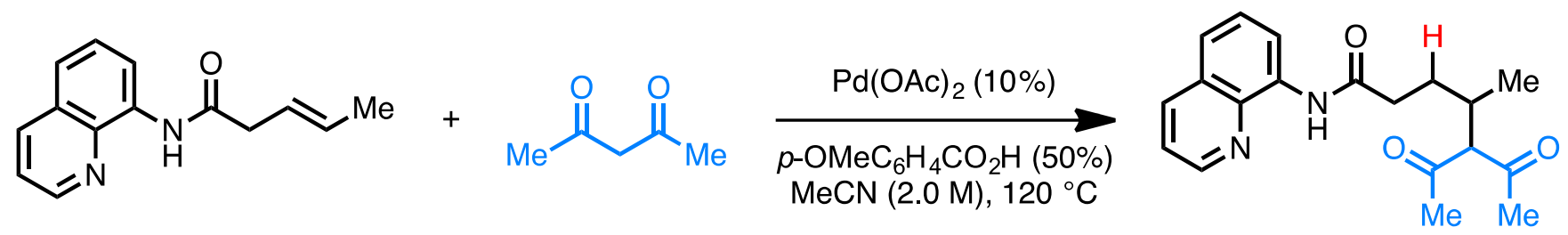

5-acetyl-4-methyl-6-oxo- $\boldsymbol{N}$-(quinolin-8-yl)heptanamide (47): The reaction was carried out according to the general procedure using alkene $\mathbf{S} \mathbf{9}(45.2 \mathrm{mg}, 0.2 \mathrm{mmol}), 2,4$-pentanedione $(30.0 \mathrm{mg}, 0.3 \mathrm{mmol}), p$-methoxybenzoic acid (15.2 mg, $0.1 \mathrm{mmol}), \mathrm{Pd}(\mathrm{OAc})_{2}(4.4 \mathrm{mg}, 0.02 \mathrm{mmol})$, and $\mathrm{MeCN}(0.1 \mathrm{~mL})$. The reaction was run for $8 \mathrm{~h}$, and then was diluted with $50 \mathrm{~mL}$ of EtOAc. The organic layer was washed with sat. $\mathrm{NaHCO}_{3}(50 \mathrm{~mL} \times 2)$, brine $(25 \mathrm{~mL})$, dried over $\mathrm{Na}_{2} \mathrm{SO}_{4}$, and concentrated under reduced pressure. The product was purified by flash column chromatography $\left(\mathrm{SiO}_{2}, 40 \%\right.$ EtOAc in Hexanes) to afford $29.1 \mathrm{mg}(45 \%)$ of $\mathbf{4 7}$ as a yellow oil. ${ }^{1} \mathbf{H} \mathbf{~ N M R}\left(600 \mathrm{MHz}, \mathrm{CDCl}_{3}\right) \delta 9.79(\mathrm{~s}, 1 \mathrm{H})$, $8.81(\mathrm{dd}, J=4.2,1.7 \mathrm{~Hz}, 1 \mathrm{H}), 8.75(\mathrm{dd}, J=7.3,1.7 \mathrm{~Hz}, 1 \mathrm{H}), 8.17(\mathrm{dd}, J=8.3,1.7 \mathrm{~Hz}, 1 \mathrm{H}), 7.57-7.49(\mathrm{~m}, 2 \mathrm{H}), 7.47(\mathrm{dd}, J$ $=8.2,4.2 \mathrm{~Hz}, 1 \mathrm{H}), 3.62(\mathrm{~d}, J=10.2 \mathrm{~Hz}, 1 \mathrm{H}), 2.66(\mathrm{ddd}, J=15.0,9.4,5.6 \mathrm{~Hz}, 1 \mathrm{H}), 2.59-2.48(\mathrm{~m}, 2 \mathrm{H}), 2.23(\mathrm{~s}, 3 \mathrm{H}), 2.19$ $(\mathrm{s}, 3 \mathrm{H}), 1.88$ (dddd, $J=13.1,9.8,6.7,3.3 \mathrm{~Hz}, 1 \mathrm{H}), 1.63-1.53(\mathrm{~m}, 1 \mathrm{H}), 0.98(\mathrm{~d}, J=6.6 \mathrm{~Hz}, 3 \mathrm{H}) .{ }^{13} \mathbf{C ~ N M R}\left(150 \mathrm{MHz}, \mathrm{CDCl}_{3}\right)$ $\delta 204.54,204.33,171.02,148.38,138.51,136.62,134.59,128.16,127.63,121.85,121.74,116.67,76.40,35.22,33.35$, 30.13, 30.05, 29.85, 17.02. HRMS calcd. for $\mathrm{C}_{19} \mathrm{H}_{23} \mathrm{~N}_{2} \mathrm{O}_{3}+[\mathrm{M}+\mathrm{H}]^{+}:$327.17032, Found: 327.1702.<smiles>[M]C=CCC(=O)Nc1cccc2cccnc12</smiles><smiles>Cn1ccc2ccccc21</smiles>
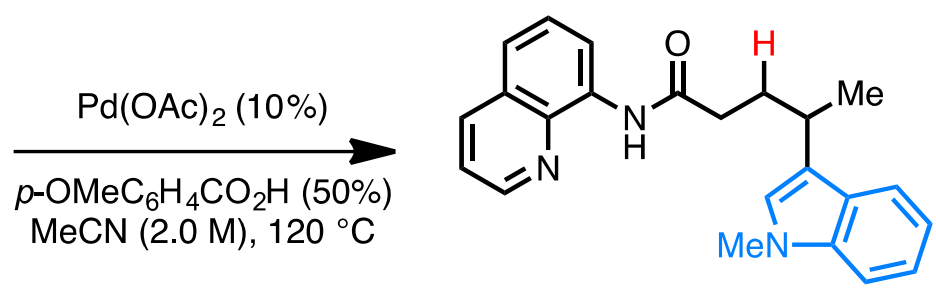

4-(1-methyl-1H-indol-3-yl)- $\boldsymbol{N}$-(quinolin-8-yl)pentanamide (48): The reaction was carried out according to the general procedure using alkene $\mathbf{S} \mathbf{9}(45.2 \mathrm{mg}, 0.2 \mathrm{mmol}), 1$-methylindole (39.3 $\mathrm{mg}, 0.3 \mathrm{mmol}$ ), $p$-methoxybenzoic acid (15.2 mg, $0.1 \mathrm{mmol}), \mathrm{Pd}(\mathrm{OAc})_{2}(4.4 \mathrm{mg}, 0.02 \mathrm{mmol})$, and $\mathrm{MeCN}(0.1 \mathrm{~mL})$. The reaction was run for $6 \mathrm{~h}$, and then was diluted with $50 \mathrm{~mL}$ of EtOAc. The organic layer was washed with sat. $\mathrm{NaHCO}_{3}(50 \mathrm{~mL} \times 2)$, brine $(25 \mathrm{~mL})$, dried over $\mathrm{Na}_{2} \mathrm{SO}_{4}$, and concentrated under reduced pressure. The product was purified by flash column chromatography $\left(\mathrm{SiO} \mathrm{O}_{2}\right.$, 25-30\% EtOAc in Hexanes) to afford $66.5 \mathrm{mg}$ (93\%) of 48 as an pale yellow oil. ${ }^{\mathbf{1}} \mathbf{H} \mathbf{~ N M R}\left(600 \mathrm{MHz}, \mathrm{CDCl}_{3}\right) \delta 9.67(\mathrm{~s}$, $1 \mathrm{H}), 8.77(\mathrm{dd}, J=7.6,1.4 \mathrm{~Hz}, 1 \mathrm{H}), 8.74(\mathrm{dd}, J=4.2,1.7 \mathrm{~Hz}, 1 \mathrm{H}), 8.14(\mathrm{dd}, J=8.2,1.7 \mathrm{~Hz}, 1 \mathrm{H}), 7.68(\mathrm{dt}, J=8.0,1.0 \mathrm{~Hz}$, $1 \mathrm{H}), 7.53(\mathrm{t}, J=7.9 \mathrm{~Hz}, 1 \mathrm{H}), 7.48(\mathrm{dd}, J=8.3,1.4 \mathrm{~Hz}, 1 \mathrm{H}), 7.43(\mathrm{dd}, J=8.2,4.2 \mathrm{~Hz}, 1 \mathrm{H}), 7.28-7.26(\mathrm{~m}, 1 \mathrm{H}), 7.19$ (ddd, $J$ 
$=8.2,7.0,1.1 \mathrm{~Hz}, 1 \mathrm{H}), 7.06(\mathrm{ddd}, J=8.0,7.0,1.1 \mathrm{~Hz}, 1 \mathrm{H}), 6.89(\mathrm{~s}, 1 \mathrm{H}), 3.73(\mathrm{~s}, 3 \mathrm{H}), 3.18(\mathrm{dq}, J=13.7,6.9 \mathrm{~Hz}, 1 \mathrm{H}), 2.57-$ $2.51(\mathrm{~m}, 2 \mathrm{H}), 2.30-2.21(\mathrm{~m}, 2 \mathrm{H}), 1.44(\mathrm{~d}, J=6.9 \mathrm{~Hz}, 3 \mathrm{H}) .{ }^{13} \mathbf{C} \mathbf{N M R}\left(150 \mathrm{MHz}, \mathrm{CDCl}_{3}\right) \delta 172.25,148.22,138.52,137.43$, 136.52, 134.81, 128.13, 127.66, 127.36, 125.59, 121.72, 121.65, 121.44, 119.79, 119.73, 118.77, 116.56, 109.41, 36.63, 33.40, 32.84, 30.90, 22.31. HRMS calcd. for $\mathrm{C}_{23} \mathrm{H}_{24} \mathrm{~N}_{3} \mathrm{O}+[\mathrm{M}+\mathrm{H}]^{+}: 358.19139$, Found: 358.1913.
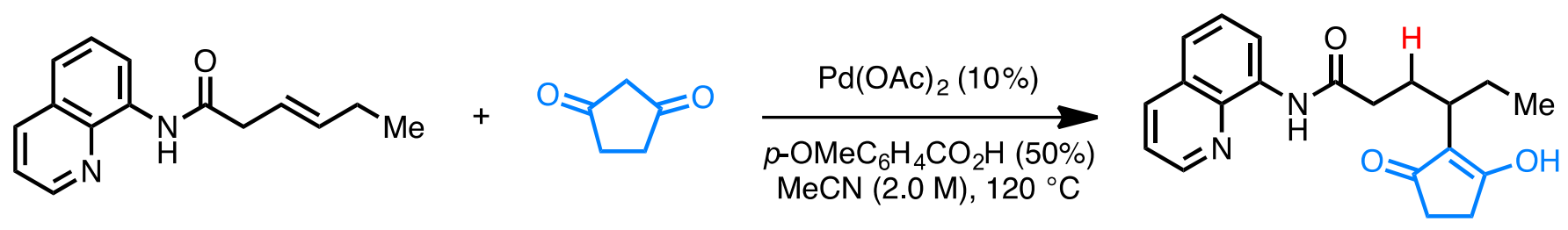

4-(2-hydroxy-5-oxocyclopent-1-en-1-yl)- $\mathbf{N}$-(quinolin-8-yl)hexanamide (49): The reaction was carried out according to the general procedure using alkene $\mathbf{S 6}$ (48.6 $\mathrm{mg}, 0.2 \mathrm{mmol}$ ), 1,3-cyclopentanedione (29.4 $\mathrm{mg}, 0.3 \mathrm{mmol}$ ), p-methoxybenzoic acid (15.2 mg, $0.1 \mathrm{mmol}), \mathrm{Pd}(\mathrm{OAc})_{2}(4.4 \mathrm{mg}, 0.02 \mathrm{mmol})$, and $\mathrm{MeCN}(0.1 \mathrm{~mL})$. The reaction was run for $3 \mathrm{~h}$ and the product was purified directly by flash column chromatography $\left(\mathrm{SiO}_{2}, 1 \% \mathrm{MeOH}\right.$ in $10 \%$ EtOAc in Hexanes) to afford $52.2 \mathrm{mg}(77 \%)$ of 49 as an orange oil. ${ }^{1} \mathbf{H}$ NMR $\left(600 \mathrm{MHz}, \mathrm{CDCl}_{3}\right) \delta 11.35(\mathrm{~s}, 1 \mathrm{H}), 10.06(\mathrm{~s}, 1 \mathrm{H})$, $8.83(\mathrm{dd}, J=4.2,1.7 \mathrm{~Hz}, 1 \mathrm{H}), 8.76(\mathrm{dd}, J=6.6,2.3 \mathrm{~Hz}, 1 \mathrm{H}), 8.20(\mathrm{dd}, J=8.2,1.7 \mathrm{~Hz}, 1 \mathrm{H}), 7.61-7.54(\mathrm{~m}, 2 \mathrm{H}), 7.49(\mathrm{dd}, J$ $=8.2,4.2 \mathrm{~Hz}, 1 \mathrm{H}), 2.69-2.52(\mathrm{~m}, 4 \mathrm{H}), 2.43(\mathrm{~d}, J=15.1 \mathrm{~Hz}, 2 \mathrm{H}), 2.33(\mathrm{td}, J=13.2,4.1 \mathrm{~Hz}, 1 \mathrm{H}), 2.20-2.09(\mathrm{~m}, 1 \mathrm{H}), 2.08-$ $2.00(\mathrm{~m}, 1 \mathrm{H}), 1.92-1.83(\mathrm{~m}, 1 \mathrm{H}), 1.52(\mathrm{tt}, J=13.0,7.4 \mathrm{~Hz}, 1 \mathrm{H}), 0.79(\mathrm{t}, J=7.4 \mathrm{~Hz}, 3 \mathrm{H}) .{ }^{13} \mathbf{C} \mathbf{N M R}\left(150 \mathrm{MHz} \mathrm{CDCl}_{3}\right) \delta$ 205.99, 187.29, 174.55, 148.71, 138.61, 136.71, 133.82, 128.16, 127.45, 122.84, 122.09, 117.55, 117.46, 36.26, 35.44, 34.23, 30.52, 27.41, 24.66, 13.01. HRMS calcd. for $\mathrm{C}_{20} \mathrm{H}_{23} \mathrm{~N}_{2} \mathrm{O}_{3}+[\mathrm{M}+\mathrm{H}]^{+}:$339.17032, Found: 339.1703 .
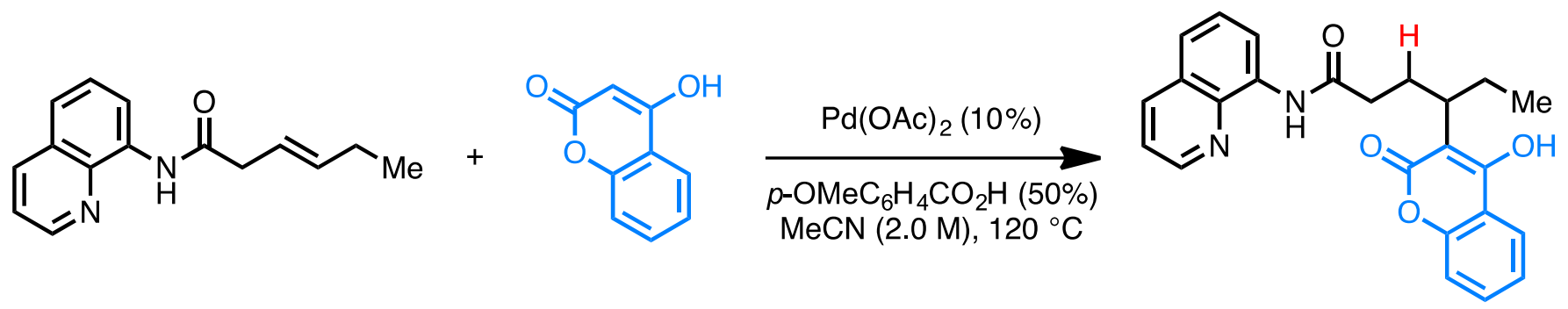

4-(4-hydroxy-2-oxo-2H-chromen-3-yl)- $\mathbf{N}$-(quinolin-8-yl)hexanamide (50): The reaction was carried out according to the general procedure using alkene $\mathbf{S 6}$ (48.6 $\mathrm{mg}, 0.2 \mathrm{mmol})$, 4-hydroxycoumarin $(46.0 \mathrm{mg}, 0.3 \mathrm{mmol}$ ), p-methoxybenzoic acid (15.2 mg, $0.1 \mathrm{mmol}), \mathrm{Pd}(\mathrm{OAc})_{2}(4.4 \mathrm{mg}, 0.02 \mathrm{mmol})$, and $\mathrm{MeCN}(0.1 \mathrm{~mL})$. The reaction was run for $4 \mathrm{~h}$, and then was diluted with $50 \mathrm{~mL}$ of EtOAc. The organic layer was washed with sat. $\mathrm{NaHCO}_{3}(50 \mathrm{~mL} \times 2)$, brine $(25 \mathrm{~mL})$, dried over $\mathrm{Na}_{2} \mathrm{SO}_{4}$, and concentrated under reduced pressure. The product was purified by flash column chromatography $\left(\mathrm{SiO}_{2}, 40 \%\right.$ EtOAc in Hexanes) to afford $62.6 \mathbf{~ m g ~ ( 7 8 \% ) ~ o f ~} \mathbf{5 0}$ as a white solid. ${ }^{\mathbf{1}} \mathbf{H}$ NMR (600 $\left.\mathrm{MHz}_{\mathrm{CDCl}}\right) \delta 11.53(\mathrm{~s}, 1 \mathrm{H}), 10.15(\mathrm{~s}, 1 \mathrm{H}), 8.84(\mathrm{dd}, J=4.2,1.6 \mathrm{~Hz}, 1 \mathrm{H}), 8.82(\mathrm{dd}, J=6.5,2.4 \mathrm{~Hz}, 1 \mathrm{H}), 8.21(\mathrm{dd}, J=8.2$, $1.8 \mathrm{~Hz}, 1 \mathrm{H}), 8.00(\mathrm{dd}, J=7.9,1.6 \mathrm{~Hz}, 1 \mathrm{H}), 7.62-7.58(\mathrm{~m}, 2 \mathrm{H}), 7.54-7.47(\mathrm{~m}, 2 \mathrm{H}), 7.33-7.25(\mathrm{~m}, 2 \mathrm{H}), 3.24-3.15(\mathrm{~m}, 1 \mathrm{H})$, 2.70 (ddd, $J=13.8,5.6,2.4 \mathrm{~Hz}, 1 \mathrm{H}), 2.51-2.42(\mathrm{~m}, 1 \mathrm{H}), 2.38(\mathrm{td}, J=13.6,3.2 \mathrm{~Hz}, 1 \mathrm{H}), 2.28-2.17(\mathrm{~m}, 1 \mathrm{H}), 1.94(\mathrm{tt}, J=$ 13.7, $2.9 \mathrm{~Hz}, 1 \mathrm{H}), 1.72-1.61(\mathrm{~m}, 1 \mathrm{H}), 0.83(\mathrm{t}, J=7.4 \mathrm{~Hz}, 3 \mathrm{H}) .{ }^{13} \mathbf{C}$ NMR $\left(150 \mathrm{MHz}, \mathrm{CDCl}_{3}\right) \delta 174.88,163.20,162.39$, 153.18, 148.78, 138.62, 136.73, 133.67, 131.47, 128.17, 127.44, 124.16, 123.77, 123.06, 122.15, 117.70, 117.34, 116.29, 105.34, 37.26, 36.04, 29.34, 25.47, 12.96. HRMS calcd. for $\mathrm{C}_{24} \mathrm{H}_{23} \mathrm{~N}_{2} \mathrm{O}_{3}+[\mathrm{M}+\mathrm{H}]^{+}:$403.16523, Found: 403.1651.
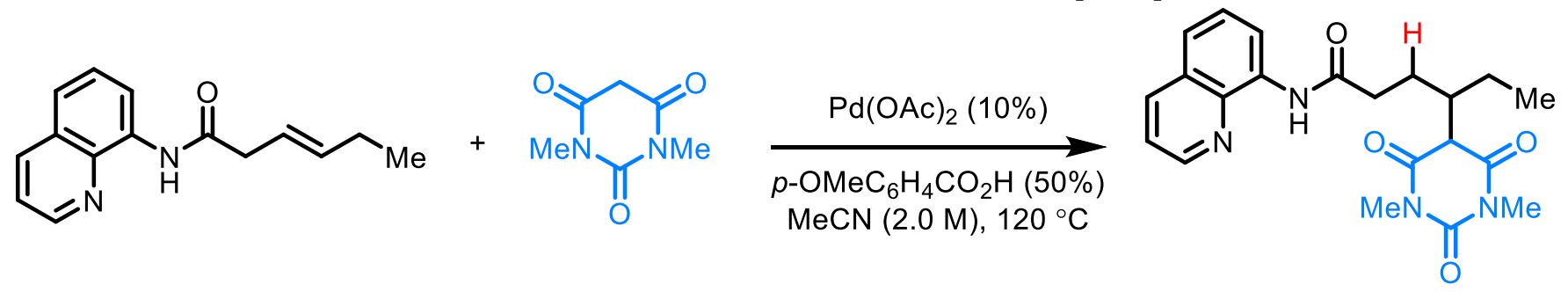

4-(6-hydroxy-1,3-dimethyl-2,4-dioxo-1,2,3,4-tetrahydropyrimidin-5-yl)- $N$-(quinolin-8-yl)hexanamide (51): The reaction was carried out according to the general procedure using alkene $\mathbf{S 6}$ (48.6 $\mathrm{mg}, 0.2 \mathrm{mmol})$, 6-hydroxy1,3-dimethylpyrimidine-2,4(1H,3H)-dione (46.8 mg, $0.3 \mathrm{mmol}), p$-methoxybenzoic acid (15.2 $\mathrm{mg}, 0.1 \mathrm{mmol}$ ), $\mathrm{Pd}(\mathrm{OAc})_{2}(4.4 \mathrm{mg}, 0.02 \mathrm{mmol})$, and $\mathrm{MeCN}(0.1 \mathrm{~mL})$. The reaction was run for $3 \mathrm{~h}$, and then was diluted with $50 \mathrm{~mL}$ of 
EtOAc. The organic layer was washed with sat. $\mathrm{NaHCO}_{3}(50 \mathrm{~mL} \times 2)$, brine $(25 \mathrm{~mL})$, dried over $\mathrm{Na}_{2} \mathrm{SO}_{4}$, and concentrated under reduced pressure. The product was purified by flash column chromatography $\left(\mathrm{SiO}_{2}, 50-70 \%\right.$ EtOAc in Hexanes) to afford $47.7 \mathrm{mg}(60 \%)$ of $\mathbf{5 1}$ as a yellow solid. The product contained a small amount of dimethylbarbituric acid which was removed by aqueous wash. ${ }^{1} \mathbf{H}$ NMR $\left(600 \mathrm{MHz}, \mathrm{CDCl}_{3}\right) \delta 9.81(\mathrm{~s}, 1 \mathrm{H}), 8.81(\mathrm{dd}, \mathrm{J}$ $=4.2,1.7 \mathrm{~Hz}, 1 \mathrm{H}), 8.73(\mathrm{dd}, \mathrm{J}=7.4,1.6 \mathrm{~Hz}, 1 \mathrm{H}), 8.16(\mathrm{dd}, \mathrm{J}=8.3,1.7 \mathrm{~Hz}, 1 \mathrm{H}), 7.56-7.48(\mathrm{~m}, 2 \mathrm{H}), 7.46$ (dd, J = 8.3, 4.2 $\mathrm{Hz}, 1 \mathrm{H}), 3.61$ (d, J = 3.2 Hz, 1H), 3.32 (s, 3H), $3.31(\mathrm{~s}, 3 \mathrm{H}), 2.67$ (td, J = 7.2, $2.5 \mathrm{~Hz}, 2 \mathrm{H}), 2.44(\mathrm{pd}, \mathrm{J}=7.2,3.3 \mathrm{~Hz}, 1 \mathrm{H})$, 2.03-1.94 (m, 2H), 1.62-1.54 (pd, 14.4, 7.3 Hz, 1H), $1.51(\mathrm{dp}, \mathrm{J}=14.4,7.3 \mathrm{~Hz}, 1 \mathrm{H}), 1.01$ (t, J = 7.3 Hz, 3H). ${ }^{13} \mathbf{C}$ NMR $\left(150 \mathrm{MHz}_{\mathrm{CDCl}}\right) \delta 170.89,168.53,151.93,148.36,138.51,136.60,134.59,128.15,127.64,121.83,121.71,116.69$, 50.72, 44.91, 35.99, 28.70, 26.86, 24.57, 12.17. HRMS calcd. for $\mathrm{C}_{21} \mathrm{H}_{25} \mathrm{~N}_{4} \mathrm{O}_{4}+[\mathrm{M}+\mathrm{H}]^{+}:$397.18703, Found: 397.1870.<smiles>CC/C=C/CC(=O)Nc1cccc2cccnc12</smiles>
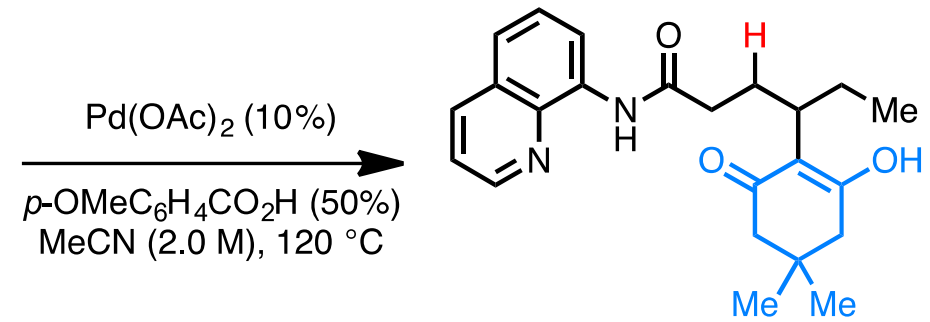

4-(2-hydroxy-4,4-dimethyl-6-oxocyclohex-1-en-1-yl)- $\boldsymbol{N}$-(quinolin-8-yl)hexanamide (52): The reaction was carried out according to the general procedure using alkene $\mathbf{S 6}$ (48.6 mg, $0.2 \mathrm{mmol}$ ), dimedone (42.0 $\mathrm{mg}, 0.3 \mathrm{mmol}$ ), p-methoxybenzoic acid (15.2 mg, $0.1 \mathrm{mmol}), \mathrm{Pd}(\mathrm{OAc})_{2}(4.4 \mathrm{mg}, 0.02 \mathrm{mmol})$, and $\mathrm{MeCN}(0.1 \mathrm{~mL})$. The reaction was run for $3 \mathrm{~h}$, and then was diluted with $50 \mathrm{~mL}$ of EtOAc. The organic layer was washed with sat. $\mathrm{NaHCO}_{3}(50 \mathrm{~mL} \times 2)$, brine $(25 \mathrm{~mL})$, dried over $\mathrm{Na}_{2} \mathrm{SO}_{4}$, and concentrated under reduced pressure. The product was purified by flash column chromatography $\left(\mathrm{SiO}_{2}, 30-40 \%\right.$ EtOAc in Hexanes) to afford $69.7 \mathrm{mg}(92 \%)$ of $\mathbf{5 2}$ as a pale yellow oil. ${ }^{\mathbf{1}} \mathbf{H}$ NMR $\left(600 \mathrm{MHz}, \mathrm{CDCl}_{3}\right) \delta 10.20(\mathrm{~s}, 1 \mathrm{H}), 10.02(\mathrm{~s}, 1 \mathrm{H}), 8.83(\mathrm{dd}, J=4.2,1.6 \mathrm{~Hz}, 1 \mathrm{H}), 8.76(\mathrm{dd}, J=6.2,2.8 \mathrm{~Hz}, 1 \mathrm{H}), 8.19$ $(\mathrm{dd}, J=8.3,1.7 \mathrm{~Hz}, 1 \mathrm{H}), 7.58-7.52(\mathrm{~m}, 2 \mathrm{H}), 7.49(\mathrm{dd}, J=8.3,4.2 \mathrm{~Hz}, 1 \mathrm{H}), 2.86-2.77(\mathrm{~m}, 1 \mathrm{H}), 2.64-2.56(\mathrm{~m}, 1 \mathrm{H}), 2.41$ $(\mathrm{d}, J=2.8 \mathrm{~Hz}, 2 \mathrm{H}), 2.36-2.23(\mathrm{~m}, 2 \mathrm{H}), 2.22(\mathrm{~s}, 2 \mathrm{H}), 2.13-2.01(\mathrm{~m}, 1 \mathrm{H}), 1.89-1.78(\mathrm{~m}, 1 \mathrm{H}), 1.58-1.47(\mathrm{~m}, 1 \mathrm{H}), 1.11(\mathrm{~s}$, 3H), $1.09(\mathrm{~s}, 3 \mathrm{H}), 0.80(\mathrm{t}, J=7.4 \mathrm{~Hz}, 3 \mathrm{H}) .{ }^{13} \mathrm{C} \mathrm{NMR}\left(150 \mathrm{MHz}, \mathrm{CDCl}_{3}\right) \delta$ 199.18, 174.76, 172.71, 148.65, 138.61, 136.68, 133.96, 128.16, 127.49, 122.63, 122.04, 117.45, 113.99, 52.23, 43.97, 36.86, 35.96, 31.49, 29.86, 29.81, 27.48, 26.12, 13.22. HRMS calcd. for $\mathrm{C}_{23} \mathrm{H}_{29} \mathrm{~N}_{2} \mathrm{O}_{3}+[\mathrm{M}+\mathrm{H}]+:$ 381.21727, Found: 381.2173 .<smiles>CC/C=C\CC(=O)Nc1cccc2cccnc12</smiles><smiles>CC1(C)CC(=O)C=C(O)C1</smiles>

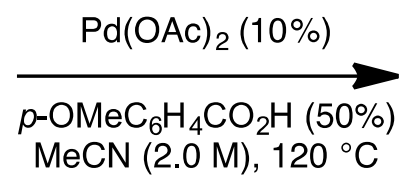<smiles>CCC(C=CCC(=O)Nc1cccc2cccnc12)C1=C(O)CC(C)(C)CC1=O</smiles>

4-(2-hydroxy-4,4-dimethyl-6-oxocyclohex-1-en-1-yl)- $\mathbf{N}$-(quinolin-8-yl)hexanamide (52): The reaction was carried out according to the general procedure using alkene $\mathbf{S 7}(48.6 \mathrm{mg}, 0.2 \mathrm{mmol})$, dimedone $(42.0 \mathrm{mg}, 0.3 \mathrm{mmol})$, p-methoxybenzoic acid (15.2 mg, $0.1 \mathrm{mmol}), \mathrm{Pd}(\mathrm{OAc})_{2}(4.4 \mathrm{mg}, 0.02 \mathrm{mmol})$, and $\mathrm{MeCN}(0.1 \mathrm{~mL})$. The reaction was run for $3 \mathrm{~h}$, and then was diluted with $50 \mathrm{~mL}$ of EtOAc. The organic layer was washed with sat. $\mathrm{NaHCO}_{3}(50 \mathrm{~mL} \times 2)$, brine $(25 \mathrm{~mL})$, dried over $\mathrm{Na}_{2} \mathrm{SO}_{4}$, and concentrated under reduced pressure. The product was purified by flash column chromatography $\left(\mathrm{SiO}_{2}, 40-50 \%\right.$ EtOAc in Hexanes) to afford $67.2 \mathrm{mg}(88 \%)$ of $\mathbf{5 2}$ as a yellow solid. Characterization data as reported above. 

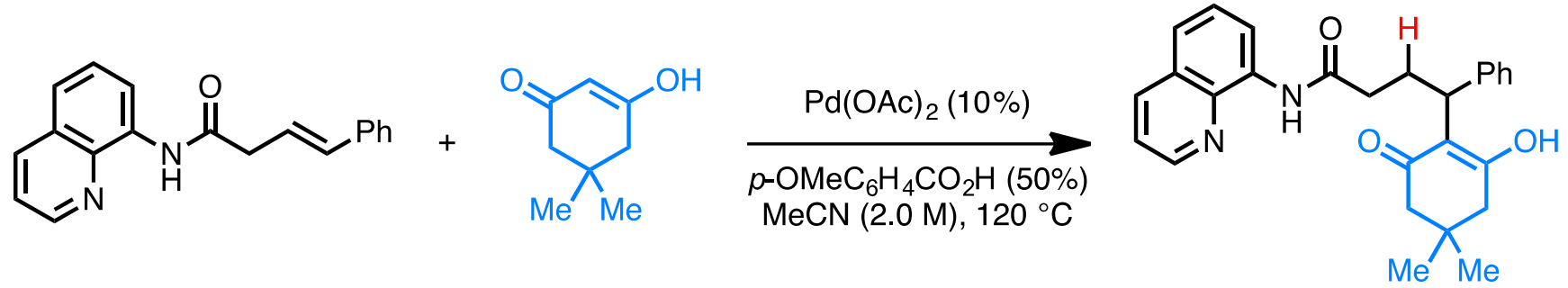

4-(2-hydroxy-4,4-dimethyl-6-oxocyclohex-1-en-1-yl)-4-phenyl- $N$-(quinolin-8-yl)butanamide (53): The reaction was carried out according to the general procedure using alkene 79 (57.7 $\mathrm{mg}, 0.2 \mathrm{mmol})$, dimedone (42.0 $\mathrm{mg}, 0.3 \mathrm{mmol}$ ), $p$-methoxybenzoic acid (15.2 mg, $0.1 \mathrm{mmol}), \mathrm{Pd}(\mathrm{OAc})_{2}(4.4 \mathrm{mg}, 0.02 \mathrm{mmol}$ ), and MeCN $(0.1 \mathrm{~mL})$. The reaction was run for $4 \mathrm{~h}$, and then was diluted with $50 \mathrm{~mL}$ of EtOAc. The organic layer was washed with sat. $\mathrm{NaHCO}_{3}$ $(50 \mathrm{~mL} \times 2)$, brine $(25 \mathrm{~mL})$, dried over $\mathrm{Na}_{2} \mathrm{SO}_{4}$, and concentrated under reduced pressure. The product was purified by flash column chromatography ( $\mathrm{SiO}_{2}, 40 \%$ EtOAc in Hexanes) to afford $70.4 \mathrm{mg}(82 \%)$ of 53 as a yellow solid. Note: The NMR spectra show an approximate 4:1 mixture of enol:keto tautomers. ${ }^{1} \mathbf{H}$ NMR for major enol tautomer (600 $\left.\mathrm{MHz}, \mathrm{CDCl}_{3}\right) \delta 1 \mathrm{H}$ NMR $\delta 10.01(\mathrm{~s}, 1 \mathrm{H}), 9.58(\mathrm{~s}, 1 \mathrm{H}), 8.81(\mathrm{dd}, \mathrm{J}=4.2,1.7 \mathrm{~Hz}, 1 \mathrm{H}), 8.77-8.70(\mathrm{~m}, 1 \mathrm{H}), 8.17(\mathrm{dd}, \mathrm{J}=8.2$, $1.7 \mathrm{~Hz}, 1 \mathrm{H}), 7.57-7.50(\mathrm{~m}, 2 \mathrm{H}), 7.47(\mathrm{dd}, \mathrm{J}=8.2,4.2 \mathrm{~Hz}, 1 \mathrm{H}), 7.38-7.31(\mathrm{~m}, 2 \mathrm{H}), 7.28-7.22(\mathrm{~m}, 2 \mathrm{H}), 7.17-7.11(\mathrm{~m}, 1 \mathrm{H})$, 4.39 (dd, J = 10.8, $4.5 \mathrm{~Hz}, 1 \mathrm{H}), 2.81-2.67(\mathrm{~m}, 2 \mathrm{H}), 2.56-2.47(\mathrm{~m}, 1 \mathrm{H}), 2.46-2.43(\mathrm{~m}, 1 \mathrm{H}), 2.43(\mathrm{~s}, 2 \mathrm{H}), 2.18(\mathrm{~d}, \mathrm{~J}=16.1$ $\mathrm{Hz}, 1 \mathrm{H}), 2.11(\mathrm{~d}, \mathrm{~J}=16.1 \mathrm{~Hz}, 1 \mathrm{H}), 1.06(\mathrm{~s}, 3 \mathrm{H}), 1.02(\mathrm{~s}, 3 \mathrm{H}) \cdot{ }^{13} \mathbf{C}$ NMR for major peaks of mixture $\left(150 \mathrm{MHz} \mathrm{CDCl}_{3}\right) \delta$ $198.10,173.89,171.42,148.59,143.48,138.57,136.65,134.00,128.66,128.22,127.92,127.47,126.09,122.52$, 122.00, 117.32, 116.12, 72.66, 53.58, 53.35, 51.43, 46.45, 43.91, 38.03, 36.10, 35.23, 31.72, 29.52, 27.54, 26.95. HRMS calcd. for $\mathrm{C}_{27} \mathrm{H}_{29} \mathrm{~N}_{2} \mathrm{O}_{3}+[\mathrm{M}+\mathrm{H}]+:$ 429.21727, Found: 429.2174 .
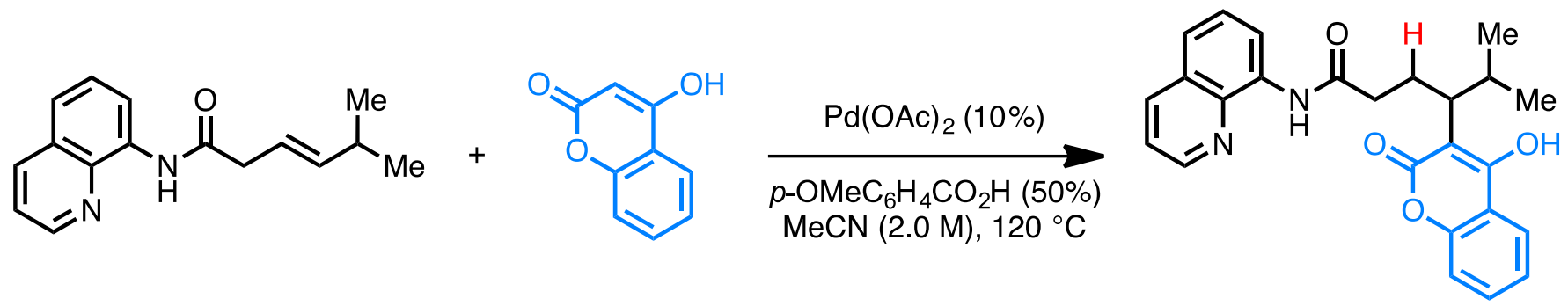

4-(4-hydroxy-2-oxo-2H-chromen-3-yl)-5-methyl- $\mathrm{N}$-(quinolin-8-yl)hexanamide (54): The reaction was carried out according to the general procedure using alkene $\mathbf{S 1 1}(50.8 \mathrm{mg}, 0.2 \mathrm{mmol}), 4$-hydroxycoumarin $(48.6 \mathrm{mg}, 0.3$ mmol), $p$-methoxybenzoic acid (15.2 mg, $0.1 \mathrm{mmol}), \mathrm{Pd}(\mathrm{OAc})_{2}(4.4 \mathrm{mg}, 0.02 \mathrm{mmol})$, and MeCN $(0.1 \mathrm{~mL})$. The reaction was run for $4 \mathrm{~h}$, and then was diluted with $50 \mathrm{~mL}$ of EtOAc. The organic layer was washed with sat. $\mathrm{NaHCO}_{3}(50 \mathrm{~mL}$ $\times 2$ ), brine $(25 \mathrm{~mL})$, dried over $\mathrm{Na}_{2} \mathrm{SO}_{4}$, and concentrated under reduced pressure. The product was purified by flash column chromatography ( $\mathrm{SiO}_{2}, 30-40 \%$ EtOAc in Hexanes) to afford $29.8 \mathrm{mg}(36 \%)$ of $\mathbf{5 4}$ as a light brown oil. ${ }^{\mathbf{1}} \mathbf{H}$ NMR $\left(600 \mathrm{MHz}, \mathrm{CDCl}_{3}\right) \delta 11.38(\mathrm{~s}, 1 \mathrm{H}), 10.14(\mathrm{~s}, 1 \mathrm{H}), 8.84(\mathrm{dd}, J=4.2,1.7 \mathrm{~Hz}, 1 \mathrm{H}), 8.81(\mathrm{dd}, J=6.6,2.3 \mathrm{~Hz}, 1 \mathrm{H}), 8.21$ $(\mathrm{dd}, J=8.3,1.7 \mathrm{~Hz}, 1 \mathrm{H}), 8.00(\mathrm{dd}, J=8.1,1.6 \mathrm{~Hz}, 1 \mathrm{H}), 7.64-7.57(\mathrm{~m}, 2 \mathrm{H}), 7.52-7.47(\mathrm{~m}, 2 \mathrm{H}), 7.33-7.26(\mathrm{~m}, 2 \mathrm{H}), 2.89-$ $2.82(\mathrm{~m}, 1 \mathrm{H}), 2.72-2.66(\mathrm{~m}, 1 \mathrm{H}), 2.58-2.49(\mathrm{~m}, 1 \mathrm{H}), 2.43-2.31(\mathrm{~m}, 2 \mathrm{H}), 2.22-2.13(\mathrm{~m}, 1 \mathrm{H}), 0.99(\mathrm{~d}, J=6.6 \mathrm{~Hz}, 3 \mathrm{H})$, $0.80(\mathrm{~d}, J=6.6 \mathrm{~Hz}, 3 \mathrm{H}) .{ }^{13} \mathrm{C}$ NMR $\left(150 \mathrm{MHz}, \mathrm{CDCl}_{3}\right) \delta{ }^{13} \mathrm{C} \mathrm{NMR}\left(151 \mathrm{MHz}, \mathrm{CDCl}_{3}\right) \delta 174.92,162.98,162.57,153.15$, $148.77,138.64,136.72,133.69,131.45,128.17,127.45,124.23,123.80,123.03,122.14,117.70,117.32,116.29$, 106.33, 43.30, 36.36, 28.88, 26.65, 22.41, 22.07. HRMS calcd. for $\mathrm{C}_{25} \mathrm{H}_{25} \mathrm{~N}_{2} \mathrm{O}_{4}+[\mathrm{M}+\mathrm{H}]^{+}:$417.18088, Found: 417.1808.
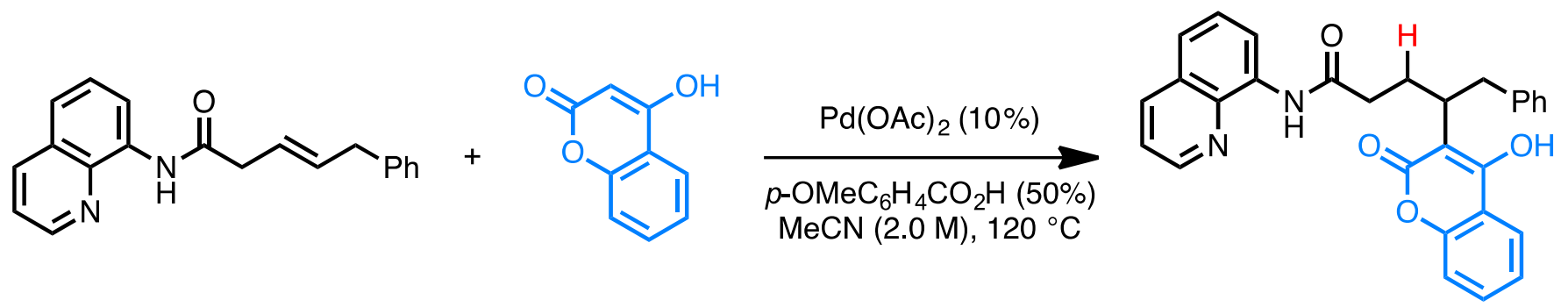

4-(4-hydroxy-2-oxo-2H-chromen-3-yl)-5-phenyl- $\mathrm{N}$-(quinolin-8-yl)pentanamide (55): The reaction was carried out according to the general procedure using alkene $\mathbf{S 1 2}$ (60.4 $\mathrm{mg}, 0.2 \mathrm{mmol}$ ), 4-hydroxycoumarin (48.6 $\mathrm{mg}$, 
$0.3 \mathrm{mmol}), p$-methoxybenzoic acid (15.2 $\mathrm{mg}, 0.1 \mathrm{mmol}), \mathrm{Pd}(\mathrm{OAc})_{2}(4.4 \mathrm{mg}, 0.02 \mathrm{mmol})$, and $\mathrm{MeCN}(0.1 \mathrm{~mL}) . \mathrm{The}$ reaction was run for $4 \mathrm{~h}$, and then was diluted with $50 \mathrm{~mL}$ of EtOAc. The organic layer was washed with sat. $\mathrm{NaHCO}_{3}$ ( $50 \mathrm{~mL} \times 2$ ), brine $(25 \mathrm{~mL})$, dried over $\mathrm{Na}_{2} \mathrm{SO}_{4}$, and concentrated under reduced pressure. The product was purified by flash column chromatography $\left(\mathrm{SiO}_{2}, 40 \%\right.$ EtOAc in Hexanes) to afford $72.5 \mathrm{mg}(78 \%)$ of $\mathbf{5 5}$ as a yellow solid. ${ }^{\mathbf{1}} \mathbf{H}$ NMR $\left(600 \mathrm{MHz} \mathrm{CDCl}_{3}\right) \delta 11.54(\mathrm{~s}, 1 \mathrm{H}), 10.10(\mathrm{~s}, 1 \mathrm{H}), 8.82(\mathrm{dd}, J=4.2,1.7 \mathrm{~Hz}, 1 \mathrm{H}), 8.80(\mathrm{dd}, J=6.3,2.6 \mathrm{~Hz}, 1 \mathrm{H}), 8.21$ (dd, $J=8.3,1.7 \mathrm{~Hz}, 1 \mathrm{H}$ ), 7.91 (dd, $J=7.9,1.5 \mathrm{~Hz}, 1 \mathrm{H}), 7.63-7.58(\mathrm{~m}, 2 \mathrm{H}), 7.50$ (dd, $J=8.2,4.2 \mathrm{~Hz}, 1 \mathrm{H}$ ), 7.47 (ddd, $J=$ 8.3, 7.2, $1.6 \mathrm{~Hz}, 1 \mathrm{H}), 7.28-7.25(\mathrm{~m}, 1 \mathrm{H}), 7.23$ (ddd, $J=8.2,7.2,1.1 \mathrm{~Hz}, 1 \mathrm{H}), 7.20-7.16(\mathrm{~m}, 2 \mathrm{H}), 7.10(\mathrm{t}, J=7.7 \mathrm{~Hz}, 2 \mathrm{H})$, 7.02-6.96 (m, 1H), 3.74-3.63 (m,1H), 3.43 (dd, $J=13.8,8.5 \mathrm{~Hz}, 1 \mathrm{H}), 3.11$ (dd, $J=13.8,6.9 \mathrm{~Hz}, 1 \mathrm{H}), 2.69$ (ddd, $J=13.9$, 5.6, $2.5 \mathrm{~Hz}, 1 \mathrm{H}), 2.59-2.50(\mathrm{~m}, 1 \mathrm{H}), 2.40(\mathrm{td}, J=13.7,3.2 \mathrm{~Hz}, 1 \mathrm{H}), 2.00(\mathrm{tt}, J=13.8,2.9 \mathrm{~Hz}, 1 \mathrm{H}) .{ }^{13} \mathrm{C} \mathrm{NMR}(150 \mathrm{MHz}$, $\left.\mathrm{CDCl}_{3}\right) \delta 174.70,163.02,162.68,153.11,148.75,140.70,138.61,136.70,133.59,131.51,128.95(\times 2), 128.40(\times 2)$, 128.15, 127.43, 126.01, 124.19, 123.73, 123.09, 122.14, 117.76, 117.16, 116.26, 105.17, 38.46, 36.78, 35.95, 28.73. HRMS calcd. for $\mathrm{C}_{29} \mathrm{H}_{25} \mathrm{~N}_{2} \mathrm{O}_{4}+[\mathrm{M}+\mathrm{H}]+:$ 465.18088, Found: 465.1810 .
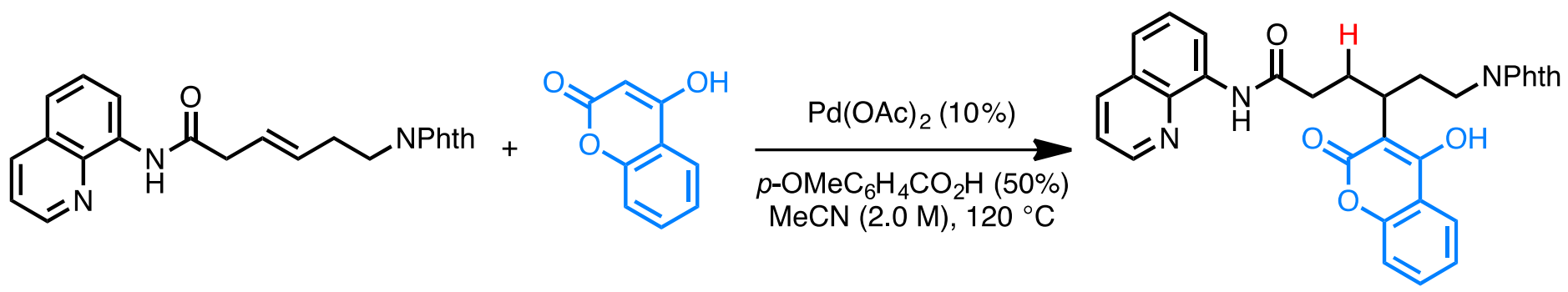

6-(1,3-dioxoisoindolin-2-yl)-4-(4-hydroxy-2-oxo-2H-chromen-3-yl)- $\mathrm{N}$-(quinolin-8-yl)hexanamide (56): The reaction was carried out according to the general procedure using alkene $\mathbf{S 1 3}$ (77.1 $\mathrm{mg}, 0.2 \mathrm{mmol}$ ), 4hydroxycoumarin (48.6 mg, $0.3 \mathrm{mmol}), p$-methoxybenzoic acid (15.2 mg, $0.1 \mathrm{mmol}$ ), Pd(OAc) 2 (4.4 mg, $0.02 \mathrm{mmol}$ ), and $\mathrm{MeCN}(0.1 \mathrm{~mL})$. The reaction was run for $3.5 \mathrm{~h}$, and then was diluted with $50 \mathrm{~mL}$ of EtOAc. The organic layer was washed with sat. $\mathrm{NaHCO}_{3}(50 \mathrm{~mL} \times 2)$, brine $(25 \mathrm{~mL})$, dried over $\mathrm{Na}_{2} \mathrm{SO}_{4}$, and concentrated under reduced pressure. The product was purified by flash column chromatography $\left(\mathrm{SiO}_{2}, 50-80 \%\right.$ EtOAc in Hexanes) to afford $65.8 \mathrm{mg}$ (60\%)

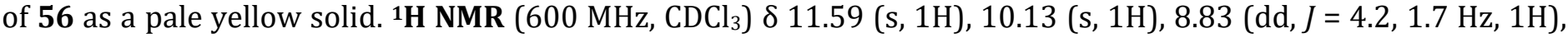
$8.72(\mathrm{dd}, J=7.6,1.4 \mathrm{~Hz}, 1 \mathrm{H}), 8.21(\mathrm{dd}, J=8.2,1.7 \mathrm{~Hz}, 1 \mathrm{H}), 7.94(\mathrm{dd}, J=8.0,1.6 \mathrm{~Hz}, 1 \mathrm{H}), 7.67-7.54(\mathrm{~m}, 6 \mathrm{H}), 7.53-7.45$ $(\mathrm{m}, 2 \mathrm{H}), 7.29-7.21(\mathrm{~m}, 2 \mathrm{H}), 3.77-3.69(\mathrm{~m}, 1 \mathrm{H}), 3.68-3.60(\mathrm{~m}, 1 \mathrm{H}), 3.34-3.27(\mathrm{~m}, 1 \mathrm{H}), 2.70-2.56(\mathrm{~m}, 2 \mathrm{H}), 2.53-2.45$ $(\mathrm{m}, 1 \mathrm{H}), 2.35(\mathrm{td}, J=13.4,3.4 \mathrm{~Hz}, 1 \mathrm{H}), 2.17(\mathrm{dq}, J=13.1,6.4 \mathrm{~Hz}, 1 \mathrm{H}), 2.11-2.03(\mathrm{~m}, 1 \mathrm{H}) .{ }^{13} \mathbf{C} \mathbf{~ N M R}\left(150 \mathrm{MHz} \mathrm{CDCl}_{3}\right) \delta$ $174.29,168.47,163.24,162.38,153.12,148.75,138.64,136.67,133.85$ (×2), 133.71, 132.19, 131.63, 128.13, 127.38, 124.22, 123.82, $123.28(\times 2), 122.97,122.11,117.78,117.27,116.29,104.60,36.97,35.72,32.88,30.97,28.73$. HRMS calcd. for $\mathrm{C}_{32} \mathrm{H}_{26} \mathrm{~N}_{3} \mathrm{O}_{6}+[\mathrm{M}+\mathrm{H}]+:$ 548.18161, Found: 548.1818 .
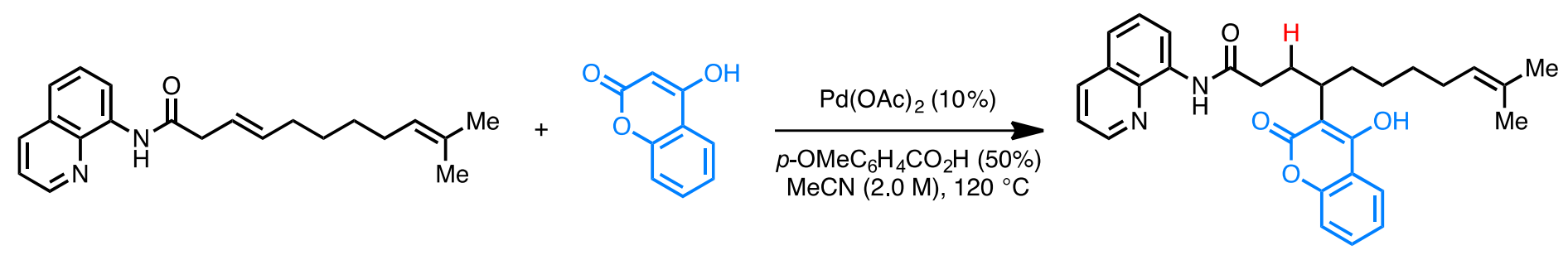

4-(4-hydroxy-2-oxo-2H-chromen-3-yl)-10-methyl- $\boldsymbol{N}$-(quinolin-8-yl) undec-9-enamide (57): The reaction was carried out according to the general procedure using alkene $\mathbf{S 1 4}$ (64.5 $\mathrm{mg}, 0.2 \mathrm{mmol}$ ), 4-hydroxycoumarin (48.6 $\mathrm{mg}$, $0.3 \mathrm{mmol}$ ), $p$-methoxybenzoic acid (15.2 $\mathrm{mg}, 0.1 \mathrm{mmol}$ ), $\mathrm{Pd}(\mathrm{OAc})_{2}(4.4 \mathrm{mg}, 0.02 \mathrm{mmol}$ ), and $\mathrm{MeCN}(0.1 \mathrm{~mL})$. The reaction was run for $4 \mathrm{~h}$, and then was diluted with $50 \mathrm{~mL}$ of EtOAc. The organic layer was washed with sat. $\mathrm{NaHCO}_{3}$ $(50 \mathrm{~mL} \times 2)$, brine $(25 \mathrm{~mL})$, dried over $\mathrm{Na}_{2} \mathrm{SO}_{4}$, and concentrated under reduced pressure. The product was purified by flash column chromatography $\left(\mathrm{SiO}_{2}, 25 \%\right.$ EtOAc in Hexanes) to afford $83.6 \mathbf{m g}(86 \%)$ of $\mathbf{5 7}$ as a yellow oil. ${ }^{\mathbf{1}} \mathbf{H}$ $\operatorname{NMR}\left(600 \mathrm{MHz}, \mathrm{CDCl}_{3}\right) \delta 11.54(\mathrm{~s}, 1 \mathrm{H}), 10.17(\mathrm{~s}, 1 \mathrm{H}), 8.86(\mathrm{dd}, J=4.2,1.7 \mathrm{~Hz}, 1 \mathrm{H}), 8.84(\mathrm{dd}, J=6.5,2.4 \mathrm{~Hz}, 1 \mathrm{H}), 8.23$ $(\mathrm{dd}, J=8.3,1.7 \mathrm{~Hz}, 1 \mathrm{H}), 8.02(\mathrm{dd}, J=7.9,1.4 \mathrm{~Hz}, 1 \mathrm{H}), 7.65-7.59(\mathrm{~m}, 2 \mathrm{H}), 7.56-7.50(\mathrm{~m}, 2 \mathrm{H}), 7.35-7.29(\mathrm{~m}, 2 \mathrm{H}), 5.06-$ $5.00(\mathrm{~m}, 1 \mathrm{H}), 3.35-3.27(\mathrm{~m}, 1 \mathrm{H}), 2.71(\mathrm{ddd}, J=13.8,5.6,2.5 \mathrm{~Hz}, 1 \mathrm{H}), 2.54-2.45(\mathrm{~m}, 1 \mathrm{H}), 2.40(\mathrm{td}, J=13.6,3.2 \mathrm{~Hz}, 1 \mathrm{H})$, 2.30-2.20 (m, 1H), 1.99-1.83 (m, 3H), 1.66-1.61 (m, 1H), $1.60(\mathrm{~s}, 3 \mathrm{H}), 1.52(\mathrm{~d}, J=1.2 \mathrm{~Hz}, 3 \mathrm{H}), 1.34-1.26(\mathrm{~m}, 2 \mathrm{H})$, 1.26-1.19 (m, 2H). ${ }^{13} \mathrm{C}$ NMR $\left(150 \mathrm{MHz}, \mathrm{CDCl}_{3}\right) \delta 174.89,162.96,162.43,153.16,148.78,138.63,136.72,133.68$, 
$131.45,131.39,128.17,127.44,124.96,124.18,123.77,123.05,122.15,117.71,117.35,116.29,105.58,36.03,35.22$, 32.37, 29.99, 29.47, 28.17, 28.16, 25.82, 17.81. HRMS calcd. for $\mathrm{C}_{30} \mathrm{H}_{33} \mathrm{~N}_{2} \mathrm{O}_{4}+[\mathrm{M}+\mathrm{H}]^{+}:$485.24348, Found: 485.2435.
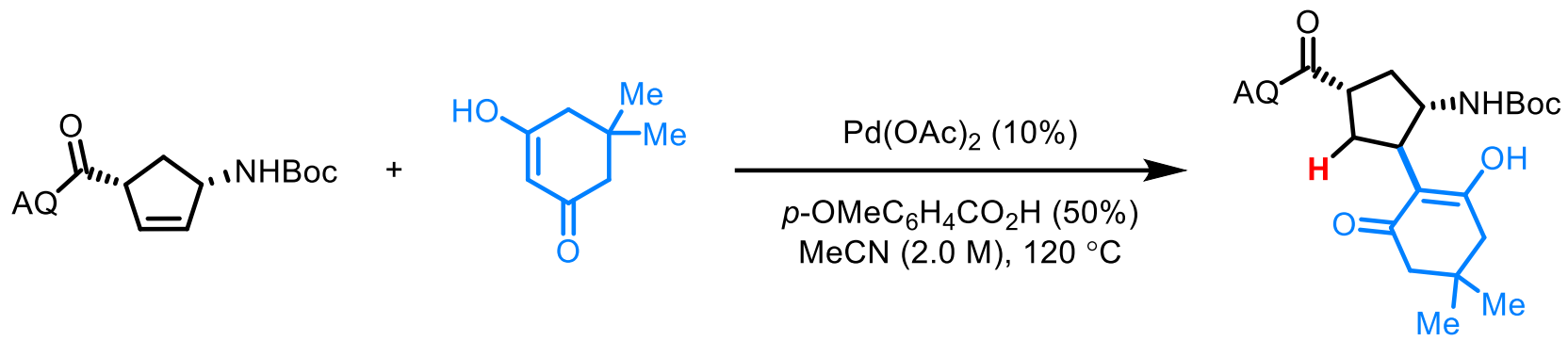

Tert-butyl((1S,2R,4S)-2-(2-hydroxy-4,4-dimethyl-6-oxocyclohex-1-en-1-yl)-4-(quinolin-8-

ylcarbamoyl)cyclopentyl)carbamate (58): The reaction was carried out according to the general procedure using alkene $\mathbf{S 1 0}$ (71.0 mg, $0.2 \mathrm{mmol}$ ), 4-hydroxycoumarin (48.6 mg, $0.3 \mathrm{mmol}$ ), $p$-methoxybenzoic acid (15.2 $\mathrm{mg}, 0.1$ $\mathrm{mmol}), \mathrm{Pd}(\mathrm{OAc})_{2}(4.4 \mathrm{mg}, 0.02 \mathrm{mmol})$, and $\mathrm{MeCN}(0.1 \mathrm{~mL})$. The reaction was run for $6 \mathrm{~h}$, and then was diluted with $50 \mathrm{~mL}$ of EtOAc. The organic layer was washed with sat. $\mathrm{NaHCO}_{3}(50 \mathrm{~mL} \times 2)$, brine $(25 \mathrm{~mL})$, dried over $\mathrm{Na}_{2} \mathrm{SO}_{4}$, and concentrated under reduced pressure. The product was purified by flash column chromatography $\left(\mathrm{SiO}_{2}, 50 \% \mathrm{EtOAc}\right.$ in Hexanes) to afford $58.9 \mathrm{mg}(60 \%)$ of $\mathbf{5 8}$ as a white solid. ${ }^{\mathbf{1}} \mathbf{H} \mathbf{~ N M R}\left(600 \mathrm{MHz}, \mathrm{CDCl}_{3}\right) \delta 10.76(\mathrm{~s}, 1 \mathrm{H}), 10.02(\mathrm{~s}, 1 \mathrm{H})$, $8.82(\mathrm{dd}, \mathrm{J}=4.2,1.6 \mathrm{~Hz}, 1 \mathrm{H}), 8.75(\mathrm{dd}, \mathrm{J}=7.2,1.7 \mathrm{~Hz}, 1 \mathrm{H}), 8.16(\mathrm{dd}, \mathrm{J}=8.2,1.7 \mathrm{~Hz}, 1 \mathrm{H}), 7.56-7.50(\mathrm{~m}, 2 \mathrm{H}), 7.46(\mathrm{dd}, \mathrm{J}$ $=8.2,4.1 \mathrm{~Hz}, 1 \mathrm{H}), 6.94(\mathrm{~d}, \mathrm{~J}=5.9 \mathrm{~Hz}, 1 \mathrm{H}), 3.84(\mathrm{q}, \mathrm{J}=9.6 \mathrm{~Hz}, 1 \mathrm{H}), 3.60(\mathrm{t}, \mathrm{J}=6.0 \mathrm{~Hz}, 1 \mathrm{H}), 3.22(\mathrm{~d}, \mathrm{~J}=8.9 \mathrm{~Hz}, 1 \mathrm{H}), 2.87$ (dd, J = 13.6, $9.9 \mathrm{~Hz}, 1 \mathrm{H}), 2.37(\mathrm{~s}, 2 \mathrm{H}), 2.26-2.09(\mathrm{~m}, 4 \mathrm{H}), 2.03(\mathrm{~d}, \mathrm{~J}=14.2 \mathrm{~Hz}, 1 \mathrm{H}), 1.48(\mathrm{~s}, 9 \mathrm{H}), 1.06(\mathrm{~d}, \mathrm{~J}=3.4 \mathrm{~Hz}, 6 \mathrm{H})$. ${ }^{13}$ C NMR $\left(150 \mathrm{MHz}, \mathrm{CDCl}_{3}\right) \delta 198.23,177.94,173.74,158.35,148.53,138.69,136.46,134.75,128.15,127.42,121.92$, 121.89, 116.64, 113.65, 80.87, 61.70, 52.41, 47.64, 44.52, 43.71, 33.86, 33.03, 31.73, 28.93, 28.66, 27.93. HRMS calcd. for $\mathrm{C}_{28} \mathrm{H}_{36} \mathrm{~N}_{3} \mathrm{O}_{5}+[\mathrm{M}+\mathrm{H}]+:$ 494.26495, Found: 494.2650 .
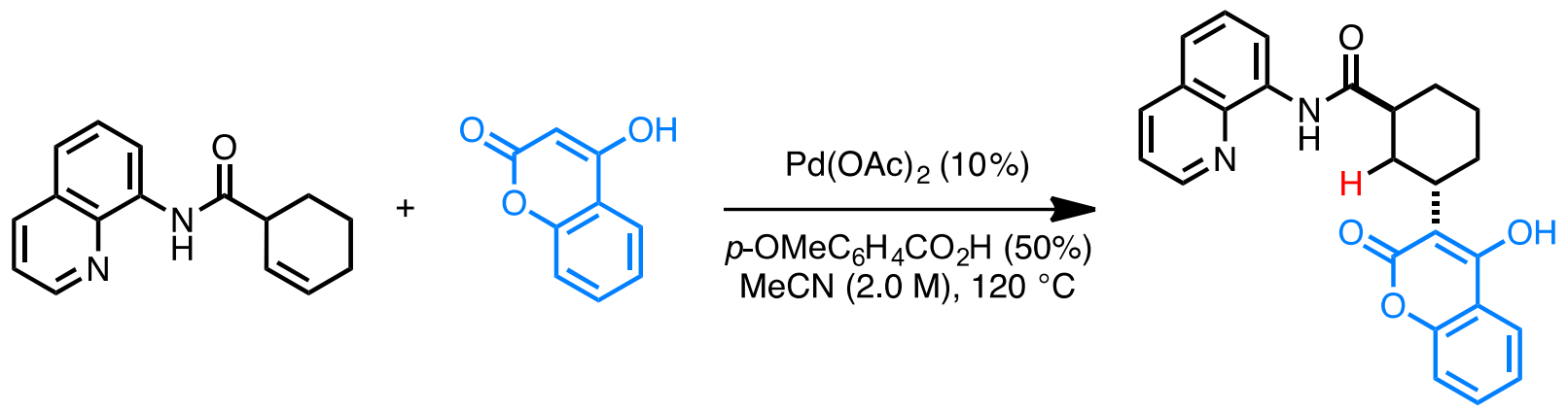

( \pm )-Trans-3-(4-hydroxy-2-oxo-2H-chromen-3-yl)- $N$-(quinolin-8-yl)cyclohexane-1-carboxamide (59): The reaction was carried out according to the general procedure using alkene S8 (50.4 $\mathrm{mg}, 0.2 \mathrm{mmol}$ ), 4hydroxycoumarin (48.6 mg, $0.3 \mathrm{mmol}), p$-methoxybenzoic acid (15.2 mg, $0.1 \mathrm{mmol}), \mathrm{Pd}(\mathrm{OAc})_{2}(4.4 \mathrm{mg}, 0.02 \mathrm{mmol})$, and $\mathrm{MeCN}(0.1 \mathrm{~mL})$. The reaction was run for $4 \mathrm{~h}$, and then was diluted with $50 \mathrm{~mL}$ of EtOAc. The organic layer was washed with sat. $\mathrm{NaHCO}_{3}(50 \mathrm{~mL} \times 2)$, brine $(25 \mathrm{~mL})$, dried over $\mathrm{Na}_{2} \mathrm{SO}_{4}$, and concentrated under reduced pressure. The product was purified by flash column chromatography $\left(\mathrm{SiO}_{2}, 40 \%\right.$ EtOAc in Hexanes) to afford $63.8 \mathrm{mg}(77 \%)$ of 59 as a yellow solid. ${ }^{1} \mathbf{H}$ NMR $\left(600 \mathrm{MHz}, \mathrm{CDCl}_{3}\right) \delta 10.31(\mathrm{~s}, 1 \mathrm{H}), 8.88-8.83(\mathrm{~m}, 2 \mathrm{H}), 8.20(\mathrm{dd}, J=8.2,1.7 \mathrm{~Hz}, 1 \mathrm{H})$, $7.97(\mathrm{dd}, J=7.8,1.1 \mathrm{~Hz}, 1 \mathrm{H}), 7.63-7.55(\mathrm{~m}, 2 \mathrm{H}), 7.53-7.47(\mathrm{~m}, 2 \mathrm{H}), 7.32-7.25(\mathrm{~m}, 3 \mathrm{H}), 3.22-3.17(\mathrm{~m}, 1 \mathrm{H}), 3.14(\mathrm{tt}, J$ $=11.9,2.5 \mathrm{~Hz}, 1 \mathrm{H}), 2.54-2.43(\mathrm{~m}, 1 \mathrm{H}), 2.40(\mathrm{~d}, J=14.5 \mathrm{~Hz}, 1 \mathrm{H}), 2.21(\mathrm{dt}, J=13.7,2.4 \mathrm{~Hz}, 1 \mathrm{H}), 2.09(\mathrm{tt}, J=14.3,4.5 \mathrm{~Hz}$, 1H), 2.00-1.90 (m, 3H), 1.55-1.44 (m, 1H). ${ }^{13}$ C NMR $\left(150 \mathrm{MHz}, \mathrm{CDCl}_{3}\right) \delta 175.76,162.29,161.29,153.17,148.75$, 138.72, 136.72, 134.08, 131.45, 128.23, 127.56, 123.78, 123.57, 122.34, 122.03, 117.11, 116.49, 116.42, 107.74, 41.92, 33.63, 32.83, 29.73, 29.65, 25.04. HRMS calcd. for $\mathrm{C}_{25} \mathrm{H}_{23} \mathrm{~N}_{2} \mathrm{O}_{4}+[\mathrm{M}+\mathrm{H}]^{+}:$415.16523, Found: 415.1654. X-ray (single-crystal) off-white needles of X-ray diffraction quality were obtained by vapor diffusion of pentane into a saturated solution of $\mathbf{5 9}$ in DCE (CCDC 1489658). 


\section{Reaction via 6-Membered Palladacycle}

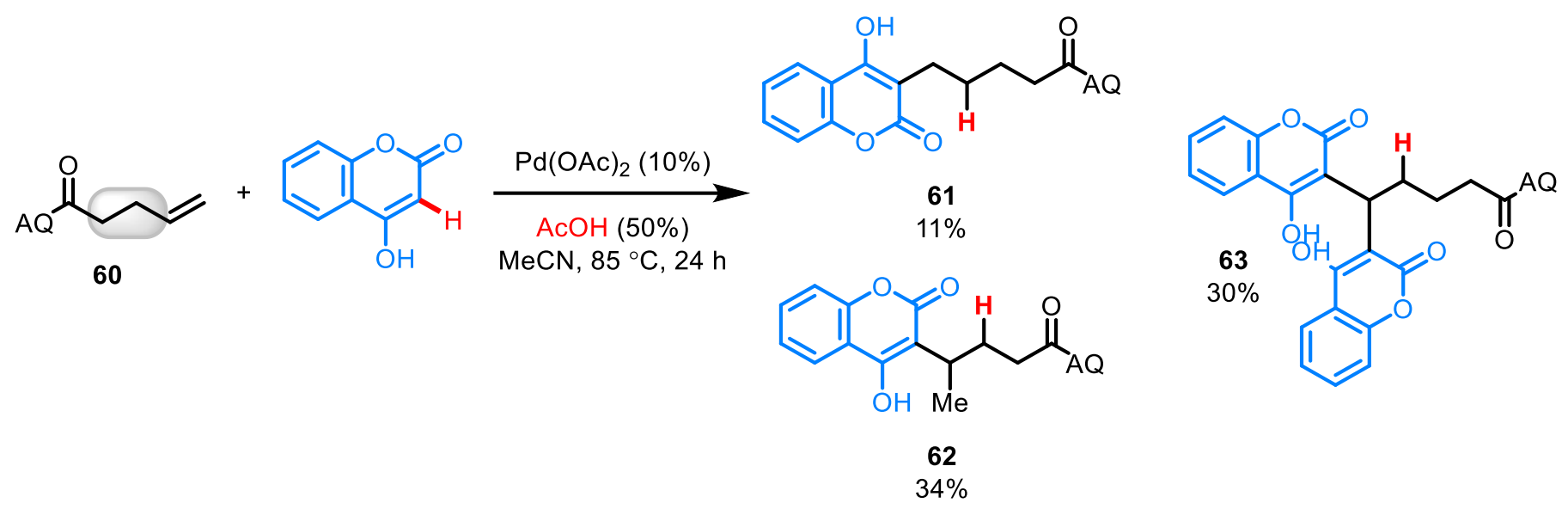

The reaction was carried out according to a modifided general procedure using alkene 60 (46.0 mg, $0.2 \mathrm{mmol}$ ), 4hydroxycoumarin (48.6 mg, $0.3 \mathrm{mmol})$, acetic acid (6.0 mg, $0.1 \mathrm{mmol}), \mathrm{Pd}(\mathrm{OAc})_{2}(4.4 \mathrm{mg}, 0.02 \mathrm{mmol})$, and $\mathrm{MeCN}(0.1$ $\mathrm{mL})$. The reaction was run for $24 \mathrm{~h}$ at $80^{\circ} \mathrm{C}$, and purified by flash column chromatography $\left(\mathrm{SiO}_{2}, 50-100 \%\right.$ EtOAc in Hexanes, then 1\% MeOH in EtOAc) to afford $8.7 \mathrm{mg} \mathrm{(11 \% )} \mathrm{of} \mathbf{6 1}$ as a white solid, $26 \mathrm{mg}(34 \%)$ of $\mathbf{6 2}$ as a white solid, and $33.0 \mathrm{mg}(30 \%)$ of 63 as a white solid.

Data for 61: ${ }^{1} \mathbf{H}$ NMR $\left(600 \mathrm{MHz}, \mathrm{CDCl}_{3}\right) \delta 10.11(\mathrm{~s}, 1 \mathrm{H}), 8.86-8.79(\mathrm{~m}, 2 \mathrm{H}), 8.20(\mathrm{dd}, \mathrm{J}=8.2,1.7 \mathrm{~Hz}, 1 \mathrm{H}), 8.00(\mathrm{dd}, \mathrm{J}=$ 7.8, $1.5 \mathrm{~Hz}, 1 \mathrm{H}), 7.61-7.56(\mathrm{~m}, 2 \mathrm{H}), 7.53-7.47(\mathrm{~m}, 2 \mathrm{H}), 7.33-7.28(\mathrm{~m}, 2 \mathrm{H}), 2.83(\mathrm{t}, \mathrm{J}=6.4 \mathrm{~Hz}, 2 \mathrm{H}), 2.76(\mathrm{t}, \mathrm{J}=7.7 \mathrm{~Hz}$, 2H), 1.95-1.88 (m, 2H), 1.84-1.76 (m, 2H). ${ }^{13}$ C NMR (150 MHz, CDCl $) \delta 173.83,164.47,161.13,152.71,148.67$, 138.56, 136.71, 133.88, 131.42, 128.17, 127.55, 123.86, 123.74, 122.70, 122.07, 117.39, 116.90, 116.48, 104.95, 37.07, 27.04, 23.37, 22.72. HRMS calcd. for $\mathrm{C}_{23} \mathrm{H}_{21} \mathrm{~N}_{2} \mathrm{O}_{4}{ }^{+}[\mathrm{M}+\mathrm{H}]^{+}: 389.14958$ Found: 389.1495

Data for 62: ${ }^{1} \mathrm{H}$ NMR $\left(600 \mathrm{MHz}, \mathrm{CDCl}_{3}\right) \delta 11.58(\mathrm{~s}, 1 \mathrm{H}), 10.16(\mathrm{~s}, 1 \mathrm{H}), 8.84(\mathrm{dd}, \mathrm{J}=4.2,1.7 \mathrm{~Hz}, 1 \mathrm{H}), 8.81(\mathrm{dd}, \mathrm{J}=6.6$, $2.3 \mathrm{~Hz}, 1 \mathrm{H}), 8.21$ (dd, J = 8.2, $1.7 \mathrm{~Hz}, 1 \mathrm{H}), 8.02-7.97(\mathrm{~m}, 1 \mathrm{H}), 7.63-7.58(\mathrm{~m}, 2 \mathrm{H}), 7.53-7.47(\mathrm{~m}, 2 \mathrm{H}), 7.32-7.25(\mathrm{~m}, 2 \mathrm{H})$, 3.45 (dtd, J = 13.7, 6.9, $3.9 \mathrm{~Hz}, 1 \mathrm{H}$ ), 2.70 (ddd, J = 13.9, 5.8, 2.4 Hz, 1H), 2.51 (dddd, J = 14.5, 11.8, 5.8, 3.1 Hz, $1 \mathrm{H}$ ), 2.37 $(\mathrm{td}, \mathrm{J}=13.6,3.1 \mathrm{~Hz}, 1 \mathrm{H}), 1.90(\mathrm{tt}, \mathrm{J}=13.7,2.9 \mathrm{~Hz}, 1 \mathrm{H}), 1.45(\mathrm{~d}, \mathrm{~J}=6.8 \mathrm{~Hz}, 3 \mathrm{H}) .{ }^{13} \mathrm{C}$ NMR $\left(150 \mathrm{MHz}, \mathrm{CDCl}_{3}\right) \delta 174.88$, 162.46, 162.03, 153.09, 148.78, 138.62, 136.72, 133.66, 131.47, 128.17, 127.44, 124.17, 123.77, 123.07, 122.16, 117.69, 116.27, 117.41, 106.90, 36.15, 30.75, 29.45, 18.69. HRMS calcd. for $\mathrm{C}_{23} \mathrm{H}_{21} \mathrm{~N}_{2} \mathrm{O}_{4}{ }^{+}[\mathrm{M}+\mathrm{H}]+$ : 389.14958 Found: 389.1495

Data for 63: ${ }^{1} \mathrm{H}$ NMR (600 MHz, $\left.\mathrm{CD}_{3} \mathrm{OD}\right) \delta 8.73(\mathrm{dd}, \mathrm{J}=4.2,1.7 \mathrm{~Hz}, 1 \mathrm{H}), 8.54(\mathrm{dd}, \mathrm{J}=7.6,1.3 \mathrm{~Hz}, 1 \mathrm{H}), 8.24(\mathrm{dd}, \mathrm{J}=8.3$, $1.7 \mathrm{~Hz}, 1 \mathrm{H}$ ), 7.93 (dd, J = 7.9, $1.6 \mathrm{~Hz}, 2 \mathrm{H}), 7.56(\mathrm{dd}, \mathrm{J}=8.3,1.3 \mathrm{~Hz}, 1 \mathrm{H}), 7.51-7.43(\mathrm{~m}, 4 \mathrm{H}), 7.25-7.17(\mathrm{~m}, 4 \mathrm{H}), 5.10(\mathrm{t}$, $\mathrm{J}=8.3 \mathrm{~Hz}, 1 \mathrm{H}), 2.64(\mathrm{t}, \mathrm{J}=7.7 \mathrm{~Hz}, 2 \mathrm{H}), 2.39(\mathrm{q}, \mathrm{J}=8.2 \mathrm{~Hz}, 2 \mathrm{H}), 1.84-1.75(\mathrm{~m}, 2 \mathrm{H}) .{ }^{13} \mathbf{C} \mathbf{N M R}\left(150 \mathrm{MHz}, \mathrm{CD}_{3} \mathrm{OD}\right) \delta 174.64$, 170.28, 168.51, 154.03, 149.84, 139.97, 137.52, 135.49, 132.01, 129.52, 127.92, 125.22, 124.35, 123.25, 122.89, 121.32, 118.27, 116.71, 105.73, 38.61, 33.20, 30.78, 25.91. HRMS calcd. for $\mathrm{C}_{32} \mathrm{H}_{25} \mathrm{~N}_{2} \mathrm{O}_{7^{+}}[\mathrm{M}+\mathrm{H}]^{+}: 549.16563$ Found: 549.1655

Tentatively assigned product distribution using 3-phenylcoumaranone as a nucleophile. 


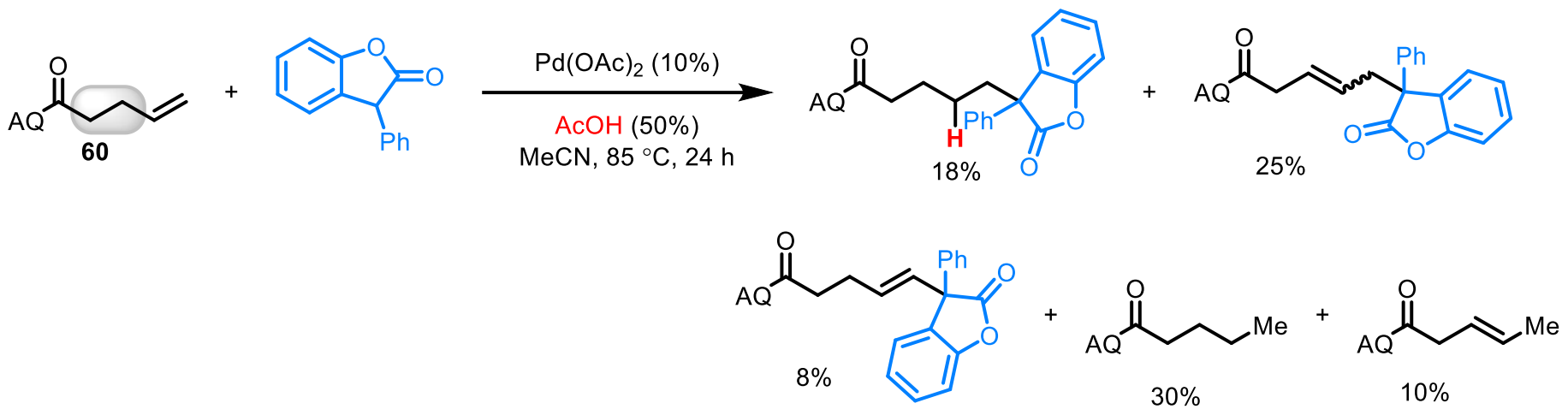




$$
\begin{aligned}
& \text { hro }=\mathrm{S}_{2}=0 \\
& =150= \\
& 3250=2450 \div 020
\end{aligned}
$$

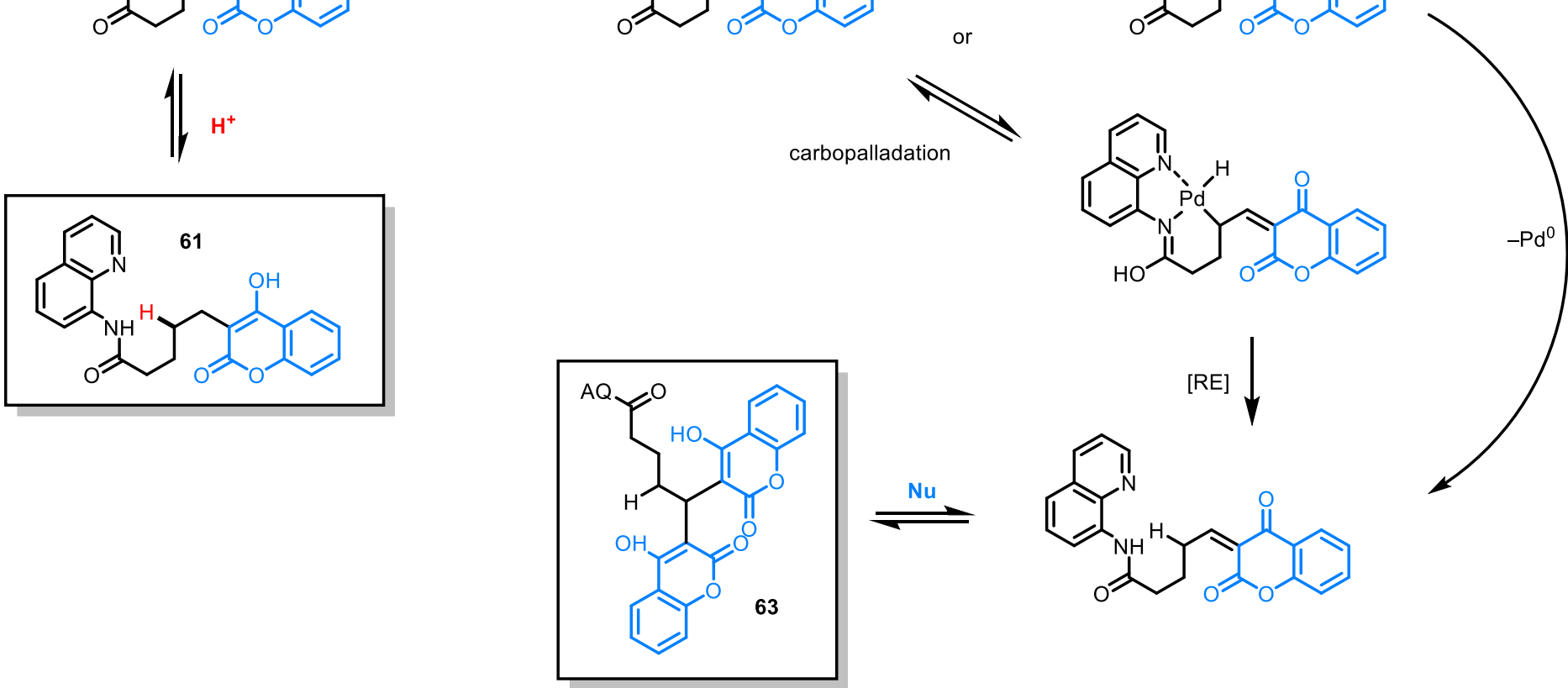


General Procedure for Evaluating Hydrofunctionalization of Dienes

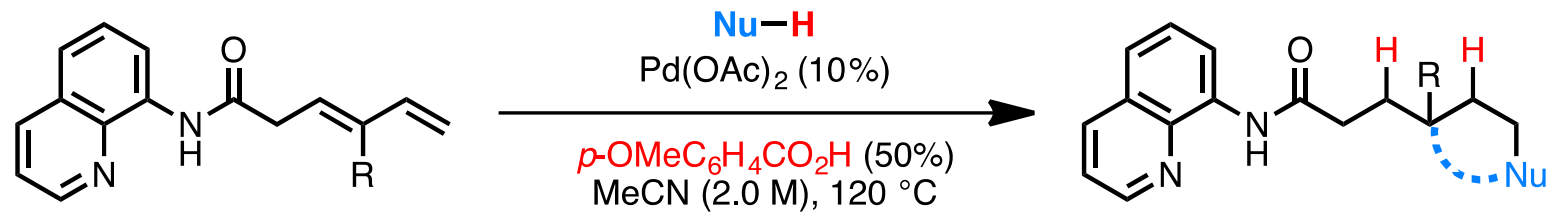

Unless otherwise stated, the procedure was as follows. To a 1 dram $(4 \mathrm{~mL})$ vial equipped with a magnetic stir bar was added $\mathrm{Pd}(\mathrm{OAc})_{2}(4.4 \mathrm{mg}, 0.02 \mathrm{mmol})$, diene 64 or $65(0.2 \mathrm{mmol}), p$-methoxybenzoic acid $(15.2 \mathrm{mg}, 0.1 \mathrm{mmol})$, carbon nucleophile $(0.3 \mathrm{mmol})$, and MeCN $(0.1 \mathrm{~mL})$. The vial was sealed with an unpunctured TFE septum-covered screw cap and placed in a heating block that was pre-heated to $120^{\circ} \mathrm{C}$. After the designated reaction time, the dark black reaction was diluted with EtOAc $(40 \mathrm{~mL})$ and washed with $\mathrm{NaHCO}_{3}(30 \mathrm{~mL}, \times 2)$ and brine $(25 \mathrm{~mL})$. The organic layer was dried over $\mathrm{Na}_{2} \mathrm{SO}_{4}$ and concentrated to afford a brown residue. Purification by flash column chromatography (EtOAc/Hexanes) produced the desired products. 
$\underbrace{(1)}_{M N}+$

HON
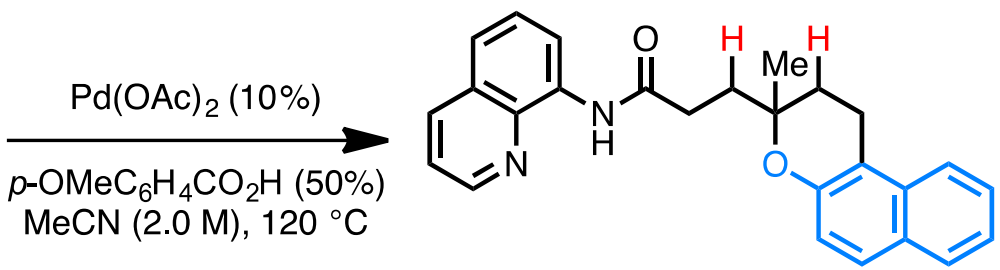

3-(3-methyl-2,3-dihydro-1H-benzo[f]chromen-3-yl)- $\mathbf{N}$-(quinolin-8-yl)propanamide (66): The reaction was carried out according to the general procedure using diene 65 (50.4 mg, $0.2 \mathrm{mmol}), 2$-naphthol ( $43.2 \mathrm{mg}, 0.3 \mathrm{mmol})$, $p$-methoxybenzoic acid (15.2 mg, $0.1 \mathrm{mmol}), \mathrm{Pd}(\mathrm{OAc})_{2}(4.4 \mathrm{mg}, 0.02 \mathrm{mmol})$, and $\mathrm{MeCN}(0.1 \mathrm{~mL})$. The reaction was run for $4 \mathrm{~h}$, and the product was purified by flash column chromatography ( $\mathrm{SiO}_{2}, 15-20 \%$ EtOAc in Hexanes) to afford $63.2 \mathrm{mg}(80 \%)$ of 66 as a yellow oil. ${ }^{1} \mathrm{H}$ NMR $\left(600 \mathrm{MHz}, \mathrm{CDCl}_{3}\right) \delta 9.85(\mathrm{~s}, 1 \mathrm{H}), 8.79-8.74(\mathrm{~m}, 2 \mathrm{H}), 8.15(\mathrm{dd}, J=8.3,1.7$ $\mathrm{Hz}, 1 \mathrm{H}), 7.82(\mathrm{dt}, J=8.6,1.0 \mathrm{~Hz}, 1 \mathrm{H}), 7.74(\mathrm{dt}, J=8.1,0.8 \mathrm{~Hz}, 1 \mathrm{H}), 7.59(\mathrm{~d}, J=8.9 \mathrm{~Hz}, 1 \mathrm{H}), 7.53(\mathrm{t}, J=7.9 \mathrm{~Hz}, 1 \mathrm{H}), 7.51-$ $7.46(\mathrm{~m}, 2 \mathrm{H}), 7.44(\mathrm{dd}, J=8.2,4.2 \mathrm{~Hz}, 1 \mathrm{H}), 7.36-7.30(\mathrm{~m}, 1 \mathrm{H}), 7.04(\mathrm{~d}, J=8.9 \mathrm{~Hz}, 1 \mathrm{H}), 3.13(\mathrm{dt}, J=16.8,6.1 \mathrm{~Hz}, 1 \mathrm{H})$, 3.09-3.01 (m, 1H), 2.84-2.78 (m, 2H), 2.29 (ddd, $J=14.2,8.9,7.1 \mathrm{~Hz}, 1 \mathrm{H}), 2.18$ (ddd, $J=14.2,8.8,7.2 \mathrm{~Hz}, 1 \mathrm{H}$ ), 2.08 (ddd, $J=14.4,8.3,6.4 \mathrm{~Hz}, 1 \mathrm{H}), 2.01(\mathrm{dt}, J=13.6,6.3 \mathrm{~Hz}, 1 \mathrm{H}), 1.40(\mathrm{~s}, 3 \mathrm{H}) .{ }^{13} \mathbf{C} \mathbf{N M R}\left(150 \mathrm{MHz}, \mathrm{CDCl}_{3}\right) \delta 171.78,151.24$, $148.31,138.54,136.55,134.77,133.23,128.98,128.58,128.15,127.97,127.65,126.44,123.25,122.07,121.80$, 121.58, 119.89, 116.63, 112.72, 75.31, 35.09, 32.52, 31.42, 23.63, 19.18. HRMS calcd. for $\mathrm{C}_{26} \mathrm{H}_{25} \mathrm{~N}_{2} \mathrm{O}_{2}+[\mathrm{M}+\mathrm{H}]^{+}$: 397.19105, Found: 397.1911.<smiles>C=CC(C)=CCC(=O)Nc1cccc2cccnc12</smiles>

3-(5-hydroxy-2-methyl-3,4-dihydro-2H-pyrano[2,3-b]pyridin-2-yl)- $N$-(quinolin-8-yl)propanamide The reaction was carried out according to the general procedure using diene 65 (50.4 mg $0.2 \mathrm{mmol})$, pyridine diol (33.3 mg, $0.3 \mathrm{mmol}), p$-methoxybenzoic acid (15.2 mg, $0.1 \mathrm{mmol}), \mathrm{Pd}(\mathrm{OAc})_{2}(4.4 \mathrm{mg}, 0.02 \mathrm{mmol})$, and $\mathrm{MeCN}(0.1$ $\mathrm{mL})$. The reaction was run for $4 \mathrm{~h}$, and the product was purified by flash column chromatography $\left(\mathrm{SiO}_{2}, 5 \% \mathrm{MeOH}\right.$ in EtOAc) to afford $51.2 \mathrm{mg}(70 \%)$ of $\mathbf{6 7}$ as a yellow oil. $1 \mathrm{H} \mathrm{NMR}\left(600 \mathrm{MHz}, \mathrm{CDCl}_{3}\right) \delta 11.81(\mathrm{~s}, 1 \mathrm{H}), 9.84(\mathrm{~s}, 1 \mathrm{H}), 8.80$ $(\mathrm{dd}, J=4.2,1.7 \mathrm{~Hz}, 1 \mathrm{H}), 8.76(\mathrm{dd}, J=7.5,1.5 \mathrm{~Hz}, 1 \mathrm{H}), 8.16(\mathrm{dd}, J=8.2,1.7 \mathrm{~Hz}, 1 \mathrm{H}), 7.58-7.48(\mathrm{~m}, 2 \mathrm{H}), 7.46(\mathrm{dd}, J=8.2$, $4.2 \mathrm{~Hz}, 1 \mathrm{H}), 7.10(\mathrm{~d}, J=7.2 \mathrm{~Hz}, 1 \mathrm{H}), 5.87(\mathrm{~d}, J=7.2 \mathrm{~Hz}, 1 \mathrm{H}), 2.74(\mathrm{t}, J=7.9 \mathrm{~Hz}, 2 \mathrm{H}), 2.65(\mathrm{dt}, J=17.4,5.8 \mathrm{~Hz}, 1 \mathrm{H}), 2.58-$ $2.49(\mathrm{~m}, 1 \mathrm{H}), 2.27-2.19(\mathrm{~m}, 1 \mathrm{H}), 2.19-2.11(\mathrm{~m}, 1 \mathrm{H}), 1.93-1.82(\mathrm{~m}, 2 \mathrm{H}), 1.36(\mathrm{~s}, 3 \mathrm{H}) .{ }^{13} \mathrm{C}$ NMR $\left(150 \mathrm{MHz} \mathrm{CDCl}_{3}\right) \delta$ 171.30, 165.58, 162.67, 148.36, 138.51, 136.62, 134.67, 132.02, 128.16, 127.66, 121.84, 121.69, 116.67, 106.79, 101.91, 77.57, 35.33, 32.33, 30.63, 23.70, 16.69. HRMS calcd. for $\mathrm{C}_{21} \mathrm{H}_{22} \mathrm{~N}_{3} \mathrm{O}_{3}+[\mathrm{M}+\mathrm{H}]^{+}:$364.16557, Found: 364.1657.

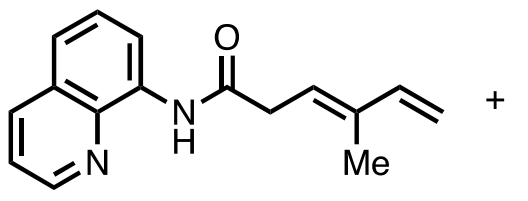<smiles>Oc1ccc2[nH]ccc2c1</smiles>

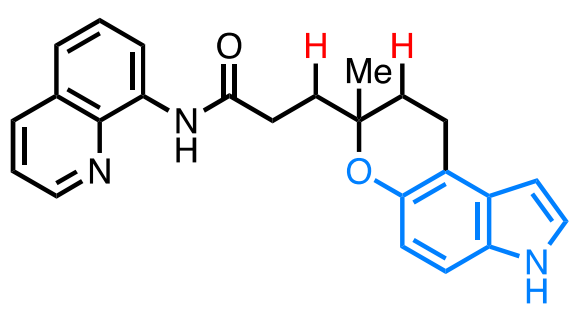

3-(7-methyl-3,7,8,9-tetrahydropyrano[3,2-e]indol-7-yl)- $\mathbf{N}$-(quinolin-8-yl)propanamide (68): The reaction was carried out according to the general procedure using diene 65 (50.4 mg, $0.2 \mathrm{mmol}), 1 \mathrm{H}$-indol-5-ol (40.0 mg, 0.3 mmol), $p$-methoxybenzoic acid (15.2 mg, $0.1 \mathrm{mmol}$ ), Pd(OAc) 2 (4.4 mg, $0.02 \mathrm{mmol}$ ), and MeCN (0.1 mL). The reaction was run for $4 \mathrm{~h}$, and the product was purified by flash column chromatography $\left(\mathrm{SiO}_{2}, 25-30 \%\right.$ EtOAc in Hexanes) to afford $29.0 \mathrm{mg}(38 \%)$ of $\mathbf{6 8}$ as a yellow solid. ${ }^{1} \mathrm{H} \mathbf{~ N M R}\left(600 \mathrm{MHz}, \mathrm{CDCl}_{3}\right) \delta 9.85(\mathrm{~s}, 1 \mathrm{H}), 8.84-8.72(\mathrm{~m}, 2 \mathrm{H}), 8.15(\mathrm{dd}, J$ $=8.3,1.7 \mathrm{~Hz}, 1 \mathrm{H}), 8.06(\mathrm{~s}, 1 \mathrm{H}), 7.53(\mathrm{t}, J=7.9 \mathrm{~Hz}, 1 \mathrm{H}), 7.49(\mathrm{dd}, J=8.2,1.5 \mathrm{~Hz}, 1 \mathrm{H}), 7.44(\mathrm{dd}, J=8.2,4.2 \mathrm{~Hz}, 1 \mathrm{H}), 7.17$ $(\mathrm{t}, J=2.8 \mathrm{~Hz}, 1 \mathrm{H}), 7.13(\mathrm{dd}, J=8.7,0.9 \mathrm{~Hz}, 1 \mathrm{H}), 6.75(\mathrm{~d}, J=8.7 \mathrm{~Hz}, 1 \mathrm{H}), 6.45$ (ddd, $J=3.1,2.1,1.0 \mathrm{~Hz}, 1 \mathrm{H}$ ), $3.04-2.93$ (m, 2H), 2.79 (ddd, $J=8.9,6.6,1.9 \mathrm{~Hz}, 2 \mathrm{H}$ ), 2.27 (ddd, $J=14.0,9.2,6.9 \mathrm{~Hz}, 1 \mathrm{H}$ ), 2.14 (ddd, $J=14.1,9.1,7.1 \mathrm{~Hz}, 1 \mathrm{H}$ ), 2.01 (ddd, $J=14.3,8.0,6.7 \mathrm{~Hz}, 1 \mathrm{H}), 1.93(\mathrm{dt}, J=13.5,6.3 \mathrm{~Hz}, 1 \mathrm{H}), 1.38(\mathrm{~s}, 3 \mathrm{H}) .{ }^{13} \mathrm{C} \mathbf{~ N M R}\left(150 \mathrm{MHz}, \mathrm{CDCl}_{3}\right) \delta 171.39$, $147.63,146.49,137.88,135.84,134.17,129.61,127.47,126.97,126.93,123.71,121.09,120.83,115.93,113.18$, 
110.55, 109.42, 99.71, 74.09, 34.44, 31.91, 30.76, 23.14, 19.19. HRMS calcd. for $\mathrm{C}_{24} \mathrm{H}_{24} \mathrm{~N}_{3} \mathrm{O}_{2}+[\mathrm{M}+\mathrm{H}]^{+}: 386.18630$, Found: 386.1864.<smiles>C=C/C=C/CC(=O)Nc1cccc2cccnc12</smiles>

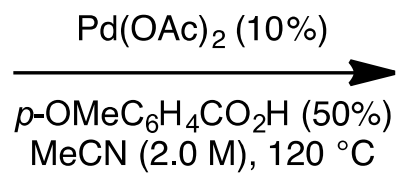<smiles>COc1cc2c(cc1OC)OC(CCC(=O)Nc1cccc3cccnc13)CC2</smiles>

3-(6,7-dimethoxychroman-2-yl)- $\mathbf{N}$-(quinolin-8-yl)propanamide (69): The reaction was carried out according to the general procedure using diene $64(47.7 \mathrm{mg}, 0.2 \mathrm{mmol}$ ), 3,4-dimethoxyphenol (46.3 $\mathrm{mg}, 0.3 \mathrm{mmol}$ ), pmethoxybenzoic acid (15.2 mg, $0.1 \mathrm{mmol}), \mathrm{Pd}(\mathrm{OAc})_{2}(4.4 \mathrm{mg}, 0.02 \mathrm{mmol})$, and $\mathrm{MeCN}(0.1 \mathrm{~mL})$. The reaction was run for $4 \mathrm{~h}$, and the product was purified by flash column chromatography ( $\mathrm{SiO}_{2}, 33 \%$ EtOAc in Hexanes) to afford 40.0 mg (51\%) of 69 as a yellow oil. ${ }^{1} \mathbf{H}$ NMR $\left(600 \mathrm{MHz}, \mathrm{CDCl}_{3}\right) \delta 9.91(\mathrm{~s}, 1 \mathrm{H}), 8.79(\mathrm{dd}, J=7.5,1.4 \mathrm{~Hz}, 1 \mathrm{H}), 8.78(\mathrm{dd}, J=$ $4.2,1.7 \mathrm{~Hz}, 1 \mathrm{H}), 8.16(\mathrm{dd}, J=8.2,1.7 \mathrm{~Hz}, 1 \mathrm{H}), 7.57-7.48(\mathrm{~m}, 2 \mathrm{H}), 7.45(\mathrm{dd}, J=8.3,4.2 \mathrm{~Hz}, 1 \mathrm{H}), 6.53(\mathrm{~s}, 1 \mathrm{H}), 6.38(\mathrm{~s}$, 1H), 4.11-4.04 (m, 1H), $3.80(\mathrm{~s}, 3 \mathrm{H}), 3.75(\mathrm{~s}, 3 \mathrm{H}), 2.90-2.76(\mathrm{~m}, 3 \mathrm{H}), 2.67$ (ddd, $J=16.2,5.7,3.0 \mathrm{~Hz}, 1 \mathrm{H}), 2.25-2.18$ (m, 1H), 2.18-2.10 (m, 1H), 2.07-2.00 (m, 1H), 1.85-1.75 (m, 1H). ${ }^{13} \mathbf{C}$ NMR $\left(150 \mathrm{MHz}, \mathrm{CDCl}_{3}\right) \delta 171.64,148.79$, $148.39,148.34,143.15,138.56,136.58,134.80,128.18,127.66,121.83,121.62,116.66,112.58,112.48,101.16$, 75.06, 56.66, 56.01, 34.18, 31.27, 28.08, 24.53. HRMS calcd. for $\mathrm{C}_{23} \mathrm{H}_{25} \mathrm{~N}_{2} \mathrm{O}_{4}+[\mathrm{M}+\mathrm{H}]+$ : 393.18088, Found: 393.1806.
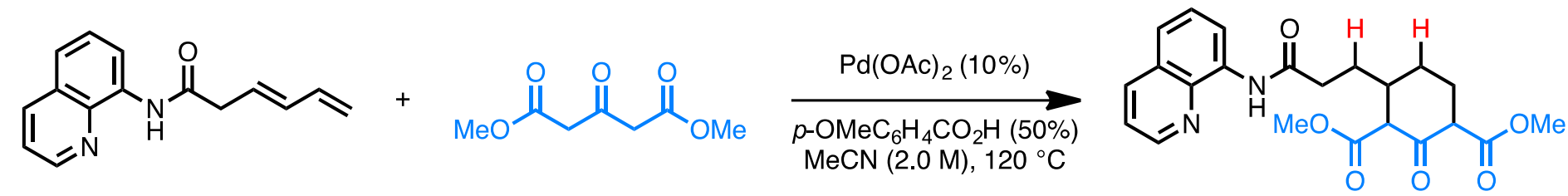<smiles>O=C(O)CC1CCCCC1</smiles><smiles>CCC(C)C(=O)O[Na]</smiles>

Dimethyl-2-oxo-4-(3-oxo-3-(quinolin-8-ylamino)propyl)cyclohexane-1,3-dicarboxylate (70): The reaction was carried out according to the general procedure using diene 64 (50.4 mg, $0.2 \mathrm{mmol}$ ), dimethyl 3oxopentanedioate ( $44.1 \mathrm{mg}, 0.3 \mathrm{mmol}), p$-methoxybenzoic acid (15.2 mg, $0.1 \mathrm{mmol}), \mathrm{Pd}(\mathrm{OAc})_{2}(4.4 \mathrm{mg}, 0.02 \mathrm{mmol}$ ), and $\mathrm{MeCN}(0.1 \mathrm{~mL})$. The reaction was run for $4 \mathrm{~h}$, and the product was purified by flash column chromatography $\left(\mathrm{SiO}_{2}, 20-30 \%\right.$ EtOAc in Hexanes) to afford $41.0 \mathrm{mg}$ (50\%) of 70 as a yellow oil. Proton and carbon revealed a complex mixture of enol/keto tautomers. HRMS calcd. for $\mathrm{C}_{20} \mathrm{H}_{23} \mathrm{~N}_{2} \mathrm{O}_{3}+[\mathrm{M}+\mathrm{H}]+:$ 413.17071, Found: 413.1709. Hydrolysis and decarboxylation gave the desired acid. ${ }^{1} \mathbf{H}$ NMR $\left(600 \mathrm{MHz}_{2} \mathrm{CDCl}_{3}\right) \delta 2.47(\mathrm{~m}, 1 \mathrm{H}), 2.44-2.35(\mathrm{~m}, 3 \mathrm{H}), 2.29(\mathrm{~m}, 1 \mathrm{H})$, 2.13-1.99 (m, 2H), $1.95(\mathrm{~m}, 1 \mathrm{H}), 1.85(\mathrm{~m}, 1 \mathrm{H}), 1.79-1.63(\mathrm{~m}, 3 \mathrm{H}), 1.38(\mathrm{~m}, 1 \mathrm{H})$. 
S36<smiles>C=C/C=C/CC(=O)Nc1cccc2cccnc12</smiles><smiles>CC1(C)CC(=O)C=C(O)C1</smiles>

$$
\underset{\substack{p-\mathrm{OMeC}_{6} \mathrm{H}_{4} \mathrm{CO}_{2} \mathrm{H}(50 \%) \\ \operatorname{MeCN}(2.0 \mathrm{M}), 120{ }^{\circ} \mathrm{C}}}{\stackrel{\mathrm{Pd}(\mathrm{OAc})_{2}(10 \%)}{\longrightarrow}}
$$<smiles></smiles>

3-(7,7-dimethyl-5-oxo-3,4,5,6,7,8-hexahydro-2H-chromen-2-yl)- $N$-(quinolin-8-yl)propanamide (71): The reaction was carried out according to the general procedure using diene 64 (47.7 $\mathrm{mg}, 0.2 \mathrm{mmol})$, dimedone (42.0 $\mathrm{mg}, 0.3 \mathrm{mmol}$ ), $p$-methoxybenzoic acid (15.2 mg, $0.1 \mathrm{mmol}$ ), $\mathrm{Pd}(\mathrm{OAc})_{2}(4.4 \mathrm{mg}, 0.02 \mathrm{mmol})$, and $\mathrm{MeCN}(0.1 \mathrm{~mL})$. The reaction was run for $4 \mathrm{~h}$, and the product was purified by flash column chromatography $\left(\mathrm{SiO}_{2}, 50 \%\right.$ EtOAc in Hexanes) to afford $40.4 \mathrm{mg}(53 \%)$ of $\mathbf{7 1}$ as a yellow solid. ${ }^{1} \mathbf{H} \mathbf{~ N M R}\left(600 \mathrm{MHz}, \mathrm{CDCl}_{3}\right) \delta 9.85(\mathrm{~s}, 1 \mathrm{H}), 8.81(\mathrm{dd}, J=4.2$, $1.7 \mathrm{~Hz}, 1 \mathrm{H}), 8.78(\mathrm{dd}, J=7.4,1.5 \mathrm{~Hz}, 1 \mathrm{H}), 8.18(\mathrm{dd}, J=8.2,1.7 \mathrm{~Hz}, 1 \mathrm{H}), 7.58-7.49(\mathrm{~m}, 2 \mathrm{H}), 7.47(\mathrm{dd}, J=8.3,4.2 \mathrm{~Hz}, 1 \mathrm{H})$, 4.11-4.05 (m, 1H), 2.82-2.73 (m, 2H), 2.47-2.39 (m,1H), 2.28-2.07 (m, 7H), 2.06-1.97 (m, 1H), 1.61 (dtd, $J=13.7$, 10.5, 5.6 Hz, 1H), $1.01(\mathrm{~d}, J=6.2 \mathrm{~Hz}, 6 \mathrm{H}) .{ }^{13} \mathbf{C}$ NMR $\left(150 \mathrm{MHz}, \mathrm{CDCl}_{3}\right) \delta 198.16,171.12,169.58,148.35,138.53,136.68$, 134.64, 128.20, 127.68, 121.87, 121.77, 116.70, 110.34, 76.77, 50.82, 42.59, 33.85, 32.18, 30.68, 29.33, 27.90, 27.03, 17.59. HRMS calcd. for $\mathrm{C}_{23} \mathrm{H}_{27} \mathrm{~N}_{2} \mathrm{O}_{3}+[\mathrm{M}+\mathrm{H}]+:$ 379.20162, Found: 379.2017. X-ray (single-crystal) off-white blocks of $\mathrm{X}$-ray diffraction quality were obtained by vapor diffusion of pentane into a saturated solution of 71 in DCM (CCDC 1489656).

Reaction of 2-methylcyclohexane-1,3-dione and Methyl Cyanoacetates with Diene<smiles>C=CC=CCC(=O)OCc1ccccc1</smiles><smiles>COC(=O)CCC(C)CC(=O)OC</smiles><smiles>COC(=O)C(C#N)CC/C=C/CC(=O)O</smiles><smiles>COC(=O)CC/C=C/CC(C#N)C(=O)OC</smiles>

$19 \%$

$4.5 \%$<smiles>C=CC=CCC(=O)OCc1ccccc1</smiles><smiles>CC1C(=O)CCCC1=O</smiles>

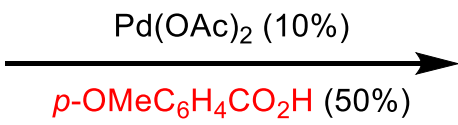<smiles>CC1(CC/C=C/CC(=O)O[Na])C(=O)CCCC1=O</smiles><smiles>CC1(C/C=C/CCC(=O)OCCCCCCCCO)C(=O)CCCC1=O</smiles>

$\mathrm{MeCN}, 120^{\circ} \mathrm{C}$

$20 \%$

$5 \%$ 


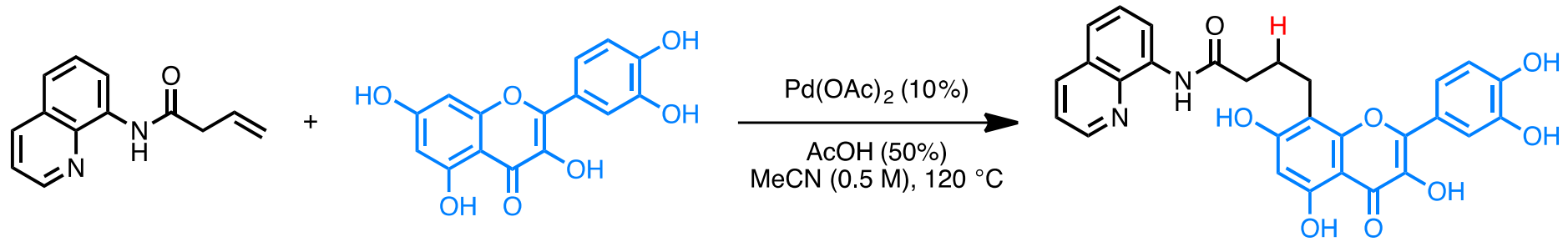

4-(2-(3,4-dihydroxyphenyl)-3,5,7-trihydroxy-4-oxo-4H-chromen-8-yl)- $\mathbf{N}$-(quinolin-8-yl)butanamide (72): To a 1 dram ( $4 \mathrm{~mL}$ ) vial equipped with a magnetic stir bar were added alkene $\mathbf{1}$ (21.2 mg, $0.1 \mathrm{mmol})$, quercetin (30.2 $\mathrm{mg}, 0.1 \mathrm{mmol})$, acetic acid (3.0 mg, $0.05 \mathrm{mmol}), \mathrm{Pd}(\mathrm{OAc})_{2}(2.2 \mathrm{mg}, 0.01 \mathrm{mmol})$, and $\mathrm{MeCN}(0.2 \mathrm{~mL})$. The reaction was run for $6 \mathrm{~h}$, and the product was purified by preparative TLC $\left(7 \% \mathrm{MeOH}\right.$ in $\left.\mathrm{CHCl}_{3}\right)$ to afford $27.2 \mathrm{mg}(53 \%)$ of 72 as a yellow solid. ${ }^{1}$ H NMR $\left(600 \mathrm{MHz}\right.$, DMSO- $\left.d_{6}\right) \delta 12.42(\mathrm{~s}, 1 \mathrm{H}), 10.00(\mathrm{~s}, 1 \mathrm{H}), 8.87(\mathrm{dd}, J=4.2,1.7 \mathrm{~Hz}, 1 \mathrm{H}), 8.59(\mathrm{~d}, J=$ $7.7 \mathrm{~Hz}, 1 \mathrm{H}), 8.38(\mathrm{dd}, J=8.2,1.7 \mathrm{~Hz}, 1 \mathrm{H}), 7.76(\mathrm{~d}, J=2.2 \mathrm{~Hz}, 1 \mathrm{H}), 7.66-7.56(\mathrm{~m}, 3 \mathrm{H}), 7.55(\mathrm{t}, J=7.9 \mathrm{~Hz}, 1 \mathrm{H}), 6.88(\mathrm{~d}, J$ $=8.5 \mathrm{~Hz}, 1 \mathrm{H}), 6.28(\mathrm{~s}, 1 \mathrm{H}), 2.87(\mathrm{t}, J=7.3 \mathrm{~Hz}, 2 \mathrm{H}), 2.63(\mathrm{t}, J=7.5 \mathrm{~Hz}, 2 \mathrm{H}), 1.97(\mathrm{p}, J=7.4 \mathrm{~Hz}, 2 \mathrm{H}) .{ }^{13} \mathrm{C}$ NMR $(150 \mathrm{MHz}$, DMSO-d6) $\delta 176.05,171.50,161.64,158.31,153.63,148.74,147.63,146.52,145.03,138.03,136.51,135.52,134.49$, $127.77,126.89,122.31,122.00,121.66,119.75,116.61,115.73,114.99,105.54,102.88,97.74,36.39,24.65,21.65$. HRMS calcd. for $\mathrm{C}_{28} \mathrm{H}_{23} \mathrm{~N}_{2} \mathrm{O}_{8}+[\mathrm{M}+\mathrm{H}]+:$ 515.14489, Found: 515.1449.
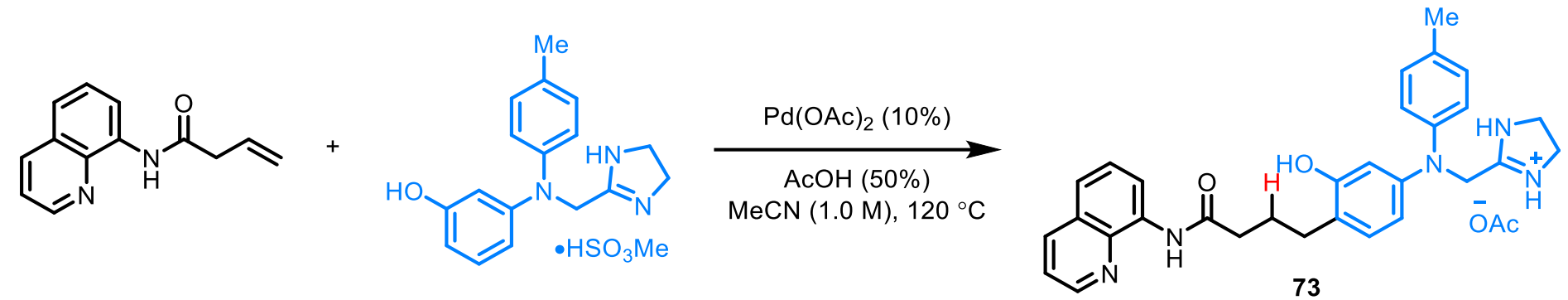

2-(((3-hydroxy-4-(4-oxo-4-(quinolin-8-ylamino)butyl)phenyl)( $p$-tolyl)amino)methyl)-4,5-dihydro-1H-

imidazol-3-ium acetate (73): To a 1 dram ( $4 \mathrm{~mL}$ ) vial equipped with a magnetic stir bar were added alkene 1 (21.2 $\mathrm{mg}, 0.1 \mathrm{mmol}$ ), phentolamine mesylate (38 mg, $0.1 \mathrm{mmol}$ ), acetic acid (3.0 mg, $0.05 \mathrm{mmol}), \mathrm{Pd}(\mathrm{OAc}) 2(2.2 \mathrm{mg}, 0.01$ mmol), and MeCN (0.1 mL). The reaction was run for $4 \mathrm{~h}$, and allowed to cool to ambient temperatures. The dark brown solution was diluted in water $(30 \mathrm{~mL})$ and $\mathrm{MeOH}(3 \mathrm{~mL})$ and then washed with a mixture of Et $\mathrm{O}_{2} \mathrm{O}$ and $\mathrm{Hexanes}$ (1:1, $20 \mathrm{~mL})$. The aqueous phase was charged with sat. $\mathrm{NaHCO}_{3}(30 \mathrm{~mL})$ and saturated with solid $\mathrm{NaCl}$. The aqueous phase was extracted with DCM $(30 \mathrm{~mL}, \times 3)$. The combined organic phases were dried over $\mathrm{Na}_{2} \mathrm{SO}_{4}$, filtered, and treated with $\mathrm{AcOH}$ (60 mg, $1.0 \mathrm{mmol}$ ). The organics were concentrated and the product was purified by preparative TLC (15\% MeOH in EtOAc) to afford $18.0 \mathrm{mg}(33 \%)$ of 73 as a brown solid. ${ }^{\mathbf{1}} \mathbf{H} \mathbf{~ N M R}\left(600 \mathrm{MHz}, \mathrm{CDCl}_{3}\right) \delta 9.85(\mathrm{~s}, 1 \mathrm{H})$, 8.82-8.65 (m, 2H), 8.29 (br s, 2H), 8.14 (dd, J = 8.2, $1.7 \mathrm{~Hz}, 1 \mathrm{H}), 7.56-7.46(\mathrm{~m}, 2 \mathrm{H}), 7.43(\mathrm{dd}, \mathrm{J}=8.3,4.2 \mathrm{~Hz}, 1 \mathrm{H}), 7.07$ $(\mathrm{d}, \mathrm{J}=8.2 \mathrm{~Hz}, 2 \mathrm{H}), 6.99-6.93(\mathrm{~m}, 2 \mathrm{H}), 6.86(\mathrm{~d}, \mathrm{~J}=8.2 \mathrm{~Hz}, 1 \mathrm{H}), 6.67$ (d, J = 2.5 Hz, 1H), 6.27 (dd, J = 8.2, $2.4 \mathrm{~Hz}, 1 \mathrm{H}), 4.52$ $(\mathrm{s}, 2 \mathrm{H}), 3.71(\mathrm{~s}, 4 \mathrm{H}), 2.66(\mathrm{t}, \mathrm{J}=7.4 \mathrm{~Hz}, 2 \mathrm{H}), 2.55(\mathrm{t}, \mathrm{J}=7.3 \mathrm{~Hz}, 2 \mathrm{H}), 2.28(\mathrm{~s}, 3 \mathrm{H}), 2.08-1.93(\mathrm{~m}, 5 \mathrm{H}) .{ }^{13} \mathbf{C ~ N M R}(150 \mathrm{MHz}$, $\left.\mathrm{CDCl}_{3}\right) \delta 177.86,172.74,169.72,156.93,148.43,146.85,145.29,136.56,134.56,133.27,130.80,130.47,130.40$, $128.14,127.57,122.64,121.84,121.81,121.32,116.87,110.67,106.07,50.65,47.24,37.27,29.09,26.26,23.62$, 20.94. HRMS calcd. for $\mathrm{C}_{30} \mathrm{H}_{32} \mathrm{~N}_{5} \mathrm{O}_{2}+[\mathrm{M}+\mathrm{H}]^{+}:$494.25505, Found: 494.2553.
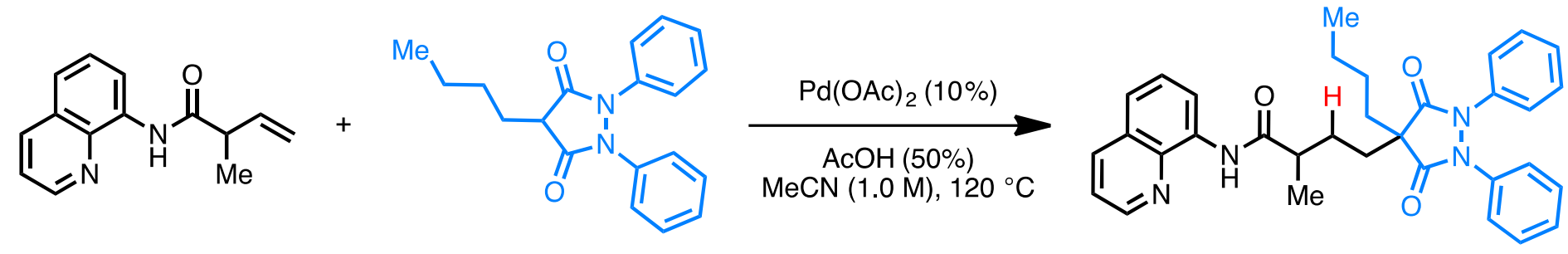
4-(4-butyl-3,5-dioxo-1,2-diphenylpyrazolidin-4-yl)-2-methyl- $N$-(quinolin-8-yl)butanamide (74): To a 1 dram $(4 \mathrm{~mL})$ vial equipped with a magnetic stir bar were added alkene 76 (22.6 mg, $0.1 \mathrm{mmol})$, phenylbutazone (30.8 mg, $0.1 \mathrm{mmol}$ ), acetic acid (3.0 mg, $0.05 \mathrm{mmol}), \mathrm{Pd}(\mathrm{OAc})_{2}(2.2 \mathrm{mg}, 0.01 \mathrm{mmol})$, and $\mathrm{MeCN}(0.1 \mathrm{~mL})$. The reaction was run for $4 \mathrm{~h}$, and the product was purified by flash column chromatography $\left(\mathrm{SiO}_{2}, 25-35 \%\right.$ EtOAc in Hexanes) to afford $48.0 \mathrm{mg}(92 \%)$ of 74 as an off-white solid. ${ }^{1} \mathbf{H} \mathbf{~ N M R}\left(600 \mathrm{MHz}, \mathrm{CDCl}_{3}\right) \delta 9.81(\mathrm{~s}, 1 \mathrm{H}), 8.79-8.75(\mathrm{~m}, 2 \mathrm{H}), 8.16(\mathrm{dd}, J=$ 8.2, $1.7 \mathrm{~Hz}, 1 \mathrm{H}), 7.56-7.48(\mathrm{~m}, 2 \mathrm{H}), 7.45(\mathrm{dd}, J=8.3,4.2 \mathrm{~Hz}, 1 \mathrm{H}), 7.35-7.32(\mathrm{~m}, 4 \mathrm{H}), 7.32-7.26(\mathrm{~m}, 4 \mathrm{H}), 7.19-7.16(\mathrm{~m}$, $1 \mathrm{H}), 7.16-7.12(\mathrm{~m}, 1 \mathrm{H}), 2.56(\mathrm{~h}, J=6.9 \mathrm{~Hz}, 1 \mathrm{H}), 2.07-1.98(\mathrm{~m}, 2 \mathrm{H}), 1.95-1.87(\mathrm{~m}, 3 \mathrm{H}), 1.63-1.56(\mathrm{~m}, 1 \mathrm{H}), 1.30(\mathrm{~d}, J=$ $6.9 \mathrm{~Hz}, 3 \mathrm{H}), 1.27$ (ddd, $J=10.1,5.4,2.3 \mathrm{~Hz}, 4 \mathrm{H}), 0.83(\mathrm{t}, J=6.9 \mathrm{~Hz}, 3 \mathrm{H}) .{ }^{13} \mathbf{C}$ NMR $\left(150 \mathrm{MHz}, \mathrm{CDCl}_{3}\right) \delta 174.28,173.53$, 173.33, 148.35, 138.65, 136.55, $135.78(\times 2), 134.64,129.21(\times 2), 129.18(\times 2), 128.13,127.63,127.00,126.95$, $122.82(\times 2), 122.80(\times 2), 121.78,121.68,116.82,54.26,42.94,36.35,34.23,29.21,27.11,22.89,18.00,13.86$. HRMS calcd. for $\mathrm{C}_{33} \mathrm{H}_{35} \mathrm{~N}_{4} \mathrm{O}_{3}+[\mathrm{M}+\mathrm{H}]+:$ 535.27037, Found: 535.2703.

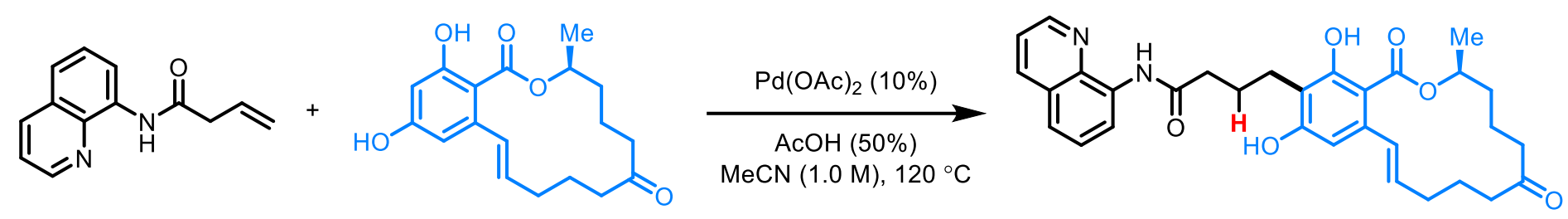

$(S, E)-4-(14,16-d i h y d r o x y-3-m e t h y l-1,7-d i o x o-3,4,5,6,7,8,9,10-o c t a h y d r o-1 H$ -

benzo $[\boldsymbol{c}][1]$ oxacyclotetradecin-15-yl)- $\boldsymbol{N}$-(quinolin-8-yl)butanamide (75): To a 1 dram (4 mL) vial equipped with a magnetic stir bar were added alkene $1(15.2 \mathrm{mg}, 0.072 \mathrm{mmol})$, zearalenone (23 $\mathrm{mg}, 0.072 \mathrm{mmol})$, acetic acid ( $2.5 \mathrm{mg}, 0.036 \mathrm{mmol}), \mathrm{Pd}(\mathrm{OAc})_{2}(1.6 \mathrm{mg}, 0.007 \mathrm{mmol})$, and $\mathrm{MeCN}(0.07 \mathrm{~mL})$. The reaction was run for $4 \mathrm{~h}$, and the product was purified by preparative TLC (40\% EtOAc in Hexanes) to afford $20.8 \mathrm{mg}(55 \%)$ of 75 as an off-white solid. ${ }^{1}$ H NMR $\left(600 \mathrm{MHz}, \mathrm{CDCl}_{3}\right) \delta 12.41(\mathrm{~s}, 1 \mathrm{H}), 10.01(\mathrm{~s}, 1 \mathrm{H}), 9.68(\mathrm{~s}, 1 \mathrm{H}), 8.83-8.82(\mathrm{~m}, 1 \mathrm{H}), 8.19$ (dd, J = 8.2, 1.7 $\mathrm{Hz}, 1 \mathrm{H}), 7.63-7.55(\mathrm{~m}, 2 \mathrm{H}), 7.49$ (dd, J = 8.2, $4.2 \mathrm{~Hz}, 1 \mathrm{H}), 7.02$ (dd, J = 15.3, $2.0 \mathrm{~Hz}, 1 \mathrm{H}), 6.57(\mathrm{~s}, 1 \mathrm{H}), 5.69$ (ddd, J = 15.3, 10.6, 3.6 Hz, 1H), 5.10-4.90 (m, 1H), 3.07-2.77 (m, 3H), 2.68-2.55 (m, 3H), 2.47-2.30 (m, 1H), 2.24-2.09 (m, 4H), 2.09-1.98 (m, 2H), 1.89-1.59 (m, 5H), 1.55-1.44 (m, 1H), $1.39(\mathrm{~d}, \mathrm{~J}=6.2 \mathrm{~Hz}, 3 \mathrm{H}) .{ }^{13} \mathbf{C} \mathbf{N M R}\left(150 \mathrm{MHz} \mathrm{CDCl}_{3}\right) \delta$ 211.40, 174.00, 172.20, 163.45, 161.15, 148.57, 141.41, 138.55, 136.70, 134.00, 133.65, 131.79, 128.16, 127.57, 122.48, 121.99, 117.47, 113.36, 109.83, 102.84, 73.37, 43.24, 36.89, 35.57, 35.01, 31.23, 24.44, 22.49, 21.76, 21.31, 21.12. HRMS calcd. for $\mathrm{C}_{31} \mathrm{H}_{35} \mathrm{~N}_{2} \mathrm{O}_{6}+[\mathrm{M}+\mathrm{H}]^{+}:$531.24896, Found: 531.2487. 
<smiles>C=CC(C)C(=O)Nc1cccc2cccnc12</smiles><smiles>Cc1c(O)cc(O)c2c1COC2=O</smiles>

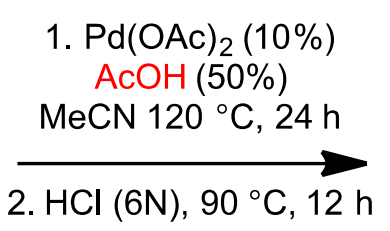<smiles>CC(Cc1c(O)c2c(c([N+]([O-])O)c1O)COC2=O)C(=O)O</smiles>

( \pm )-Euparvic acid $0.83 \mathrm{~g}$

( \pm )-Euparvic acid (78): Core 77 was synthesized as reported by Anderson and Duan (6-7). Alkene 76 (1.256 g, 5.55 $\mathrm{mmol}$ ), 77 (1.00 g, $5.55 \mathrm{mmol}$ ), acetic acid (166.5 mg, $2.27 \mathrm{mmol}), \mathrm{Pd}(\mathrm{OAc})_{2}(124.0 \mathrm{mg}, 0.55 \mathrm{mmol})$, and MeCN (3.7 $\mathrm{mL}$ ) were charged to a $48 \mathrm{~mL}$ sealed tube. The resulting light yellow suspension was stirred for 1 min before being submerged into a pre-heated $120^{\circ} \mathrm{C}$ silicon oil bath. After $24 \mathrm{~h}$, the reaction was cooled to ambient temperature. The cap was replaced with a septa and the solvent was removed in vacuo. The tube was charged with $6 \mathrm{~N} \mathrm{HCl}(25 \mathrm{~mL})$, sealed, and submerged into a $90{ }^{\circ} \mathrm{C}$ silicon oil bath where it was heated for $12 \mathrm{~h}$. After cooling to ambient temperatures, the solution was diluted with water $(100 \mathrm{~mL})$, and transferred to a $250 \mathrm{~mL}$ separatory funnel. The aqueous layer was extracted with EtOAc $(75 \mathrm{~mL}, \times 3)$. The combined organics were extracted with sat. $\mathrm{NaHCO}_{3}(75$ $\mathrm{mL}, \times 2)$. The combined aqueous layer was then washed with DCM $(75 \mathrm{~mL}, \times 2)$ before being acidified to $\mathrm{pH}<2 \mathrm{with}$ the dropwise addition of conc. $\mathrm{HCl}$. The aqueous layer was saturated with the addition of solid $\mathrm{NaCl}$, and extracted with DCM $(75 \mathrm{~mL}, \times 5)$. The combined organics were dried over $\mathrm{Na}_{2} \mathrm{SO}_{4}$, concentrated, and the product was purified by flash column chromatography $\left(\mathrm{SiO}_{2}, 25-45 \%\right.$ EtOAc in Hexanes with $\left.0.5 \% \mathrm{AcOH}\right)$ to afford $834 \mathrm{mg}(54 \%)$ of 78 as a white solid. Spectra data matches previous report (8). ${ }^{1} \mathbf{H}$ NMR (600 MHz, Acetone-d6) $\delta 5.26$ (s, $2 \mathrm{H}$ ), 2.79 (ddd, $\mathrm{J}=13.6,9.8,6.4 \mathrm{~Hz}, 2 \mathrm{H}), 2.72$ (ddd, J = 13.5, 9.9, 5.4 Hz, 1H), 2.57-2.46 (m, 1H), 2.12 (s, 3H), 1.86 (dddd, J = 13.4, 9.8, 7.9, $5.4 \mathrm{~Hz}, 1 \mathrm{H}), 1.66(\mathrm{ddt}, \mathrm{J}=13.5,9.9,6.2 \mathrm{~Hz}, 1 \mathrm{H}), 1.23(\mathrm{~d}, \mathrm{~J}=7.1 \mathrm{~Hz}, 3 \mathrm{H}) .{ }^{13}$ C NMR (150 MHz, Acetone-d6) $\delta 178.74$, $173.22,161.24,154.05,145.77,115.65,111.02,103.36,70.32,39.39,33.27,21.16,17.44,11.31$. HRMS calcd. for $\mathrm{C}_{14} \mathrm{H}_{17} \mathrm{O}_{6}+[\mathrm{M}+\mathrm{H}]^{+}:$281.10196, Found: 281.1020 .
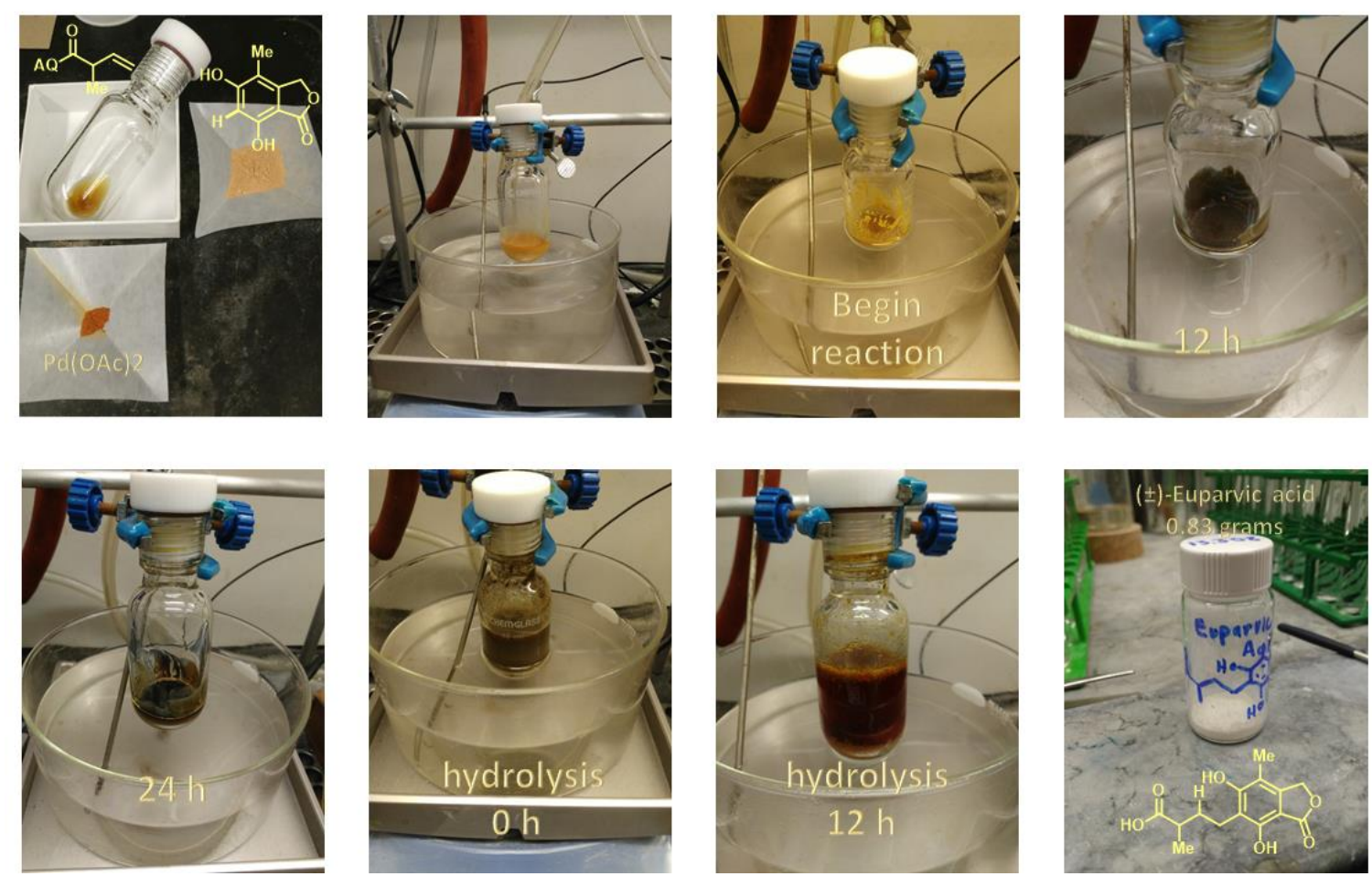

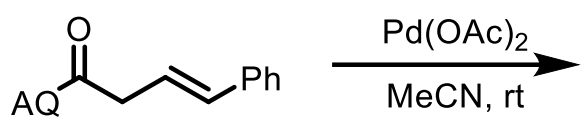

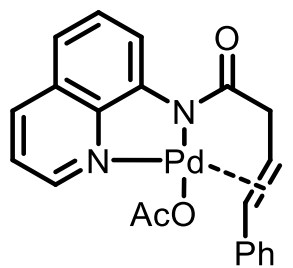

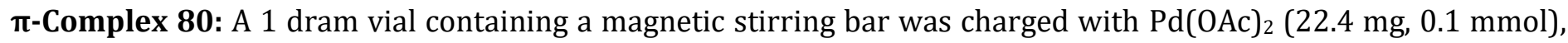
alkene 79 (28.8 $\mathrm{mg}, 0.1 \mathrm{mmol})$, and $\mathrm{MeCN}(1 \mathrm{~mL})$. The resulting orange solution was stirred at ambient temperature for $6 \mathrm{~h}$, during which a yellow precipitate crashed out. The reaction mixture was filtered, and the filter cake was collected and dried in vacuo to afford $20 \mathrm{mg}(42 \%)$ of complex 80 as a yellow powder. ${ }^{1} \mathbf{H} \mathbf{~ N M R}\left(600 \mathbf{M H z} \mathrm{CDCl}_{3}\right) \delta$ $8.84(\mathrm{dd}, \mathrm{J}=7.9,1.2 \mathrm{~Hz}, 1 \mathrm{H}), 8.39(\mathrm{dd}, \mathrm{J}=8.3,1.5 \mathrm{~Hz}, 1 \mathrm{H}), 8.30(\mathrm{dd}, \mathrm{J}=5.0,1.5 \mathrm{~Hz}, 1 \mathrm{H}), 7.66-7.61(\mathrm{~m}, 2 \mathrm{H}), 7.59(\mathrm{t}, \mathrm{J}=$ $8.0 \mathrm{~Hz}, 1 \mathrm{H}$ ), 7.51-7.44 (m, 3H), 7.44-7.38 (m, 2H), 7.31 (d, J = 15.0 Hz, 1H), 6.72 (ddd, J = 15.1, 6.8, 3.2 Hz, 1H), 3.87 $(\mathrm{dd}, \mathrm{J}=17.6,7.1 \mathrm{~Hz}, 1 \mathrm{H}), 3.27(\mathrm{~d}, \mathrm{~J}=17.4 \mathrm{~Hz}, 1 \mathrm{H}), 1.70(\mathrm{~s}, 3 \mathrm{H}) .{ }^{13} \mathbf{C}$ NMR $\left(150 \mathrm{MHz}, \mathrm{CDCl}_{3}\right) \delta 177.45,176.81,146.82$, 145.89, 145.75, 139.71, 133.36, 130.15, 129.49, 129.35, 128.62, 128.38, 122.19, 120.18, 119.85, 113.97, 85.32, 39.71, 22.72. X-ray (single-crystal) orange blocks of X-ray diffraction quality were obtained by vapor diffusion of hexanes into a saturated solution of $\mathbf{8 0}$ in EtOAc (CCDC 1489655).
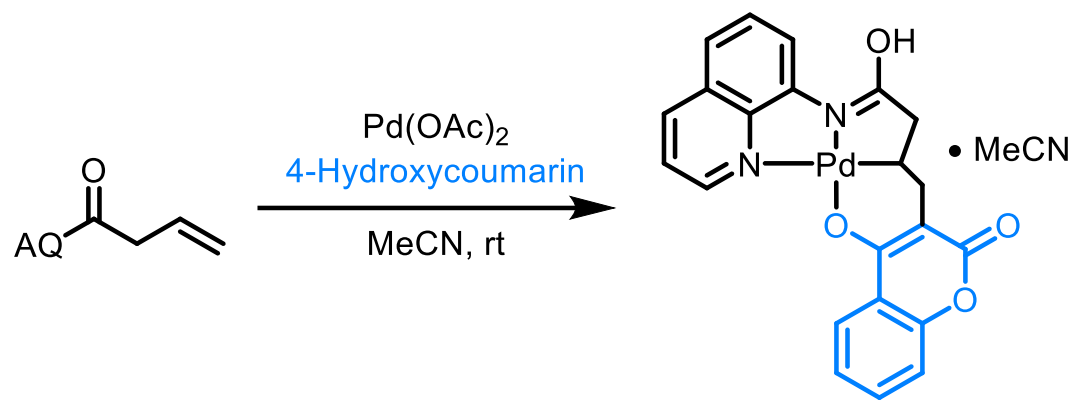

Alkylpalladium complex 81: A $20 \mathrm{~mL}$ scintillation vial containing a magnetic stirring bar was charged with $\mathrm{Pd}(\mathrm{OAc})_{2}(112 \mathrm{mg}, 0.5 \mathrm{mmol})$, alkene $1(112 \mathrm{mg}, 0.53 \mathrm{mmol}), 4$-hydroxycoumarin (80.0 mg, $\left.0.5 \mathrm{mmol}\right)$, and MeCN $(12 \mathrm{~mL})$. The resulting mixture was stirred at ambient temperature for $4 \mathrm{~h}$, during which a yellow precipitate crashed out. The reaction mixture was filtered, and the filter cake was rinsed with MeCN $(5 \mathrm{~mL}, \times 2)$, collected, and dried in vacuo to afford pallacycle $\mathbf{8 1}$ as a yellow powder. ${ }^{1} \mathbf{H}$ NMR $(600 \mathrm{MHz}$, DMSO-d6) $\delta 11.49(\mathrm{~s}, 1 \mathrm{H}), 9.10(\mathrm{dd}, \mathrm{J}=4.8,1.6$ $\mathrm{Hz}, 1 \mathrm{H}), 8.85(\mathrm{dd}, \mathrm{J}=7.8,1.3 \mathrm{~Hz}, 1 \mathrm{H}), 8.54(\mathrm{dd}, \mathrm{J}=8.3,1.7 \mathrm{~Hz}, 1 \mathrm{H}), 8.02(\mathrm{dd}, \mathrm{J}=8.2,1.5 \mathrm{~Hz}, 1 \mathrm{H}), 7.69$ (dd, $\mathrm{J}=8.3,4.7$ $\mathrm{Hz}, 1 \mathrm{H}), 7.61$ (ddd, J = 8.6, 7.2, $1.5 \mathrm{~Hz}, 1 \mathrm{H}), 7.53(\mathrm{t}, \mathrm{J}=7.9 \mathrm{~Hz}, 1 \mathrm{H}), 7.46(\mathrm{dd}, \mathrm{J}=8.1,1.3 \mathrm{~Hz}, 1 \mathrm{H}), 7.40-7.34(\mathrm{~m}, 2 \mathrm{H})$, 3.23 (dd, J = 13.7, 11.9 Hz, 1H), 3.02 (dd, J = 16.3, 7.6 Hz, 1H), 2.71 (d, J = 12.6 Hz, 1H), 2.07 (s, 3H), 2.07-2.01 (m, 1H), 1.95 (d, J = 16.8 Hz, 1H). ${ }^{13}$ C NMR (150 MHz, DMSO-d6) $\delta 182.65,163.41,160.28,151.76,149.70,145.59,144.32$, 138.91, 131.59, 129.60, 128.37, 123.91, 123.19, 121.62, 118.76, 118.56, 118.06, 116.23, 116.20, 103.86, 47.61, 30.90, 26.16, 1.15. HRMS calcd. for $\mathrm{C}_{22} \mathrm{H}_{17} \mathrm{~N}_{2} \mathrm{O}_{4} \mathrm{Pd}+[\mathrm{M}+\mathrm{H}]^{+}:$479.02177, Found: 479.0224. A single-crystal green cluster of X-ray diffraction quality were obtained when $5 \mathrm{mg}$ of $\mathbf{8 1}$ was allowed to sit in $0.3 \mathrm{~mL}$ DMF over 2 days (CCDC: 1489657).<smiles></smiles>

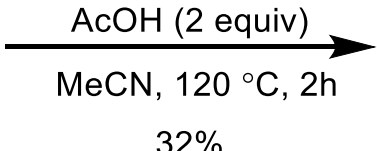

$32 \%$

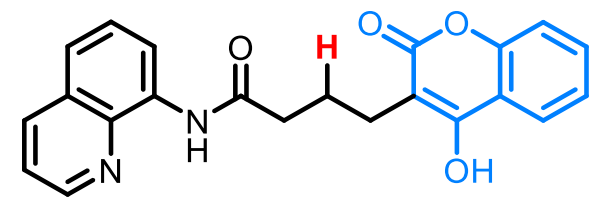

4-(4-hydroxy-2-oxo-2H-chromen-3-yl)-N-(quinolin-8-yl)butanamide (39): In a 1 dram vial was charged palladacycle 81 (47.8 mg, $0.1 \mathrm{mmol}$ ), AcOH (12 mg, $0.2 \mathrm{mmol}$ ), and MeCN (0.5 mL). The vial was sealed with a Teflon 
coated cap, and placed into a pre-heated $120{ }^{\circ} \mathrm{C}$ heating block. After $2 \mathrm{~h}$, the reaction was cooled to ambient temperature, and 1,4-butanedithiol (61 $\mathrm{mg}, 0.5 \mathrm{mmol}$ ) was added. The reaction was allowed to stir at ambient temperature for $20 \mathrm{~min}$ before filtering through a plug of silica. The product was purified by preparative TLC (40\% EtOAc in Hexanes) to afford $12.0 \mathrm{mg}$ (32\%) of $\mathbf{3 9}$ as a white solid. Spectroscopic data as reported above.
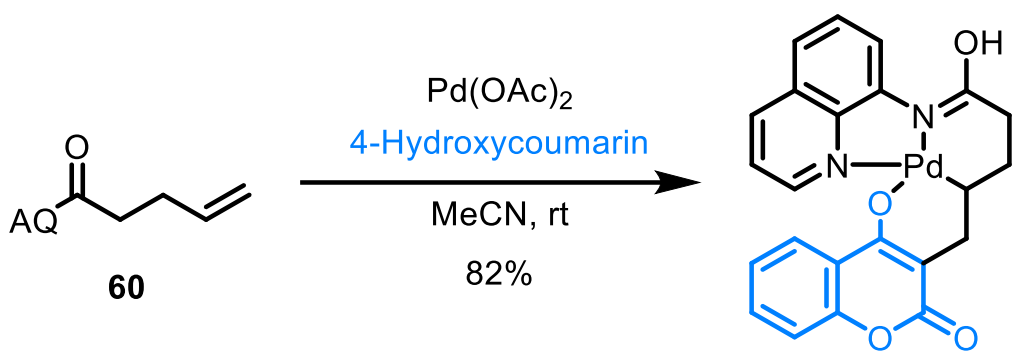

Alkylpalladium complex 82: A $20 \mathrm{~mL}$ scintillation vial containing a magnetic stirring bar was charged with $\mathrm{Pd}(\mathrm{OAc})_{2}$ (112 mg, $0.5 \mathrm{mmol}$ ), alkene 60 (118 mg, $0.53 \mathrm{mmol}$ ), 4-hydroxycoumarin ( $\left.80.0 \mathrm{mg}, 0.5 \mathrm{mmol}\right)$, and MeCN $(6 \mathrm{~mL})$. The resulting mixture was stirred at ambient temperature for $6 \mathrm{~h}$, during which, a pale precipitate crashed out. The reaction mixture was filtered, and the filter cake was rinsed with MeCN ( $5 \mathrm{~mL}, \times 1)$, collected, and dried in vacuo to afford $202 \mathrm{mg}(82 \%)$ of palladacycle 82 as a tan powder. ${ }^{1} \mathbf{H}$ NMR (600 MHz, DMSO-d6) $\delta 8.99$ (dd, J $=4.5$, $1.6 \mathrm{~Hz}, 1 \mathrm{H}), 8.61(\mathrm{dd}, \mathrm{J}=8.4,1.6 \mathrm{~Hz}, 1 \mathrm{H}), 8.50(\mathrm{~d}, \mathrm{~J}=7.9 \mathrm{~Hz}, 1 \mathrm{H}), 8.14(\mathrm{dd}, \mathrm{J}=7.8,1.7 \mathrm{~Hz}, 1 \mathrm{H}), 7.87-7.74(\mathrm{~m}, 2 \mathrm{H}), 7.67$ $(\mathrm{t}, \mathrm{J}=8.0 \mathrm{~Hz}, 1 \mathrm{H}), 7.47(\mathrm{ddd}, \mathrm{J}=8.5,7.2,1.7 \mathrm{~Hz}, 1 \mathrm{H}), 7.35-7.24(\mathrm{~m}, 1 \mathrm{H}), 7.20(\mathrm{~d}, \mathrm{~J}=8.1 \mathrm{~Hz}, 1 \mathrm{H}), 2.93(\mathrm{dd}, \mathrm{J}=16.2,5.4$ $\mathrm{Hz}, 1 \mathrm{H}), 2.69-2.57(\mathrm{~m}, 1 \mathrm{H}), 2.21(\mathrm{dd}, \mathrm{J}=17.0,12.9 \mathrm{~Hz}, 1 \mathrm{H}), 2.16-2.08(\mathrm{~m}, 1 \mathrm{H}), 1.68(\mathrm{~d}, \mathrm{~J}=15.1 \mathrm{~Hz}, 1 \mathrm{H}), 1.44(\mathrm{q}, \mathrm{J}=$ 12.7, $11.8 \mathrm{~Hz}, 1 \mathrm{H}), 1.25-1.05$ (m, 1H). ${ }^{13} \mathrm{C}$ NMR (125 MHz, DMSO-d6) $\delta 175.02,167.32,164.02,151.60,146.58$, 144.26, 142.26, 138.11, 129.94, 128.94, 127.55, 124.80, 124.07, 123.43, 122.63, 122.40, 120.43, 115.39, 100.36, 38.12, 36.34, 31.80, 29.79. . HRMS calcd. for $\mathrm{C}_{23} \mathrm{H}_{19} \mathrm{~N}_{2} \mathrm{O}_{4} \mathrm{Pd}+[\mathrm{M}+\mathrm{H}]+:$ 493.03742, Found: 493.03742 .
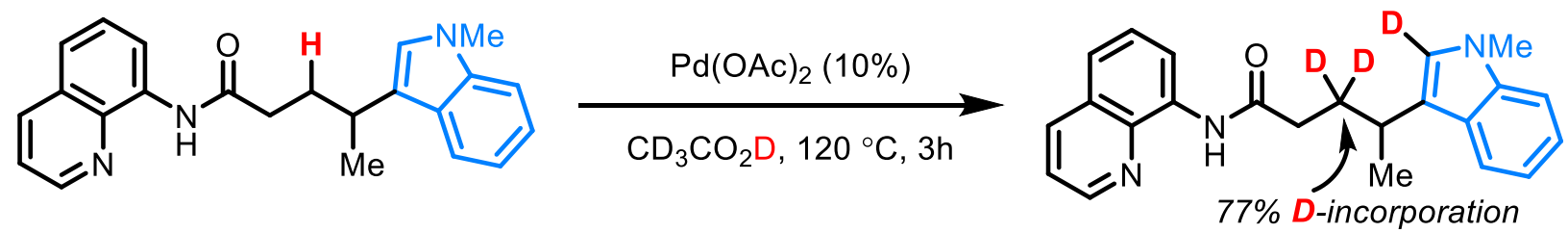

3,3-dideutero-4-(1-methyl-1H-indol-3-yl-2-d)-N-(quinolin-8-yl)pentanamide (83): In a 1 dram vial was charged 48 (30 mg, $0.09 \mathrm{mmol}), \mathrm{CD}_{3} \mathrm{COOD}(1 \mathrm{~mL})$, and $\mathrm{Pd}(\mathrm{OAc})_{2}(2.0 \mathrm{mg}, 0.009 \mathrm{mmol})$. The vial was sealed with a Teflon coated cap, and placed into a pre-heated $120^{\circ} \mathrm{C}$ heating block. After $3 \mathrm{~h}$, the reaction was cooled to ambient temperature, and the product was purified by preparative TLC (30\% EtOAc in Hexanes) to afford $24.0 \mathrm{mg}(80 \%)$ of 83 as a yellow oil. . 1 H NMR $\left(600 \mathrm{MHz}, \mathrm{CDCl}_{3}\right) \delta 9.67(\mathrm{~s}, 1 \mathrm{H}), 8.77(\mathrm{dd}, \mathrm{J}=7.7,1.3 \mathrm{~Hz}, 1 \mathrm{H}), 8.74(\mathrm{dd}, \mathrm{J}=4.2,1.7 \mathrm{~Hz}$, $1 \mathrm{H}), 8.13(\mathrm{dd}, \mathrm{J}=8.3,1.7 \mathrm{~Hz}, 1 \mathrm{H}), 7.67(\mathrm{dt}, \mathrm{J}=7.9,1.0 \mathrm{~Hz}, 1 \mathrm{H}), 7.52(\mathrm{t}, \mathrm{J}=7.9 \mathrm{~Hz}, 1 \mathrm{H}), 7.47(\mathrm{dd}, \mathrm{J}=8.2,1.4 \mathrm{~Hz}, 1 \mathrm{H})$, $7.42(\mathrm{dd}, \mathrm{J}=8.2,4.2 \mathrm{~Hz}, 1 \mathrm{H}), 7.26(\mathrm{~d}, \mathrm{~J}=8.1 \mathrm{~Hz}, 1 \mathrm{H}), 7.22-7.16(\mathrm{~m}, 1 \mathrm{H}), 7.06(\mathrm{t}, \mathrm{J}=7.4 \mathrm{~Hz}, 1 \mathrm{H}), 3.72(\mathrm{~s}, 3 \mathrm{H}), 3.17(\mathrm{q}, \mathrm{J}$ $=6.8 \mathrm{~Hz}, 1 \mathrm{H}), 2.58-2.48(\mathrm{~m}, 2 \mathrm{H}), 1.43(\mathrm{dd}, \mathrm{J}=7.0,1.1 \mathrm{~Hz}, 3 \mathrm{H}) .{ }^{13} \mathbf{C}$ NMR $\left(150 \mathrm{MHz}, \mathrm{CDCl}_{3}\right) \delta 172.26,148.20,138.50$, 137.39, 136.51, 134.79, 128.11, 127.64, 127.36, 125.32 (t, J = 27.4 Hz), 121.71, 121.62, 121.44, 119.71, 119.62, 118.76, 116.54, 109.4, 36.57-36.34 (m), 33.01 (t, J = 19.3 Hz), 32.78, 30.81-30.62 (m), 22.63-21.87 (m). HRMS calcd. for $\mathrm{C}_{23} \mathrm{H}_{21} \mathrm{D}_{3} \mathrm{~N}_{3} \mathrm{O}+[\mathrm{M}+\mathrm{H}]^{+}: 361.21022$ Found: 361.2102
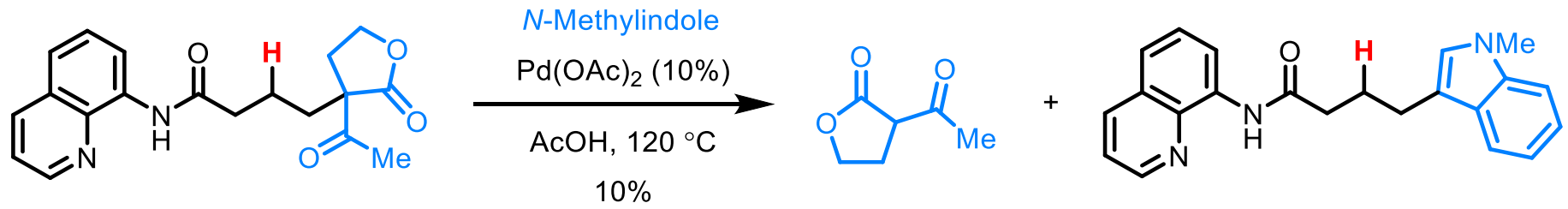

4-(1-methyl-1H-indol-3-yl)-N-(quinolin-8-yl)butanamide (30): In a 1 dram vial was charged 17 (34 mg, 0.1 mmol), 1-methylindole (26.2 mg, $0.2 \mathrm{mmol}), \mathrm{Pd}(\mathrm{OAc})_{2}(2.2 \mathrm{mg}, 0.01 \mathrm{mmol})$, and $\mathrm{MeCN}(0.1 \mathrm{~mL})$. The vial was sealed with a Teflon coated cap, and placed into a pre-heated $120^{\circ} \mathrm{C}$ heating block. After $24 \mathrm{~h}$, the reaction was cooled to 
ambient temperature, and the product was purified by preparative TLC (30\% EtOAc in Hexanes) to afford $6.8 \mathrm{mg}$ $(10 \%)$ of $\mathbf{3 0}$ as a yellow oil. Spectroscopic data as reported above.

Control: The same procedure was carried out in the absence of $\mathrm{Pd}(\mathrm{OAc})_{2}$. After $24 \mathrm{~h}$, no product was detected from crude NMR analysis. 


\section{References:}

1. Gurak, J. A. Jr.; Yang, K. S.; Liu, Z.; Engle, K. M. J. Am. Chem. Soc. 2016, 138, 5805-5808.

2. Kawamata, Y.; Hashimoto, T.; Maruoka, K. J. Am. Chem. Soc. 2016, 138, 5206-5209.

3. Affron, D. P.; Bull, J. A. Eur. J. Org. Chem. 2016, 139-149.

4. Brodney, M. A.; O'Leary, J. P.; Hansen, J. A.; Giguere, R. J. Synth. Commun. 1995, 25, 521-531.

5. Schlosser, M.; Zellner, A.; Leroux, F. Synthesis 2001, 1830-1836.

6. Makara, G. M.; Klubek, K.; Anderson, W. K. Synth. Commun. 1996, 26, 1935-1942.

7. Zuo, L.; Yao, S.; Wang, W.; Duan, W. Tetrahedron Lett. 2008, 49, 4054-4056.

8. Habib, E.; León, F.; Bauer, J. D.; Hill, R. A.; Carvalho, P.; Cutler, H. G.; Cutler, S. J. J. Nat. Prod. 2008, 71, 1915-1918. 


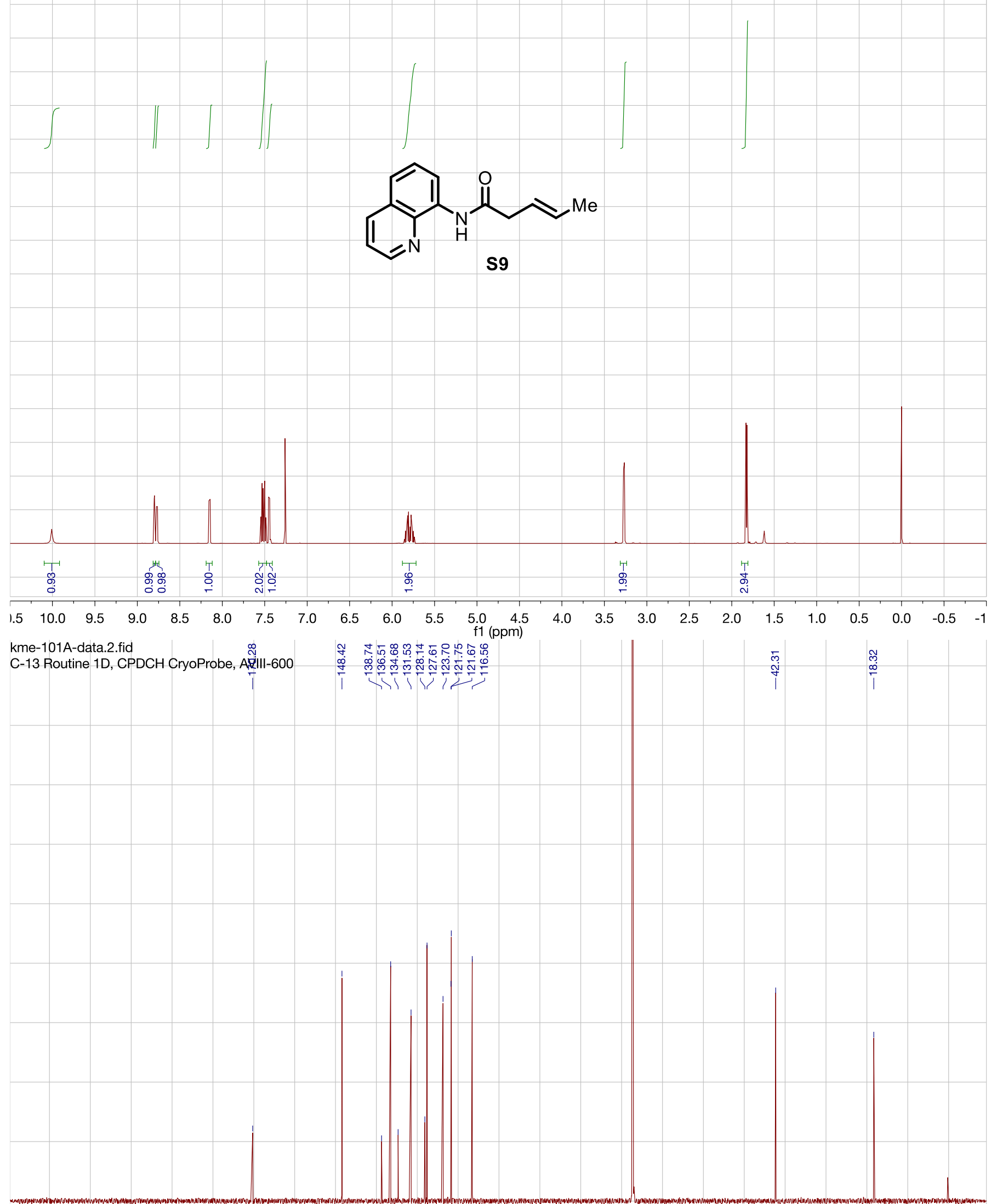

$\begin{array}{lllllllllllllllllllllll}220 & 210 & 200 & 190 & 180 & 170 & 160 & 150 & 140 & 130 & 120 & \begin{array}{c}110 \\ \mathrm{f} 1(\mathrm{ppm})\end{array} & 100 & 90 & 80 & 70 & 60 & 50 & 40 & 30 & 20 & 10 & 0\end{array}$


ky-n3-jg-styrene.1.fid

H-1 Routine. CPQCI, AVIII-600, 5-12-2016
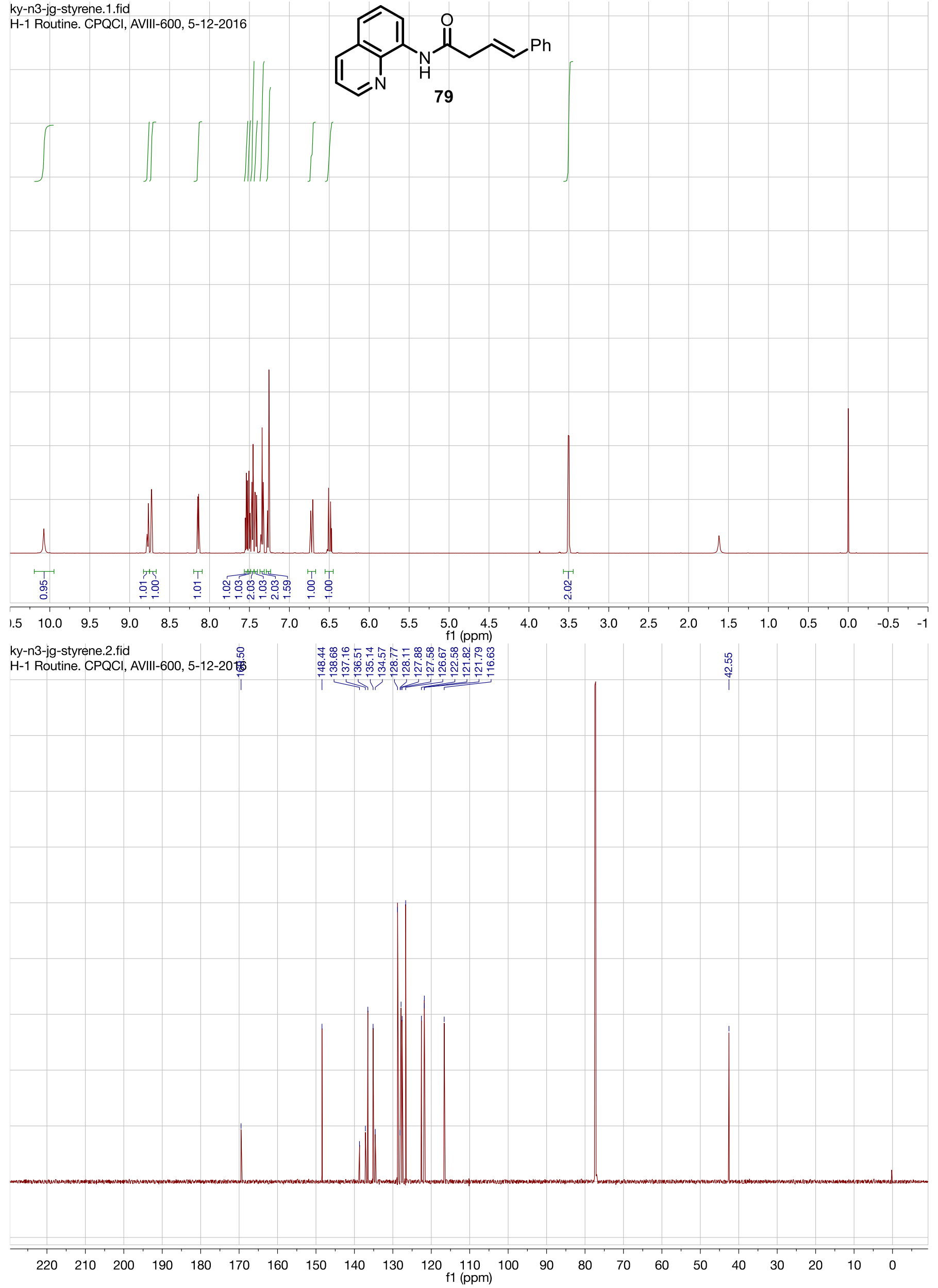
ky-n3-boc-cypent.1.fid

H-1 Routine. CPQCl, AVIII-600, 5-12-2016

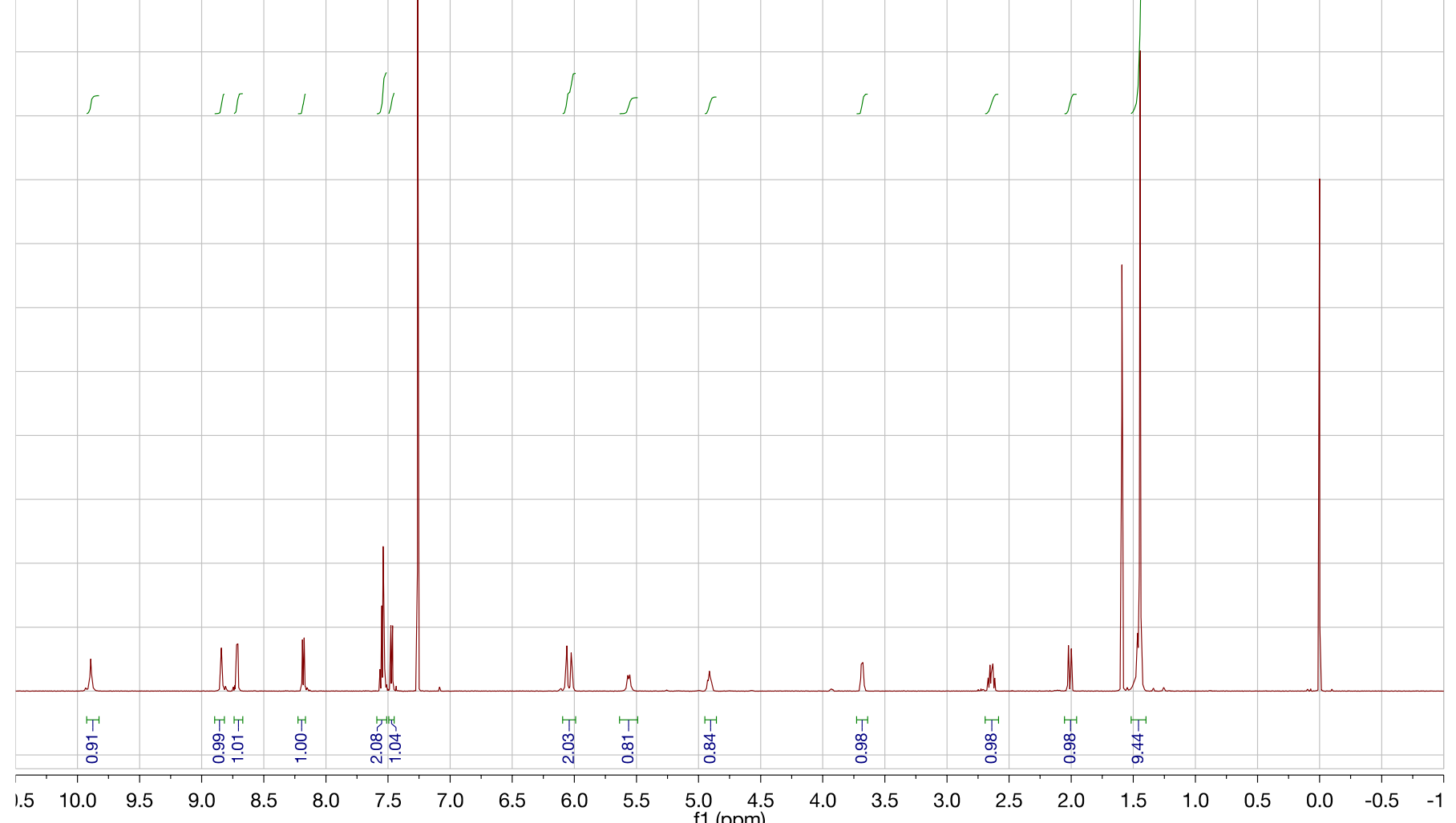

ky-n3-boc-cypent.2.fid

H-1 Routine. CPQCI, AVIII-600, 5-12-22Q16

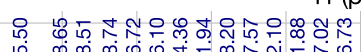

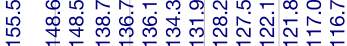

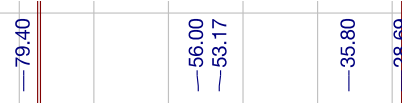

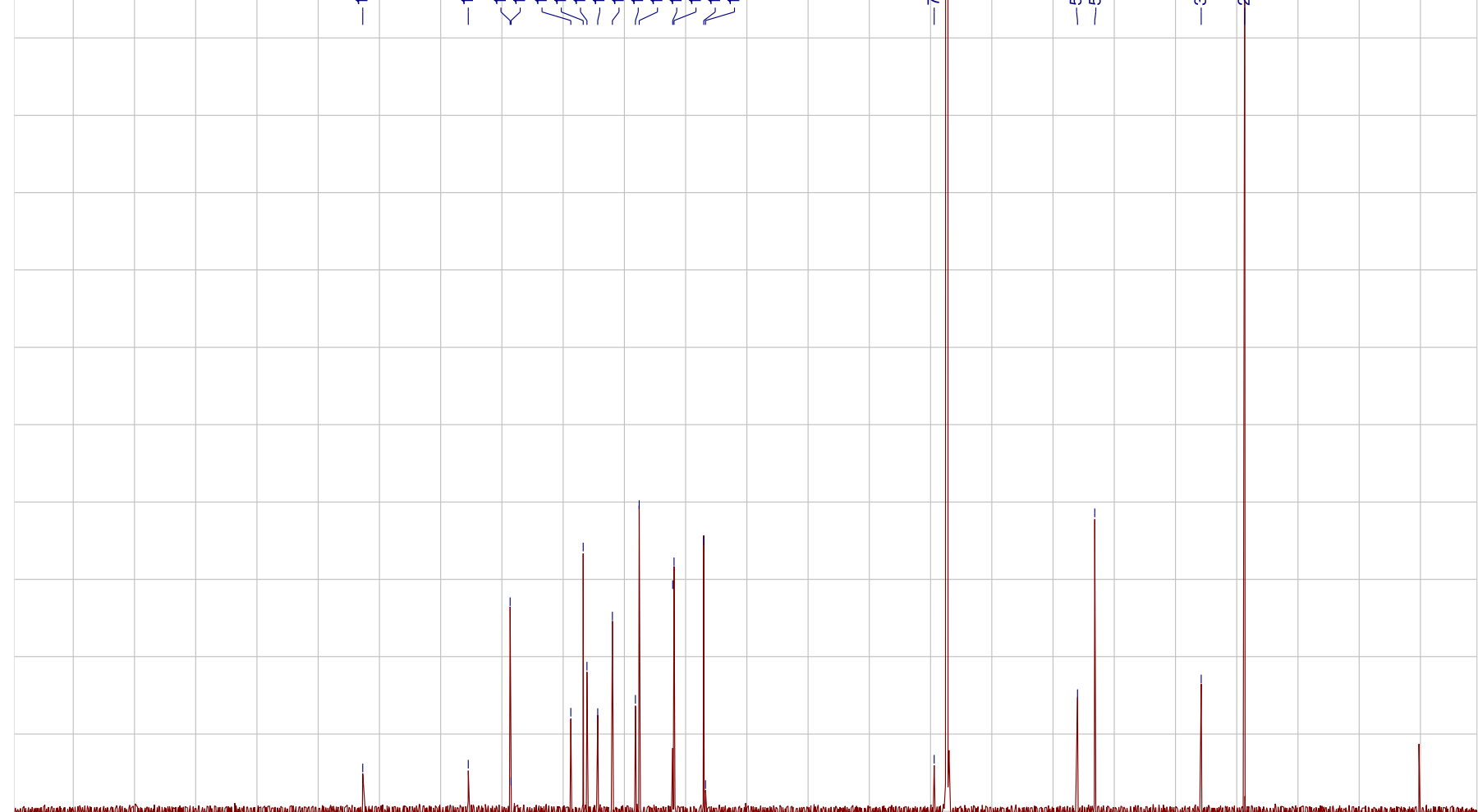

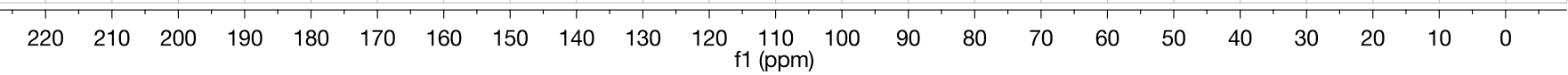


ky-n3-046-data.1.fid

C-13 Routine 1D, CPDCH CryoProbe, AVIII-600<smiles>CC(C)C=CCC(=O)Nc1cccc2cccnc12</smiles>

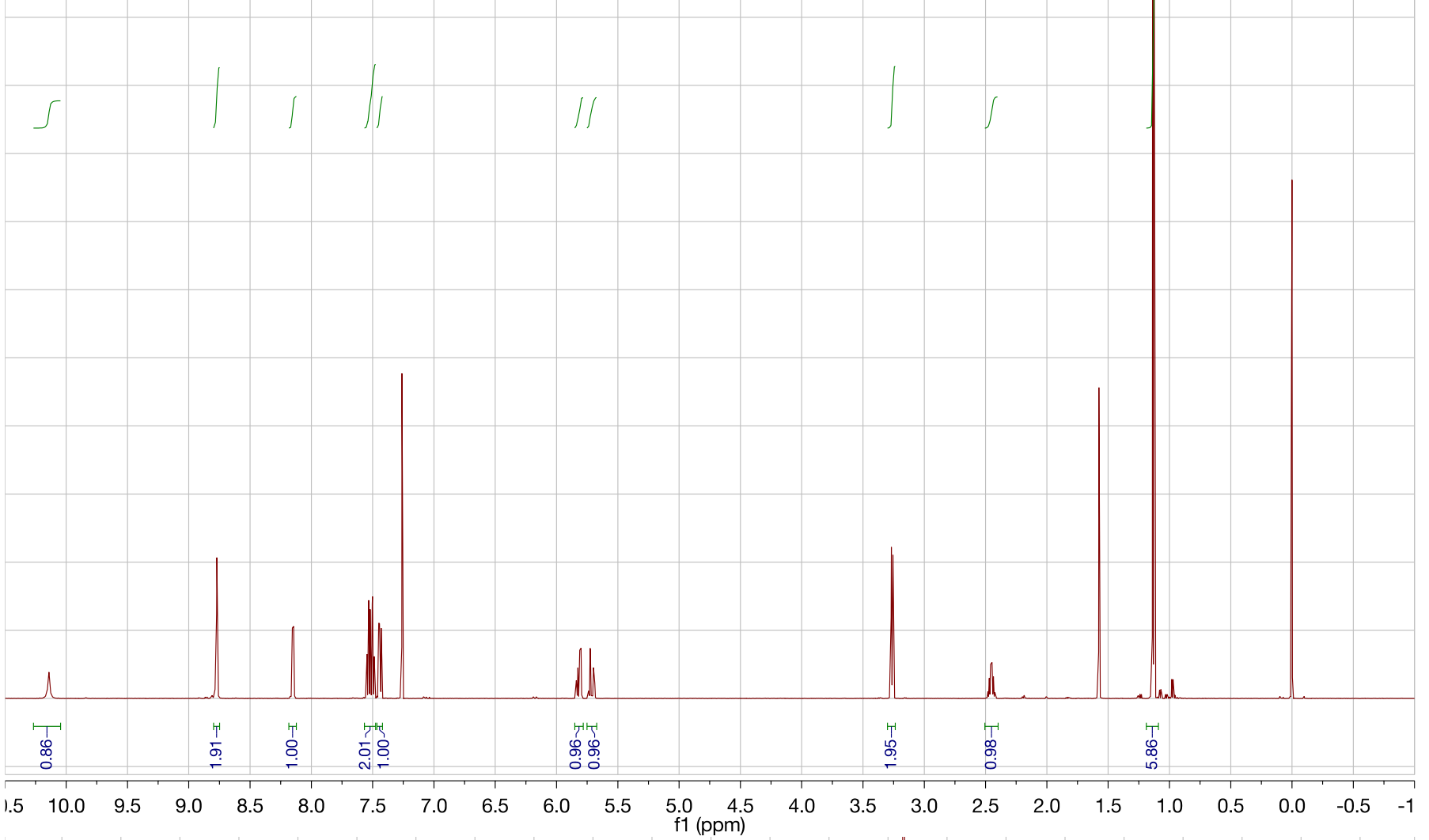
ky-n3-046-data.2.fid $\mid$ C-13 Routine 1D, CPDCH CryoProbe, A A 
kme-98A-data.1.fid

C-13 Routine 1D, CPDCH CryoProbe, AVIII-600

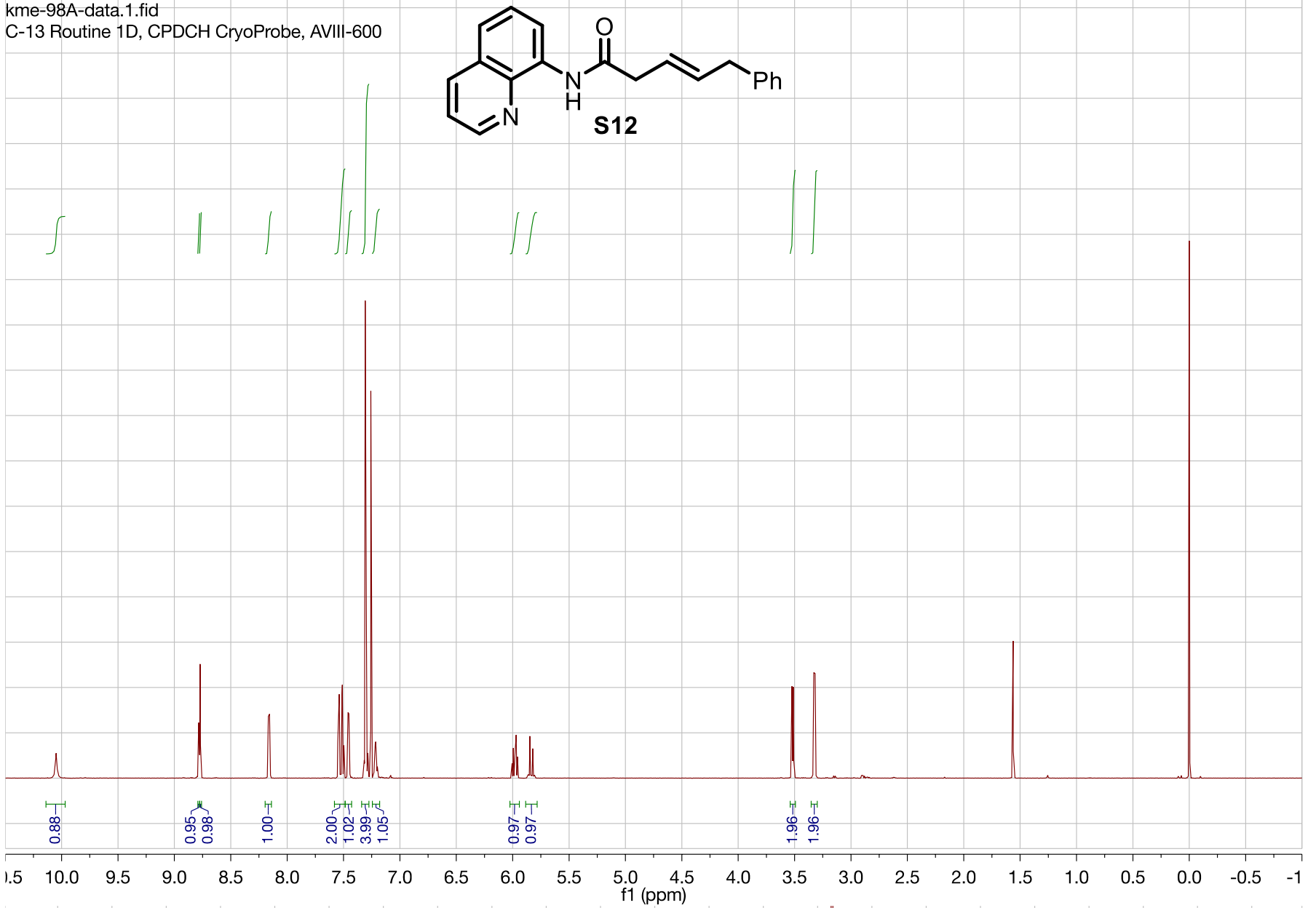

kme-98A-data.2.fid I

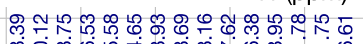
C-13 Routine 1D, CPDCH CryoProbe, Agill-600 
kme-110A-data.1.fid

C-13 Routine 1D, CPDCH CryoProbe, AVIII-600

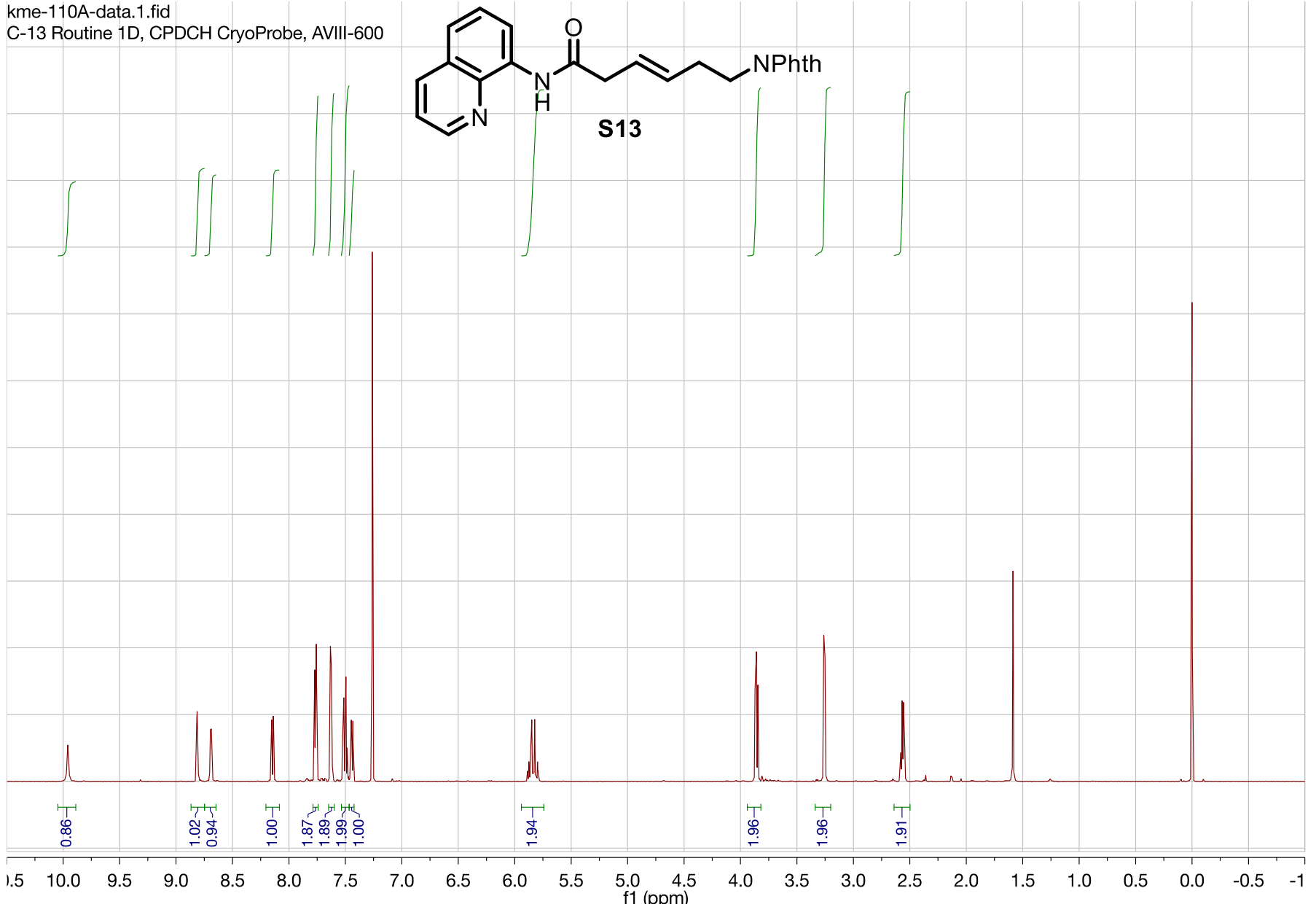

kme-110A-data.2.fid

我

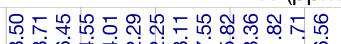
C-13 Routine 1D, CPDCH CryoProbe, Aqi|l|g-600 -

งู่

Ұभर्ण

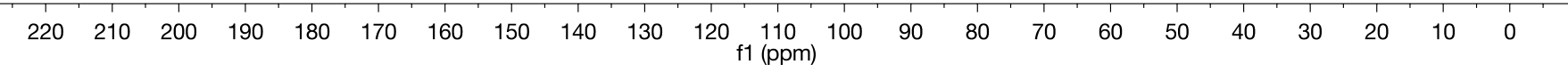




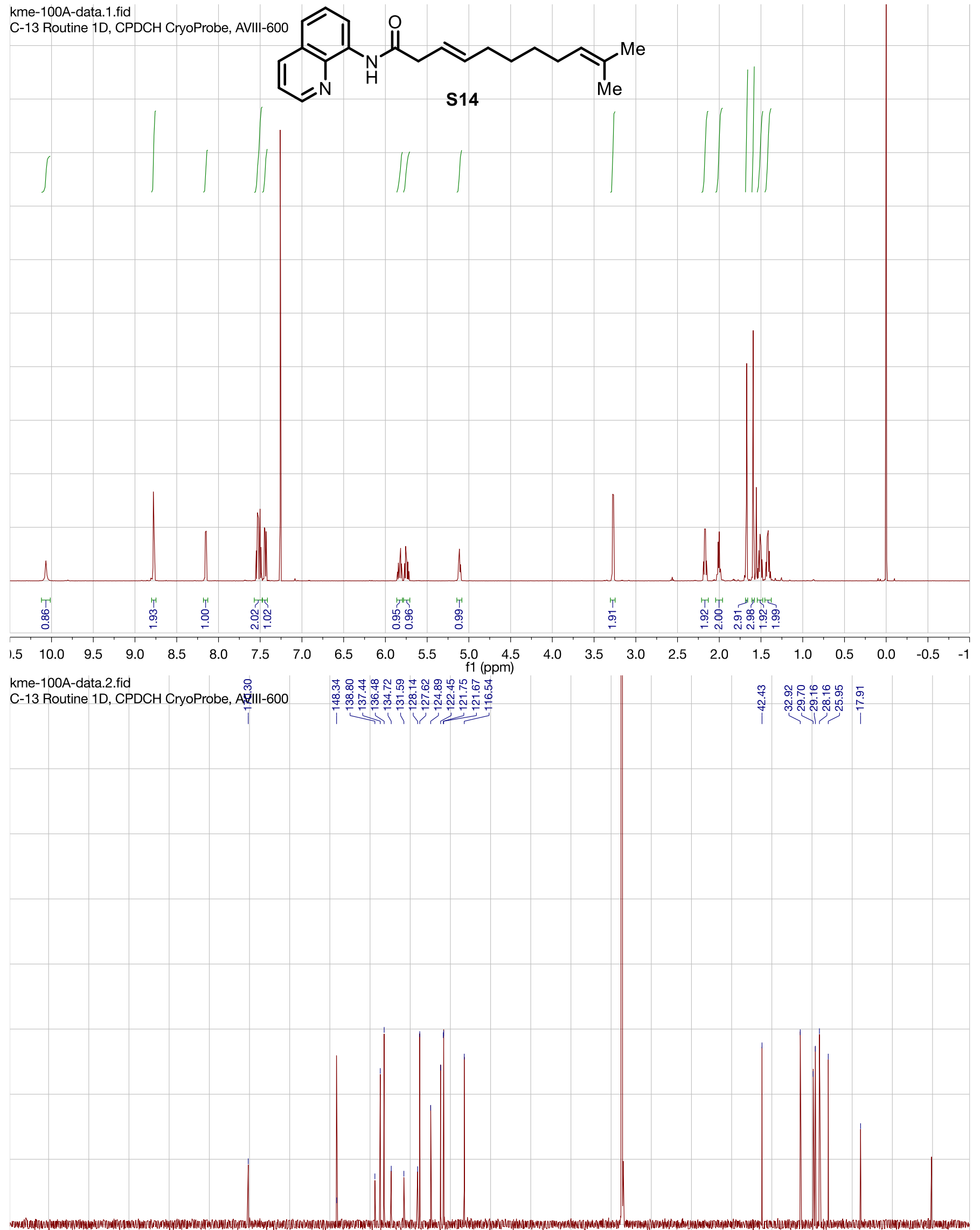

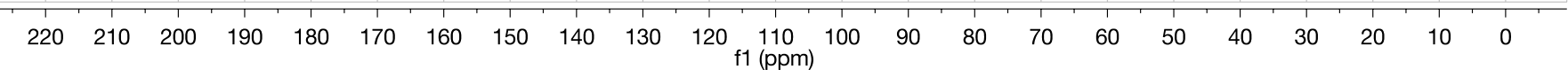


KME-A-068_CHAR 1.1.fid<smiles>C=CCC(=O)NC(C)(C)c1ccccn1</smiles>

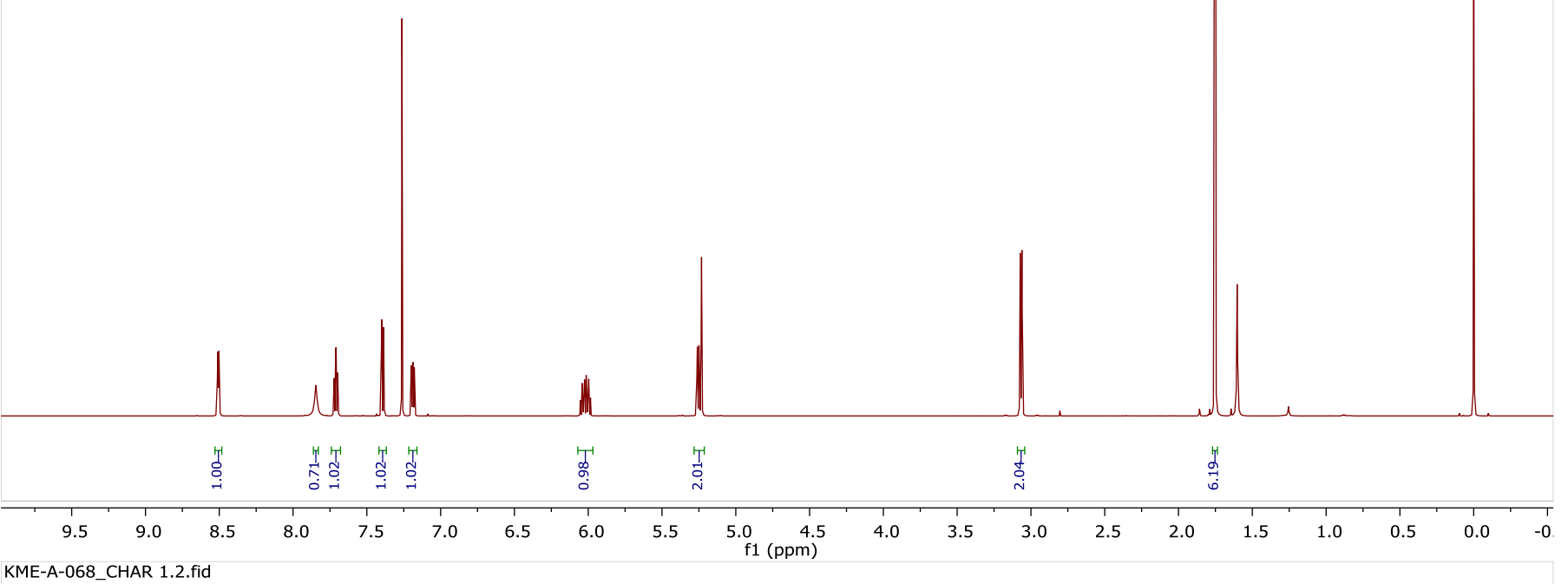
KME-A-068_CHAR 1.2.fid
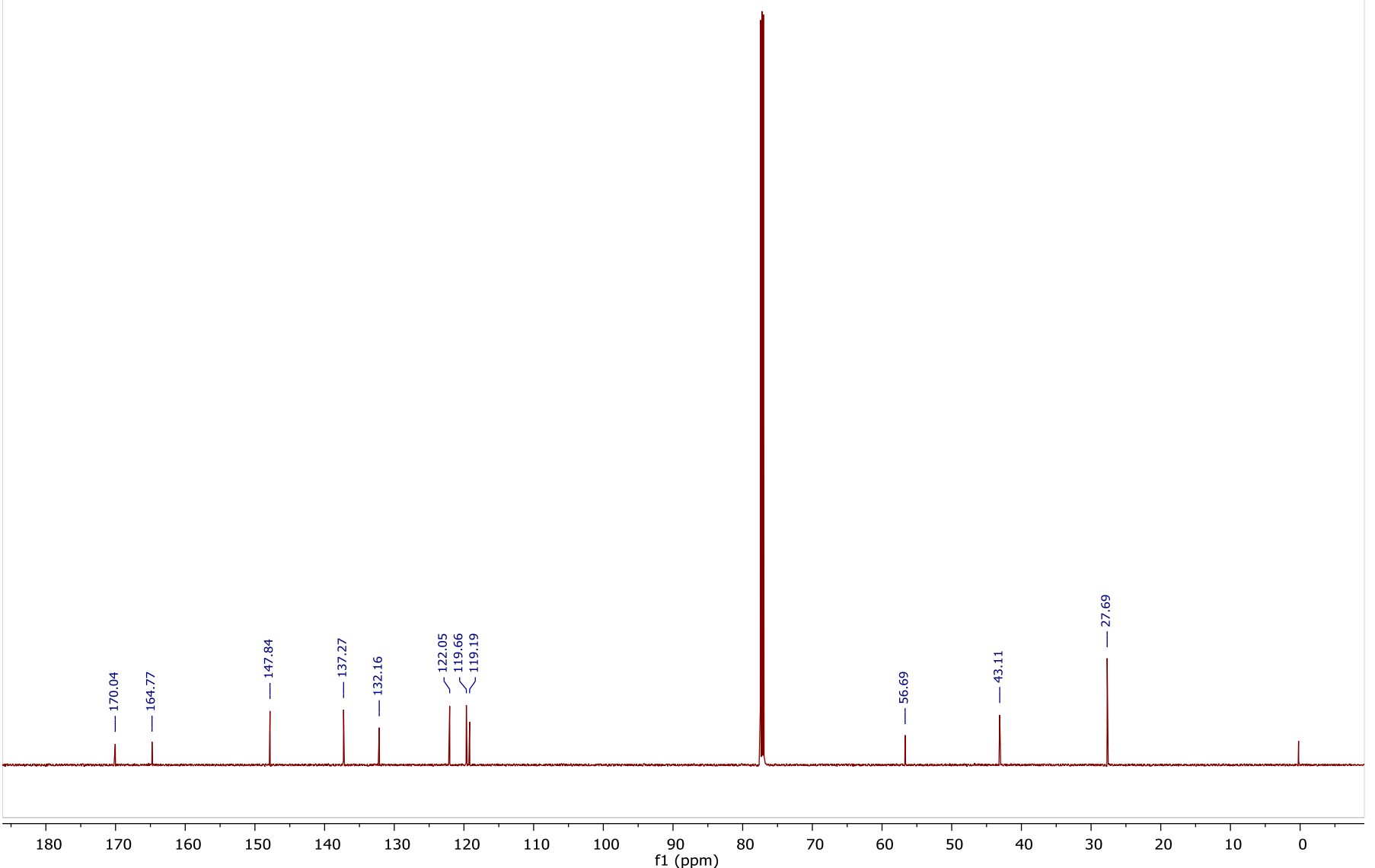
ky-n3-kme-121A.1.fid

H-1 Routine. CPQCI, AVIII-600, 5-12-2016
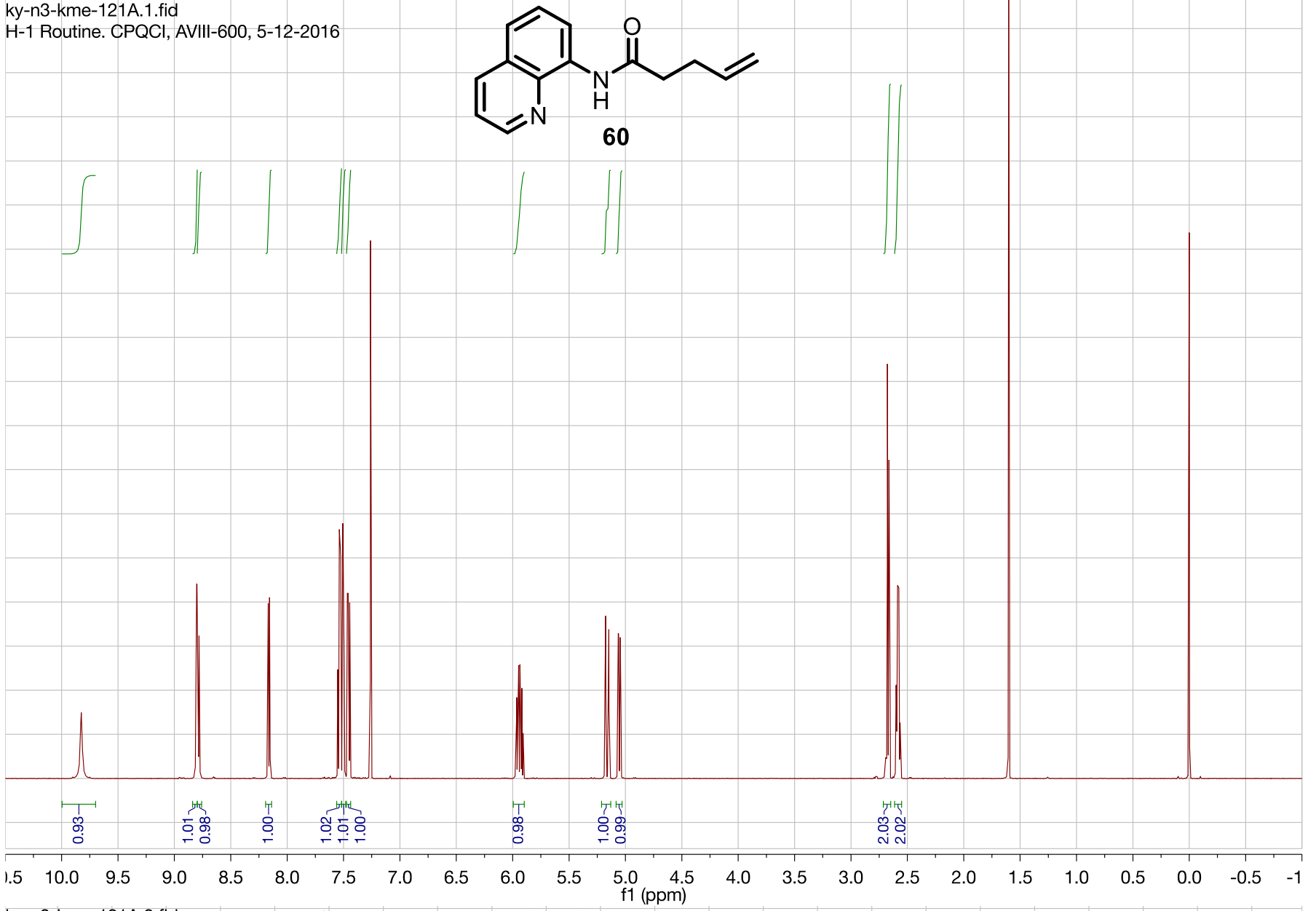

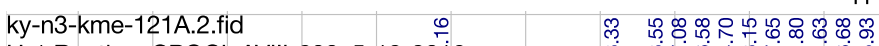

H-1 Routine. CPQCl, AVIII-600, 5-12-2Qj6 
ky-n2-diene-data.1.fid

H-1 Routine. CPQCI, AVIII-600, 5-12-2016
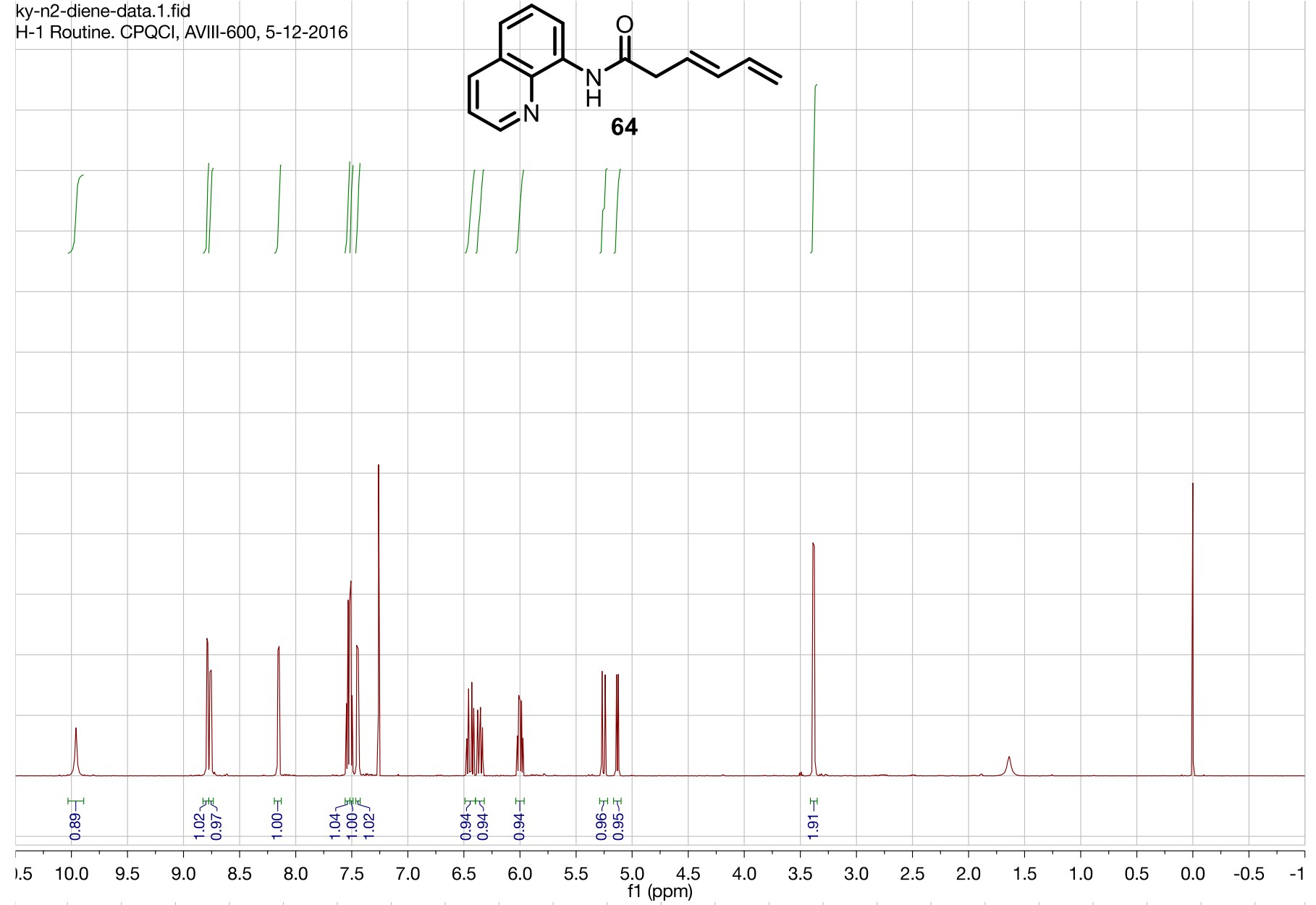

ky-n2-diene-data.2.fid

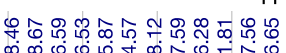
$\mathrm{H}-1$ Routine. CPQCl, AVIII-600, 5-12-2010 
ky-n3-077-diene-data.1.fid

H-1 Routine. CPQCI, AVIII-600, 5-12-2016<smiles>C=C/C(C)=C/CC(=O)Nc1cccc2cccnc12</smiles>

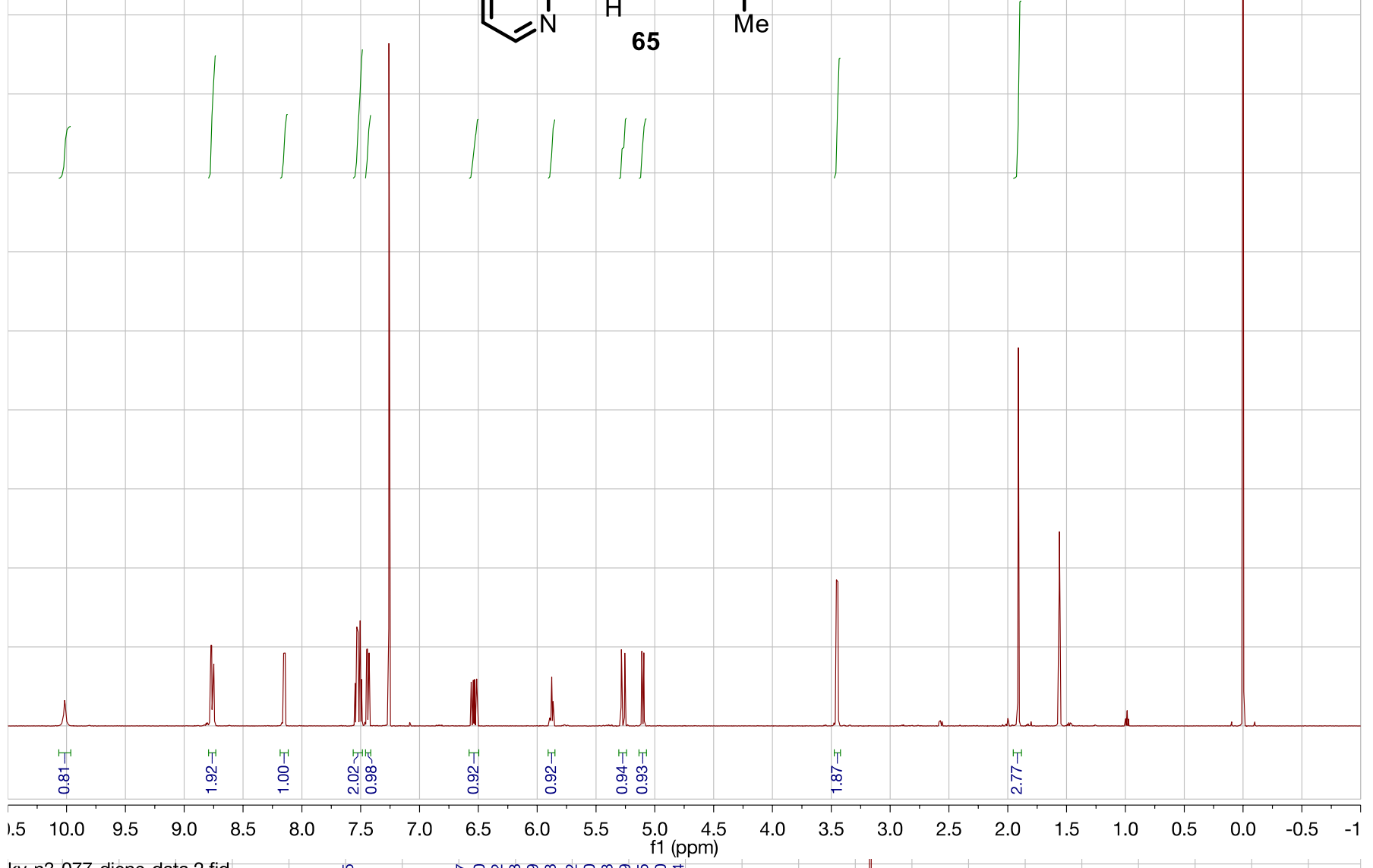

ky-n3-077-diene-data.2.fid t.

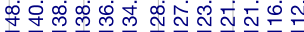

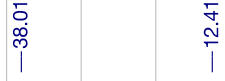

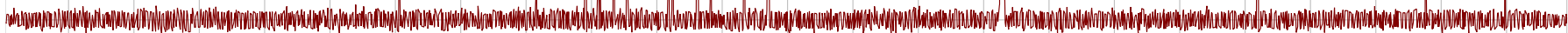

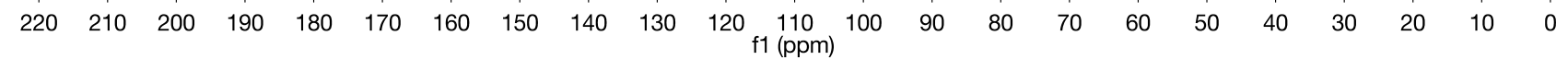


ky-n3-007-A-data.1.fid

H-1 Routine. CPQCI, AVIII-600, 12-20-2013<smiles>CC(=O)C(CCC(=O)Nc1cccc2cccnc12)C(C)=O</smiles>

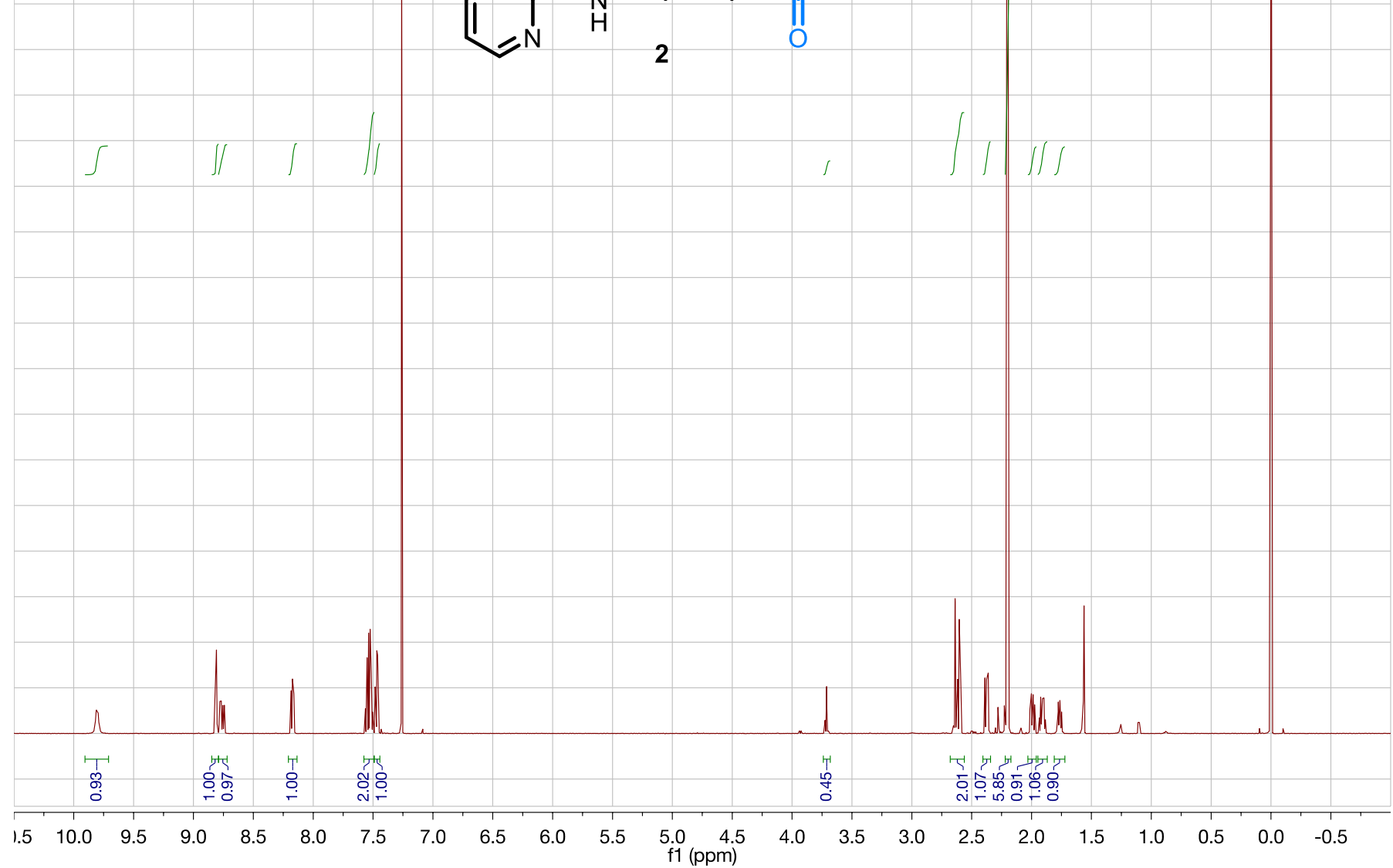

ky-n3-007-A-dater. 2. fid i

\%

等

$\underset{\substack{1 \\ \infty}}{\substack{1 \\ 0}}$

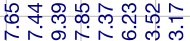

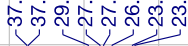




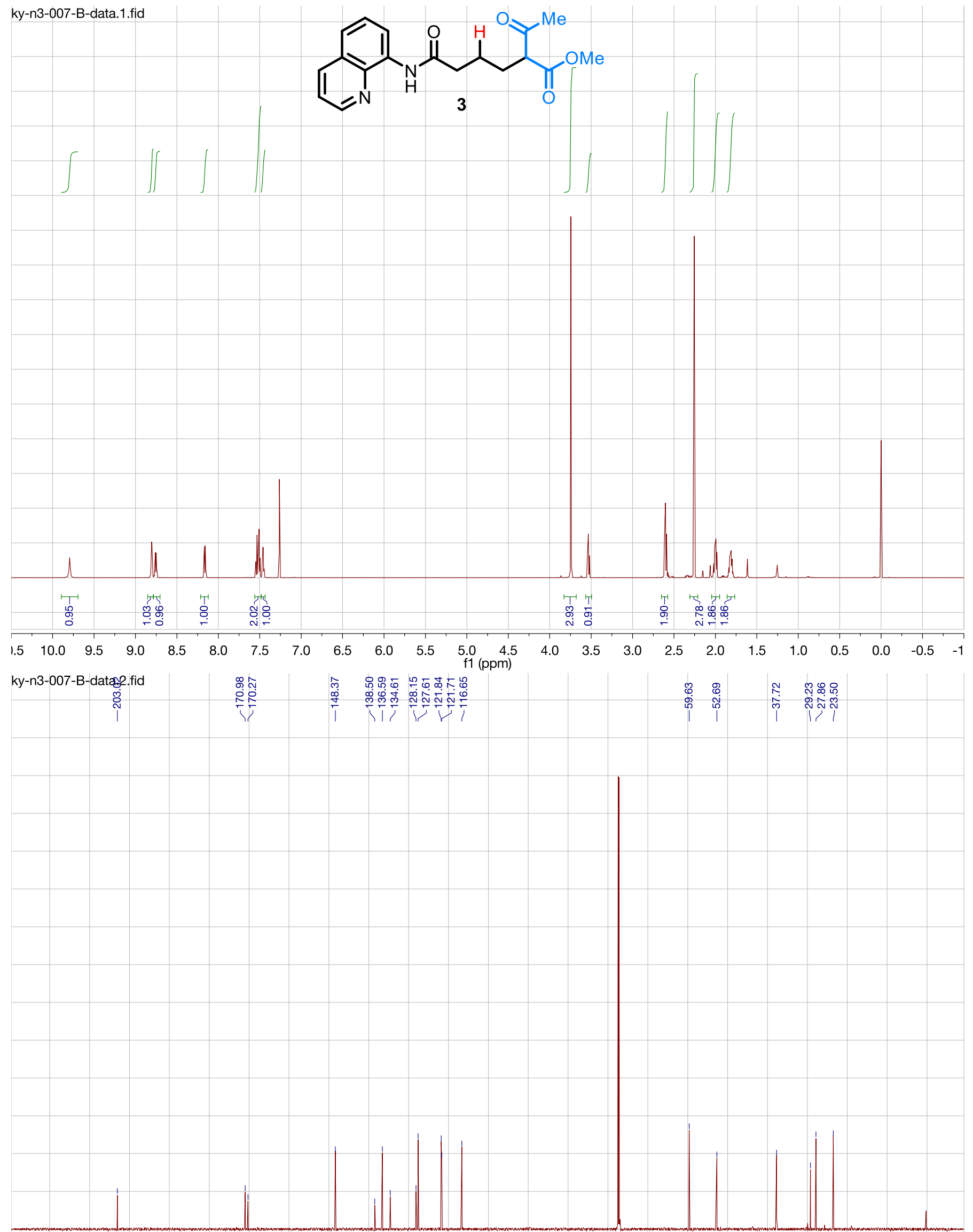




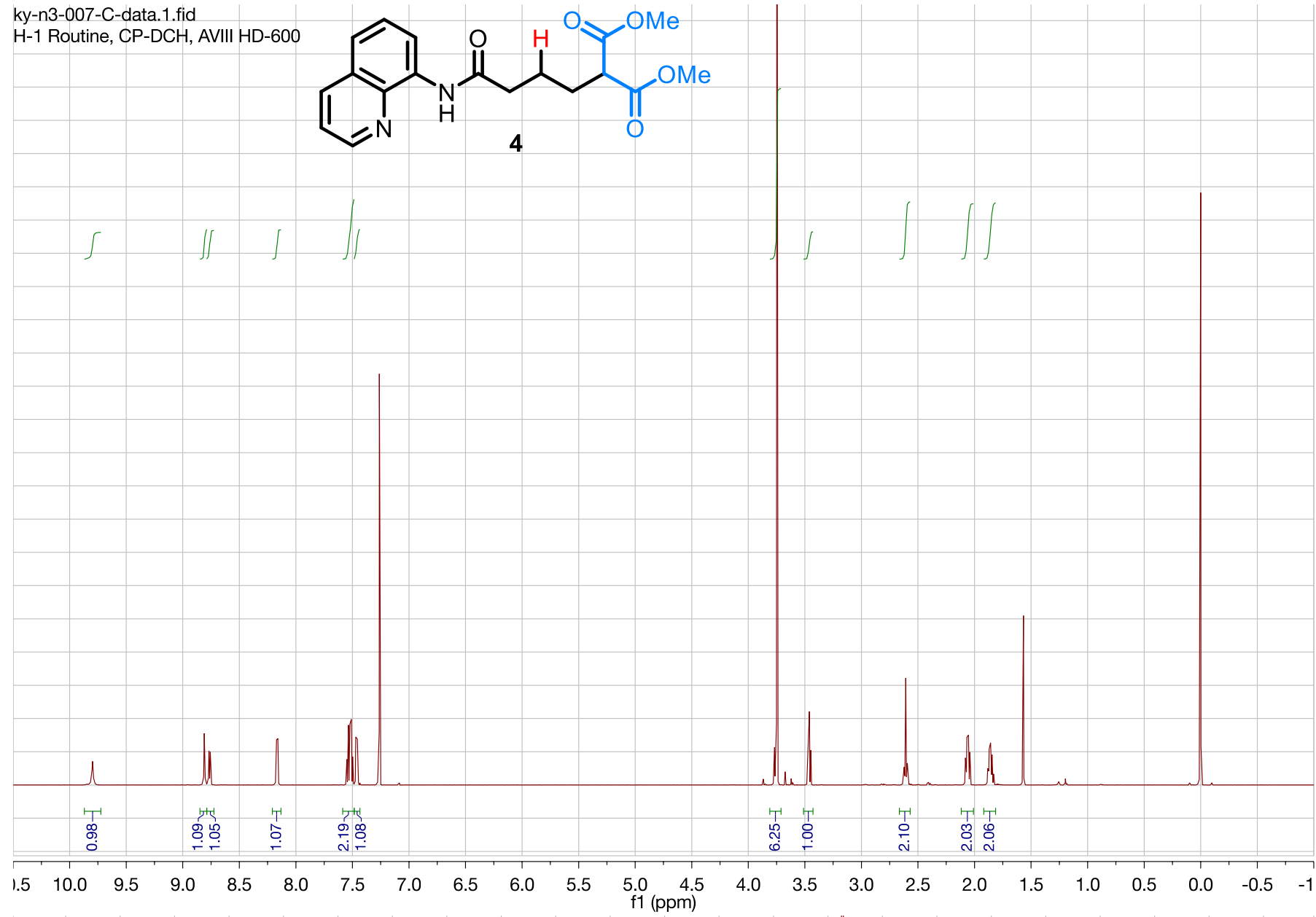
ky-n3-007-C-data.2.fid

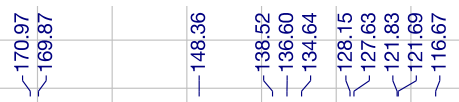

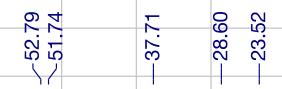


ky-n3-015-data.1.fid

H-1 Routine. CPQCI, AVIII-600, 12-20-2013<smiles>CC1(C)CC(=O)C(CCC(=O)Nc2cccc3cccnc23)=C(O)C1</smiles>

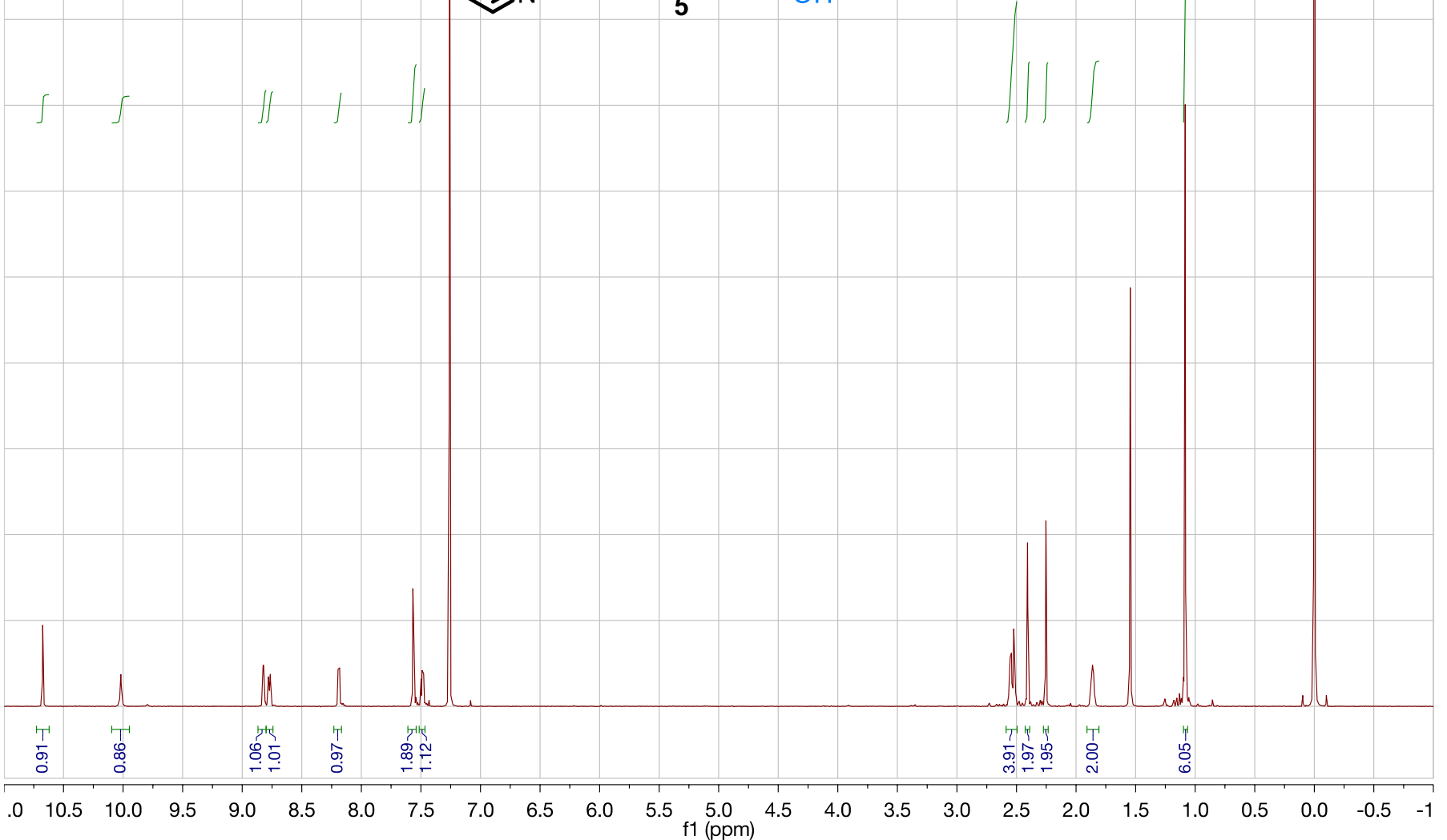

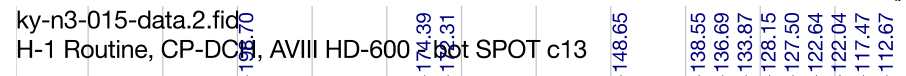

मे.

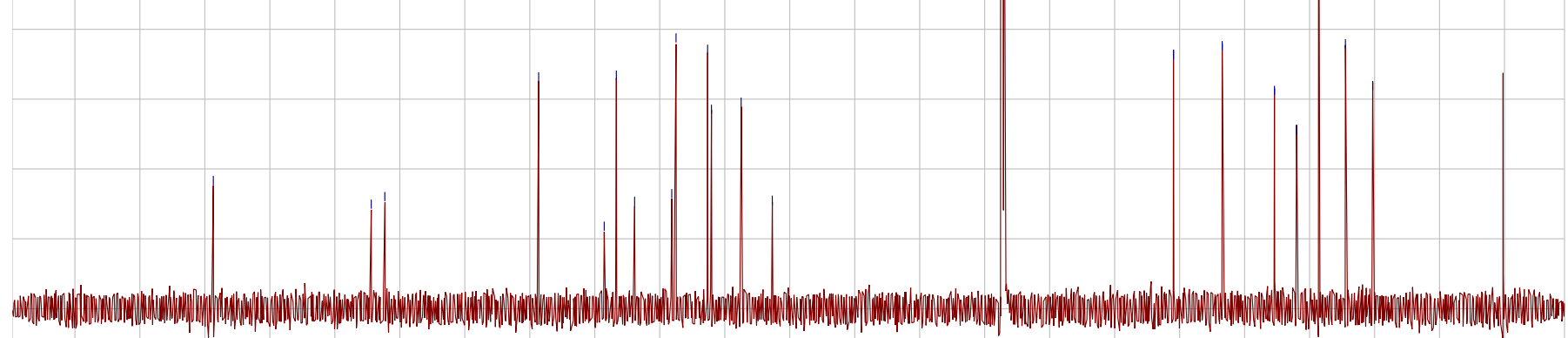


ky-n3-017-C-data.3.fid

H-1 Routine. CPQCI, AVIII-600, 12-20-2013<smiles>O=C(CCC1C(=O)CCCCC1=O)Nc1cccc2cccnc12</smiles>

(1)

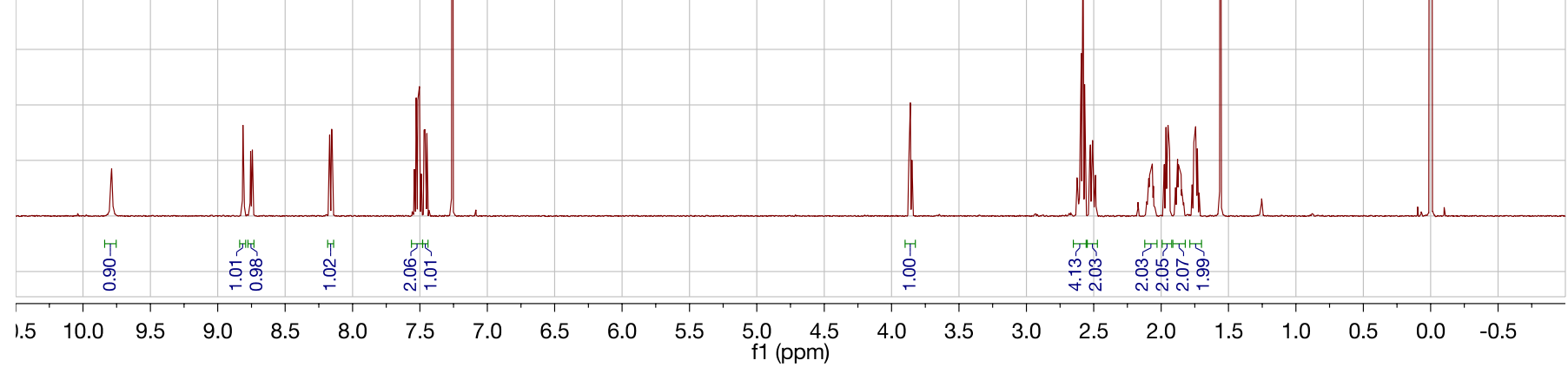

ky-n3-017-C-data.2.fid

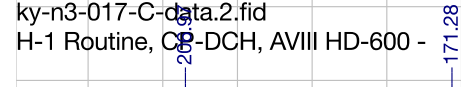

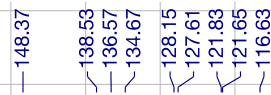

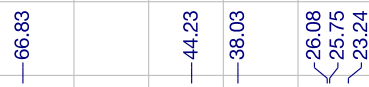




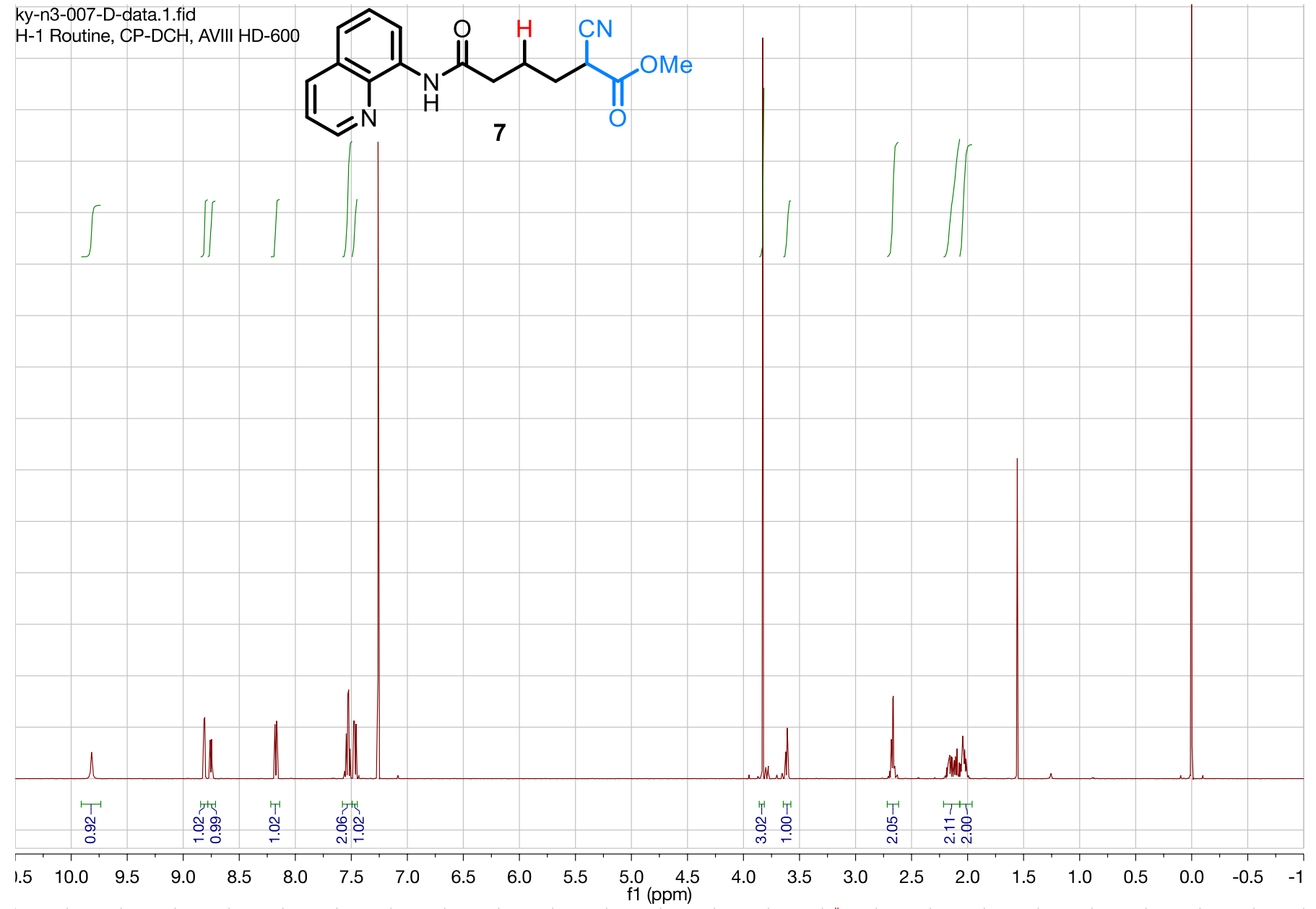

ky-n3-007-D-data.2 fid

H-1 Routine, CP-DCH, AVIII HD-600

T广 T 
ky-jg-malononitrile-data.1.fid

H-1 Routine, CPOCI, AVIII-600, 5-12-2016
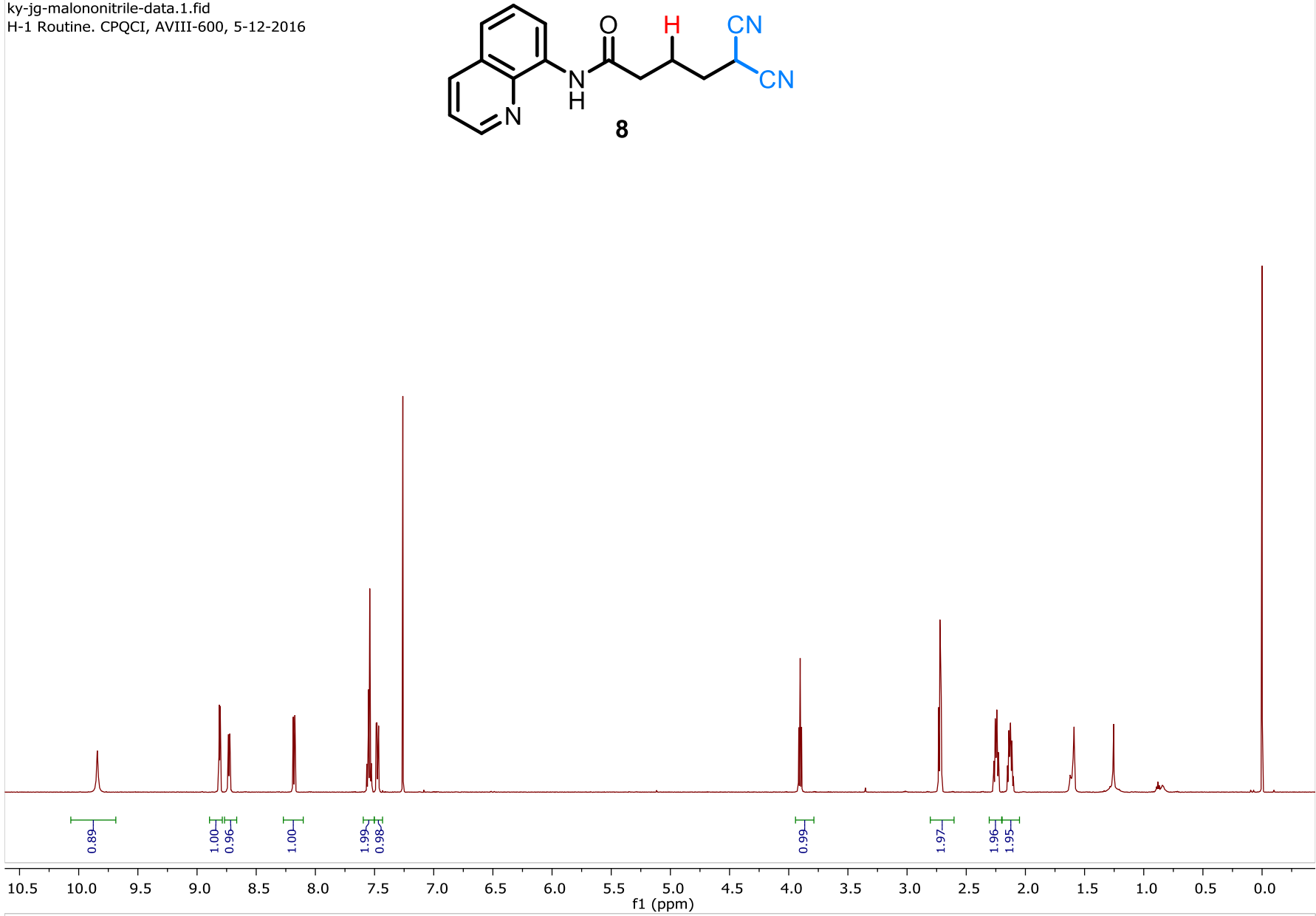

ky-jg-malononitrile-data.2.fid

H-1 Routine. CPQCI, AVIII-600, 5-12-2016
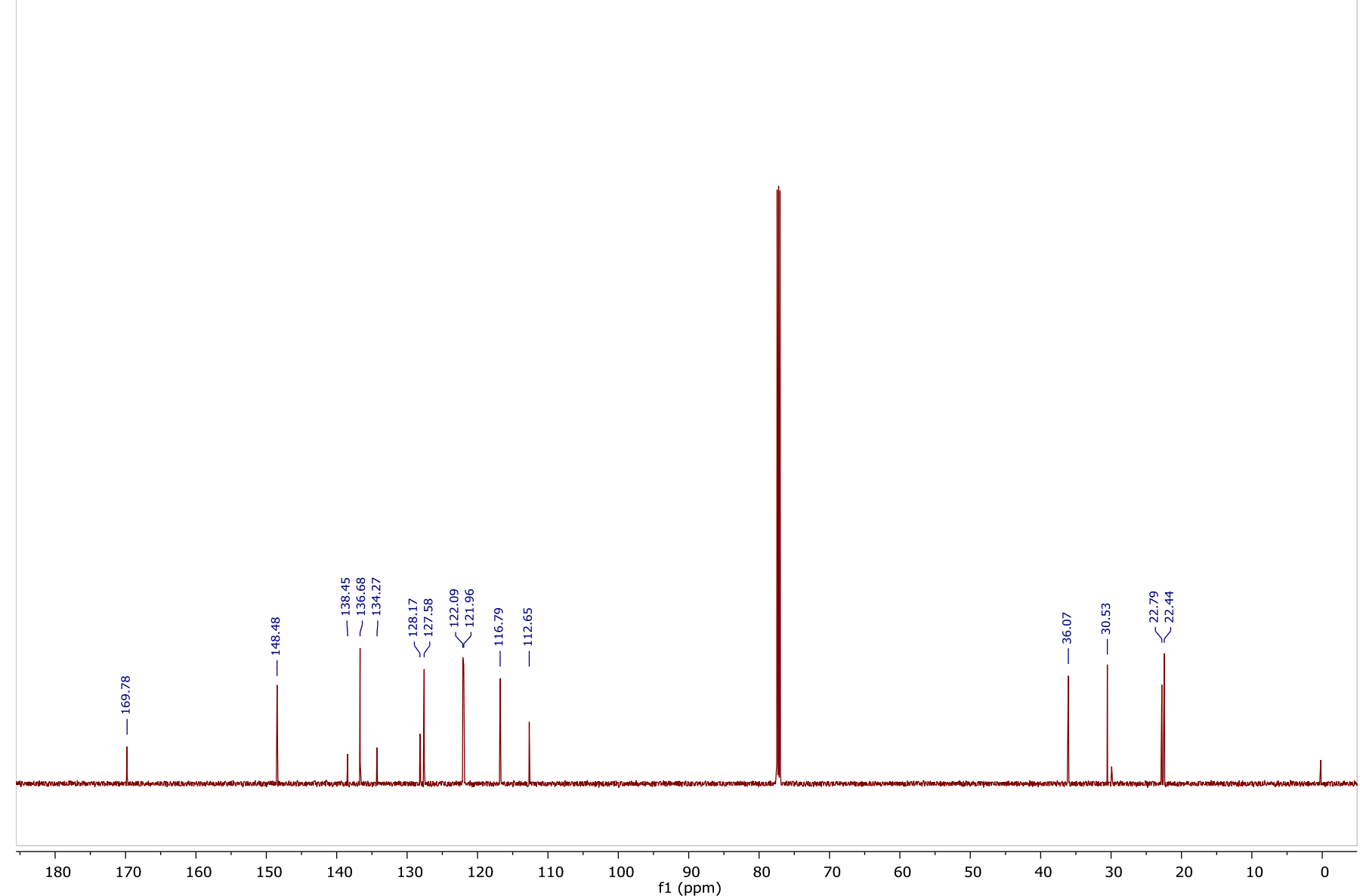
ky-n3-jg-cyanphos.1.fid H-1 Routine, CP-DCH, AVIII HD-600 -<smiles>CCOP(=O)(OCC)C(C#N)CCC(=O)Nc1cccc2cccnc12</smiles>

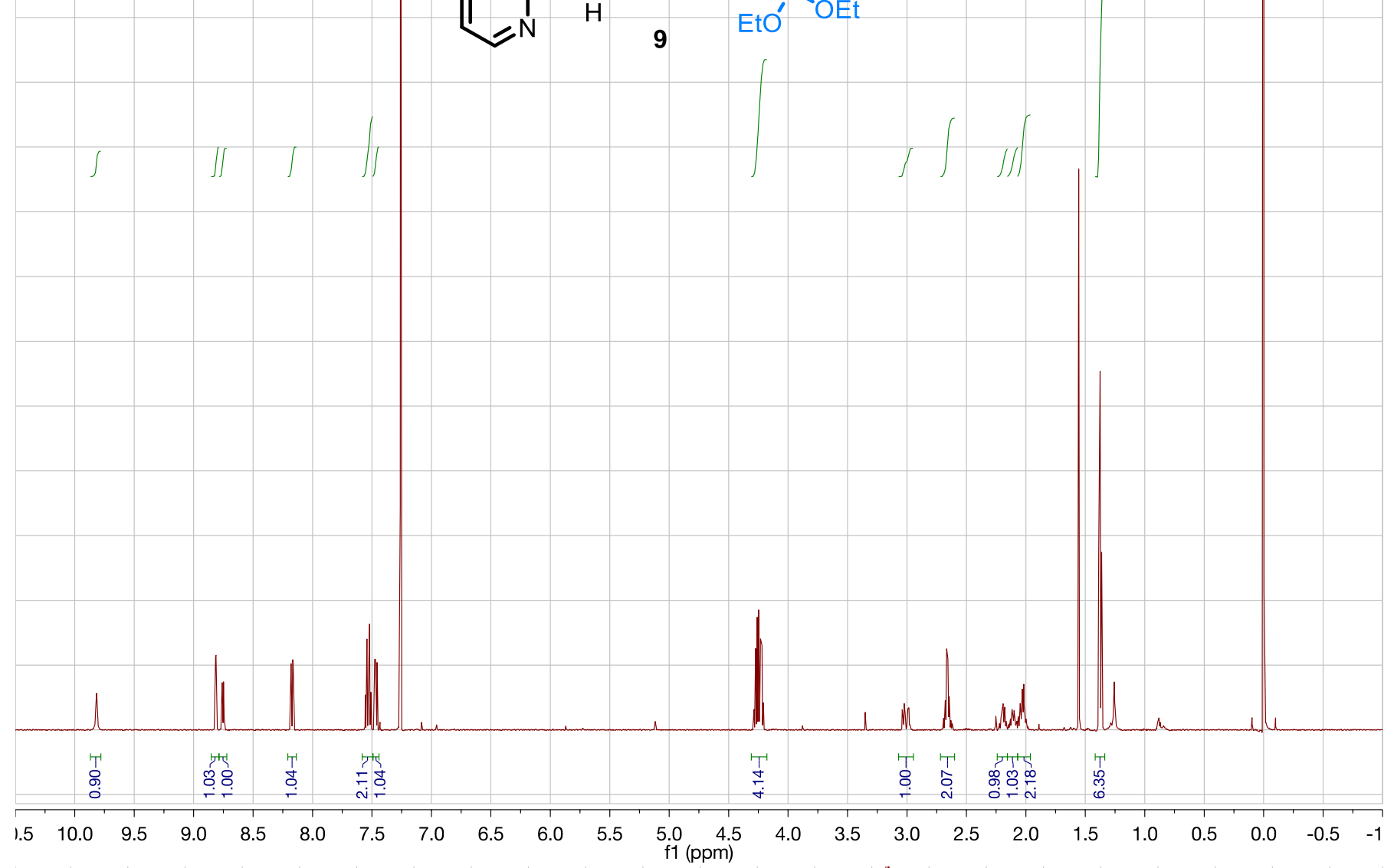

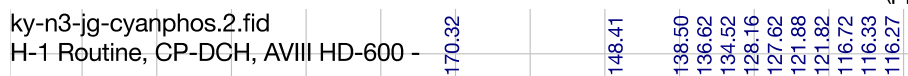

H-1 Routine, CP-DCH, AVIII HD-600 - ? 
ky-n3-017-A-data.3.fid

H-1 Routine. CPQCI, AVIII-600, 12-20-2013<smiles>COC(=O)C(CCC(=O)Nc1cccc2cccnc12)S(=O)(=O)c1ccccc1</smiles>

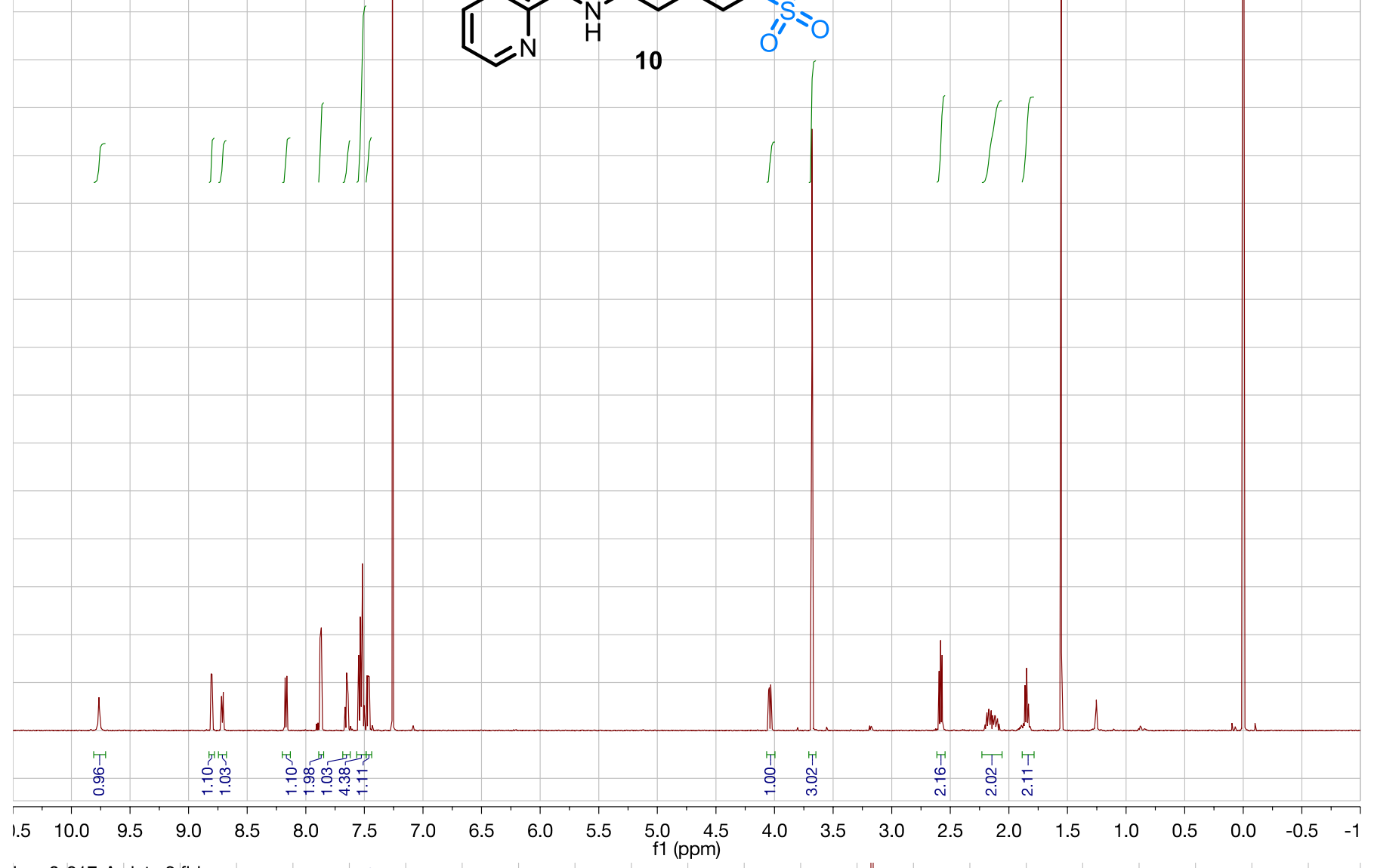

ky-n3-017-A-data.2.fid

$\begin{array}{cc}0 & 0 \\ 0 & 0 \\ 0 & 0 \\ 0 & 0\end{array}$

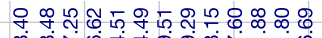

我

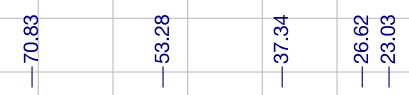


ky-n3-016-B-data.1.fid

H-1 Routine, CP-DCH, AVIII HD-600 -<smiles>CCOC(=O)C(N)(CCC(=O)Nc1cccc2cccnc12)C(=O)OCC</smiles>

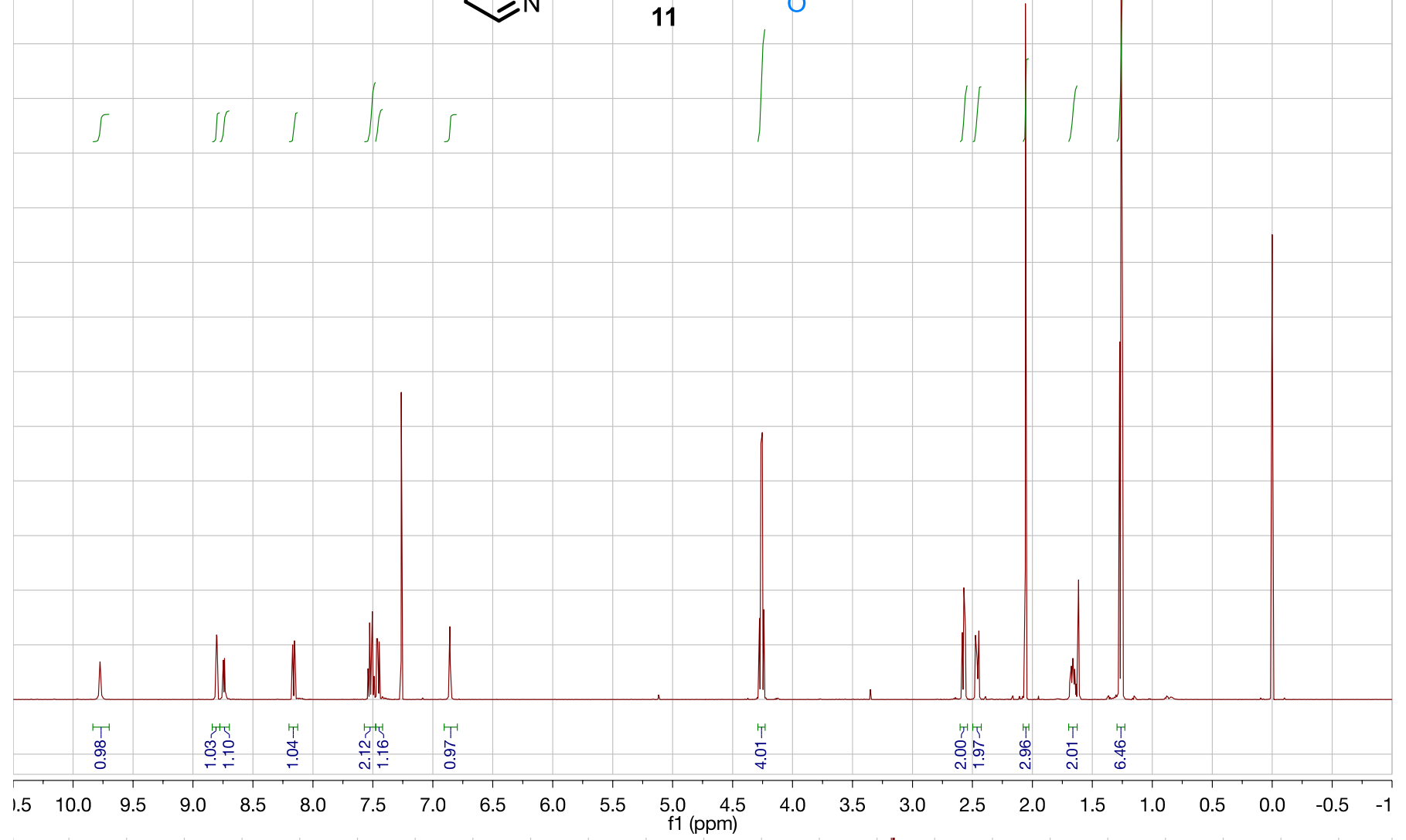

\section{ky-n3-016-B-data.2.fid}

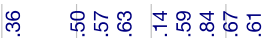

H-1 Routine, CP-DCH, AVIII HD-600 -

要

ธே

\&

N

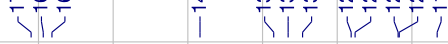

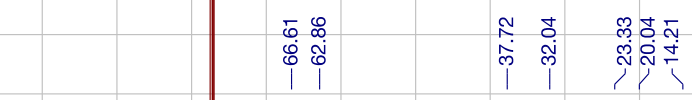


ky-n3-jg-no2-me-data.1.fid

H-1 Routine, CP-DCH, AVIII HD-600 -
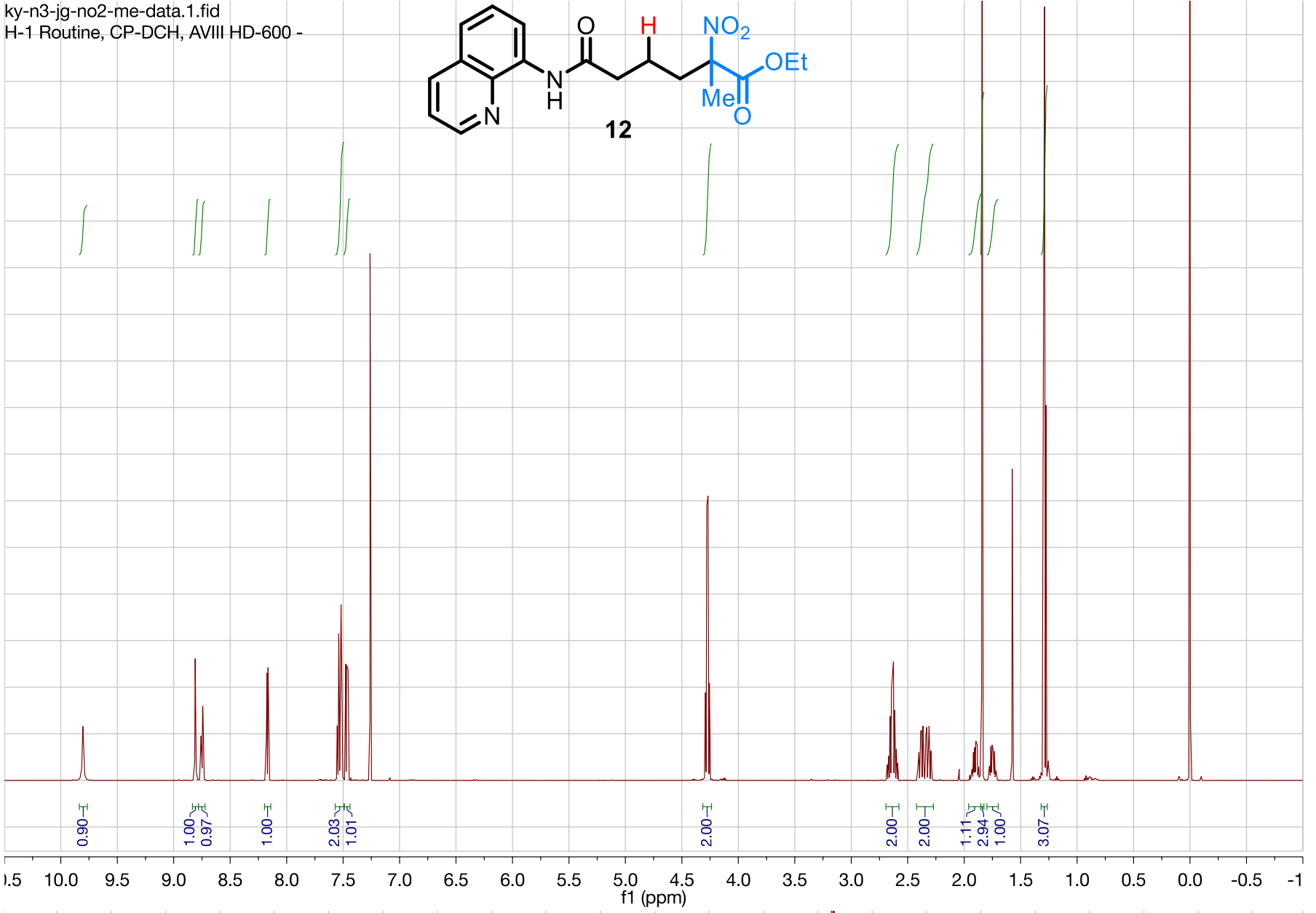

ky-n3-jg-no2-me-data.2.fid

$\mathrm{H}-1$ Routine, CP-DCH, AVIII HD-600 - 웡

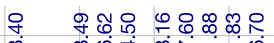

索

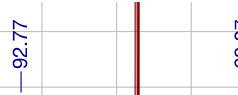

ij 
ky-n3-040-A-data.1.fid

C-13 Routine 1D, CPDCH CryoProbe, AVIII-600<smiles>CCOC(=O)C(F)(CCCC(=O)Nc1cccc2cccnc12)C(C)=O</smiles>

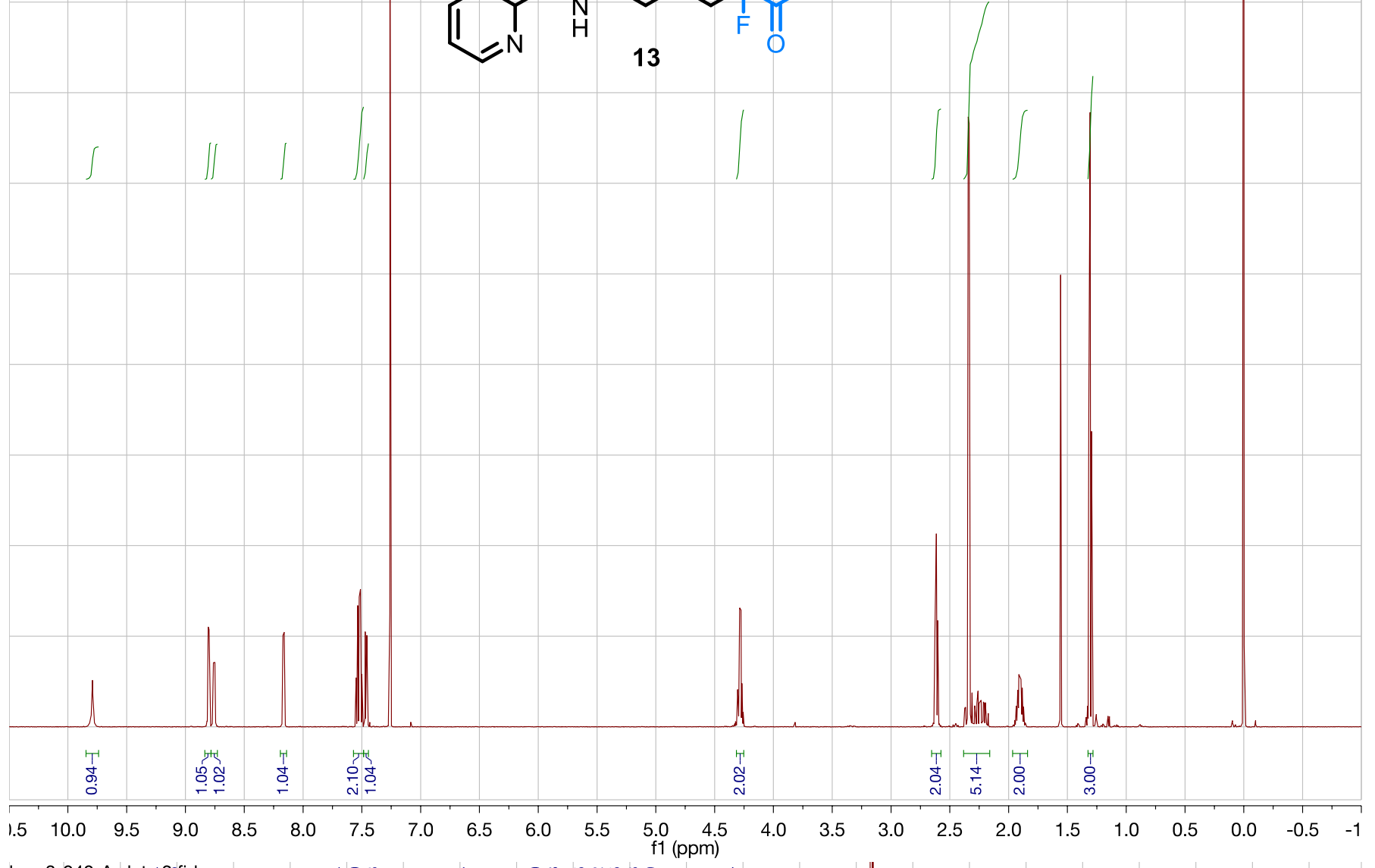

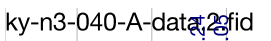
C-13 Routine 1D gofidDCH CryoProbe,

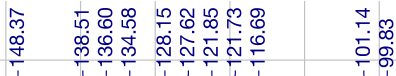
广广 $\bar{\top}$ 
ky-n3-057-B-data.1.fid

C-13 Routine 1D, CPDCH CryoProbe, AVIII-600

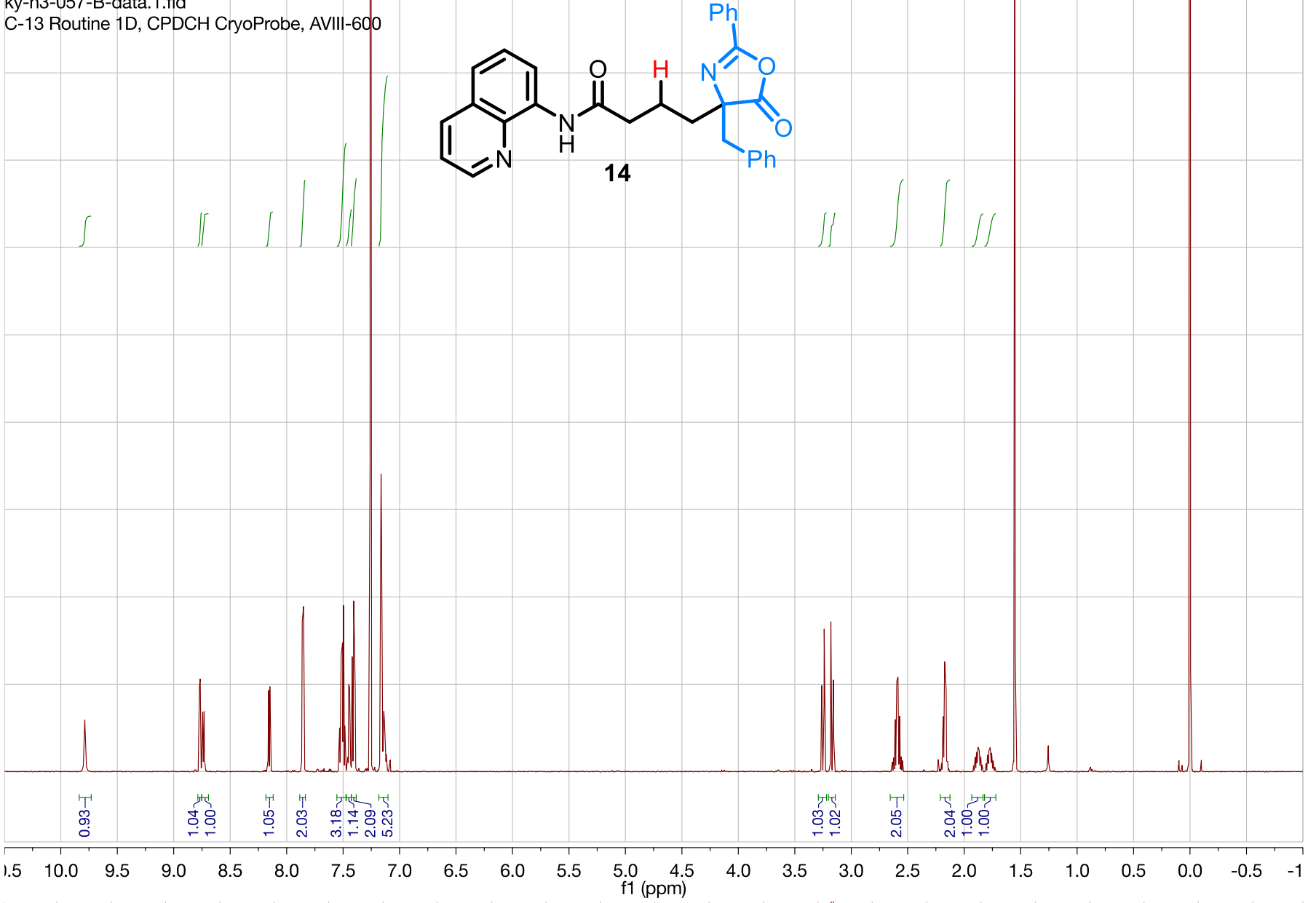

ky-n3-057-B-data. 2 fid

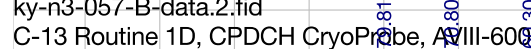

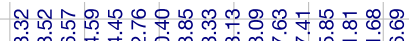

।

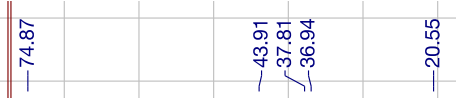


ky-n3-066-A-data.1.fid

C-13 Routine 1D, CPDCH CryoProbe, AVIII-600<smiles>CC(=O)C(C#N)(CC(CC(=O)Nc1cccc2cccnc12)c1ccccc1)c1ccccc1</smiles>
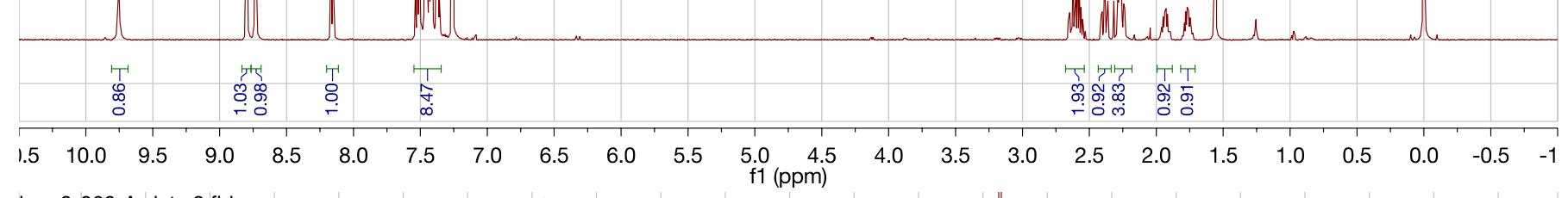

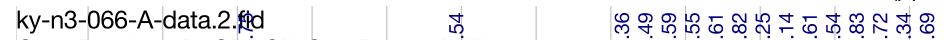

C-13 Routine 1D, CPDCH CryoProbe, A

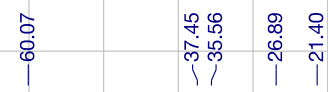


ky-n3-018-D-data.1.fid

H-1 Routine. Loaner CPTXI, AVIII-600, 2-23-2016<smiles>CC(=O)C1(CCC(=O)Nc2cccc3cccnc23)CCCC1=O</smiles>

$\underbrace{}_{16}$

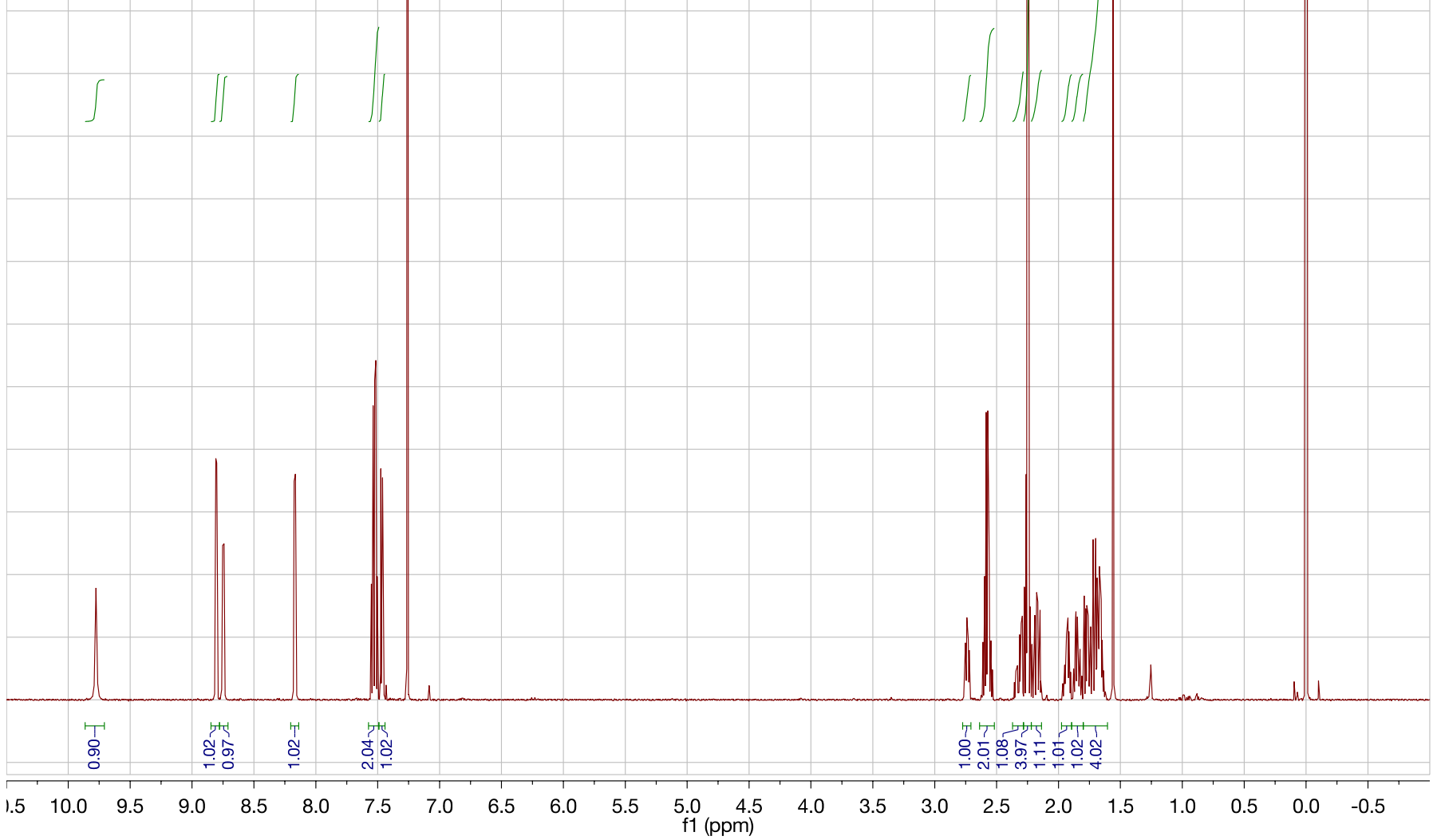

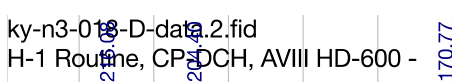

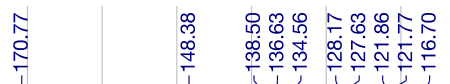

i

广

@্

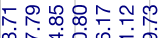

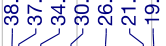

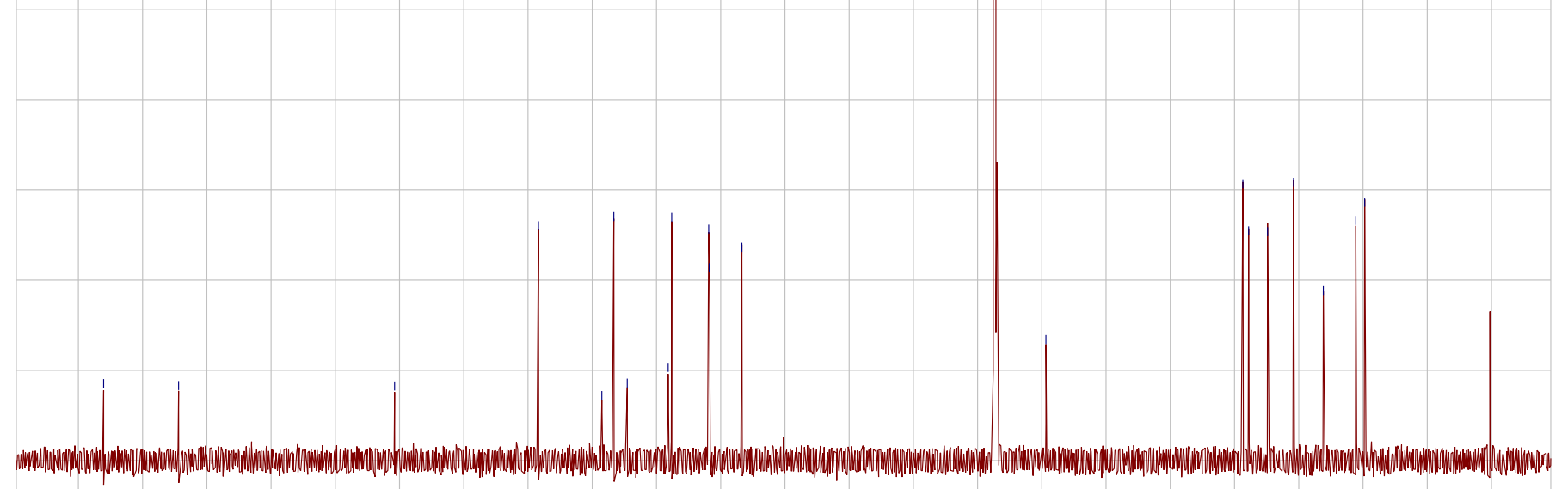


ky-n3-016-A-data.1.fid H-1 Routine, CP-DCH, AVIII HD-600 -<smiles>CC(=O)C1(CCC(=O)Nc2cccc3cccnc23)CCOC1=O</smiles>
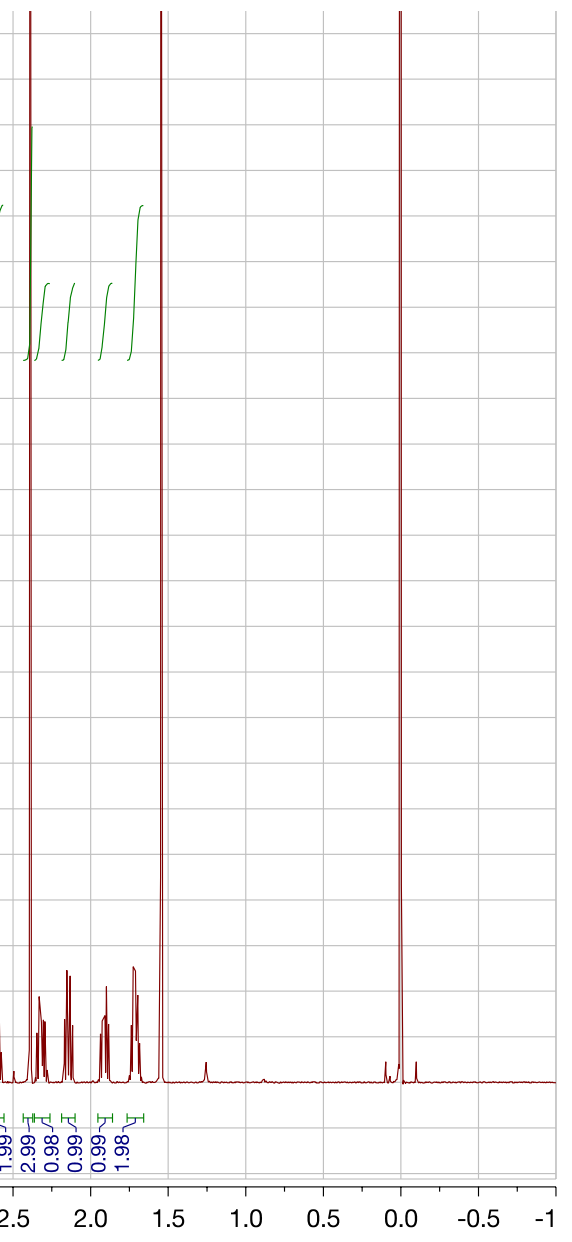

$\begin{array}{lllllllllllll}1.5 & 10.0 & 9.5 & 9.0 & 8.5 & 8.0 & 7.5 & 7.0 & 6.5 & 6.0 & 5.5 & 5.0 & 4.5 \\ \mathrm{f} 1(\mathrm{ppm})\end{array}$

ky-n3-016-A-datage fid 1 fid

$\mathrm{H}-1$ Routine, CP-

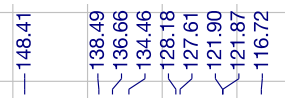

$\begin{array}{lll}0 & 2 \\ 0 & 0 \\ 0 & 0 \\ 1 & 1\end{array}$

กิ ชิ

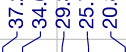

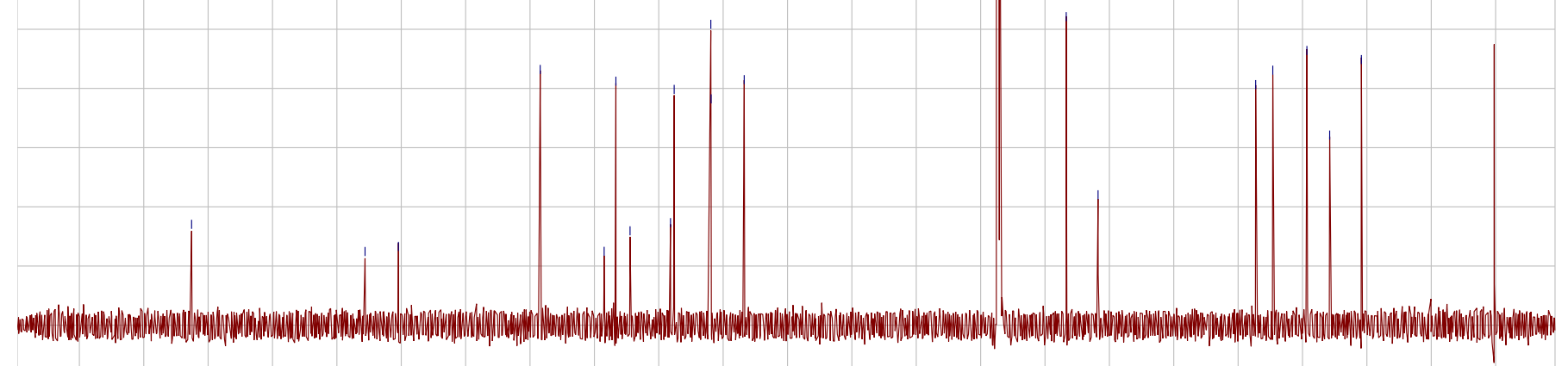


ky-n3-021-C-data.3.fid

H-1 Routine. CPQCI, AVIII-600, 12-20-2013
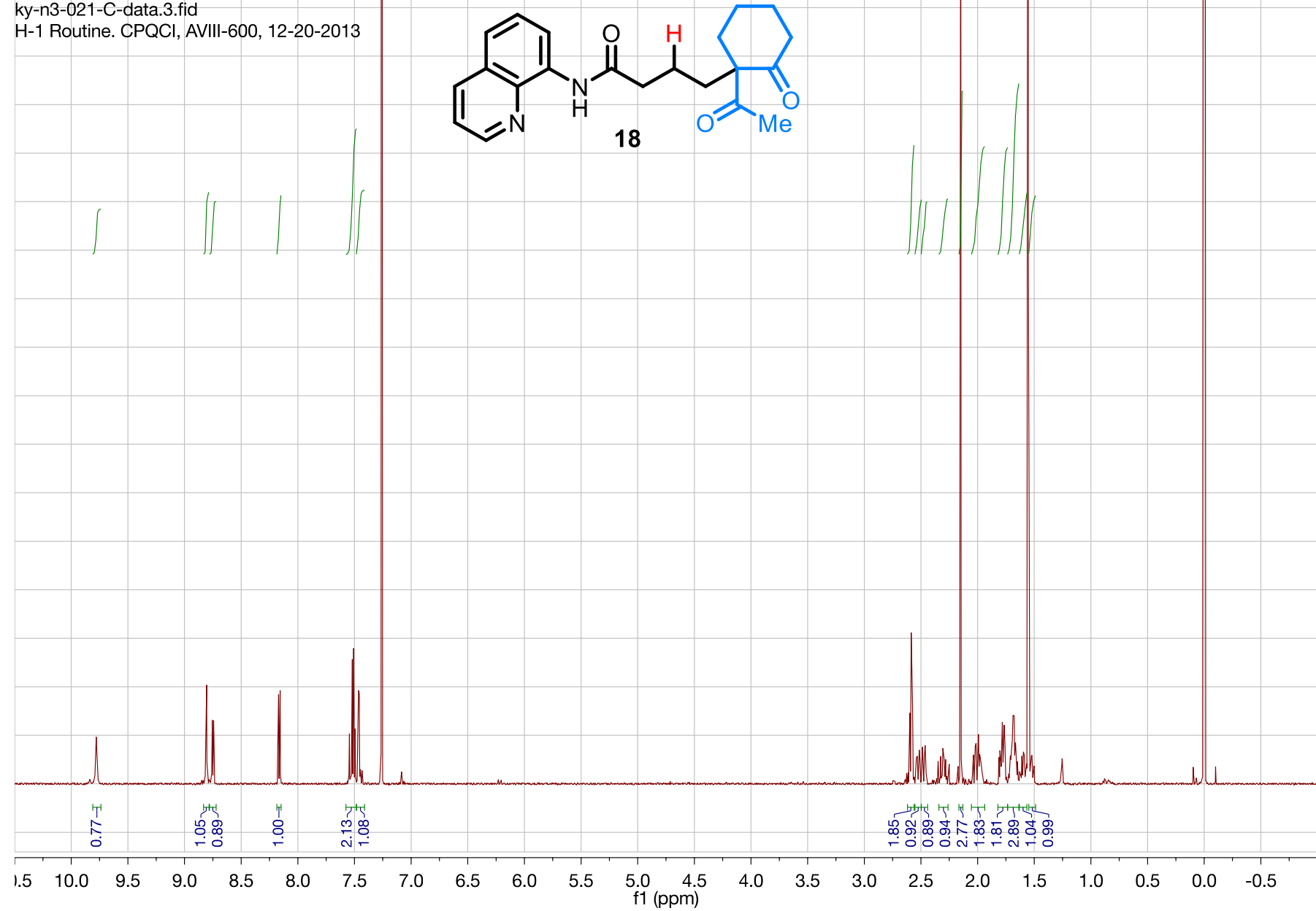

\section{ky-n3-021-Ce्edgata.2.fid}

H-1 Routine, icge

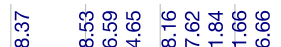

|

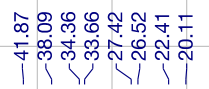


ky-n3-jg-ac-tetra-data.1.fid

C-13 Routine 1D, CPDCH CryoProbe, AVIII-600

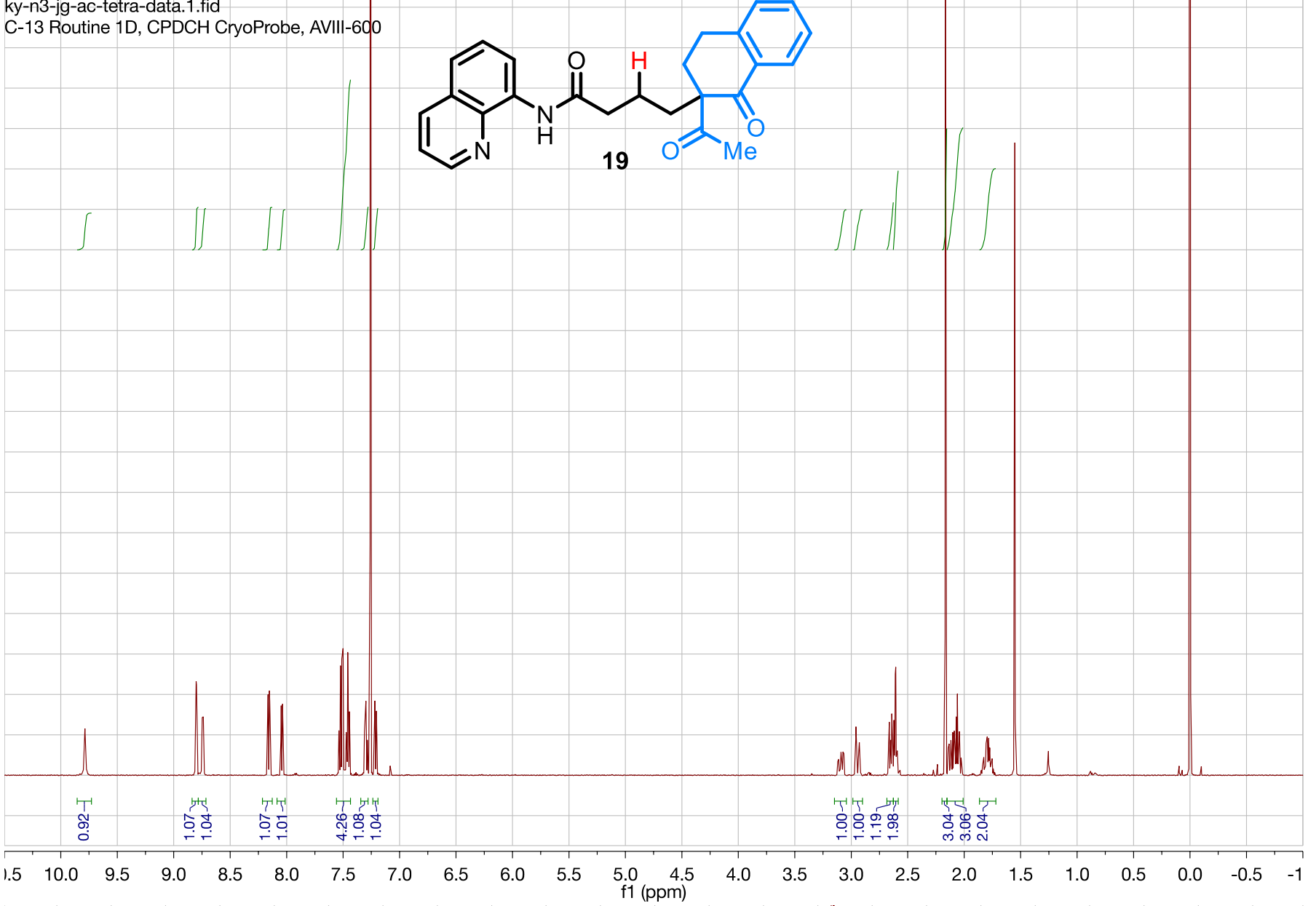

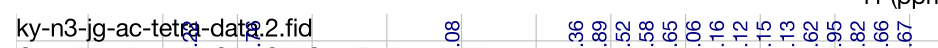

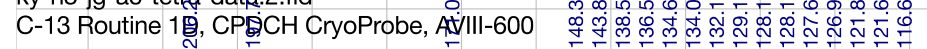

$\underset{\substack{0 \\ \dot{0} \\ 0}}{1}$

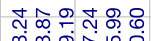

एंग

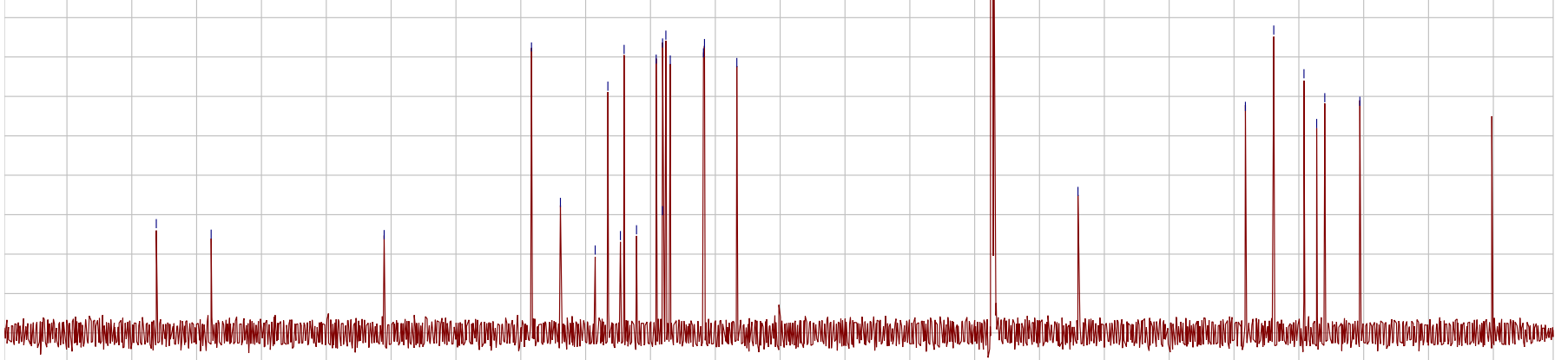


ky-n3-016-C-data.1.fid

H-1 Routine, CP-DCH, AVIII HD-600 -<smiles>O=C(CCC1C(=O)CCc2ccccc21)Nc1cccc2cccnc12</smiles>

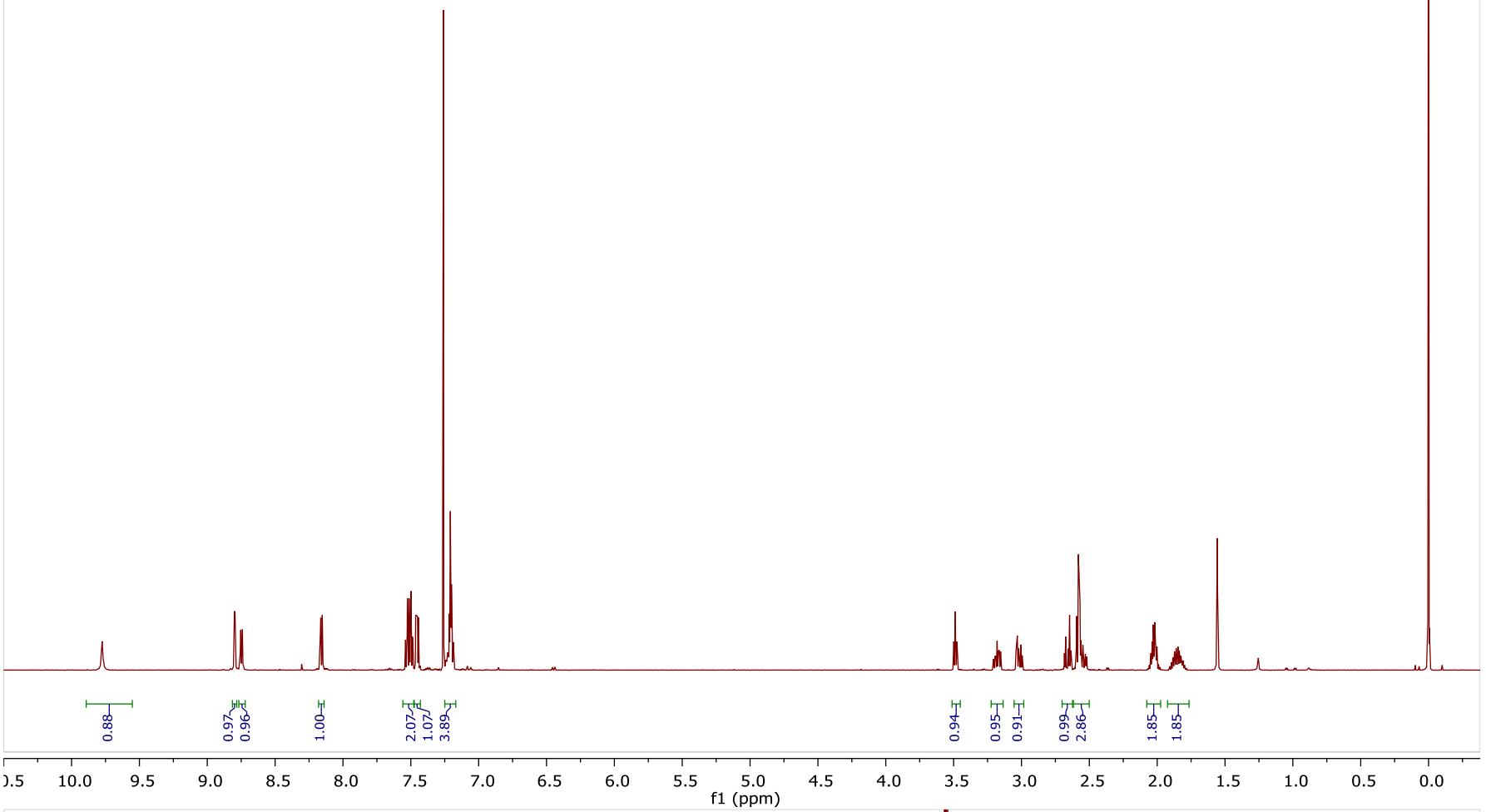

ky-n3-016-C-data.2.fid

H-1 Routine, CP-DCH, AVIII HD-600 -

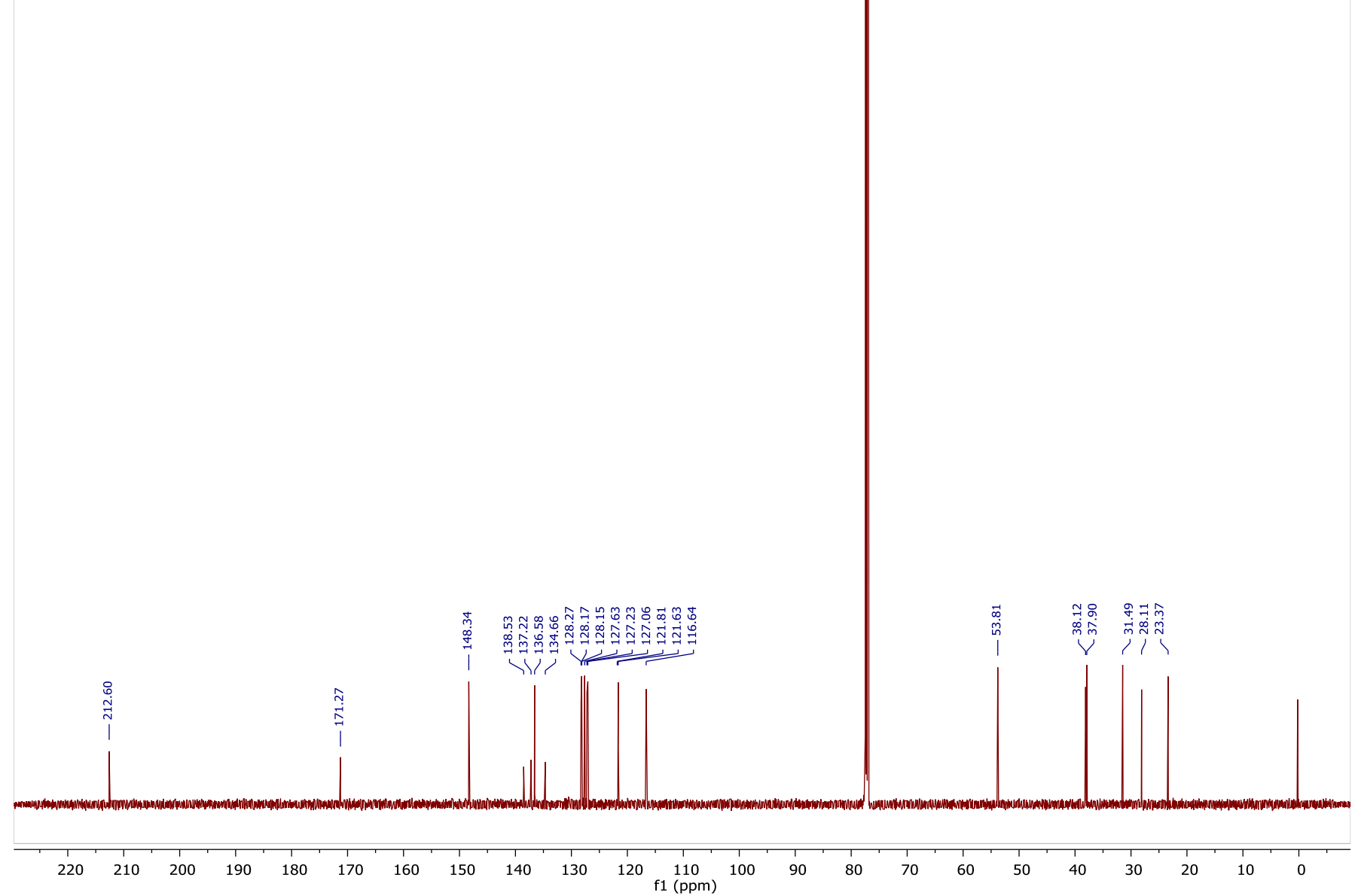


ky-n3-037-A-data.1.fid

C-13 Routine 1D, CPDCH CryoProbe, AVIII-600

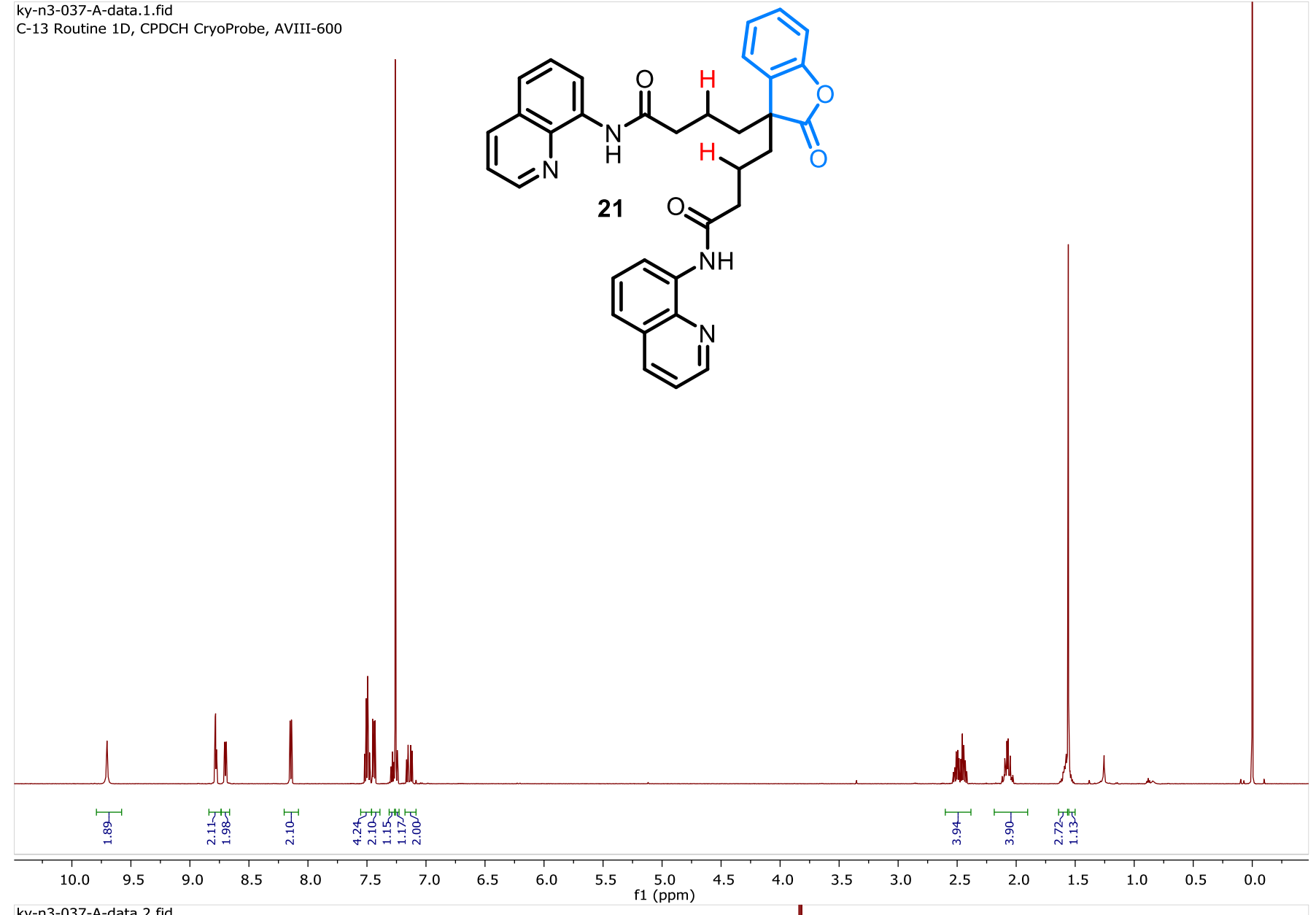

ky-n3-037-A-data.2.fid

C-13 Routine 1D, CPDCH CryoProbe, AVIII-600
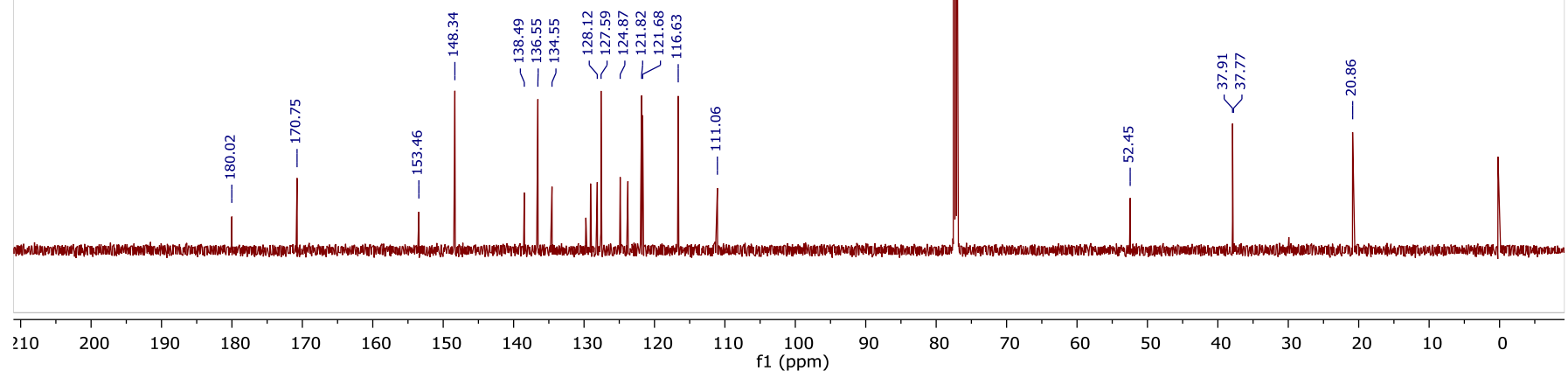
ky-n3-043-C-data.1.fid

C-13 Routine 1D, CPDCH CryoProbe, AVIII-600

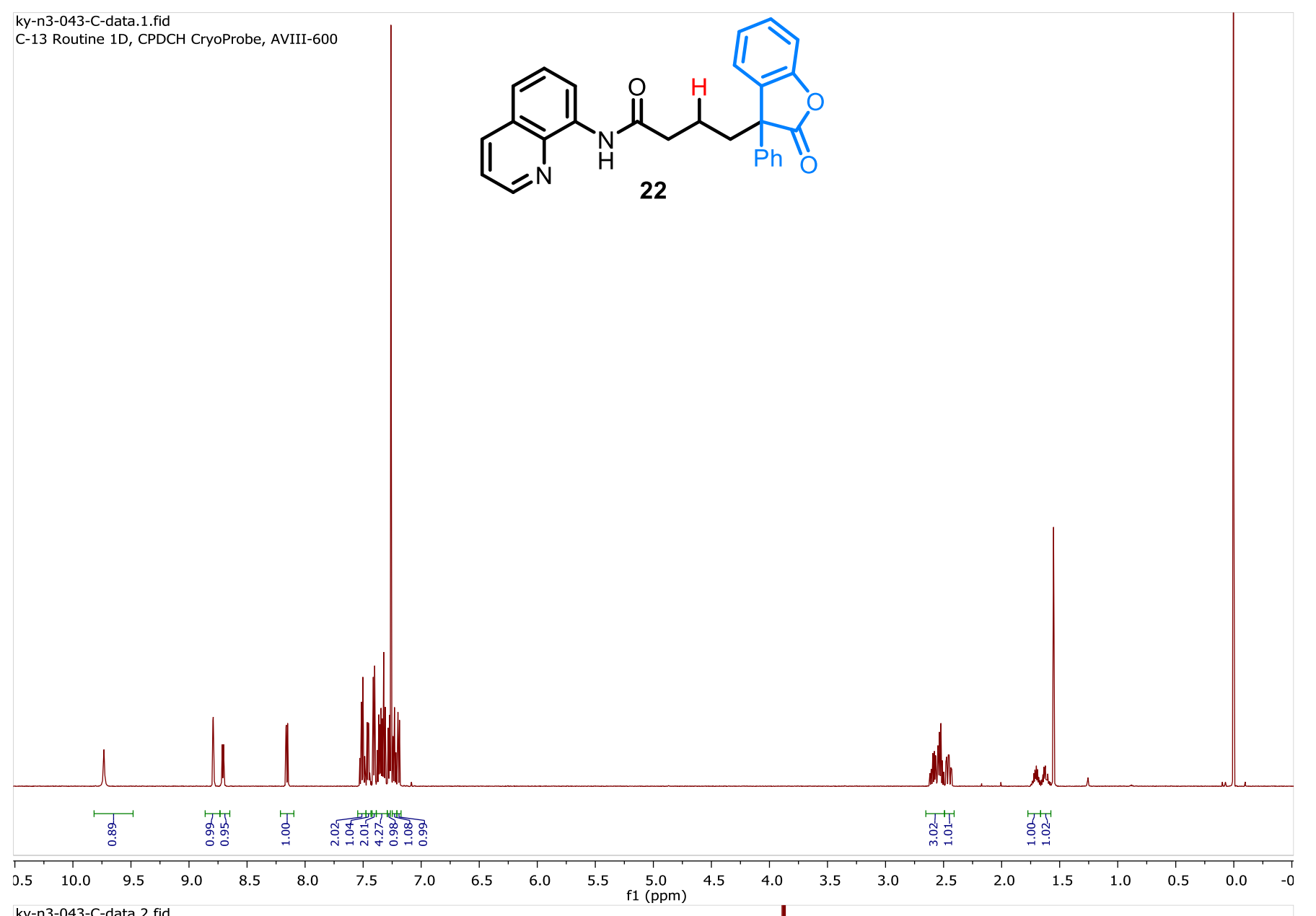

ky-n3-043-C-data.2.fid

C-13 Routine 1D, CPDCH CryoProbe, AVIII-600

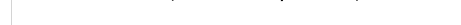


ky-n3-043-A-data.1.fid

C-13 Routine 1D, CPDCH CryoProbe, AVIII-600<smiles>CCC1(CCCC(=O)Nc2cccc3cccnc23)C(=O)Oc2ccccc21</smiles>

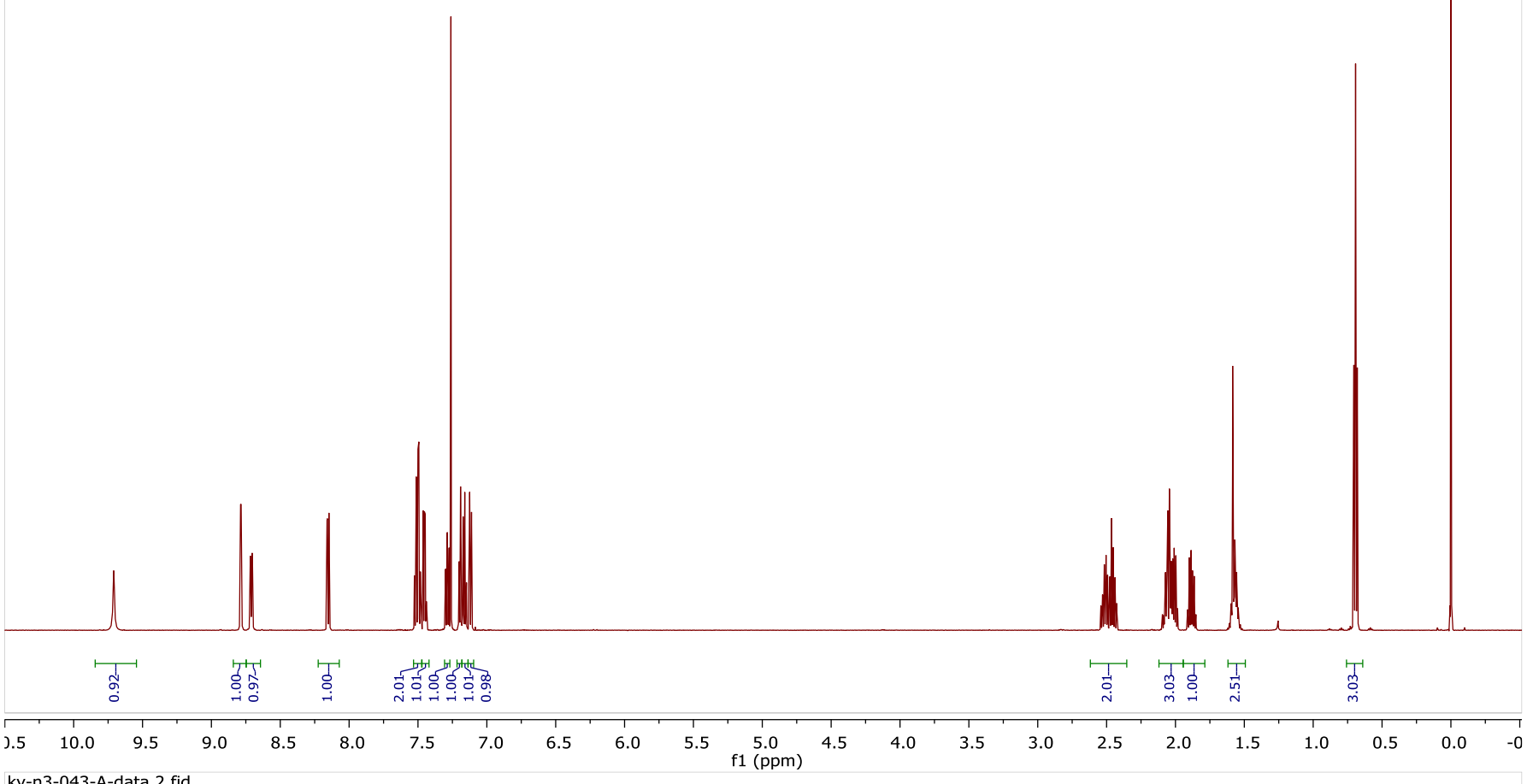

ky-n3-043-A-data.2.fid

C-13 Routine 1D, CPDCH CryoProbe, AVIII-600

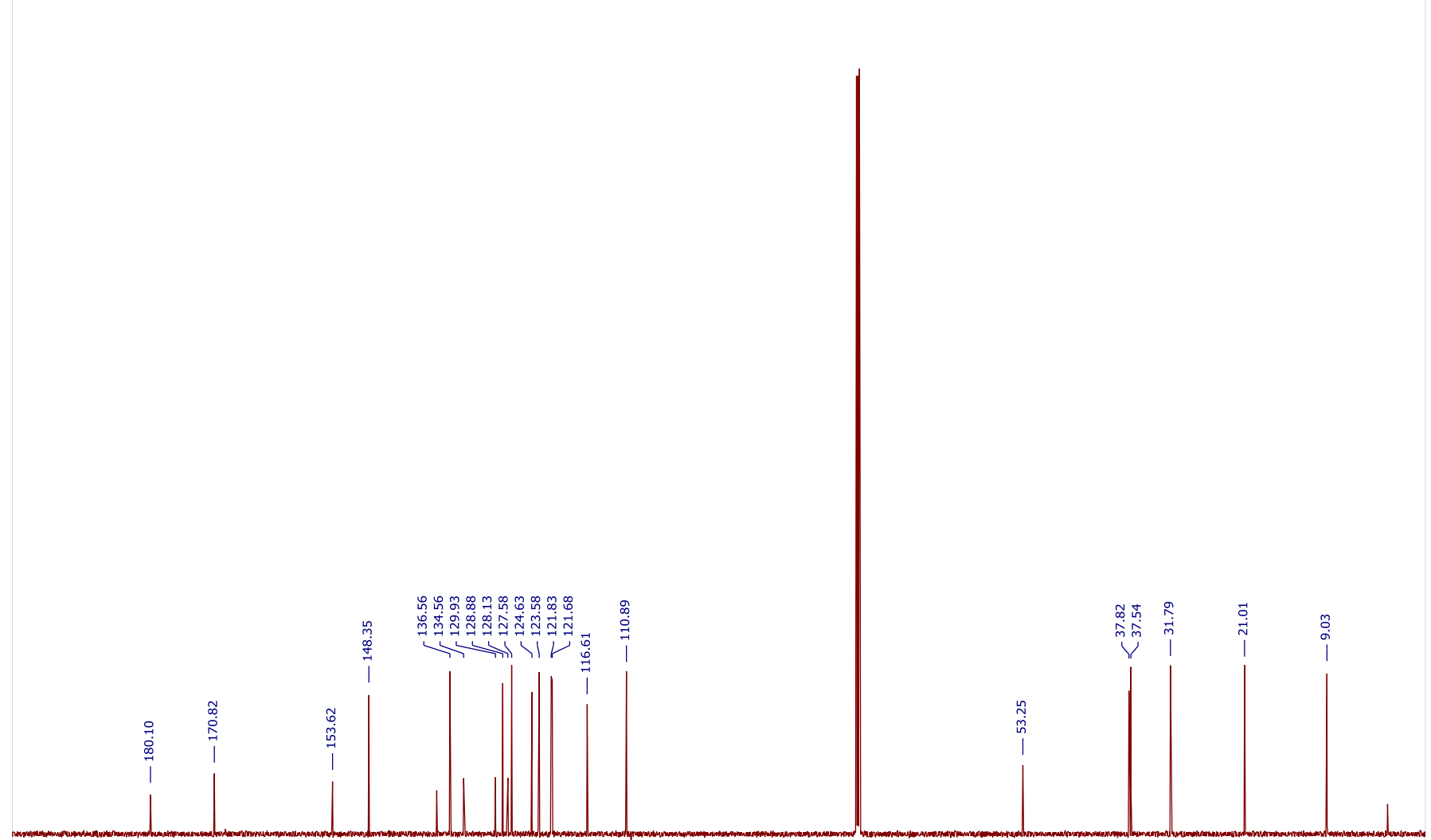


ky-n3-057-A-data.1.fid

ky-n3-057-A-data.1.fid
C-13 Routine 1D, CPDCH CryoProbe, AVIII-600

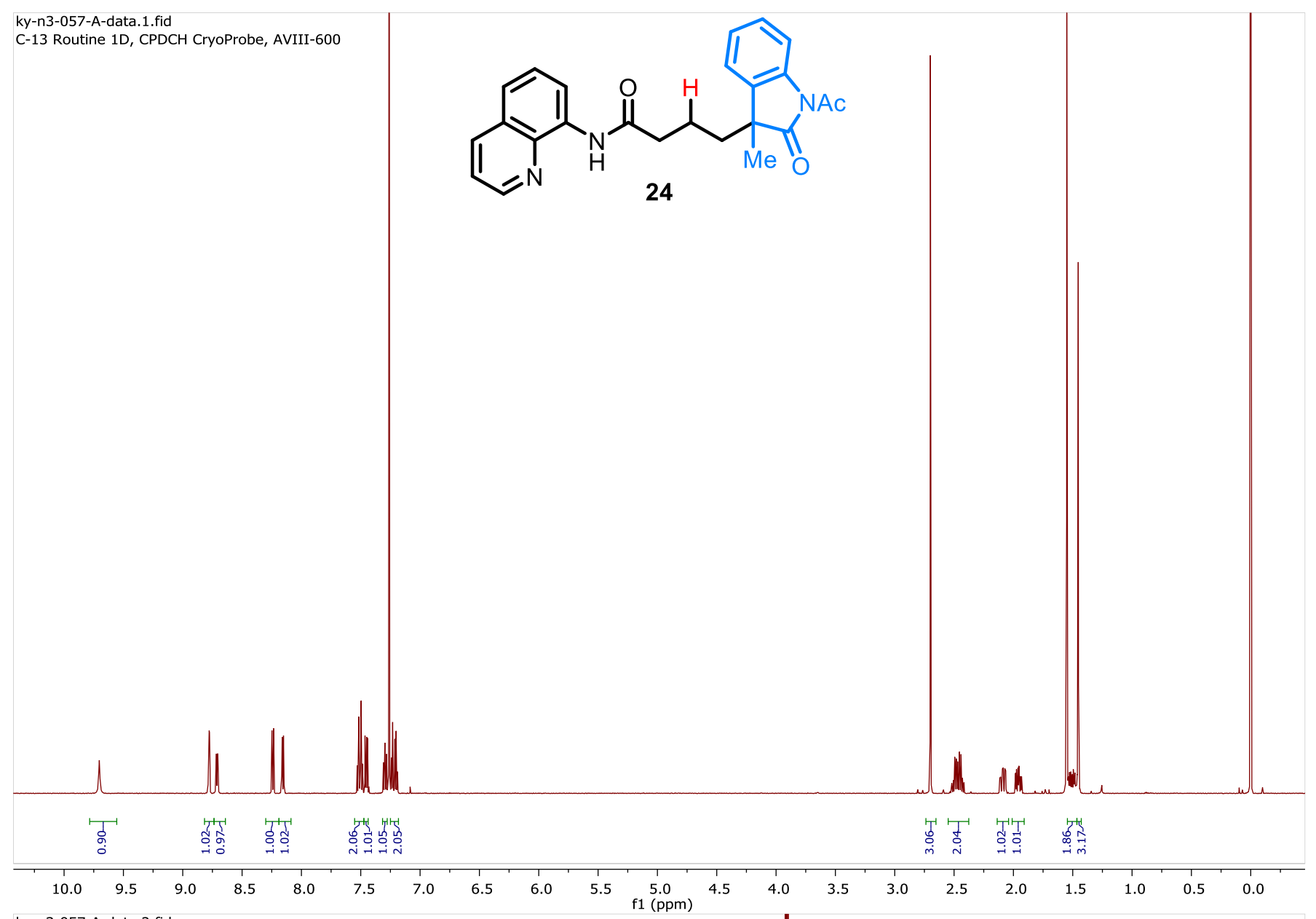

ky-n3-057-A-data.2.fid

ky-n3-057-A-data.2.fid
C-13 Routine 1D, CPDCH CryoProbe, AVIII-600
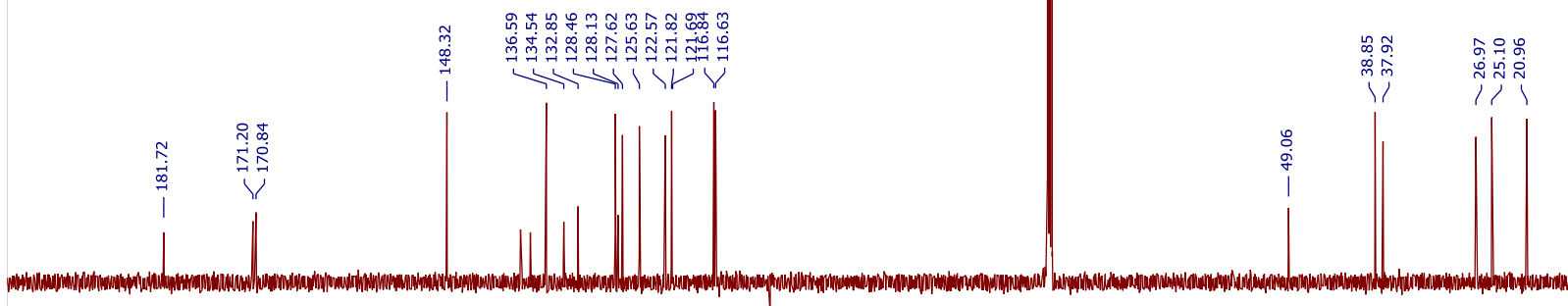
ky-n3-jg-pyr-et.1.fid

H-1 Routine, CP-DCH, AVIII HD-600 -<smiles>CCOC(=O)C(CCC(=O)Nc1cccc2cccnc12)c1ccccn1</smiles>

25

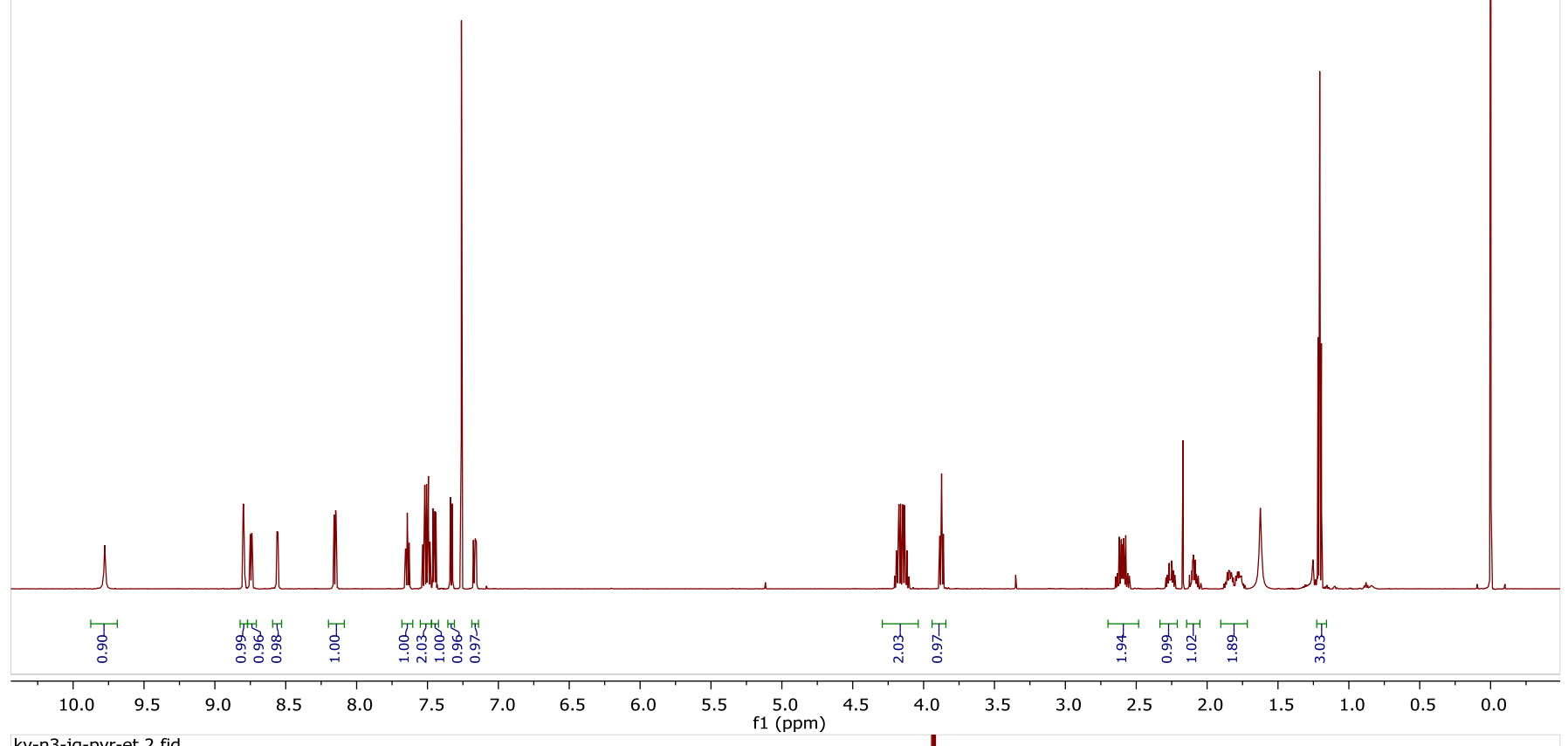

ky-n3-jg-pyr-et.2.fid

H-1 Routine, CP-DCH, AVIII HD-600 -
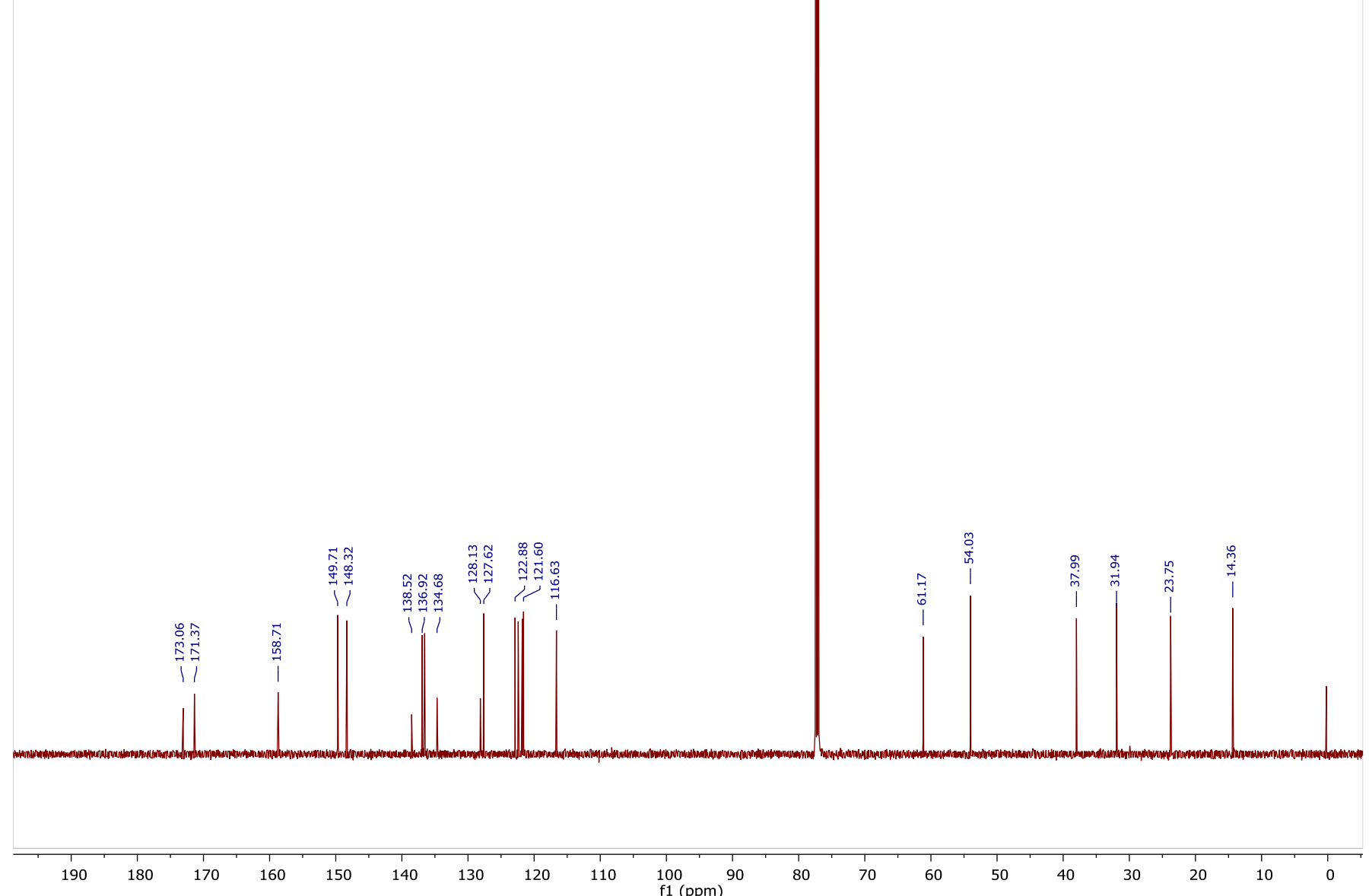

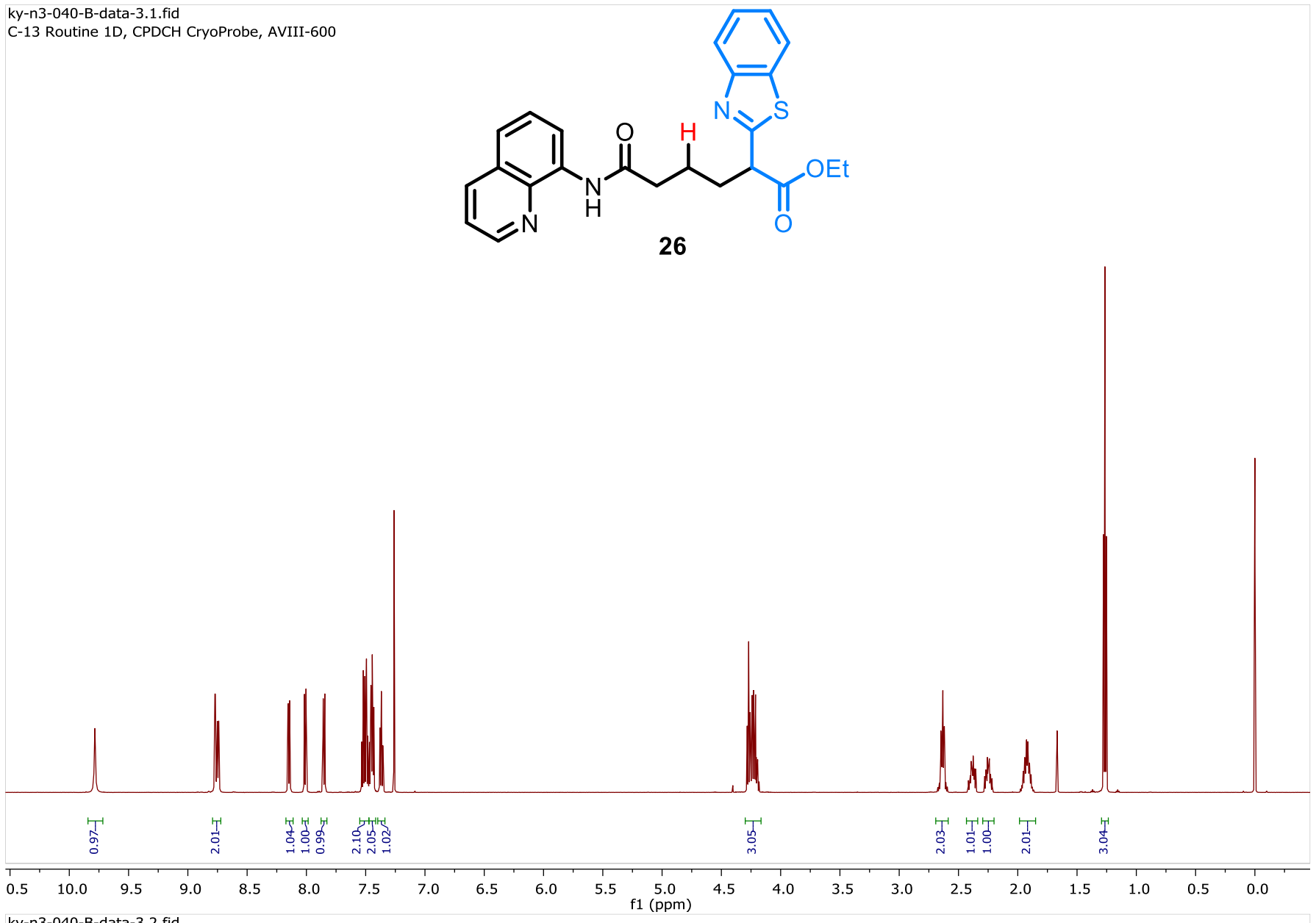

ky-n3-040-B-data-3.2.fid

C-13 Routine 1D, CPDCH CryoProbe, AVIII-600

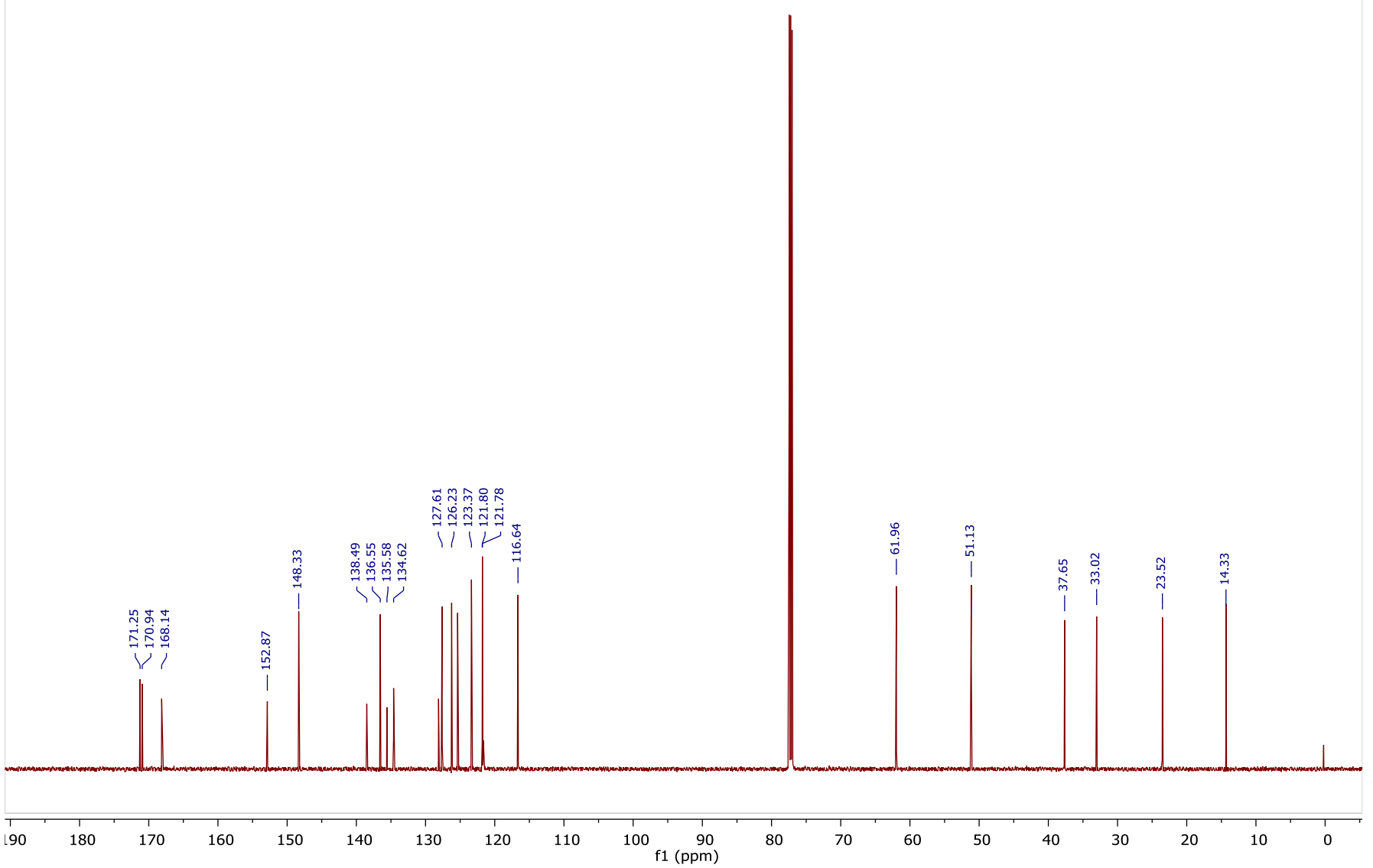


ky-n3-jg-ph-ph.1.fid

H-1 Routine, CP-DCH, AVIII HD-600<smiles>[Z7]CC(CC(=O)Nc1cccc2cccnc12)CC(C(=O)c1ccccc1)c1ccccc1</smiles>

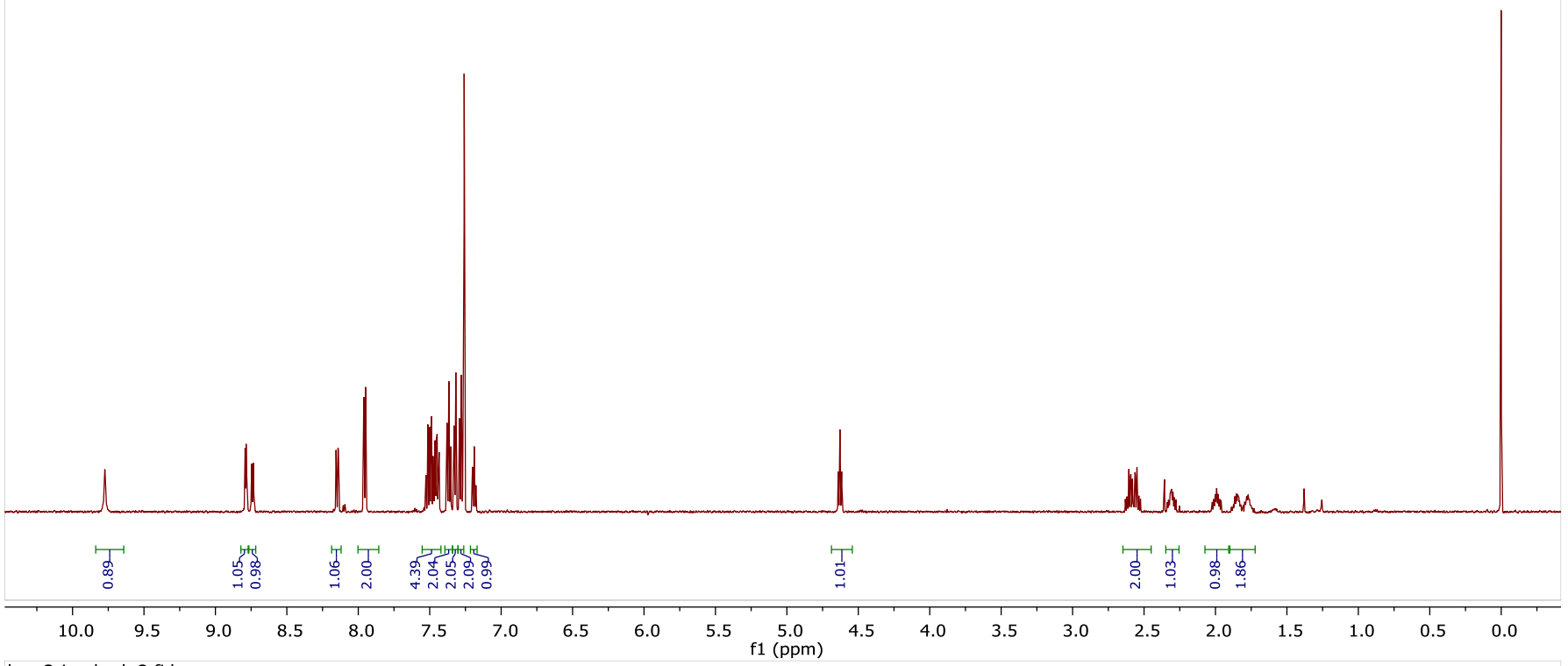

ky-n3-jg-ph-ph.2.fid

$\mathrm{H}-1$ Routine, CP-DCH, AVIII HD-600

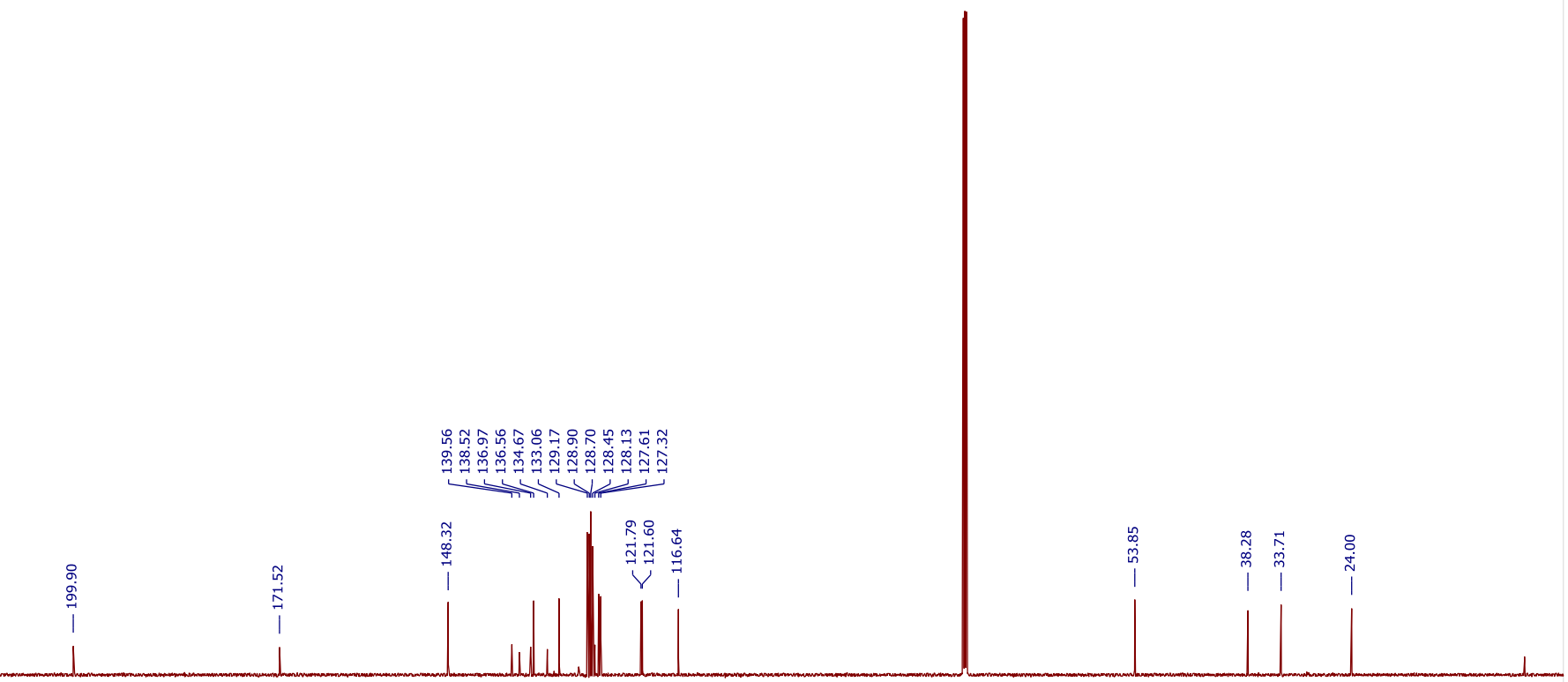


ky-n3-018-E-data.1.fid

H-1 Routine, CP-DCH, AVIII HD-600 -<smiles>O=C(CCc1c[nH]c2ccccc12)Nc1cccc2cccnc12</smiles>

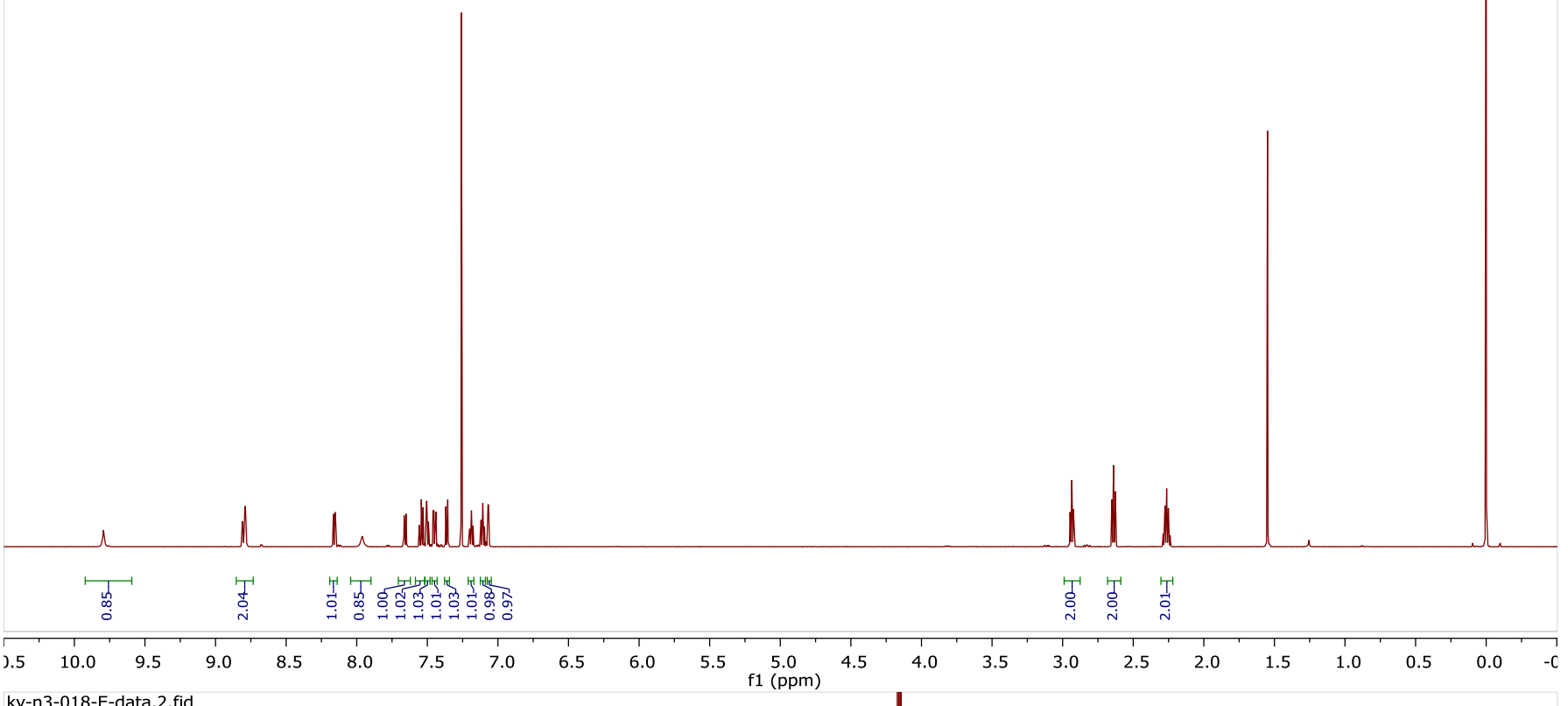

ky-n3-018-E-data.2.fid

H-1 Routine, CP-DCH, AVIII HD-600 -

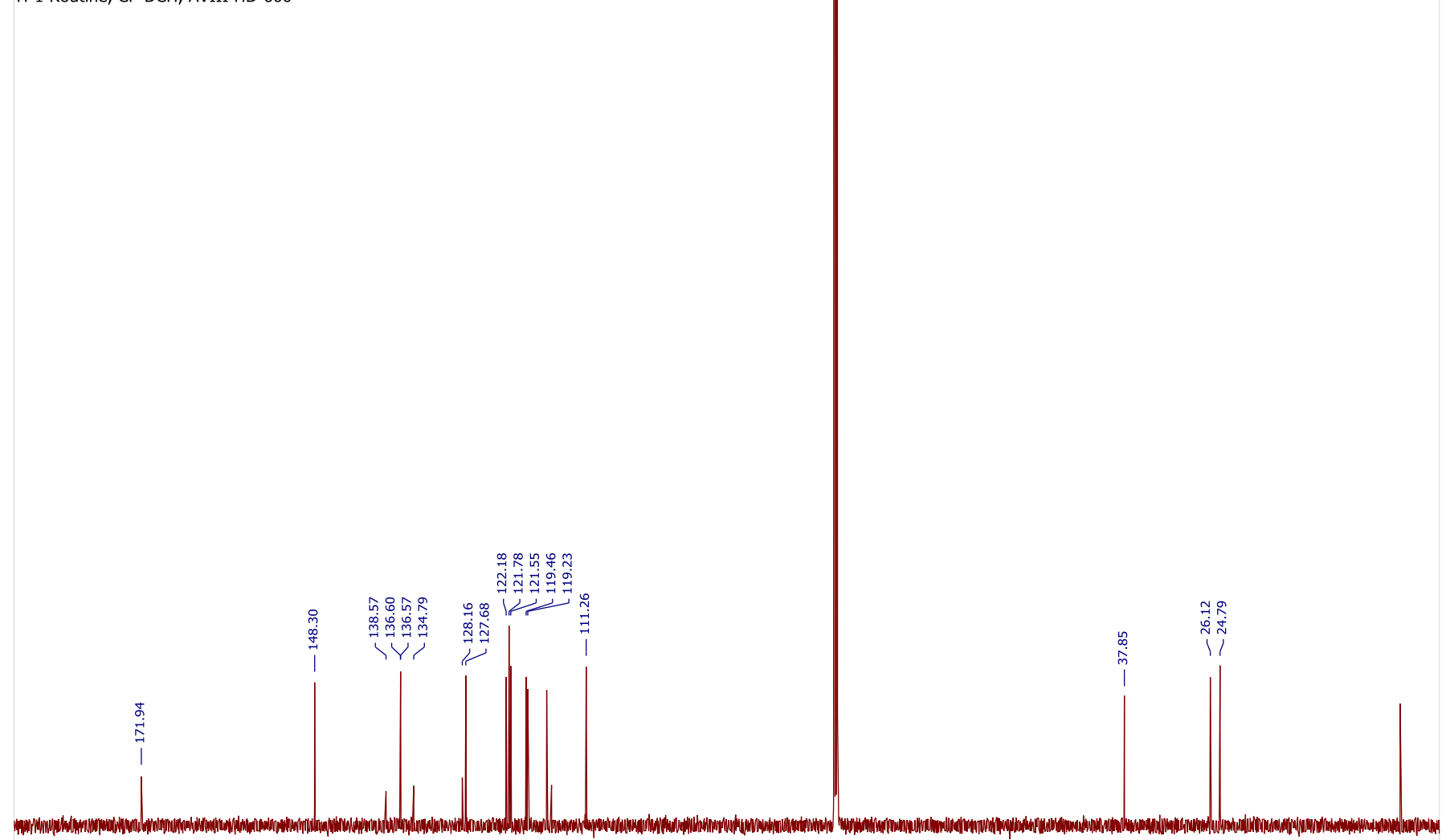


ky-n3-018-A-data.3.fid

$\mathrm{H}-1$ Routine. CPOCI, AVIII-600, 12-20-2013<smiles>Cn1cc(CCC(=O)Nc2cccc3cccnc23)c2ccccc21</smiles>

(1)

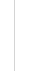

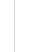

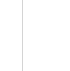

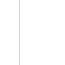

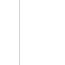

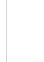

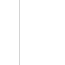

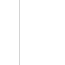

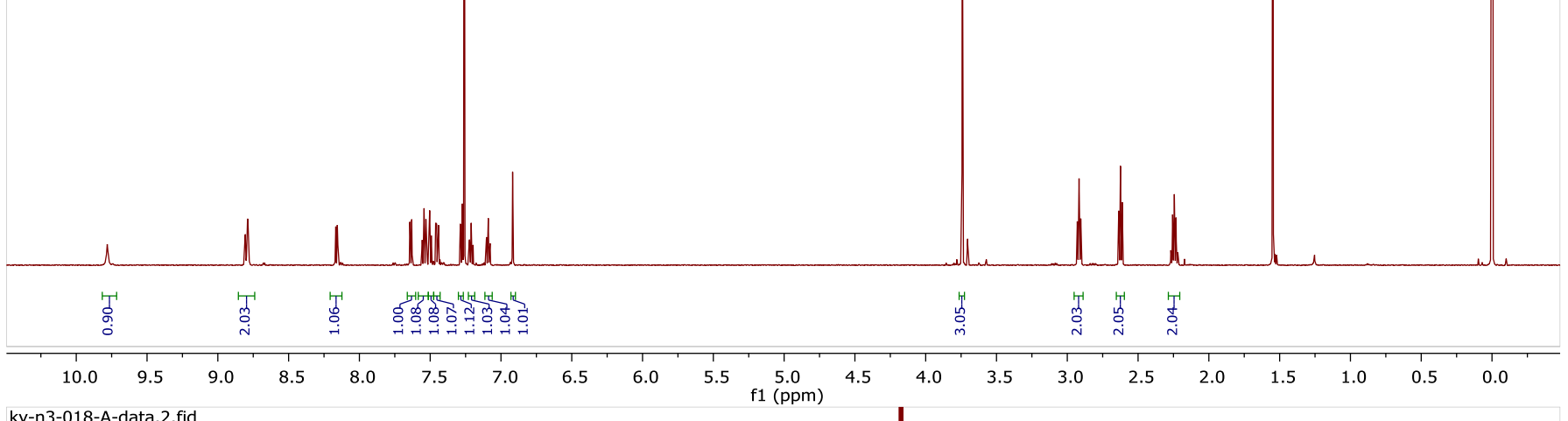

ky-n3-018-A-data.2.fid

H-1 Routine, CP-DCH, AVIII HD-600 -

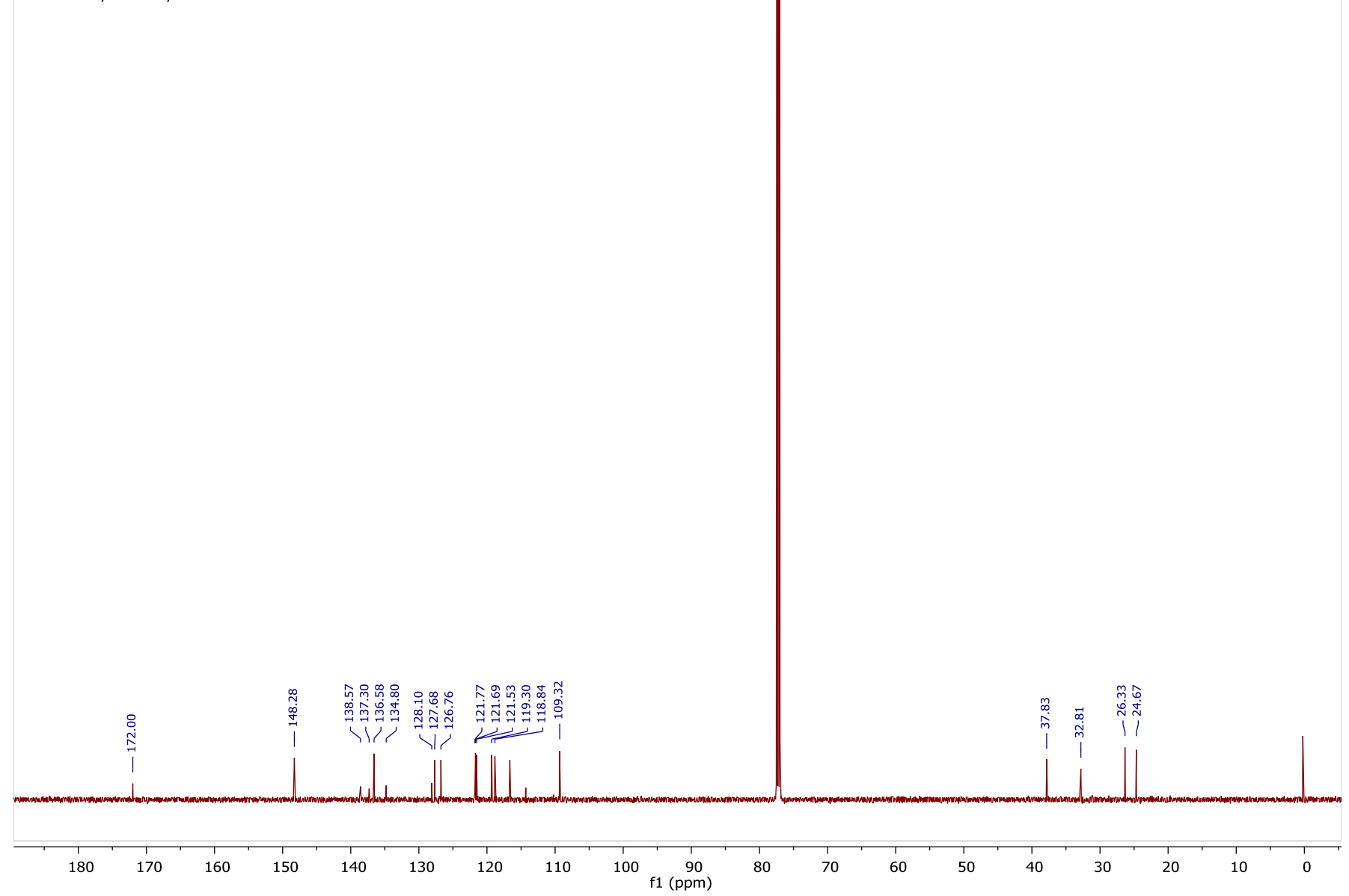


ky-n3-036-A-data-2.1.fid

C-13 Routine 1D, CPDCH CryoProbe, AVIII-600<smiles>CN(C)c1ccc(CCC(=O)Nc2cccc3cccnc23)c(O)c1</smiles>

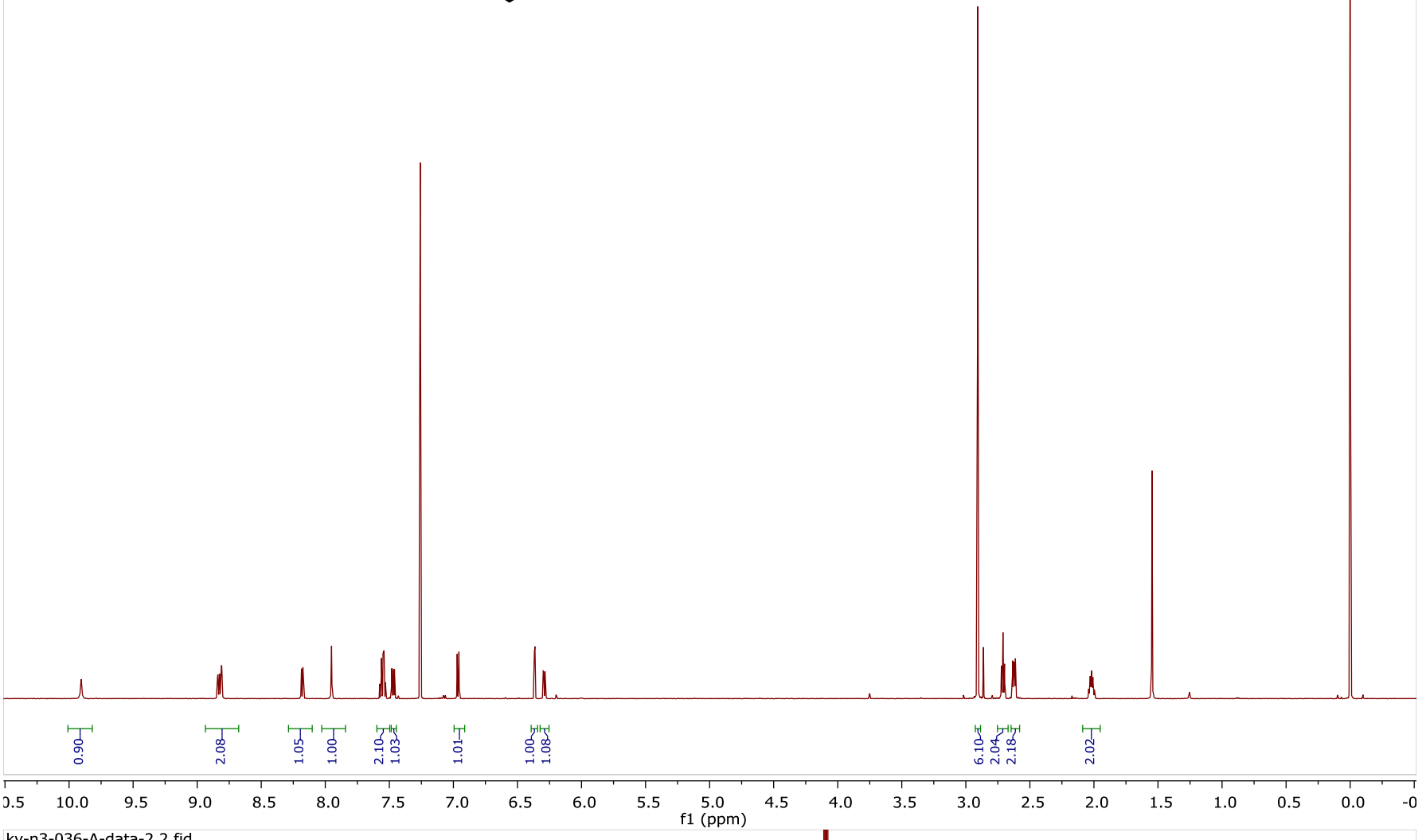

ky-n3-036-A-data-2.2.fid

C-13 Routine 1D, CPDCH CryoProbe, AVIII-600

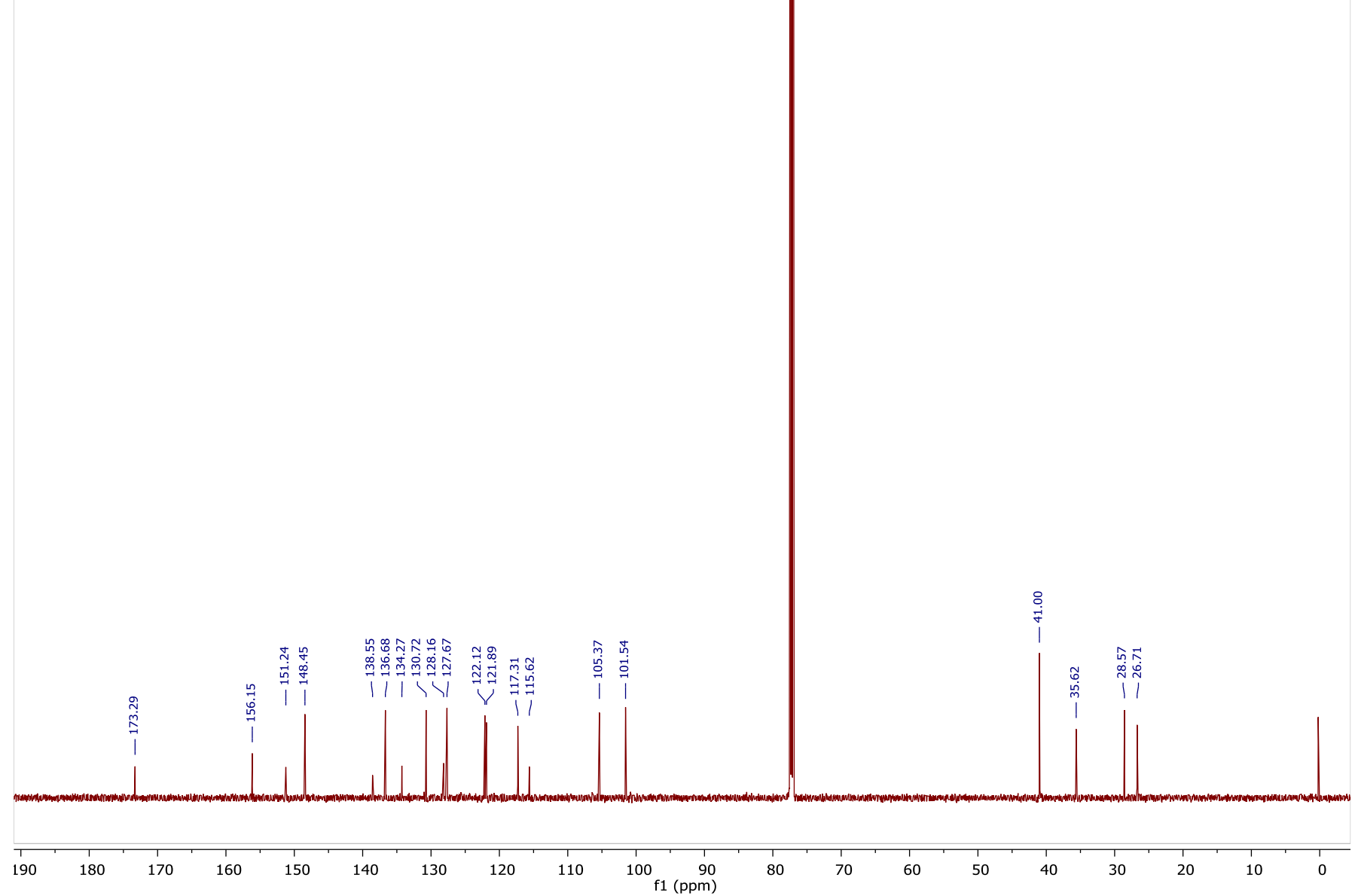


<smiles>O=Cc1ccc(O)c(CCC(=O)Nc2cccc3cccnc23)c1O</smiles>

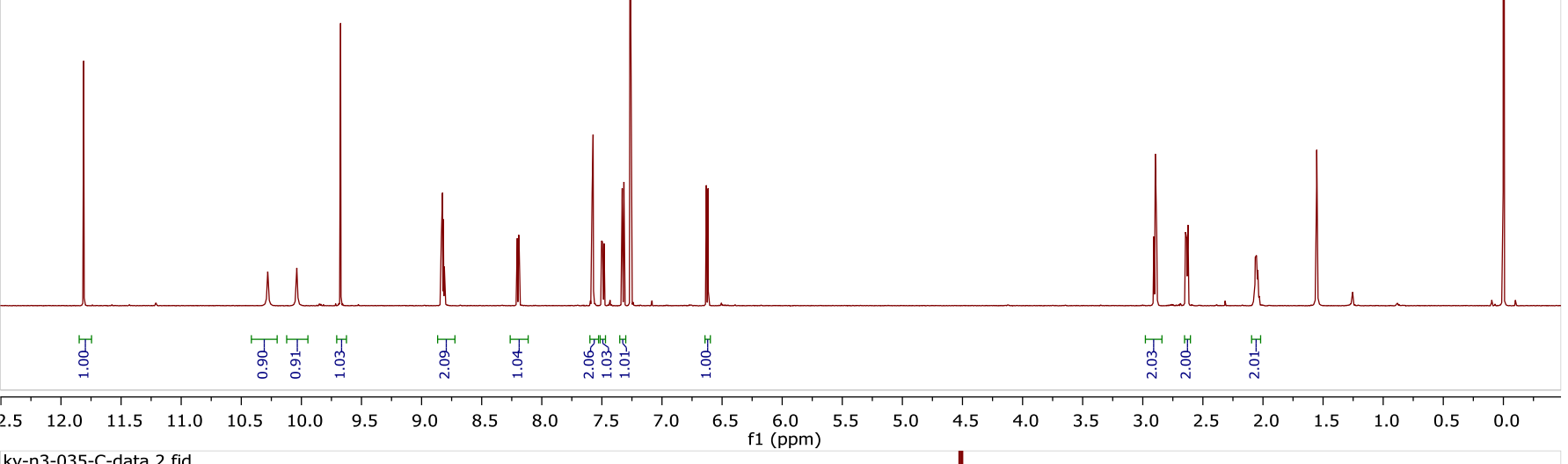

ky-n3-035-C-data. 2. fid

H-1 Routine, CP-DCH, AVIII HD-600 -

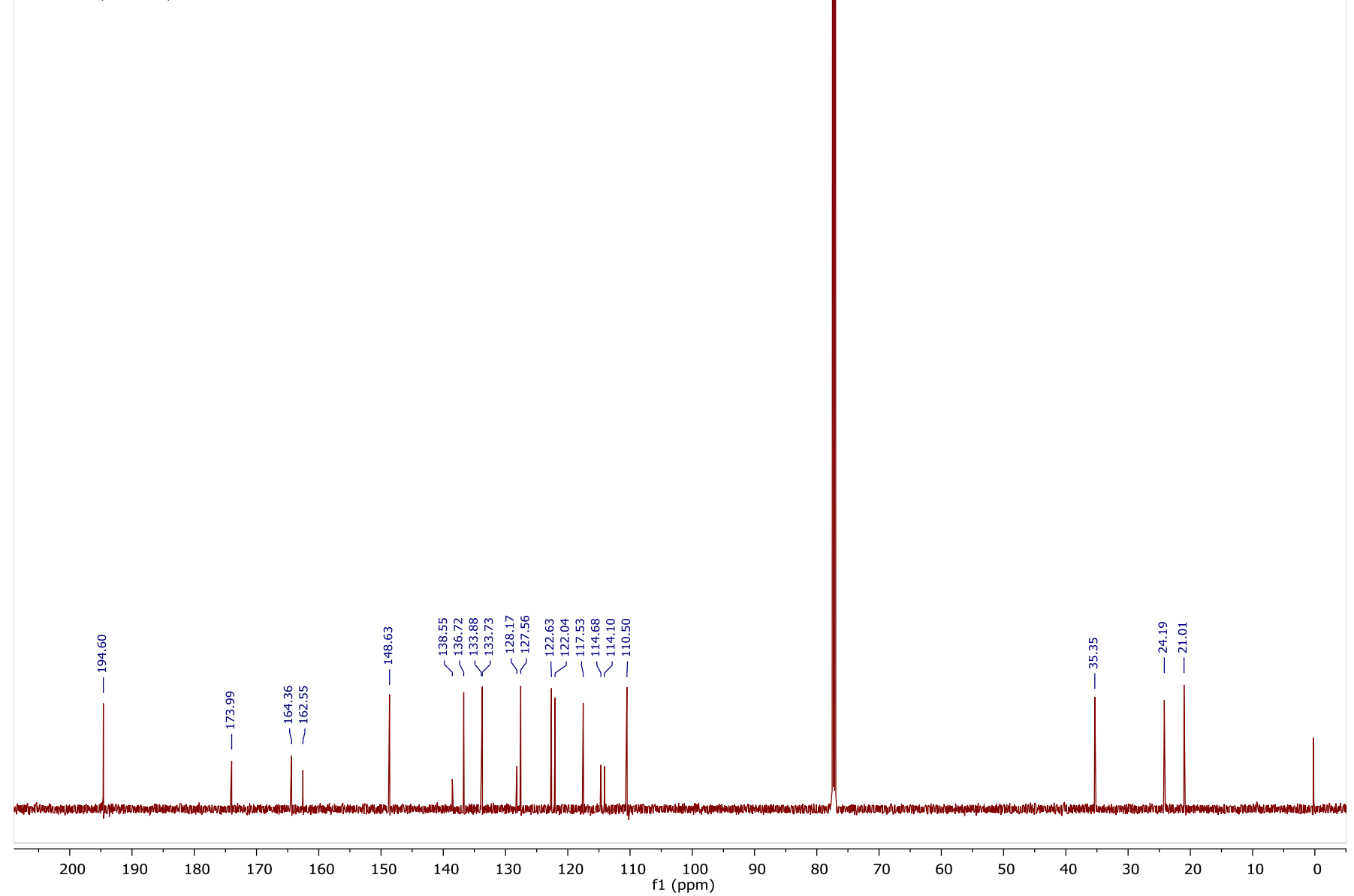


ky-n3-036-B-data.1.fid

C-13 Routine 1D, CPDCH CryoProbe, AVIII-600

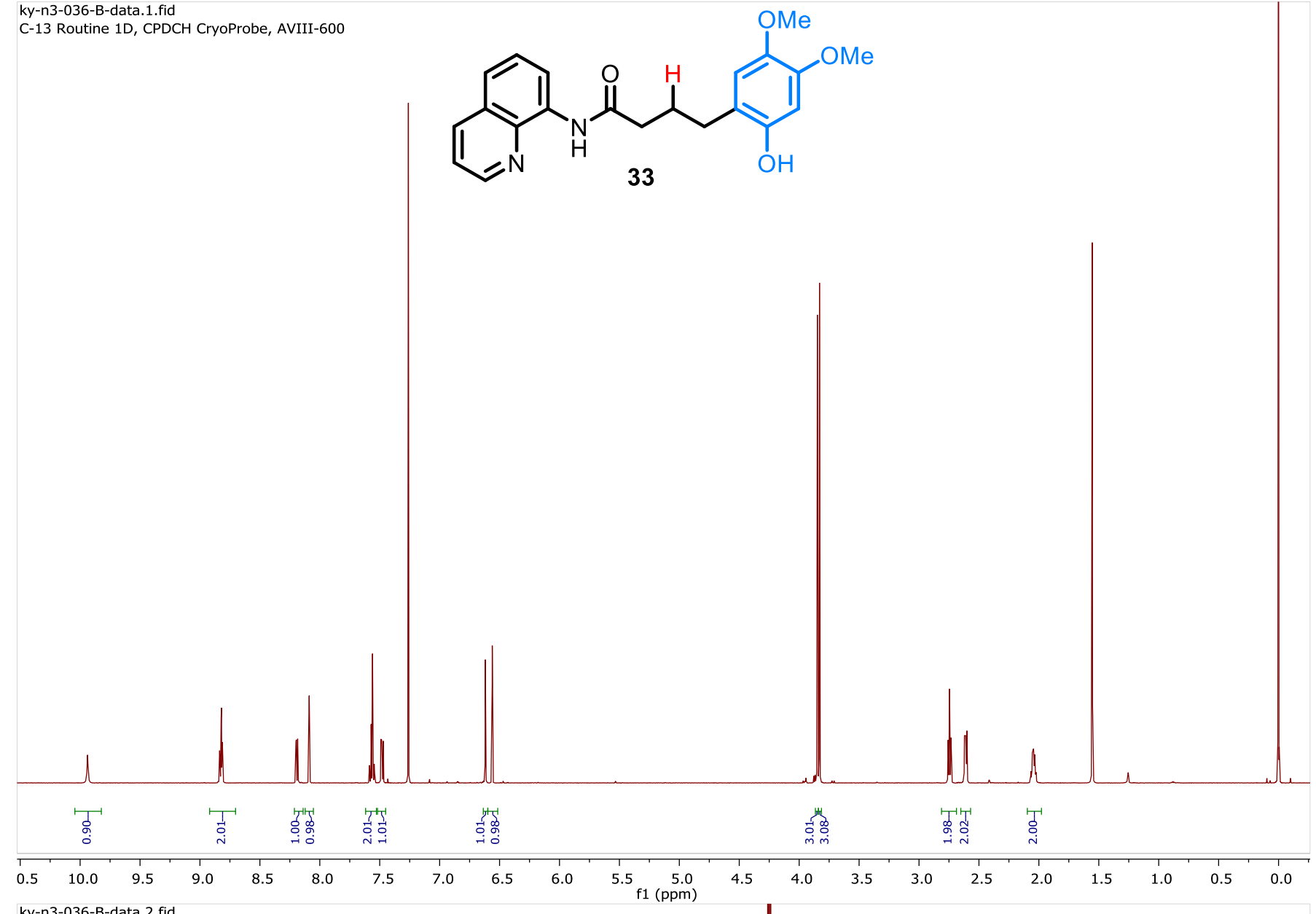

ky-n3-036-B-data.2.fid

C-13 Routine 1D, CPDCH CryoProbe, AVIII-600

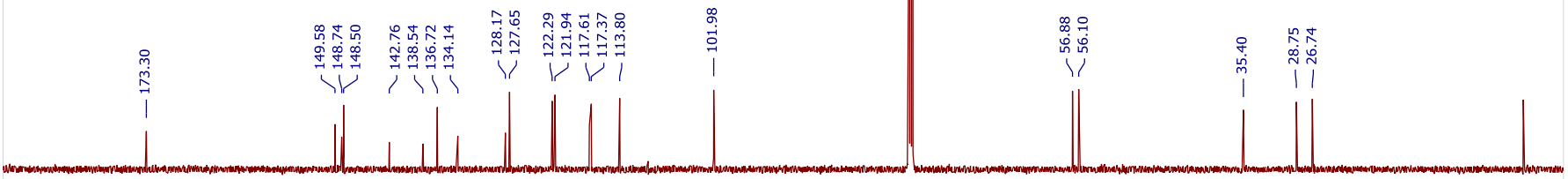


ky-n3-074-data.1.fid

$\mathrm{H}-1$ Routine, CP-DCH, AVIII HD-600<smiles>O=C(CCCc1c(O)ccc2ccccc12)Nc1cccc2cccnc12</smiles>

(1)

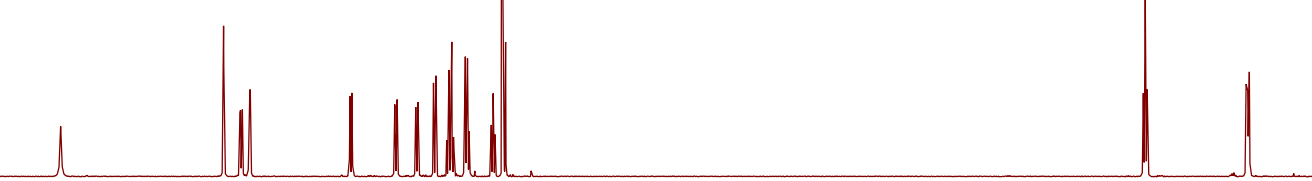

\begin{tabular}{|c|c|c|c|c|c|c|c|c|c|c|c|c|c|c|c|c|c|c|c|c|}
\hline $\begin{array}{l}\underset{d}{d} \\
\substack{0 \\
0}\end{array}$ & & 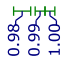 & & 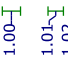 & 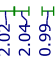 & & & & & & & & & & গ్రু & $\begin{array}{l}T \\
\stackrel{T}{0} \\
\stackrel{i}{ }\end{array}$ & & & & \\
\hline 10.0 & 9.5 & 9.0 & 8.5 & 8.0 & 7.5 & 7.0 & 6.5 & 6.0 & 5.5 & $\begin{array}{c}5.0 \\
\text { f1 }(p p m)\end{array}$ & 4.5 & 4.0 & 3.5 & 3.0 & 2.5 & 2.0 & 1.5 & 1.0 & 0.5 & 0.0 \\
\hline
\end{tabular}

ky-n3-074-data.2.fid

H-1 Routine, CP-DCH, AVIII HD-600
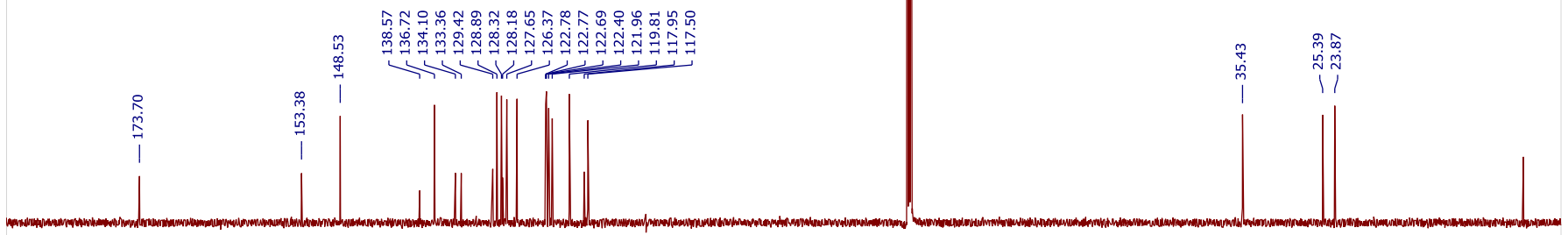
ky-n3-jg-br-nap-data.1.fid

H-1 Routine, CP-DCH, AVIII HD-600

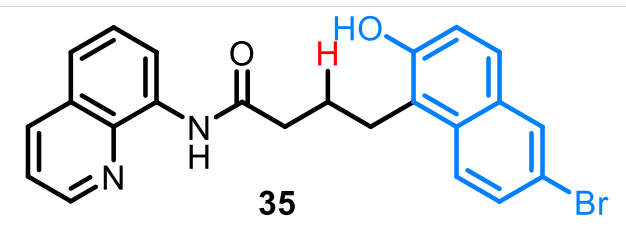

(n)
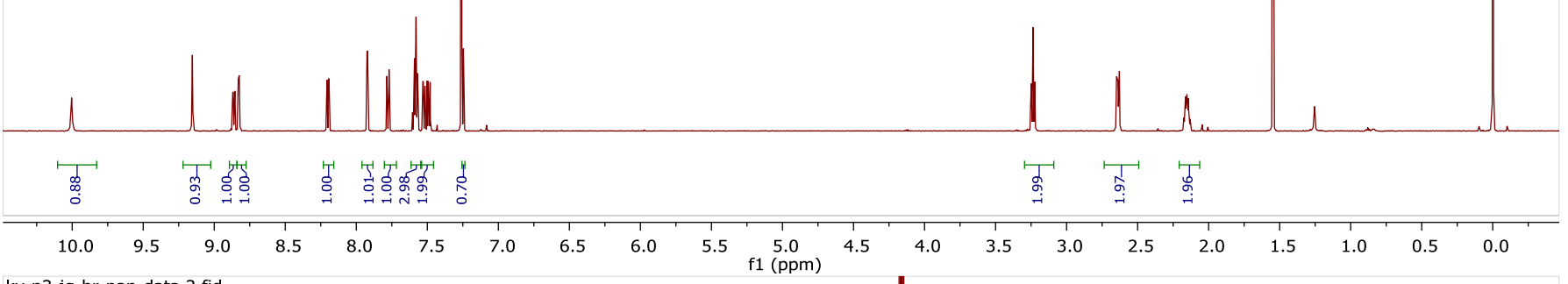

ky-n3-jg-br-nap-data.2.fid

H-1 Routine, CP-DCH, AVIII HD-600

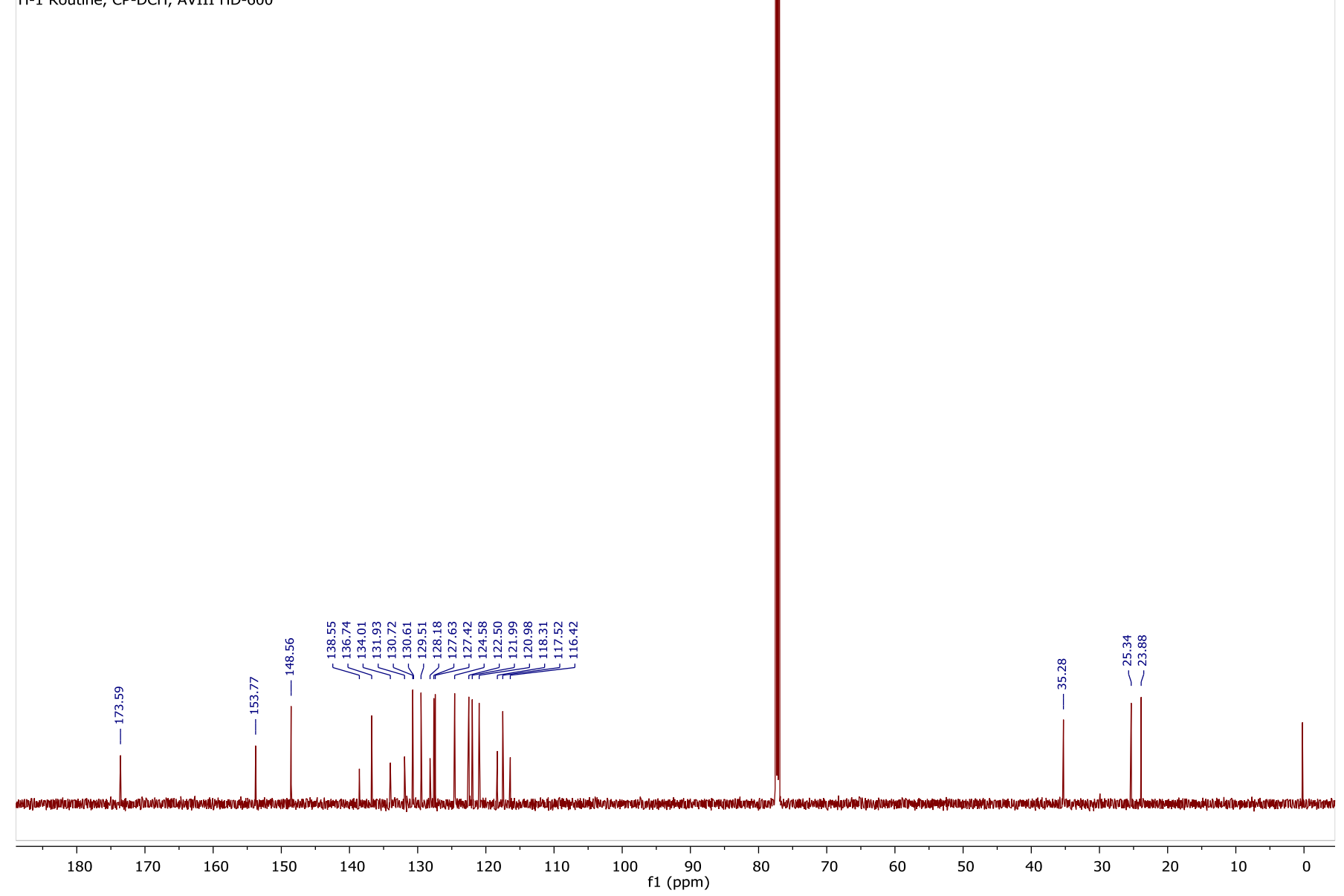




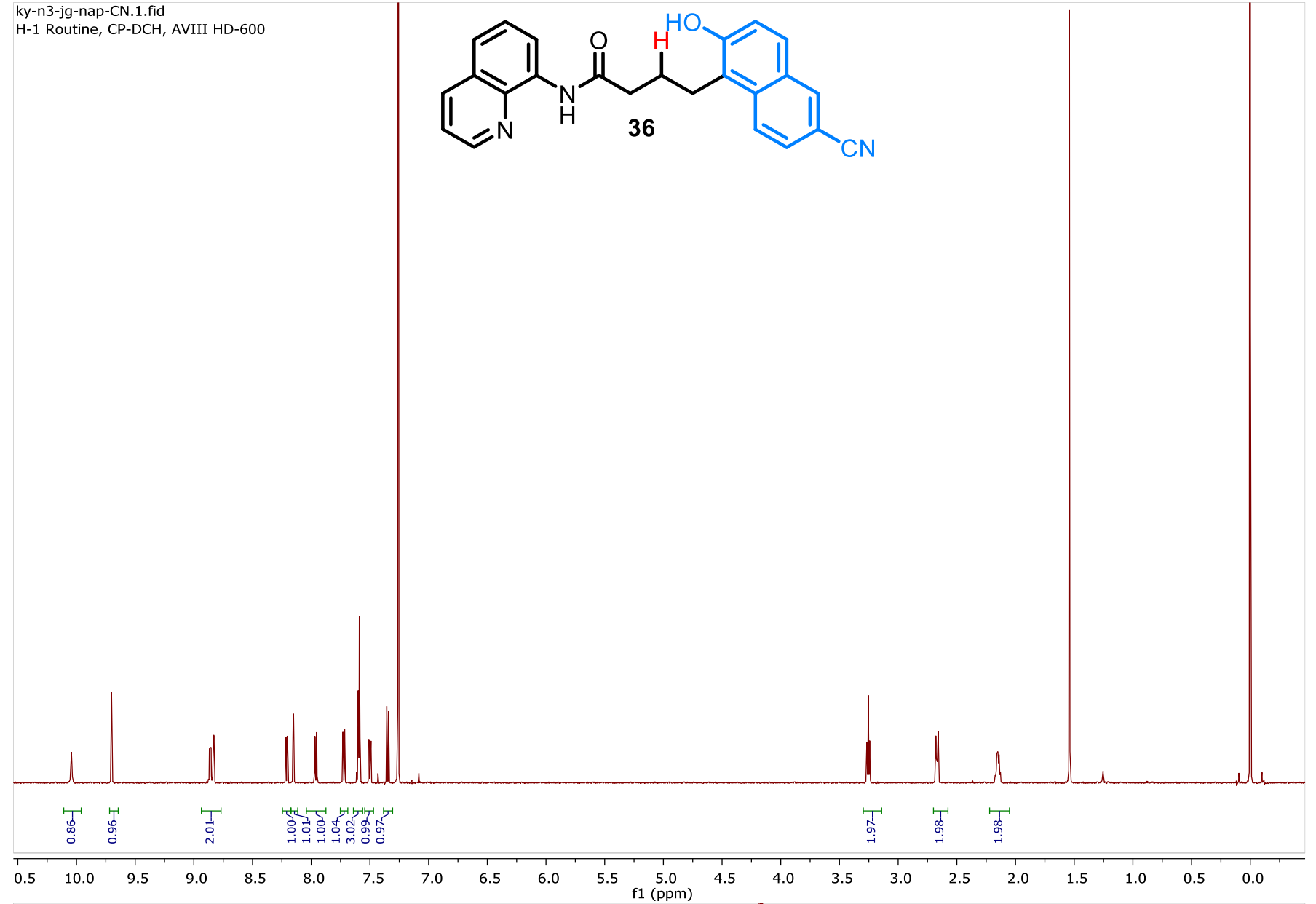

ky-n3-jg-nap-CN.2.fid

H-1 Routine, CP-DCH, AVIII HD-600
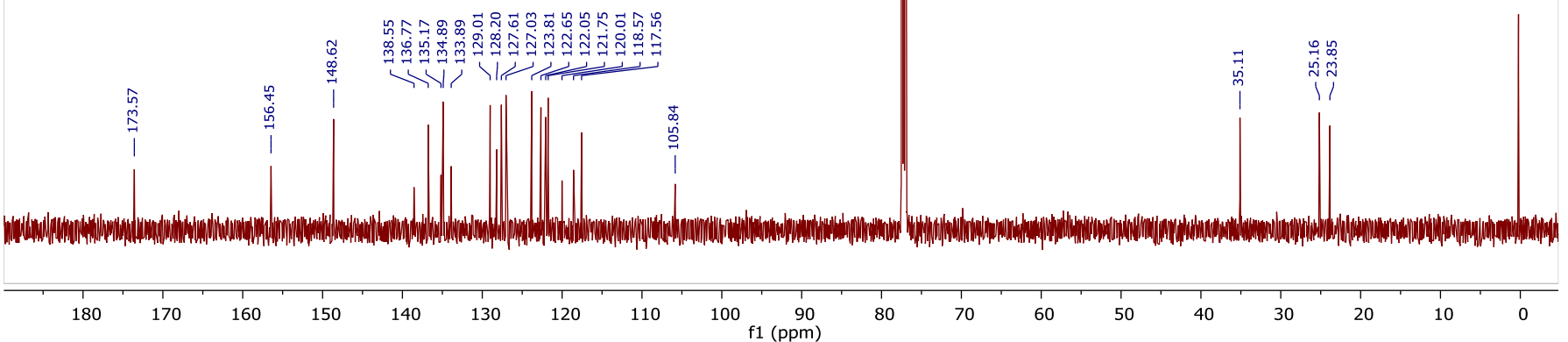
ky-n3-jg-nap-cl.1.fid

H-1 Routine, CP-DCH, AVIII HD-600

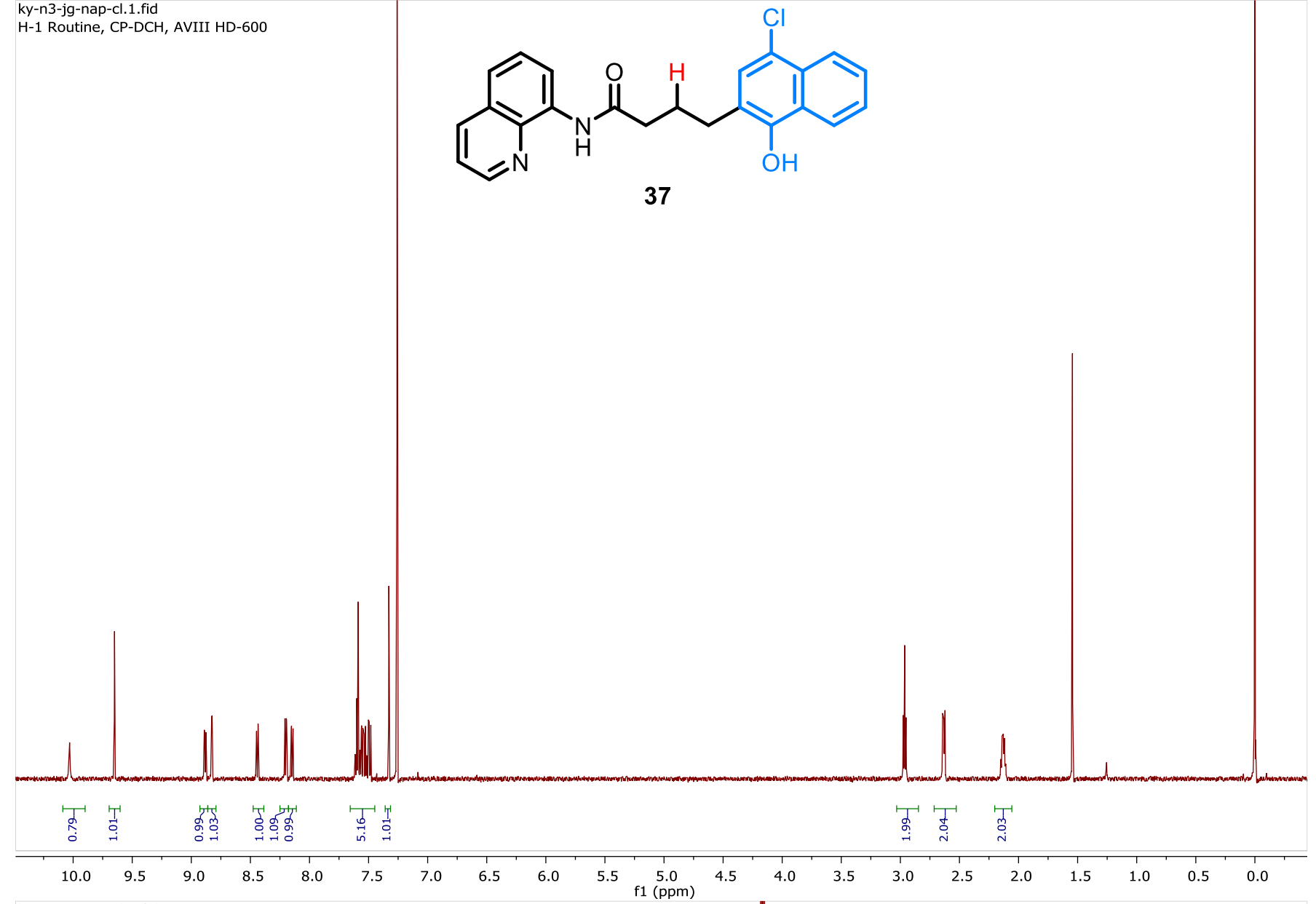

37

ky-n3-jg-nap-cl.2.fid

H-1 Routine, CP-DCH, AVIII HD-600
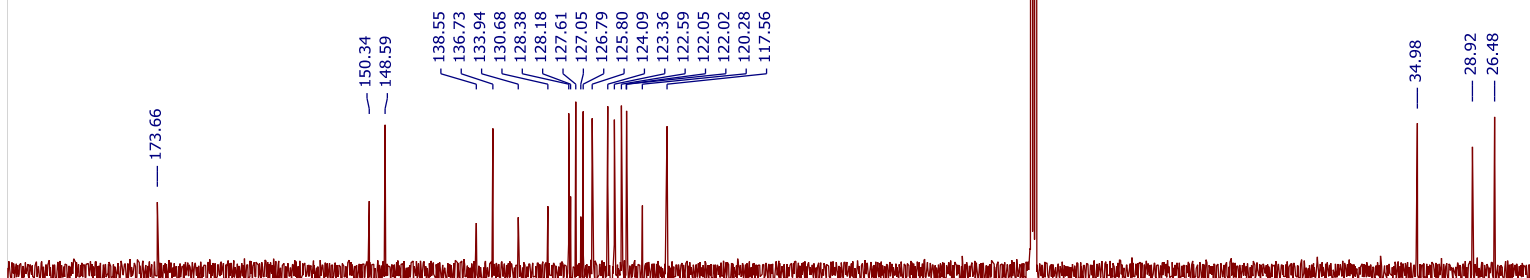
ky-n3-jg-hyd-quin.1.fid

H-1 Routine. CPQCI, AVIII-600, 5-12-2016<smiles>O=C(CCCc1c(O)ccc2cnccc12)Nc1cccc2cccnc12</smiles>

(1)

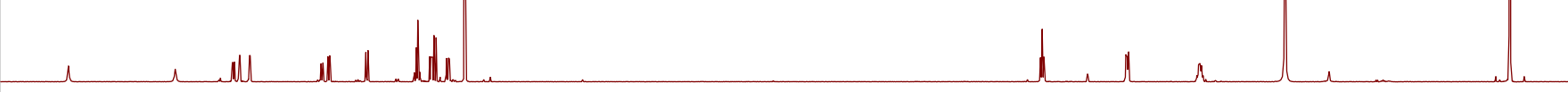

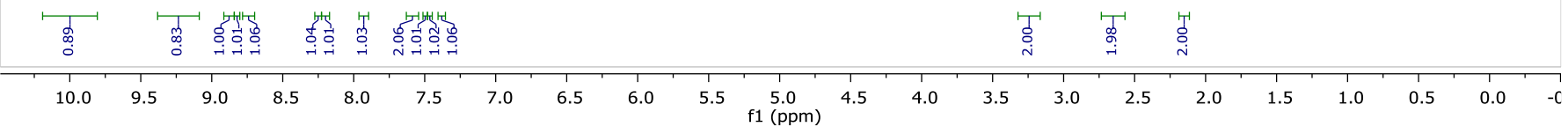

ky-n3-jg-hyd-quin.2.fid

H-1 Routine. CPQCI, AVIII-600, 5-12-2016

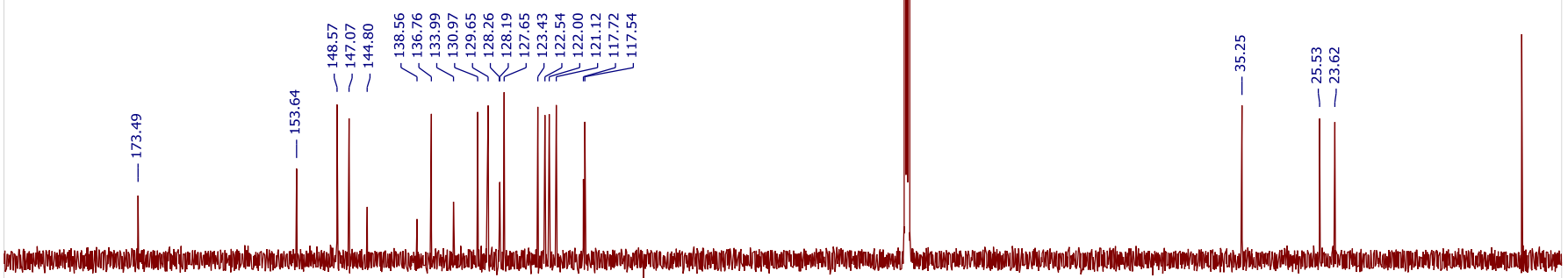




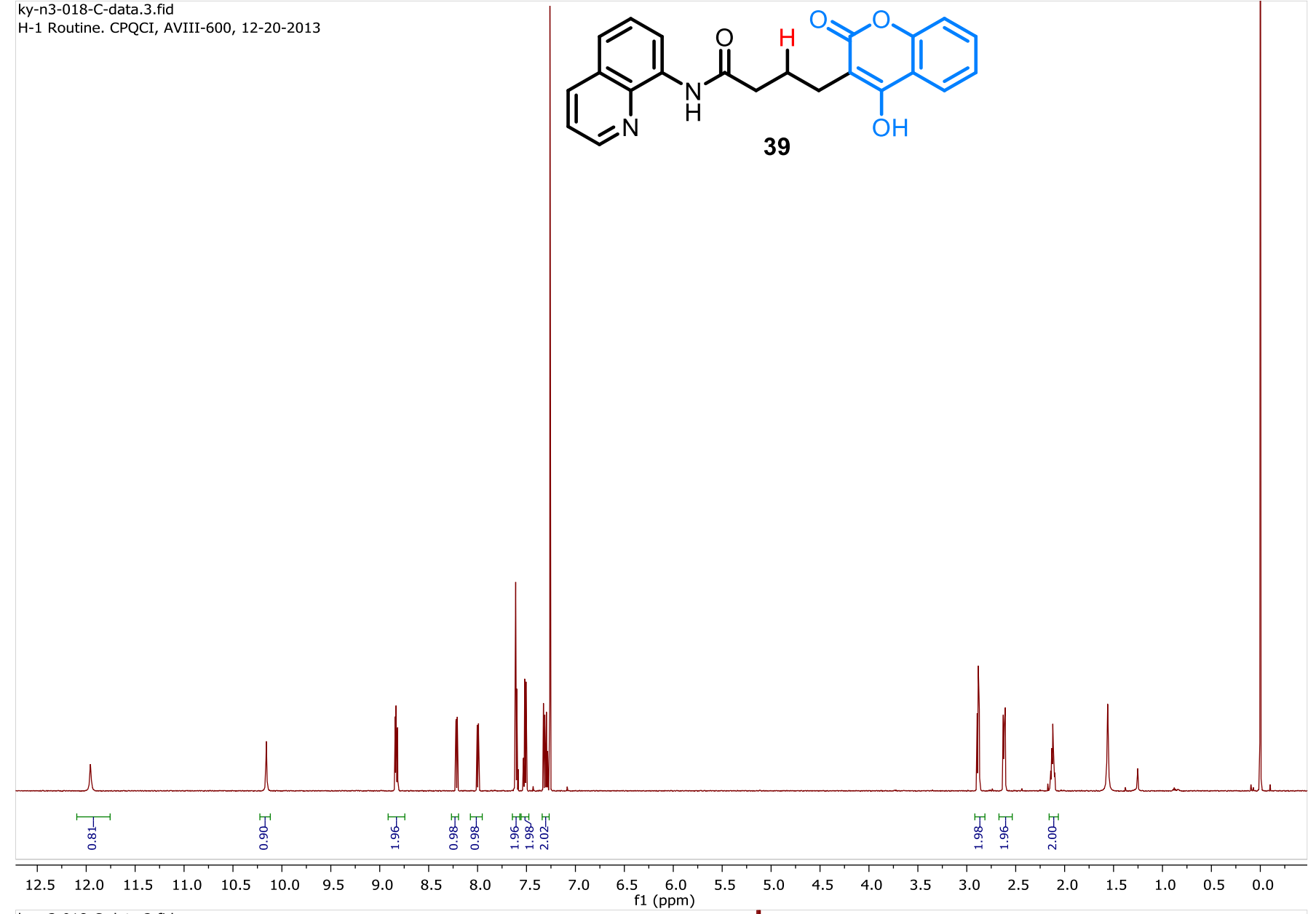

39 ky-n3-018-C-data.2.fid H-1 Routine, CP-DCH, AVIII HD-600 -

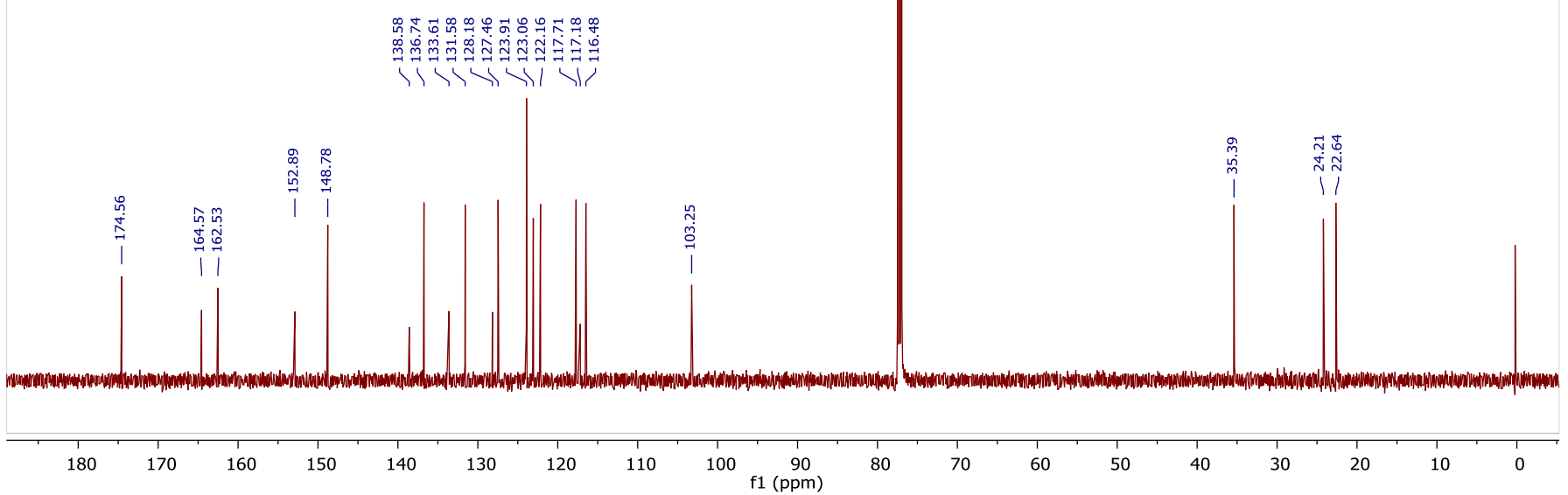


ky-n3-035-D-data.1.fid H-1 Routine, CP-DCH, AVIII HD-600 -

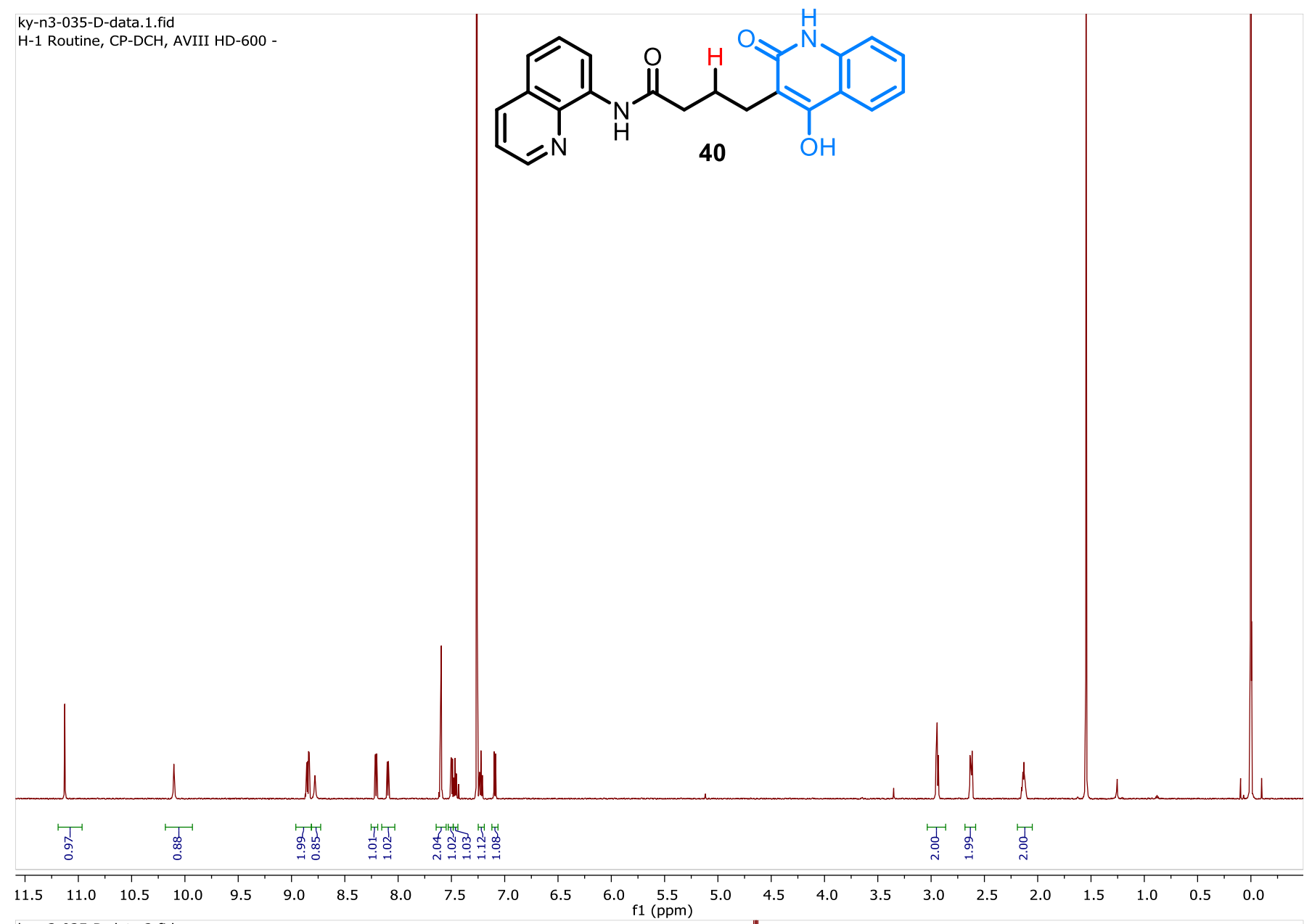
ky-n3-035-D-data.2.fid C-13 Routine 1D, CPDCH CryoProbe, AVIII-600

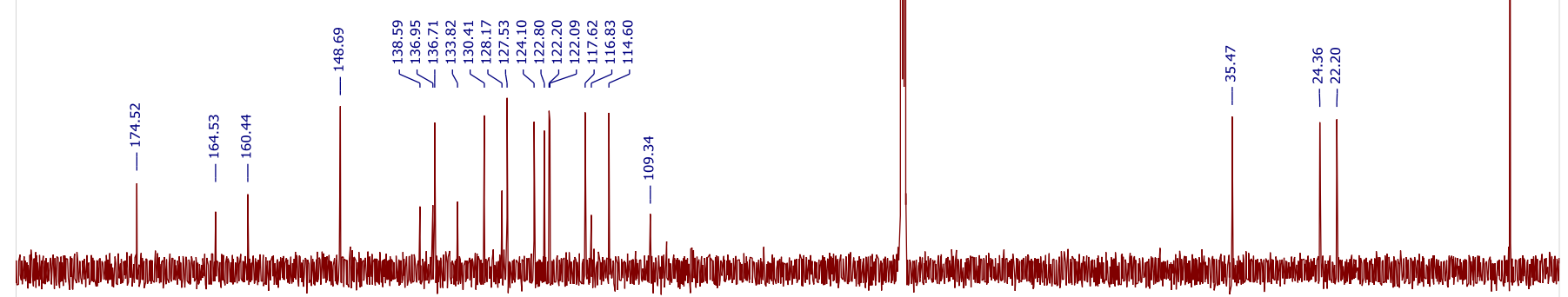


ky-n3-jg-phoh-data.1.fid

H-1 Routine. CPQCI, AVIII-600, 5-12-2016<smiles>[14CH3]CC(=O)Nc1cccc2cccnc12</smiles>

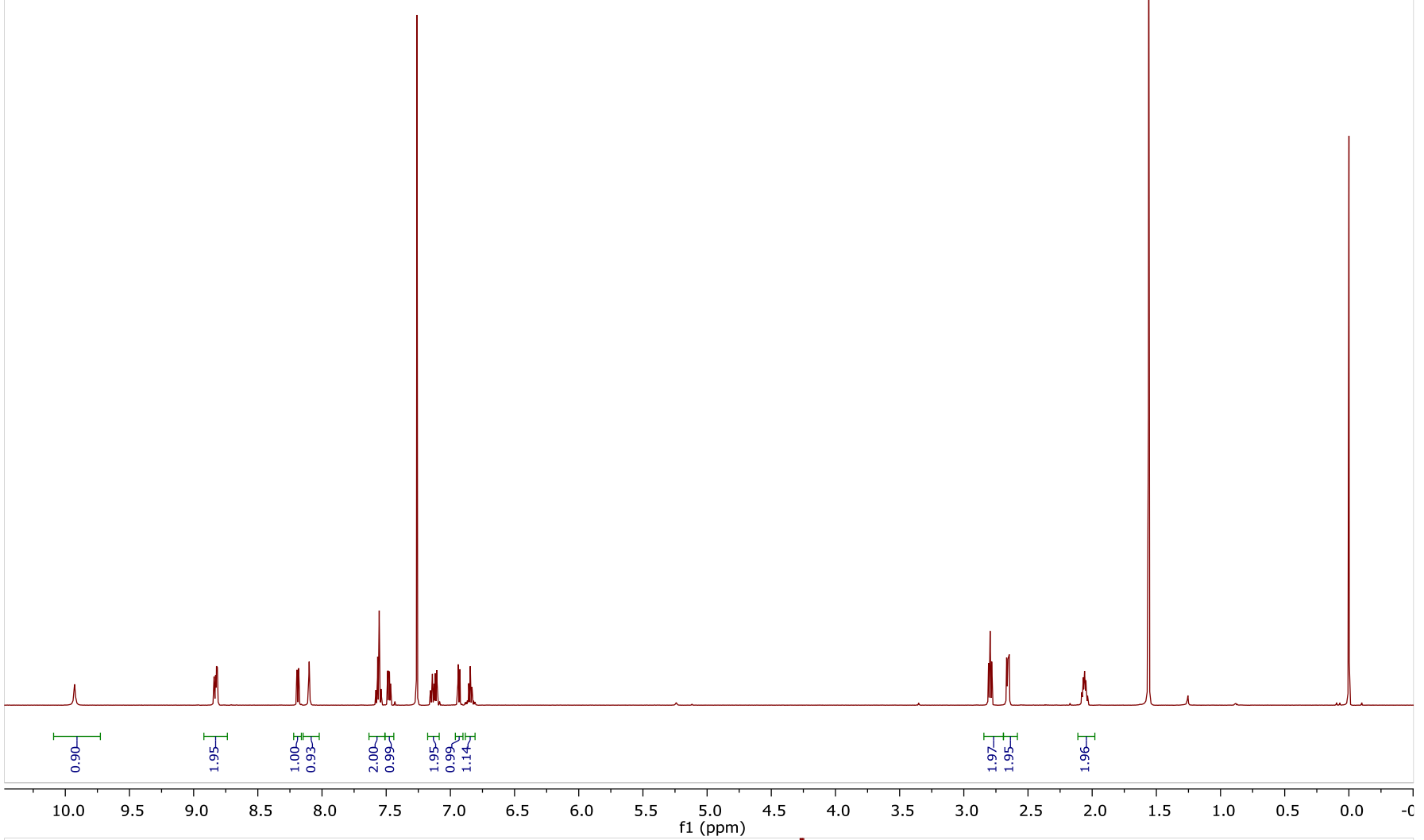

ky-n3-jg-phoh-data.2.fid

H-1 Routine. CPQCI, AVIII-600, 5-12-2016

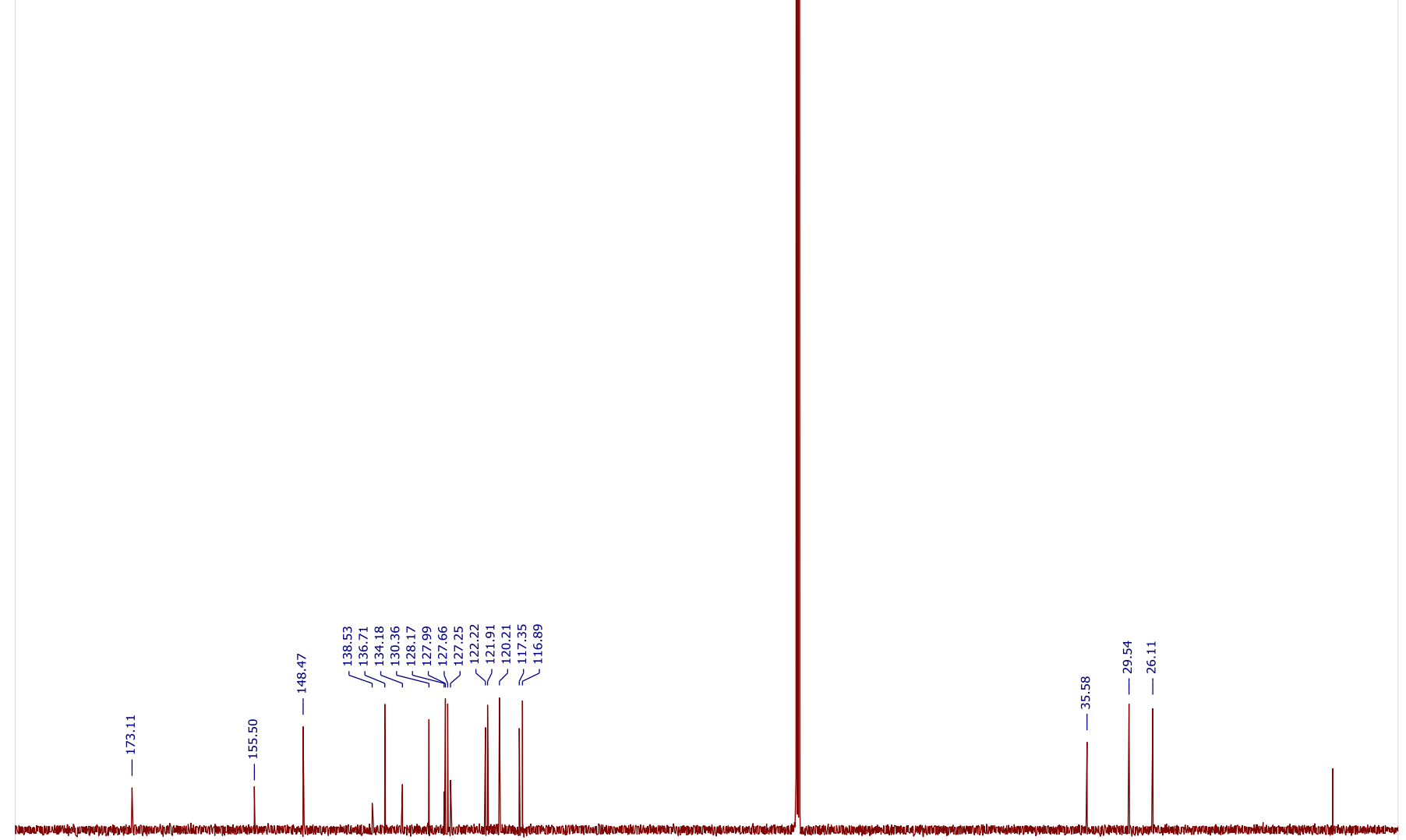

f1 $(\mathrm{ppm})$
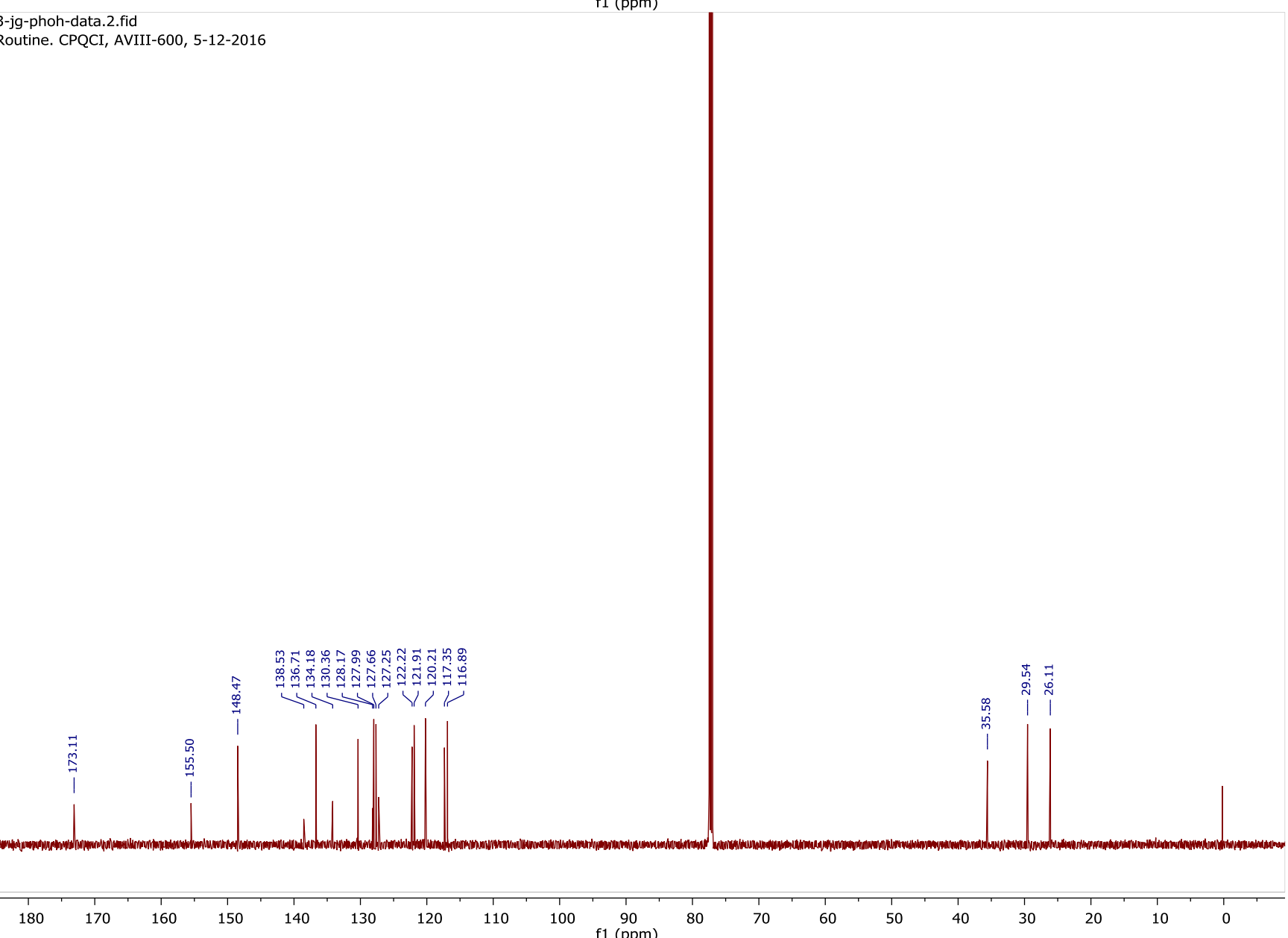
ky-n3-034-D-data.1.fid

C-13 Routine 1D, CPDCH CryoProbe, AVIII-600

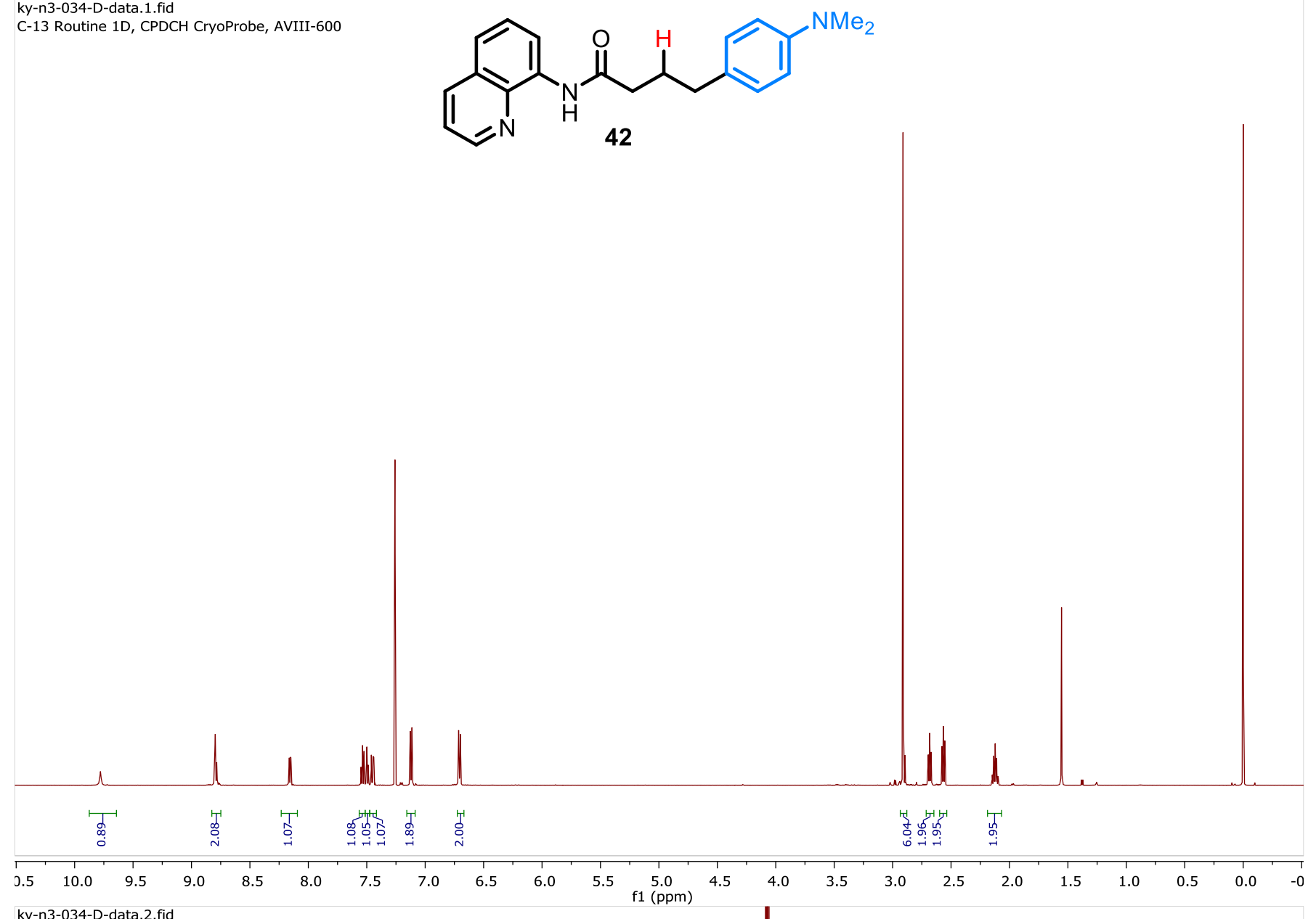

ky-n3-034-D-data.2.fid

C-13 Routine 1D, CPDCH CryoProbe, AVIII-600
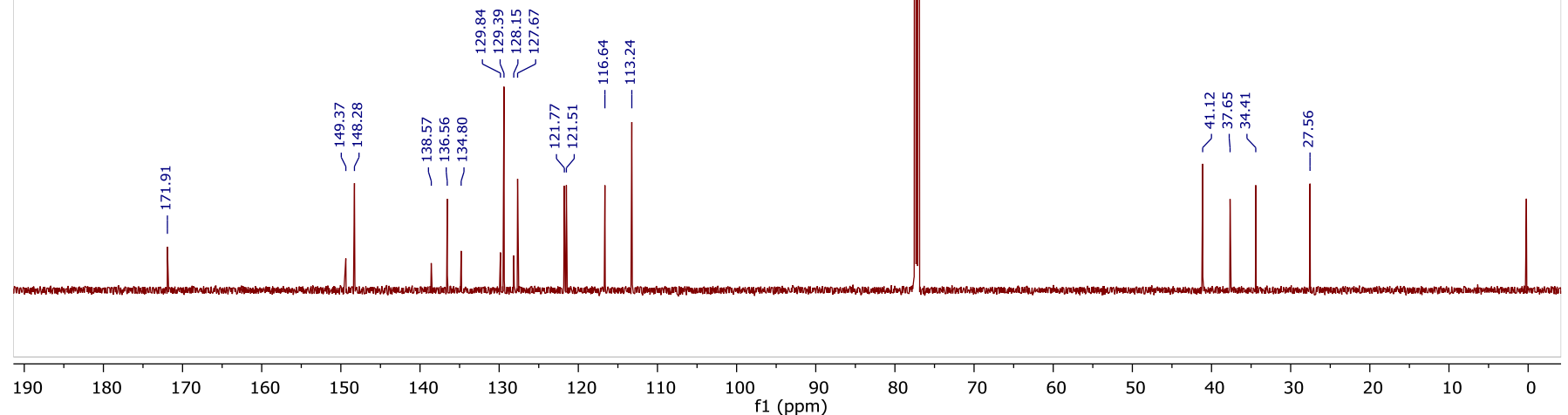
ky-n3-024-A-data.1.fid

H-1 Routine, CP-DCH, AVIII HD-600 -<smiles>CC(=O)C(CC=C(C(=O)Nc1cccc2cccnc12)C(C)C)C(C)=O</smiles>

43

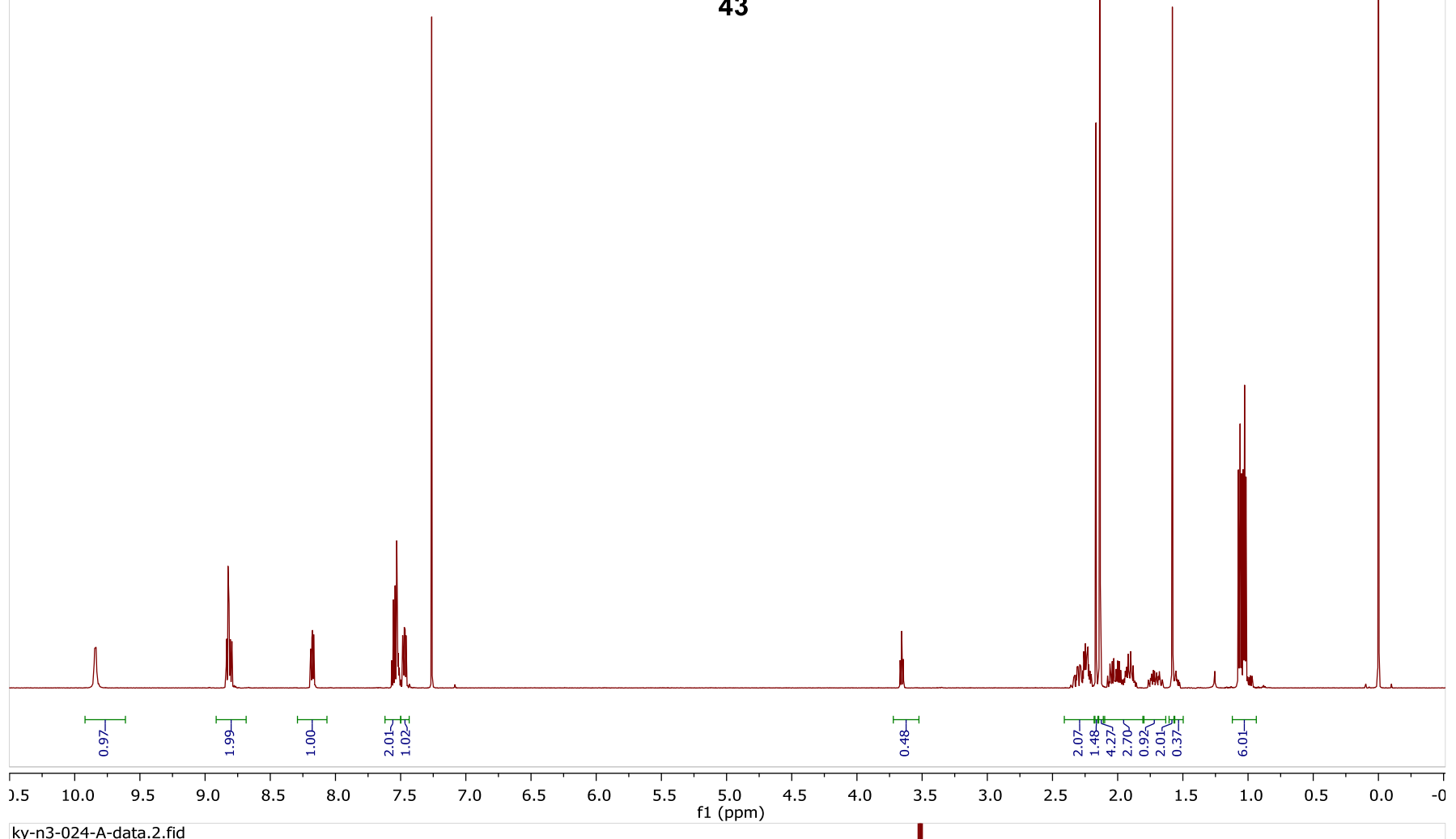

ky-n3-024-A-data.2.fid

H-1 Routine, CP-DCH, AVIII HD-600 -

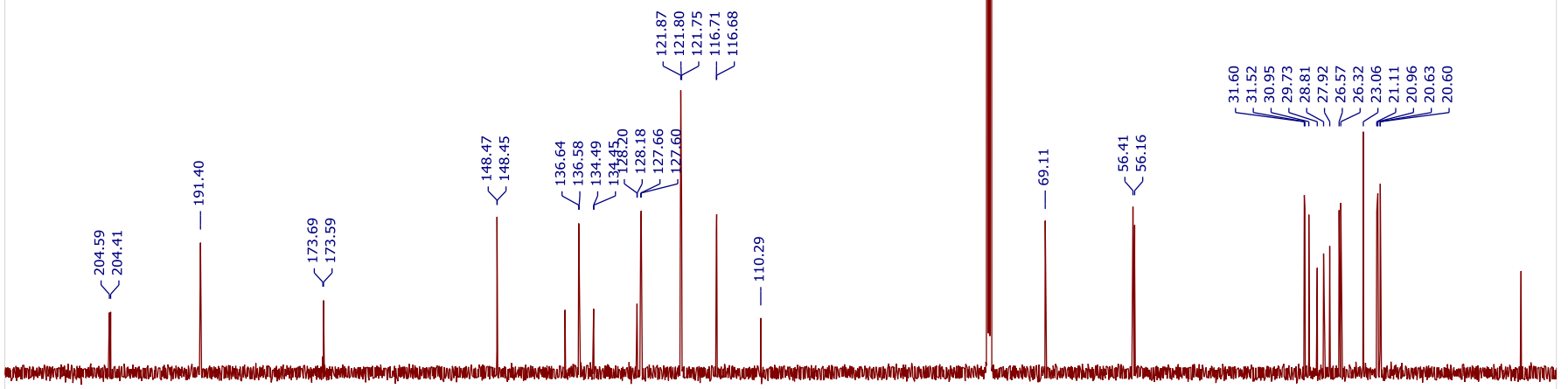


ky-n3-024-C-data.1.fid

H-1 Routine, CP-DCH, AVIII HD-600 -<smiles>CC(=O)C(CCC(C)(C)C(=O)Nc1cccc2cccnc12)C(C)=O</smiles>

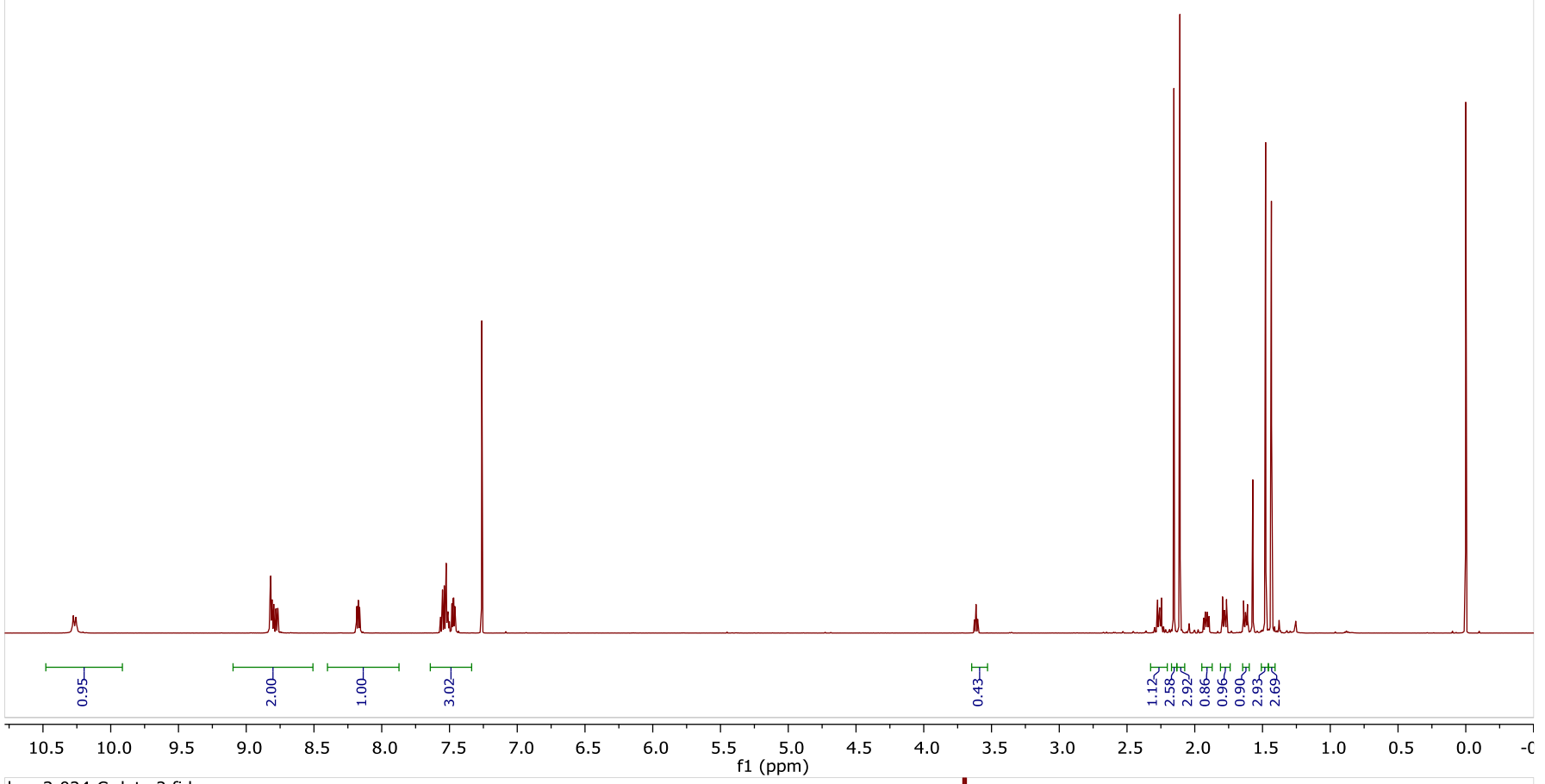

ky-n3-024-C-data.2.fid

H-1 Routine, CP-DCH, AVIII HD-600 -
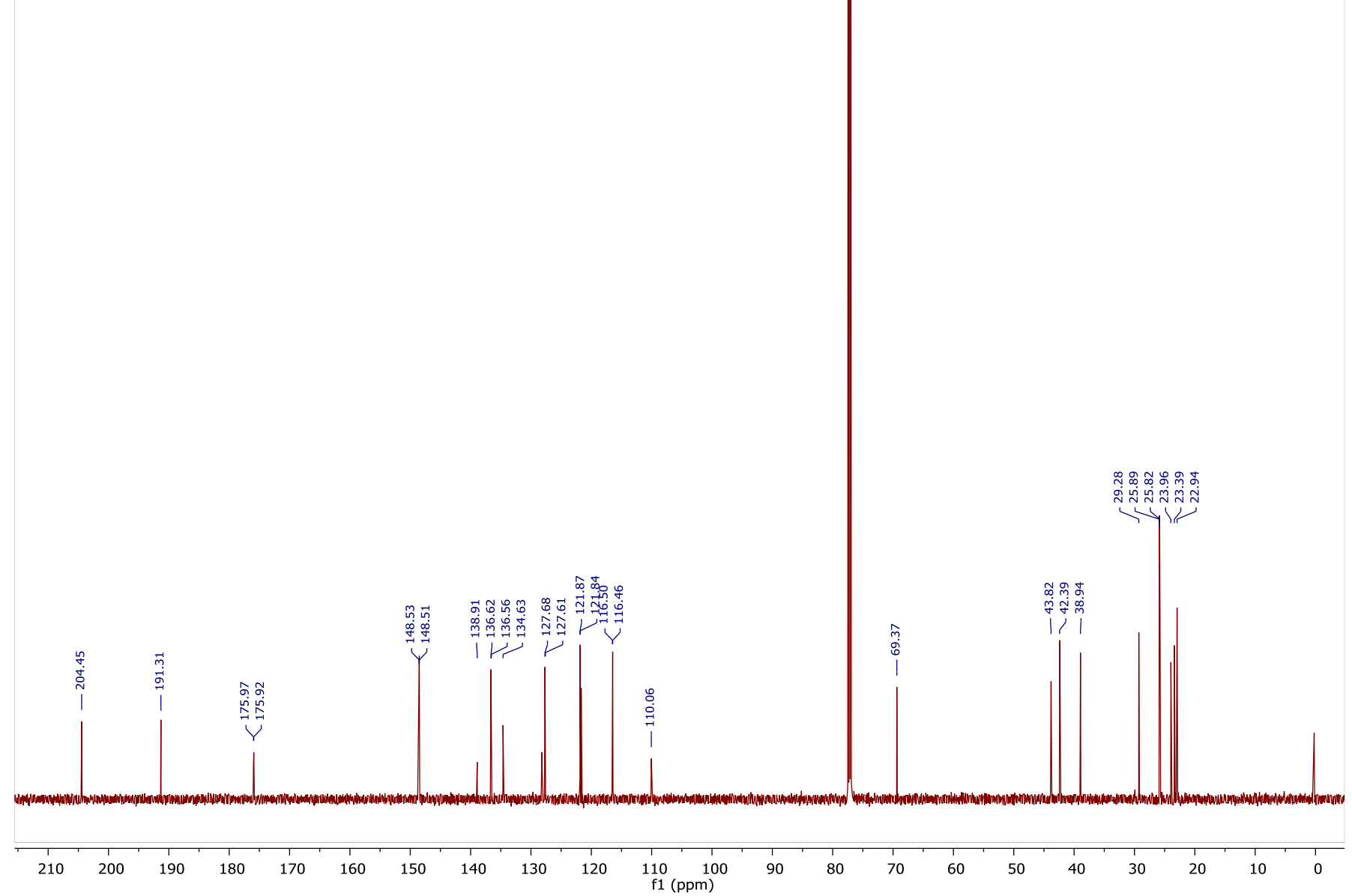
ky-n3-024-D-data.1.fid

H-1 Routine, CP-DCH, AVIII HD-600 -<smiles>C=C(C)CCC(CCC(C(C)=O)C(C)=O)C(=O)Nc1cccc2cccnc12</smiles>

$\underbrace{M}_{45}$

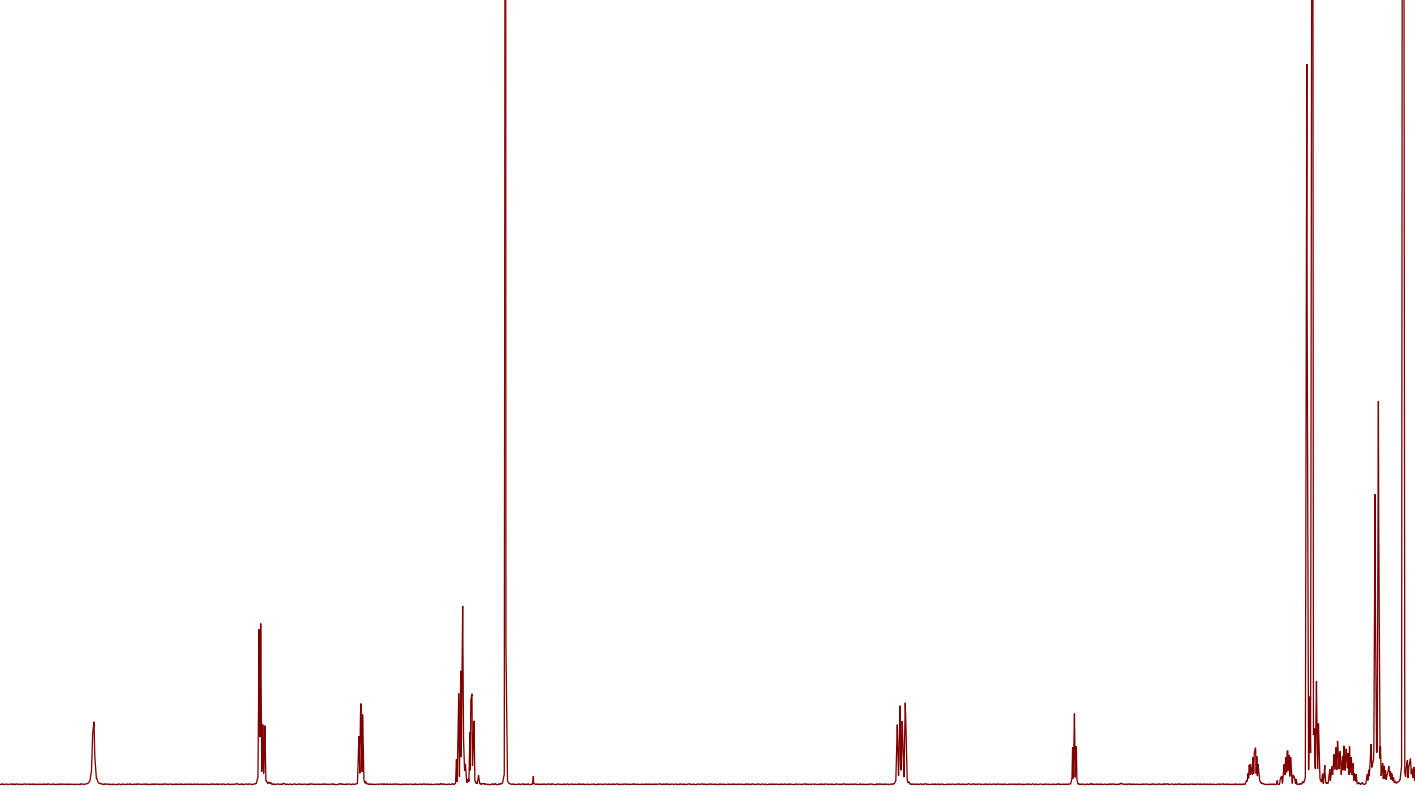

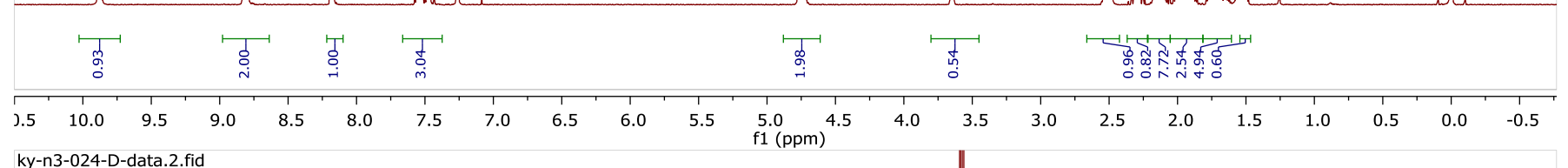

ky-n3-024-D-data.2.fid

H-1 Routine, CP-DCH, AVIII HD-600 -

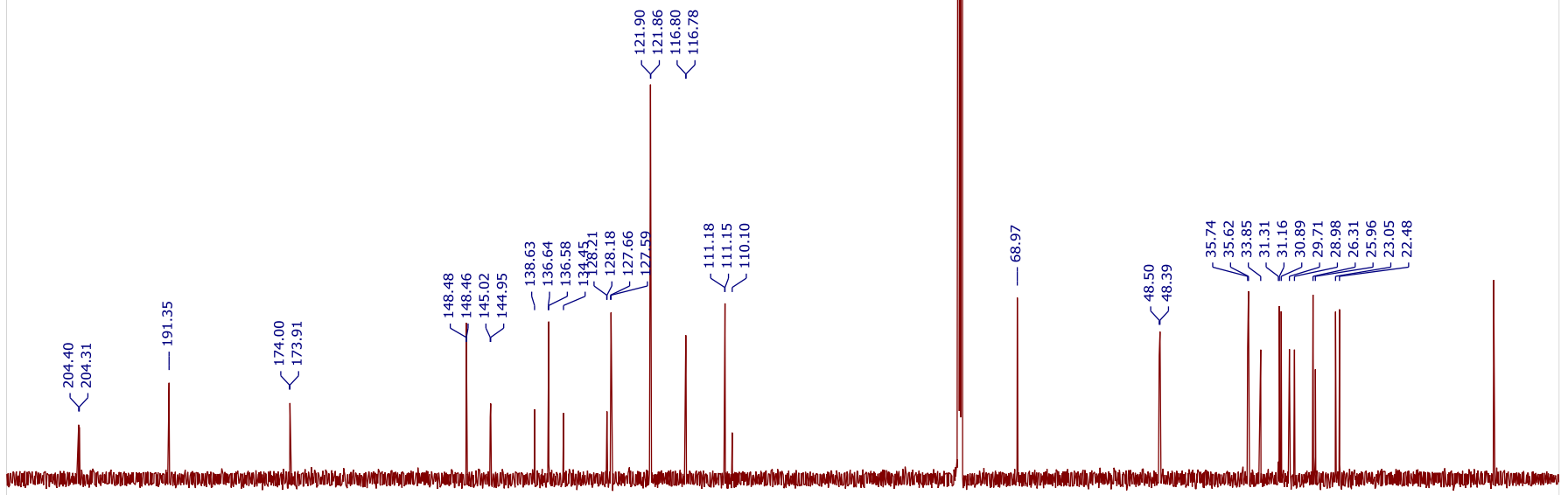


ky-n3-024-B-data.1.fid

H-1 Routine, CP-DCH, AVIII HD-600 -<smiles>CC(=O)C(CC=C(C(=O)Nc1cccc2cccnc12)C(C)(C)O)C(C)=O</smiles>

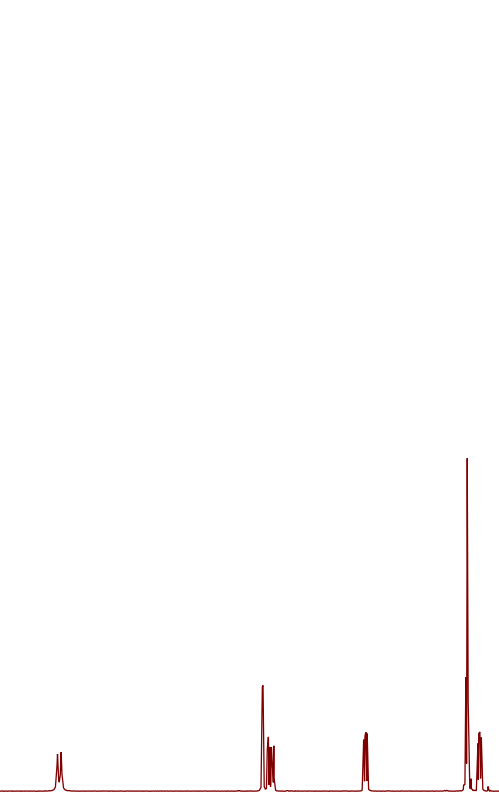

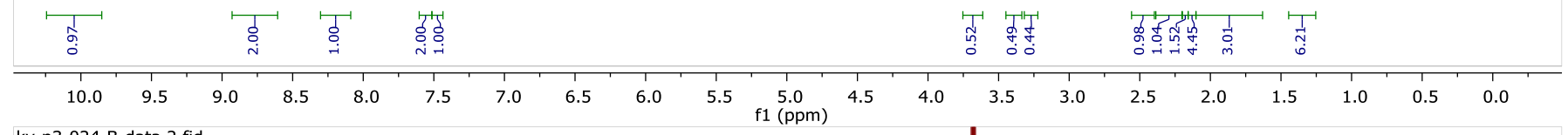

\section{ky-n3-024-B-data.2.fid}

H-1 Routine, CP-DCH, AVIII HD-600 -

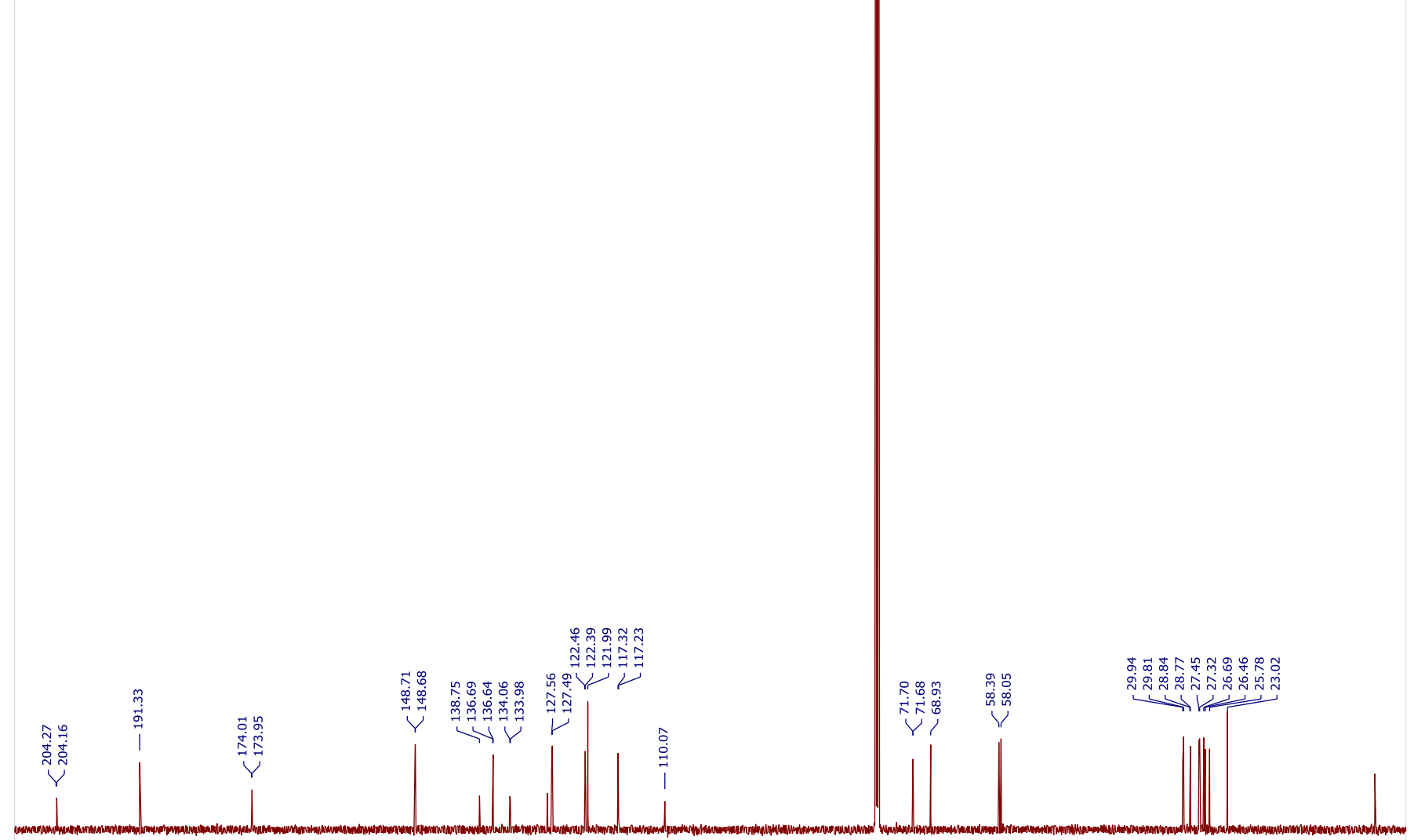

$$
\stackrel{T}{10}
$$


ky-n3-055-B-data.1.fid

C-13 Routine 1D, CPDCH CryoProbe, AVIII-600<smiles>CC(=O)C(C(C)=O)C(C)CCC(=O)Nc1cccc2cccnc12</smiles>

(-13 Routine 1D, CPDCH cryoprobe, AVIII-600

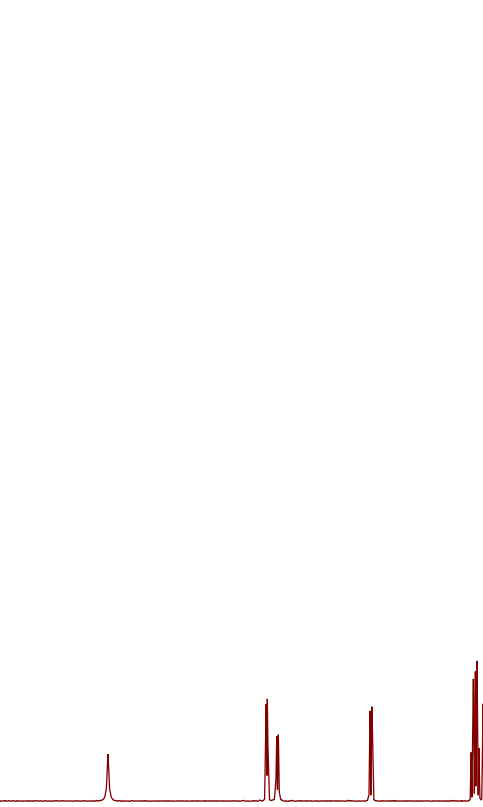

\begin{tabular}{|c|c|c|c|c|c|c|c|c|c|c|c|c|c|c|c|c|c|c|c|c|}
\hline d & & & & tri & 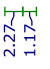 & & & & & & & & $\frac{1}{6}$ & & 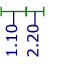 & 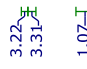 & 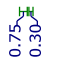 & $\underset{\text { N }}{\stackrel{T}{N}}$ & & \\
\hline 10.0 & 9.5 & 9.0 & 8.5 & 8.0 & 7.5 & 7.0 & 6.5 & 6.0 & 5.5 & $\begin{array}{c}5.0 \\
\text { f1 }(\mathrm{ppm})\end{array}$ & 4.5 & 4.0 & 3.5 & 3.0 & 2.5 & 2.0 & 1.5 & 1.0 & 0.5 & 0.0 \\
\hline
\end{tabular}
ky-n3-055-B-data.2.fid

C-13 Routine 1D, CPDCH CryoProbe, AVIII-600
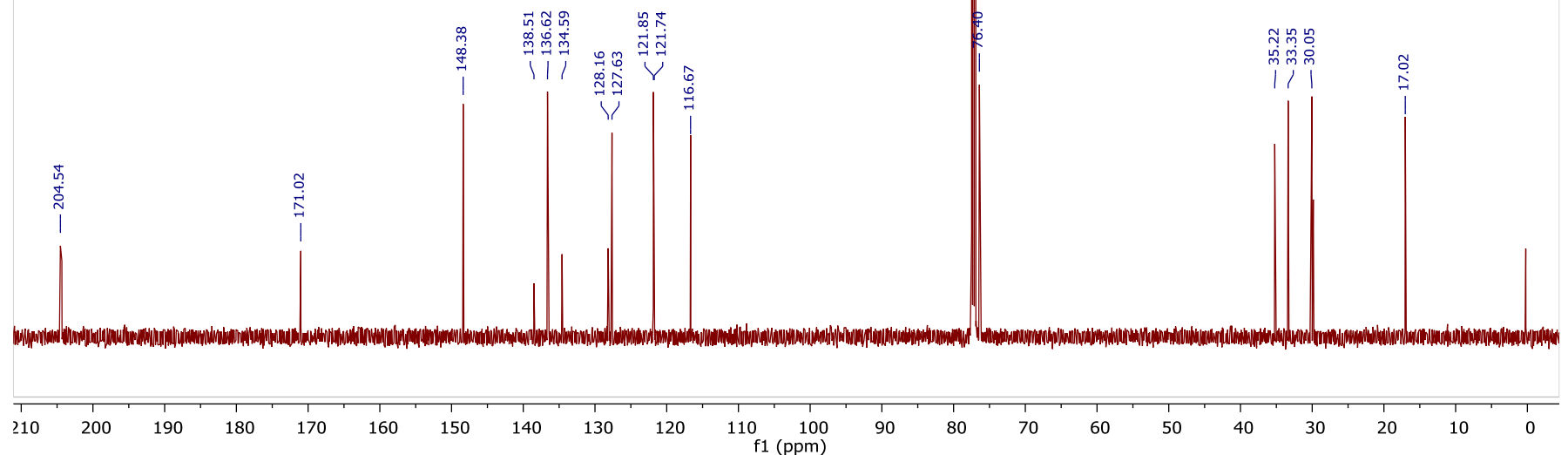
ky-n3-055-A-data.1.fid

C-13 Routine 1D, CPDCH CryoProbe, AVIII-600

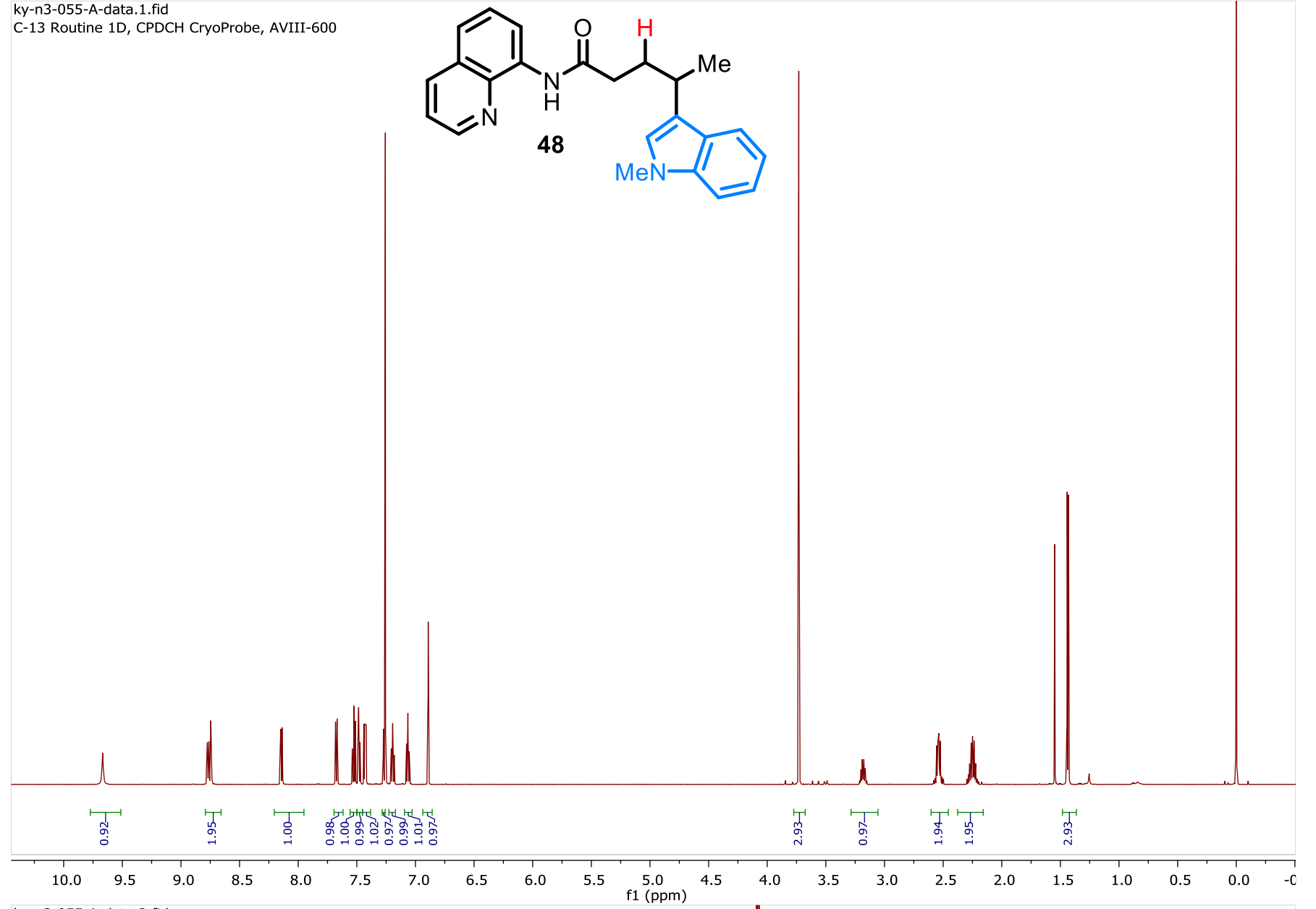

ky-n3-055-A-data.2.fid

C-13 Routine 1D, CPDCH CryoProbe, AVIII-600
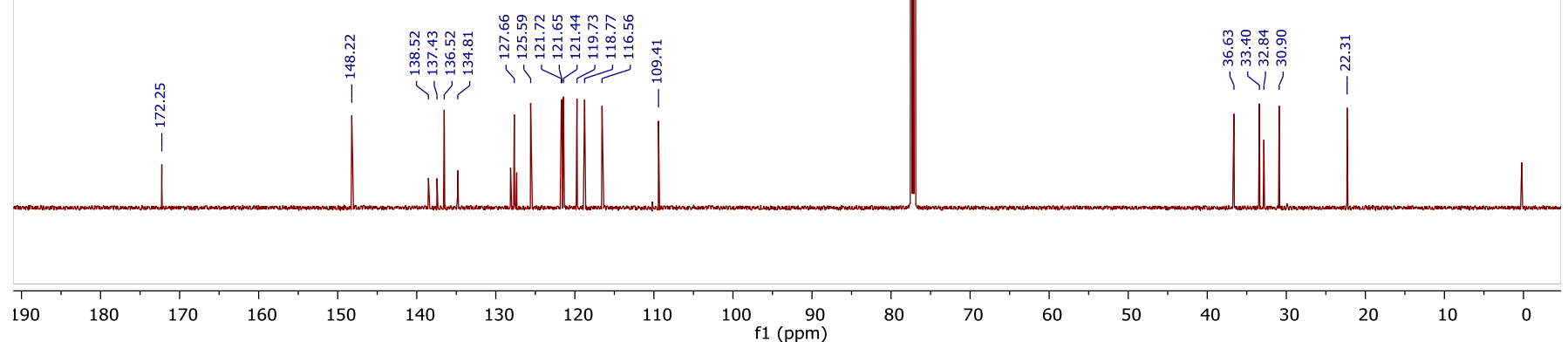


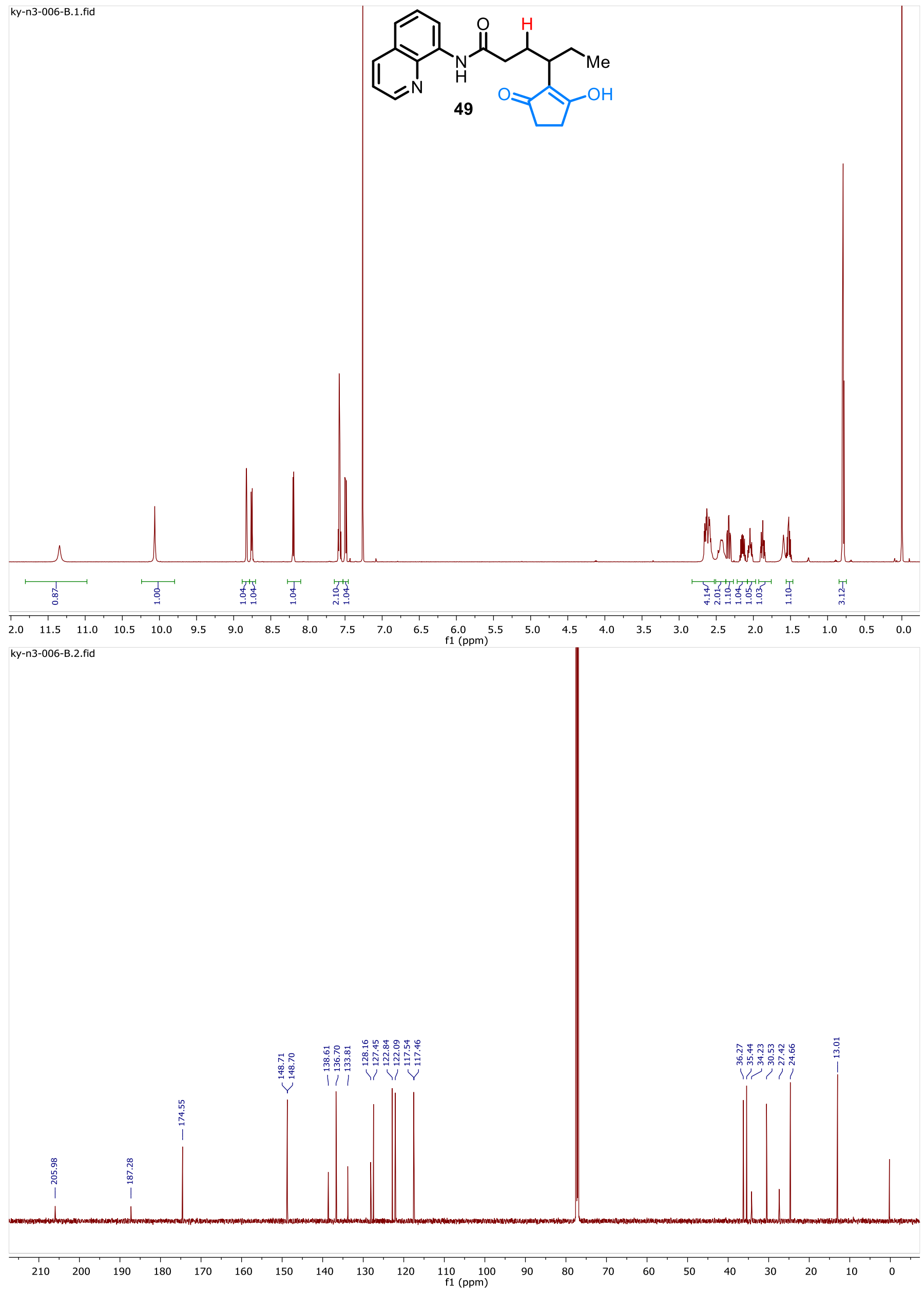


ky-n3-013-B-data.1.fid

$\mathrm{H}-1$ Routine. CPOCI, AVIII-600, 12-20-2013<smiles>CCC(CCC(=O)Nc1cccc2cccnc12)C(CC)c1c(O)c2ccccc2oc1=O</smiles>

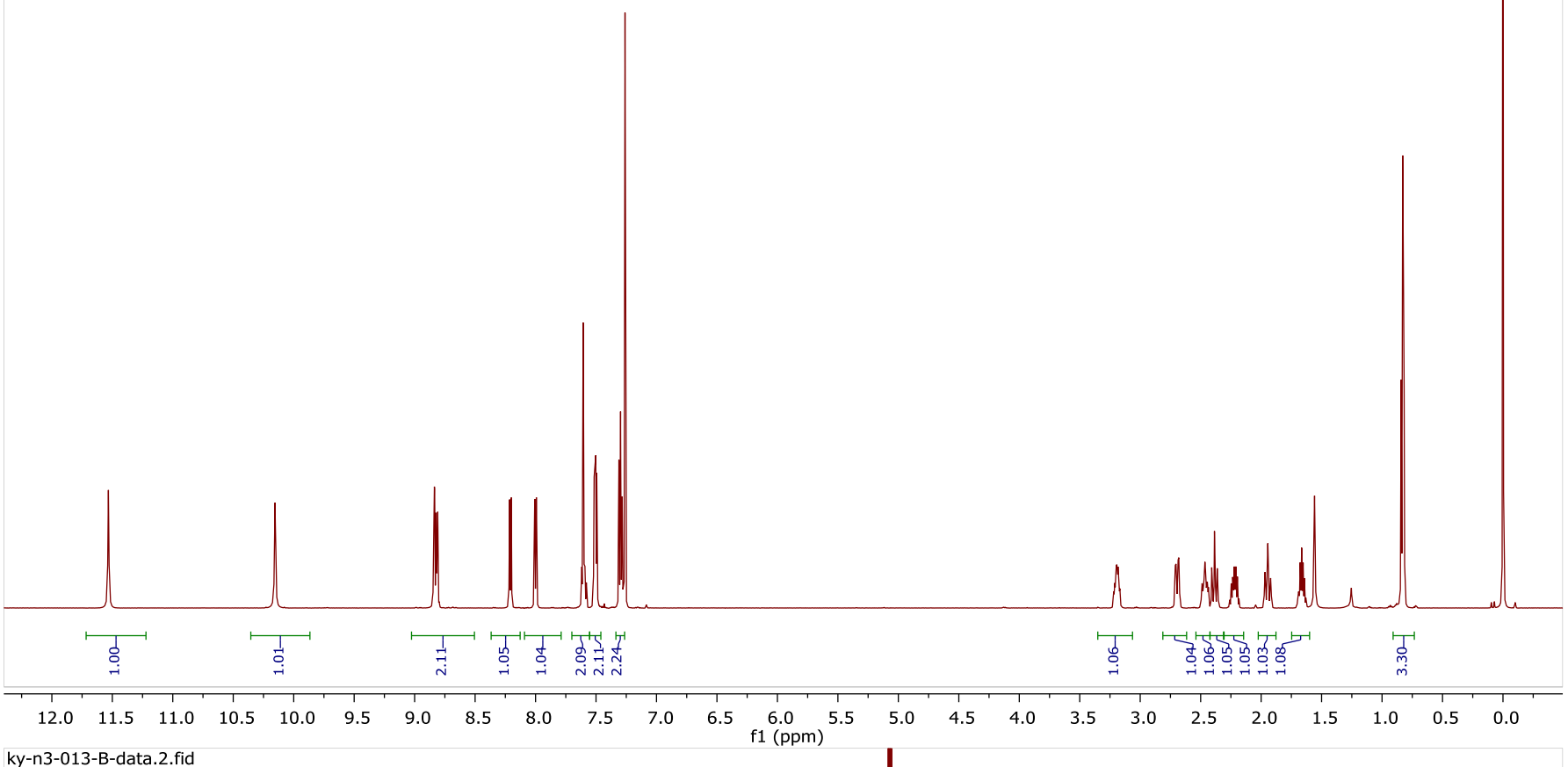

ky-n3-013-B-data.2.fid

H-1 Routine, CP-DCH, AVIII HD-600 - bot SPOT C13
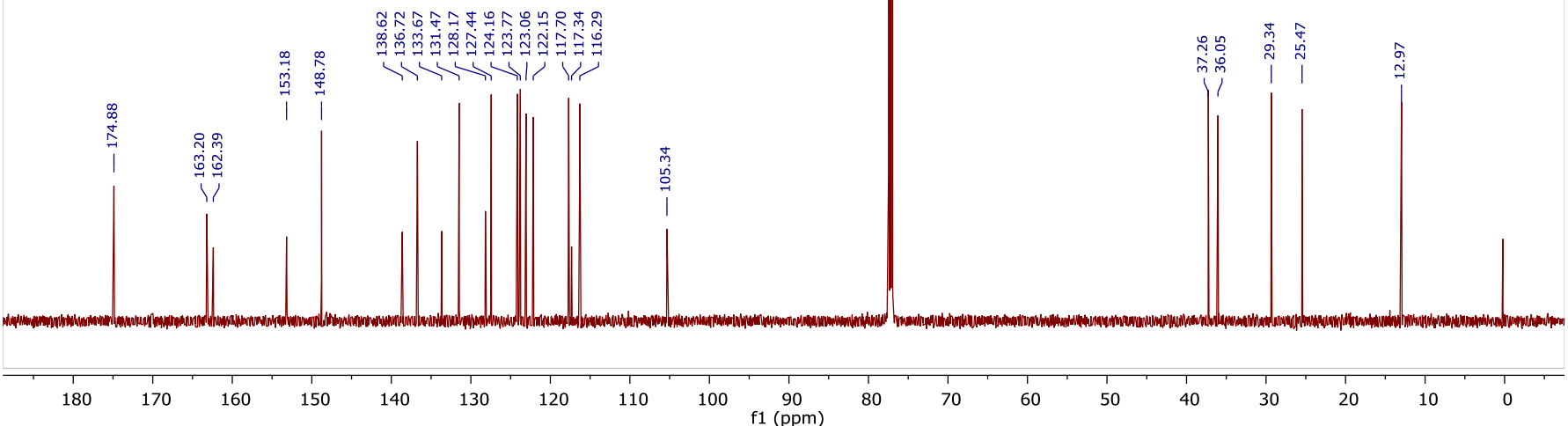


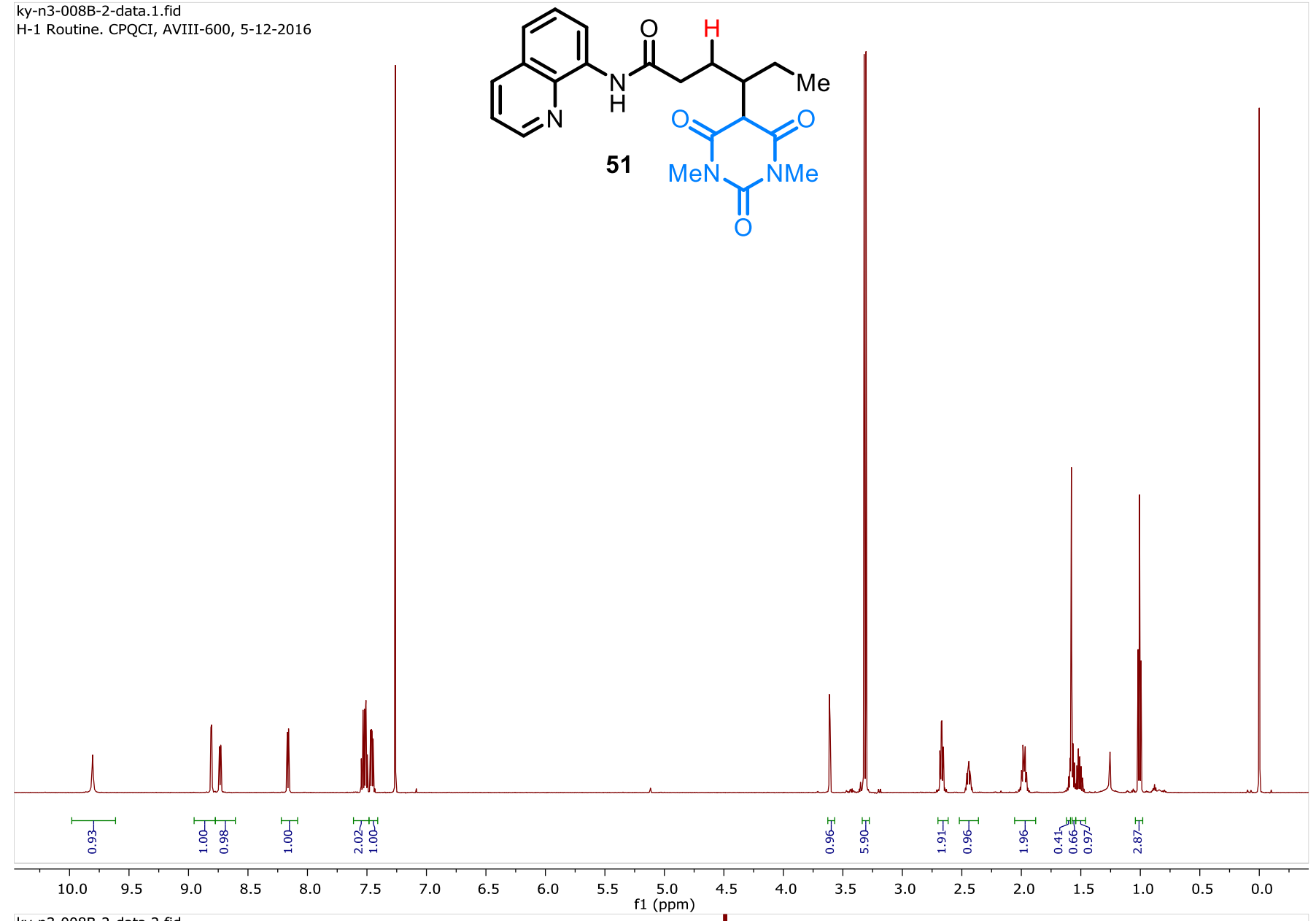

ky-n3-008B-2-data.2.fid

H-1 Routine. CPQCI, AVIII-600, 5-12-2016
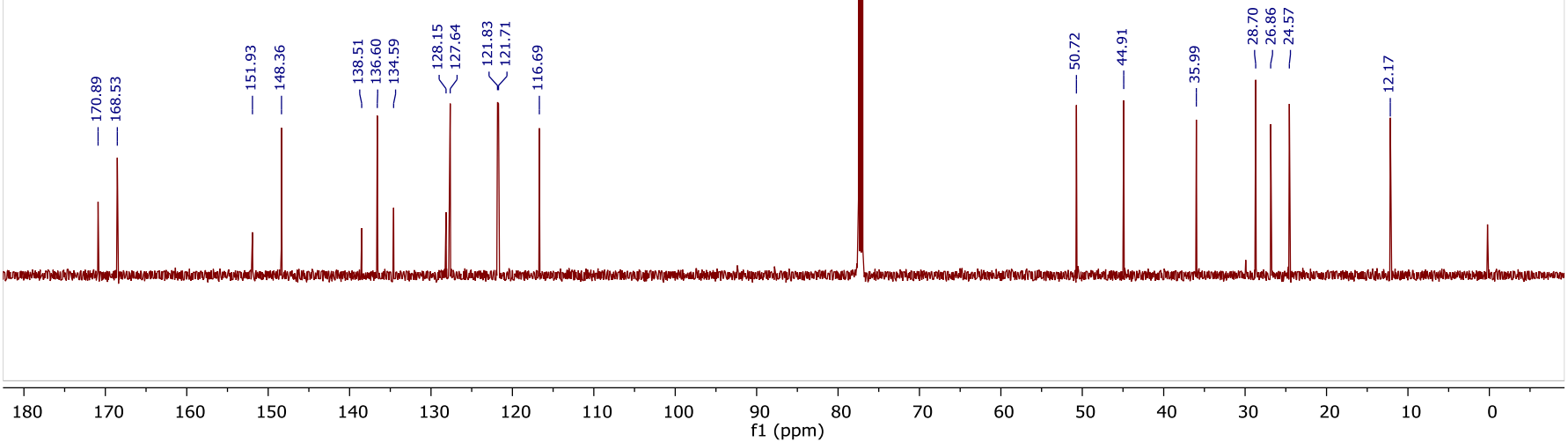
ky-n3-009-C-data.1.fid

H-1 Routine, CP-DCH, AVIII HD-600<smiles>CCC(C=CCC(=O)Nc1cccc2cccnc12)C1=C(O)CC(C)(C)CC1=O</smiles>

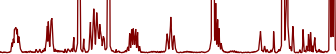

\begin{tabular}{|c|c|c|c|c|c|c|c|c|c|c|c|c|c|c|c|c|c|c|c|c|}
\hline & $\begin{array}{l}1 \\
0 \\
0 \\
0 \\
0\end{array}$ & & 帘 & & 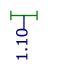 & 架带 & & & & & & & & & 管员 & 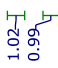 & 'T & 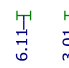 & & \\
\hline 10.5 & 10.0 & 9.5 & 9.0 & 8.5 & 8.0 & 7.5 & 7.0 & 6.5 & 6.0 & $\begin{array}{rr}5.5 & 5.0 \\
f 1(\mathrm{ppm})\end{array}$ & 4.5 & 4.0 & 3.5 & 3.0 & 2.5 & 2.0 & 1.5 & 1.0 & 0.5 & 0.0 \\
\hline
\end{tabular}

ky-n3-009-C-data.2.fid

H-1 Routine, CP-DCH, AVIII HD-600

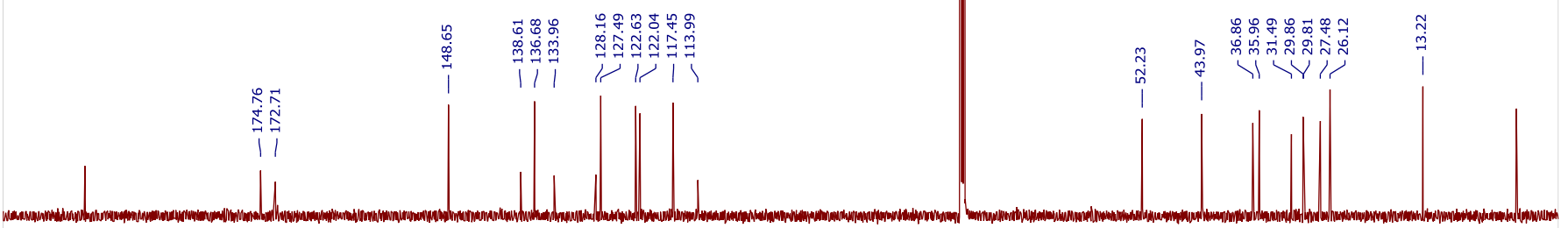




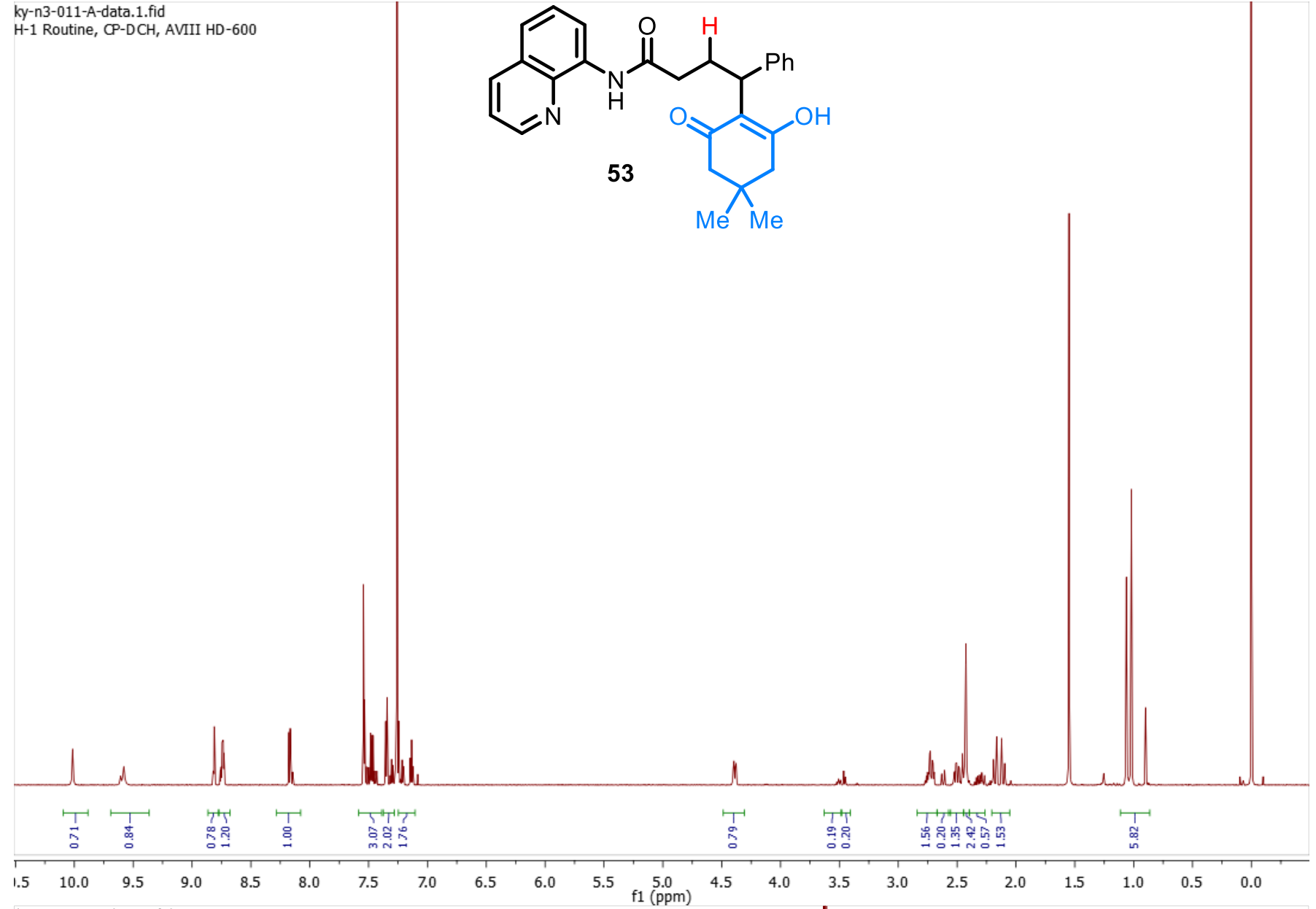

ky-n3-011-A-data.2.fid

H-1 Routine, CP-DCH, AVIII HD-600

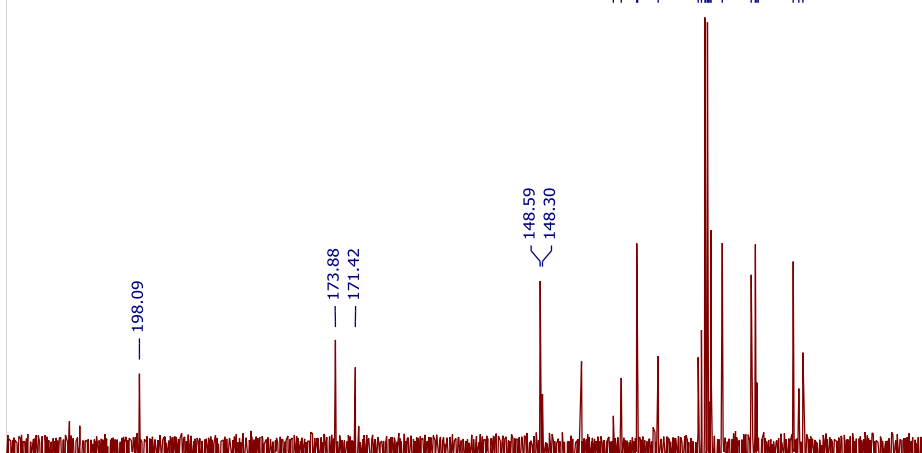

ing


ky-n3-050-B-data.1.fid

C-13 Routine 1D, CPDCH CryoProbe, AVIII-600

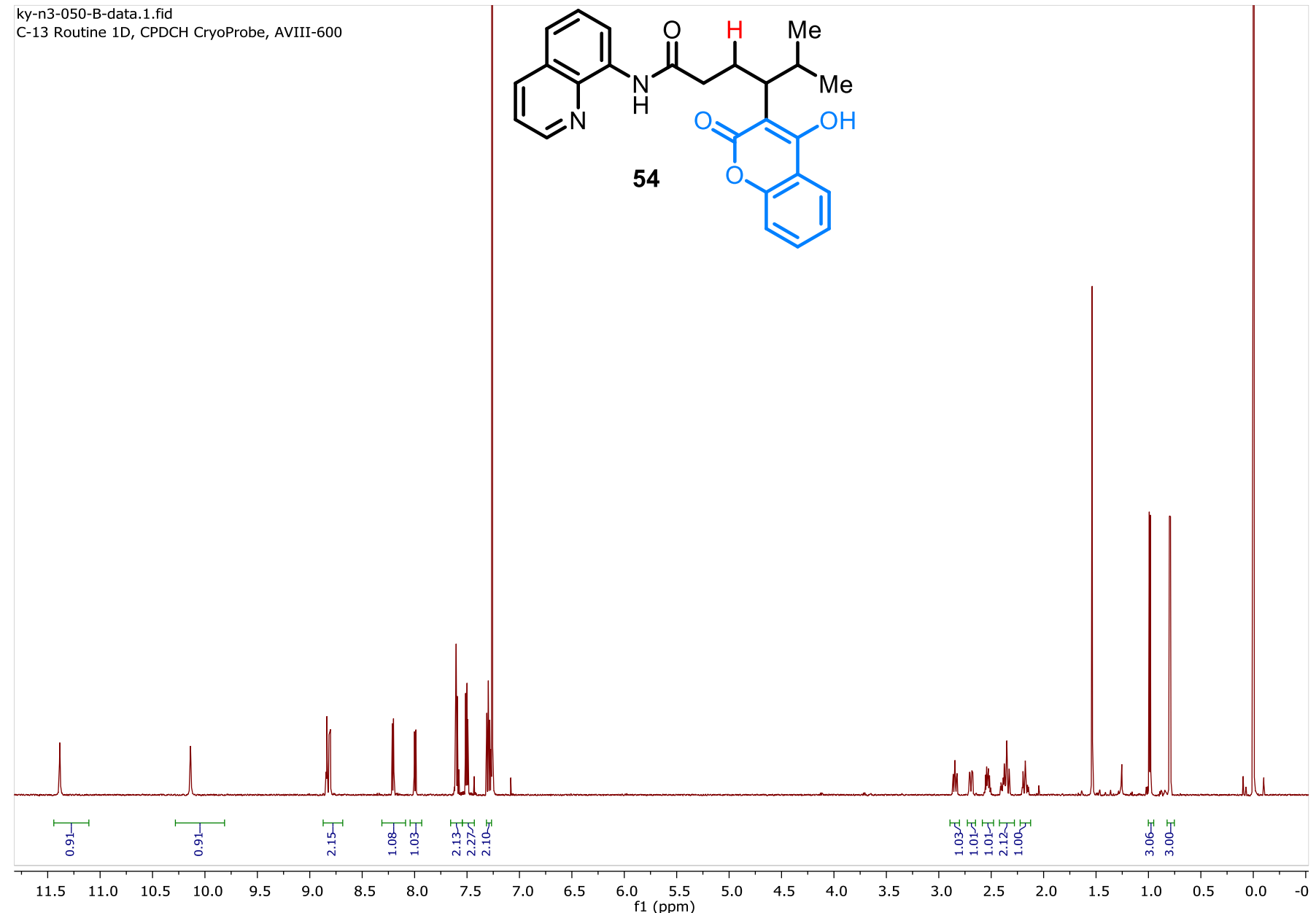

ky-n3-050-B-data.2.fid

C-13 Routine 1D, CPDCH CryoProbe, AVIII-600

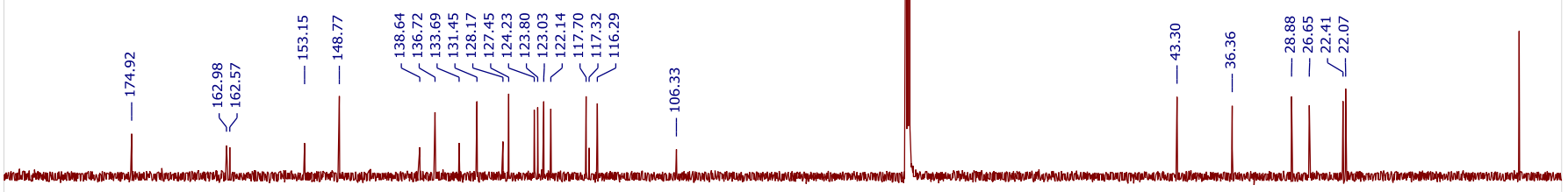


ky-n3-054-C-data.1.fid

C-13 Routine 1D, CPDCH CryoProbe, AVIII-600

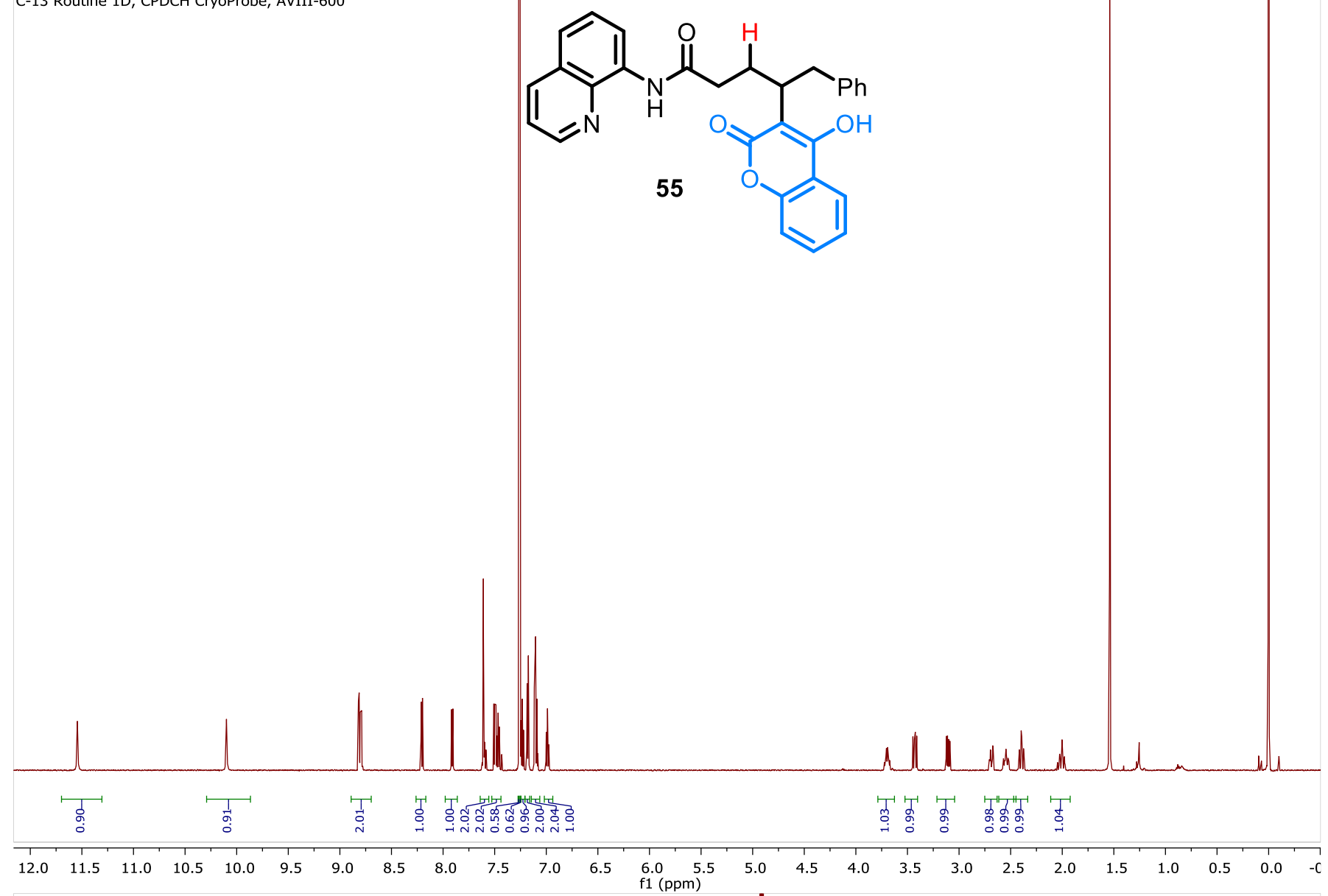

ky-n3-054-C-data.2.fid

C-13 Routine 1D, CPDCH CryoProbe, AVIII-600
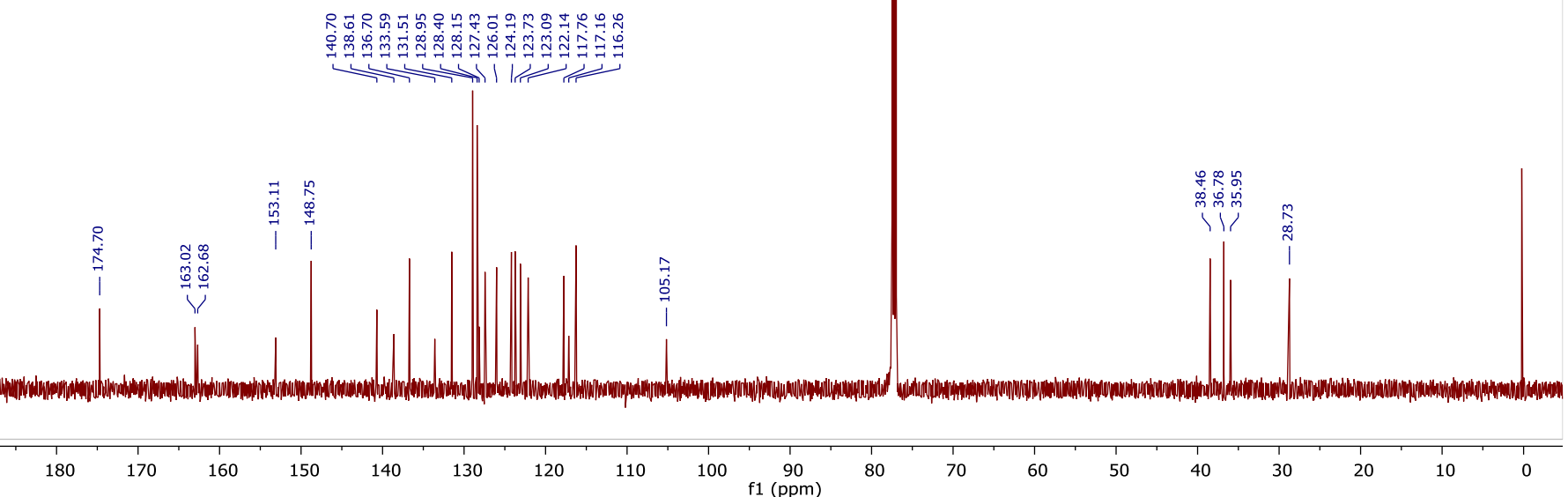
ky-n3-065-data.1.fid

C-13 Routine 1D, CPDCH CryoProbe, AVIII-600

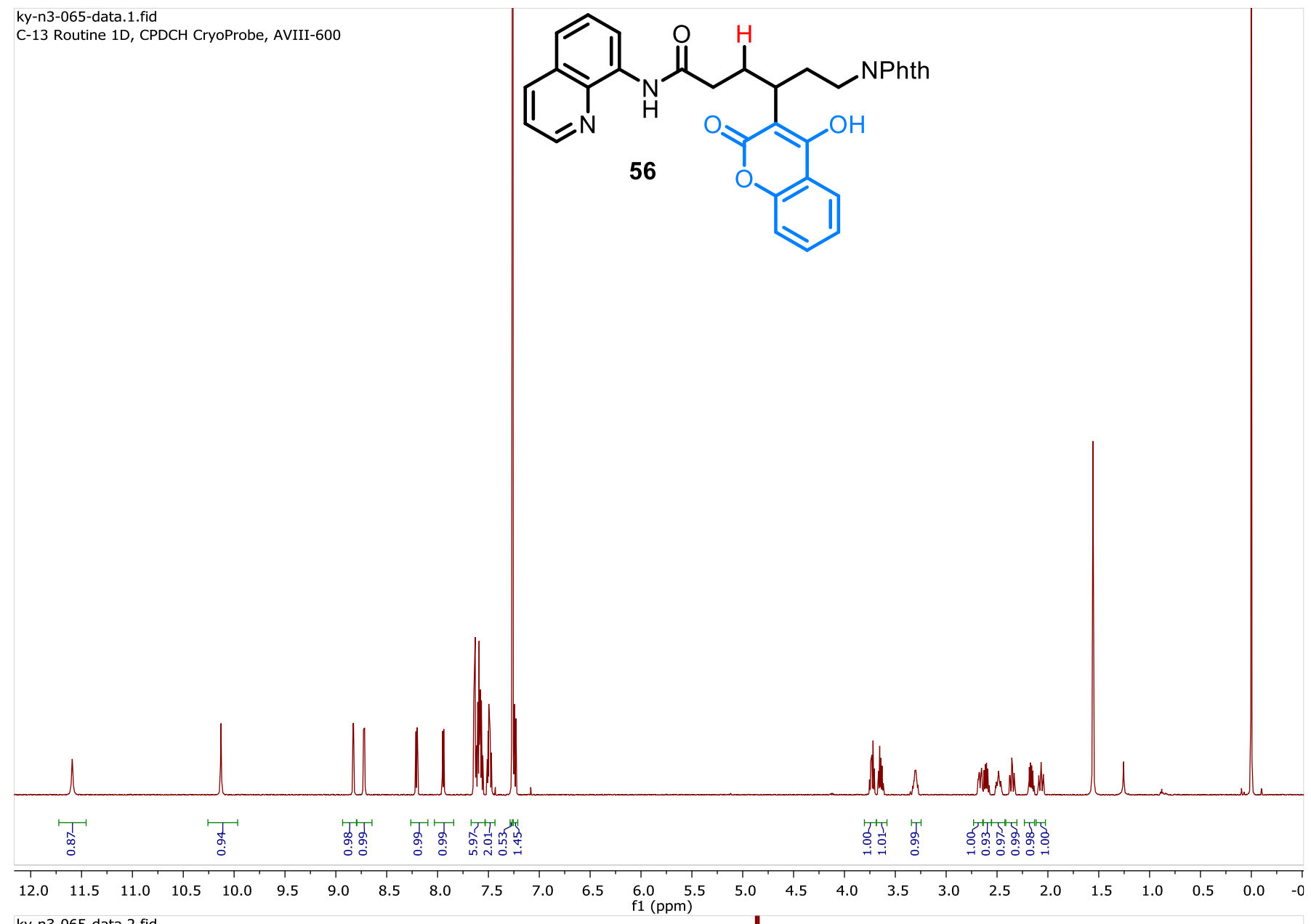

ky-n3-065-data.2.fid

C-13 Routine 1D, CPDCH CryoProbe, AVIII-600

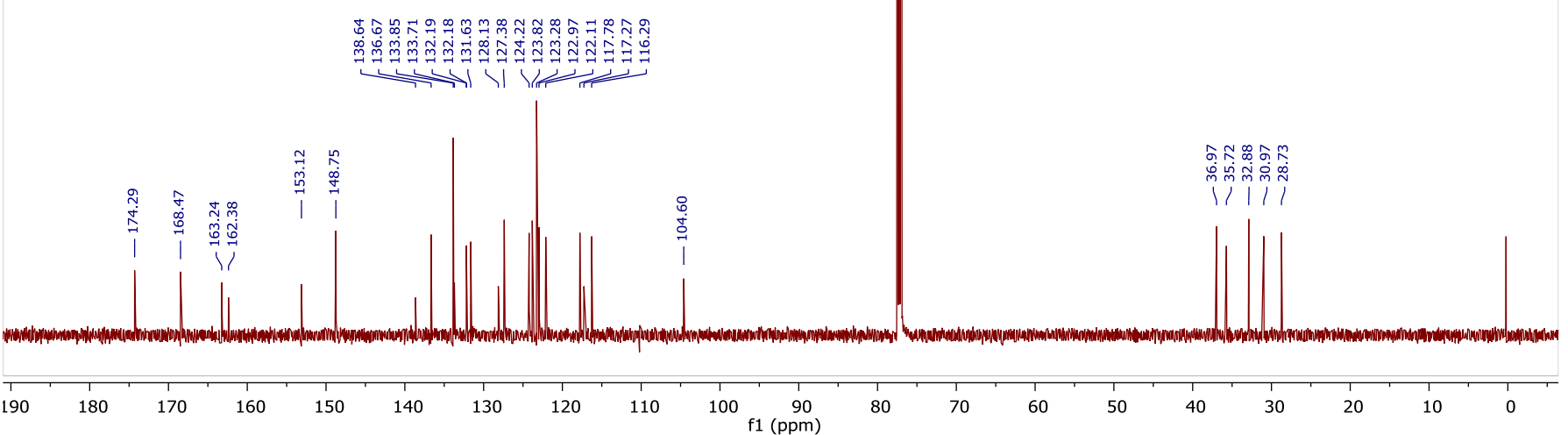




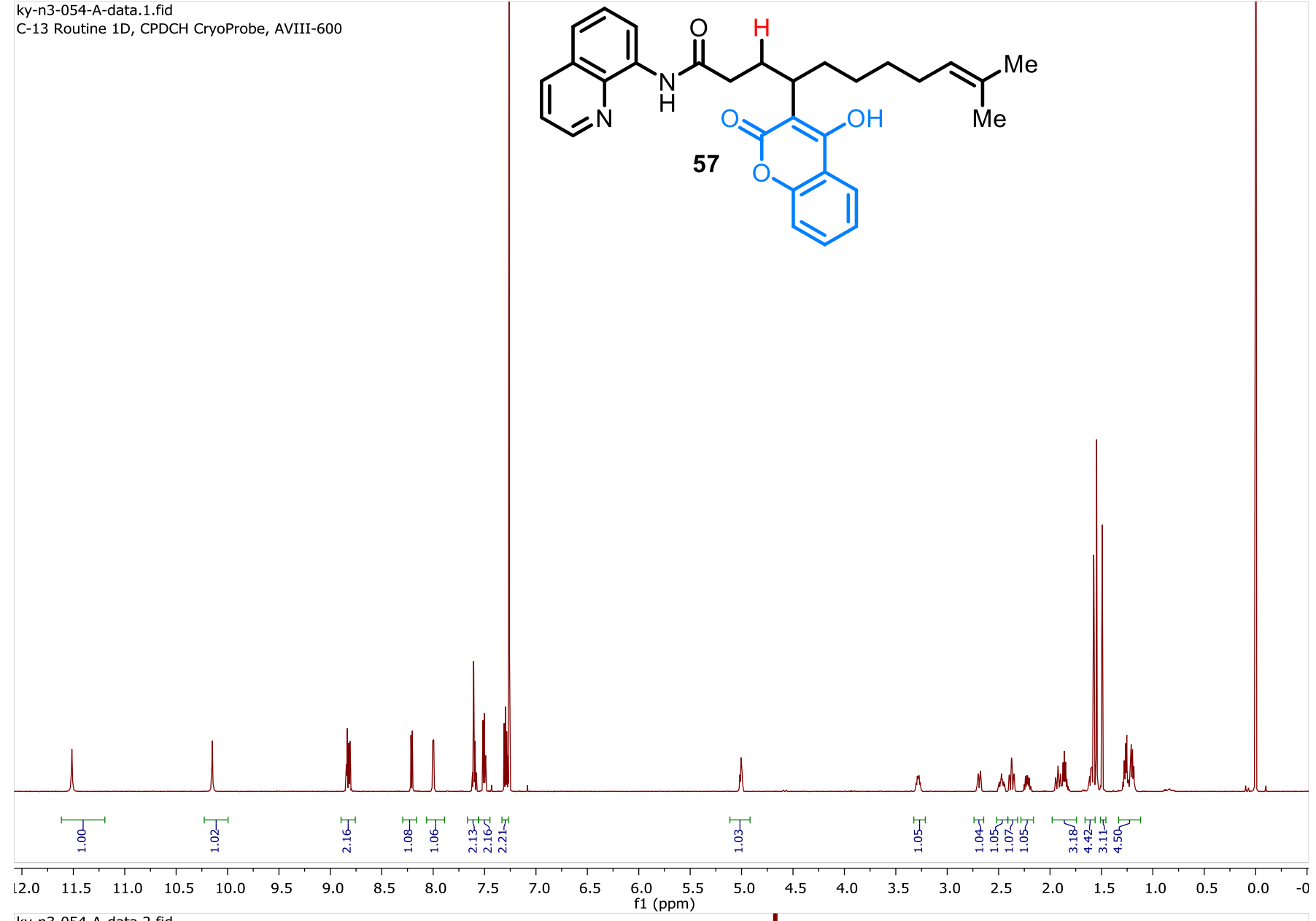

ky-n3-054-A-data.2.fid

C-13 Routine 1D, CPDCH CryoProbe, AVIII-600
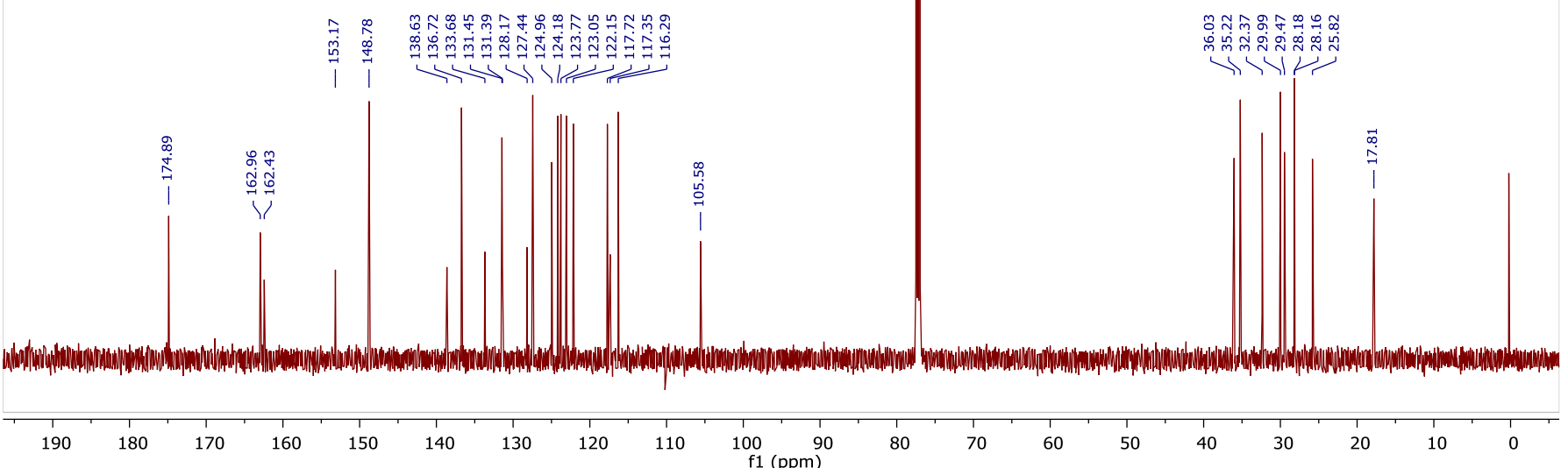
ky-n3-022-C-data.1.fid

H-1 Routine Loaner CPTXI, AVIII-600, 2-23-2016

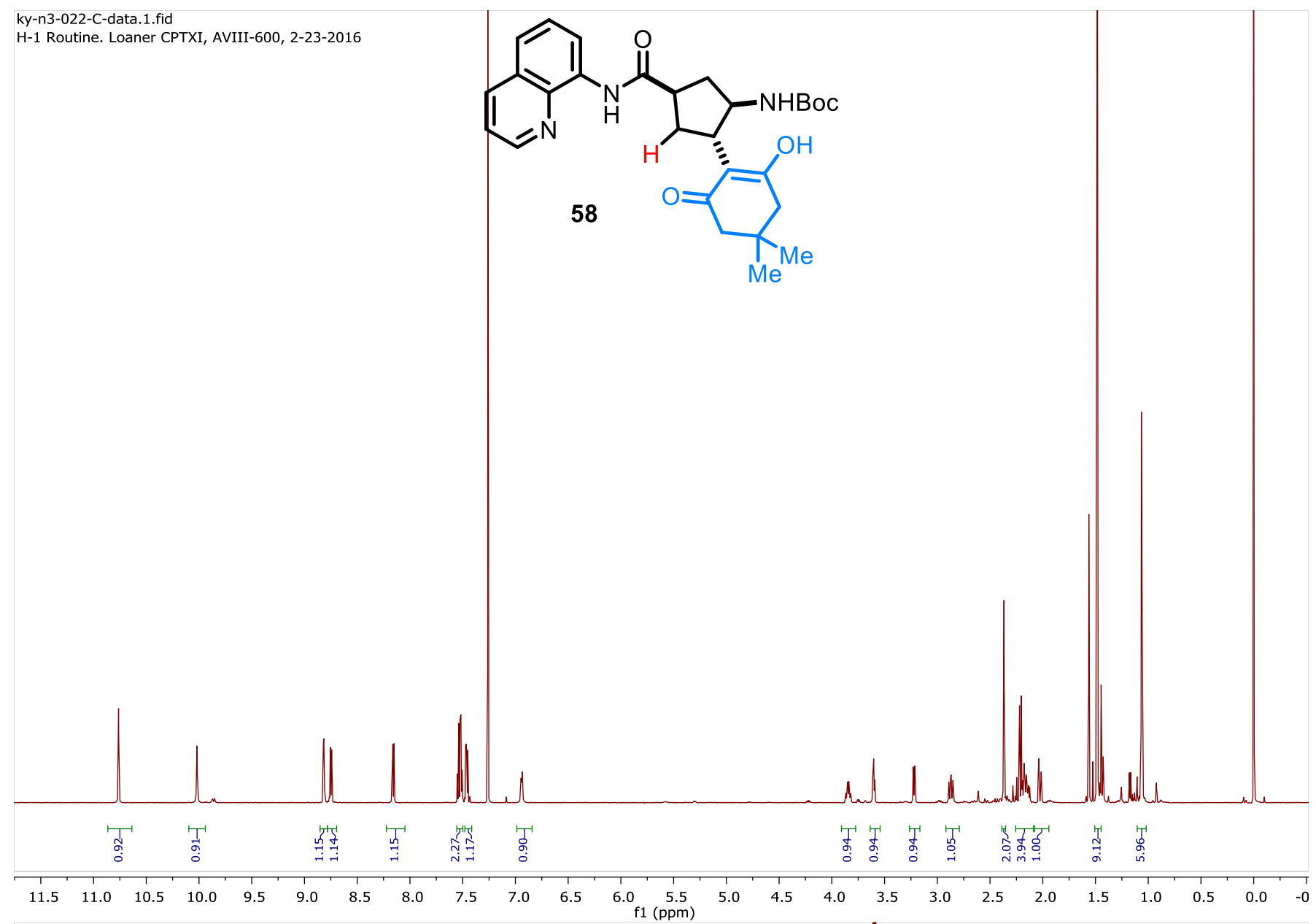

ky-n3-022-C-data.2.fid

H-1 Routine, CP-DCH, AVIII HD-600 -
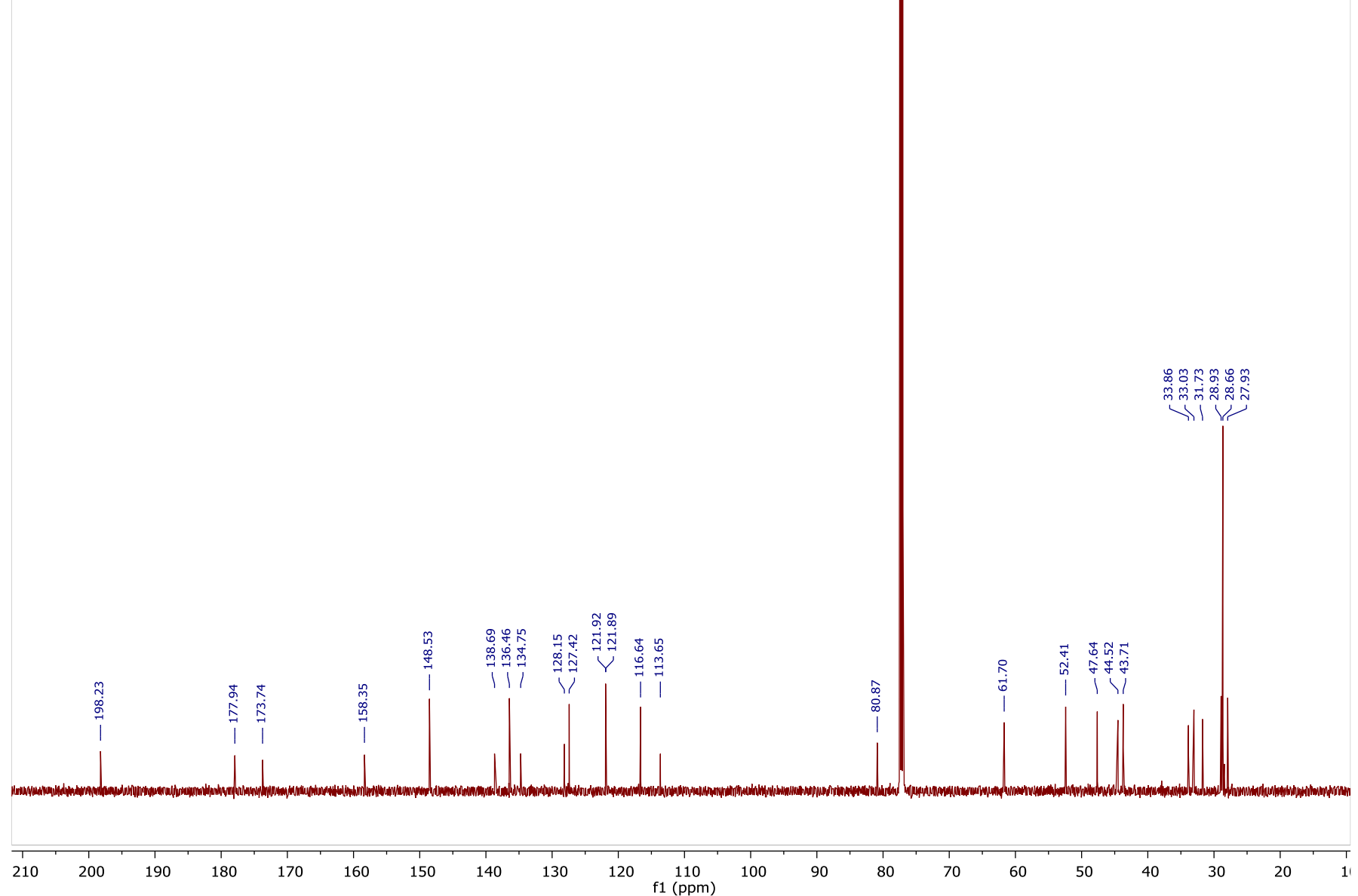
ky-n3-014-A-data.1.fid

H-1 Routine, CP-DCH, AVIII HD-600 - bot SPOT c13

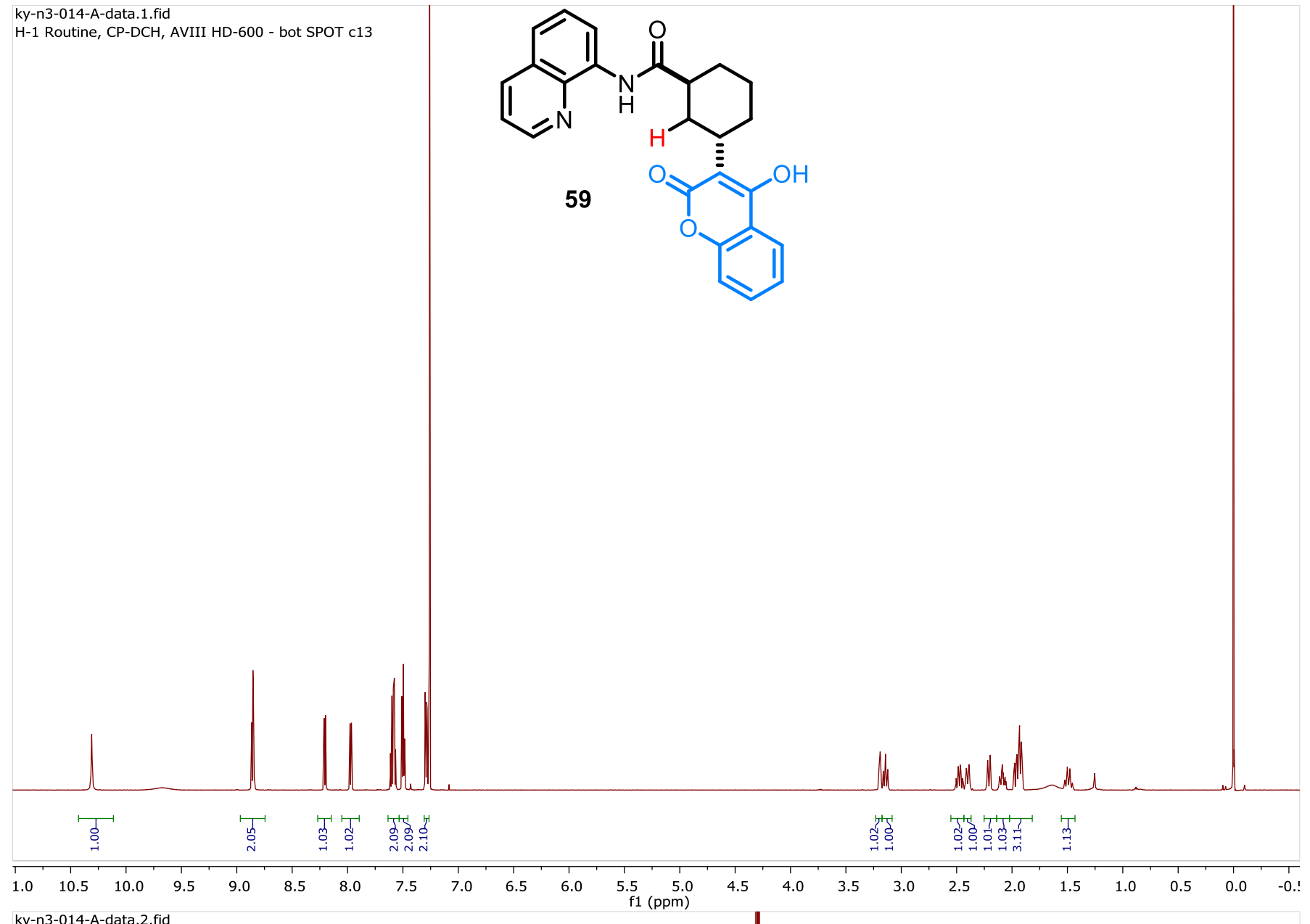

ky-n3-014-A-data.2.fid

H-1 Routine, CP-DCH, AVIII HD-600 -
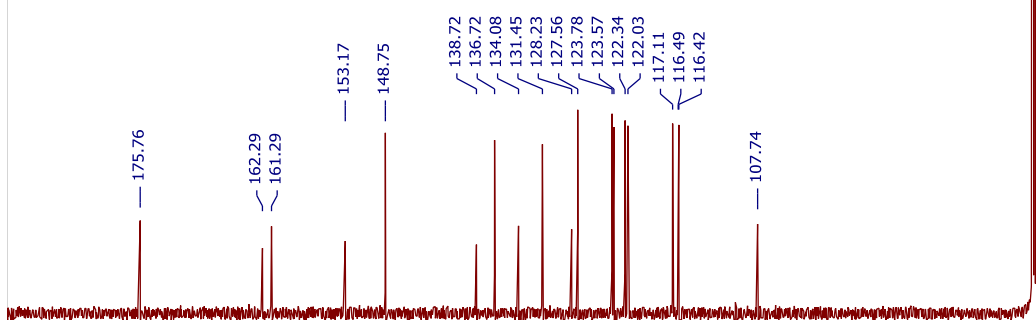

$$
90
$$

$180 \quad 170 \quad 160$

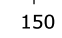

140

130

$120 \quad 110$

100 f1 (ppm)

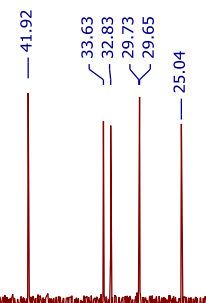


ky-n3-094B-data.1.fid

H-1 Routine. CPQCI, AVIII-600, 5-12-2016<smiles>O=C(CCCc1c(O)c2ccccc2oc1=O)Nc1cccc2cccnc12</smiles>

61

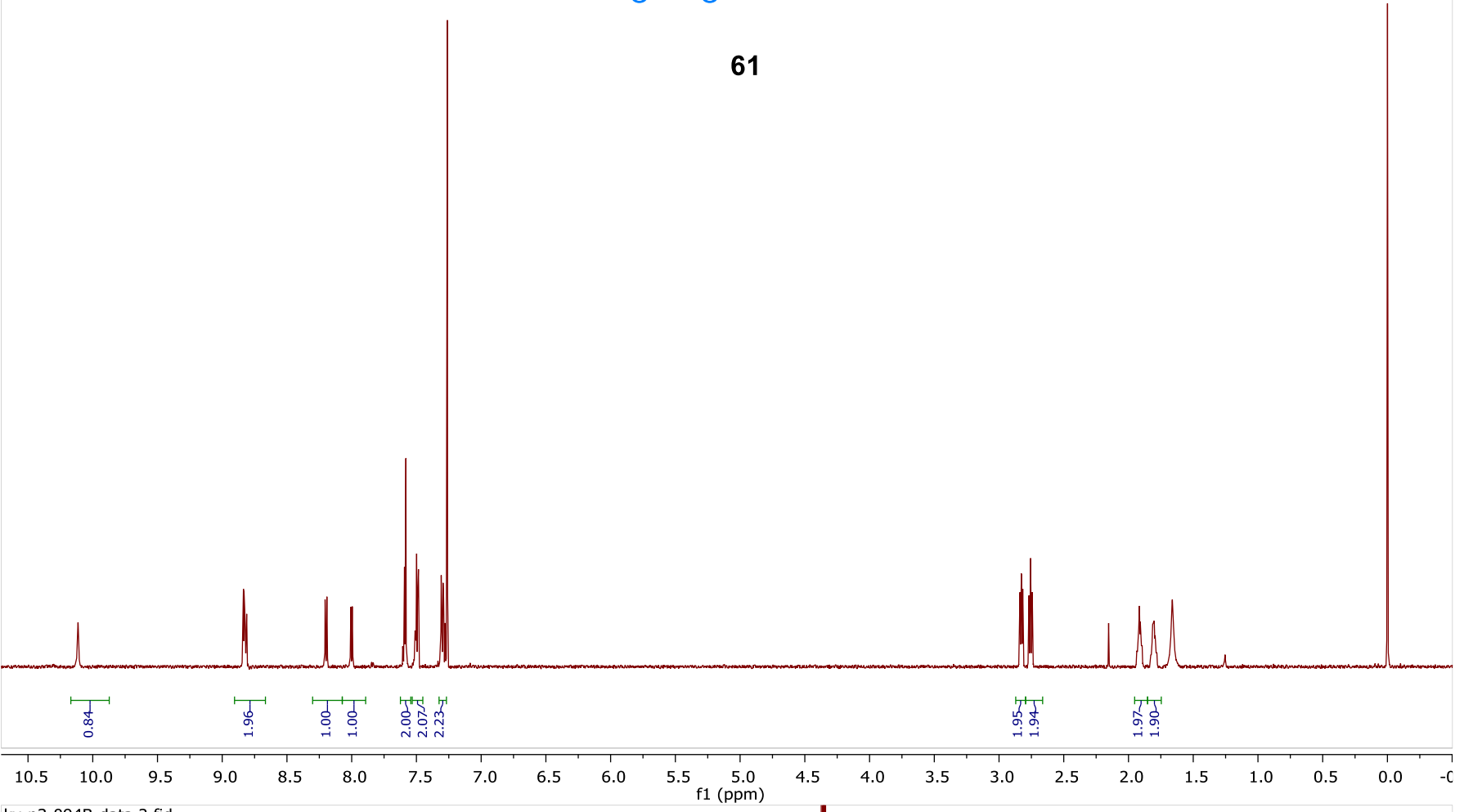

ky-n3-094B-data.2.fid

H-1 Routine. CPQCI, AVIII-600, 5-12-2016
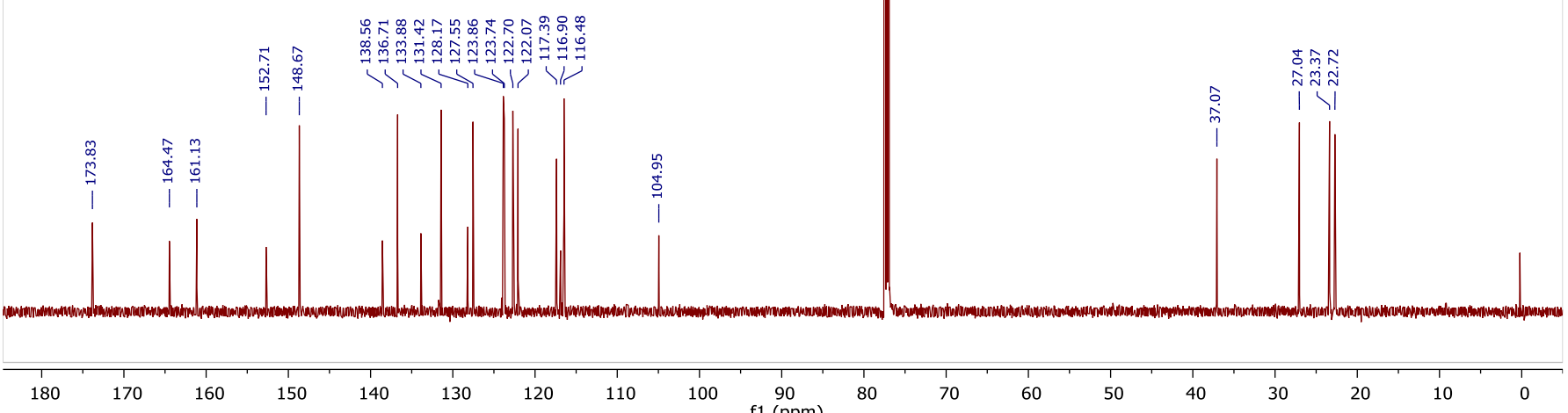


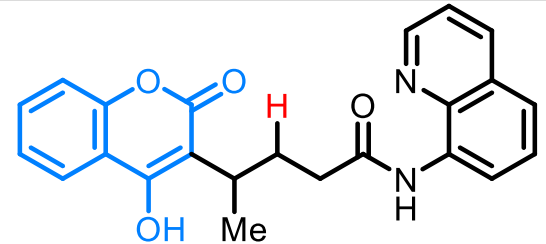

62
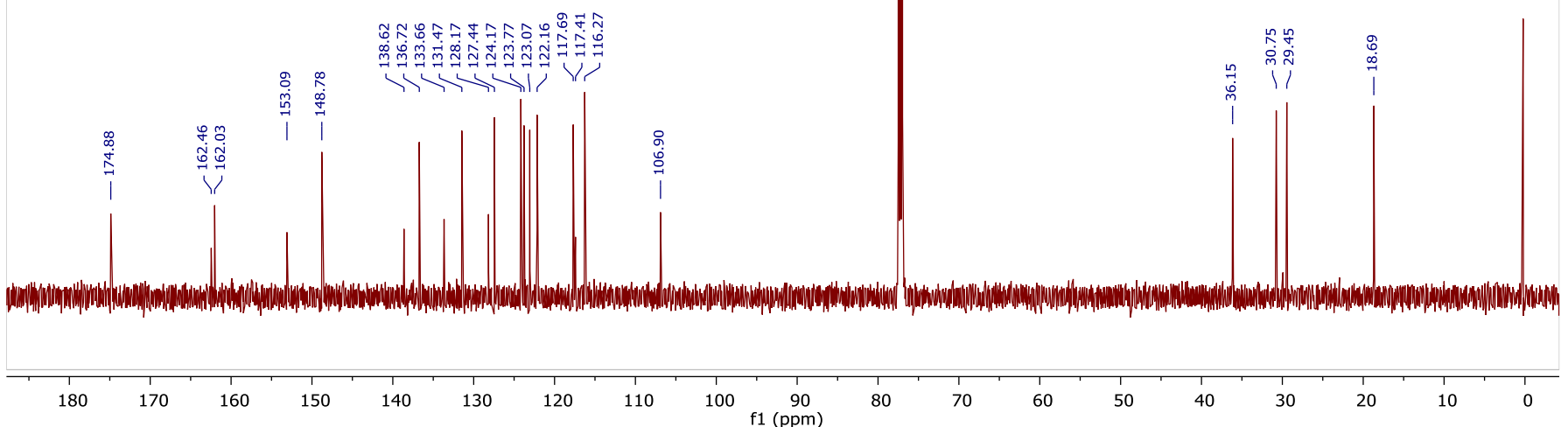

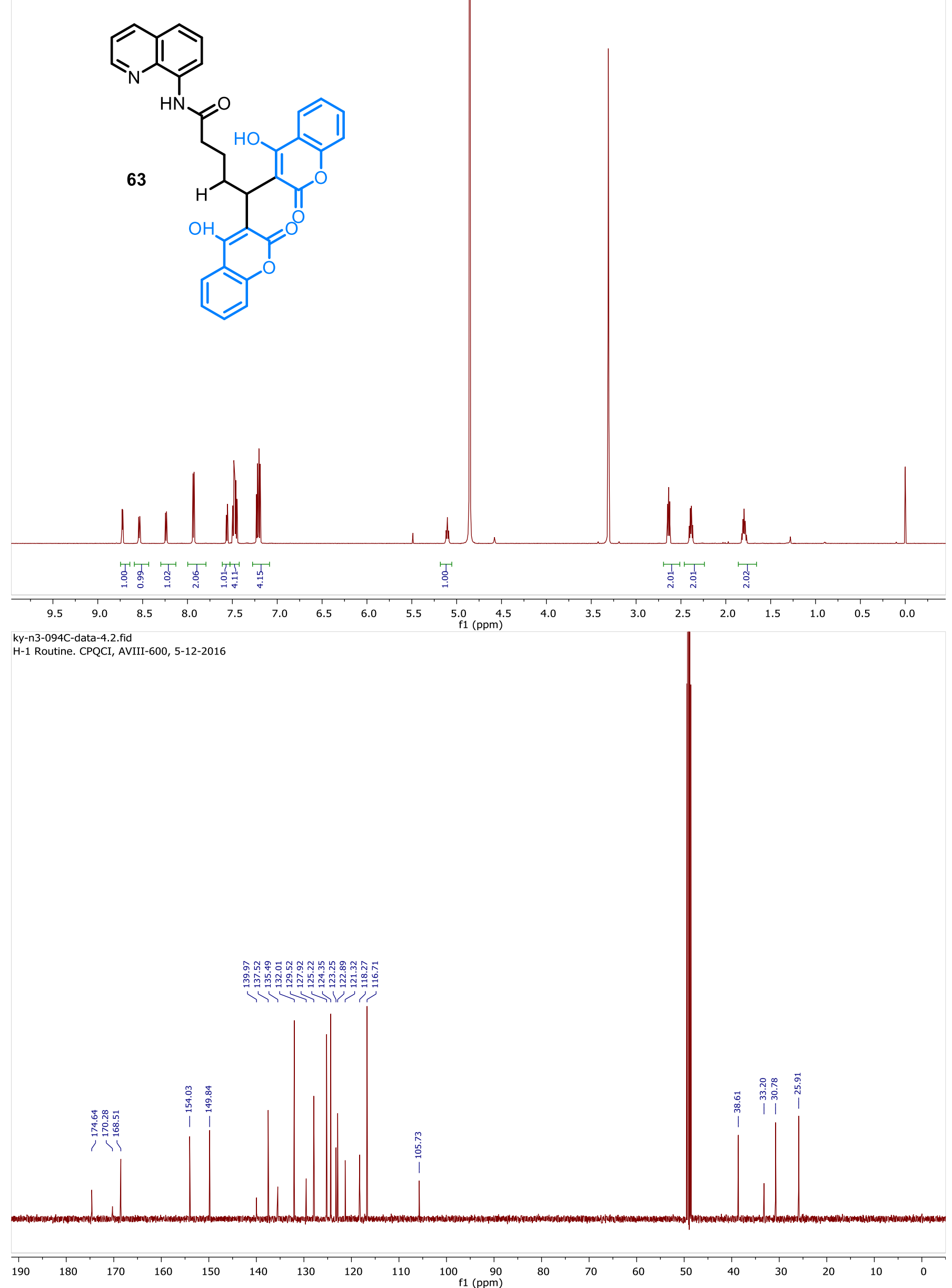
ky-n3-079-B-data.1.fid

H-1 Routine, CP-DCH, AVIII HD-600
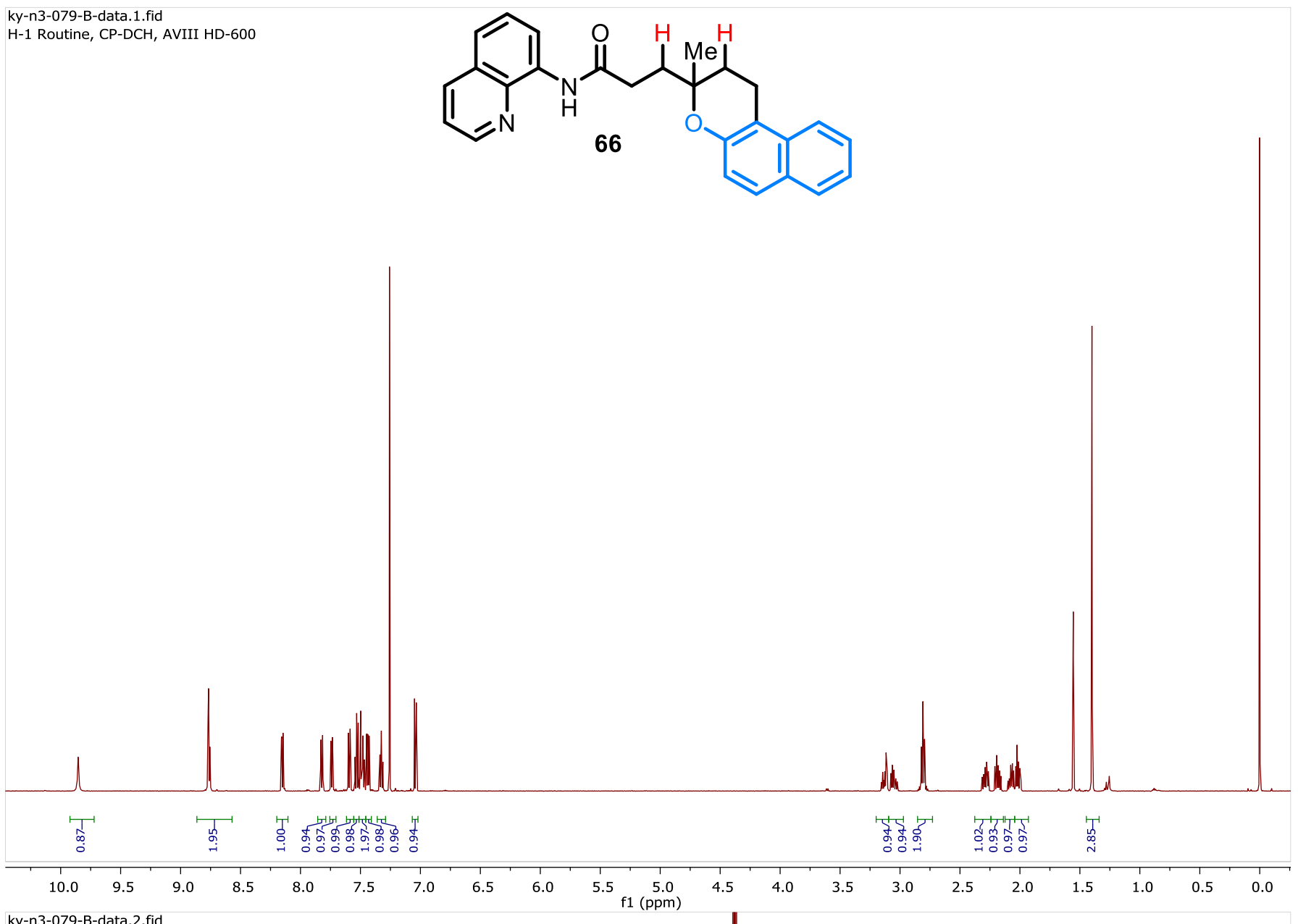

ky-n3-079-B-data.2.fid

H-1 Routine, CP-DCH, AVIII HD-600
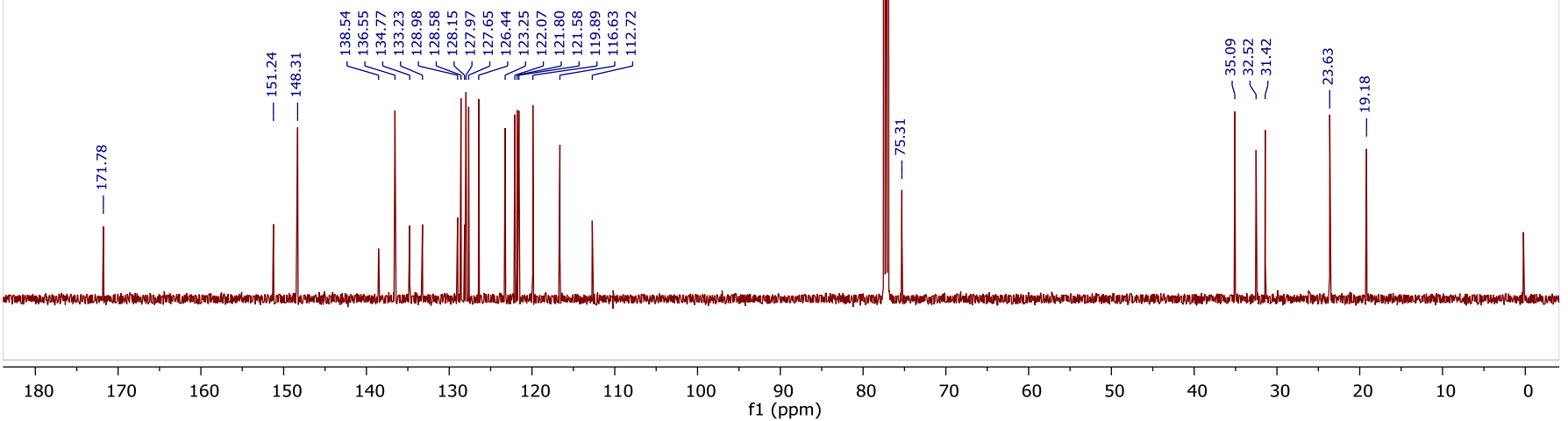
ky-n3-083-C-data.1.fid

H-1 Routine, CP-DCH, AVIII HD-600
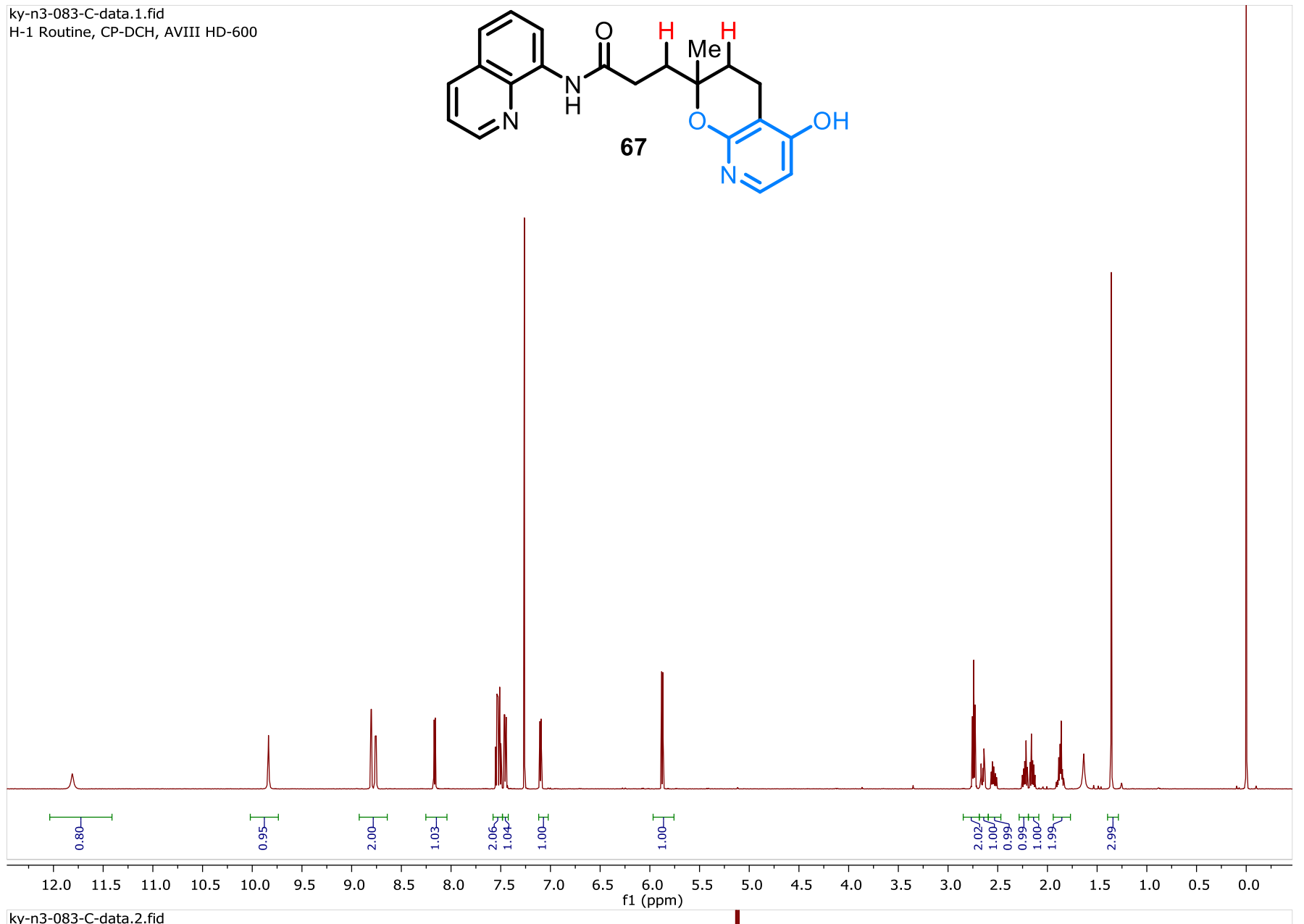

ky-n3-083-C-data.2.fid

H-1 Routine, CP-DCH, AVIII HD-600
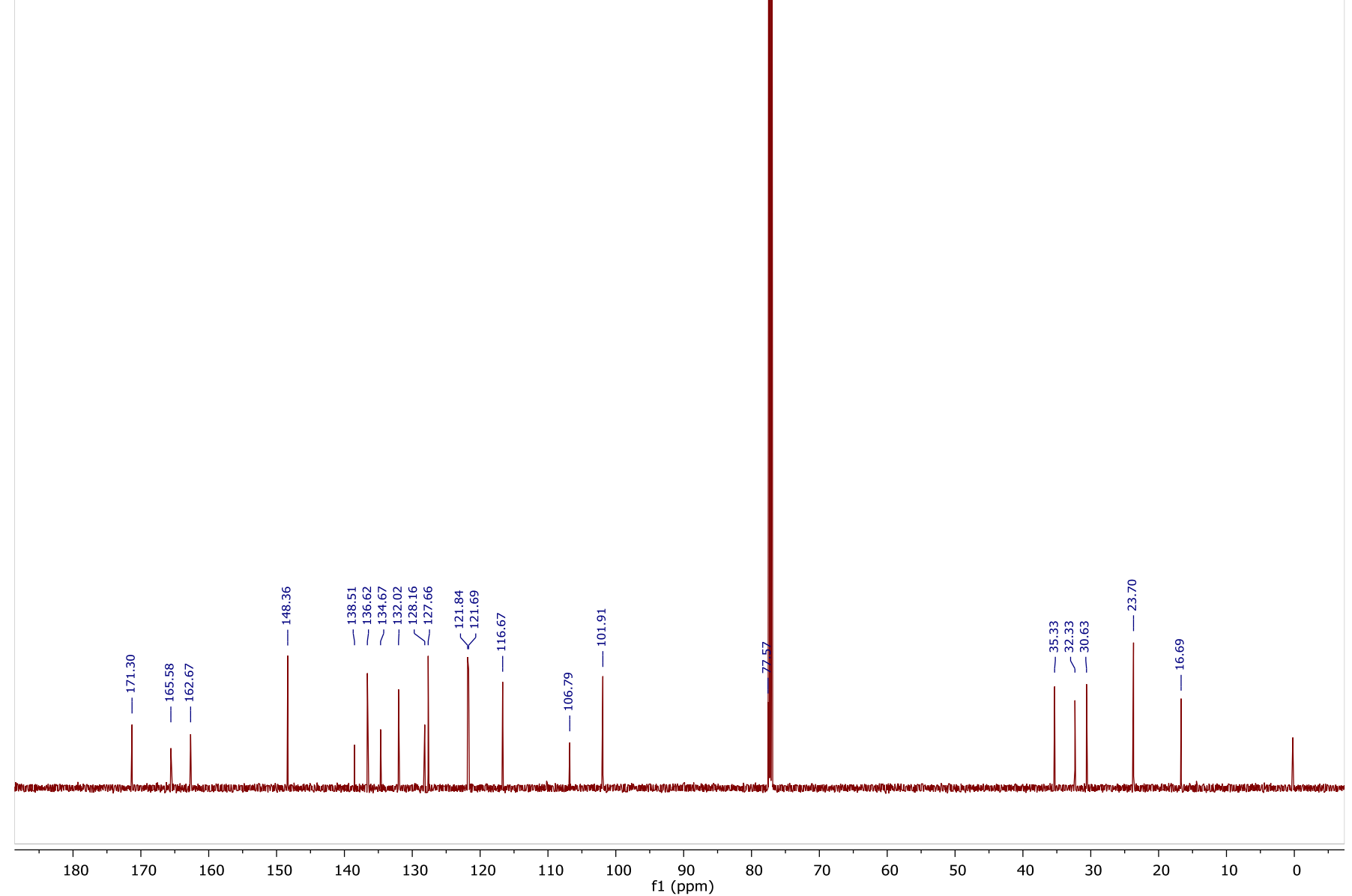


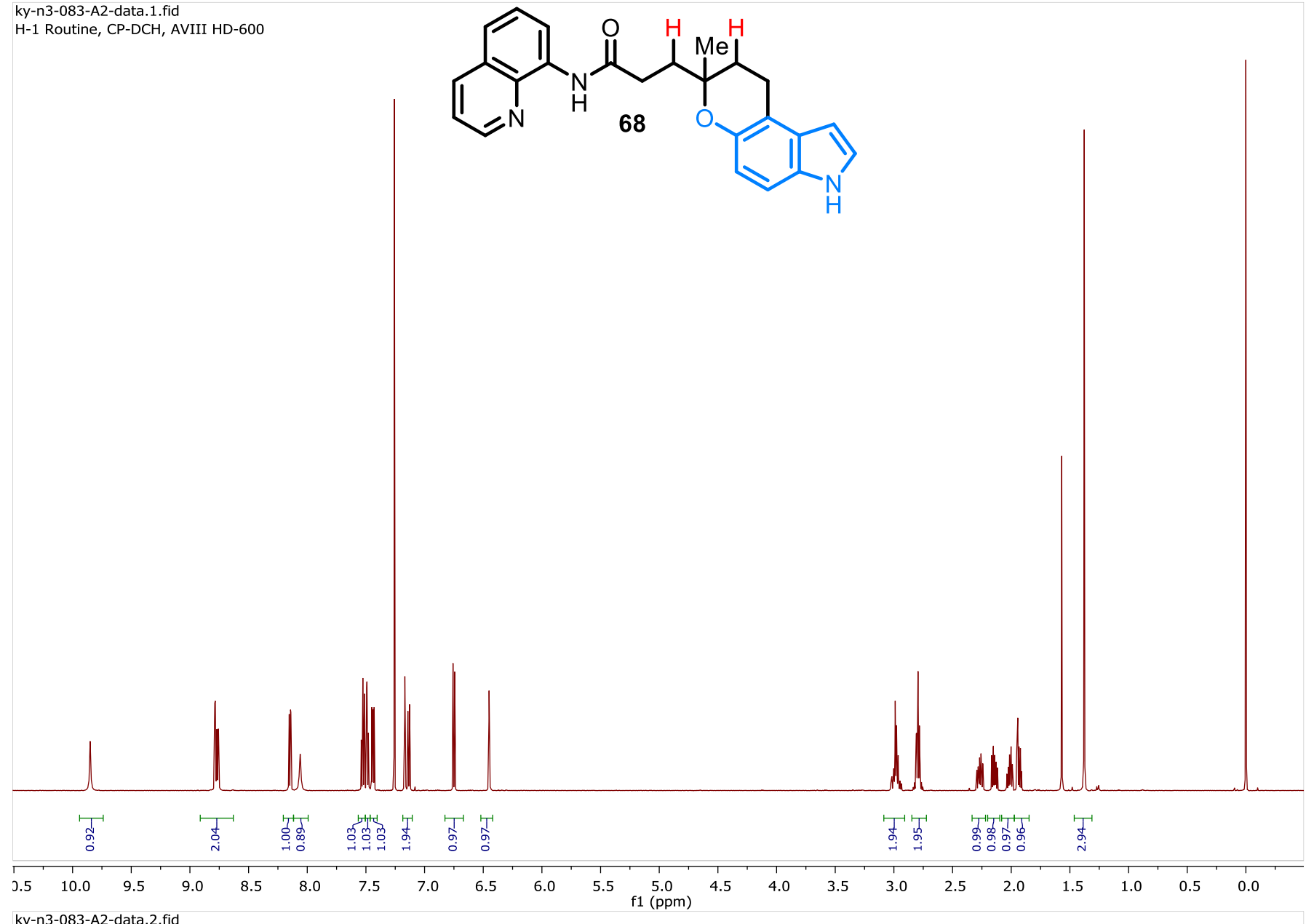

ky-n3-083-A2-data.2.fid

$\mathrm{H}-1$ Routine, CP-DCH, AVIII HD-600

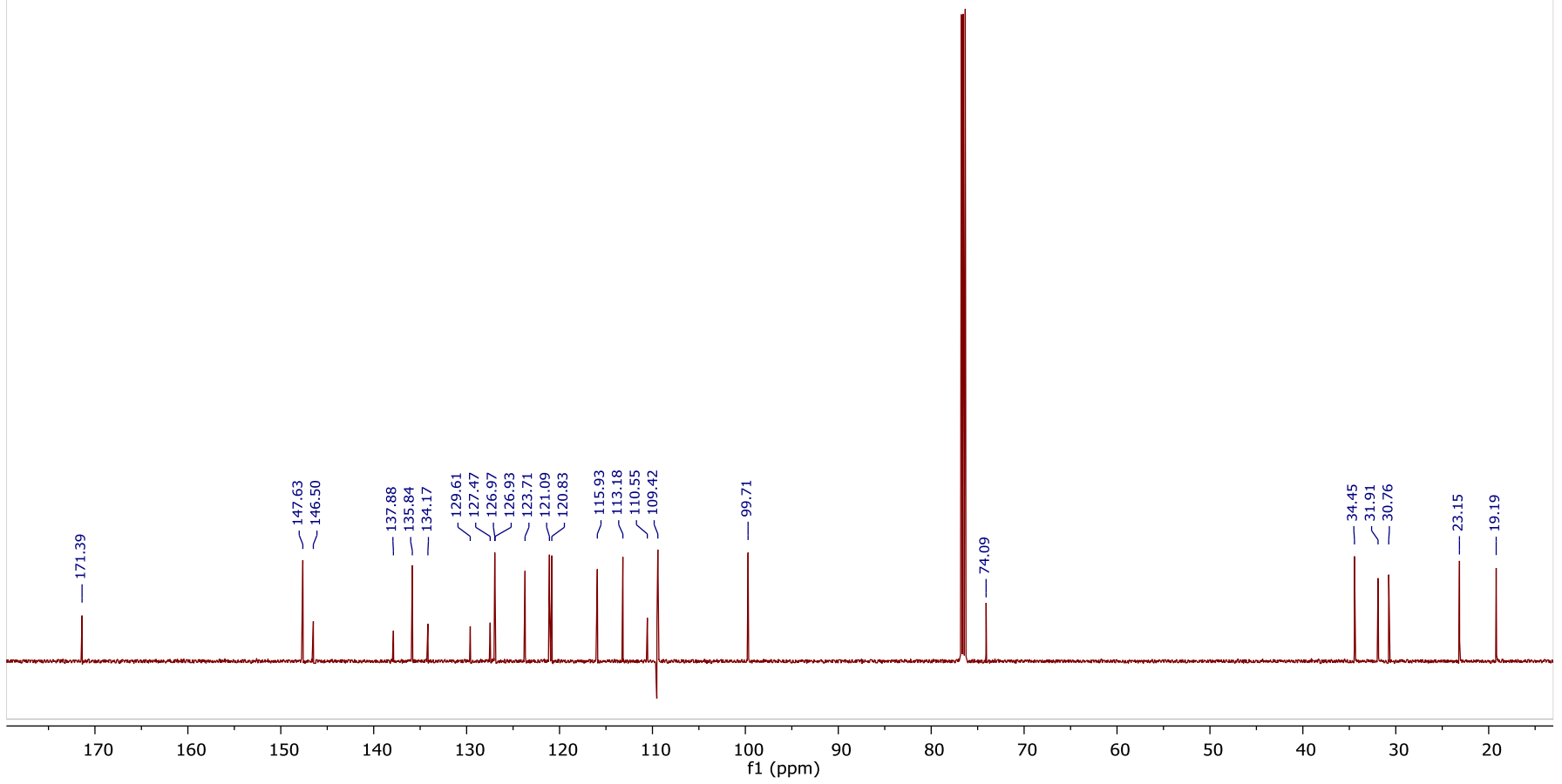




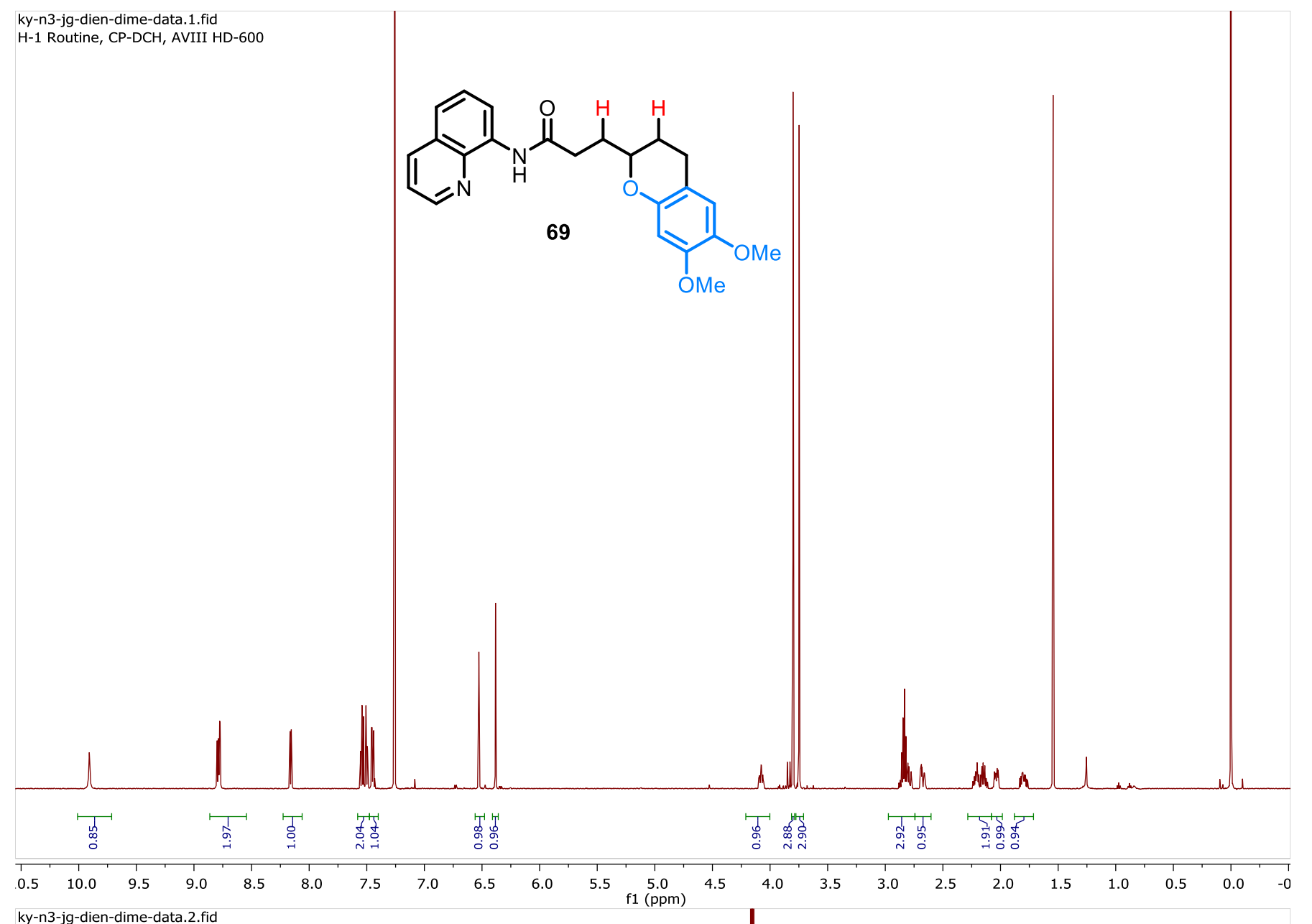

ky-n3-jg-dien-dime-data.2.fid H-1 Routine, CP-DCH, AVIII HD-600
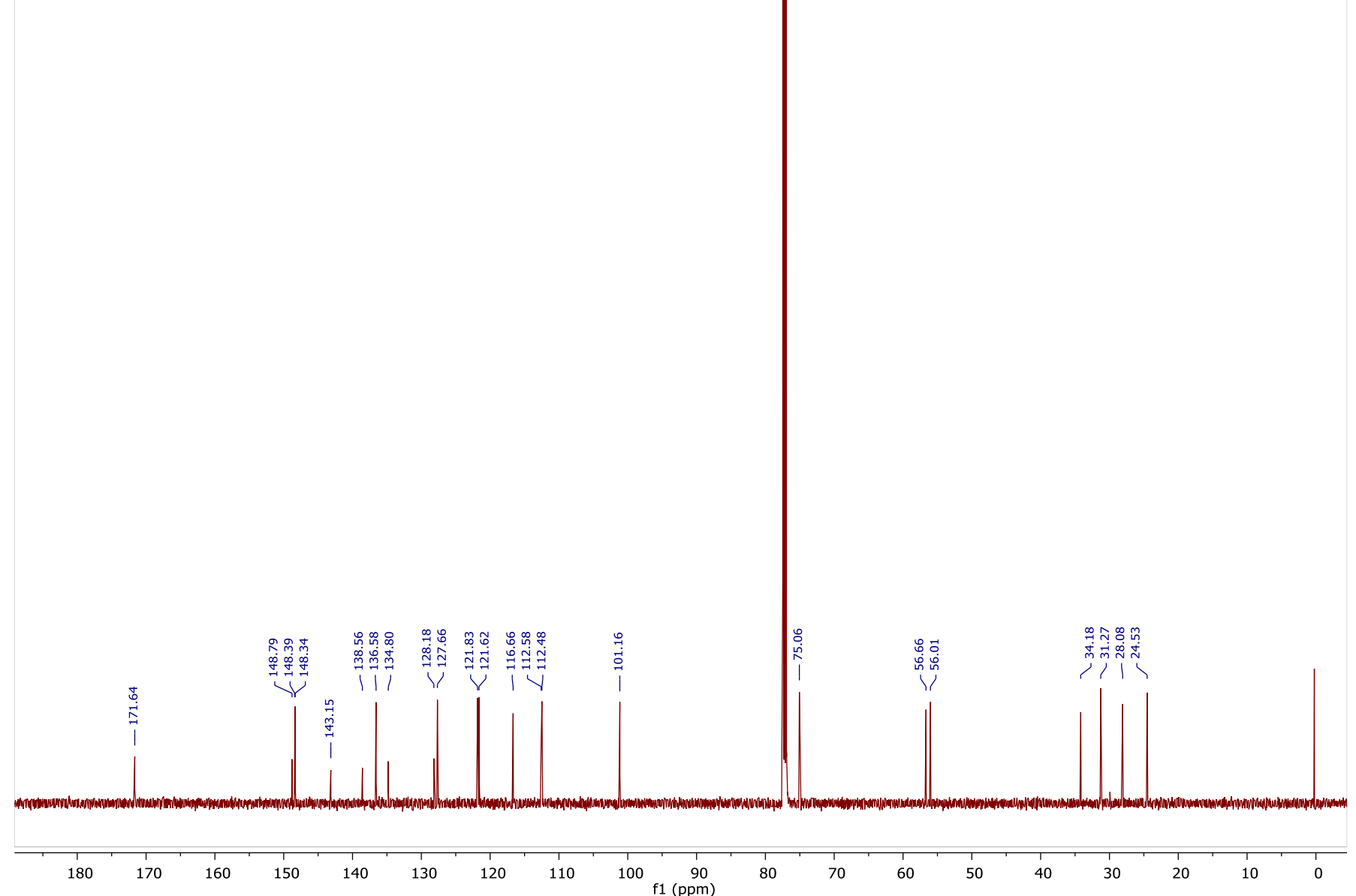
ky-n3-067-data.1.fid

$\mathrm{H}-1$ Routine, CP-DCH, AVIII HD-600
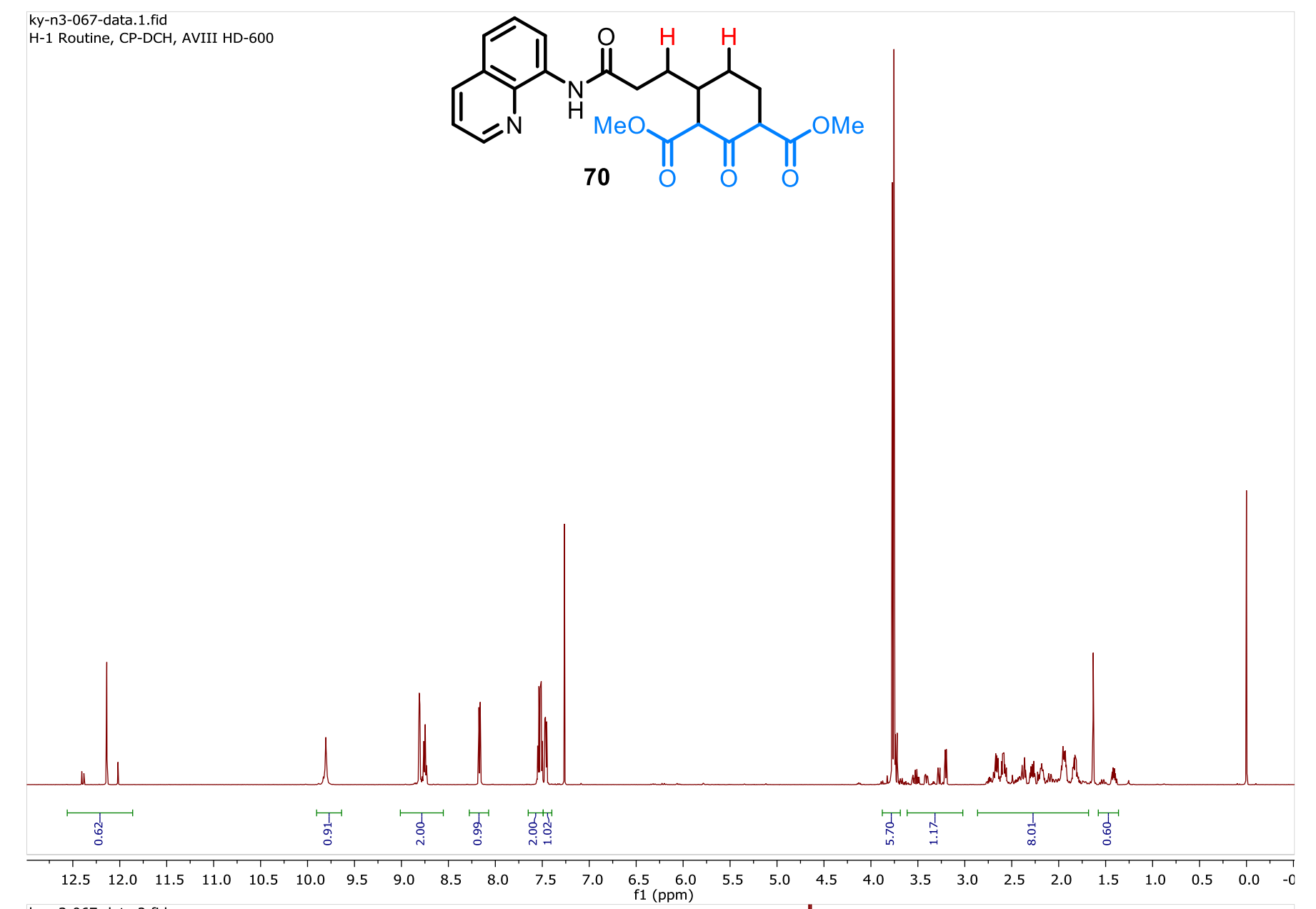

(n)

ky-n3-067-data.2.fid

H-1 Routine, CP-DCH, AVIII HD-600
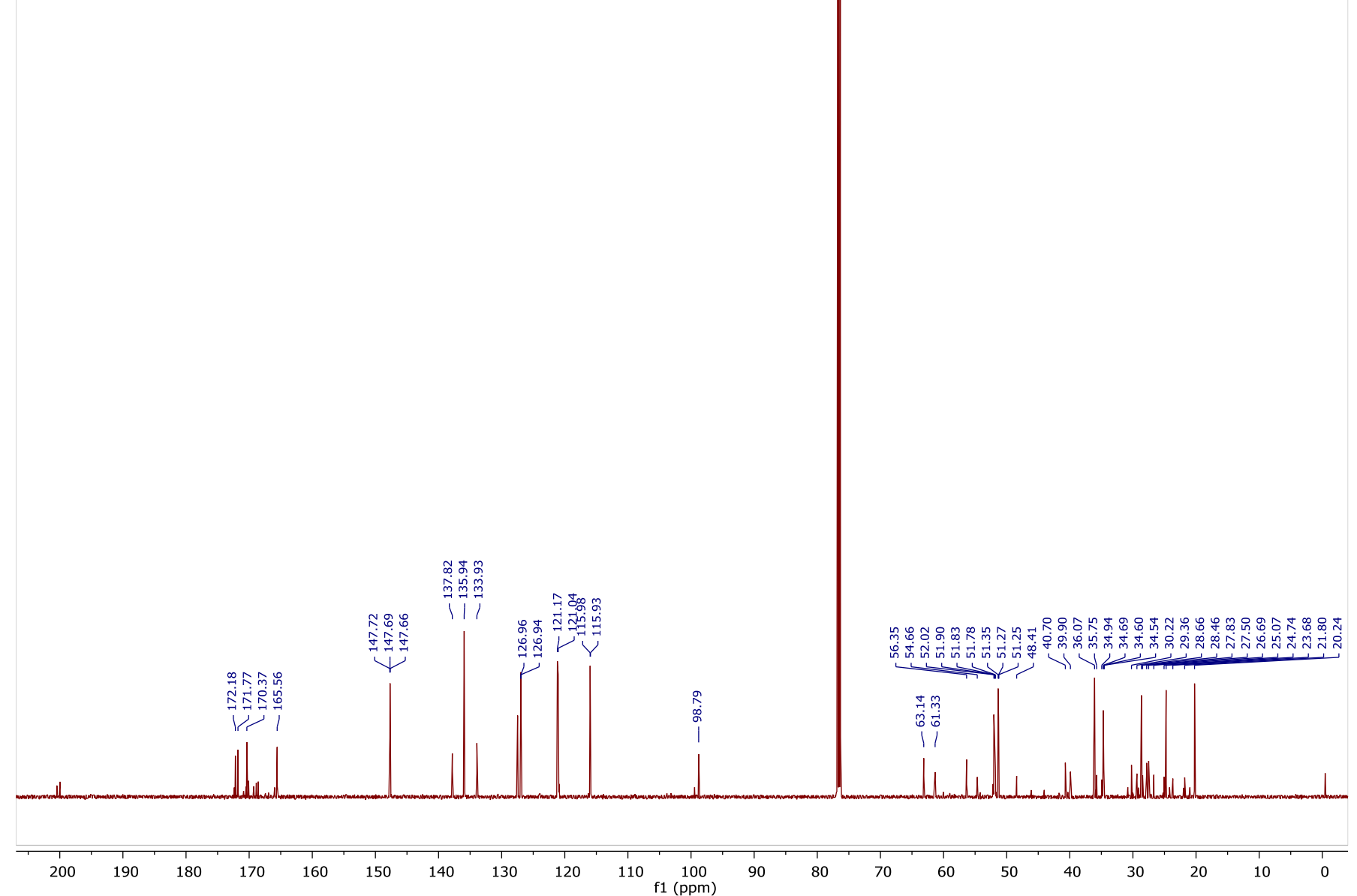

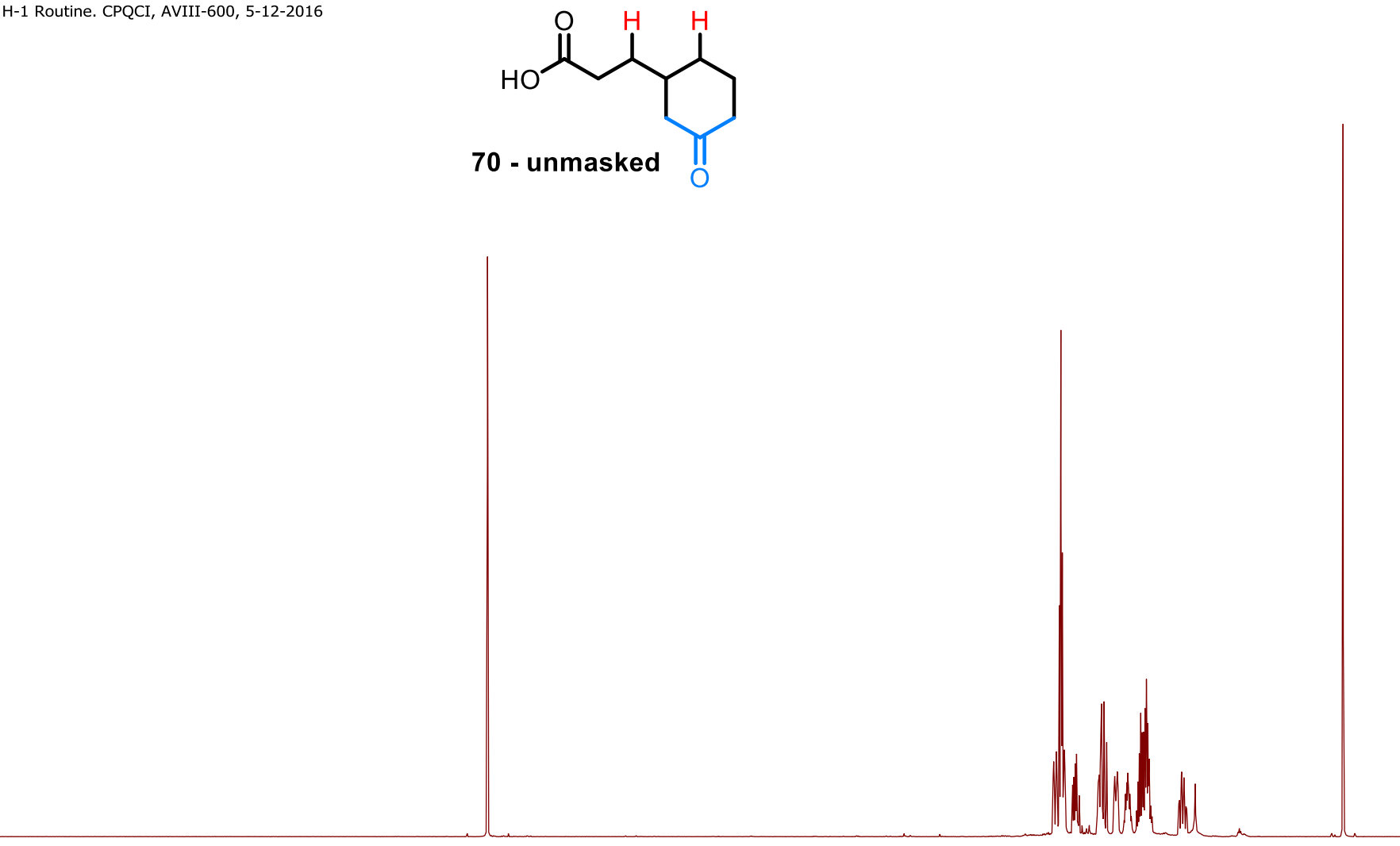

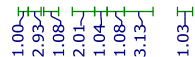

\begin{tabular}{|c|c|c|c|c|c|c|c|c|c|c|c|c|c|c|c|c|c|c|c|c|c|c|c|c|c|c|c|c|}
\hline 11.0 & 10.5 & 10. & & 9.5 & 9.0 & 8.5 & 8.0 & 7.5 & 7.0 & 6.5 & 6.0 & $\begin{array}{c}5.5 \\
\mathrm{f} 1(\mathrm{ppm})\end{array}$ & )$^{5.0}$ & 4. & 5 & 4.0 & 3.5 & & .0 & 2.5 & & 2.0 & 1.5 & 1.0 & & 0.5 & 0.0 & -0 \\
\hline
\end{tabular}

ky-n3-067-acid-decarb-cr.1.fid

H-1 Routine. CPQCI, AVIII-600, 5-12-2016

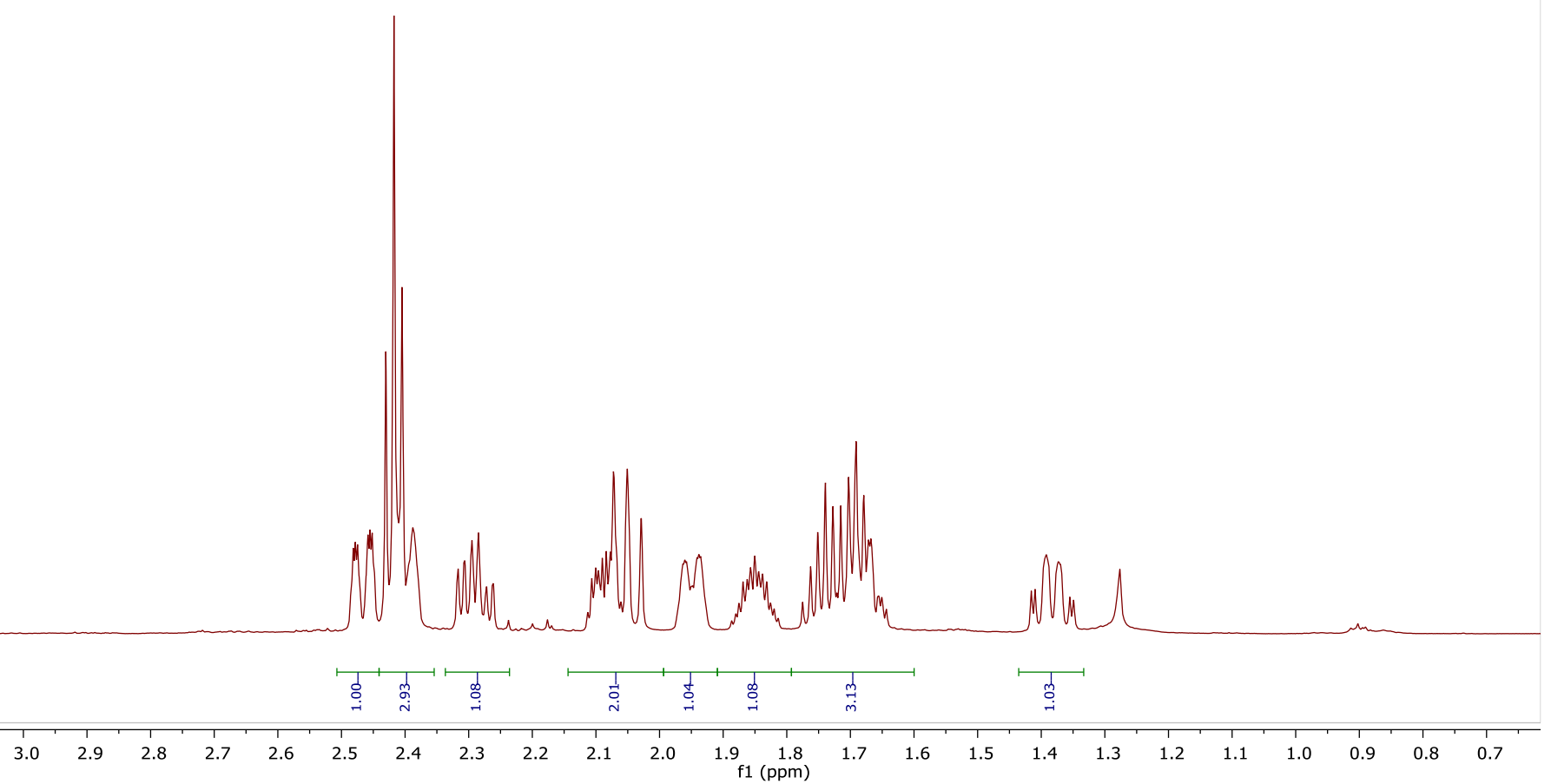


ky-n3-011-B-data.1.fid

H-1 Routine, CP-DCH, AVIII HD-600

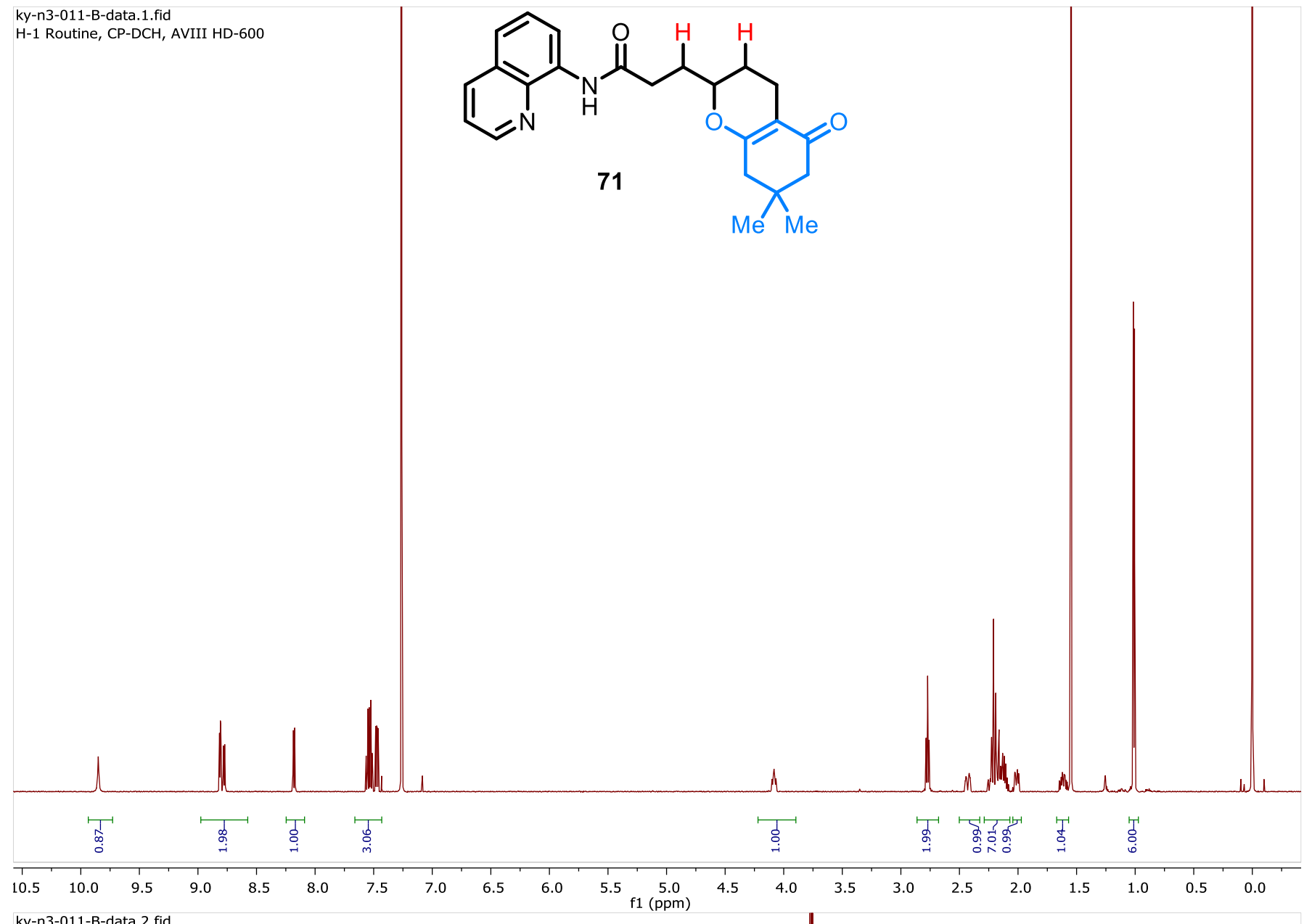

ky-n3-011-B-data.2.fid

H-1 Routine, CP-DCH, AVIII HD-600
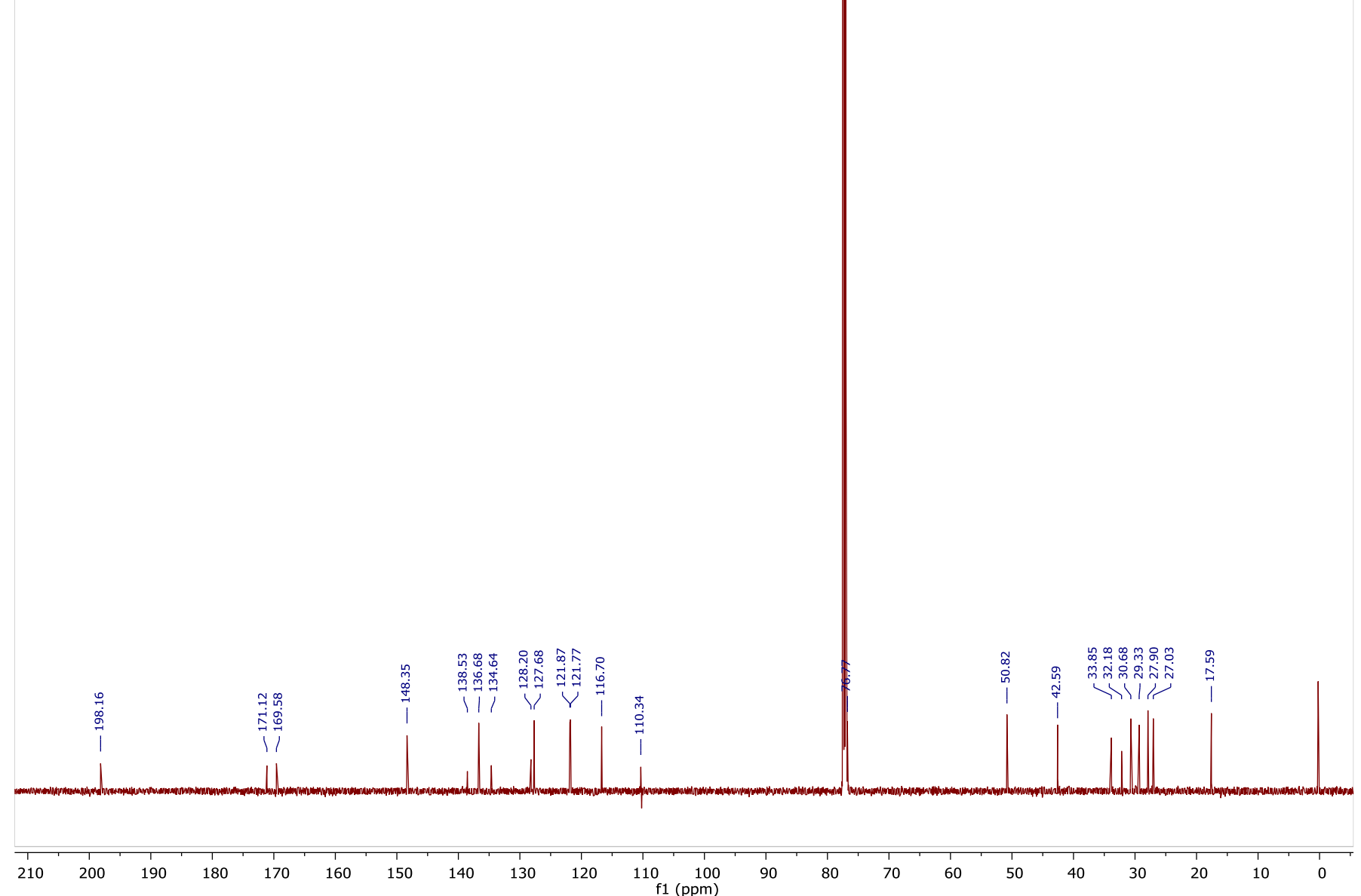


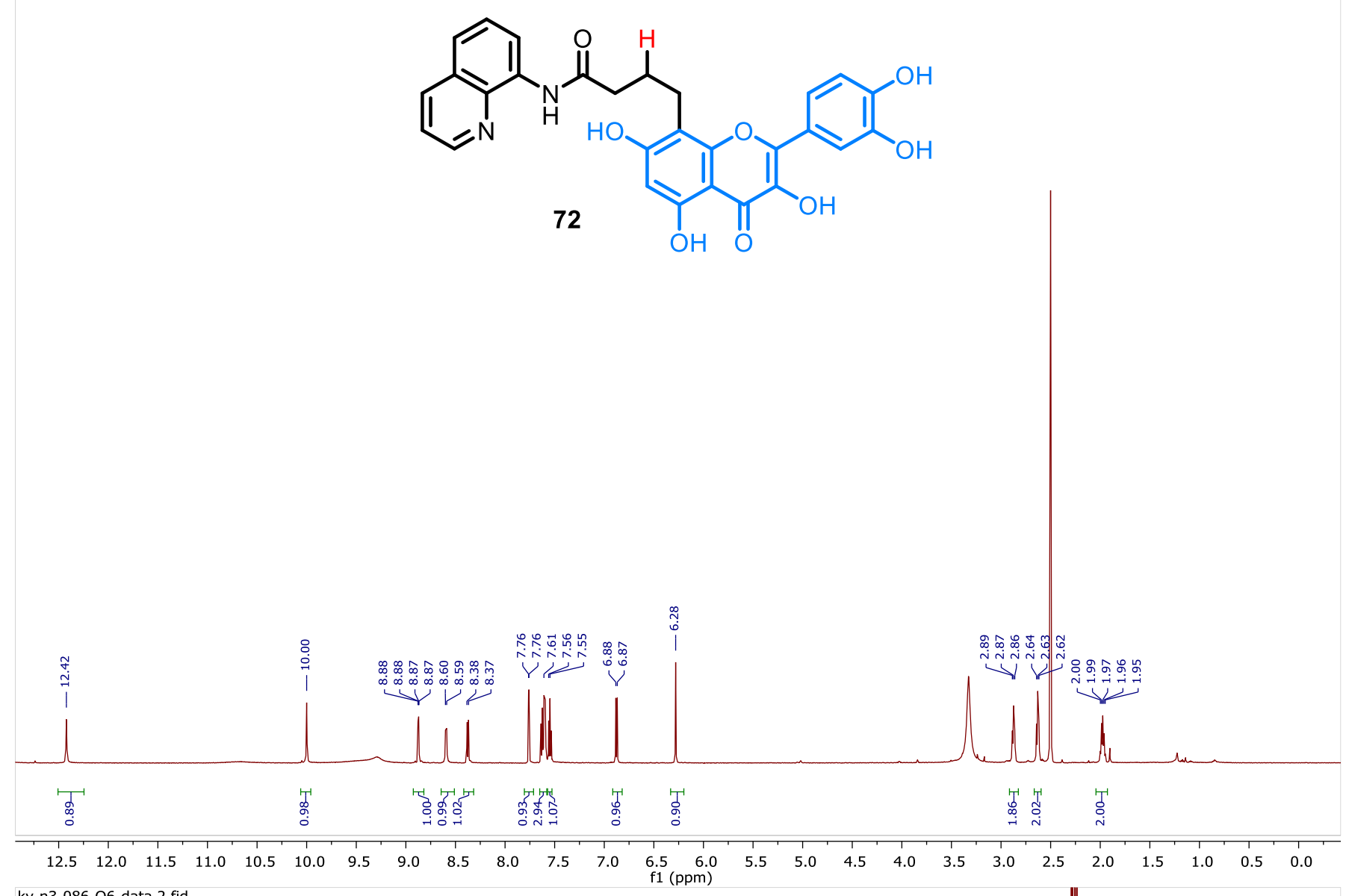
ky-n3-086-Q6-data.2.fid H-1 Routine. CPQCI, AVIII-600, 5-12-2016
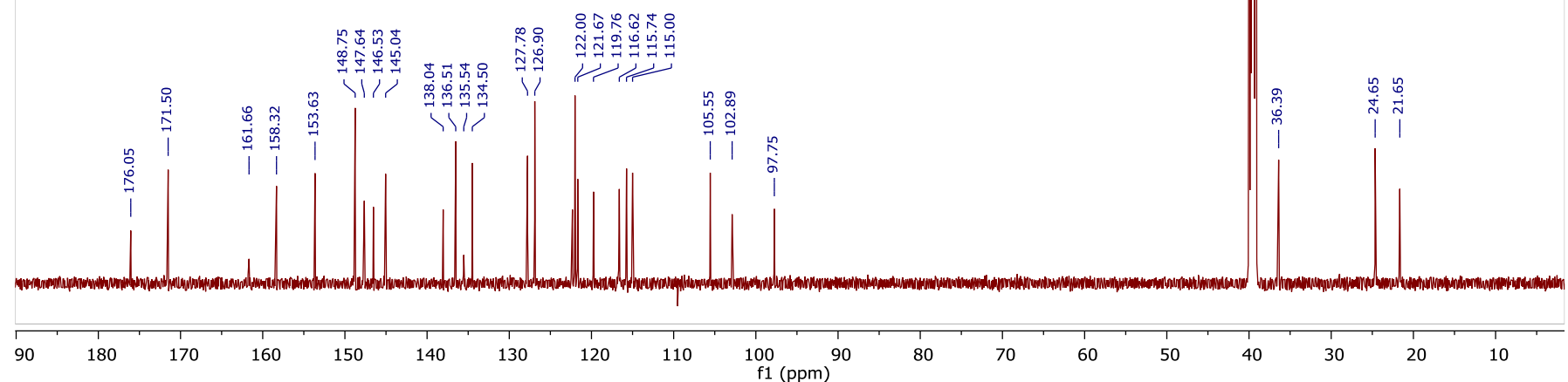

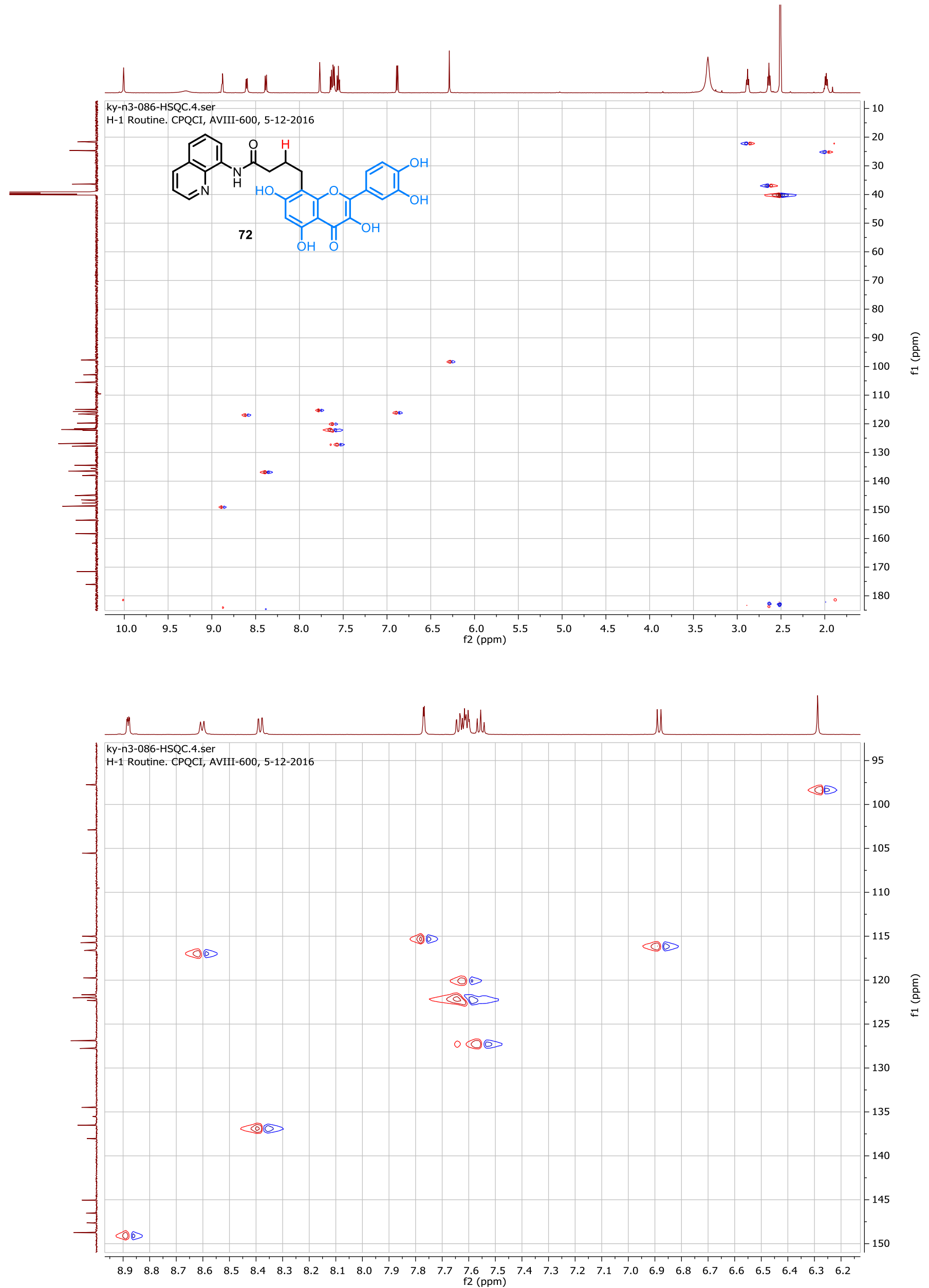

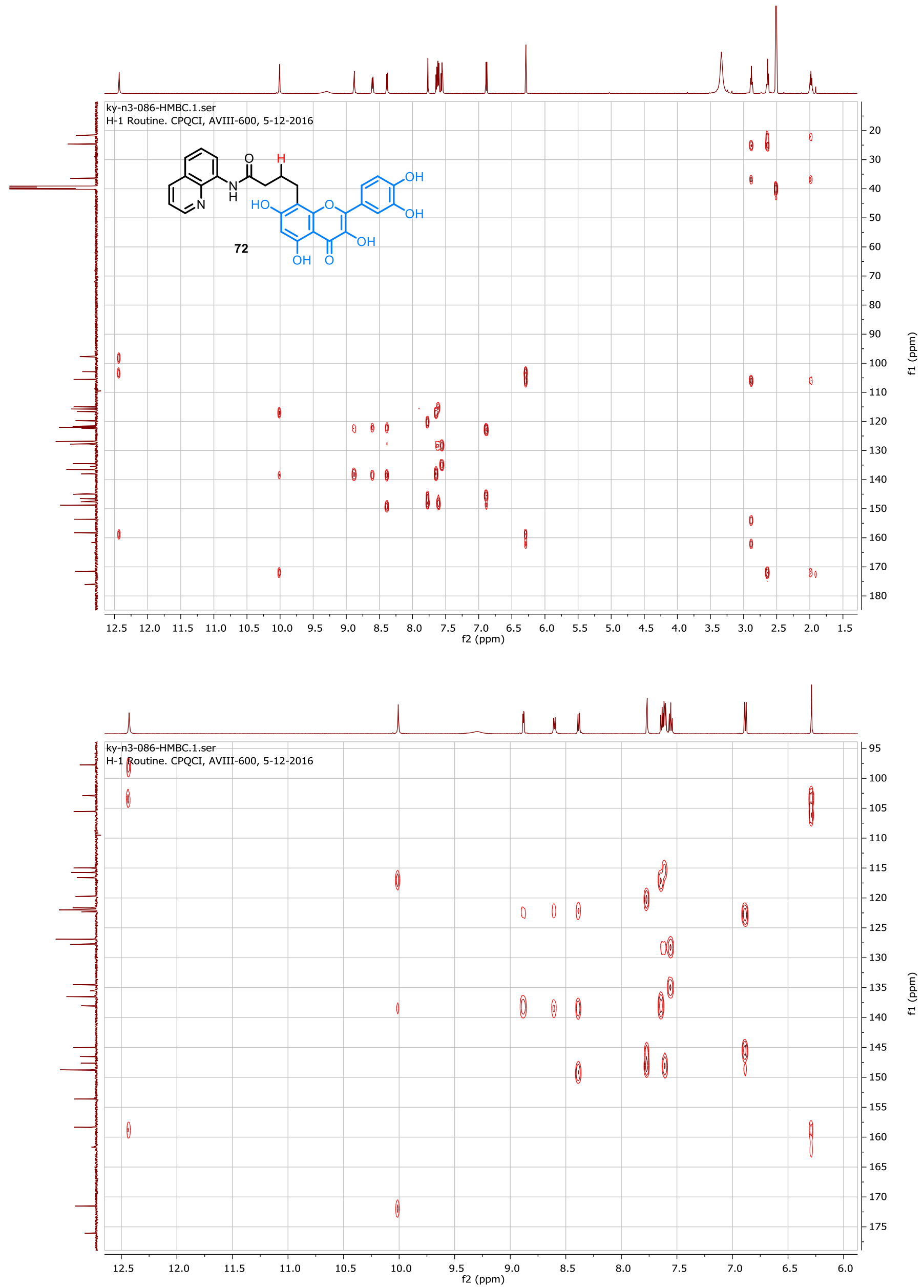
ky-n3-088-PA-data.1.fid

H-1 Routine, CP-DCH, AVIII HD-600<smiles>CC(=O)OCCN(c1ccc(C)cc1)c1ccc(CC(C)CC(=O)Nc2cccc3cccnc23)c(O)c1</smiles>

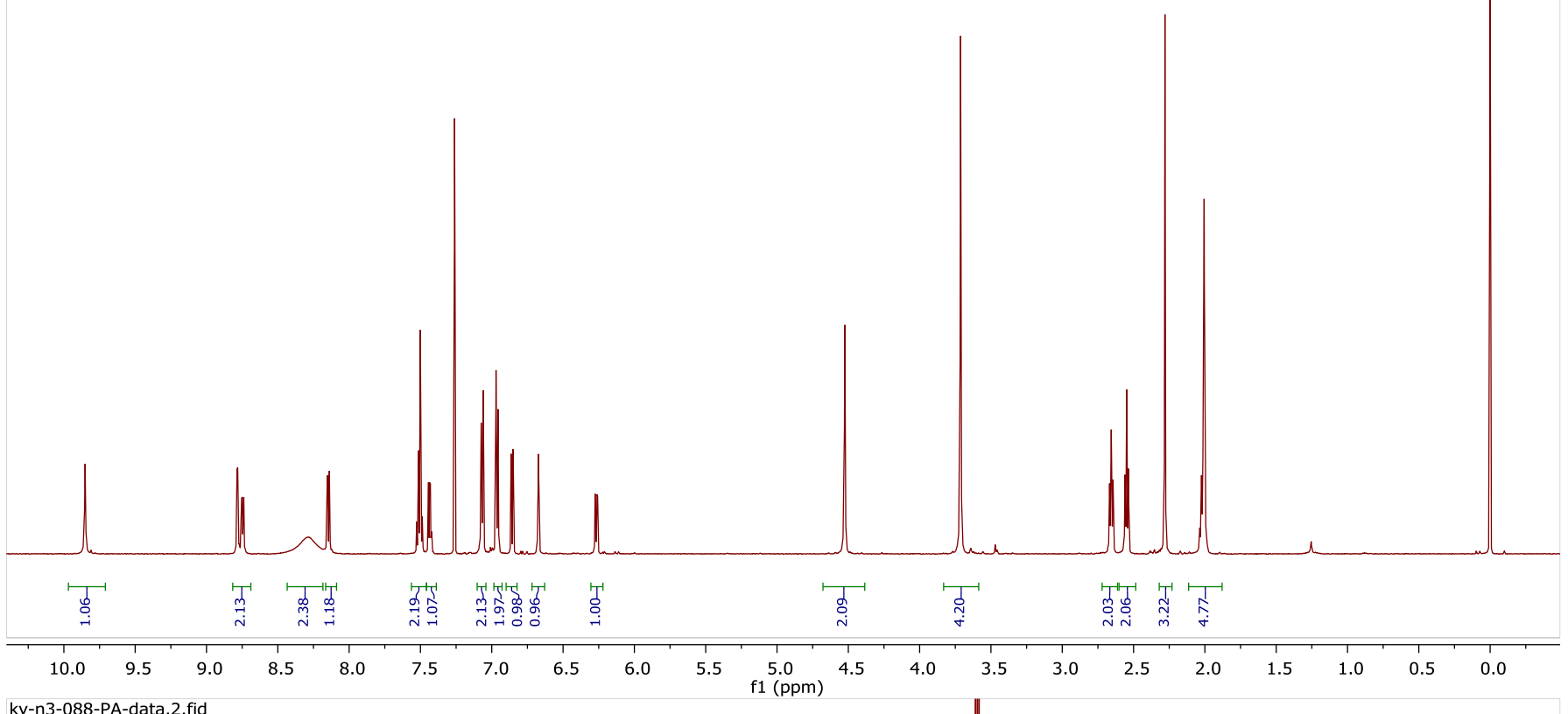

ky-n3-088-PA-data.2.fid

H-1 Routine, CP-DCH, AVIII HD-600
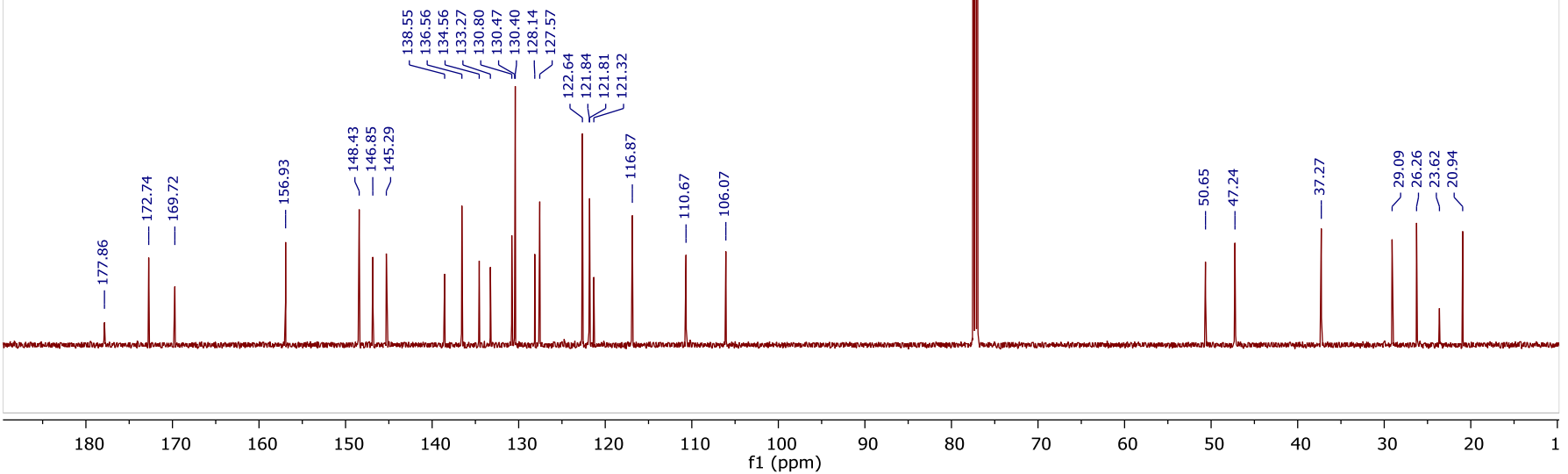
ky-n3-096-data.1.fid

$\mathrm{H}-1$ Routine. CPQCI, AVIII-600, 5-12-2016<smiles>[Y4]CC(CCCC)C(C)C(C)C(=O)Nc1cccc2cccnc12</smiles>

74

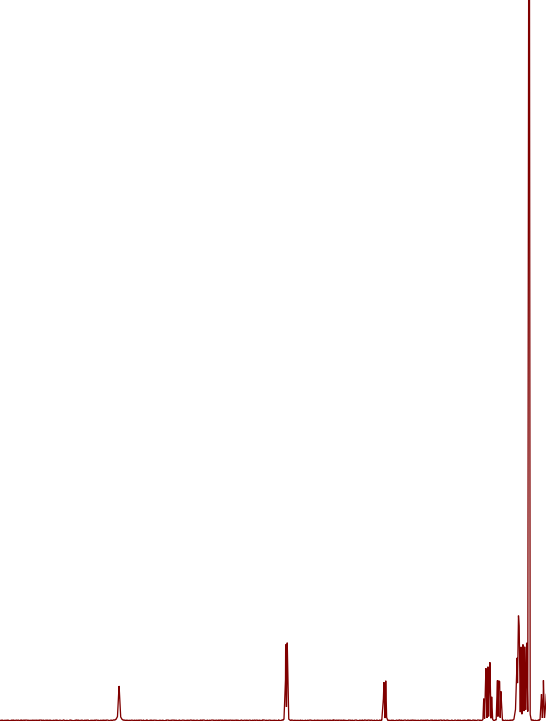

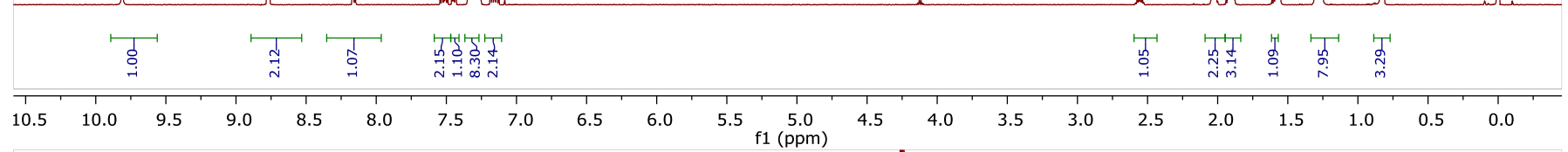

ky-n3-096-data.2.fid

H-1 Routine. CPQCI, AVIII-600, 5-12-2016
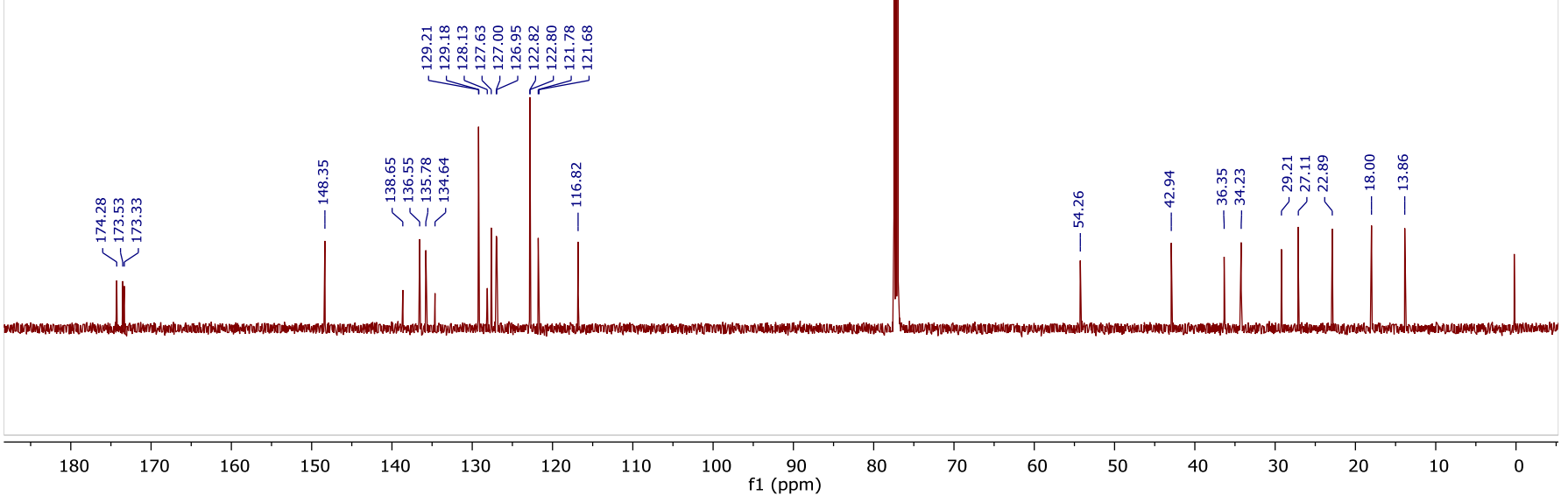


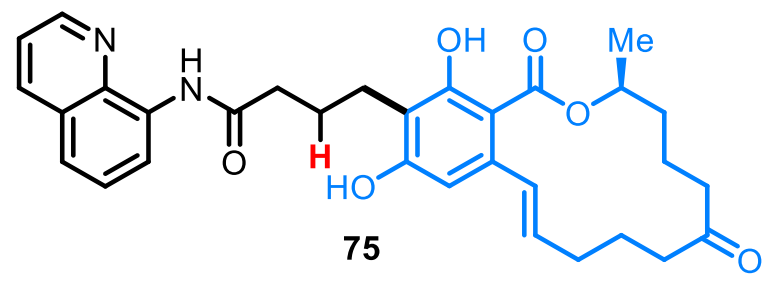

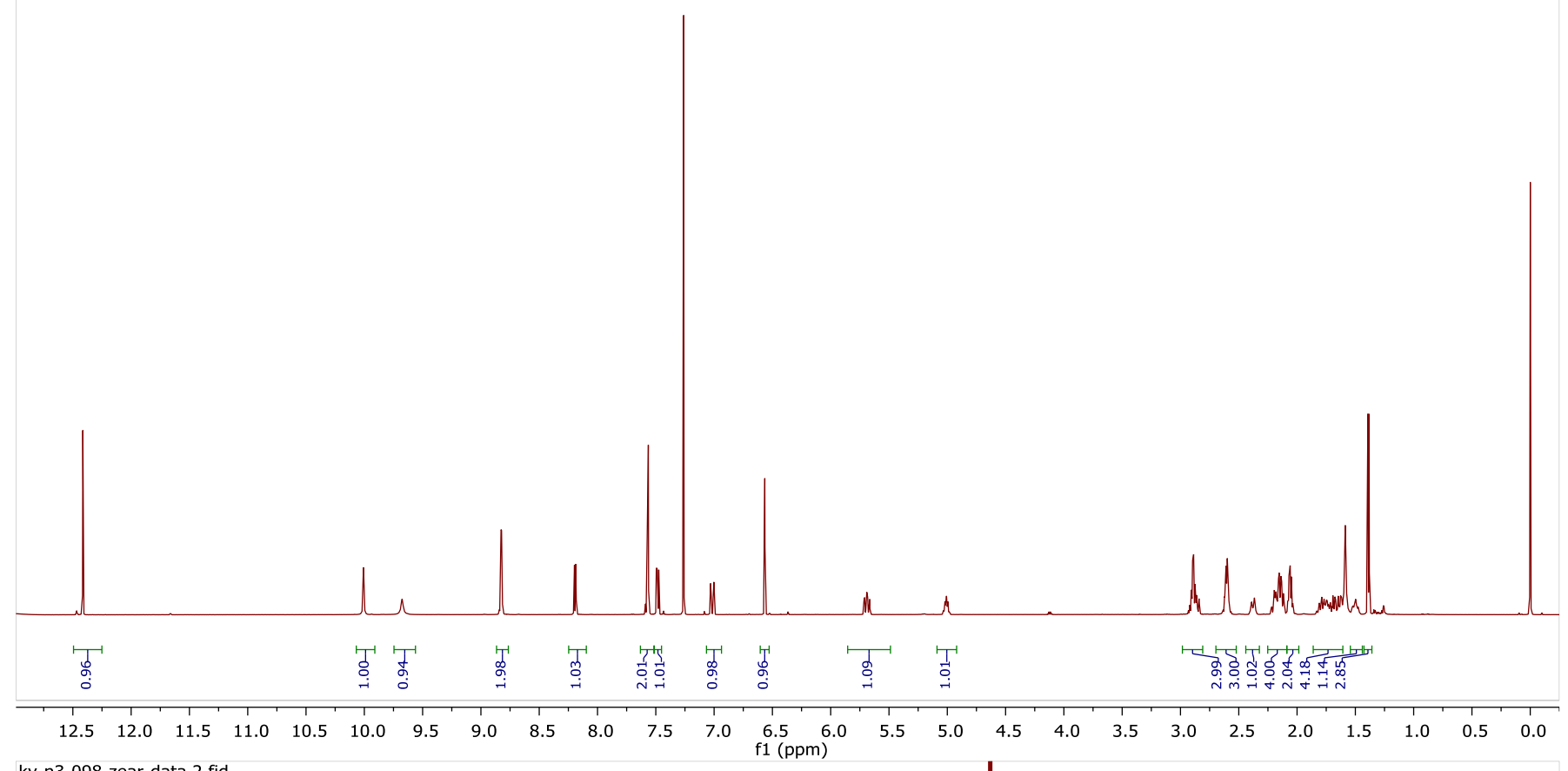
ky-n3-098-zear-data.2.fid H-1 Routine. CPQCI, AVIII-600, 5-12-2016
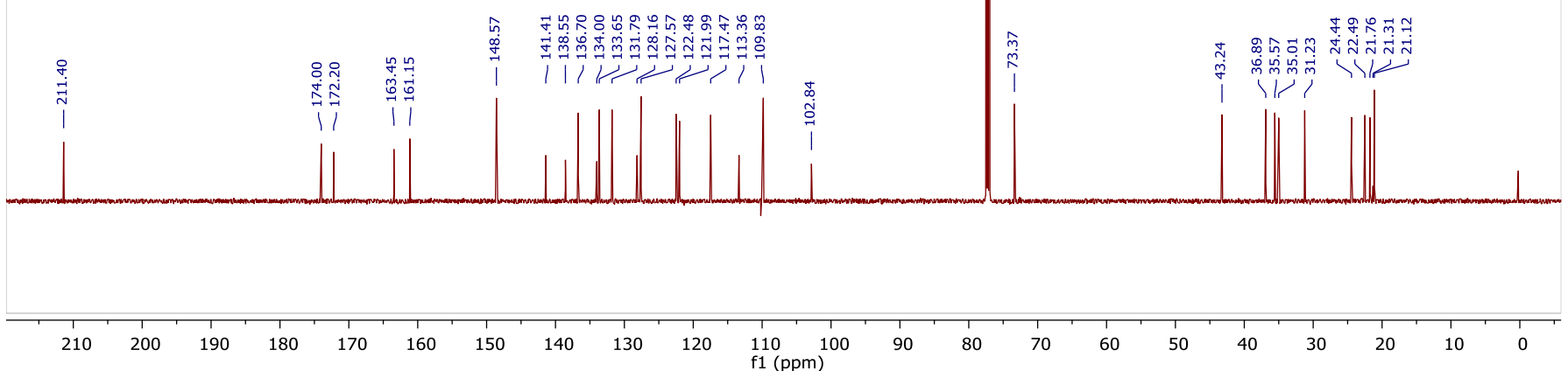


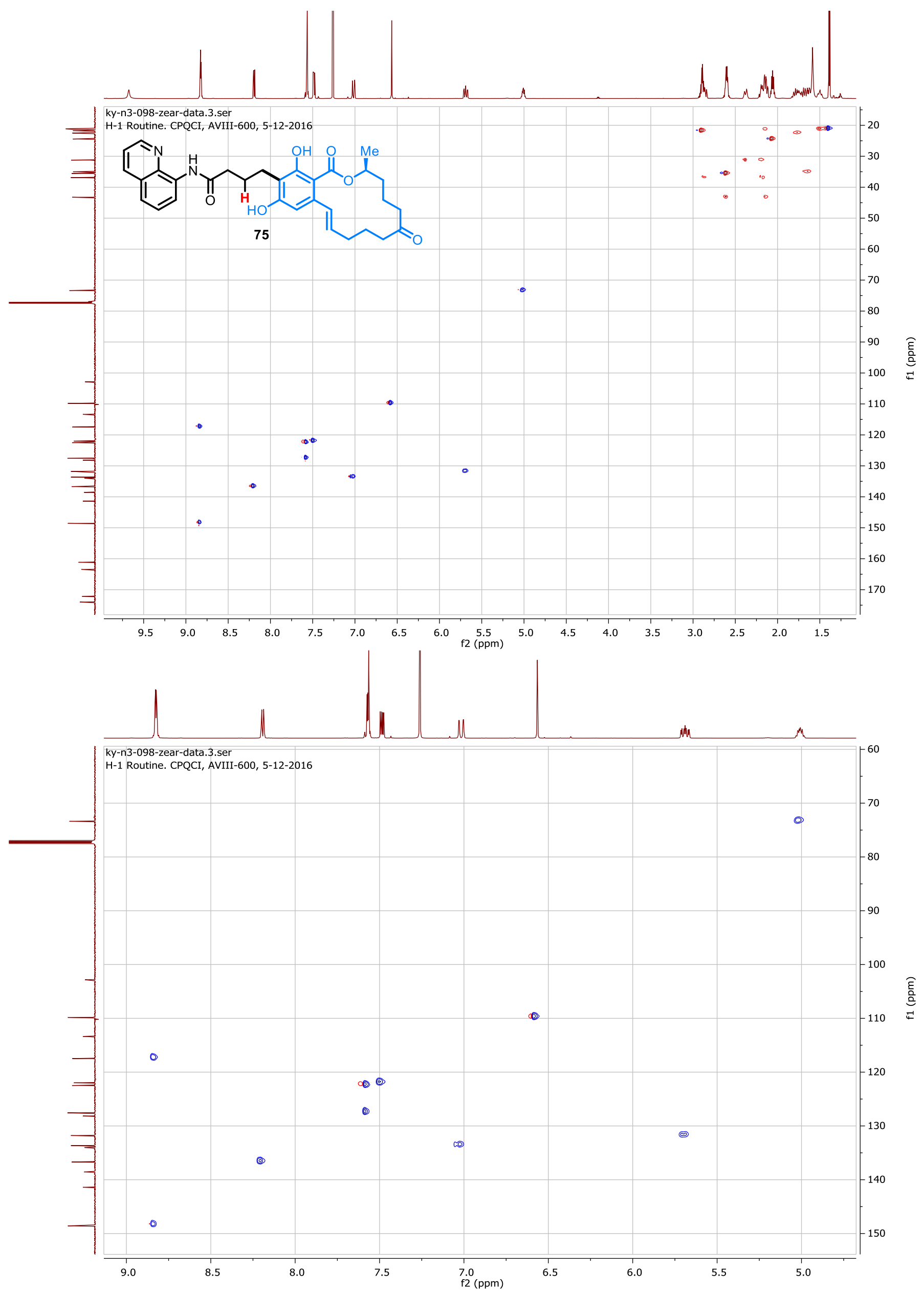




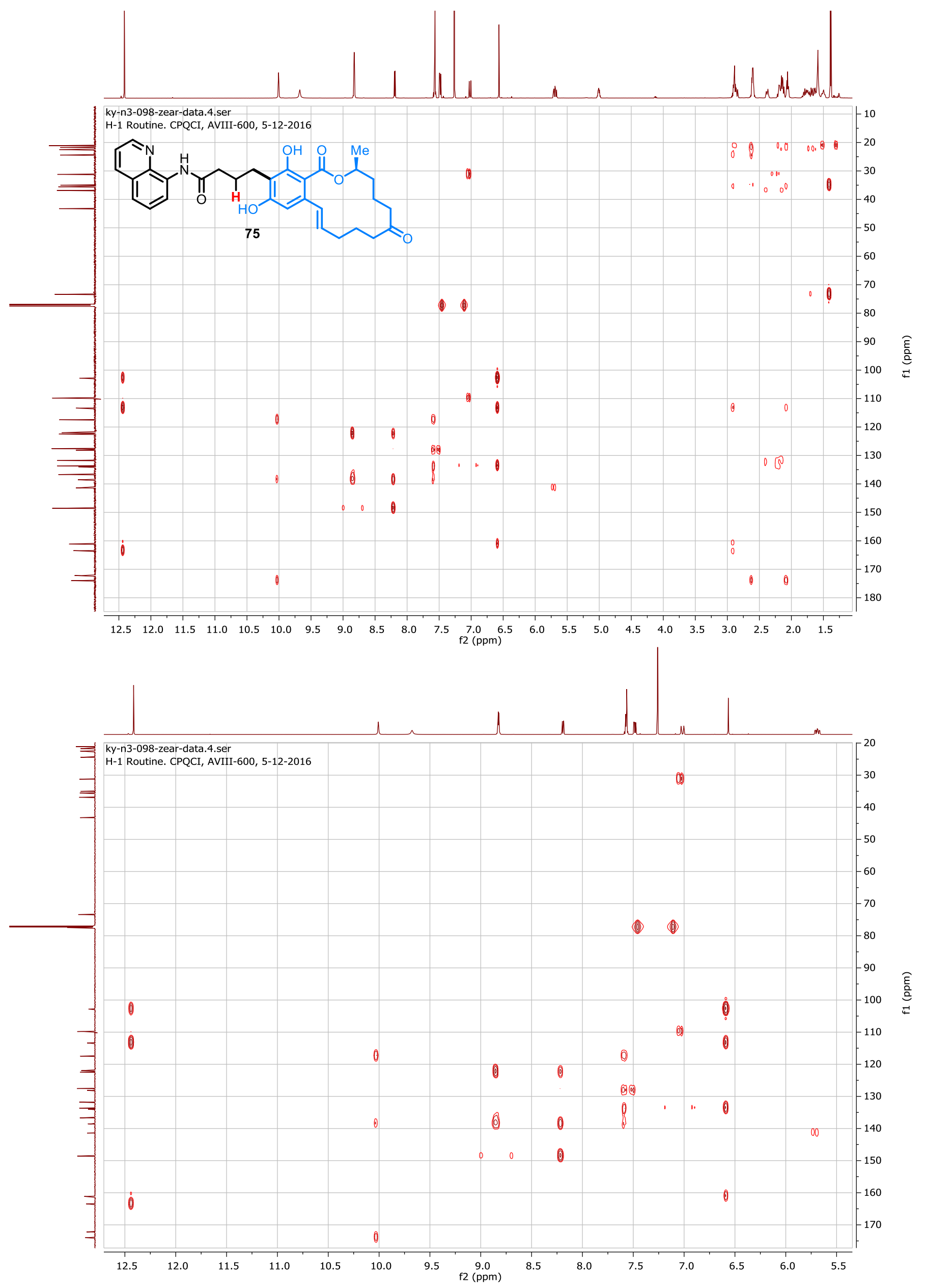


ky-n3-92-data.1.fid

H-1 Routine. CPQCI, AVIII-600, 5-12-2016<smiles>Cc1c(O)c(CC(C)C(C)C(=O)O)c(O)c2c1COC2=O</smiles>

(士)-Euparvic acid

78

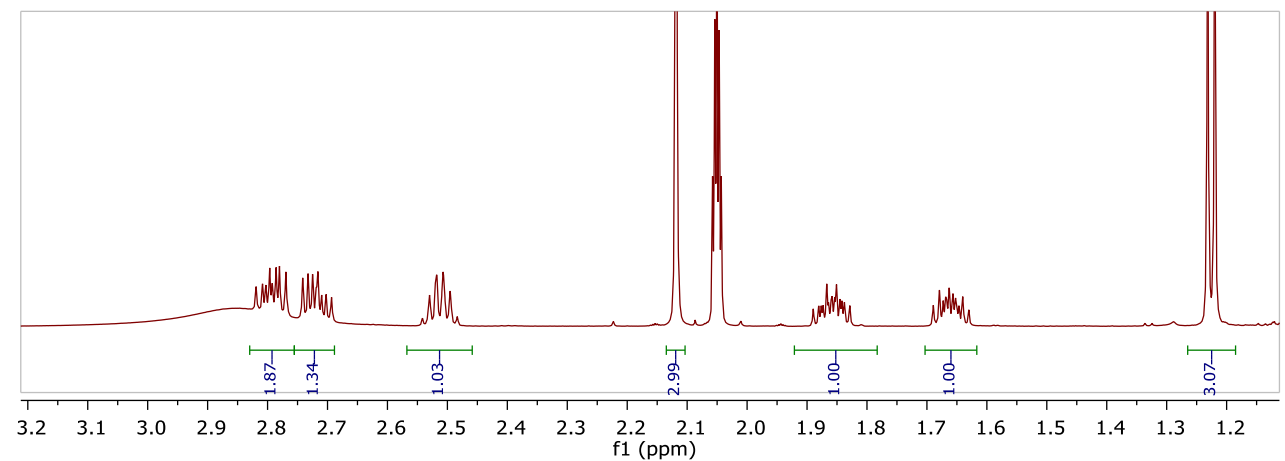

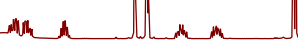

\begin{tabular}{|c|c|c|c|c|c|c|c|c|c|c|c|c|c|c|c|c|c|c|c|c|}
\hline & & & & & & & & & & & & & & & 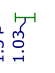 & $\begin{array}{l}\stackrel{4}{\alpha} \\
\stackrel{N}{N}\end{array}$ & 'す่' & & $\begin{array}{l}T \\
\\
\\
m\end{array}$ & \\
\hline 0.0 & 9.5 & 9.0 & 8.5 & 8.0 & 7.5 & 7.0 & 6.5 & 6.0 & 5.5 & f1 (ppm) & 4.5 & 4.0 & 3.5 & 3.0 & 2.5 & 2.0 & & 1.5 & 1.0 & 0.5 \\
\hline
\end{tabular}

ky-n3-p2-data.2.fid

H-1 Routine. CPQCI, AVIII-600, 5-12-2016
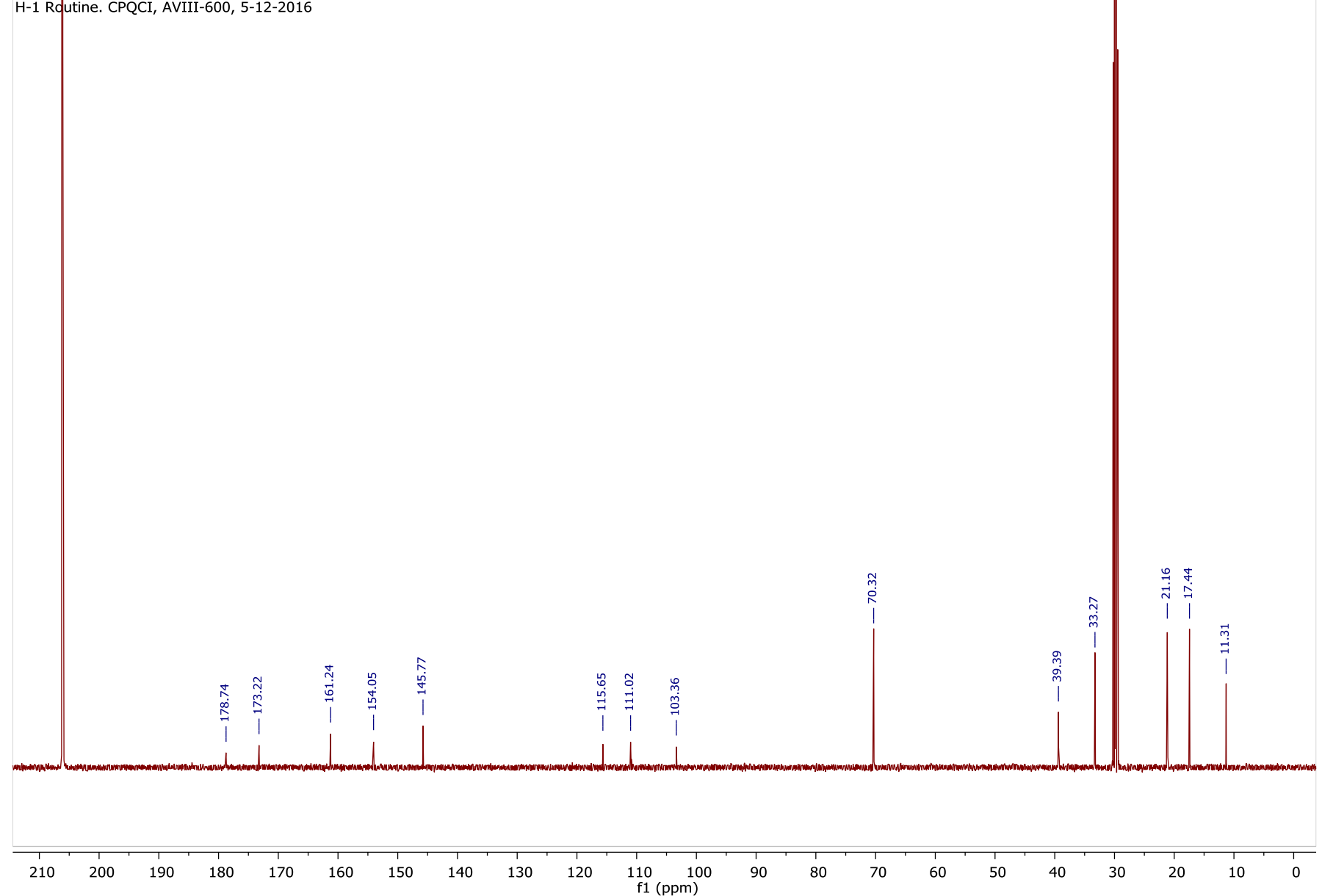
ky-n3-styrene-complex.1.fid

H-1 Routine. CPQCI, AVIII-600, 5-12-2016
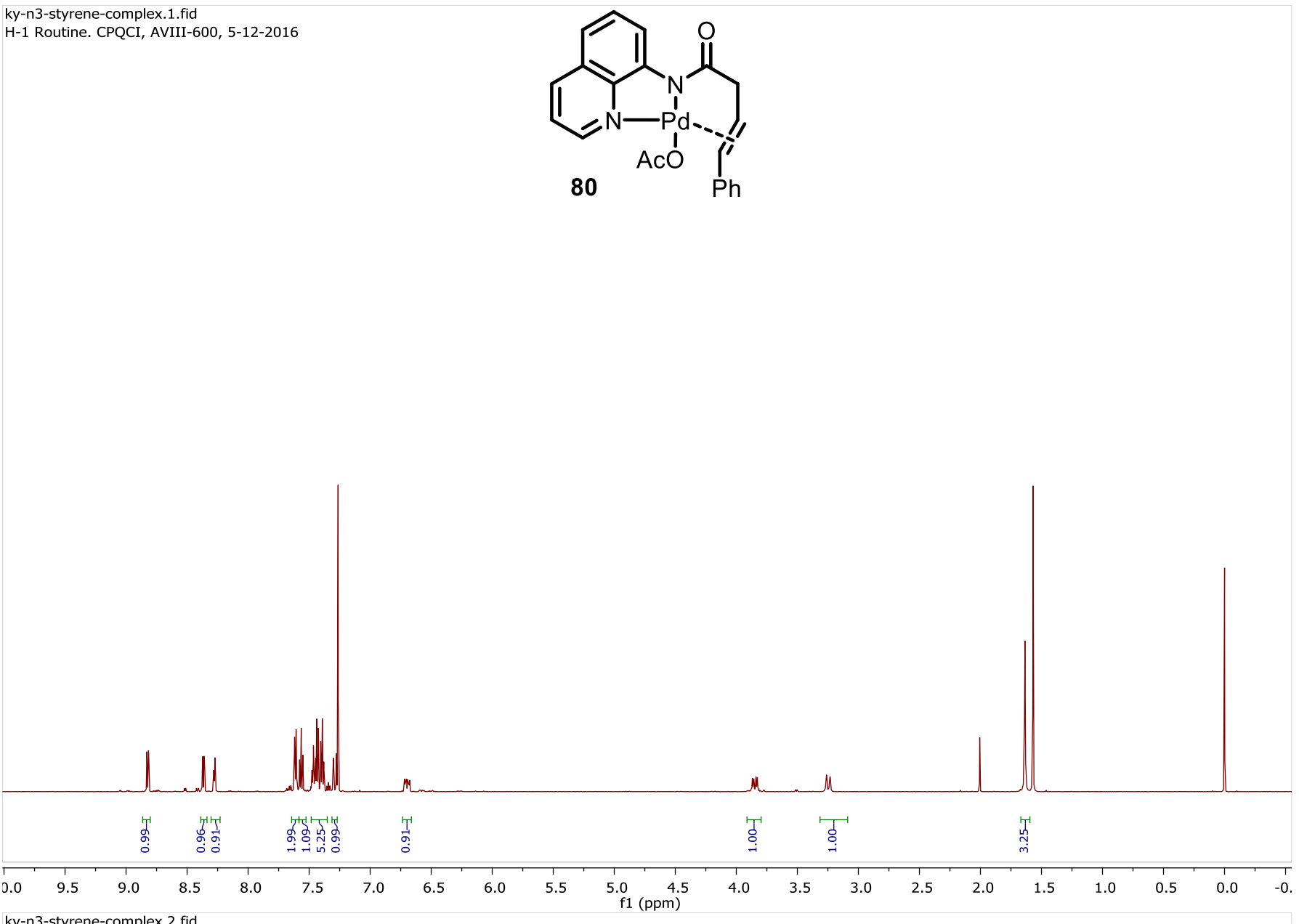

ky-n3-styrene-complex.2.fid

H-1 Routine. CPQCI, AVIII-600, 5-12-2016

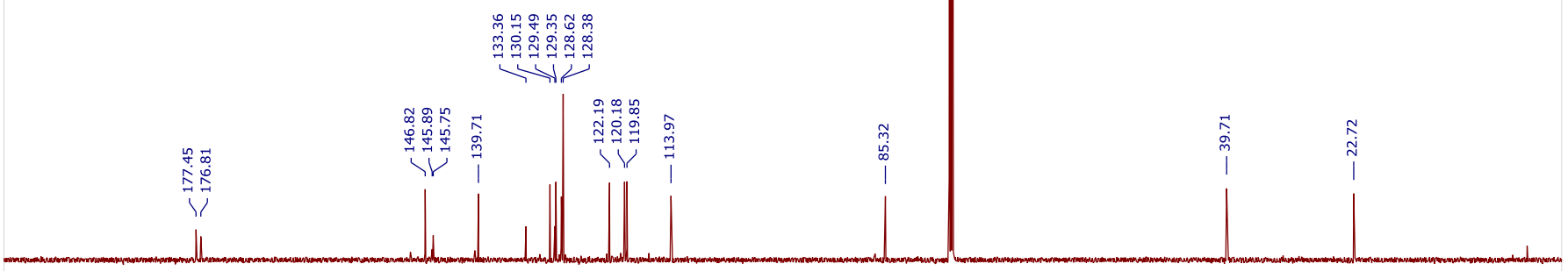


<smiles></smiles>

(1)

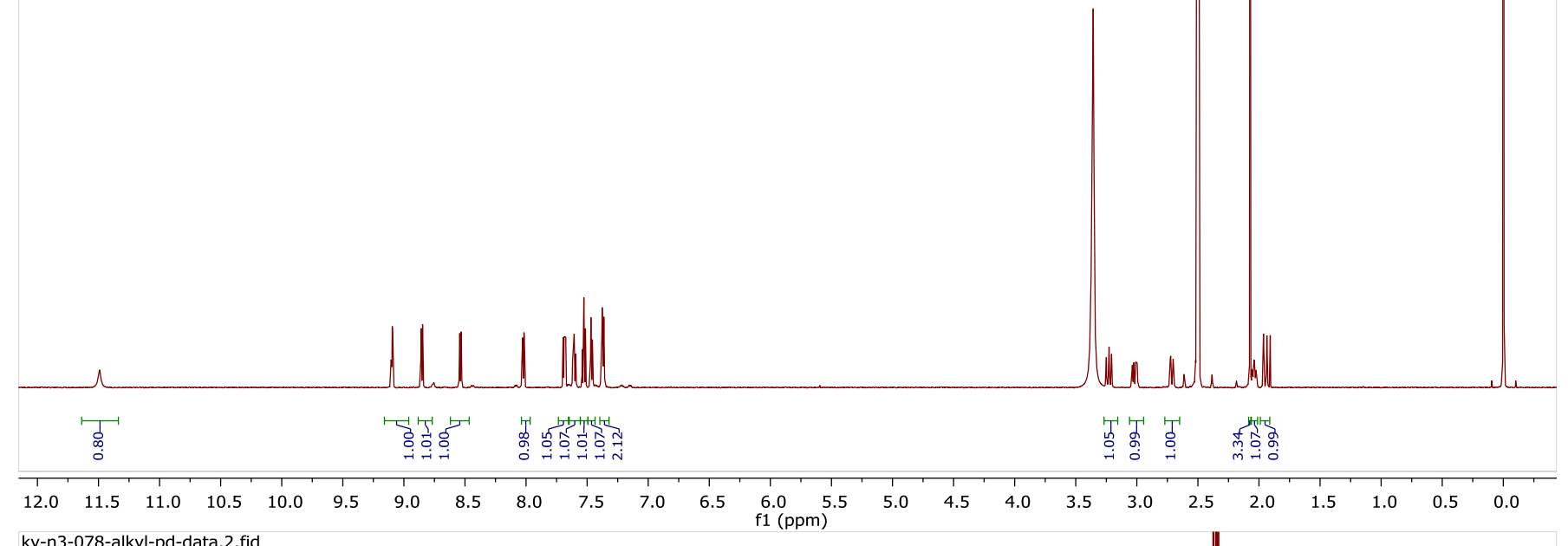
ky-n3-078-alkyl-pd-data.2.fid H-1 Routine, CP-DCH, AVIII HD-600

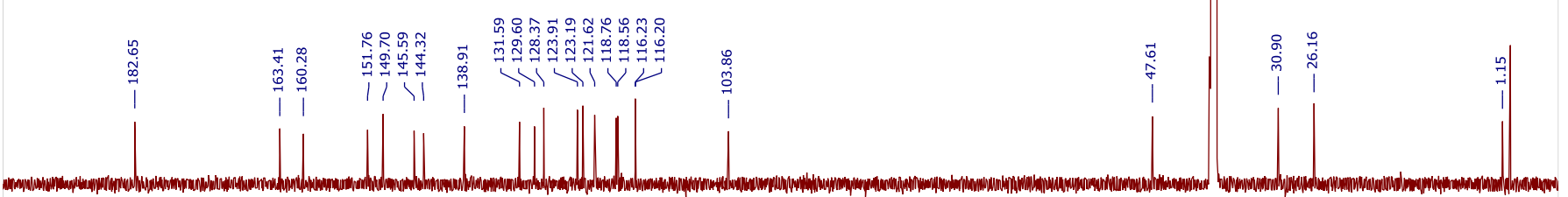


<smiles></smiles>

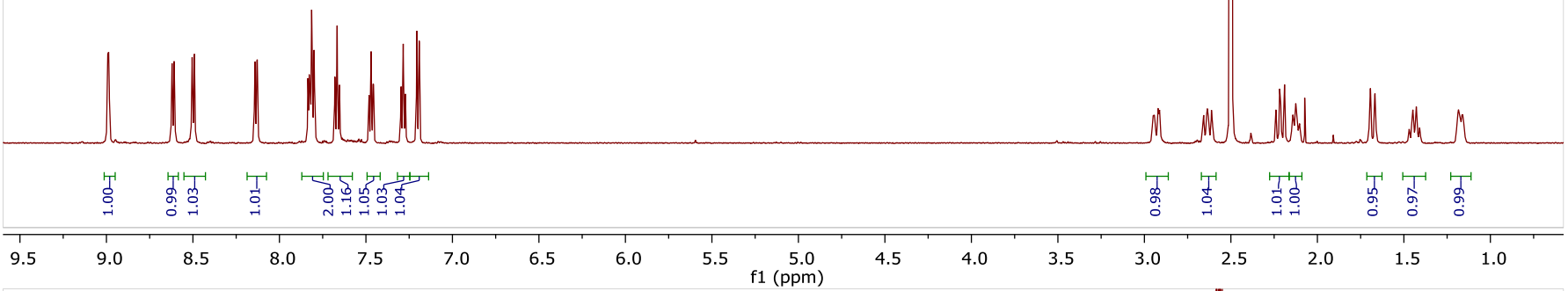

ky-n3-099-pd-6mem.2.fid

H-1 Routine. CPQCI, AVIII-600, 5-12-2016

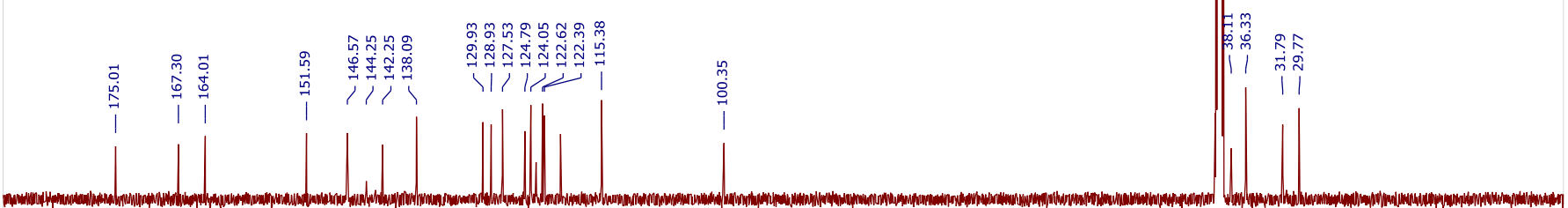

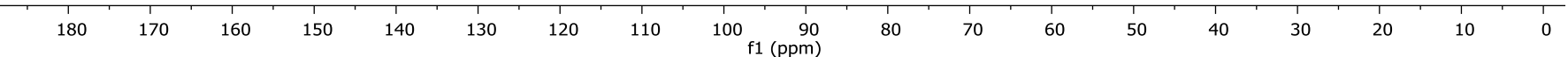




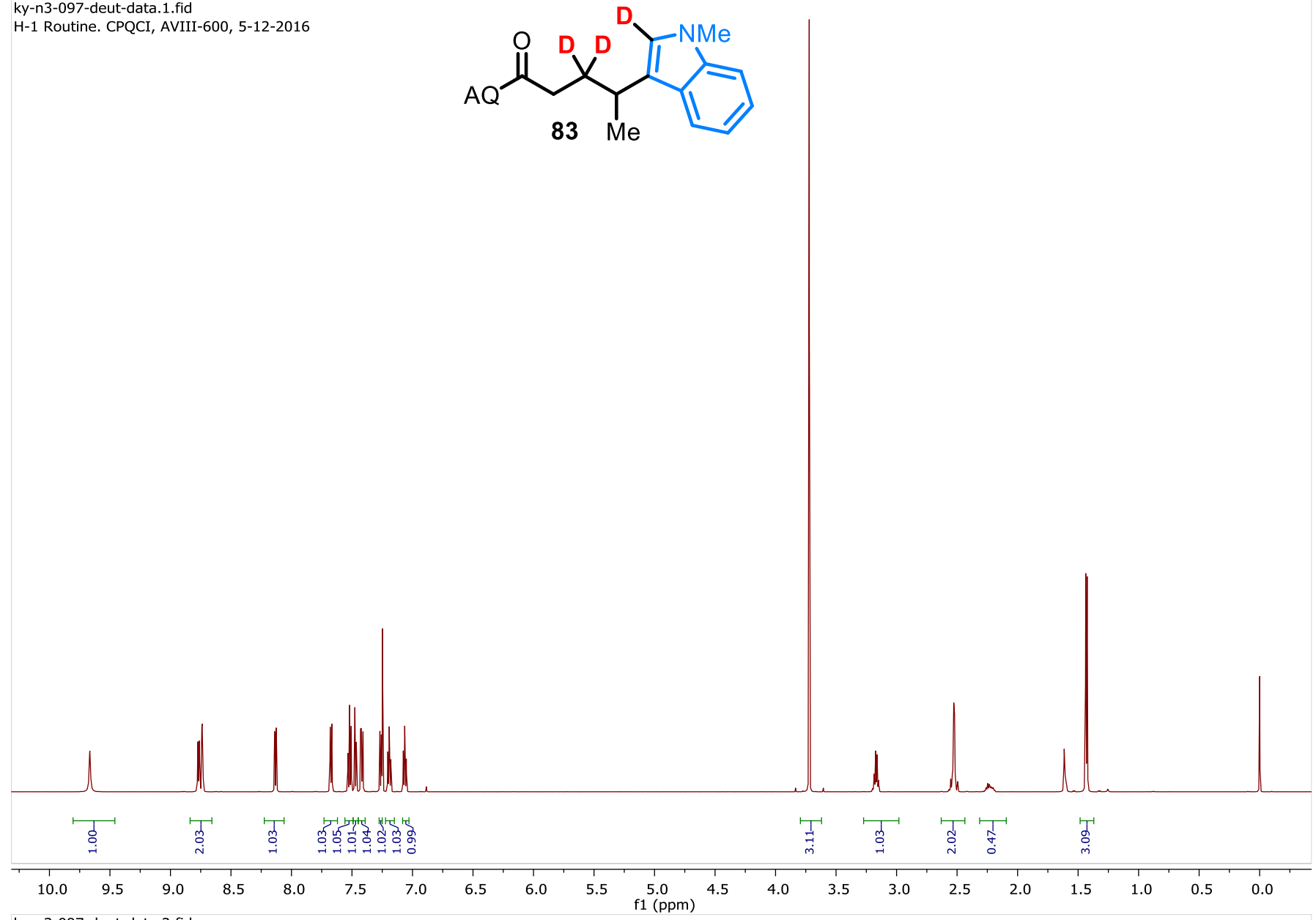

83

ky-n3-097-deut-data.2.fid

H-1 Routine. CPQCI, AVIII-600, 5-12-2016
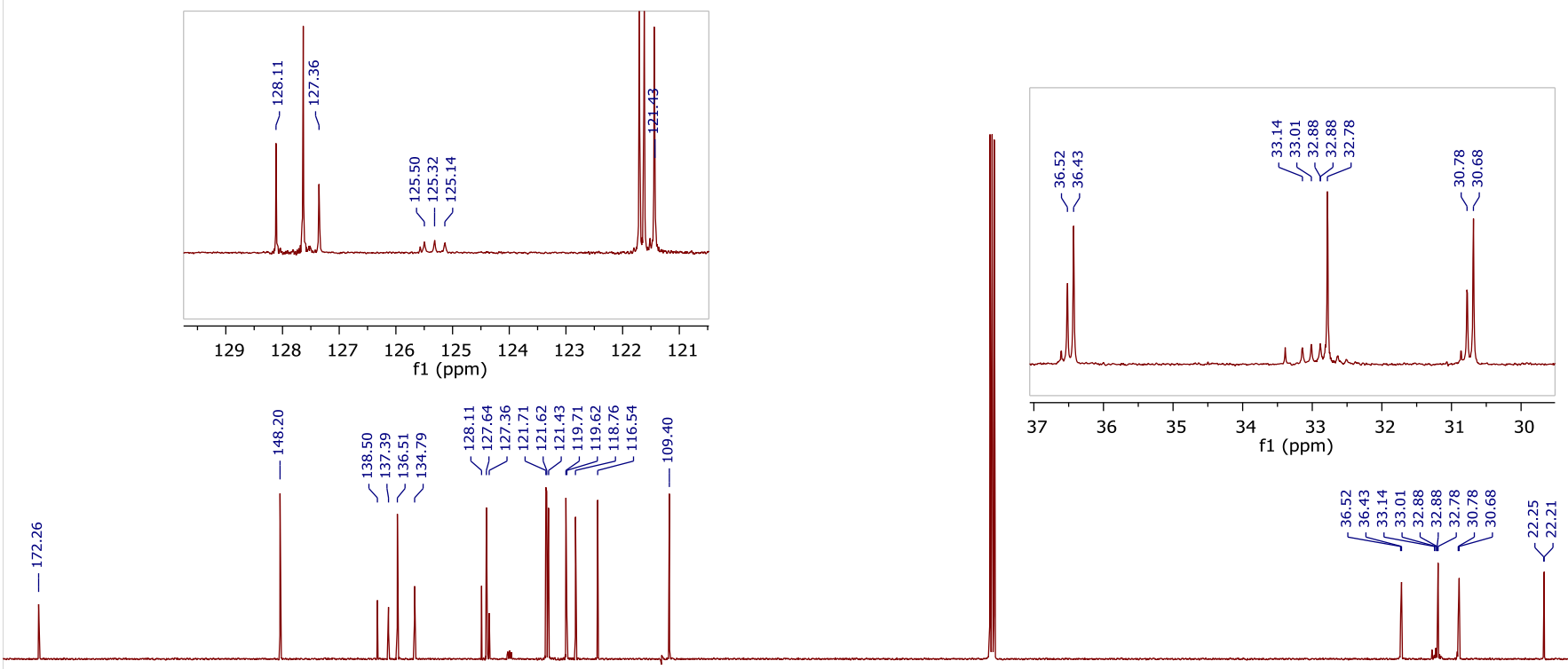

กุ̧ 히씼
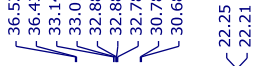

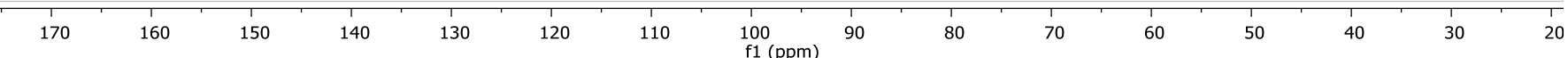




\section{Crystal Data}

\section{Experimental Summary for Crystal of 59}

The single crystal X-ray diffraction studies were carried out on a Bruker Platinum 135 CCD diffractometer equipped with $\mathrm{Cu} \mathrm{K}$ radiation $(\lambda=1.54178)$. Crystals of the subject compound were used as received (grown from DCE/Penthane) . A $0.25 \times 0.20 \times 20 \mathrm{~mm}$ light colorless block was mounted on a Cryoloop with Paratone oil.

Data were collected in a nitrogen gas stream at 100(2) K using $\phi$ and $\varpi$ scans. Crystal-todetector distance was $45 \mathrm{~mm}$ using exposure time 2 and $4 \mathrm{~s}$, with a scan width of $1.25^{\circ}$. Data collection was $99.9 \%$ complete to $67.500^{\circ}$ in $\theta$. A total of 27960 reflections were collected covering the indices, $-16<=\mathrm{h}<=16,-8<=\mathrm{k}<=8,-29<=\mathrm{l}<=29.4123$ reflections were found to be symmetry independent, with a $\mathrm{R}_{\text {int }}$ of 0.0522 . Indexing and unit cell refinement indicated a primitive, Monoclinic lattice. The space group was found to be $\boldsymbol{P 2} \mathbf{2}_{\mathbf{1}} / \mathbf{n}$. The data were integrated using the Bruker SAINT software program and scaled using the SADABS software program. Solution by direct methods (SHELXT) produced a complete phasing model consistent with the proposed structure.

All nonhydrogen atoms were refined anisotropically by full-matrix least-squares (SHELXL2014). All carbon bonded hydrogen atoms were placed using a riding model. Their positions were constrained relative to their parent atom using the appropriate HFIX command in SHELXL-2014.

. All other hydrogen atoms (H-bonding) were located in the difference map. There relative positions were restrained using AFIX commands and their thermals refined using riding model. Crystallographic data are summarized in Table S2.

Notes: X-ray Structure agrees to suggested model

Total molecule disorder approx. 93/7\% SAME and EADP applied.

Solvent molecule removed using squeeze procedure.

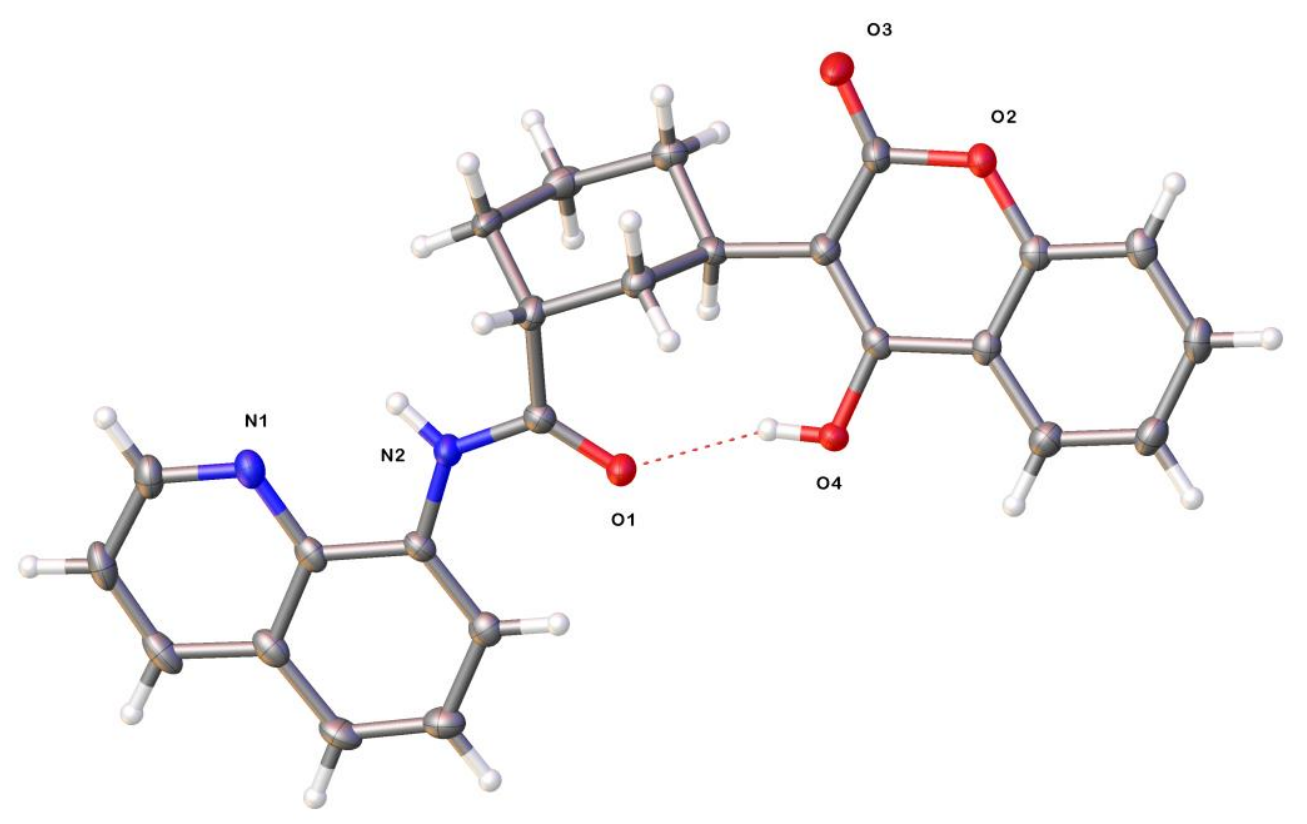




\section{Table S2}

Report date

Identification code

Empirical formula

Molecular formula

Formula weight

Temperature

Wavelength

Crystal system

Space group

Unit cell dimensions

Volume

Z

Density (calculated)

Absorption coefficient

$\mathrm{F}(000)$

Crystal size

Crystal color, habit

Theta range for data collection

Index ranges

Reflections collected

Independent reflections

Completeness to theta $=67.500^{\circ}$

Absorption correction

Max. and min. transmission

Refinement method

Data / restraints / parameters

Goodness-of-fit on $\mathrm{F}^{2}$

Final R indices [I $>2$ sigma(I)]

$\mathrm{R}$ indices (all data)

Extinction coefficient

Largest diff. peak and hole
2016-06-25

Engle14-KY14A

C25 H22 N2 04

C25 H22 N2 04

414.44

$100.0 \mathrm{~K}$

$1.54178 \AA$

Monoclinic

P 1 21/n 1

$\mathrm{a}=13.7635(4) \AA$

$\mathrm{b}=6.7495(2) \AA$

$\mathrm{c}=24.5387(7) \AA$

$\alpha=90^{\circ}$.

$\beta=99.3900(10)^{\circ}$.

$\gamma=90^{\circ}$.

2249.02(11) $\AA^{3}$

4

$1.224 \mathrm{Mg} / \mathrm{m}^{3}$

$0.680 \mathrm{~mm}^{-1}$

872

$0.25 \times 0.2 \times 0.2 \mathrm{~mm}^{3}$

colorless block

3.462 to $68.347^{\circ}$.

$-16<=\mathrm{h}<=16,-8<=\mathrm{k}<=8,-29<=\mathrm{l}<=29$

27960

$4123[\mathrm{R}(\mathrm{int})=0.0522]$

$99.9 \%$

Semi-empirical from equivalents

0.3201 and 0.2078

Full-matrix least-squares on $\mathrm{F}^{2}$

4123 / 86 / 376

1.092

$\mathrm{R} 1=0.0654, \mathrm{wR} 2=0.1623$

$\mathrm{R} 1=0.0686, \mathrm{wR} 2=0.1645$

$\mathrm{n} / \mathrm{a}$

0.470 and -0.373 e. $\AA^{-3}$ 
Table S3. Atomic coordinates $\left(\mathrm{x} \mathrm{10}^{4}\right)$ and equivalent isotropic displacement parameters $\left(\AA^{2} \times 10^{3}\right)$ for Engle14-KY14A. U(eq) is defined as one third of the trace of the orthogonalized $\mathrm{U}^{\mathrm{ij}}$ tensor.

\begin{tabular}{|c|c|c|c|c|}
\hline & $\mathrm{X}$ & $\mathrm{y}$ & $\mathrm{z}$ & $\mathrm{U}(\mathrm{eq})$ \\
\hline $\mathrm{O}(1)$ & $7511(1)$ & $6523(3)$ & 7038(1) & $24(1)$ \\
\hline $\mathrm{O}(2)$ & $5826(2)$ & $7228(3)$ & $9243(1)$ & $22(1)$ \\
\hline $\mathrm{O}(3)$ & $4587(2)$ & $7431(7)$ & $8554(1)$ & $38(1)$ \\
\hline$O(4)$ & $7854(1)$ & $6732(3)$ & $8191(1)$ & $21(1)$ \\
\hline$N(1)$ & $6422(2)$ & $6652(4)$ & $5048(1)$ & $24(1)$ \\
\hline$N(2)$ & $6829(2)$ & $6580(4)$ & $6130(1)$ & $22(1)$ \\
\hline$C(1)$ & $6193(2)$ & $6639(5)$ & $4503(1)$ & $27(1)$ \\
\hline $\mathrm{C}(2)$ & $6883(2)$ & $6837(5)$ & $4146(1)$ & $30(1)$ \\
\hline$C(3)$ & $7852(2)$ & $7025(4)$ & $4358(1)$ & $29(1)$ \\
\hline$C(4)$ & $8141(2)$ & $7049(4)$ & $4940(1)$ & $23(1)$ \\
\hline$C(5)$ & $9130(2)$ & $7234(5)$ & $5206(1)$ & $27(1)$ \\
\hline$C(6)$ & $9342(2)$ & $7248(4)$ & $5769(1)$ & $26(1)$ \\
\hline$C(7)$ & $8602(2)$ & $7066(4)$ & $6103(1)$ & $20(1)$ \\
\hline$C(8)$ & $7642(2)$ & $6865(4)$ & $5857(1)$ & $18(1)$ \\
\hline $\mathrm{C}(9)$ & $7389(2)$ & $6855(4)$ & $5267(1)$ & $20(1)$ \\
\hline$C(10)$ & $6786(2)$ & $6341(4)$ & $6672(1)$ & $21(1)$ \\
\hline $\mathrm{C}(11)$ & $5761(2)$ & $5744(4)$ & $6783(1)$ & $22(1)$ \\
\hline$C(12)$ & $5770(2)$ & $5510(4)$ & $7404(1)$ & $22(1)$ \\
\hline$C(13)$ & $5879(2)$ & $7562(4)$ & $7687(1)$ & $19(1)$ \\
\hline$C(14)$ & $4987(2)$ & $8838(5)$ & $7469(1)$ & $25(1)$ \\
\hline$C(15)$ & $4957(2)$ & $9130(5)$ & $6849(1)$ & $27(1)$ \\
\hline$C(16)$ & $4929(2)$ & $7180(6)$ & $6541(1)$ & $26(1)$ \\
\hline$C(17)$ & $6179(2)$ & 7327(7) & $8304(1)$ & $21(1)$ \\
\hline$C(18)$ & $5477(2)$ & $7357(11)$ & $8681(1)$ & $26(1)$ \\
\hline$C(19)$ & $6806(2)$ & $7017(4)$ & $9447(1)$ & $19(1)$ \\
\hline$C(20)$ & $7077(2)$ & $6992(5)$ & $10018(1)$ & $22(1)$ \\
\hline$C(21)$ & $8059(2)$ & $6785(5)$ & $10237(1)$ & $26(1)$ \\
\hline$C(22)$ & $8767(2)$ & $6592(4)$ & 9898(1) & $26(1)$ \\
\hline$C(23)$ & $8493(2)$ & $6596(4)$ & $9332(1)$ & $24(1)$ \\
\hline$C(24)$ & $7501(2)$ & $6833(4)$ & $9095(1)$ & $18(1)$ \\
\hline$C(25)$ & $7148(2)$ & $6933(4)$ & $8509(1)$ & $17(1)$ \\
\hline $\mathrm{O}(1 \mathrm{~A})$ & $7499(12)$ & $8230(40)$ & $7037(7)$ & $24(1)$ \\
\hline$O(2 A)$ & $5820(13)$ & $7770(60)$ & $9231(8)$ & $22(1)$ \\
\hline$O(3 A)$ & $4620(20)$ & $7880(130)$ & $8521(15)$ & $38(1)$ \\
\hline$O(4 A)$ & $7829(13)$ & $8240(40)$ & $8182(7)$ & $21(1)$ \\
\hline $\mathrm{N}(1 \mathrm{~A})$ & $6485(12)$ & $8170(40)$ & $5042(7)$ & $24(1)$ \\
\hline $\mathrm{N}(2 \mathrm{~A})$ & $6820(12)$ & $8210(40)$ & $6131(6)$ & $22(1)$ \\
\hline$C(1 \mathrm{~A})$ & $6296(15)$ & $7960(60)$ & $4497(7)$ & $27(1)$ \\
\hline$C(2 A)$ & $7032(18)$ & $8030(60)$ & $4162(7)$ & $30(1)$ \\
\hline$C(3 A)$ & $7976(17)$ & $7680(70)$ & $4401(7)$ & $29(1)$ \\
\hline$C(4 A)$ & 8221(13) & $7720(60)$ & $4985(7)$ & $23(1)$ \\
\hline$C(5 A)$ & $9201(13)$ & $7770(90)$ & $5272(10)$ & $27(1)$ \\
\hline$C(6 A)$ & $9378(13)$ & $7810(80)$ & $5835(10)$ & $26(1)$ \\
\hline
\end{tabular}




$\begin{array}{lrlrl}\mathrm{C}(7 \mathrm{~A}) & 8593(13) & 7860(60) & 6142(8) & 20(1) \\ \mathrm{C}(8 \mathrm{~A}) & 7661(11) & 8270(40) & 5868(6) & 18(1) \\ \mathrm{C}(9 \mathrm{~A}) & 7427(12) & 7780(50) & 5289(6) & 20(1) \\ \mathrm{C}(10 \mathrm{~A}) & 6763(11) & 8090(40) & 6676(6) & 21(1) \\ \mathrm{C}(11 \mathrm{~A}) & 5729(11) & 8620(30) & 6789(7) & 22(1) \\ \mathrm{C}(12 \mathrm{~A}) & 5765(17) & 8880(30) & 7411(7) & 22(1) \\ \mathrm{C}(13 \mathrm{~A}) & 5892(16) & 6840(30) & 7699(6) & 19(1) \\ \mathrm{C}(14 \mathrm{~A}) & 4986(17) & 5570(40) & 7503(8) & 25(1) \\ \mathrm{C}(15 \mathrm{~A}) & 4920(20) & 5230(40) & 6882(8) & 27(1) \\ \mathrm{C}(16 \mathrm{~A}) & 4913(19) & 7140(50) & 6558(9) & 26(1) \\ \mathrm{C}(17 \mathrm{~A}) & 6207(14) & 7150(70) & 8315(6) & 21(1) \\ \mathrm{C}(18 \mathrm{~A}) & 5478(13) & 7410(110) & 8675(8) & 26(1) \\ \mathrm{C}(19 \mathrm{~A}) & 6806(13) & 7780(60) & 9438(6) & 19(1) \\ \mathrm{C}(20 \mathrm{~A}) & 7070(15) & 7840(60) & 10008(7) & 22(1) \\ \mathrm{C}(21 \mathrm{~A}) & 8049(16) & 8110(60) & 10226(7) & 26(1) \\ \mathrm{C}(22 \mathrm{~A}) & 8762(14) & 8200(50) & 9888(7) & 26(1) \\ \mathrm{C}(23 \mathrm{~A}) & 8495(12) & 8190(50) & 9322(7) & 24(1) \\ \mathrm{C}(24 \mathrm{~A}) & 7498(12) & 8040(50) & 9087(6) & 18(1) \\ \mathrm{C}(25 \mathrm{~A}) & 7134(13) & 7890(50) & 8501(6) & 17(1) \\ & & & & \end{array}$


Table S4. Bond lengths $[\AA ̊]$ and angles $\left[{ }^{\circ}\right]$ for Engle14-KY14A.

\begin{tabular}{|c|c|c|c|}
\hline $\mathrm{O}(1)-\mathrm{C}(10)$ & $1.235(3)$ & $\mathrm{C}(20)-\mathrm{H}(20)$ & 0.9500 \\
\hline $\mathrm{O}(2)-\mathrm{C}(18)$ & $1.388(3)$ & $C(20)-C(21)$ & $1.378(4)$ \\
\hline$O(2)-C(19)$ & $1.368(3)$ & $\mathrm{C}(21)-\mathrm{H}(21)$ & 0.9500 \\
\hline $\mathrm{O}(3)-\mathrm{C}(18)$ & $1.215(3)$ & $C(21)-C(22)$ & $1.387(4)$ \\
\hline $\mathrm{O}(4)-\mathrm{H}(4)$ & 0.8400 & $\mathrm{C}(22)-\mathrm{H}(22)$ & 0.9500 \\
\hline$O(4)-C(25)$ & $1.348(3)$ & $C(22)-C(23)$ & $1.379(4)$ \\
\hline $\mathrm{N}(1)-\mathrm{C}(1)$ & $1.323(4)$ & $\mathrm{C}(23)-\mathrm{H}(23)$ & 0.9500 \\
\hline $\mathrm{N}(1)-\mathrm{C}(9)$ & $1.359(4)$ & $C(23)-C(24)$ & $1.402(4)$ \\
\hline $\mathrm{N}(2)-\mathrm{H}(2)$ & 0.8800 & $C(24)-C(25)$ & $1.442(3)$ \\
\hline $\mathrm{N}(2)-\mathrm{C}(8)$ & $1.408(3)$ & $O(1 A)-C(10 A)$ & $1.236(10)$ \\
\hline$N(2)-C(10)$ & $1.350(3)$ & $\mathrm{O}(2 \mathrm{~A})-\mathrm{C}(18 \mathrm{~A})$ & $1.391(10)$ \\
\hline $\mathrm{C}(1)-\mathrm{H}(1)$ & 0.9500 & $O(2 A)-C(19 A)$ & $1.369(9)$ \\
\hline $\mathrm{C}(1)-\mathrm{C}(2)$ & $1.400(4)$ & $\mathrm{O}(3 \mathrm{~A})-\mathrm{C}(18 \mathrm{~A})$ & $1.217(11)$ \\
\hline $\mathrm{C}(2)-\mathrm{H}(2 \mathrm{~A})$ & 0.9500 & $\mathrm{O}(4 \mathrm{~A})-\mathrm{H}(4 \mathrm{~A})$ & 0.8400 \\
\hline$C(2)-C(3)$ & $1.356(5)$ & $O(4 A)-C(25 A)$ & $1.352(10)$ \\
\hline $\mathrm{C}(3)-\mathrm{H}(3)$ & 0.9500 & $N(1 A)-C(1 A)$ & $1.327(10)$ \\
\hline$C(3)-C(4)$ & $1.418(4)$ & $N(1 A)-C(9 A)$ & $1.364(10)$ \\
\hline$C(4)-C(5)$ & $1.416(4)$ & $\mathrm{N}(2 \mathrm{~A})-\mathrm{H}(2 \mathrm{AA})$ & 0.8800 \\
\hline$C(4)-C(9)$ & $1.415(4)$ & $N(2 A)-C(8 A)$ & $1.416(10)$ \\
\hline $\mathrm{C}(5)-\mathrm{H}(5)$ & 0.9500 & $N(2 A)-C(10 A)$ & $1.353(10)$ \\
\hline$C(5)-C(6)$ & $1.364(4)$ & $\mathrm{C}(1 \mathrm{~A})-\mathrm{H}(1 \mathrm{~A})$ & 0.9500 \\
\hline $\mathrm{C}(6)-\mathrm{H}(6)$ & 0.9500 & $C(1 A)-C(2 A)$ & $1.405(11)$ \\
\hline$C(6)-C(7)$ & $1.414(4)$ & $\mathrm{C}(2 \mathrm{~A})-\mathrm{H}(2 \mathrm{AB})$ & 0.9500 \\
\hline $\mathrm{C}(7)-\mathrm{H}(7)$ & 0.9500 & $C(2 A)-C(3 A)$ & $1.356(10)$ \\
\hline$C(7)-C(8)$ & $1.367(4)$ & $\mathrm{C}(3 \mathrm{~A})-\mathrm{H}(3 \mathrm{~A})$ & 0.9500 \\
\hline$C(8)-C(9)$ & $1.432(4)$ & $C(3 A)-C(4 A)$ & $1.418(10)$ \\
\hline$C(10)-C(11)$ & $1.534(4)$ & $C(4 A)-C(5 A)$ & $1.415(10)$ \\
\hline $\mathrm{C}(11)-\mathrm{H}(11)$ & 1.0000 & $C(4 A)-C(9 A)$ & $1.420(9)$ \\
\hline$C(11)-C(12)$ & $1.530(3)$ & $\mathrm{C}(5 \mathrm{~A})-\mathrm{H}(5 \mathrm{~A})$ & 0.9500 \\
\hline$C(11)-C(16)$ & $1.543(5)$ & $C(5 A)-C(6 A)$ & $1.363(10)$ \\
\hline $\mathrm{C}(12)-\mathrm{H}(12 \mathrm{~A})$ & 0.9900 & $\mathrm{C}(6 \mathrm{~A})-\mathrm{H}(6 \mathrm{~A})$ & 0.9500 \\
\hline $\mathrm{C}(12)-\mathrm{H}(12 \mathrm{~B})$ & 0.9900 & $C(6 A)-C(7 A)$ & $1.415(10)$ \\
\hline$C(12)-C(13)$ & $1.545(4)$ & $\mathrm{C}(7 \mathrm{~A})-\mathrm{H}(7 \mathrm{~A})$ & 0.9500 \\
\hline $\mathrm{C}(13)-\mathrm{H}(13)$ & 1.0000 & $C(7 A)-C(8 A)$ & $1.375(10)$ \\
\hline $\mathrm{C}(13)-\mathrm{C}(14)$ & $1.523(4)$ & $C(8 A)-C(9 A)$ & $1.443(10)$ \\
\hline$C(13)-C(17)$ & $1.512(3)$ & $C(10 A)-C(11 A)$ & $1.537(10)$ \\
\hline $\mathrm{C}(14)-\mathrm{H}(14 \mathrm{~A})$ & 0.9900 & $\mathrm{C}(11 \mathrm{~A})-\mathrm{H}(11 \mathrm{~A})$ & 1.0000 \\
\hline $\mathrm{C}(14)-\mathrm{H}(14 \mathrm{~B})$ & 0.9900 & $C(11 \mathrm{~A})-\mathrm{C}(12 \mathrm{~A})$ & $1.528(10)$ \\
\hline$C(14)-C(15)$ & $1.530(4)$ & $C(11 A)-C(16 A)$ & $1.538(11)$ \\
\hline $\mathrm{C}(15)-\mathrm{H}(15 \mathrm{~A})$ & 0.9900 & $\mathrm{C}(12 \mathrm{~A})-\mathrm{H}(12 \mathrm{C})$ & 0.9900 \\
\hline $\mathrm{C}(15)-\mathrm{H}(15 \mathrm{~B})$ & 0.9900 & $\mathrm{C}(12 \mathrm{~A})-\mathrm{H}(12 \mathrm{D})$ & 0.9900 \\
\hline$C(15)-C(16)$ & $1.514(5)$ & $C(12 A)-C(13 A)$ & $1.547(11)$ \\
\hline $\mathrm{C}(16)-\mathrm{H}(16 \mathrm{~A})$ & 0.9900 & $\mathrm{C}(13 \mathrm{~A})-\mathrm{H}(13 \mathrm{~A})$ & 1.0000 \\
\hline $\mathrm{C}(16)-\mathrm{H}(16 \mathrm{~B})$ & 0.9900 & $C(13 A)-C(14 A)$ & $1.524(10)$ \\
\hline $\mathrm{C}(17)-\mathrm{C}(18)$ & $1.442(4)$ & $C(13 A)-C(17 A)$ & $1.518(9)$ \\
\hline$C(17)-C(25)$ & $1.373(4)$ & $\mathrm{C}(14 \mathrm{~A})-\mathrm{H}(14 \mathrm{C})$ & 0.9900 \\
\hline$C(19)-C(20)$ & $1.389(4)$ & $\mathrm{C}(14 \mathrm{~A})-\mathrm{H}(14 \mathrm{D})$ & 0.9900 \\
\hline$C(19)-C(24)$ & $1.395(4)$ & $C(14 A)-C(15 A)$ & $1.528(11)$ \\
\hline
\end{tabular}




\begin{tabular}{|c|c|c|c|}
\hline $\mathrm{C}(15 \mathrm{~A})-\mathrm{H}(15 \mathrm{C})$ & 0.9900 & $\mathrm{~N}(2)-\mathrm{C}(10)-\mathrm{C}(11)$ & $113.4(2)$ \\
\hline$C(15 A)-H(15 D)$ & 0.9900 & $\mathrm{C}(10)-\mathrm{C}(11)-\mathrm{H}(11)$ & 107.5 \\
\hline$C(15 A)-C(16 A)$ & $1.513(12)$ & $C(10)-C(11)-C(16)$ & $114.3(2)$ \\
\hline $\mathrm{C}(16 \mathrm{~A})-\mathrm{H}(16 \mathrm{C})$ & 0.9900 & $C(12)-C(11)-C(10)$ & $110.2(2)$ \\
\hline$C(16 A)-H(16 D)$ & 0.9900 & $\mathrm{C}(12)-\mathrm{C}(11)-\mathrm{H}(11)$ & 107.5 \\
\hline$C(17 A)-C(18 A)$ & $1.450(9)$ & $C(12)-C(11)-C(16)$ & $109.6(2)$ \\
\hline$C(17 A)-C(25 A)$ & $1.375(10)$ & $\mathrm{C}(16)-\mathrm{C}(11)-\mathrm{H}(11)$ & 107.5 \\
\hline$C(19 A)-C(20 A)$ & $1.387(10)$ & $\mathrm{C}(11)-\mathrm{C}(12)-\mathrm{H}(12 \mathrm{~A})$ & 109.7 \\
\hline$C(19 A)-C(24 A)$ & $1.396(9)$ & $\mathrm{C}(11)-\mathrm{C}(12)-\mathrm{H}(12 \mathrm{~B})$ & 109.7 \\
\hline $\mathrm{C}(20 \mathrm{~A})-\mathrm{H}(20 \mathrm{~A})$ & 0.9500 & $C(11)-C(12)-C(13)$ & $109.8(2)$ \\
\hline$C(20 A)-C(21 A)$ & $1.379(10)$ & $\mathrm{H}(12 \mathrm{~A})-\mathrm{C}(12)-\mathrm{H}(12 \mathrm{~B})$ & 108.2 \\
\hline$C(21 A)-H(21 A)$ & 0.9500 & $\mathrm{C}(13)-\mathrm{C}(12)-\mathrm{H}(12 \mathrm{~A})$ & 109.7 \\
\hline$C(21 A)-C(22 A)$ & $1.387(10)$ & $\mathrm{C}(13)-\mathrm{C}(12)-\mathrm{H}(12 \mathrm{~B})$ & 109.7 \\
\hline $\mathrm{C}(22 \mathrm{~A})-\mathrm{H}(22 \mathrm{~A})$ & 0.9500 & $\mathrm{C}(12)-\mathrm{C}(13)-\mathrm{H}(13)$ & 105.7 \\
\hline$C(22 A)-C(23 A)$ & $1.378(10)$ & $C(14)-C(13)-C(12)$ & $109.6(2)$ \\
\hline $\mathrm{C}(23 \mathrm{~A})-\mathrm{H}(23 \mathrm{~A})$ & 0.9500 & $\mathrm{C}(14)-\mathrm{C}(13)-\mathrm{H}(13)$ & 105.7 \\
\hline$C(23 A)-C(24 A)$ & $1.403(10)$ & $C(17)-C(13)-C(12)$ & $110.2(3)$ \\
\hline \multirow[t]{2}{*}{$C(24 A)-C(25 A)$} & $1.447(9)$ & $\mathrm{C}(17)-\mathrm{C}(13)-\mathrm{H}(13)$ & 105.7 \\
\hline & & $C(17)-C(13)-C(14)$ & $118.8(2)$ \\
\hline$C(19)-O(2)-C(18)$ & $122.1(2)$ & $\mathrm{C}(13)-\mathrm{C}(14)-\mathrm{H}(14 \mathrm{~A})$ & 110.1 \\
\hline $\mathrm{C}(25)-\mathrm{O}(4)-\mathrm{H}(4)$ & 109.5 & $\mathrm{C}(13)-\mathrm{C}(14)-\mathrm{H}(14 \mathrm{~B})$ & 110.1 \\
\hline$C(1)-N(1)-C(9)$ & $117.3(2)$ & $C(13)-C(14)-C(15)$ & $108.1(2)$ \\
\hline $\mathrm{C}(8)-\mathrm{N}(2)-\mathrm{H}(2)$ & 114.7 & $\mathrm{H}(14 \mathrm{~A})-\mathrm{C}(14)-\mathrm{H}(14 \mathrm{~B})$ & 108.4 \\
\hline $\mathrm{C}(10)-\mathrm{N}(2)-\mathrm{H}(2)$ & 114.7 & $C(15)-C(14)-H(14 A)$ & 110.1 \\
\hline $\mathrm{C}(10)-\mathrm{N}(2)-\mathrm{C}(8)$ & $130.7(2)$ & $\mathrm{C}(15)-\mathrm{C}(14)-\mathrm{H}(14 \mathrm{~B})$ & 110.1 \\
\hline $\mathrm{N}(1)-\mathrm{C}(1)-\mathrm{H}(1)$ & 118.0 & $\mathrm{C}(14)-\mathrm{C}(15)-\mathrm{H}(15 \mathrm{~A})$ & 109.1 \\
\hline$N(1)-C(1)-C(2)$ & $123.9(3)$ & $\mathrm{C}(14)-\mathrm{C}(15)-\mathrm{H}(15 \mathrm{~B})$ & 109.1 \\
\hline $\mathrm{C}(2)-\mathrm{C}(1)-\mathrm{H}(1)$ & 118.0 & $H(15 A)-C(15)-H(15 B)$ & 107.9 \\
\hline $\mathrm{C}(1)-\mathrm{C}(2)-\mathrm{H}(2 \mathrm{~A})$ & 120.2 & $C(16)-C(15)-C(14)$ & $112.3(3)$ \\
\hline$C(3)-C(2)-C(1)$ & $119.5(3)$ & $\mathrm{C}(16)-\mathrm{C}(15)-\mathrm{H}(15 \mathrm{~A})$ & 109.1 \\
\hline $\mathrm{C}(3)-\mathrm{C}(2)-\mathrm{H}(2 \mathrm{~A})$ & 120.2 & $C(16)-C(15)-H(15 B)$ & 109.1 \\
\hline $\mathrm{C}(2)-\mathrm{C}(3)-\mathrm{H}(3)$ & 120.5 & $\mathrm{C}(11)-\mathrm{C}(16)-\mathrm{H}(16 \mathrm{~A})$ & 108.9 \\
\hline$C(2)-C(3)-C(4)$ & $119.0(3)$ & $\mathrm{C}(11)-\mathrm{C}(16)-\mathrm{H}(16 \mathrm{~B})$ & 108.9 \\
\hline $\mathrm{C}(4)-\mathrm{C}(3)-\mathrm{H}(3)$ & 120.5 & $C(15)-C(16)-C(11)$ & $113.3(2)$ \\
\hline$C(5)-C(4)-C(3)$ & $123.8(3)$ & $\mathrm{C}(15)-\mathrm{C}(16)-\mathrm{H}(16 \mathrm{~A})$ & 108.9 \\
\hline$C(9)-C(4)-C(3)$ & $117.3(3)$ & $\mathrm{C}(15)-\mathrm{C}(16)-\mathrm{H}(16 \mathrm{~B})$ & 108.9 \\
\hline$C(9)-C(4)-C(5)$ & $118.9(3)$ & $\mathrm{H}(16 \mathrm{~A})-\mathrm{C}(16)-\mathrm{H}(16 \mathrm{~B})$ & 107.7 \\
\hline $\mathrm{C}(4)-\mathrm{C}(5)-\mathrm{H}(5)$ & 120.1 & $C(18)-C(17)-C(13)$ & $122.6(2)$ \\
\hline$C(6)-C(5)-C(4)$ & $119.9(2)$ & $C(25)-C(17)-C(13)$ & $118.5(2)$ \\
\hline $\mathrm{C}(6)-\mathrm{C}(5)-\mathrm{H}(5)$ & 120.1 & $C(25)-C(17)-C(18)$ & $118.7(3)$ \\
\hline$C(5)-C(6)-H(6)$ & 118.9 & $O(2)-C(18)-C(17)$ & $118.5(2)$ \\
\hline$C(5)-C(6)-C(7)$ & $122.1(3)$ & $\mathrm{O}(3)-\mathrm{C}(18)-\mathrm{O}(2)$ & $115.4(3)$ \\
\hline $\mathrm{C}(7)-\mathrm{C}(6)-\mathrm{H}(6)$ & 118.9 & $\mathrm{O}(3)-\mathrm{C}(18)-\mathrm{C}(17)$ & $126.1(3)$ \\
\hline $\mathrm{C}(6)-\mathrm{C}(7)-\mathrm{H}(7)$ & 120.4 & $O(2)-C(19)-C(20)$ & $117.2(2)$ \\
\hline$C(8)-C(7)-C(6)$ & $119.2(3)$ & $O(2)-C(19)-C(24)$ & $121.2(2)$ \\
\hline $\mathrm{C}(8)-\mathrm{C}(7)-\mathrm{H}(7)$ & 120.4 & $C(20)-C(19)-C(24)$ & $121.7(2)$ \\
\hline$N(2)-C(8)-C(9)$ & $113.6(2)$ & $\mathrm{C}(19)-\mathrm{C}(20)-\mathrm{H}(20)$ & 120.7 \\
\hline $\mathrm{C}(7)-\mathrm{C}(8)-\mathrm{N}(2)$ & $126.0(2)$ & $C(21)-C(20)-C(19)$ & $118.7(3)$ \\
\hline$C(7)-C(8)-C(9)$ & $120.4(2)$ & $\mathrm{C}(21)-\mathrm{C}(20)-\mathrm{H}(20)$ & 120.7 \\
\hline$N(1)-C(9)-C(4)$ & $122.9(2)$ & $\mathrm{C}(20)-\mathrm{C}(21)-\mathrm{H}(21)$ & 119.5 \\
\hline$N(1)-C(9)-C(8)$ & $117.5(2)$ & $C(20)-C(21)-C(22)$ & $121.0(3)$ \\
\hline$C(4)-C(9)-C(8)$ & $119.5(2)$ & $\mathrm{C}(22)-\mathrm{C}(21)-\mathrm{H}(21)$ & 119.5 \\
\hline $\mathrm{O}(1)-\mathrm{C}(10)-\mathrm{N}(2)$ & $123.0(2)$ & $\mathrm{C}(21)-\mathrm{C}(22)-\mathrm{H}(22)$ & 120.0 \\
\hline $\mathrm{O}(1)-\mathrm{C}(10)-\mathrm{C}(11)$ & $123.6(2)$ & $C(23)-C(22)-C(21)$ & $120.1(3)$ \\
\hline
\end{tabular}




\begin{tabular}{|c|c|}
\hline $\mathrm{C}(23)-\mathrm{C}(22)-\mathrm{H}(22)$ & 120.0 \\
\hline $\mathrm{C}(22)-\mathrm{C}(23)-\mathrm{H}(23)$ & 119.8 \\
\hline$C(22)-C(23)-C(24)$ & $120.3(3)$ \\
\hline $\mathrm{C}(24)-\mathrm{C}(23)-\mathrm{H}(23)$ & 119.8 \\
\hline$C(19)-C(24)-C(23)$ & $118.2(2)$ \\
\hline$C(19)-C(24)-C(25)$ & $117.3(2)$ \\
\hline$C(23)-C(24)-C(25)$ & $124.4(2)$ \\
\hline$O(4)-C(25)-C(17)$ & $123.8(2)$ \\
\hline$O(4)-C(25)-C(24)$ & $114.5(2)$ \\
\hline$C(17)-C(25)-C(24)$ & $121.6(2)$ \\
\hline$C(19 A)-O(2 A)-C(18 A)$ & $121.4(12)$ \\
\hline $\mathrm{C}(25 \mathrm{~A})-\mathrm{O}(4 \mathrm{~A})-\mathrm{H}(4 \mathrm{~A})$ & 109.5 \\
\hline$C(1 A)-N(1 A)-C(9 A)$ & $116.3(12)$ \\
\hline $\mathrm{C}(8 \mathrm{~A})-\mathrm{N}(2 \mathrm{~A})-\mathrm{H}(2 \mathrm{AA})$ & 115.3 \\
\hline $\mathrm{C}(10 \mathrm{~A})-\mathrm{N}(2 \mathrm{~A})-\mathrm{H}(2 \mathrm{AA})$ & 115.3 \\
\hline$C(10 A)-N(2 A)-C(8 A)$ & $129.5(12)$ \\
\hline$N(1 A)-C(1 A)-H(1 A)$ & 118.6 \\
\hline $\mathrm{N}(1 \mathrm{~A})-\mathrm{C}(1 \mathrm{~A})-\mathrm{C}(2 \mathrm{~A})$ & $122.9(14)$ \\
\hline$C(2 A)-C(1 A)-H(1 A)$ & 118.6 \\
\hline $\mathrm{C}(1 \mathrm{~A})-\mathrm{C}(2 \mathrm{~A})-\mathrm{H}(2 \mathrm{AB})$ & 120.9 \\
\hline$C(3 A)-C(2 A)-C(1 A)$ & $118.1(13)$ \\
\hline $\mathrm{C}(3 \mathrm{~A})-\mathrm{C}(2 \mathrm{~A})-\mathrm{H}(2 \mathrm{AB})$ & 120.9 \\
\hline $\mathrm{C}(2 \mathrm{~A})-\mathrm{C}(3 \mathrm{~A})-\mathrm{H}(3 \mathrm{~A})$ & 120.4 \\
\hline$C(2 A)-C(3 A)-C(4 A)$ & $119.2(12)$ \\
\hline $\mathrm{C}(4 \mathrm{~A})-\mathrm{C}(3 \mathrm{~A})-\mathrm{H}(3 \mathrm{~A})$ & 120.4 \\
\hline$C(3 A)-C(4 A)-C(9 A)$ & $117.0(10)$ \\
\hline$C(5 A)-C(4 A)-C(3 A)$ & $123.6(12)$ \\
\hline$C(5 A)-C(4 A)-C(9 A)$ & $119.4(10)$ \\
\hline $\mathrm{C}(4 \mathrm{~A})-\mathrm{C}(5 \mathrm{~A})-\mathrm{H}(5 \mathrm{~A})$ & 119.9 \\
\hline$C(6 A)-C(5 A)-C(4 A)$ & $120.2(12)$ \\
\hline $\mathrm{C}(6 \mathrm{~A})-\mathrm{C}(5 \mathrm{~A})-\mathrm{H}(5 \mathrm{~A})$ & 119.9 \\
\hline$C(5 A)-C(6 A)-H(6 A)$ & 119.6 \\
\hline$C(5 A)-C(6 A)-C(7 A)$ & $120.9(13)$ \\
\hline$C(7 A)-C(6 A)-H(6 A)$ & 119.6 \\
\hline$C(6 A)-C(7 A)-H(7 A)$ & 120.7 \\
\hline$C(8 A)-C(7 A)-C(6 A)$ & $118.5(12)$ \\
\hline $\mathrm{C}(8 \mathrm{~A})-\mathrm{C}(7 \mathrm{~A})-\mathrm{H}(7 \mathrm{~A})$ & 120.7 \\
\hline$N(2 A)-C(8 A)-C(9 A)$ & $112.0(11)$ \\
\hline$C(7 A)-C(8 A)-N(2 A)$ & $122.6(13)$ \\
\hline$C(7 A)-C(8 A)-C(9 A)$ & $118.5(12)$ \\
\hline$N(1 A)-C(9 A)-C(4 A)$ & $121.9(12)$ \\
\hline$N(1 A)-C(9 A)-C(8 A)$ & $115.9(12)$ \\
\hline$C(4 A)-C(9 A)-C(8 A)$ & $117.0(11)$ \\
\hline $\mathrm{O}(1 \mathrm{~A})-\mathrm{C}(10 \mathrm{~A})-\mathrm{N}(2 \mathrm{~A})$ & $122.1(13)$ \\
\hline$O(1 \mathrm{~A})-\mathrm{C}(10 \mathrm{~A})-\mathrm{C}(11 \mathrm{~A})$ & $121.8(13)$ \\
\hline$N(2 A)-C(10 A)-C(11 A)$ & $111.7(11)$ \\
\hline $\mathrm{C}(10 \mathrm{~A})-\mathrm{C}(11 \mathrm{~A})-\mathrm{H}(11 \mathrm{~A})$ & 107.3 \\
\hline$C(10 A)-C(11 A)-C(16 A)$ & $115.1(14)$ \\
\hline$C(12 A)-C(11 A)-C(10 A)$ & $109.1(11)$ \\
\hline $\mathrm{C}(12 \mathrm{~A})-\mathrm{C}(11 \mathrm{~A})-\mathrm{H}(11 \mathrm{~A})$ & 107.3 \\
\hline$C(12 A)-C(11 A)-C(16 A)$ & $110.3(11)$ \\
\hline$C(16 A)-C(11 A)-H(11 A)$ & 107.3 \\
\hline $\mathrm{C}(11 \mathrm{~A})-\mathrm{C}(12 \mathrm{~A})-\mathrm{H}(12 \mathrm{C})$ & 109.8 \\
\hline $\mathrm{C}(11 \mathrm{~A})-\mathrm{C}(12 \mathrm{~A})-\mathrm{H}(12 \mathrm{D})$ & 109.8 \\
\hline
\end{tabular}

\begin{tabular}{|c|c|}
\hline$C(11 A)-C(12 A)-C(13 A)$ & $109.6(11)$ \\
\hline $\mathrm{H}(12 \mathrm{C})-\mathrm{C}(12 \mathrm{~A})-\mathrm{H}(12 \mathrm{D})$ & 108.2 \\
\hline $\mathrm{C}(13 \mathrm{~A})-\mathrm{C}(12 \mathrm{~A})-\mathrm{H}(12 \mathrm{C})$ & 109.8 \\
\hline$C(13 A)-C(12 A)-H(12 D)$ & 109.8 \\
\hline$C(12 A)-C(13 A)-H(13 A)$ & 106.5 \\
\hline$C(14 A)-C(13 A)-C(12 A)$ & $109.3(13)$ \\
\hline$C(14 A)-C(13 A)-H(13 A)$ & 106.5 \\
\hline$C(17 A)-C(13 A)-C(12 A)$ & $108.8(14)$ \\
\hline $\mathrm{C}(17 \mathrm{~A})-\mathrm{C}(13 \mathrm{~A})-\mathrm{H}(13 \mathrm{~A})$ & 106.5 \\
\hline$C(17 A)-C(13 A)-C(14 A)$ & $118.6(12)$ \\
\hline $\mathrm{C}(13 \mathrm{~A})-\mathrm{C}(14 \mathrm{~A})-\mathrm{H}(14 \mathrm{C})$ & 110.1 \\
\hline $\mathrm{C}(13 \mathrm{~A})-\mathrm{C}(14 \mathrm{~A})-\mathrm{H}(14 \mathrm{D})$ & 110.1 \\
\hline$C(13 A)-C(14 A)-C(15 A)$ & $108.1(12)$ \\
\hline $\mathrm{H}(14 \mathrm{C})-\mathrm{C}(14 \mathrm{~A})-\mathrm{H}(14 \mathrm{D})$ & 108.4 \\
\hline $\mathrm{C}(15 \mathrm{~A})-\mathrm{C}(14 \mathrm{~A})-\mathrm{H}(14 \mathrm{C})$ & 110.1 \\
\hline$C(15 A)-C(14 A)-H(14 D)$ & 110.1 \\
\hline $\mathrm{C}(14 \mathrm{~A})-\mathrm{C}(15 \mathrm{~A})-\mathrm{H}(15 \mathrm{C})$ & 109.0 \\
\hline$C(14 A)-C(15 A)-H(15 D)$ & 109.0 \\
\hline $\mathrm{H}(15 \mathrm{C})-\mathrm{C}(15 \mathrm{~A})-\mathrm{H}(15 \mathrm{D})$ & 107.8 \\
\hline$C(16 A)-C(15 A)-C(14 A)$ & $112.9(13)$ \\
\hline$C(16 A)-C(15 A)-H(15 C)$ & 109.0 \\
\hline$C(16 A)-C(15 A)-H(15 D)$ & 109.0 \\
\hline$C(11 A)-C(16 A)-H(16 C)$ & 108.6 \\
\hline$C(11 A)-C(16 A)-H(16 D)$ & 108.6 \\
\hline$C(15 A)-C(16 A)-C(11 A)$ & $114.6(12)$ \\
\hline$C(15 A)-C(16 A)-H(16 C)$ & 108.6 \\
\hline$C(15 A)-C(16 A)-H(16 D)$ & 108.6 \\
\hline $\mathrm{H}(16 \mathrm{C})-\mathrm{C}(16 \mathrm{~A})-\mathrm{H}(16 \mathrm{D})$ & 107.6 \\
\hline$C(18 A)-C(17 A)-C(13 A)$ & $120.7(12)$ \\
\hline$C(25 A)-C(17 A)-C(13 A)$ & $118.6(11)$ \\
\hline$C(25 A)-C(17 A)-C(18 A)$ & $116.9(14)$ \\
\hline$O(2 A)-C(18 A)-C(17 A)$ & $117.5(11)$ \\
\hline$O(3 A)-C(18 A)-O(2 A)$ & $114.3(19)$ \\
\hline$O(3 A)-C(18 A)-C(17 A)$ & $125(2)$ \\
\hline$O(2 A)-C(19 A)-C(20 A)$ & $117.1(11)$ \\
\hline$O(2 A)-C(19 A)-C(24 A)$ & $120.4(12)$ \\
\hline$C(20 A)-C(19 A)-C(24 A)$ & $121.8(10)$ \\
\hline$C(19 A)-C(20 A)-H(20 A)$ & 120.8 \\
\hline$C(21 A)-C(20 A)-C(19 A)$ & $118.3(12)$ \\
\hline $\mathrm{C}(21 \mathrm{~A})-\mathrm{C}(20 \mathrm{~A})-\mathrm{H}(20 \mathrm{~A})$ & 120.8 \\
\hline$C(20 A)-C(21 A)-H(21 A)$ & 119.5 \\
\hline$C(20 A)-C(21 A)-C(22 A)$ & $121.1(12)$ \\
\hline $\mathrm{C}(22 \mathrm{~A})-\mathrm{C}(21 \mathrm{~A})-\mathrm{H}(21 \mathrm{~A})$ & 119.5 \\
\hline $\mathrm{C}(21 \mathrm{~A})-\mathrm{C}(22 \mathrm{~A})-\mathrm{H}(22 \mathrm{~A})$ & 119.8 \\
\hline$C(23 A)-C(22 A)-C(21 A)$ & $120.3(12)$ \\
\hline $\mathrm{C}(23 \mathrm{~A})-\mathrm{C}(22 \mathrm{~A})-\mathrm{H}(22 \mathrm{~A})$ & 119.8 \\
\hline $\mathrm{C}(22 \mathrm{~A})-\mathrm{C}(23 \mathrm{~A})-\mathrm{H}(23 \mathrm{~A})$ & 120.1 \\
\hline$C(22 A)-C(23 A)-C(24 A)$ & $119.8(11)$ \\
\hline $\mathrm{C}(24 \mathrm{~A})-\mathrm{C}(23 \mathrm{~A})-\mathrm{H}(23 \mathrm{~A})$ & 120.1 \\
\hline$C(19 A)-C(24 A)-C(23 A)$ & $118.4(10)$ \\
\hline$C(19 A)-C(24 A)-C(25 A)$ & $116.4(10)$ \\
\hline$C(23 A)-C(24 A)-C(25 A)$ & $124.8(11)$ \\
\hline$O(4 A)-C(25 A)-C(17 A)$ & $125.3(12)$ \\
\hline$O(4 A)-C(25 A)-C(24 A)$ & $113.5(11)$ \\
\hline
\end{tabular}


$\mathrm{C}(17 \mathrm{~A})-\mathrm{C}(25 \mathrm{~A})-\mathrm{C}(24 \mathrm{~A}) \quad 120.5(11)$ 
Table S5. Anisotropic displacement parameters $\left(\AA^{2} \times 10^{3}\right)$ for Engle14-KY14A. The anisotropic displacement factor exponent takes the form: $-2 \pi^{2}\left[h^{2} a^{* 2} U^{11}+\ldots+2 h \mathrm{k}^{*} b^{*} U^{12}\right]$

\begin{tabular}{|c|c|c|c|c|c|c|}
\hline & $\mathrm{U}^{11}$ & $\mathrm{U}^{22}$ & $\mathrm{U}^{33}$ & $\mathrm{U}^{23}$ & $\mathrm{U}^{13}$ & $\mathrm{U}^{12}$ \\
\hline $\mathrm{O}(1)$ & $18(1)$ & $37(1)$ & $16(1)$ & $-2(1)$ & $2(1)$ & $0(1)$ \\
\hline$O(2)$ & $21(1)$ & $31(2)$ & $14(1)$ & $-1(1)$ & $3(1)$ & $0(1)$ \\
\hline$O(3)$ & $17(1)$ & $77(4)$ & $19(1)$ & $-3(1)$ & $4(1)$ & $-1(1)$ \\
\hline$O(4)$ & $16(1)$ & $32(1)$ & $15(1)$ & $-1(1)$ & $4(1)$ & $3(1)$ \\
\hline $\mathrm{N}(1)$ & $28(1)$ & $26(1)$ & $17(1)$ & $-3(1)$ & $4(1)$ & $-1(1)$ \\
\hline $\mathrm{N}(2)$ & $18(1)$ & $34(1)$ & $14(1)$ & $-1(1)$ & $2(1)$ & $-1(1)$ \\
\hline $\mathrm{C}(1)$ & $34(2)$ & $28(2)$ & $17(1)$ & $-4(1)$ & $1(1)$ & $0(1)$ \\
\hline$C(2)$ & $46(2)$ & $30(2)$ & $14(1)$ & $-2(1)$ & $6(1)$ & $0(1)$ \\
\hline$C(3)$ & $45(2)$ & $23(2)$ & $23(1)$ & $-2(1)$ & $19(1)$ & $-2(1)$ \\
\hline$C(4)$ & $31(2)$ & $18(2)$ & $23(1)$ & $-2(1)$ & $11(1)$ & $-1(1)$ \\
\hline $\mathrm{C}(5)$ & $26(1)$ & $29(2)$ & $29(2)$ & $0(1)$ & $16(1)$ & $-1(1)$ \\
\hline$C(6)$ & $20(1)$ & $28(2)$ & $32(2)$ & $-1(1)$ & $8(1)$ & $0(1)$ \\
\hline$C(7)$ & $23(1)$ & $17(1)$ & $21(1)$ & $-2(1)$ & $4(1)$ & $0(1)$ \\
\hline$C(8)$ & $20(1)$ & $20(1)$ & $17(1)$ & $-1(1)$ & $7(1)$ & 1(1) \\
\hline C(9) & $27(1)$ & $15(1)$ & $19(1)$ & $-3(1)$ & $8(1)$ & $0(1)$ \\
\hline$C(10)$ & $19(1)$ & $25(1)$ & $20(1)$ & $-2(1)$ & $4(1)$ & 1(1) \\
\hline $\mathrm{C}(11)$ & $19(1)$ & $28(1)$ & $17(1)$ & $-2(1)$ & $1(1)$ & $-3(1)$ \\
\hline$C(12)$ & $18(1)$ & $26(1)$ & 21(1) & $2(1)$ & $3(1)$ & $-2(1)$ \\
\hline $\mathrm{C}(13)$ & $16(1)$ & $24(2)$ & $18(1)$ & $-2(1)$ & $5(1)$ & $0(1)$ \\
\hline$C(14)$ & $21(1)$ & $31(2)$ & $24(1)$ & $-2(1)$ & $3(1)$ & $3(1)$ \\
\hline$C(15)$ & $20(1)$ & $32(2)$ & $29(2)$ & $8(1)$ & $3(1)$ & $3(1)$ \\
\hline$C(16)$ & $15(1)$ & $45(2)$ & $17(1)$ & $0(1)$ & $2(1)$ & $2(1)$ \\
\hline $\mathrm{C}(17)$ & $19(1)$ & $27(2)$ & $17(1)$ & $-2(1)$ & $4(1)$ & $0(1)$ \\
\hline$C(18)$ & $23(1)$ & $37(2)$ & $16(1)$ & $-3(1)$ & $3(1)$ & $-1(1)$ \\
\hline C(19) & $22(1)$ & $17(1)$ & $18(1)$ & $1(1)$ & $3(1)$ & $-1(1)$ \\
\hline$C(20)$ & $31(1)$ & $19(1)$ & $18(1)$ & $0(1)$ & $3(1)$ & $-1(1)$ \\
\hline$C(21)$ & $34(2)$ & $26(2)$ & $16(1)$ & $1(1)$ & $-2(1)$ & $-1(1)$ \\
\hline$C(22)$ & $24(1)$ & $30(2)$ & $23(1)$ & $0(1)$ & $-4(1)$ & 1(1) \\
\hline$C(23)$ & $22(1)$ & $26(1)$ & $22(1)$ & $0(1)$ & $1(1)$ & 1(1) \\
\hline$C(24)$ & $22(1)$ & $16(1)$ & $17(1)$ & $0(1)$ & $1(1)$ & $0(1)$ \\
\hline$C(25)$ & $21(1)$ & $14(1)$ & $17(1)$ & $-2(1)$ & $5(1)$ & $0(1)$ \\
\hline$O(1 \mathrm{~A})$ & $18(1)$ & $37(1)$ & $16(1)$ & $-2(1)$ & $2(1)$ & $0(1)$ \\
\hline $\mathrm{O}(2 \mathrm{~A})$ & $21(1)$ & $31(2)$ & $14(1)$ & $-1(1)$ & $3(1)$ & $0(1)$ \\
\hline $0(3 A)$ & $17(1)$ & $77(4)$ & $19(1)$ & $-3(1)$ & $4(1)$ & $-1(1)$ \\
\hline $\mathrm{O}(4 \mathrm{~A})$ & $16(1)$ & $32(1)$ & $15(1)$ & $-1(1)$ & $4(1)$ & $3(1)$ \\
\hline$N(1 \mathrm{~A})$ & $28(1)$ & $26(1)$ & $17(1)$ & $-3(1)$ & $4(1)$ & $-1(1)$ \\
\hline $\mathrm{N}(2 \mathrm{~A})$ & $18(1)$ & $34(1)$ & $14(1)$ & $-1(1)$ & $2(1)$ & $-1(1)$ \\
\hline$C(1 \mathrm{~A})$ & $34(2)$ & $28(2)$ & $17(1)$ & $-4(1)$ & 1(1) & $0(1)$ \\
\hline $\mathrm{C}(2 \mathrm{~A})$ & $46(2)$ & $30(2)$ & $14(1)$ & $-2(1)$ & $6(1)$ & $0(1)$ \\
\hline$C(3 A)$ & $45(2)$ & $23(2)$ & $23(1)$ & $-2(1)$ & $19(1)$ & $-2(1)$ \\
\hline$C(4 A)$ & $31(2)$ & $18(2)$ & $23(1)$ & $-2(1)$ & $11(1)$ & $-1(1)$ \\
\hline$C(5 A)$ & $26(1)$ & $29(2)$ & $29(2)$ & $0(1)$ & $16(1)$ & $-1(1)$ \\
\hline$C(6 A)$ & $20(1)$ & $28(2)$ & $32(2)$ & $-1(1)$ & $8(1)$ & $0(1)$ \\
\hline$C(7 A)$ & $23(1)$ & $17(1)$ & 21(1) & $-2(1)$ & $4(1)$ & $0(1)$ \\
\hline $\mathrm{C}(8 \mathrm{~A})$ & $20(1)$ & $20(1)$ & $17(1)$ & $-1(1)$ & $7(1)$ & 1(1) \\
\hline $\mathrm{C}(9 \mathrm{~A})$ & $27(1)$ & $15(1)$ & $19(1)$ & $-3(1)$ & $8(1)$ & $0(1)$ \\
\hline $\mathrm{C}(10 \mathrm{~A})$ & $19(1)$ & $25(1)$ & $20(1)$ & $-2(1)$ & $4(1)$ & $1(1)$ \\
\hline $\mathrm{C}(11 \mathrm{~A})$ & $19(1)$ & $28(1)$ & $17(1)$ & $-2(1)$ & $1(1)$ & $-3(1)$ \\
\hline $\mathrm{C}(12 \mathrm{~A})$ & $18(1)$ & $26(1)$ & 21(1) & $2(1)$ & $3(1)$ & $-2(1)$ \\
\hline
\end{tabular}




\begin{tabular}{lllllll}
$\mathrm{C}(13 \mathrm{~A})$ & $16(1)$ & $24(2)$ & $18(1)$ & $-2(1)$ & $5(1)$ & $0(1)$ \\
$\mathrm{C}(14 \mathrm{~A})$ & $21(1)$ & $31(2)$ & $24(1)$ & $-2(1)$ & $3(1)$ & $3(1)$ \\
$\mathrm{C}(15 \mathrm{~A})$ & $20(1)$ & $32(2)$ & $29(2)$ & $8(1)$ & $3(1)$ & $3(1)$ \\
$\mathrm{C}(16 \mathrm{~A})$ & $15(1)$ & $45(2)$ & $17(1)$ & $0(1)$ & $2(1)$ & $2(1)$ \\
$\mathrm{C}(17 \mathrm{~A})$ & $19(1)$ & $27(2)$ & $17(1)$ & $-2(1)$ & $4(1)$ & $0(1)$ \\
$\mathrm{C}(18 \mathrm{~A})$ & $23(1)$ & $37(2)$ & $16(1)$ & $-3(1)$ & $3(1)$ & $-1(1)$ \\
$\mathrm{C}(19 \mathrm{~A})$ & $22(1)$ & $17(1)$ & $18(1)$ & $1(1)$ & $3(1)$ & $-1(1)$ \\
$\mathrm{C}(20 \mathrm{~A})$ & $31(1)$ & $19(1)$ & $18(1)$ & $0(1)$ & $3(1)$ & $-1(1)$ \\
$\mathrm{C}(21 \mathrm{~A})$ & $34(2)$ & $26(2)$ & $16(1)$ & $1(1)$ & $-2(1)$ & $-1(1)$ \\
$\mathrm{C}(22 \mathrm{~A})$ & $24(1)$ & $30(2)$ & $23(1)$ & $0(1)$ & $-4(1)$ & $1(1)$ \\
$\mathrm{C}(23 \mathrm{~A})$ & $22(1)$ & $26(1)$ & $22(1)$ & $0(1)$ & $1(1)$ & $1(1)$ \\
$\mathrm{C}(24 \mathrm{~A})$ & $22(1)$ & $16(1)$ & $17(1)$ & $0(1)$ & $1(1)$ & $0(1)$ \\
$\mathrm{C}(25 \mathrm{~A})$ & $21(1)$ & $14(1)$ & $17(1)$ & $-2(1)$ & $5(1)$ & $0(1)$ \\
\hline
\end{tabular}


Table S6. Hydrogen coordinates ( $\left.\times 10^{4}\right)$ and isotropic displacement parameters $\left(\AA^{2} \times 10^{3}\right)$ for Engle14-KY14A.

\begin{tabular}{|c|c|c|c|c|}
\hline & $\mathrm{x}$ & $\mathrm{y}$ & $\mathrm{z}$ & $\mathrm{U}(\mathrm{eq})$ \\
\hline $\mathrm{H}(4)$ & 7591 & 6661 & 7858 & 31 \\
\hline $\mathrm{H}(2)$ & 6255 & 6552 & 5912 & 26 \\
\hline $\mathrm{H}(1)$ & 5520 & 6484 & 4344 & 32 \\
\hline $\mathrm{H}(2 \mathrm{~A})$ & 6674 & 6841 & 3757 & 36 \\
\hline $\mathrm{H}(3)$ & 8330 & 7140 & 4121 & 35 \\
\hline $\mathrm{H}(5)$ & 9645 & 7348 & 4993 & 32 \\
\hline $\mathrm{H}(6)$ & 10008 & 7384 & 5942 & 32 \\
\hline $\mathrm{H}(7)$ & 8769 & 7083 & 6495 & 24 \\
\hline $\mathrm{H}(11)$ & 5603 & 4420 & 6609 & 26 \\
\hline $\mathrm{H}(12 \mathrm{~A})$ & 6325 & 4646 & 7565 & 26 \\
\hline $\mathrm{H}(12 \mathrm{~B})$ & 5150 & 4878 & 7469 & 26 \\
\hline$H(13)$ & 6451 & 8211 & 7554 & 23 \\
\hline $\mathrm{H}(14 \mathrm{~A})$ & 5039 & 10137 & 7660 & 30 \\
\hline $\mathrm{H}(14 \mathrm{~B})$ & 4377 & 8177 & 7539 & 30 \\
\hline$H(15 A)$ & 4368 & 9918 & 6698 & 32 \\
\hline$H(15 B)$ & 5546 & 9887 & 6787 & 32 \\
\hline$H(16 A)$ & 4980 & 7449 & 6150 & 31 \\
\hline$H(16 B)$ & 4286 & 6533 & 6549 & 31 \\
\hline $\mathrm{H}(20)$ & 6594 & 7114 & 10252 & 27 \\
\hline $\mathrm{H}(21)$ & 8254 & 6776 & 10627 & 31 \\
\hline $\mathrm{H}(22)$ & 9441 & 6456 & 10056 & 32 \\
\hline $\mathrm{H}(23)$ & 8978 & 6439 & 9101 & 28 \\
\hline $\mathrm{H}(4 \mathrm{~A})$ & 7570 & 8218 & 7848 & 31 \\
\hline $\mathrm{H}(2 \mathrm{AA})$ & 6251 & 8263 & 5909 & 26 \\
\hline $\mathrm{H}(1 \mathrm{~A})$ & 5634 & 7741 & 4326 & 32 \\
\hline $\mathrm{H}(2 \mathrm{AB})$ & 6873 & 8324 & 3779 & 36 \\
\hline $\mathrm{H}(3 \mathrm{~A})$ & 8466 & 7415 & 4180 & 35 \\
\hline $\mathrm{H}(5 \mathrm{~A})$ & 9735 & 7778 & 5071 & 32 \\
\hline $\mathrm{H}(6 \mathrm{~A})$ & 10038 & 7803 & 6023 & 32 \\
\hline $\mathrm{H}(7 \mathrm{~A})$ & 8708 & 7624 & 6528 & 24 \\
\hline $\mathrm{H}(11 \mathrm{~A})$ & 5548 & 9928 & 6611 & 26 \\
\hline $\mathrm{H}(12 \mathrm{C})$ & 5149 & 9512 & 7482 & 26 \\
\hline$H(12 D)$ & 6323 & 9755 & 7561 & 26 \\
\hline $\mathrm{H}(13 \mathrm{~A})$ & 6457 & 6173 & 7563 & 23 \\
\hline $\mathrm{H}(14 \mathrm{C})$ & 4386 & 6257 & 7579 & 30 \\
\hline $\mathrm{H}(14 \mathrm{D})$ & 5040 & 4288 & 7700 & 30 \\
\hline $\mathrm{H}(15 \mathrm{C})$ & 5491 & 4418 & 6817 & 32 \\
\hline$H(15 D)$ & 4315 & 4476 & 6745 & 32 \\
\hline$H(16 C)$ & 4267 & 7791 & 6550 & 31 \\
\hline$H(16 D)$ & 4981 & 6814 & 6173 & 31 \\
\hline$H(20 A)$ & 6588 & 7696 & 10242 & 27 \\
\hline $\mathrm{H}(21 \mathrm{~A})$ & 8237 & 8237 & 10615 & 31 \\
\hline $\mathrm{H}(22 \mathrm{~A})$ & 9438 & 8280 & 10048 & 32 \\
\hline $\mathrm{H}(23 \mathrm{~A})$ & 8985 & 8282 & 9091 & 28 \\
\hline
\end{tabular}


Table S7. Hydrogen bonds for Engle14-KY14A [Å and $\left.{ }^{\circ}\right]$.

\begin{tabular}{lcccc}
\hline $\mathrm{D}-\mathrm{H} . . . \mathrm{A}$ & $\mathrm{d}(\mathrm{D}-\mathrm{H})$ & $\mathrm{d}(\mathrm{H} \ldots \mathrm{A})$ & $\mathrm{d}(\mathrm{D} \ldots \mathrm{A})$ & $<(\mathrm{DHA})$ \\
\hline $\mathrm{O}(4)-\mathrm{H}(4) \ldots \mathrm{O}(1)$ & 0.84 & 2.00 & $2.795(3)$ & 158.0
\end{tabular}




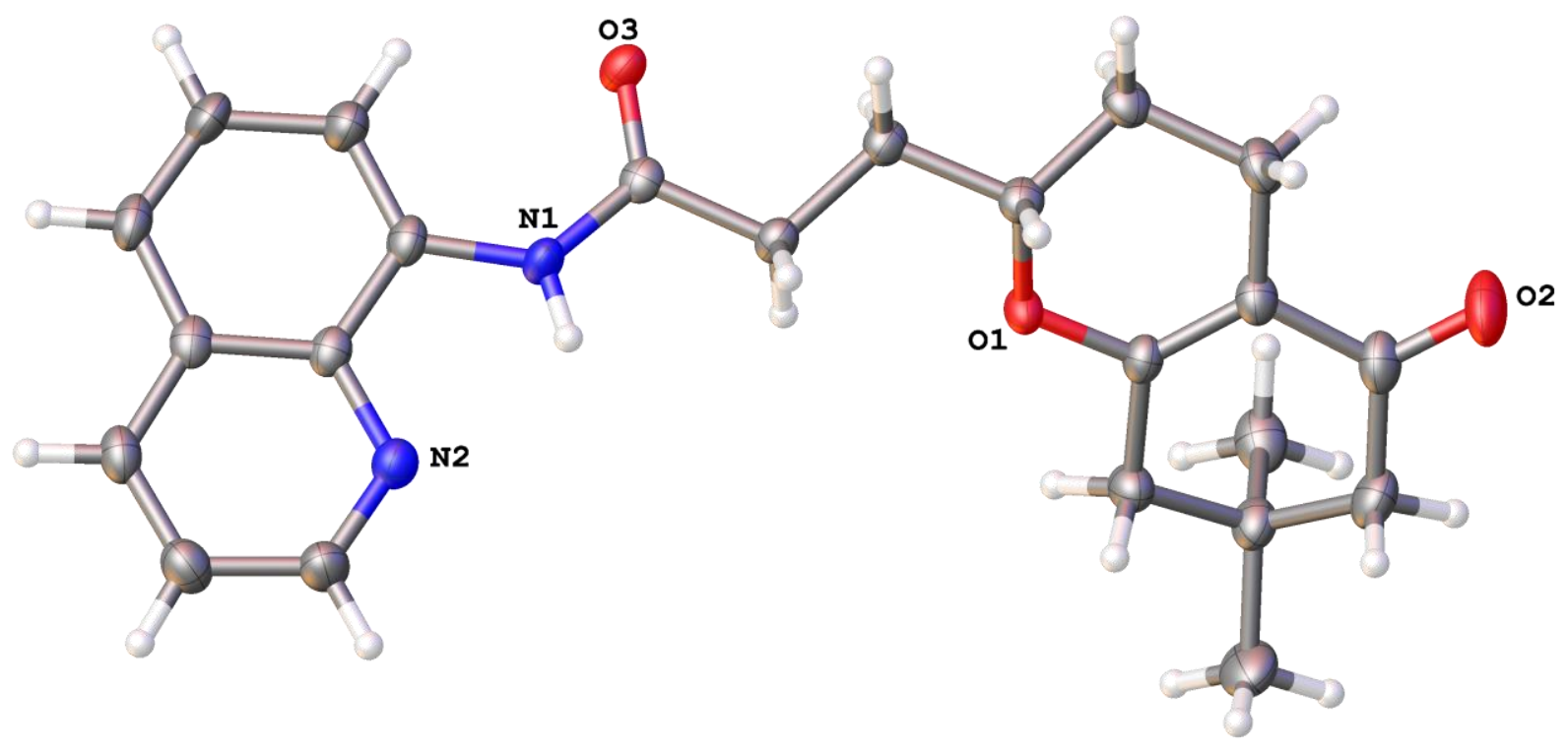

Table S8. Crystal data and structure refinement for engle12.

Identification code

Empirical formula

Formula weight

Temperature

Wavelength

Crystal system

Space group

Unit cell dimensions

Volume

Z

Density (calculated)

Absorption coefficient

F(000)

Crystal size

Theta range for data collection

Index ranges

Reflections collected

Independent reflections

Completeness to theta $=25.242^{\circ}$

Absorption correction

Max. and min. transmission

Refinement method

Data / restraints / parameters

Goodness-of-fit on $\mathrm{F}^{2}$

Final R indices [I>2sigma(I)]

$\mathrm{R}$ indices (all data)

Extinction coefficient

Largest diff. peak and hole
KY11B

C23 H26 N2 03

378.46

$100.0 \mathrm{~K}$

$0.71073 \AA$

Monoclinic

P 21/c

$\mathrm{a}=13.9598(8) \AA$

$\alpha=90^{\circ}$.

$\beta=90.919(2)^{\circ}$.

$\mathrm{b}=14.3552(8) \AA$

$c=9.9380(4) \AA$

1991.28(18) $\AA^{3}$

4

$1.262 \mathrm{Mg} / \mathrm{m}^{3}$

$0.084 \mathrm{~mm}^{-1}$

808

$0.33 \times 0.22 \times 0.22 \mathrm{~mm}^{3}$

2.035 to $26.403^{\circ}$.

$-17<=\mathrm{h}<=17,-17<=\mathrm{k}<=17,-10<=\mathrm{l}<=12$

13906

$4063[\mathrm{R}$ (int) $=0.0576]$

$99.9 \%$

Semi-empirical from equivalents

0.1660 and 0.1381

Full-matrix least-squares on $\mathrm{F}^{2}$

4063 / 0 / 255

1.019

$\mathrm{R} 1=0.0469, \mathrm{wR} 2=0.1048$

$\mathrm{R} 1=0.0712, \mathrm{wR} 2=0.1186$

$\mathrm{n} / \mathrm{a}$

0.207 and -0.212 e. $\AA^{-3}$ 
Table S9. Atomic coordinates $\left(\times 10^{4}\right)$ and equivalent isotropic displacement parameters $\left(\AA^{2} \times 10^{3}\right)$ for engle12. $U(\mathrm{eq})$ is defined as one third of the trace of the orthogonalized $U^{i j}$ tensor.

\begin{tabular}{|c|c|c|c|}
\hline & $\mathrm{x}$ & $\mathrm{y}$ & $\mathrm{z}$ \\
\hline $\mathrm{O}(1)$ & $8116(1)$ & $903(1)$ & $3248(1)$ \\
\hline $\mathrm{O}(2)$ & $10130(1)$ & $-1728(1)$ & $2916(2)$ \\
\hline$O(3)$ & $6472(1)$ & $3434(1)$ & $1018(1)$ \\
\hline$N(1)$ & $6674(1)$ & $4186(1)$ & $3012(1)$ \\
\hline$N(2)$ & $6510(1)$ & $5265(1)$ & $5159(1)$ \\
\hline$C(1)$ & $7556(1)$ & $-1797(1)$ & $4556(2)$ \\
\hline $\mathrm{C}(2)$ & $8284(1)$ & $-1221(1)$ & $5354(2)$ \\
\hline $\mathrm{C}(3)$ & $8153(1)$ & $-175(1)$ & $5036(2)$ \\
\hline$C(4)$ & $8515(1)$ & $92(1)$ & $3678(2)$ \\
\hline$C(5)$ & $8556(1)$ & $1346(1)$ & $2082(2)$ \\
\hline$C(6)$ & $7856(1)$ & $2077(1)$ & $1589(2)$ \\
\hline$C(7)$ & $7575(1)$ & $2779(1)$ & $2653(2)$ \\
\hline$C(8)$ & $6858(1)$ & $3489(1)$ & $2129(2)$ \\
\hline$C(9)$ & $6022(1)$ & $4928(1)$ & $2900(2)$ \\
\hline$C(10)$ & $5956(1)$ & $5496(1)$ & $4069(2)$ \\
\hline$C(11)$ & 5321(1) & $6269(1)$ & $4064(2)$ \\
\hline$C(12)$ & $5276(1)$ & $6804(1)$ & $5253(2)$ \\
\hline$C(13)$ & $5829(1)$ & $6560(1)$ & $6335(2)$ \\
\hline$C(14)$ & $8138(2)$ & $-1386(2)$ & $6860(2)$ \\
\hline$C(15)$ & $8785(1)$ & $636(1)$ & $1026(2)$ \\
\hline$C(16)$ & $9449(1)$ & $-118(1)$ & $1578(2)$ \\
\hline$C(17)$ & $9155(1)$ & $-399(1)$ & $2971(2)$ \\
\hline$C(18)$ & $9564(1)$ & $-1246(1)$ & $3552(2)$ \\
\hline$C(19)$ & $9304(1)$ & $-1496(1)$ & $4976(2)$ \\
\hline$C(20)$ & $6438(1)$ & 5781(1) & $6246(2)$ \\
\hline$C(21)$ & $4758(1)$ & $6451(1)$ & $2898(2)$ \\
\hline$C(22)$ & $4831(1)$ & $5891(1)$ & $1801(2)$ \\
\hline$C(23)$ & $5462(1)$ & $5124(1)$ & $1787(2)$ \\
\hline
\end{tabular}

$$
\begin{aligned}
& U(e q) \\
& 24(1) \\
& 47(1) \\
& 27(1) \\
& 26(1) \\
& 28(1) \\
& 38(1) \\
& 30(1) \\
& 29(1) \\
& 23(1) \\
& 24(1) \\
& 24(1) \\
& 23(1) \\
& 21(1) \\
& 24(1) \\
& 23(1) \\
& 24(1) \\
& 29(1) \\
& 34(1) \\
& 45(1) \\
& 28(1) \\
& 31(1) \\
& 26(1) \\
& 31(1) \\
& 35(1) \\
& 32(1) \\
& 29(1) \\
& 33(1) \\
& 31(1)
\end{aligned}
$$


Table S10. Bond lengths $[\AA ̊]$ and angles $\left[{ }^{\circ}\right]$ for engle12.

\begin{tabular}{|c|c|}
\hline $\mathrm{O}(1)-\mathrm{C}(4)$ & $1.3555(19)$ \\
\hline $\mathrm{O}(1)-\mathrm{C}(5)$ & $1.4660(19)$ \\
\hline $\mathrm{O}(2)-\mathrm{C}(18)$ & $1.232(2)$ \\
\hline $\mathrm{O}(3)-\mathrm{C}(8)$ & $1.2241(19)$ \\
\hline $\mathrm{N}(1)-\mathrm{H}(1)$ & 0.8800 \\
\hline$N(1)-C(8)$ & $1.357(2)$ \\
\hline $\mathrm{N}(1)-\mathrm{C}(9)$ & $1.406(2)$ \\
\hline$N(2)-C(10)$ & $1.362(2)$ \\
\hline$N(2)-C(20)$ & $1.315(2)$ \\
\hline $\mathrm{C}(1)-\mathrm{H}(1 \mathrm{~A})$ & 0.9800 \\
\hline $\mathrm{C}(1)-\mathrm{H}(1 \mathrm{~B})$ & 0.9800 \\
\hline $\mathrm{C}(1)-\mathrm{H}(1 \mathrm{C})$ & 0.9800 \\
\hline$C(1)-C(2)$ & $1.523(3)$ \\
\hline$C(2)-C(3)$ & $1.544(2)$ \\
\hline$C(2)-C(14)$ & $1.533(2)$ \\
\hline$C(2)-C(19)$ & $1.530(3)$ \\
\hline $\mathrm{C}(3)-\mathrm{H}(3 \mathrm{~A})$ & 0.9900 \\
\hline $\mathrm{C}(3)-\mathrm{H}(3 \mathrm{~B})$ & 0.9900 \\
\hline$C(3)-C(4)$ & $1.499(2)$ \\
\hline$C(4)-C(17)$ & $1.346(2)$ \\
\hline $\mathrm{C}(5)-\mathrm{H}(5)$ & 1.0000 \\
\hline$C(5)-C(6)$ & $1.510(2)$ \\
\hline$C(5)-C(15)$ & $1.501(2)$ \\
\hline $\mathrm{C}(6)-\mathrm{H}(6 \mathrm{~A})$ & 0.9900 \\
\hline $\mathrm{C}(6)-\mathrm{H}(6 \mathrm{~B})$ & 0.9900 \\
\hline$C(6)-C(7)$ & $1.517(2)$ \\
\hline $\mathrm{C}(7)-\mathrm{H}(7 \mathrm{~A})$ & 0.9900 \\
\hline $\mathrm{C}(7)-\mathrm{H}(7 \mathrm{~B})$ & 0.9900 \\
\hline$C(7)-C(8)$ & $1.514(2)$ \\
\hline $\mathrm{C}(9)-\mathrm{C}(10)$ & $1.423(2)$ \\
\hline$C(9)-C(23)$ & $1.374(2)$ \\
\hline$C(10)-C(11)$ & $1.420(2)$ \\
\hline$C(11)-C(12)$ & $1.411(2)$ \\
\hline$C(11)-C(21)$ & $1.414(2)$ \\
\hline $\mathrm{C}(12)-\mathrm{H}(12)$ & 0.9500 \\
\hline$C(12)-C(13)$ & $1.360(3)$ \\
\hline $\mathrm{C}(13)-\mathrm{H}(13)$ & 0.9500 \\
\hline$C(13)-C(20)$ & $1.408(3)$ \\
\hline $\mathrm{C}(14)-\mathrm{H}(14 \mathrm{~A})$ & 0.9800 \\
\hline $\mathrm{C}(14)-\mathrm{H}(14 \mathrm{~B})$ & 0.9800 \\
\hline $\mathrm{C}(14)-\mathrm{H}(14 \mathrm{C})$ & 0.9800 \\
\hline $\mathrm{C}(15)-\mathrm{H}(15 \mathrm{~A})$ & 0.9900 \\
\hline $\mathrm{C}(15)-\mathrm{H}(15 \mathrm{~B})$ & 0.9900 \\
\hline$C(15)-C(16)$ & $1.521(2)$ \\
\hline $\mathrm{C}(16)-\mathrm{H}(16 \mathrm{~A})$ & 0.9900 \\
\hline $\mathrm{C}(16)-\mathrm{H}(16 \mathrm{~B})$ & 0.9900 \\
\hline$C(16)-C(17)$ & $1.505(2)$ \\
\hline$C(17)-C(18)$ & $1.459(2)$ \\
\hline$C(18)-C(19)$ & $1.509(3)$ \\
\hline $\mathrm{C}(19)-\mathrm{H}(19 \mathrm{~A})$ & 0.9900 \\
\hline $\mathrm{C}(19)-\mathrm{H}(19 \mathrm{~B})$ & 0.9900 \\
\hline $\mathrm{C}(20)-\mathrm{H}(20)$ & 0.9500 \\
\hline
\end{tabular}




\begin{tabular}{|c|c|}
\hline $\mathrm{C}(21)-\mathrm{H}(21)$ & 0.9500 \\
\hline$C(21)-C(22)$ & $1.360(2)$ \\
\hline $\mathrm{C}(22)-\mathrm{H}(22)$ & 0.9500 \\
\hline$C(22)-C(23)$ & $1.409(2)$ \\
\hline $\mathrm{C}(23)-\mathrm{H}(23)$ & 0.9500 \\
\hline$C(4)-O(1)-C(5)$ & $116.56(12)$ \\
\hline $\mathrm{C}(8)-\mathrm{N}(1)-\mathrm{H}(1)$ & 115.2 \\
\hline$C(8)-N(1)-C(9)$ & $129.51(14)$ \\
\hline $\mathrm{C}(9)-\mathrm{N}(1)-\mathrm{H}(1)$ & 115.2 \\
\hline$C(20)-N(2)-C(10)$ & $117.78(15)$ \\
\hline $\mathrm{H}(1 \mathrm{~A})-\mathrm{C}(1)-\mathrm{H}(1 \mathrm{~B})$ & 109.5 \\
\hline $\mathrm{H}(1 \mathrm{~A})-\mathrm{C}(1)-\mathrm{H}(1 \mathrm{C})$ & 109.5 \\
\hline $\mathrm{H}(1 \mathrm{~B})-\mathrm{C}(1)-\mathrm{H}(1 \mathrm{C})$ & 109.5 \\
\hline$C(2)-C(1)-H(1 A)$ & 109.5 \\
\hline $\mathrm{C}(2)-\mathrm{C}(1)-\mathrm{H}(1 \mathrm{~B})$ & 109.5 \\
\hline $\mathrm{C}(2)-\mathrm{C}(1)-\mathrm{H}(1 \mathrm{C})$ & 109.5 \\
\hline$C(1)-C(2)-C(3)$ & $110.22(15)$ \\
\hline$C(1)-C(2)-C(14)$ & $109.03(16)$ \\
\hline$C(1)-C(2)-C(19)$ & $110.35(15)$ \\
\hline$C(14)-C(2)-C(3)$ & $109.48(15)$ \\
\hline$C(19)-C(2)-C(3)$ & $107.96(15)$ \\
\hline$C(19)-C(2)-C(14)$ & $109.78(16)$ \\
\hline $\mathrm{C}(2)-\mathrm{C}(3)-\mathrm{H}(3 \mathrm{~A})$ & 109.0 \\
\hline $\mathrm{C}(2)-\mathrm{C}(3)-\mathrm{H}(3 \mathrm{~B})$ & 109.0 \\
\hline $\mathrm{H}(3 \mathrm{~A})-\mathrm{C}(3)-\mathrm{H}(3 \mathrm{~B})$ & 107.8 \\
\hline$C(4)-C(3)-C(2)$ & $113.11(14)$ \\
\hline $\mathrm{C}(4)-\mathrm{C}(3)-\mathrm{H}(3 \mathrm{~A})$ & 109.0 \\
\hline $\mathrm{C}(4)-\mathrm{C}(3)-\mathrm{H}(3 \mathrm{~B})$ & 109.0 \\
\hline $\mathrm{O}(1)-\mathrm{C}(4)-\mathrm{C}(3)$ & $111.17(14)$ \\
\hline$C(17)-C(4)-O(1)$ & $123.90(15)$ \\
\hline$C(17)-C(4)-C(3)$ & $124.93(15)$ \\
\hline $\mathrm{O}(1)-\mathrm{C}(5)-\mathrm{H}(5)$ & 108.9 \\
\hline$O(1)-C(5)-C(6)$ & $106.32(13)$ \\
\hline $\mathrm{O}(1)-\mathrm{C}(5)-\mathrm{C}(15)$ & $110.73(13)$ \\
\hline $\mathrm{C}(6)-\mathrm{C}(5)-\mathrm{H}(5)$ & 108.9 \\
\hline $\mathrm{C}(15)-\mathrm{C}(5)-\mathrm{H}(5)$ & 108.9 \\
\hline$C(15)-C(5)-C(6)$ & $112.91(14)$ \\
\hline$C(5)-C(6)-H(6 A)$ & 108.7 \\
\hline$C(5)-C(6)-H(6 B)$ & 108.7 \\
\hline$C(5)-C(6)-C(7)$ & $114.14(13)$ \\
\hline$H(6 A)-C(6)-H(6 B)$ & 107.6 \\
\hline$C(7)-C(6)-H(6 A)$ & 108.7 \\
\hline $\mathrm{C}(7)-\mathrm{C}(6)-\mathrm{H}(6 \mathrm{~B})$ & 108.7 \\
\hline$C(6)-C(7)-H(7 A)$ & 109.1 \\
\hline $\mathrm{C}(6)-\mathrm{C}(7)-\mathrm{H}(7 \mathrm{~B})$ & 109.1 \\
\hline$H(7 A)-C(7)-H(7 B)$ & 107.8 \\
\hline$C(8)-C(7)-C(6)$ & $112.63(13)$ \\
\hline $\mathrm{C}(8)-\mathrm{C}(7)-\mathrm{H}(7 \mathrm{~A})$ & 109.1 \\
\hline$C(8)-C(7)-H(7 B)$ & 109.1 \\
\hline $\mathrm{O}(3)-\mathrm{C}(8)-\mathrm{N}(1)$ & $123.06(15)$ \\
\hline $\mathrm{O}(3)-\mathrm{C}(8)-\mathrm{C}(7)$ & $123.08(15)$ \\
\hline $\mathrm{N}(1)-\mathrm{C}(8)-\mathrm{C}(7)$ & $113.86(13)$ \\
\hline$N(1)-C(9)-C(10)$ & $114.82(14)$ \\
\hline
\end{tabular}




$\begin{array}{ll}\mathrm{C}(23)-\mathrm{C}(9)-\mathrm{N}(1) & 125.42(15) \\ \mathrm{C}(23)-\mathrm{C}(9)-\mathrm{C}(10) & 119.75(16) \\ \mathrm{N}(2)-\mathrm{C}(10)-\mathrm{C}(9) & 117.80(15) \\ \mathrm{N}(2)-\mathrm{C}(10)-\mathrm{C}(11) & 122.64(15) \\ \mathrm{C}(11)-\mathrm{C}(10)-\mathrm{C}(9) & 119.56(15) \\ \mathrm{C}(12)-\mathrm{C}(11)-\mathrm{C}(10) & 117.29(15) \\ \mathrm{C}(12)-\mathrm{C}(11)-\mathrm{C}(21) & 123.64(16) \\ \mathrm{C}(21)-\mathrm{C}(11)-\mathrm{C}(10) & 119.05(15) \\ \mathrm{C}(11)-\mathrm{C}(12)-\mathrm{H}(12) & 120.3 \\ \mathrm{C}(13)-\mathrm{C}(12)-\mathrm{C}(11) & 119.31(16) \\ \mathrm{C}(13)-\mathrm{C}(12)-\mathrm{H}(12) & 120.3 \\ \mathrm{C}(12)-\mathrm{C}(13)-\mathrm{H}(13) & 120.3 \\ \mathrm{C}(12)-\mathrm{C}(13)-\mathrm{C}(20) & 119.37(17) \\ \mathrm{C}(20)-\mathrm{C}(13)-\mathrm{H}(13) & 120.3 \\ \mathrm{C}(2)-\mathrm{C}(14)-\mathrm{H}(14 \mathrm{~A}) & 109.5 \\ \mathrm{C}(2)-\mathrm{C}(14)-\mathrm{H}(14 \mathrm{~B}) & 109.5 \\ \mathrm{C}(2)-\mathrm{C}(14)-\mathrm{H}(14 \mathrm{C}) & 109.5 \\ \mathrm{H}(14 \mathrm{~A})-\mathrm{C}(14)-\mathrm{H}(14 \mathrm{~B}) & 109.5 \\ \mathrm{H}(14 \mathrm{~A})-\mathrm{C}(14)-\mathrm{H}(14 \mathrm{C}) & 109.5 \\ \mathrm{H}(14 \mathrm{~B})-\mathrm{C}(14)-\mathrm{H}(14 \mathrm{C}) & 109.5 \\ \mathrm{C}(5)-\mathrm{C}(15)-\mathrm{H}(15 \mathrm{~A}) & 109.3 \\ \mathrm{C}(5)-\mathrm{C}(15)-\mathrm{H}(15 \mathrm{~B}) & 109.3 \\ \mathrm{C}(5)-\mathrm{C}(15)-\mathrm{C}(16) & 111.50(14) \\ \mathrm{H}(15 \mathrm{~A})-\mathrm{C}(15)-\mathrm{H}(15 \mathrm{~B}) & 108.0 \\ \mathrm{C}(16)-\mathrm{C}(15)-\mathrm{H}(15 \mathrm{~A}) & 109.3 \\ \mathrm{C}(16)-\mathrm{C}(15)-\mathrm{H}(15 \mathrm{~B}) & 109.3 \\ \mathrm{C}(15)-\mathrm{C}(16)-\mathrm{H}(16 \mathrm{~A}) & 109.6 \\ \mathrm{C}(15)-\mathrm{C}(16)-\mathrm{H}(16 \mathrm{~B}) & 109.6 \\ \mathrm{H}(16 \mathrm{~A})-\mathrm{C}(16)-\mathrm{H}(16 \mathrm{~B}) & 108.1 \\ \mathrm{C}(17)-\mathrm{C}(16)-\mathrm{C}(15) & 110.44(14) \\ \mathrm{C}(17)-\mathrm{C}(16)-\mathrm{H}(16 \mathrm{~A}) & 109.6 \\ \mathrm{C}(17)-\mathrm{C}(16)-\mathrm{H}(16 \mathrm{~B}) & 109.6 \\ \mathrm{C}(4)-\mathrm{C}(17)-\mathrm{C}(16) & 122.19(15) \\ \mathrm{C}(4)-\mathrm{C}(17)-\mathrm{C}(18) & 119.29(16) \\ \mathrm{C}(18)-\mathrm{C}(17)-\mathrm{C}(16) & 118.50(15) \\ \mathrm{O}(2)-\mathrm{C}(18)-\mathrm{C}(17) & 120.98(17) \\ \mathrm{O}(2)-\mathrm{C}(18)-\mathrm{C}(19) & 120.94(16) \\ \mathrm{C}(17)-\mathrm{C}(18)-\mathrm{C}(19) & 118.04(15) \\ \mathrm{C}(2)-\mathrm{C}(19)-\mathrm{H}(19 \mathrm{~A}) & 108.7 \\ \mathrm{C}(2)-\mathrm{C}(19)-\mathrm{H}(19 \mathrm{~B}) & 108.7 \\ \mathrm{C}(18)-\mathrm{C}(19)-\mathrm{C}(2) & 114.08(15) \\ \mathrm{C}(18)-\mathrm{C}(19)-\mathrm{H}(19 \mathrm{~A}) & 108.7 \\ \mathrm{C}(18)-\mathrm{C}(19)-\mathrm{H}(19 \mathrm{~B}) & 108.7 \\ \mathrm{H}(19 \mathrm{~A})-\mathrm{C}(19)-\mathrm{H}(19 \mathrm{~B}) & 107.6 \\ \mathrm{~N}(2)-\mathrm{C}(20)-\mathrm{C}(13) & 123.60(17) \\ \mathrm{C}(11)-\mathrm{C}(20)-\mathrm{H}(20) & 118.2 \\ \mathrm{C}(22)-\mathrm{C}(21)-\mathrm{H}(21)-\mathrm{C}(11) & 118.2 \\ \mathrm{C}(22)-\mathrm{C}(21)-\mathrm{H}(21) & 120.0 \\ \mathrm{C}(21)-\mathrm{C}(22)-\mathrm{H}(22) & 120.00(16) \\ \mathrm{C}(21)-\mathrm{C}(22)-\mathrm{C}(23) & 121.66(16) \\ \mathrm{C}(23)-\mathrm{C}(22)-\mathrm{H}(22) & 119.2 \\ \mathrm{C}(9)-\mathrm{C}(23)-\mathrm{C}(22) & 119.97(16) \\ & \end{array}$


$\mathrm{C}(9)-\mathrm{C}(23)-\mathrm{H}(23) \quad 120.0$

$\mathrm{C}(22)-\mathrm{C}(23)-\mathrm{H}(23) \quad 120.0$

Symmetry transformations used to generate equivalent atoms: 
Table S11. Anisotropic displacement parameters $\left(\AA^{2} \times 1^{3}\right)$ for engle12. The anisotropic displacement factor exponent takes the form: $-2 \pi^{2}\left[h^{2} a^{* 2} U^{11}+\ldots+2 h k^{*} b^{*} U^{12}\right]$

\begin{tabular}{lcccccc}
\hline & $\mathrm{U}^{11}$ & $\mathrm{U}^{22}$ & $\mathrm{U}^{33}$ & $\mathrm{U}^{23}$ & $\mathrm{U}^{13}$ & $\mathrm{U}$ \\
& & & & & & \\
$\mathrm{O}(1)$ & $28(1)$ & $20(1)$ & $25(1)$ & $3(1)$ & $7(1)$ & $5(1)$ \\
$\mathrm{O}(2)$ & $41(1)$ & $38(1)$ & $64(1)$ & $7(1)$ & $18(1)$ & $19(1)$ \\
$\mathrm{O}(3)$ & $30(1)$ & $24(1)$ & $27(1)$ & $2(1)$ & $-6(1)$ & $3(1)$ \\
$\mathrm{N}(1)$ & $28(1)$ & $25(1)$ & $26(1)$ & $-1(1)$ & $-7(1)$ & $7(1)$ \\
$\mathrm{N}(2)$ & $26(1)$ & $27(1)$ & $29(1)$ & $1(1)$ & $-1(1)$ & $2(1)$ \\
$\mathrm{C}(1)$ & $36(1)$ & $28(1)$ & $48(1)$ & $3(1)$ & $11(1)$ & $0(1)$ \\
$\mathrm{C}(2)$ & $33(1)$ & $25(1)$ & $32(1)$ & $6(1)$ & $5(1)$ & $6(1)$ \\
$\mathrm{C}(3)$ & $35(1)$ & $25(1)$ & $27(1)$ & $1(1)$ & $4(1)$ & $4(1)$ \\
$\mathrm{C}(4)$ & $23(1)$ & $18(1)$ & $28(1)$ & $1(1)$ & $0(1)$ & $2(1)$ \\
$\mathrm{C}(5)$ & $24(1)$ & $24(1)$ & $24(1)$ & $3(1)$ & $4(1)$ & $0(1)$ \\
$\mathrm{C}(6)$ & $23(1)$ & $25(1)$ & $25(1)$ & $3(1)$ & $1(1)$ & $2(1)$ \\
$\mathrm{C}(7)$ & $24(1)$ & $21(1)$ & $24(1)$ & $1(1)$ & $-3(1)$ & $1(1)$ \\
$\mathrm{C}(8)$ & $21(1)$ & $18(1)$ & $25(1)$ & $4(1)$ & $2(1)$ & $-3(1)$ \\
$\mathrm{C}(9)$ & $24(1)$ & $19(1)$ & $30(1)$ & $5(1)$ & $2(1)$ & $3(1)$ \\
$\mathrm{C}(10)$ & $21(1)$ & $19(1)$ & $29(1)$ & $4(1)$ & $1(1)$ & $-1(1)$ \\
$\mathrm{C}(11)$ & $20(1)$ & $21(1)$ & $32(1)$ & $5(1)$ & $6(1)$ & $-1(1)$ \\
$\mathrm{C}(12)$ & $28(1)$ & $22(1)$ & $38(1)$ & $1(1)$ & $6(1)$ & $4(1)$ \\
$\mathrm{C}(13)$ & $34(1)$ & $33(1)$ & $33(1)$ & $-6(1)$ & $2(1)$ & $3(1)$ \\
$\mathrm{C}(14)$ & $61(2)$ & $38(1)$ & $38(1)$ & $15(1)$ & $8(1)$ & $10(1)$ \\
$\mathrm{C}(15)$ & $28(1)$ & $28(1)$ & $27(1)$ & $1(1)$ & $8(1)$ & $0(1)$ \\
$\mathrm{C}(16)$ & $29(1)$ & $28(1)$ & $35(1)$ & $0(1)$ & $10(1)$ & $4(1)$ \\
$\mathrm{C}(17)$ & $24(1)$ & $22(1)$ & $32(1)$ & $1(1)$ & $4(1)$ & $2(1)$ \\
$\mathrm{C}(18)$ & $24(1)$ & $25(1)$ & $45(1)$ & $1(1)$ & $5(1)$ & $4(1)$ \\
$\mathrm{C}(19)$ & $36(1)$ & $28(1)$ & $42(1)$ & $9(1)$ & $-2(1)$ & $7(1)$ \\
$\mathrm{C}(20)$ & $33(1)$ & $34(1)$ & $29(1)$ & $-4(1)$ & $-4(1)$ & $5(1)$ \\
$\mathrm{C}(21)$ & $27(1)$ & $24(1)$ & $37(1)$ & $8(1)$ & $4(1)$ & $8(1)$ \\
$\mathrm{C}(22)$ & $35(1)$ & $30(1)$ & $32(1)$ & $7(1)$ & $-6(1)$ & $10(1)$ \\
$\mathrm{C}(23)$ & $37(1)$ & $27(1)$ & $30(1)$ & $1(1)$ & $-3(1)$ & $7(1)$ \\
& & & & & & \\
\hline & & & & & &
\end{tabular}


Table S12. Hydrogen coordinates $\left(\times 10^{4}\right)$ and isotropic displacement parameters $\left(\AA^{2} \times 10^{3}\right)$ for engle12.

\begin{tabular}{|c|c|c|c|c|}
\hline & $\mathrm{x}$ & $\mathrm{y}$ & $\mathrm{z}$ & $\mathrm{U}(\mathrm{eq})$ \\
\hline $\mathrm{H}(1)$ & 7014 & 4170 & 3766 & 32 \\
\hline $\mathrm{H}(1 \mathrm{~A})$ & 7652 & -1702 & 3591 & 56 \\
\hline $\mathrm{H}(1 \mathrm{~B})$ & 6907 & -1601 & 4789 & 56 \\
\hline $\mathrm{H}(1 \mathrm{C})$ & 7641 & -2458 & 4774 & 56 \\
\hline $\mathrm{H}(3 \mathrm{~A})$ & 7464 & -17 & 5082 & 35 \\
\hline $\mathrm{H}(3 \mathrm{~B})$ & 8496 & 195 & 5732 & 35 \\
\hline $\mathrm{H}(5)$ & 9163 & 1660 & 2381 & 29 \\
\hline $\mathrm{H}(6 \mathrm{~A})$ & 7270 & 1762 & 1247 & 29 \\
\hline$H(6 B)$ & 8143 & 2415 & 826 & 29 \\
\hline $\mathrm{H}(7 \mathrm{~A})$ & 7295 & 2444 & 3424 & 28 \\
\hline $\mathrm{H}(7 \mathrm{~B})$ & 8156 & 3108 & 2982 & 28 \\
\hline $\mathrm{H}(12)$ & 4864 & 7329 & 5297 & 35 \\
\hline $\mathrm{H}(13)$ & 5805 & 6911 & 7144 & 40 \\
\hline $\mathrm{H}(14 \mathrm{~A})$ & 7474 & -1240 & 7088 & 68 \\
\hline $\mathrm{H}(14 \mathrm{~B})$ & 8574 & -985 & 7381 & 68 \\
\hline $\mathrm{H}(14 \mathrm{C})$ & 8272 & -2040 & 7075 & 68 \\
\hline $\mathrm{H}(15 \mathrm{~A})$ & 9094 & 949 & 258 & 33 \\
\hline$H(15 B)$ & 8184 & 347 & 691 & 33 \\
\hline $\mathrm{H}(16 \mathrm{~A})$ & 9426 & -667 & 976 & 37 \\
\hline $\mathrm{H}(16 \mathrm{~B})$ & 10116 & 117 & 1607 & 37 \\
\hline $\mathrm{H}(19 \mathrm{~A})$ & 9760 & -1185 & 5603 & 42 \\
\hline$H(19 B)$ & 9378 & -2177 & 5096 & 42 \\
\hline $\mathrm{H}(20)$ & 6818 & 5620 & 7012 & 38 \\
\hline $\mathrm{H}(21)$ & 4329 & 6965 & 2881 & 35 \\
\hline $\mathrm{H}(22)$ & 4448 & 6019 & 1025 & 39 \\
\hline $\mathrm{H}(23)$ & 5499 & 4744 & 1007 & 37 \\
\hline
\end{tabular}


Experimental Summary for Crystal of 80

Table S13. Crystal data and structure refinement for engle01_c.

Identification code

Empirical formula

Formula weight

Temperature

Wavelength

Crystal system

Space group

Unit cell dimensions

Volume

$\mathrm{Z}$

Density (calculated)

Absorption coefficient

$\mathrm{F}(000)$

Crystal size

Theta range for data collection

Index ranges

Reflections collected

Independent reflections

Completeness to theta $=25.000^{\circ}$

Absorption correction

Max. and min. transmission

Refinement method

Data / restraints / parameters

Goodness-of-fit on $\mathrm{F}^{2}$

Final R indices [I $>2$ sigma(I)]

$\mathrm{R}$ indices (all data)

Absolute structure parameter

Extinction coefficient

Largest diff. peak and hole engle01_c

C21 H18 N2 03 Pd

452.77

$100.0 \mathrm{~K}$

$0.71073 \AA$

Monoclinic

C 1 c 1

$\mathrm{a}=13.718(3) \AA$

$\mathrm{b}=5.1398(11) \AA$

$c=24.816(5) \AA$

$1707.2(6) \AA^{3}$

4

$1.762 \mathrm{Mg} / \mathrm{m}^{3}$

$1.113 \mathrm{~mm}^{-1}$

912

$0.26 \times 0.2 \times 0.05 \mathrm{~mm}^{3}$

1.682 to $26.455^{\circ}$.

$-16<=\mathrm{h}<=16,-6<=\mathrm{k}<=6,-29<=\mathrm{l}<=31$

7374

$3164[\mathrm{R}(\mathrm{int})=0.0205]$

$99.9 \%$

Semi-empirical from equivalents

0.0932 and 0.0661

Full-matrix least-squares on $\mathrm{F}^{2}$

3164 / 2 / 252

1.088

$\mathrm{R} 1=0.0201, \mathrm{wR} 2=0.0455$

$\mathrm{R} 1=0.0209, \mathrm{wR} 2=0.0459$

$-0.005(19)$

$\mathrm{n} / \mathrm{a}$

0.253 and -0.240 e. $\AA^{-3}$ $\alpha=90^{\circ}$.

$\beta=102.657(6)^{\circ}$.

$\gamma=90^{\circ}$. 
Table S14. Atomic coordinates $\left(x 1^{4}\right)$ and equivalent isotropic displacement parameters $\left(\AA^{2} \times 10^{3}\right)$ for engle01_c. $U(e q)$ is defined as one third of the trace of the orthogonalized $U i j$ tensor.

\begin{tabular}{|c|c|c|c|}
\hline & $\mathrm{x}$ & $\mathrm{y}$ & $\mathrm{z}$ \\
\hline $\operatorname{Pd}(1)$ & $4868(1)$ & $7561(1)$ & $5106(1)$ \\
\hline$O(1)$ & $6395(2)$ & $9247(6)$ & $4458(1)$ \\
\hline $\mathrm{O}(2)$ & $5314(2)$ & $6002(5)$ & $4457(1)$ \\
\hline $\mathrm{O}(3)$ & $3668(2)$ & $11569(6)$ & $6246(1)$ \\
\hline$N(1)$ & $5781(2)$ & $5084(6)$ & $5622(1)$ \\
\hline$N(2)$ & $4539(3)$ & $8724(6)$ & $5808(1)$ \\
\hline$C(1)$ & $6341(3)$ & $3221(7)$ & $5488(2)$ \\
\hline$C(2)$ & $6927(3)$ & $1610(8)$ & $5893(2)$ \\
\hline$C(3)$ & $6917(3)$ & $1953(8)$ & $6433(2)$ \\
\hline$C(4)$ & $6305(3)$ & 3896(8) & $6592(2)$ \\
\hline$C(5)$ & $6216(4)$ & $4363(11)$ & $7134(2)$ \\
\hline$C(6)$ & $5599(5)$ & $6263(13)$ & $7239(2)$ \\
\hline$C(7)$ & $4997(4)$ & 7772(9) & $6813(2)$ \\
\hline $\mathrm{C}(8)$ & $5058(3)$ & $7373(7)$ & $6276(2)$ \\
\hline C(9) & $5726(3)$ & 5431(7) & $6167(2)$ \\
\hline$C(10)$ & $3888(3)$ & $10704(7)$ & $5832(2)$ \\
\hline$C(11)$ & $3456(3)$ & 11814(8) & $5268(2)$ \\
\hline$C(14)$ & $3657(4)$ & 8731(11) & $3879(2)$ \\
\hline$C(15)$ & $3145(5)$ & $6669(13)$ & $3630(3)$ \\
\hline$C(16)$ & $3082(5)$ & $6174(11)$ & $3081(2)$ \\
\hline C(17) & $3532(6)$ & 7741(10) & $2767(2)$ \\
\hline $\mathrm{C}(18)$ & $4041(5)$ & $9856(13)$ & $3022(2)$ \\
\hline$C(19)$ & $4095(5)$ & $10335(11)$ & $3574(2)$ \\
\hline$C(20)$ & $5974(3)$ & $7247(9)$ & $4261(2)$ \\
\hline$C(21)$ & $6186(4)$ & 5983(13) & $3747(2)$ \\
\hline$C(12)$ & $3935(5)$ & 10981(11) & $4796(2)$ \\
\hline$C(13)$ & $3519(5)$ & $8962(12)$ & $4463(3)$ \\
\hline $\mathrm{C}\left(12^{\prime}\right)$ & $3462(9)$ & $9790(20)$ & $4802(5)$ \\
\hline$C\left(13^{\prime}\right)$ & $3996(8)$ & $10270(20)$ & $4440(4)$ \\
\hline
\end{tabular}

$U($ eq)
$21(1)$
$37(1)$
$29(1)$
$31(1)$
$23(1)$
$24(1)$
$26(1)$
$31(1)$
$32(1)$
$34(1)$
$55(2)$
$64(2)$
$44(1)$
$27(1)$
$25(1)$
$23(1)$
$29(1)$
$48(1)$
$65(2)$
$64(2)$
$57(2)$
$68(2)$
$60(2)$
$33(1)$
$53(1)$
$21(1)$
$24(1)$
$24(1)$
$21(1)$

$\mathrm{U}(\mathrm{eq})$

21(1)

37(1)

(1)

23(1)

24(1)

31(1)

32(1)

34(1)

$55(2)$

64(2)

44(1)

27(1)

25(1)

23(1)

29(1)

48(1)

65(2)

$57(2)$

68(2)

33(1)

53(1)

21(1)

24(1)

21(1) 
Table S15. Bond lengths $[\AA ̊]$ and angles $\left[{ }^{\circ}\right]$ for engle01_c.

\begin{tabular}{|c|c|}
\hline $\operatorname{Pd}(1)-O(2)$ & $2.012(3)$ \\
\hline $\operatorname{Pd}(1)-N(1)$ & $2.031(3)$ \\
\hline $\mathrm{Pd}(1)-\mathrm{N}(2)$ & $1.984(4)$ \\
\hline $\operatorname{Pd}(1)-C(12)$ & $2.211(6)$ \\
\hline $\operatorname{Pd}(1)-C(13)$ & $2.279(6)$ \\
\hline $\mathrm{Pd}(1)-\mathrm{C}\left(12^{\prime}\right)$ & $2.229(10)$ \\
\hline $\operatorname{Pd}(1)-C\left(13^{\prime}\right)$ & $2.290(10)$ \\
\hline $\mathrm{O}(1)-\mathrm{C}(20)$ & $1.227(5)$ \\
\hline $\mathrm{O}(2)-\mathrm{C}(20)$ & $1.286(6)$ \\
\hline $\mathrm{O}(3)-\mathrm{C}(10)$ & $1.216(5)$ \\
\hline$N(1)-C(1)$ & $1.315(5)$ \\
\hline $\mathrm{N}(1)-\mathrm{C}(9)$ & $1.383(5)$ \\
\hline $\mathrm{N}(2)-\mathrm{C}(8)$ & $1.406(5)$ \\
\hline$N(2)-C(10)$ & $1.364(5)$ \\
\hline $\mathrm{C}(1)-\mathrm{H}(1)$ & 0.9500 \\
\hline $\mathrm{C}(1)-\mathrm{C}(2)$ & $1.409(6)$ \\
\hline $\mathrm{C}(2)-\mathrm{H}(2)$ & 0.9500 \\
\hline$C(2)-C(3)$ & $1.354(6)$ \\
\hline $\mathrm{C}(3)-\mathrm{H}(3)$ & 0.9500 \\
\hline$C(3)-C(4)$ & $1.415(6)$ \\
\hline$C(4)-C(5)$ & $1.399(6)$ \\
\hline$C(4)-C(9)$ & $1.414(5)$ \\
\hline $\mathrm{C}(5)-\mathrm{H}(5)$ & 0.9500 \\
\hline$C(5)-C(6)$ & $1.354(7)$ \\
\hline $\mathrm{C}(6)-\mathrm{H}(6)$ & 0.9500 \\
\hline$C(6)-C(7)$ & $1.422(7)$ \\
\hline $\mathrm{C}(7)-\mathrm{H}(7)$ & 0.9500 \\
\hline$C(7)-C(8)$ & $1.370(6)$ \\
\hline$C(8)-C(9)$ & $1.420(6)$ \\
\hline$C(10)-C(11)$ & $1.509(5)$ \\
\hline $\mathrm{C}(11)-\mathrm{H}(11 \mathrm{~A})$ & 0.9900 \\
\hline $\mathrm{C}(11)-\mathrm{H}(11 \mathrm{~B})$ & 0.9900 \\
\hline $\mathrm{C}(11)-\mathrm{H}(11 \mathrm{C})$ & 0.9900 \\
\hline $\mathrm{C}(11)-\mathrm{H}(11 \mathrm{D})$ & 0.9900 \\
\hline $\mathrm{C}(11)-\mathrm{C}(12)$ & $1.524(8)$ \\
\hline$C(11)-C\left(12^{\prime}\right)$ & $1.556(12)$ \\
\hline$C(14)-C(15)$ & $1.345(9)$ \\
\hline$C(14)-C(19)$ & $1.346(8)$ \\
\hline$C(14)-C(13)$ & $1.508(8)$ \\
\hline$C(14)-C\left(13^{\prime}\right)$ & $1.580(12)$ \\
\hline $\mathrm{C}(15)-\mathrm{H}(15)$ & 0.9500 \\
\hline$C(15)-C(16)$ & $1.370(8)$ \\
\hline $\mathrm{C}(16)-\mathrm{H}(16)$ & 0.9500 \\
\hline$C(16)-C(17)$ & $1.360(9)$ \\
\hline $\mathrm{C}(17)-\mathrm{H}(17)$ & 0.9500 \\
\hline $\mathrm{C}(17)-\mathrm{C}(18)$ & $1.369(8)$ \\
\hline $\mathrm{C}(18)-\mathrm{H}(18)$ & 0.9500 \\
\hline $\mathrm{C}(18)-\mathrm{C}(19)$ & $1.378(7)$ \\
\hline $\mathrm{C}(19)-\mathrm{H}(19)$ & 0.9500 \\
\hline$C(20)-C(21)$ & $1.517(6)$ \\
\hline $\mathrm{C}(21)-\mathrm{H}(21 \mathrm{~A})$ & 0.9800 \\
\hline $\mathrm{C}(21)-\mathrm{H}(21 \mathrm{~B})$ & 0.9800 \\
\hline
\end{tabular}




\begin{tabular}{|c|c|}
\hline $\mathrm{C}(21)-\mathrm{H}(21 \mathrm{C})$ & 0.9800 \\
\hline $\mathrm{C}(12)-\mathrm{H}(12)$ & 1.0000 \\
\hline$C(12)-C(13)$ & $1.371(9)$ \\
\hline $\mathrm{C}(13)-\mathrm{H}(13)$ & 1.0000 \\
\hline $\mathrm{C}\left(12^{\prime}\right)-\mathrm{H}\left(12^{\prime}\right)$ & 1.0000 \\
\hline $\mathrm{C}\left(12^{\prime}\right)-\mathrm{C}\left(13^{\prime}\right)$ & $1.302(17$ \\
\hline $\mathrm{C}\left(13^{\prime}\right)-\mathrm{H}\left(13^{\prime}\right)$ & 1.0000 \\
\hline $\mathrm{O}(2)-\mathrm{Pd}(1)-\mathrm{N}(1)$ & $90.09(13)$ \\
\hline $\mathrm{O}(2)-\mathrm{Pd}(1)-\mathrm{C}(12)$ & 107.01(19) \\
\hline$O(2)-P d(1)-C(13)$ & 84.73(19) \\
\hline $\mathrm{O}(2)-\mathrm{Pd}(1)-\mathrm{C}\left(12^{\prime}\right)$ & $109.0(3)$ \\
\hline $\mathrm{O}(2)-\mathrm{Pd}(1)-\mathrm{C}\left(13^{\prime}\right)$ & $82.3(3)$ \\
\hline$N(1)-P d(1)-C(12)$ & $161.37(18)$ \\
\hline$N(1)-P d(1)-C(13)$ & 158.91(18) \\
\hline$N(1)-P d(1)-C\left(12^{\prime}\right)$ & $154.7(3)$ \\
\hline $\mathrm{N}(1)-\mathrm{Pd}(1)-\mathrm{C}\left(13^{\prime}\right)$ & $171.6(3)$ \\
\hline$N(2)-P d(1)-O(2)$ & $172.20(15)$ \\
\hline$N(2)-P d(1)-N(1)$ & $82.14(14)$ \\
\hline$N(2)-P d(1)-C(12)$ & $80.79(19)$ \\
\hline$N(2)-P d(1)-C(13)$ & $102.3(2)$ \\
\hline$N(2)-P d(1)-C\left(12^{\prime}\right)$ & $78.2(3)$ \\
\hline$N(2)-P d(1)-C(13 ')$ & $105.5(3)$ \\
\hline$C(12)-P d(1)-C(13)$ & $35.5(2)$ \\
\hline$C\left(12^{\prime}\right)-P d(1)-C\left(13^{\prime}\right)$ & $33.4(4)$ \\
\hline$C(20)-O(2)-P d(1)$ & $117.8(3)$ \\
\hline$C(1)-N(1)-P d(1)$ & $127.7(3)$ \\
\hline $\mathrm{C}(1)-\mathrm{N}(1)-\mathrm{C}(9)$ & $120.1(3)$ \\
\hline$C(9)-N(1)-P d(1)$ & $112.2(2)$ \\
\hline $\mathrm{C}(8)-\mathrm{N}(2)-\mathrm{Pd}(1)$ & $113.8(3)$ \\
\hline $\mathrm{C}(10)-\mathrm{N}(2)-\mathrm{Pd}(1)$ & $122.8(3)$ \\
\hline $\mathrm{C}(10)-\mathrm{N}(2)-\mathrm{C}(8)$ & $123.4(4)$ \\
\hline $\mathrm{N}(1)-\mathrm{C}(1)-\mathrm{H}(1)$ & 119.3 \\
\hline$N(1)-C(1)-C(2)$ & $121.4(4)$ \\
\hline $\mathrm{C}(2)-\mathrm{C}(1)-\mathrm{H}(1)$ & 119.3 \\
\hline $\mathrm{C}(1)-\mathrm{C}(2)-\mathrm{H}(2)$ & 120.0 \\
\hline$C(3)-C(2)-C(1)$ & $120.0(4)$ \\
\hline $\mathrm{C}(3)-\mathrm{C}(2)-\mathrm{H}(2)$ & 120.0 \\
\hline $\mathrm{C}(2)-\mathrm{C}(3)-\mathrm{H}(3)$ & 119.9 \\
\hline$C(2)-C(3)-C(4)$ & $120.2(4)$ \\
\hline $\mathrm{C}(4)-\mathrm{C}(3)-\mathrm{H}(3)$ & 119.9 \\
\hline$C(5)-C(4)-C(3)$ & $124.9(4)$ \\
\hline$C(5)-C(4)-C(9)$ & $117.9(4)$ \\
\hline$C(9)-C(4)-C(3)$ & $117.2(4)$ \\
\hline $\mathrm{C}(4)-\mathrm{C}(5)-\mathrm{H}(5)$ & 120.0 \\
\hline$C(6)-C(5)-C(4)$ & $120.0(4)$ \\
\hline $\mathrm{C}(6)-\mathrm{C}(5)-\mathrm{H}(5)$ & 120.0 \\
\hline $\mathrm{C}(5)-\mathrm{C}(6)-\mathrm{H}(6)$ & 118.8 \\
\hline$C(5)-C(6)-C(7)$ & $122.4(5)$ \\
\hline $\mathrm{C}(7)-\mathrm{C}(6)-\mathrm{H}(6)$ & 118.8 \\
\hline $\mathrm{C}(6)-\mathrm{C}(7)-\mathrm{H}(7)$ & 120.2 \\
\hline $\mathrm{C}(8)-\mathrm{C}(7)-\mathrm{C}(6)$ & $119.5(4)$ \\
\hline $\mathrm{C}(8)-\mathrm{C}(7)-\mathrm{H}(7)$ & 120.2 \\
\hline $\mathrm{N}(2)-\mathrm{C}(8)-\mathrm{C}(9)$ & $114.9(4)$ \\
\hline
\end{tabular}




\begin{tabular}{|c|c|}
\hline$C(7)-C(8)-N(2)$ & $127.0(4)$ \\
\hline$C(7)-C(8)-C(9)$ & $118.0(4)$ \\
\hline$N(1)-C(9)-C(4)$ & $121.0(4)$ \\
\hline$N(1)-C(9)-C(8)$ & $116.9(3)$ \\
\hline$C(4)-C(9)-C(8)$ & $122.1(4)$ \\
\hline $\mathrm{O}(3)-\mathrm{C}(10)-\mathrm{N}(2)$ & $126.7(4)$ \\
\hline $\mathrm{O}(3)-\mathrm{C}(10)-\mathrm{C}(11)$ & $121.7(3)$ \\
\hline$N(2)-C(10)-C(11)$ & $111.6(3)$ \\
\hline$C(10)-C(11)-H(11 A)$ & 108.0 \\
\hline $\mathrm{C}(10)-\mathrm{C}(11)-\mathrm{H}(11 \mathrm{~B})$ & 108.0 \\
\hline $\mathrm{C}(10)-\mathrm{C}(11)-\mathrm{H}(11 \mathrm{C})$ & 109.2 \\
\hline$C(10)-C(11)-H(11 D)$ & 109.2 \\
\hline$C(10)-C(11)-C(12)$ & $117.3(4)$ \\
\hline$C(10)-C(11)-C(12)$ & $111.9(5)$ \\
\hline$H(11 A)-C(11)-H(11 B)$ & 107.2 \\
\hline $\mathrm{H}(11 \mathrm{C})-\mathrm{C}(11)-\mathrm{H}(11 \mathrm{D})$ & 107.9 \\
\hline$C(12)-C(11)-H(11 A)$ & 108.0 \\
\hline $\mathrm{C}(12)-\mathrm{C}(11)-\mathrm{H}(11 \mathrm{~B})$ & 108.0 \\
\hline $\mathrm{C}\left(12^{\prime}\right)-\mathrm{C}(11)-\mathrm{H}(11 \mathrm{C})$ & 109.2 \\
\hline $\mathrm{C}\left(12^{\prime}\right)-\mathrm{C}(11)-\mathrm{H}(11 \mathrm{D})$ & 109.2 \\
\hline$C(15)-C(14)-C(19)$ & $118.5(5)$ \\
\hline$C(15)-C(14)-C(13)$ & $109.9(6)$ \\
\hline$C(15)-C(14)-C\left(13^{\prime}\right)$ & $145.4(7)$ \\
\hline$C(19)-C(14)-C(13)$ & $131.2(5)$ \\
\hline$C(19)-C(14)-C\left(13^{\prime}\right)$ & $96.1(6)$ \\
\hline $\mathrm{C}(14)-\mathrm{C}(15)-\mathrm{H}(15)$ & 119.5 \\
\hline$C(14)-C(15)-C(16)$ & $121.0(6)$ \\
\hline $\mathrm{C}(16)-\mathrm{C}(15)-\mathrm{H}(15)$ & 119.5 \\
\hline$C(15)-C(16)-H(16)$ & 119.2 \\
\hline$C(17)-C(16)-C(15)$ & $121.5(5)$ \\
\hline $\mathrm{C}(17)-\mathrm{C}(16)-\mathrm{H}(16)$ & 119.2 \\
\hline $\mathrm{C}(16)-\mathrm{C}(17)-\mathrm{H}(17)$ & 121.5 \\
\hline$C(16)-C(17)-C(18)$ & $117.0(5)$ \\
\hline $\mathrm{C}(18)-\mathrm{C}(17)-\mathrm{H}(17)$ & 121.5 \\
\hline $\mathrm{C}(17)-\mathrm{C}(18)-\mathrm{H}(18)$ & 119.6 \\
\hline$C(17)-C(18)-C(19)$ & $120.9(6)$ \\
\hline $\mathrm{C}(19)-\mathrm{C}(18)-\mathrm{H}(18)$ & 119.6 \\
\hline$C(14)-C(19)-C(18)$ & $121.1(5)$ \\
\hline $\mathrm{C}(14)-\mathrm{C}(19)-\mathrm{H}(19)$ & 119.5 \\
\hline $\mathrm{C}(18)-\mathrm{C}(19)-\mathrm{H}(19)$ & 119.5 \\
\hline $\mathrm{O}(1)-\mathrm{C}(20)-\mathrm{O}(2)$ & $125.2(4)$ \\
\hline$O(1)-C(20)-C(21)$ & $122.2(5)$ \\
\hline$O(2)-C(20)-C(21)$ & $112.6(4)$ \\
\hline$C(20)-C(21)-H(21 A)$ & 109.5 \\
\hline $\mathrm{C}(20)-\mathrm{C}(21)-\mathrm{H}(21 \mathrm{~B})$ & 109.5 \\
\hline$C(20)-C(21)-H(21 C)$ & 109.5 \\
\hline$H(21 A)-C(21)-H(21 B)$ & 109.5 \\
\hline$H(21 A)-C(21)-H(21 C)$ & 109.5 \\
\hline$H(21 B)-C(21)-H(21 C)$ & 109.5 \\
\hline $\mathrm{Pd}(1)-\mathrm{C}(12)-\mathrm{H}(12)$ & 116.5 \\
\hline$C(11)-C(12)-P d(1)$ & $105.7(3)$ \\
\hline $\mathrm{C}(11)-\mathrm{C}(12)-\mathrm{H}(12)$ & 116.5 \\
\hline$C(13)-C(12)-P d(1)$ & $75.0(3)$ \\
\hline $\mathrm{C}(13)-\mathrm{C}(12)-\mathrm{C}(11)$ & $118.9(5)$ \\
\hline
\end{tabular}




$\begin{array}{ll}\mathrm{C}(13)-\mathrm{C}(12)-\mathrm{H}(12) & 116.5 \\ \mathrm{Pd}(1)-\mathrm{C}(13)-\mathrm{H}(13) & 115.0 \\ \mathrm{C}(14)-\mathrm{C}(13)-\mathrm{Pd}(1) & 113.2(4) \\ \mathrm{C}(14)-\mathrm{C}(13)-\mathrm{H}(13) & 115.0 \\ \mathrm{C}(12)-\mathrm{C}(13)-\mathrm{Pd}(1) & 69.5(3) \\ \mathrm{C}(12)-\mathrm{C}(13)-\mathrm{C}(14) & 121.0(6) \\ \mathrm{C}(12)-\mathrm{C}(13)-\mathrm{H}(13) & 115.0 \\ \mathrm{Pd}(1)-\mathrm{C}\left(12^{\prime}\right)-\mathrm{H}\left(12^{\prime}\right) & 116.8 \\ \mathrm{C}(11)-\mathrm{C}\left(12^{\prime}\right)-\mathrm{Pd}(1) & 103.7(5) \\ \mathrm{C}(11)-\mathrm{C}\left(12^{\prime}\right)-\mathrm{H}\left(12^{\prime}\right) & 116.8 \\ \mathrm{C}\left(13^{\prime}\right)-\mathrm{C}\left(12^{\prime}\right)-\mathrm{Pd}(1) & 75.8(7) \\ \mathrm{C}\left(13^{\prime}\right)-\mathrm{C}\left(12^{\prime}\right)-\mathrm{C}(11) & 118.8(10) \\ \mathrm{C}\left(13^{\prime}\right)-\mathrm{C}\left(12^{\prime}\right)-\mathrm{H}\left(12^{\prime}\right) & 116.8 \\ \mathrm{Pd}(1)-\mathrm{C}\left(13^{\prime}\right)-\mathrm{H}\left(13^{\prime}\right) & 117.7 \\ \mathrm{C}(14)-\mathrm{C}\left(13^{\prime}\right)-\mathrm{Pd}(1) & 109.7(6) \\ \mathrm{C}(14)-\mathrm{C}\left(13^{\prime}\right)-\mathrm{H}\left(13^{\prime}\right) & 117.7 \\ \mathrm{C}\left(12^{\prime}\right)-\mathrm{C}\left(13^{\prime}\right)-\mathrm{Pd}(1) & 70.7(6) \\ \mathrm{C}\left(12^{\prime}\right)-\mathrm{C}\left(13^{\prime}\right)-\mathrm{C}(14) & 114.3(10) \\ \mathrm{C}\left(12^{\prime}\right)-\mathrm{C}\left(13^{\prime}\right)-\mathrm{H}\left(13^{\prime}\right) & 117.7\end{array}$

Symmetry transformations used to generate equivalent atoms: 
Table S16. Anisotropic displacement parameters $\left(\AA^{2} \times 1^{3}\right)$ for engle01_c. The anisotropic displacement factor exponent takes the form: $-2 \pi^{2}\left[h^{2} a^{* 2} U^{11}+\ldots+2 h k^{*} b^{*} U^{12}\right]$

\begin{tabular}{|c|c|c|c|c|c|c|}
\hline & $\mathrm{U}^{11}$ & $\mathrm{U}^{22}$ & $\mathrm{U}^{33}$ & $\mathrm{U}^{23}$ & U13 & $\mathrm{U}^{12}$ \\
\hline $\operatorname{Pd}(1)$ & $25(1)$ & $19(1)$ & $21(1)$ & $0(1)$ & $7(1)$ & $1(1)$ \\
\hline $\mathrm{O}(1)$ & $27(2)$ & $55(2)$ & $31(2)$ & $-8(1)$ & $10(1)$ & $-9(1)$ \\
\hline $\mathrm{O}(2)$ & $32(2)$ & $29(2)$ & $26(2)$ & $-4(1)$ & $7(1)$ & $4(1)$ \\
\hline $\mathrm{O}(3)$ & $36(2)$ & $29(1)$ & $30(2)$ & $-2(1)$ & $12(1)$ & $4(1)$ \\
\hline $\mathrm{N}(1)$ & $20(2)$ & $21(2)$ & $28(2)$ & $-2(1)$ & $6(1)$ & $-2(1)$ \\
\hline$N(2)$ & $29(2)$ & $23(2)$ & $20(2)$ & $2(1)$ & $8(1)$ & $2(1)$ \\
\hline $\mathrm{C}(1)$ & $21(2)$ & $24(2)$ & $33(2)$ & $-2(2)$ & $7(2)$ & $-3(2)$ \\
\hline $\mathrm{C}(2)$ & $26(2)$ & $22(2)$ & $45(3)$ & $0(2)$ & $7(2)$ & $2(2)$ \\
\hline$C(3)$ & $27(2)$ & $28(2)$ & $40(2)$ & $12(2)$ & $3(2)$ & $2(2)$ \\
\hline$C(4)$ & $35(2)$ & $34(2)$ & $32(2)$ & $5(2)$ & $7(2)$ & $2(2)$ \\
\hline $\mathrm{C}(5)$ & $67(4)$ & $62(3)$ & $33(3)$ & $20(2)$ & $6(3)$ & $25(3)$ \\
\hline$C(6)$ & $96(5)$ & $79(4)$ & $17(2)$ & $12(3)$ & $15(3)$ & $37(4)$ \\
\hline$C(7)$ & $51(3)$ & $54(3)$ & $28(2)$ & $2(2)$ & $13(2)$ & $19(2)$ \\
\hline$C(8)$ & $30(2)$ & $27(2)$ & $25(2)$ & $4(2)$ & $7(2)$ & $-2(2)$ \\
\hline $\mathrm{C}(9)$ & $24(2)$ & $25(2)$ & $26(2)$ & $4(2)$ & $8(2)$ & $-1(2)$ \\
\hline$C(10)$ & $22(2)$ & $21(2)$ & $25(2)$ & $-4(2)$ & $6(2)$ & $-1(1)$ \\
\hline $\mathrm{C}(11)$ & $30(2)$ & $25(2)$ & $31(2)$ & $-1(2)$ & $8(2)$ & $9(2)$ \\
\hline$C(14)$ & $60(3)$ & $55(3)$ & $28(2)$ & $0(2)$ & $3(2)$ & $34(3)$ \\
\hline$C(15)$ & $75(4)$ & $71(4)$ & $49(3)$ & $14(3)$ & $12(3)$ & $4(4)$ \\
\hline$C(16)$ & $87(5)$ & $46(3)$ & $50(3)$ & $-6(3)$ & $-6(3)$ & $-15(3)$ \\
\hline $\mathrm{C}(17)$ & $93(5)$ & $52(3)$ & $21(2)$ & $-8(2)$ & $2(3)$ & $11(3)$ \\
\hline$C(18)$ & $90(5)$ & $73(4)$ & $41(3)$ & $6(3)$ & $14(3)$ & $-30(4)$ \\
\hline$C(19)$ & $76(4)$ & $46(3)$ & $49(3)$ & $-13(3)$ & $-6(3)$ & $-7(3)$ \\
\hline$C(20)$ & $20(2)$ & $51(3)$ & $26(2)$ & $-3(2)$ & $3(2)$ & $7(2)$ \\
\hline $\mathrm{C}(21)$ & $40(3)$ & $86(4)$ & $36(3)$ & $-22(3)$ & $14(2)$ & $1(3)$ \\
\hline $\mathrm{C}(12)$ & $24(3)$ & $14(2)$ & $25(3)$ & $4(2)$ & $6(2)$ & $0(2)$ \\
\hline$C(13)$ & $22(2)$ & $23(3)$ & $26(3)$ & $2(2)$ & $1(3)$ & $1(2)$ \\
\hline$C\left(12^{\prime}\right)$ & $22(2)$ & $23(3)$ & $26(3)$ & $2(2)$ & $1(3)$ & $1(2)$ \\
\hline$C\left(13^{\prime}\right)$ & $24(3)$ & $14(2)$ & $25(3)$ & $4(2)$ & $6(2)$ & $0(2)$ \\
\hline
\end{tabular}


Table S17. Hydrogen coordinates $\left(\times 1^{4}\right)$ and isotropic displacement parameters $\left(\AA^{2} \times 10^{3}\right)$ for engle01_c.

\begin{tabular}{|c|c|c|c|c|}
\hline & $\mathrm{x}$ & $\mathrm{y}$ & $\mathrm{Z}$ & $\mathrm{U}(\mathrm{eq})$ \\
\hline $\mathrm{H}(1)$ & 6351 & 2955 & 5110 & 31 \\
\hline $\mathrm{H}(2)$ & 7329 & 282 & 5787 & 37 \\
\hline $\mathrm{H}(3)$ & 7323 & 887 & 6705 & 39 \\
\hline $\mathrm{H}(5)$ & 6588 & 3348 & 7429 & 66 \\
\hline $\mathrm{H}(6)$ & 5568 & 6599 & 7612 & 76 \\
\hline $\mathrm{H}(7)$ & 4555 & 9049 & 6900 & 52 \\
\hline $\mathrm{H}(11 \mathrm{~A})$ & 3494 & 13734 & 5295 & 34 \\
\hline $\mathrm{H}(11 \mathrm{~B})$ & 2739 & 11342 & 5167 & 34 \\
\hline $\mathrm{H}(11 \mathrm{C})$ & 3849 & 13359 & 5206 & 34 \\
\hline $\mathrm{H}(11 \mathrm{D})$ & 2761 & 12386 & 5251 & 34 \\
\hline $\mathrm{H}(15)$ & 2821 & 5538 & 3838 & 78 \\
\hline $\mathrm{H}(16)$ & 2715 & 4703 & 2916 & 77 \\
\hline $\mathrm{H}(17)$ & 3496 & 7384 & 2387 & 68 \\
\hline $\mathrm{H}(18)$ & 4360 & 11007 & 2815 & 81 \\
\hline $\mathrm{H}(19)$ & 4448 & 11820 & 3742 & 72 \\
\hline$H(21 A)$ & 6262 & 4101 & 3804 & 79 \\
\hline $\mathrm{H}(21 \mathrm{~B})$ & 6804 & 6704 & 3671 & 79 \\
\hline $\mathrm{H}(21 \mathrm{C})$ & 5630 & 6327 & 3432 & 79 \\
\hline$H(12)$ & 4260 & 12383 & 4618 & 25 \\
\hline $\mathrm{H}(13)$ & 2858 & 8349 & 4521 & 29 \\
\hline $\mathrm{H}\left(12^{\prime}\right)$ & 2838 & 8755 & 4673 & 29 \\
\hline $\mathrm{H}\left(13^{\prime}\right)$ & 4253 & 12084 & 4421 & 25 \\
\hline
\end{tabular}




\section{Experimental Summary for Crystal of 81}

The single crystal X-ray diffraction studies were carried out on a Bruker Kappa APEX-II CCD diffractometer equipped with Mo $\mathrm{K}_{\alpha}$ radiation $(\lambda=0.71073)$. Crystals of the subject compound were used as received (grown from DMF). A $0.22 \times 0.10 \times 008$ mm light yellow needle was mounted on a Cryoloop with Paratone oil.

Data were collected in a nitrogen gas stream at 100(2) K using $\phi$ and $\varpi$ scans. Crystal-to-detector distance was $40 \mathrm{~mm}$ using exposure time 2 , with a scan width of $0.7^{\circ}$. Data collection was $99.9 \%$ complete to $25.242^{\circ}$ in $\theta$. A total of 26864 reflections were collected covering the indices, $-15<=\mathrm{h}<=15,-27<=\mathrm{k}<=27,-8<=\mathrm{l}<=8$. 3999 reflections were found to be symmetry independent, with a $\mathrm{R}_{\text {int }}$ of 0.0281 . Indexing and unit cell refinement indicated a primitive, Monoclinic lattice. The space group was found to be $\boldsymbol{P 2} \mathbf{2}_{\mathbf{1}} / \mathbf{c}$. The data were integrated using the Bruker SAINT software program and scaled using the SADABS software program. Solution by direct methods (SHELXT) produced a complete phasing model consistent with the proposed structure.

All nonhydrogen atoms were refined anisotropically by full-matrix least-squares (SHELXL-2014). All carbon bonded hydrogen atoms were placed using a riding model. Their positions were constrained relative to their parent atom using the appropriate HFIX command in SHELXL-2014.

. All other hydrogen atoms (H-bonding) were located in the difference map. There relative positions were restrained using AFIX commands and their thermals refined using riding model. Crystallographic data are summarized in Table S18.

Notes: X-ray Structure agrees to suggested model Minor disorder on the C11 \& C12 "bridge" RIGU and EADP applied.

One molecule of DMF forms hydrogen bond with O-H group details in Table S23 


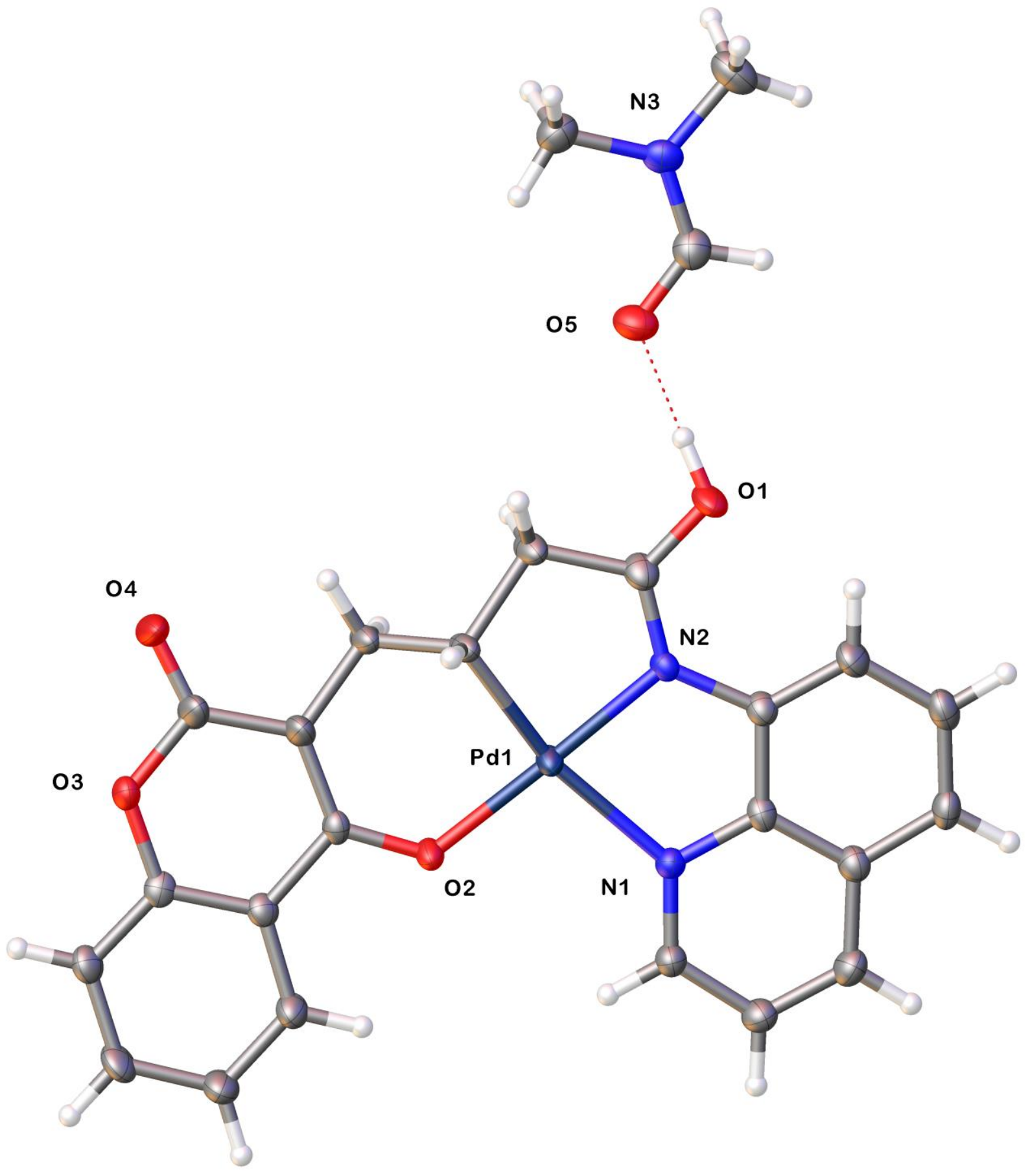


Table S18. Crystal data and structure refinement for Engle17.

\section{Report date}

Identification code

Empirical formula

Molecular formula

Formula weight

Temperature

Wavelength

Crystal system

Space group

Unit cell dimensions

Volume

Z

Density (calculated)

Absorption coefficient

$\mathrm{F}(000)$

Crystal size

Crystal color, habit

Theta range for data collection

Index ranges

Reflections collected

Independent reflections

Completeness to theta $=25.242^{\circ}$

Absorption correction

Max. and min. transmission

Refinement method

Data / restraints / parameters

Goodness-of-fit on $\mathrm{F}^{2}$

Final R indices [I $>2$ sigma(I)]

$\mathrm{R}$ indices (all data)

Extinction coefficient

Largest diff. peak and hole
2016-05-27

engle17

C25 H23 N3 O5 Pd

C22 H16 N2 O4 Pd, C3 H7 N O

551.86

$100.0 \mathrm{~K}$

$0.71073 \AA$

Monoclinic

P 1 21/c 1

$\mathrm{a}=13.1532(7) \AA$

$\mathrm{b}=22.9508(13) \AA$

$\mathrm{c}=7.4256(4) \AA$

$\alpha=90^{\circ}$.

$\beta=102.080(2)^{\circ}$.

$\gamma=90^{\circ}$.

4

$1.672 \mathrm{Mg} / \mathrm{m}^{3}$

$0.891 \mathrm{~mm}^{-1}$

1120

$0.22 \times 0.1 \times 0.08 \mathrm{~mm}^{3}$

light yellow needle

1.775 to $25.348^{\circ}$.

$-15<=\mathrm{h}<=15,-27<=\mathrm{k}<=27,-8<=\mathrm{l}<=8$

26864

3999 [R(int) $=0.0281]$

$99.9 \%$

Semi-empirical from equivalents

0.4903 and 0.4548

Full-matrix least-squares on $\mathrm{F}^{2}$

3999 / 30 / 319

1.419

$\mathrm{R} 1=0.0439, \mathrm{wR} 2=0.1009$

$\mathrm{R} 1=0.0450, \mathrm{wR} 2=0.1013$

$\mathrm{n} / \mathrm{a}$

0.737 and -0.894 e. $\AA^{-3}$ 
Table 19. Atomic coordinates $\left(x 1^{4}\right)$ and equivalent isotropic displacement parameters $\left(\AA^{2} \times 10^{3}\right)$ for Engle17. $U(\mathrm{eq})$ is defined as one third of the trace of the orthogonalized $U i j$ tensor.

\begin{tabular}{|c|c|c|c|c|}
\hline & $\mathrm{x}$ & $\mathrm{y}$ & $\mathrm{z}$ & $\mathrm{U}(\mathrm{eq})$ \\
\hline $\operatorname{Pd}(1)$ & $10404(1)$ & $1529(1)$ & $7648(1)$ & $20(1)$ \\
\hline$O(1)$ & $7694(3)$ & $847(2)$ & $8544(6)$ & $34(1)$ \\
\hline $\mathrm{O}(2)$ & $11835(2)$ & $1567(1)$ & $7054(5)$ & $25(1)$ \\
\hline$O(3)$ & $13778(2)$ & $236(1)$ & $6216(5)$ & $23(1)$ \\
\hline$O(4)$ & $12588(3)$ & $-428(2)$ & $6273(7)$ & $51(1)$ \\
\hline$N(1)$ & $10070(3)$ & $2436(2)$ & $7780(5)$ & $20(1)$ \\
\hline $\mathrm{N}(2)$ & 8991(3) & $1476(2)$ & $8156(5)$ & $17(1)$ \\
\hline $\mathrm{C}(1)$ & $10612(4)$ & $2902(2)$ & $7556(7)$ & $23(1)$ \\
\hline $\mathrm{C}(2)$ & $10249(4)$ & $3473(2)$ & $7663(7)$ & $27(1)$ \\
\hline$C(3)$ & $9290(4)$ & $3555(2)$ & $8039(7)$ & $28(1)$ \\
\hline $\mathrm{C}(4)$ & $8677(4)$ & $3072(2)$ & $8293(7)$ & $24(1)$ \\
\hline $\mathrm{C}(5)$ & $7669(4)$ & $3128(2)$ & $8688(7)$ & $26(1)$ \\
\hline$C(6)$ & $7134(4)$ & $2640(2)$ & $8952(7)$ & $28(1)$ \\
\hline C(7) & $7543(4)$ & $2080(2)$ & $8850(7)$ & $28(1)$ \\
\hline $\mathrm{C}(8)$ & $8508(4)$ & $2009(2)$ & $8425(6)$ & $22(1)$ \\
\hline$C(9)$ & $9095(3)$ & $2513(2)$ & $8160(6)$ & $21(1)$ \\
\hline$C(10)$ & $8617(4)$ & $949(2)$ & $8207(7)$ & $27(1)$ \\
\hline $\mathrm{C}(11)$ & $9305(4)$ & $466(2)$ & $7826(8)$ & $29(1)$ \\
\hline$C(14)$ & $12086(4)$ & $545(2)$ & $6733(8)$ & $27(1)$ \\
\hline$C(15)$ & $12384(3)$ & $1119(2)$ & $6792(6)$ & $18(1)$ \\
\hline$C(16)$ & $13428(4)$ & $1255(2)$ & $6498(6)$ & $20(1)$ \\
\hline $\mathrm{C}(17)$ & $13804(4)$ & $1826(2)$ & $6528(7)$ & $27(1)$ \\
\hline $\mathrm{C}(18)$ & $14801(4)$ & $1936(2)$ & $6287(8)$ & $32(1)$ \\
\hline$C(19)$ & $15437(4)$ & $1471(2)$ & $6019(7)$ & $31(1)$ \\
\hline$C(20)$ & $15085(4)$ & $904(2)$ & $5971(6)$ & $24(1)$ \\
\hline$C(21)$ & $14085(4)$ & $803(2)$ & $6223(6)$ & $20(1)$ \\
\hline$C(22)$ & $12772(4)$ & $91(2)$ & $6400(8)$ & $29(1)$ \\
\hline$C(12)$ & $10476(6)$ & $672(3)$ & $8002(13)$ & $24(2)$ \\
\hline $\mathrm{C}(13)$ & $10985(5)$ & $364(3)$ & $6635(13)$ & $25(2)$ \\
\hline$C(12 B)$ & 10168(13) & $657(7)$ & $7070(30)$ & $24(2)$ \\
\hline$C(13 B)$ & $11151(12)$ & $317(7)$ & $7720(30)$ & $25(2)$ \\
\hline$O(5)$ & $7185(3)$ & $-236(2)$ & $8370(5)$ & $36(1)$ \\
\hline $\mathrm{N}(3)$ & $6019(3)$ & $-930(2)$ & $8704(5)$ & $25(1)$ \\
\hline $\mathrm{C}(23)$ & $6320(4)$ & $-387(2)$ & $8586(7)$ & $29(1)$ \\
\hline$C(24)$ & $4964(4)$ & $-1070(3)$ & $8883(8)$ & $38(1)$ \\
\hline$C(25)$ & $6713(4)$ & $-1415(2)$ & $8596(8)$ & $34(1)$ \\
\hline
\end{tabular}


Table 20. Bond lengths $[\AA \AA]$ and angles $\left[^{\circ}\right]$ for Engle17.

\begin{tabular}{|c|c|c|c|}
\hline $\mathrm{Pd}(1)-\mathrm{O}(2)$ & $2.023(3)$ & $\mathrm{C}(12)-\mathrm{H}(12)$ & 1.0000 \\
\hline $\operatorname{Pd}(1)-N(1)$ & $2.134(4)$ & $C(12)-C(13)$ & $1.503(12)$ \\
\hline $\operatorname{Pd}(1)-\mathrm{N}(2)$ & $1.976(4)$ & $C(13)-H(13 A)$ & 0.9900 \\
\hline $\operatorname{Pd}(1)-C(12)$ & $1.986(7)$ & $\mathrm{C}(13)-\mathrm{H}(13 \mathrm{~B})$ & 0.9900 \\
\hline $\mathrm{Pd}(1)-\mathrm{C}(12 \mathrm{~B})$ & $2.058(17)$ & $\mathrm{C}(12 \mathrm{~B})-\mathrm{H}(12 \mathrm{~B})$ & 1.0000 \\
\hline $\mathrm{O}(1)-\mathrm{C}(10)$ & $1.311(6)$ & $C(12 B)-C(13 B)$ & $1.50(2)$ \\
\hline $\mathrm{O}(1)-\mathrm{H}(1)$ & $0.90(7)$ & $\mathrm{C}(13 \mathrm{~B})-\mathrm{H}(13 \mathrm{C})$ & 0.9900 \\
\hline $\mathrm{O}(2)-\mathrm{C}(15)$ & $1.293(5)$ & $C(13 B)-H(13 D)$ & 0.9900 \\
\hline$O(3)-C(21)$ & $1.364(6)$ & $\mathrm{O}(5)-\mathrm{C}(23)$ & $1.231(6)$ \\
\hline$O(3)-C(22)$ & $1.398(6)$ & $N(3)-C(23)$ & $1.315(7)$ \\
\hline$O(4)-C(22)$ & $1.214(6)$ & $\mathrm{N}(3)-\mathrm{C}(24)$ & $1.458(6)$ \\
\hline$N(1)-C(1)$ & $1.313(6)$ & $N(3)-C(25)$ & $1.453(6)$ \\
\hline $\mathrm{N}(1)-\mathrm{C}(9)$ & $1.381(6)$ & $\mathrm{C}(23)-\mathrm{H}(23)$ & 0.9500 \\
\hline $\mathrm{N}(2)-\mathrm{C}(8)$ & $1.411(6)$ & $\mathrm{C}(24)-\mathrm{H}(24 \mathrm{~A})$ & 0.9800 \\
\hline$N(2)-C(10)$ & $1.310(6)$ & $\mathrm{C}(24)-\mathrm{H}(24 \mathrm{~B})$ & 0.9800 \\
\hline $\mathrm{C}(1)-\mathrm{H}(1 \mathrm{~A})$ & 0.9500 & $\mathrm{C}(24)-\mathrm{H}(24 \mathrm{C})$ & 0.9800 \\
\hline $\mathrm{C}(1)-\mathrm{C}(2)$ & $1.404(7)$ & $\mathrm{C}(25)-\mathrm{H}(25 \mathrm{~A})$ & 0.9800 \\
\hline $\mathrm{C}(2)-\mathrm{H}(2)$ & 0.9500 & $\mathrm{C}(25)-\mathrm{H}(25 \mathrm{~B})$ & 0.9800 \\
\hline$C(2)-C(3)$ & $1.360(7)$ & $\mathrm{C}(25)-\mathrm{H}(25 \mathrm{C})$ & 0.9800 \\
\hline $\mathrm{C}(3)-\mathrm{H}(3)$ & 0.9500 & & \\
\hline$C(3)-C(4)$ & $1.406(7)$ & $\mathrm{O}(2)-\mathrm{Pd}(1)-\mathrm{N}(1)$ & $100.28(13)$ \\
\hline$C(4)-C(5)$ & $1.422(7)$ & $\mathrm{O}(2)-\mathrm{Pd}(1)-\mathrm{C}(12 \mathrm{~B})$ & $95.9(5)$ \\
\hline$C(4)-C(9)$ & $1.407(7)$ & $\mathrm{N}(2)-\mathrm{Pd}(1)-\mathrm{O}(2)$ & $178.09(15)$ \\
\hline $\mathrm{C}(5)-\mathrm{H}(5)$ & 0.9500 & $N(2)-P d(1)-N(1)$ & $80.86(15)$ \\
\hline$C(5)-C(6)$ & $1.358(7)$ & $N(2)-P d(1)-C(12)$ & $86.2(2)$ \\
\hline $\mathrm{C}(6)-\mathrm{H}(6)$ & 0.9500 & $N(2)-P d(1)-C(12 B)$ & $82.7(5)$ \\
\hline$C(6)-C(7)$ & $1.402(7)$ & $C(12)-P d(1)-O(2)$ & $92.8(2)$ \\
\hline $\mathrm{C}(7)-\mathrm{H}(7)$ & 0.9500 & $\mathrm{C}(12)-\mathrm{Pd}(1)-\mathrm{N}(1)$ & $164.9(2)$ \\
\hline $\mathrm{C}(7)-\mathrm{C}(8)$ & $1.381(7)$ & $C(12 B)-P d(1)-N(1)$ & $159.3(5)$ \\
\hline $\mathrm{C}(8)-\mathrm{C}(9)$ & $1.429(6)$ & $\mathrm{C}(10)-\mathrm{O}(1)-\mathrm{H}(1)$ & $113(4)$ \\
\hline$C(10)-C(11)$ & $1.495(7)$ & $C(15)-O(2)-P d(1)$ & $125.0(3)$ \\
\hline $\mathrm{C}(11)-\mathrm{H}(11 \mathrm{~A})$ & 0.9900 & $C(21)-O(3)-C(22)$ & $120.8(4)$ \\
\hline $\mathrm{C}(11)-\mathrm{H}(11 \mathrm{~B})$ & 0.9900 & $C(1)-N(1)-P d(1)$ & $131.7(3)$ \\
\hline $\mathrm{C}(11)-\mathrm{H}(11 \mathrm{C})$ & 0.9900 & $\mathrm{C}(1)-\mathrm{N}(1)-\mathrm{C}(9)$ & $118.2(4)$ \\
\hline $\mathrm{C}(11)-\mathrm{H}(11 \mathrm{D})$ & 0.9900 & $C(9)-N(1)-P d(1)$ & $110.0(3)$ \\
\hline $\mathrm{C}(11)-\mathrm{C}(12)$ & $1.590(9)$ & $C(8)-N(2)-P d(1)$ & $116.3(3)$ \\
\hline $\mathrm{C}(11)-\mathrm{C}(12 \mathrm{~B})$ & $1.437(17)$ & $\mathrm{C}(10)-\mathrm{N}(2)-\mathrm{Pd}(1)$ & $115.9(3)$ \\
\hline$C(14)-C(15)$ & $1.373(7)$ & $\mathrm{C}(10)-\mathrm{N}(2)-\mathrm{C}(8)$ & $127.8(4)$ \\
\hline$C(14)-C(22)$ & $1.434(7)$ & $N(1)-C(1)-H(1 A)$ & 118.2 \\
\hline$C(14)-C(13)$ & $1.493(8)$ & $\mathrm{N}(1)-\mathrm{C}(1)-\mathrm{C}(2)$ & $123.6(4)$ \\
\hline$C(14)-C(13 B)$ & $1.644(17)$ & $\mathrm{C}(2)-\mathrm{C}(1)-\mathrm{H}(1 \mathrm{~A})$ & 118.2 \\
\hline$C(15)-C(16)$ & $1.468(6)$ & $\mathrm{C}(1)-\mathrm{C}(2)-\mathrm{H}(2)$ & 120.7 \\
\hline$C(16)-C(17)$ & $1.399(7)$ & $C(3)-C(2)-C(1)$ & $118.7(5)$ \\
\hline$C(16)-C(21)$ & $1.391(6)$ & $\mathrm{C}(3)-\mathrm{C}(2)-\mathrm{H}(2)$ & 120.7 \\
\hline $\mathrm{C}(17)-\mathrm{H}(17)$ & 0.9500 & $\mathrm{C}(2)-\mathrm{C}(3)-\mathrm{H}(3)$ & 119.9 \\
\hline$C(17)-C(18)$ & $1.383(7)$ & $C(2)-C(3)-C(4)$ & $120.2(5)$ \\
\hline $\mathrm{C}(18)-\mathrm{H}(18)$ & 0.9500 & $\mathrm{C}(4)-\mathrm{C}(3)-\mathrm{H}(3)$ & 119.9 \\
\hline $\mathrm{C}(18)-\mathrm{C}(19)$ & $1.396(7)$ & $C(3)-C(4)-C(5)$ & $122.9(4)$ \\
\hline $\mathrm{C}(19)-\mathrm{H}(19)$ & 0.9500 & $C(3)-C(4)-C(9)$ & $117.6(4)$ \\
\hline$C(19)-C(20)$ & $1.380(7)$ & $C(9)-C(4)-C(5)$ & $119.5(4)$ \\
\hline $\mathrm{C}(20)-\mathrm{H}(20)$ & 0.9500 & $\mathrm{C}(4)-\mathrm{C}(5)-\mathrm{H}(5)$ & 120.4 \\
\hline$C(20)-C(21)$ & $1.386(7)$ & $C(6)-C(5)-C(4)$ & $119.3(4)$ \\
\hline
\end{tabular}




$\begin{array}{ll}\mathrm{C}(6)-\mathrm{C}(5)-\mathrm{H}(5) & 120.4 \\ \mathrm{C}(5)-\mathrm{C}(6)-\mathrm{H}(6) & 118.9 \\ \mathrm{C}(5)-\mathrm{C}(6)-\mathrm{C}(7) & 122.2(5) \\ \mathrm{C}(7)-\mathrm{C}(6)-\mathrm{H}(6) & 118.9 \\ \mathrm{C}(6)-\mathrm{C}(7)-\mathrm{H}(7) & 119.9 \\ \mathrm{C}(8)-\mathrm{C}(7)-\mathrm{C}(6) & 120.1(5) \\ \mathrm{C}(8)-\mathrm{C}(7)-\mathrm{H}(7) & 119.9 \\ \mathrm{~N}(2)-\mathrm{C}(8)-\mathrm{C}(9) & 114.2(4) \\ \mathrm{C}(7)-\mathrm{C}(8)-\mathrm{N}(2) & 126.7(4) \\ \mathrm{C}(7)-\mathrm{C}(8)-\mathrm{C}(9) & 119.0(4) \\ \mathrm{N}(1)-\mathrm{C}(9)-\mathrm{C}(4) & 121.6(4) \\ \mathrm{N}(1)-\mathrm{C}(9)-\mathrm{C}(8) & 118.5(4) \\ \mathrm{C}(4)-\mathrm{C}(9)-\mathrm{C}(8) & 119.9(4) \\ \mathrm{O}(1)-\mathrm{C}(10)-\mathrm{C}(11) & 121.7(4) \\ \mathrm{N}(2)-\mathrm{C}(10)-\mathrm{O}(1) & 122.6(4) \\ \mathrm{N}(2)-\mathrm{C}(10)-\mathrm{C}(11) & 115.7(4) \\ \mathrm{C}(10)-\mathrm{C}(11)-\mathrm{H}(11 \mathrm{~A}) & 109.2 \\ \mathrm{C}(10)-\mathrm{C}(11)-\mathrm{H}(11 \mathrm{~B}) & 109.2 \\ \mathrm{C}(10)-\mathrm{C}(11)-\mathrm{H}(11 \mathrm{C}) & 108.8 \\ \mathrm{C}(10)-\mathrm{C}(11)-\mathrm{H}(11 \mathrm{D}) & 108.8 \\ \mathrm{C}(10)-\mathrm{C}(11)-\mathrm{C}(12) & 112.2(5) \\ \mathrm{H}(11 \mathrm{~A})-\mathrm{C}(11)-\mathrm{H}(11 \mathrm{~B}) & 107.9 \\ \mathrm{H}(11 \mathrm{C})-\mathrm{C}(11)-\mathrm{H}(11 \mathrm{D}) & 107.7 \\ \mathrm{C}(12)-\mathrm{C}(11)-\mathrm{H}(11 \mathrm{~A}) & 109.2 \\ \mathrm{C}(12)-\mathrm{C}(11)-\mathrm{H}(11 \mathrm{~B}) & 109.2 \\ \mathrm{C}(12 \mathrm{~B})-\mathrm{C}(11)-\mathrm{C}(10) & 113.9(8) \\ \mathrm{C}(12 \mathrm{~B})-\mathrm{C}(11)-\mathrm{H}(11 \mathrm{C}) & 108.8 \\ \mathrm{C}(12 \mathrm{~B})-\mathrm{C}(11)-\mathrm{H}(11 \mathrm{D}) & 108.8 \\ \mathrm{C}(15)-\mathrm{C}(14)-\mathrm{C}(22) & 121.2(4) \\ \mathrm{C}(15)-\mathrm{C}(14)-\mathrm{C}(13) & 122.4(5) \\ \mathrm{C}(15)-\mathrm{C}(14)-\mathrm{C}(13 \mathrm{~B}) & 121.8(7) \\ \mathrm{C}(22)-\mathrm{C}(14)-\mathrm{C}(13) & 115.4(5) \\ \mathrm{C}(22)-\mathrm{C}(14)-\mathrm{C}(13 \mathrm{~B}) & 113.7(7) \\ \mathrm{O}(2)-\mathrm{C}(15)-\mathrm{C}(14) & 127.0(4) \\ \mathrm{O}(2)-\mathrm{C}(15)-\mathrm{C}(16) & 115.0(4) \\ \mathrm{C}(14)-\mathrm{C}(15)-\mathrm{C}(16) & 117.9(4) \\ \mathrm{C}(17)-\mathrm{C}(16)-\mathrm{C}(15) & 122.3(4) \\ \mathrm{C}(21)-\mathrm{C}(16)-\mathrm{C}(15) & 119.5(4) \\ \mathrm{C}(21)-\mathrm{C}(16)-\mathrm{C}(17) & 118.1(4) \\ \mathrm{C}(16)-\mathrm{C}(17)-\mathrm{H}(17) & 119.6 \\ \mathrm{C}(18)-\mathrm{C}(17)-\mathrm{C}(16) & 120.7(5) \\ \mathrm{C}(18)-\mathrm{C}(17)-\mathrm{H}(17) & 119.6 \\ \mathrm{C}(17)-\mathrm{C}(18)-\mathrm{H}(18) & 120.3 \\ \mathrm{C}(17)-\mathrm{C}(18)-\mathrm{C}(19) & 119.4(5) \\ \mathrm{C}(19)-\mathrm{C}(18)-\mathrm{H}(18) & 120.3 \\ \mathrm{C}(18)-\mathrm{C}(19)-\mathrm{H}(19) & 119.4 \\ \mathrm{C}(20)-\mathrm{C}(19)-\mathrm{C}(18) & 121.1(5) \\ \mathrm{C}(20)-\mathrm{C}(19)-\mathrm{H}(19) & 119.4 \\ \mathrm{C}(19)-\mathrm{C}(20)-\mathrm{H}(20) & 120.8 \\ \mathrm{C}(19)-\mathrm{C}(20)-\mathrm{C}(21) & 118.5(4) \\ \mathrm{C}(21)-\mathrm{C}(20)-\mathrm{H}(20) & 120.8 \\ \mathrm{O}(3)-\mathrm{C}(21)-\mathrm{C}(16)-\mathrm{C}(21)-\mathrm{C}(20) & 121.3(4) \\ \mathrm{C}(20)-\mathrm{C}(21)-\mathrm{C}(16) & 116.6(4) \\ & 122.1(4)\end{array}$

\begin{tabular}{|c|c|}
\hline $\mathrm{O}(3)-\mathrm{C}(22)-\mathrm{C}(14)$ & 119.1(4) \\
\hline $\mathrm{O}(4)-\mathrm{C}(22)-\mathrm{O}(3)$ & $113.7(4)$ \\
\hline$O(4)-C(22)-C(14)$ & $127.2(5)$ \\
\hline $\mathrm{Pd}(1)-\mathrm{C}(12)-\mathrm{H}(12)$ & 109.0 \\
\hline$C(11)-C(12)-P d(1)$ & $105.4(4)$ \\
\hline $\mathrm{C}(11)-\mathrm{C}(12)-\mathrm{H}(12)$ & 109.0 \\
\hline$C(13)-C(12)-P d(1)$ & $113.0(6)$ \\
\hline$C(13)-C(12)-C(11)$ & $111.4(7)$ \\
\hline $\mathrm{C}(13)-\mathrm{C}(12)-\mathrm{H}(12)$ & 109.0 \\
\hline$C(14)-C(13)-C(12)$ & $113.7(7)$ \\
\hline $\mathrm{C}(14)-\mathrm{C}(13)-\mathrm{H}(13 \mathrm{~A})$ & 108.8 \\
\hline $\mathrm{C}(14)-\mathrm{C}(13)-\mathrm{H}(13 \mathrm{~B})$ & 108.8 \\
\hline $\mathrm{C}(12)-\mathrm{C}(13)-\mathrm{H}(13 \mathrm{~A})$ & 108.8 \\
\hline $\mathrm{C}(12)-\mathrm{C}(13)-\mathrm{H}(13 \mathrm{~B})$ & 108.8 \\
\hline $\mathrm{H}(13 \mathrm{~A})-\mathrm{C}(13)-\mathrm{H}(13 \mathrm{~B})$ & 107.7 \\
\hline $\mathrm{Pd}(1)-\mathrm{C}(12 \mathrm{~B})-\mathrm{H}(12 \mathrm{~B})$ & 107.7 \\
\hline$C(11)-C(12 B)-P d(1)$ & $108.2(10)$ \\
\hline $\mathrm{C}(11)-\mathrm{C}(12 \mathrm{~B})-\mathrm{H}(12 \mathrm{~B})$ & 107.7 \\
\hline$C(11)-C(12 B)-C(13 B)$ & $114.3(14)$ \\
\hline $\mathrm{C}(13 \mathrm{~B})-\mathrm{C}(12 \mathrm{~B})-\mathrm{Pd}(1)$ & $110.9(12)$ \\
\hline $\mathrm{C}(13 \mathrm{~B})-\mathrm{C}(12 \mathrm{~B})-\mathrm{H}(12 \mathrm{~B})$ & 107.7 \\
\hline$C(14)-C(13 B)-H(13 C)$ & 109.3 \\
\hline $\mathrm{C}(14)-\mathrm{C}(13 \mathrm{~B})-\mathrm{H}(13 \mathrm{D})$ & 109.3 \\
\hline$C(12 B)-C(13 B)-C(14)$ & $111.4(13)$ \\
\hline $\mathrm{C}(12 \mathrm{~B})-\mathrm{C}(13 \mathrm{~B})-\mathrm{H}(13 \mathrm{C})$ & 109.3 \\
\hline$C(12 B)-C(13 B)-H(13 D)$ & 109.3 \\
\hline $\mathrm{H}(13 \mathrm{C})-\mathrm{C}(13 \mathrm{~B})-\mathrm{H}(13 \mathrm{D})$ & 108.0 \\
\hline$C(23)-N(3)-C(24)$ & $121.5(5)$ \\
\hline$C(23)-N(3)-C(25)$ & $121.3(4)$ \\
\hline$C(25)-N(3)-C(24)$ & $117.2(4)$ \\
\hline$O(5)-C(23)-N(3)$ & $125.2(5)$ \\
\hline $\mathrm{O}(5)-\mathrm{C}(23)-\mathrm{H}(23)$ & 117.4 \\
\hline $\mathrm{N}(3)-\mathrm{C}(23)-\mathrm{H}(23)$ & 117.4 \\
\hline $\mathrm{N}(3)-\mathrm{C}(24)-\mathrm{H}(24 \mathrm{~A})$ & 109.5 \\
\hline $\mathrm{N}(3)-\mathrm{C}(24)-\mathrm{H}(24 \mathrm{~B})$ & 109.5 \\
\hline $\mathrm{N}(3)-\mathrm{C}(24)-\mathrm{H}(24 \mathrm{C})$ & 109.5 \\
\hline $\mathrm{H}(24 \mathrm{~A})-\mathrm{C}(24)-\mathrm{H}(24 \mathrm{~B})$ & 109.5 \\
\hline $\mathrm{H}(24 \mathrm{~A})-\mathrm{C}(24)-\mathrm{H}(24 \mathrm{C})$ & 109.5 \\
\hline $\mathrm{H}(24 \mathrm{~B})-\mathrm{C}(24)-\mathrm{H}(24 \mathrm{C})$ & 109.5 \\
\hline $\mathrm{N}(3)-\mathrm{C}(25)-\mathrm{H}(25 \mathrm{~A})$ & 109.5 \\
\hline $\mathrm{N}(3)-\mathrm{C}(25)-\mathrm{H}(25 \mathrm{~B})$ & 109.5 \\
\hline $\mathrm{N}(3)-\mathrm{C}(25)-\mathrm{H}(25 \mathrm{C})$ & 109.5 \\
\hline $\mathrm{H}(25 \mathrm{~A})-\mathrm{C}(25)-\mathrm{H}(25 \mathrm{~B})$ & 109.5 \\
\hline $\mathrm{H}(25 \mathrm{~A})-\mathrm{C}(25)-\mathrm{H}(25 \mathrm{C})$ & 109.5 \\
\hline $\mathrm{H}(25 \mathrm{~B})-\mathrm{C}(25)-\mathrm{H}(25 \mathrm{C})$ & 109.5 \\
\hline
\end{tabular}


Table 21. Anisotropic displacement parameters $\left(\AA^{2} \times 1^{3}\right)$ for Engle17. The anisotropic displacement factor exponent takes the form: $-2 \pi^{2}\left[h^{2} a^{* 2} U^{11}+\ldots+2 h k^{*} b^{*} U^{12}\right]$

\begin{tabular}{lcccccc}
\hline & $\mathrm{U}^{11}$ & $\mathrm{U}^{22}$ & $\mathrm{U}^{33}$ & $\mathrm{U}^{23}$ & $\mathrm{U}$ & $\mathrm{U}$ \\
& & & & & & \\
\hline $\mathrm{Pd}(1)$ & $18(1)$ & $16(1)$ & $30(1)$ & $5(1)$ & $11(1)$ & $2(1)$ \\
$\mathrm{O}(1)$ & $24(2)$ & $26(2)$ & $57(3)$ & $6(2)$ & $21(2)$ & $-3(2)$ \\
$\mathrm{O}(2)$ & $21(2)$ & $17(2)$ & $40(2)$ & $4(1)$ & $15(2)$ & $2(1)$ \\
$\mathrm{O}(3)$ & $17(2)$ & $21(2)$ & $32(2)$ & $-3(1)$ & $6(1)$ & $3(1)$ \\
$\mathrm{O}(4)$ & $25(2)$ & $20(2)$ & $113(4)$ & $-13(2)$ & $23(2)$ & $-2(2)$ \\
$\mathrm{N}(1)$ & $18(2)$ & $18(2)$ & $23(2)$ & $-1(2)$ & $4(2)$ & $1(2)$ \\
$\mathrm{N}(2)$ & $13(2)$ & $20(2)$ & $19(2)$ & $2(2)$ & $2(1)$ & $1(2)$ \\
$\mathrm{C}(1)$ & $21(2)$ & $21(2)$ & $29(3)$ & $0(2)$ & $8(2)$ & $1(2)$ \\
$\mathrm{C}(2)$ & $25(3)$ & $21(2)$ & $34(3)$ & $2(2)$ & $7(2)$ & $0(2)$ \\
$\mathrm{C}(3)$ & $28(3)$ & $21(2)$ & $36(3)$ & $0(2)$ & $7(2)$ & $4(2)$ \\
$\mathrm{C}(4)$ & $21(2)$ & $25(2)$ & $25(3)$ & $2(2)$ & $5(2)$ & $2(2)$ \\
$\mathrm{C}(5)$ & $23(2)$ & $25(2)$ & $29(3)$ & $-2(2)$ & $6(2)$ & $7(2)$ \\
$\mathrm{C}(6)$ & $20(2)$ & $35(3)$ & $29(3)$ & $-4(2)$ & $8(2)$ & $3(2)$ \\
$\mathrm{C}(7)$ & $24(3)$ & $29(3)$ & $33(3)$ & $0(2)$ & $15(2)$ & $0(2)$ \\
$\mathrm{C}(8)$ & $20(2)$ & $22(2)$ & $23(2)$ & $4(2)$ & $7(2)$ & $2(2)$ \\
$\mathrm{C}(9)$ & $18(2)$ & $25(2)$ & $19(2)$ & $1(2)$ & $5(2)$ & $3(2)$ \\
$\mathrm{C}(10)$ & $25(3)$ & $26(3)$ & $30(3)$ & $8(2)$ & $11(2)$ & $2(2)$ \\
$\mathrm{C}(11)$ & $20(2)$ & $20(2)$ & $48(3)$ & $10(2)$ & $10(2)$ & $2(2)$ \\
$\mathrm{C}(14)$ & $15(2)$ & $24(2)$ & $44(3)$ & $-6(2)$ & $6(2)$ & $-1(2)$ \\
$\mathrm{C}(15)$ & $16(2)$ & $21(2)$ & $18(2)$ & $-1(2)$ & $2(2)$ & $4(2)$ \\
$\mathrm{C}(16)$ & $21(2)$ & $23(2)$ & $19(2)$ & $1(2)$ & $7(2)$ & $1(2)$ \\
$\mathrm{C}(17)$ & $26(3)$ & $23(2)$ & $36(3)$ & $2(2)$ & $16(2)$ & $1(2)$ \\
$\mathrm{C}(18)$ & $28(3)$ & $25(3)$ & $46(3)$ & $2(2)$ & $18(2)$ & $-3(2)$ \\
$\mathrm{C}(19)$ & $25(3)$ & $35(3)$ & $38(3)$ & $5(2)$ & $19(2)$ & $0(2)$ \\
$\mathrm{C}(20)$ & $23(2)$ & $28(3)$ & $24(3)$ & $0(2)$ & $10(2)$ & $2(2)$ \\
$\mathrm{C}(21)$ & $23(2)$ & $22(2)$ & $14(2)$ & $2(2)$ & $4(2)$ & $3(2)$ \\
$\mathrm{C}(22)$ & $15(2)$ & $24(3)$ & $46(3)$ & $-7(2)$ & $2(2)$ & $1(2)$ \\
$\mathrm{C}(12)$ & $15(3)$ & $17(3)$ & $39(5)$ & $5(3)$ & $6(3)$ & $0(2)$ \\
$\mathrm{C}(13)$ & $15(3)$ & $17(3)$ & $42(5)$ & $3(3)$ & $6(3)$ & $1(2)$ \\
$\mathrm{C}(12 \mathrm{~B})$ & $15(3)$ & $17(3)$ & $39(5)$ & $5(3)$ & $6(3)$ & $0(2)$ \\
$\mathrm{C}(13 \mathrm{~B})$ & $15(3)$ & $17(3)$ & $42(5)$ & $3(3)$ & $6(3)$ & $1(2)$ \\
$\mathrm{O}(5)$ & $32(2)$ & $32(2)$ & $45(2)$ & $3(2)$ & $11(2)$ & $-7(2)$ \\
$\mathrm{N}(3)$ & $23(2)$ & $28(2)$ & $21(2)$ & $-1(2)$ & $-1(2)$ & $-5(2)$ \\
$\mathrm{C}(23)$ & $31(3)$ & $30(3)$ & $27(3)$ & $3(2)$ & $6(2)$ & $3(2)$ \\
$\mathrm{C}(24)$ & $26(3)$ & $50(4)$ & $38(3)$ & $2(3)$ & $8(2)$ & $-6(3)$ \\
$\mathrm{C}(25)$ & $36(3)$ & $28(3)$ & $38(3)$ & $-3(2)$ & $7(2)$ & $-1(2)$ \\
& & & & & &
\end{tabular}


Table 22. Hydrogen coordinates $\left(x 1^{4}\right)$ and isotropic displacement parameters $\left(\AA^{2} \times 1^{3}\right)$ for Engle17.

\begin{tabular}{|c|c|c|c|}
\hline & $\mathrm{x}$ & $\mathrm{y}$ & $\mathrm{z}$ \\
\hline $\mathrm{H}(1 \mathrm{~A})$ & 11285 & 2849 & 7307 \\
\hline $\mathrm{H}(2)$ & 10665 & 3797 & 7477 \\
\hline $\mathrm{H}(3)$ & 9035 & 3938 & 8130 \\
\hline $\mathrm{H}(5)$ & 7373 & 3502 & 8766 \\
\hline $\mathrm{H}(6)$ & 6461 & 2680 & 9215 \\
\hline $\mathrm{H}(7)$ & 7154 & 1749 & 9074 \\
\hline $\mathrm{H}(11 \mathrm{~A})$ & 9037 & 315 & 6567 \\
\hline $\mathrm{H}(11 \mathrm{~B})$ & 9284 & 143 & 8704 \\
\hline $\mathrm{H}(11 \mathrm{C})$ & 9576 & 254 & 8988 \\
\hline $\mathrm{H}(11 \mathrm{D})$ & 8883 & 188 & 6956 \\
\hline$H(17)$ & 13369 & 2141 & 6715 \\
\hline $\mathrm{H}(18)$ & 15050 & 2325 & 6304 \\
\hline H(19) & 16124 & 1547 & 5867 \\
\hline $\mathrm{H}(20)$ & 15517 & 590 & 5769 \\
\hline $\mathrm{H}(12)$ & 10874 & 584 & 9274 \\
\hline$H(13 A)$ & 10965 & -61 & 6851 \\
\hline $\mathrm{H}(13 \mathrm{~B})$ & 10578 & 442 & 5378 \\
\hline $\mathrm{H}(12 \mathrm{~B})$ & 9966 & 614 & 5700 \\
\hline$H(13 C)$ & 11016 & -101 & 7437 \\
\hline$H(13 D)$ & 11375 & 357 & 9073 \\
\hline $\mathrm{H}(23)$ & 5833 & -89 & 8674 \\
\hline $\mathrm{H}(24 \mathrm{~A})$ & 4550 & -712 & 8791 \\
\hline $\mathrm{H}(24 \mathrm{~B})$ & 4983 & -1254 & 10081 \\
\hline $\mathrm{H}(24 \mathrm{C})$ & 4649 & -1339 & 7897 \\
\hline $\mathrm{H}(25 \mathrm{~A})$ & 7416 & -1267 & 8635 \\
\hline$H(25 B)$ & 6468 & -1626 & 7440 \\
\hline$H(25 C)$ & 6723 & -1678 & 9638 \\
\hline$H(1)$ & $7500(50)$ & $470(30)$ & $8390(90)$ \\
\hline
\end{tabular}


Table 23. Hydrogen bonds for Engle17 [Å and $\left.{ }^{\circ}\right]$.

\begin{tabular}{lcccc}
\hline $\mathrm{D}-\mathrm{H} \ldots \mathrm{A}$ & $\mathrm{d}(\mathrm{D}-\mathrm{H})$ & $\mathrm{d}(\mathrm{H} . . . \mathrm{A})$ & $\mathrm{d}(\mathrm{D} \ldots \mathrm{A})$ & $<(\mathrm{DHA})$ \\
\hline $\mathrm{O}(1)-\mathrm{H}(1) \ldots \mathrm{O}(5)$ & $0.90(7)$ & $1.67(7)$ & $2.569(5)$ & $174(6)$
\end{tabular}

